\section{Pacific Northwest}

National Laboratory

Operated by Battelle for the

U.S. Department of Energy

\title{
Assessment of the HV-C2 Stack Sampling Probe Location
}

\author{
J. A. Glissmeyer \\ J. G. Droppo
}

August 2007

Prepared for the U.S. Department of Energy under Contract DE-AC05-76RL01830 


\title{
DISCLAIMER
}

This report was prepared as an account of work sponsored by an agency of the United States Government. Neither the United States Government nor any agency thereof, nor Battelle Memorial Institute, nor any of their employees, makes any warranty, express or implied, or assumes any legal liability or responsibility for the accuracy, completeness, or usefulness of any information, apparatus, product, or process disclosed, or represents that its use would not infringe privately owned rights. Reference herein to any specific commercial product, process, or service by trade name, trademark, manufacturer, or otherwise does not necessarily constitute or imply its endorsement, recommendation, or favoring by the United States Government or any agency thereof, or Battelle Memorial Institute. The views and opinions of authors expressed herein do not necessarily state or reflect those of the United States Government or any agency thereof.

\author{
PACIFIC NORTHWEST NATIONAL LABORATORY \\ operated by \\ BATTELLE \\ for the \\ UNITED STATES DEPARTMENT OF ENERGY \\ under Contract DE-ACO5-76RL01830
}

Printed in the United States of America
Available to DOE and DOE contractors from the
Office of Scientific and Technical Information,
P.O. Box 62, Oak Ridge, TN 37831-0062;
ph: (865) 576-8401
fax: (865) 5765728
email: reports@adonis.osti.gov

\footnotetext{
Available to the public from the National Technical Information Service, U.S. Department of Commerce, 5285 Port Royal Rd., Springfield, VA 22161 ph: (800) 553-6847 fax: (703) 605-6900

email: orders@nits.fedworld.gov online ordering: http://www.ntis.gov/ordering.htm
} 


\title{
Assessment of the HV-C2 STACK Sampling Probe Location
}

\author{
J. A. Glissmeyer \\ J. G. Droppo
}

August 2007

Test Plan: TP-RPP-WTP-441

Test Plan ICN: ICN-TP-RPP-WTP-441.1

Statement of Work: N13.1-1999 Stack Monitor Scale Model Testing and Qualification, Feb. 5, 2007, Rev. 0

Pacific Northwest National Laboratory

Richland, Washington 99352 


\section{Completeness of Testing}

This report describes the results of work and testing specified by test plan TP-RPPWTP-441, Rev. 0, and ICN-TP-RPP-WTP-441.1. The work and any associated testing followed the quality assurance requirements outlined in the test specification/plan. The descriptions provided in this test report are an accurate account of both the conduct of the work and the data collected. Test plan results are reported. Also reported are any unusual or anomalous occurrences that are different from expected results. The test results and this report have been reviewed and verified.

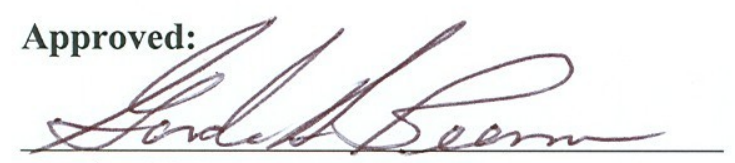

Gordon H. Beeman, Manager RPP-WTP Support Program

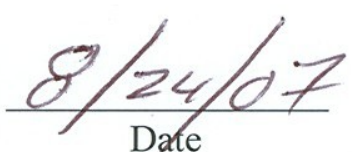




\section{Contents}

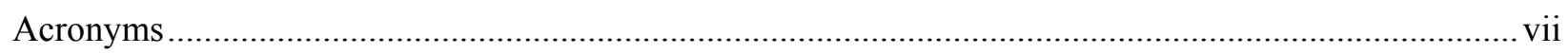

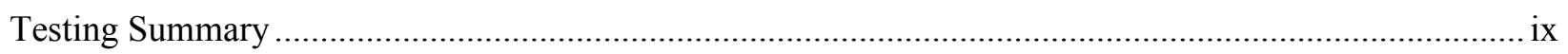

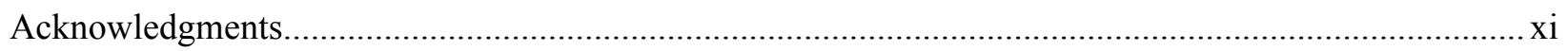

1.0 Introduction: Qualification of HV-C2 Air Monitoring Location.................................................. 1.1

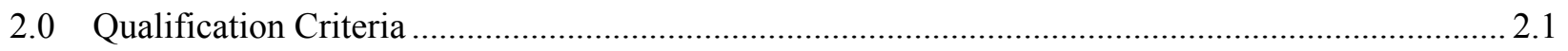

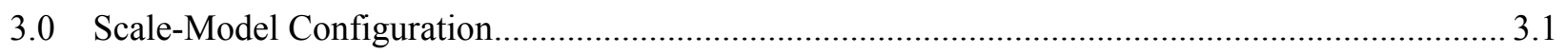

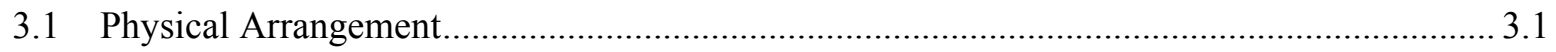

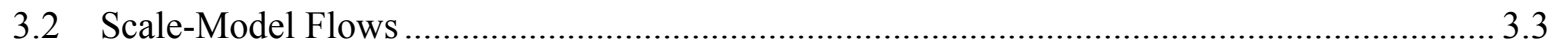

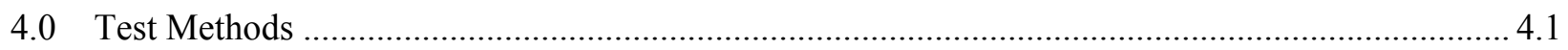

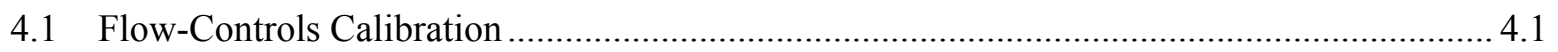

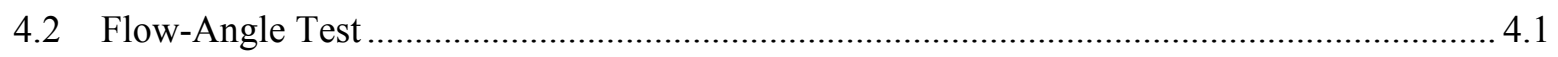

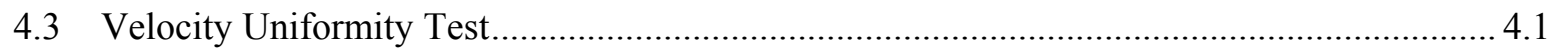

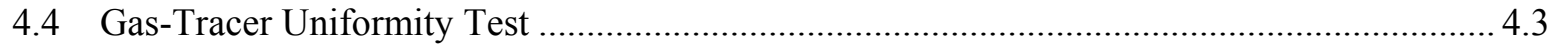

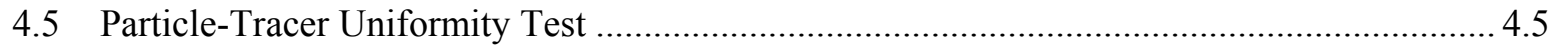

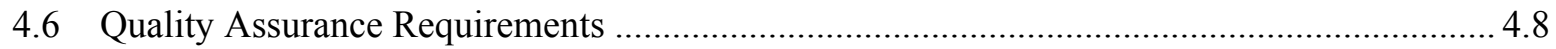

4.6.1 Application of RPP-WTP Quality Assurance Requirements................................... 4.8

4.6.2 Conduct of Experimental and Analytical Work .................................................... 4.8

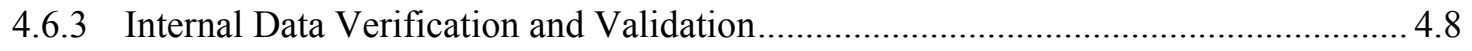

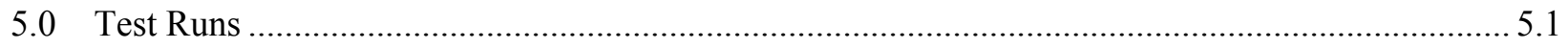

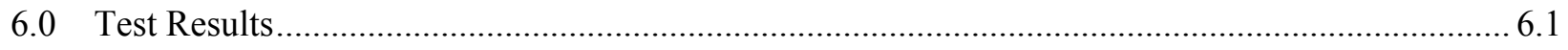

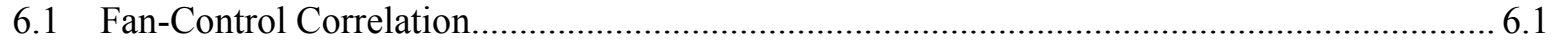

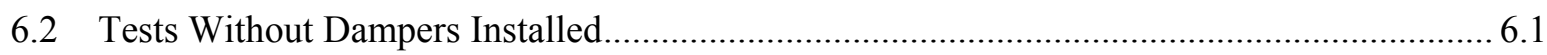

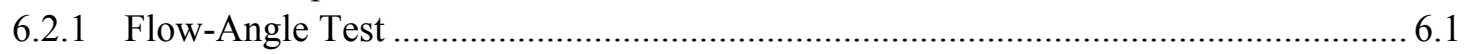

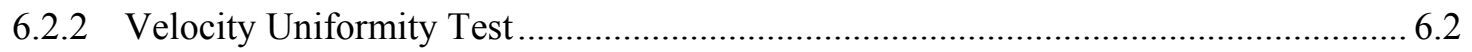

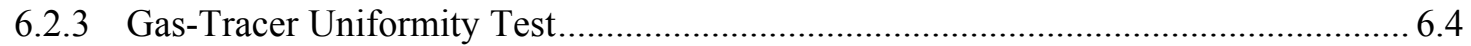

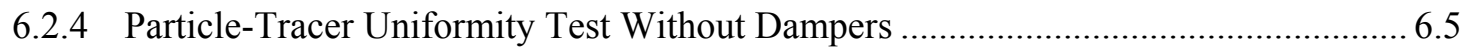

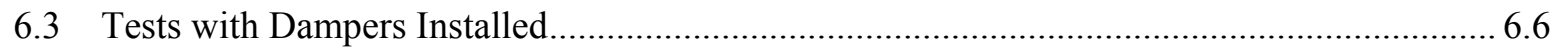

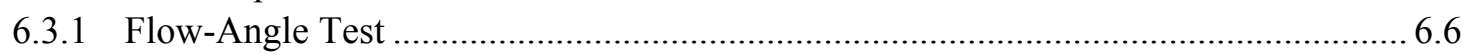

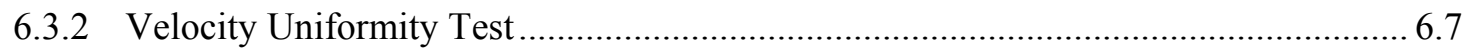

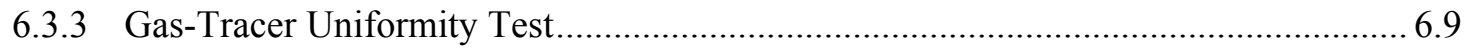

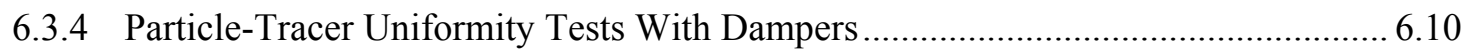




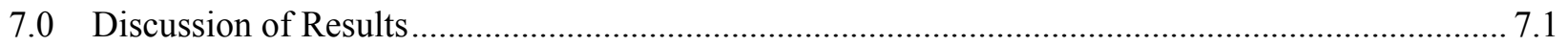

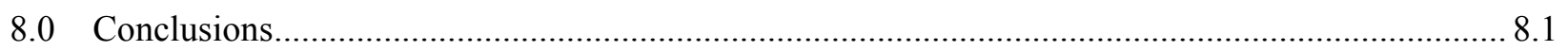

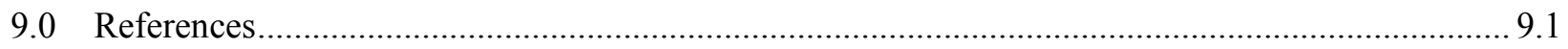

Appendix A: Dimensional Calculations for Scale Model WTP HV-C2 Stack ................................... A.1

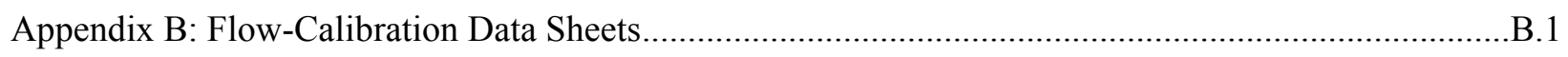

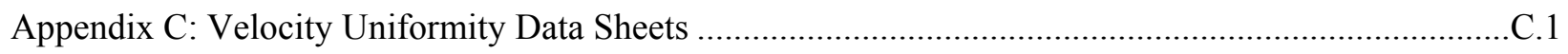

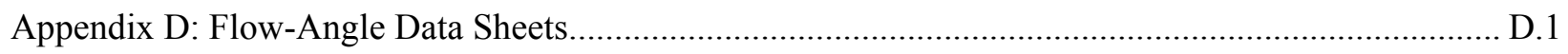

Appendix E: Tracer-Gas Uniformity Data Sheets ….......................................................................

Appendix F: Particle-Tracer Uniformity Data Sheets.................................................................... 


\section{Figures}

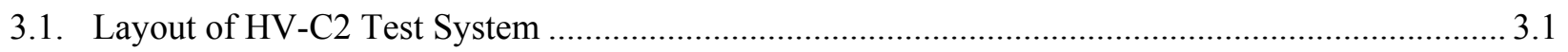

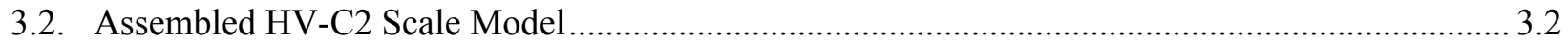

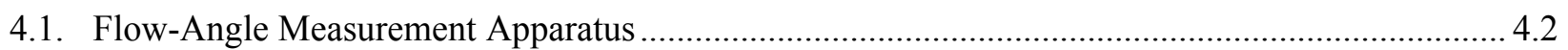

4.2. View of Type-S Pitot Tube Inserted into Model Stack................................................................. 4.2

4.3. Electronic Manometer and Pitot Tube …................................................................................... 4.3

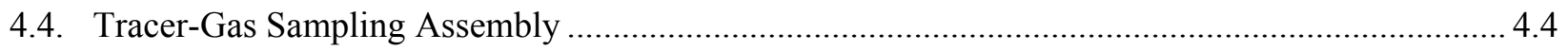

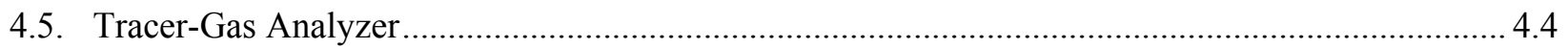

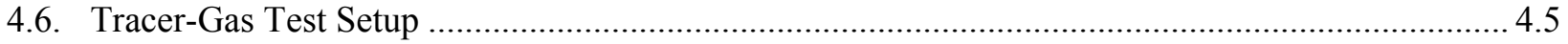

4.7. Particle Counter Connected to Probe ................................................................................................ 4.6

4.8. Particle Tracer Generator Apparatus (HVC2 not shown) ......................................................... 4.7

6.1. Plot of Scale-Model Flow-Angle Results, which are shown in degrees, with Both Fans

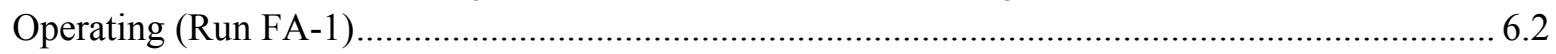

6.2. Scale-Model Velocity Profile with Fans A \& B Operating (Run VT-1) ...................................... 6.3

6.3. Plot of Gas-Tracer Results for Injection in Center at Port A with both Fans Operating

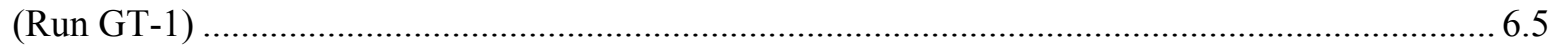

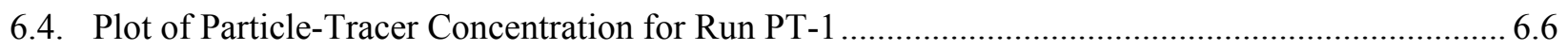

6.5. Plot of Flow-Angle Results, which are shown in degrees, with both Fans Operating (Run FA-14).

6.6. Scale-Model Velocity Profile with Fan A Operating and Dampers Set at 45 Degrees

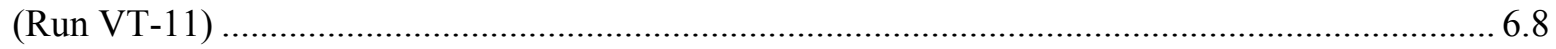

6.7. Plot of Gas-Tracer Results for Injection in Center at Port A with Fans A \& B Operating

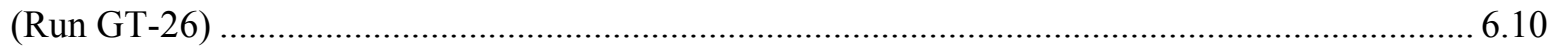

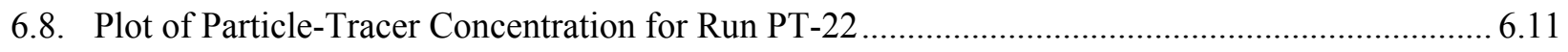

7.1. Flow-Angle Test Results Comparison (on left without dampers and on right with dampers).........7.1

7.2. Velocity Uniformity Results (on left without dampers and on right with dampers) ...................... 7.2

7.3. Gas Mixing \% COV Scatter Plot with and Without Dampers Installed ....................................... 7.2

7.4. Gas Mixing \% Deviation Scatter Plot with and Without Dampers Installed .................................. 7.3

7.5. Particle Mixing \% COV Scatter Plot with and Without Dampers Installed ................................. 7.3 


\section{Tables}

3.1. Key Dimensions of Actual and Scale-Model Stacks ..................................................................... 3.2

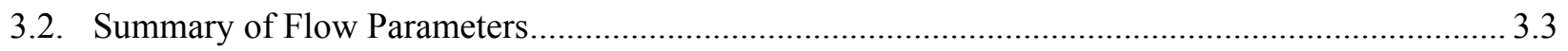

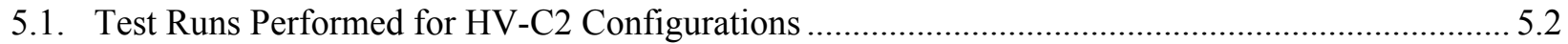

6.1. Flowrates Measured at Test Port 1 During Control Correlation ...................................................... 6.1

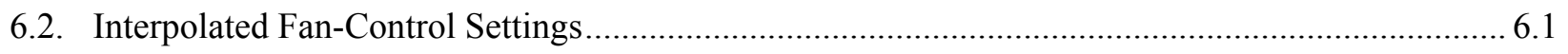

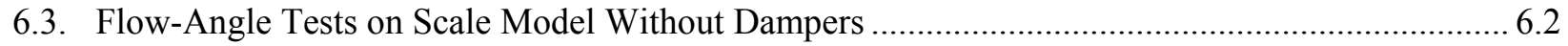

6.4. Velocity Uniformity Tests on Scale Model at Test Port 1 Without Dampers ................................ 6.3

6.5. Summarized Results of Gas-Tracer Uniformity Tests Without Dampers ..................................... 6.4

6.6. Particle-Tracer Uniformity Tests Without Dampers................................................................... 6.6

6.7. Flow-Angle Results on Scale Model with Dampers Installed .................................................... 6.7

6.8. Velocity Uniformity Tests on Scale Model at Air-Sampling-Probe Location................................ 6.8

6.9. Summarized Results of Gas-Tracer Uniformity Tests with Dampers ........................................... 6.9

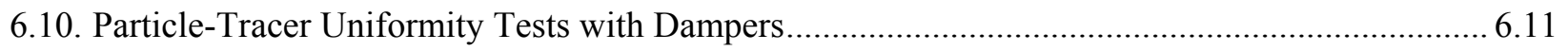




\section{Acronyms}

$\begin{array}{ll}\text { AD } & \text { aerodynamic diameter } \\ \text { BNI } & \text { Bechtel National, Inc. } \\ \text { COV } & \text { coefficient of variance } \\ \text { EPA } & \text { U.S. Environmental Protection Agency } \\ \text { HEPA } & \text { high-efficiency particulate air (filter) } \\ \text { OPC } & \text { optical particle counter } \\ \text { Q/D } & \text { ratio of flowrate (Q) to stack diameter (D) }\end{array}$





\section{Testing Summary}

Tests were performed to evaluate the location of the air-sampling probe in the proposed design for the Waste Treatment Plant's HV-C2 air exhaust stack. The evaluation criteria come from ANSI/HPS N13.11999, "Sampling and Monitoring Releases of Airborne Radioactive Substances from the Stacks and Ducts of Nuclear Facilities." Pacific Northwest National Laboratory conducted the tests on a 3.67:1 scale model of the stack. Limited confirmatory tests on the actual stack will need to be conducted during cold startup of the High Level Waste Treatment Facility. The tests documented here assessed the capability of the airmonitoring probe to extract a sample representative of the effluent stream in accordance with criteria in ANSI/HPS N13.1. The test parameters covered the expected range of system flowrates with both one and two operating fans.

The current stack design calls for the sampling probe to be located about 10 diameters downstream of the junction of the duct from Fan A with the stack. In accordance with the statement of work and the test plan, the test measurements were made at that location and also at one point upstream and another downstream. An adjustment was made for the distance between a typical sampling probe inlet and the centerline of its mounting flange. Thus, the test measurements were made at three positions designated as Test Port 1, 2, and 3, respectively.

The designed HV-C2 exhaust system includes dampers on the fan discharges. Custom-scale model dampers were fabricated to simulate the same number and configuration of damper blades shown in the design documents received from BNI. A subset of the test runs was run without the dampers to determine whether the dampers should be included in future tests on scale models.

The testing addressed the following criteria from ANSI/HPS N13.1-1999:

1. Angular Flow-The purpose is to determine whether the velocity vector is aligned with the sampling nozzle. The average yaw angle relative to the nozzle axis should not be more than $20^{\circ}$. The measured average values ranged from 3.73 to 6.15 degrees, regardless of the test port and presence of the dampers. These same types of flow angle measurements will have to be made on the constructed stack during cold startup to show that the mean flow angle is $<20^{\circ}$. There is no criterion for agreement with the scale-model flow-angle results.

2. Uniform Air Velocity-The gas momentum across the stack cross section where the sample is extracted should be well mixed or uniform. The uniformity is expressed as the variability of the measurements about the mean, the coefficient of variance (COV). It is calculated as the standard deviation divided by the mean and expressed as a percentage - the lower the COV value, the more uniform the velocity. The acceptance criterion is that the COV of the air velocity must be $\leq 20 \%$ across the center two-thirds of the area of the stack. The maximum result was $12.8 \% \mathrm{COV}$, indicating that this criterion is met regardless of test port, fan configuration, and damper presence. The values declined with increasing distance from the duct junction. To confirm the validity of these scale-model tests, air-velocity uniformity measurements will have to be made on the constructed stack during cold startup to check for agreement with the velocity-uniformity results presented here for the same relative probe location. The agreement must be within $5 \% \mathrm{COV}$. 
3. Uniform Concentration of Tracer Gases-A uniform contaminant concentration in the sampling plane enables the extraction of samples that represent the true concentration. This was first tested using a tracer gas to represent gaseous effluents. The fan is a good mixer, so injecting the tracer downstream of the fans provides worst-case results. The two acceptance criteria are that 1) the COV of the measured tracer-gas concentration is $\leq 20 \%$ across the center two-thirds of the sampling plane and 2 ) at no point in the sampling plane does the concentration vary from the mean by $>30 \%$. With the dampers installed on the scale model, the results met the criteria at Test Ports 2 and 3. The maximum values observed were $10 \% \mathrm{COV}$ and $28.3 \%$ maximum deviation, both at Test Port 2. At Test Port 3, the maxima were $3.9 \%$ COV and $9.1 \%$ maximum deviation. Without the dampers, the results were only acceptable at Test Port 3.

4. Uniform Concentration of Tracer Particles-Uniformity in contaminant concentration at the sampling probe was further demonstrated using tracer particles large enough to exhibit inertial effects.

Particles of $10-\mu \mathrm{m}$ aerodynamic diameter were used. The acceptance criterion is that the COV of particle concentration is $\leq 20 \%$ across the center two-thirds of the sampling plane. The data were normalized to account for drift in the particle generator. With the dampers installed on the scale model, the results met the criterion at Test Ports 2 and 3. The maximum values observed were $13.75 \% \mathrm{COV}$ and $9.72 \% \mathrm{COV}$ at Test Port 2 and Test Port 3, respectively. Without the dampers, the results were still acceptable at both Test Ports 2 and 3.

Based on these tests, the location of the air-sampling probe in the current HV-C2 stack design (with dampers), namely 10 duct diameters downstream of the duct junction, meets the requirements of the ANSI/HPS N13.1-1999 standard. There was one gas tracer test that was borderline acceptable at 28.3\% maximum deviation compared to the $30 \%$ criterion. 


\section{Acknowledgments}

The author would like to express his appreciation to James G. Droppo, Mikhail S. Pekour, and Brad G. Fritz of Pacific Northwest National Laboratory for performing most of the testing required for the work described in this report. Wayne Cosby formatted and edited the report. 


\subsection{Introduction: Qualification of HV-C2 Air Monitoring Location}

This report documents a series of tests conducted to assess how the proposed air-monitoring system for the HV-C2 Stack will meet the applicable regulatory criteria regarding the placement of the air-sampling probe. Pacific Northwest National Laboratory conducted the tests on a scale model of the stack as it is currently designed. The objective was to determine the suitability of the probe location before the stack design is finalized. The standard governing the performance of the tests, test methods, and acceptance criteria is ANSI/HPS N13.1-1999, Sampling and Monitoring Releases of Airborne Radioactive Substances from the Stacks and Ducts of Nuclear Facilities. 


\subsection{Qualification Criteria}

The qualification criteria for the location of an air-monitoring probe, which are given in ANSI/HPS N13.1-1999, address four main characteristics.

1. Angular Flow-Sampling nozzles are usually aligned with the axis of the stack. If the air transits the stack in a cyclonic fashion, the air-velocity vector approaching the nozzle could be misaligned with the sampling nozzles enough to impair the extraction of particles. Consequently, the flow angle is measured in the stack at the location of the sampling nozzle. The average flow angle must not deviate from the axis of the sampling nozzle by more than 20 degrees.

2. Uniform Air Velocity-The gas momentum across the stack cross section where the sample is extracted should be well mixed or uniform. Consequently, the velocity is measured at several points in the stack at the location of the sampling nozzle. The uniformity is expressed as the variability of the measurements about the mean. This is expressed using the coefficient of variance (COV), which is the standard deviation divided by the mean and expressed as a percentage - the lower the COV value, the more uniform the velocity. The acceptance criterion is that the COV of the air velocity must be $\leq 20 \%$ across the center two-thirds of the area of the stack.

3. Uniform Concentration of Tracer Gases-A uniform contaminant concentration at the probe location enables the extraction of samples that represent the true concentration. This is first tested with a tracer gas to represent gaseous effluents. The fan is a good mixer, so injecting the tracer downstream of the fan provides worst-case results. The two acceptance criteria are that 1) the COV of the measured tracer gas concentration is $\leq 20 \%$ across the center two-thirds of the sampling plane and 2) at no point in the sampling plane does the concentration vary from the mean by $>30 \%$.

4. Uniform Concentration of Tracer Particles - Uniformity in contaminant concentration at the sampling probe is further demonstrated using tracer particles large enough to exhibit inertial effects. Particles of 10- $\mu \mathrm{m}$ aerodynamic diameter (AD) are used by default unless it is known that larger particles are expected in the actual airstream. The acceptance criterion is that the COV of particle concentration is $\leq 20 \%$ across the center two-thirds of the sampling plane.

The tests to assess compliance with Criteria 1 to 4 can be performed on the actual stack or with a scale model. The ANSI/HPS N13.1-1999 standard sets acceptance criteria for the use of a scale model as a substitute for the actual stack.

- The scale model and its sampling location must be geometrically similar to the actual stack.

- The product of the model's mean air velocity times the hydraulic diameter will be within a factor of six of the actual stack.

- The Reynolds number for the prototype and model stacks must $>10,000$. 
The scale model results are considered valid if:

- The velocity profile in the actual stack meets the uniformity criterion.

- The velocity uniformity COV for the actual and model stacks agree within 5\% COV.

This validation step will be performed during cold-startup testing on the constructed stack. If the scalemodel results are not validated on the constructed stack, the qualification testing is then performed on the constructed stack. 


\subsection{Scale-Model Configuration}

This section describes the configuration for the scale model and the flows in the scale model.

\subsection{Physical Arrangement}

ANSI N13.1 requires that the model be geometrically similar to the actual stack. Figure 3.1 shows the layout of the test system with the scale-model HV-C2 exhauster and heaters and filters on the inlet air. Also shown are the tracer injection ports $\mathrm{A}$ and $\mathrm{B}$ and the pairs of Test Ports 1,2, and 3. Figure 3.2 is a picture of the assembled model taken before conducting tests. The picture shows the model with caps on the air intakes and on the exhaust opening. During tests, these caps were removed, and pre-filters were placed on the air intakes.

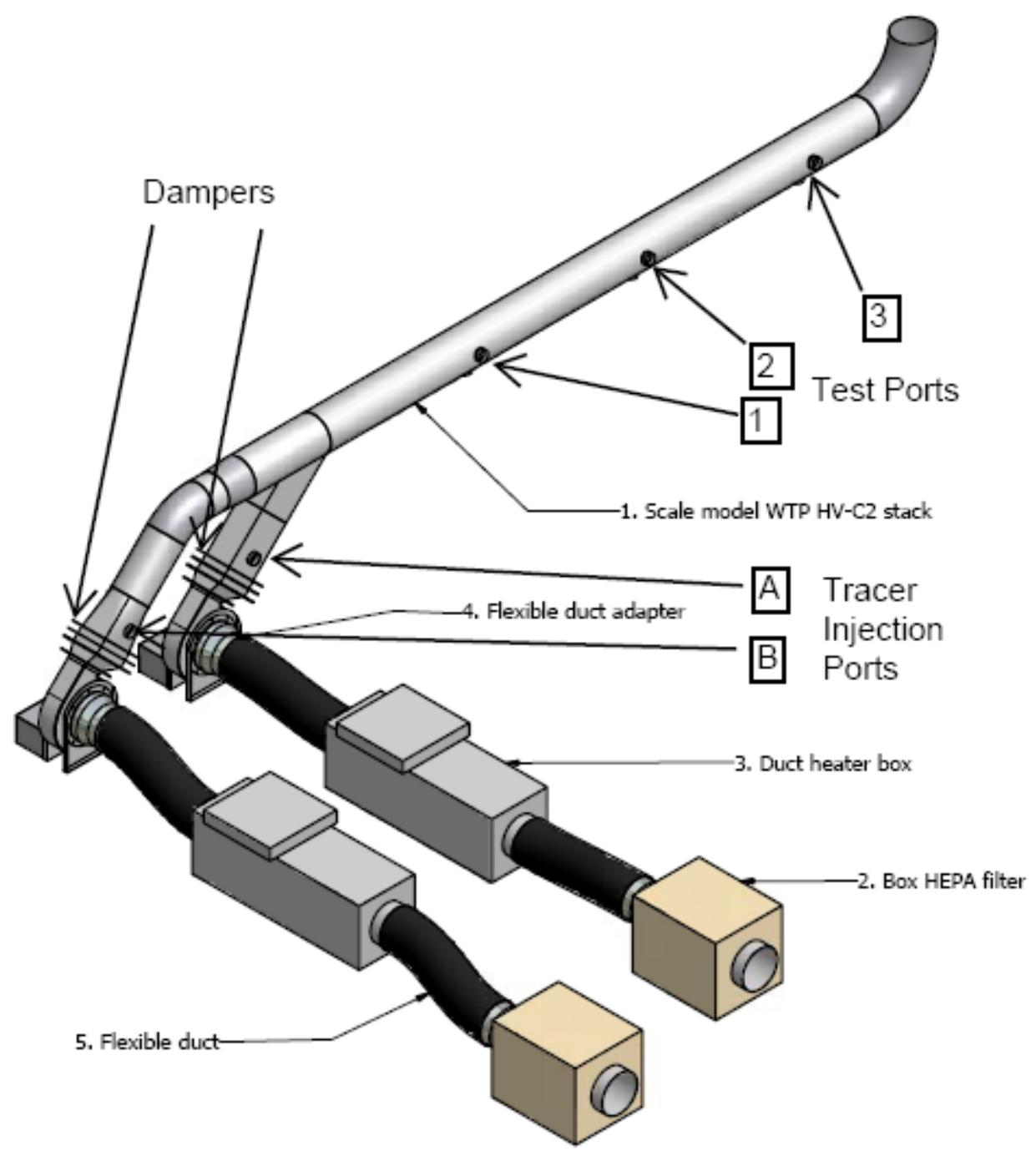

Figure 3.1. Layout of HV-C2 Test System 
At each fan outlet is a control damper and a backdraft damper. Scale-model dampers and blank duct sections were interchanged to test the effect of the dampers on the test results.

The ratio of prototype dimensions to scale-model dimensions was determined by the ratio of the designed stack diameter (44 in.) to the scale-model stack diameter (12 in.), or $44 / 12=3.667$. Appendix A details the assumptions and calculations that were the basis of the scale-model dimensions. These were approved by Bechtel National, Inc. (BNI) staff. Appendix A also includes the referenced BNI design documents used for the scale-model parameters.

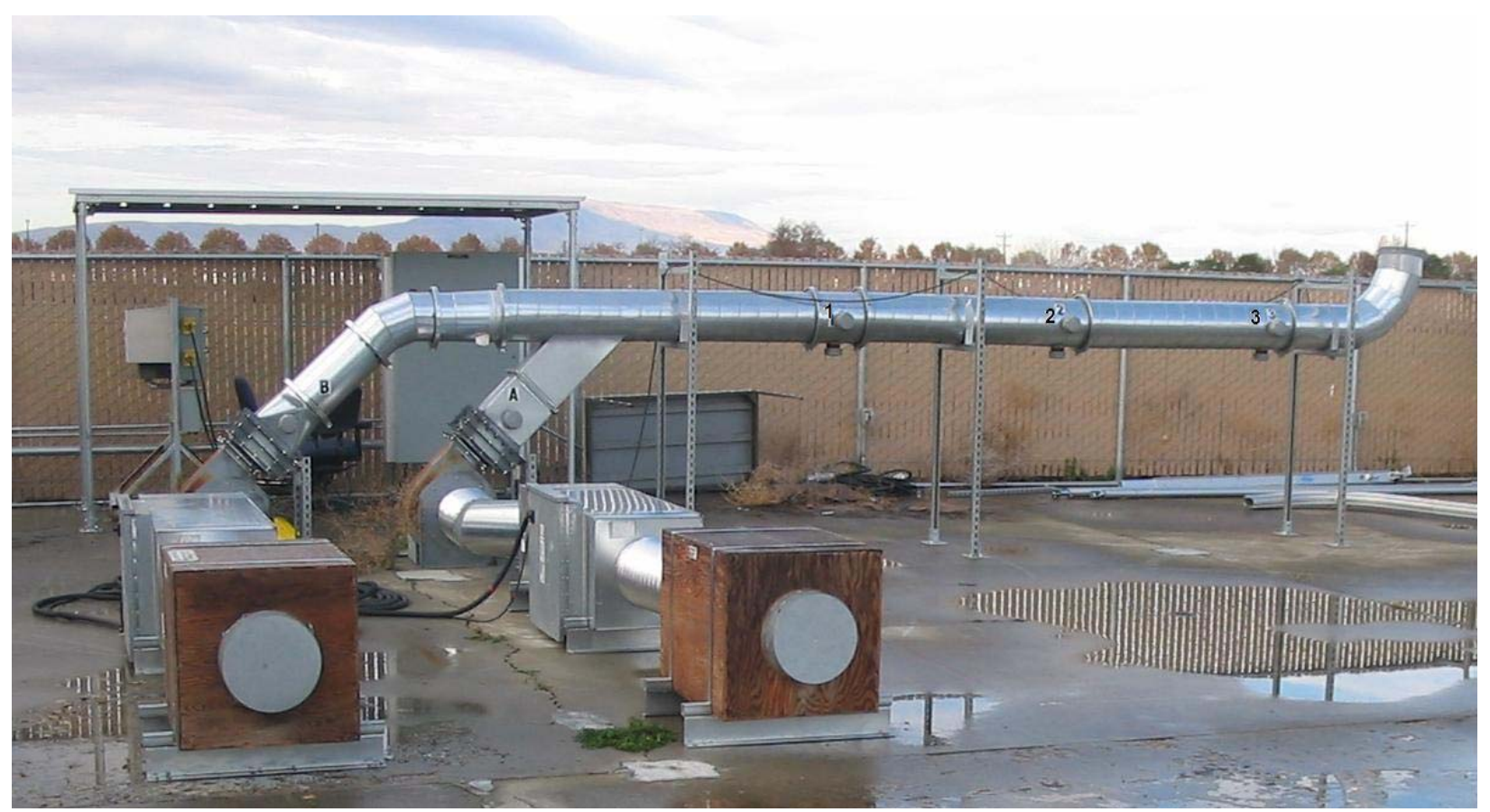

Figure 3.2. Assembled HV-C2 Scale Model

Table 3.1 lists the key dimensions along the run of the duct for HV-C2. Deviations in key length dimensions of the scale model arising from scaling and fabrication errors were acceptable. Test Ports 1 and 3 were simply located five duct diameters $(5 \mathrm{D})$ in both directions from the planned sampling probe.

Table 3.1. Key Dimensions of Actual and Scale-Model Stacks

\begin{tabular}{|c|c|c|}
\hline Key Dimensions & Current HV-C2 Design & Scale Model, As-Built \\
\hline Junction of Fan A outlet and stack to $90^{\circ}$ bend & Not given & 201.25 in. $(16.77 \mathrm{D})$ \\
\hline Test Port 3 to start of $90^{\circ}$ bend & Not given & 27 in. $(2.25 \mathrm{D})$ \\
\hline $\begin{array}{l}\text { Expansion of duct from Fan B to junction of Fan A } \\
\text { outlet and stack }\end{array}$ & 99 in. $(2.25 \mathrm{D})$ & 28.5 in. $(2.38 \mathrm{D})$ \\
\hline Junction of Fan A outlet and stack to Test Port 1 & N.A. & 53.5 in. $(4.45 \mathrm{D})$ \\
\hline $\begin{array}{l}\text { Junction of Fan A outlet and stack to sampling } \\
\text { probe and Test Port } 2\end{array}$ & 418 in. $(9.5 \mathrm{D})$ & 113.75 in. $(9.47 \mathrm{D})$ \\
\hline $\begin{array}{l}\text { Junction of Fan A outlet and stack to Test Port } 3 \\
\end{array}$ & N.A. & 174.25 in. $(14.52 \mathrm{D})$ \\
\hline
\end{tabular}




\subsection{Scale-Model Flows}

The test parameters should bracket the range of expected design flowrates and the two main flow conditions - one and two operating fans. The normal flowrate with two fans operating should be about $40,000 \mathrm{cfm} .{ }^{\text {(a) }}$ For the maximum flowrate, we assumed that the new system with clean ventilation filters may operate at $15 \%$ above normal, or $46,000 \mathrm{cfm}$. Garcia and Love ${ }^{(\mathrm{b})}$ predicted that with one fan operating, the system operating pressure will decrease. Thus, the one fan will actually provide more than half the normal flowrate (actually about $70 \%$ of normal). For the purposes of these tests, it was assumed that the scaled version of 20,000 and $46,000 \mathrm{cfm}$ adequately bracketed the range of conditions.

The ANSI/HPS N13.1-1999 standard requires that the scale model's product of mean velocity $\times$ hydraulic diameter be within a factor of six of the actual stack. For stacks with a circular cross section, this is equivalent to requiring that the ratio of flowrate to stack diameter $(\mathrm{Q} / \mathrm{D})$ be within a factor of six of the actual stack. The standard also requires that the Reynolds number for the prototype and model stacks must $>10,000$.

Table 3.2 summarizes the prototype and scale-model flow parameters that satisfy the scaling requirements where the scale-model stack has a 12-in. diameter. The flowrate and velocity values for the scale model were treated as minimum target values.

Table 3.2. Summary of Flow Parameters

\begin{tabular}{||l|c|c|c|c|c|c||}
\hline & \multicolumn{2}{|c|}{ Air Flow cfm } & \multicolumn{2}{c|}{ Air Velocity fpm } & \multicolumn{2}{c||}{ Reynolds Number } \\
\hline & $\begin{array}{c}\text { Actual } \\
\text { Stack }\end{array}$ & $\begin{array}{c}\text { Scale } \\
\text { Model Min. }\end{array}$ & $\begin{array}{c}\text { Actual } \\
\text { Stack }\end{array}$ & $\begin{array}{c}\text { Scale } \\
\text { Model Min. }\end{array}$ & $\begin{array}{c}\text { Actual } \\
\text { Stack }\end{array}$ & $\begin{array}{c}\text { Scale } \\
\text { Model Min. }\end{array}$ \\
\hline $\begin{array}{l}\text { Two fans- } \\
\text { max. flow }\end{array}$ & 46,000 & 2,091 & 4,356 & 2,662 & $1.7 \mathrm{E}+06$ & $2.8 \mathrm{E}+05$ \\
\hline $\begin{array}{l}\text { Two fans- } \\
\text { normal flow }\end{array}$ & 40,000 & 1,818 & 3,788 & 2,315 & $1.4 \mathrm{E}+06$ & $2.4 \mathrm{E}+05$ \\
\hline $\begin{array}{l}\text { Single fan- } \\
\text { minimum flow }\end{array}$ & 20,000 & 909 & 1,894 & 1,158 & $7.2 \mathrm{E}+05$ & $1.2 \mathrm{E}+05$ \\
\hline
\end{tabular}

The air temperature in the operating stack did not need to be simulated because it has a negligible effect on the turbulence produced by the geometry of the system. However, the air temperatures in the model were maintained above $55^{\circ} \mathrm{F}$ during the particle uniformity test so that the optical particle counters would provide reliable data.

(a) G. M. Gaulden. Exhaust Stack Design Standardization Data. RPP WTP Memorandum, March 23, 2005, CCN: 116112.

(b) G. Garcia, and B. Love. C2 Exhaust Fan Redundancy Criteria. RPP WTP Memorandum, August 3, 2002, CCN: 038045. 


\subsection{Test Methods}

This section describes the flow-angle and velocity uniformity tests, the flow-controls calibration, and the gas-tracer and particle-tracer uniformity tests. The test quality assurance measures taken are also summarized. The detailed procedures are given in the appendices.

\subsection{Flow-Controls Calibration}

The first test performed with the scale model was to obtain an approximate calibration of the fan flow controls. Each fan has its own speed control, which is a variable frequency drive with settings from 0 to $60 \mathrm{~Hz}$. The first step was to measure the flowrate of each fan separately with the speed control set at $30 \mathrm{~Hz}$. Then a point with approximately average velocity is identified. A calibrated air-velocity meter is used to take readings at that point while the speed settings are varied from 5 to $60 \mathrm{~Hz}$. Procedure EMS-JAG-03, "Test to Calibrate Ventilation Flow Controller," was used for this preliminary test. The results provide the approximate settings to achieve the flowrate desired for the balance of the tests. The data sheets are given in Appendix B.

\subsection{Flow-Angle Test}

The air-velocity vector approaching the sample nozzle should be aligned with the axis of the nozzle, within an acceptable angle, so sample-extraction performance is not degraded. The test method is based on 40 CFR 60, Appendix A, Method 1, Section 2.4, "Verification of the Absence of Cyclonic Flow."

The term "flow angle" is the angle between the air-velocity vector and the axis of the sampling nozzle. The flow angle was measured at a grid of points in a cross section of the stack at the scaled elevation of the actual sampling probe. The grid was an array of points in an x-pattern in the cross section of the duct. One line of points was aligned in the same direction as the proposed sampling probe. The other line was perpendicular to that. The number and distance between measurement points was based on the U.S. Environmental Protection Agency (EPA) procedure 40 CFR 60, Appendix A, Method 1.

Measurements were made using a type-S pitot tube attached by flexible tubes to a slant-tube manometer, and an angle-indicating device was attached to the pitot tube as shown in Figure 4.1. Figure 4.2 shows the angle-indicating device and S-type pitot mounted on a test port. Procedure EMS-JAG-05, "Test to Determine Flow Angle at the Elevation of a Sampler Probe," provides the general procedure to determine the mean flow angle. The data sheets are provided in Appendix C.

\subsection{Velocity Uniformity Test}

The uniformity of air velocity where the air sample is being extracted verifies that the air momentum in the stack is well mixed. To determine uniformity, air velocity was measured at the same grid of points used for the angular flow test. The method used was based on 40 CFR 60, Appendix A, Method 2. 


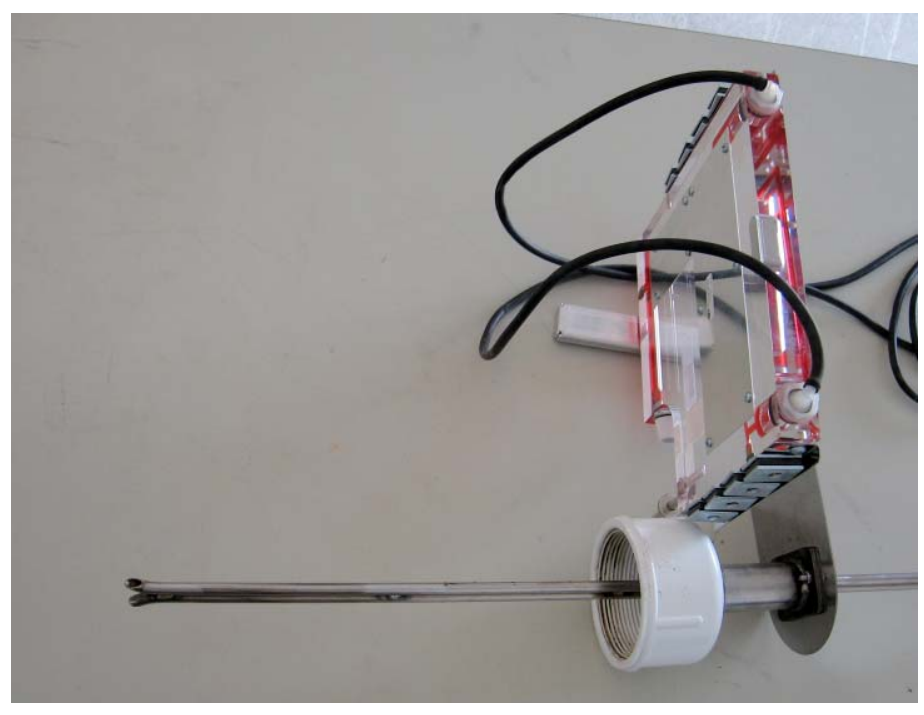

Figure 4.1. Flow-Angle Measurement Apparatus

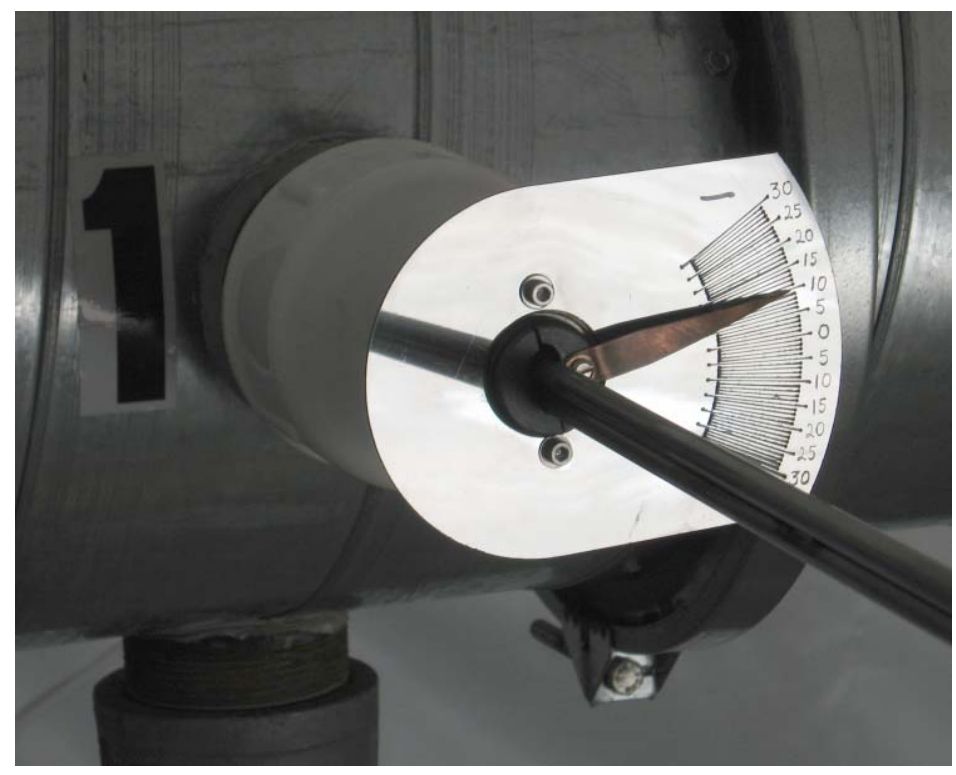

Figure 4.2. View of Type-S Pitot Tube Inserted into Model Stack

The air velocity was measured three times at each grid point, and each measurement was recorded. The measurements at each grid point were averaged to determine the average velocity at each grid point. The average values for each grid point in the center two-thirds of the stack were used to calculate the overall mean and standard deviation of velocity for the sampling location. The COV (also called the percent relative standard deviation) was calculated as 100 times the standard deviation divided by the mean. This value should be less than or equal to $20 \%$.

The equipment used included a standard pitot tube (Prandtl type) and a calibrated electronic manometer and air-velocity meter. Figure 4.3 shows the setup. Procedure EMS-JAG-04, "Test to Determine 
Uniformity of Gas Velocity at the Elevation of a Sampler Probe," was used for this test. The data sheets are provided in Appendix D.

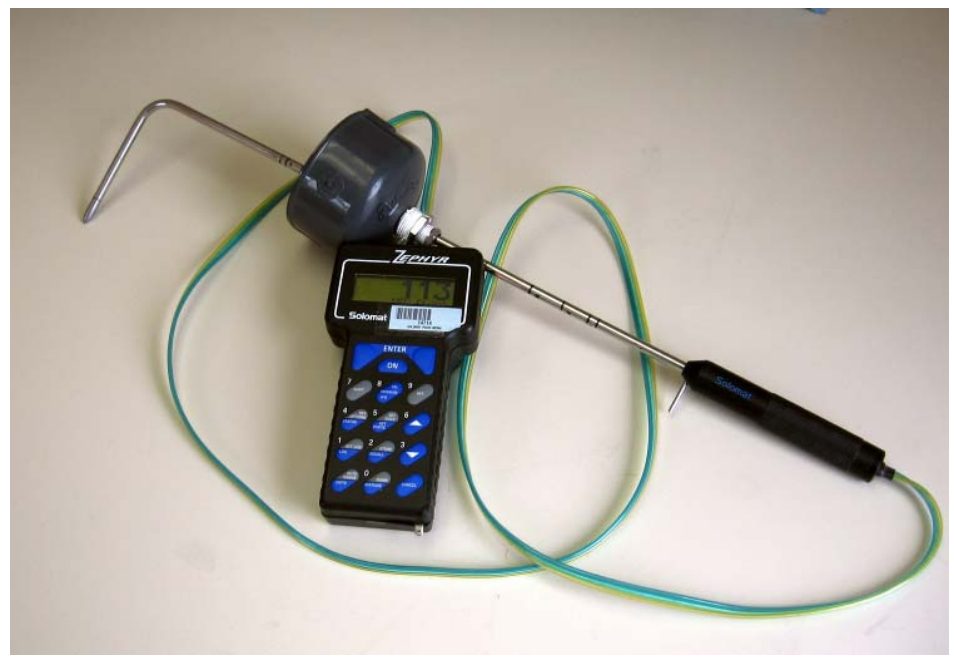

Figure 4.3. Electronic Manometer and Pitot Tube

\subsection{Gas-Tracer Uniformity Test}

The gaseous-contaminant-concentration uniformity was demonstrated using sulfur hexafluoride as a tracer gas. The tracer gas was injected into the air downstream of a fan through one of the injection ports shown in Figure 3.1 and Figure 3.2. A probe was used to inject the tracer along the centerline of the duct and at four points near the corners of the duct. One injection point at a time was tested.

For each injection position, the tracer concentration was measured at the sampling location using the same measurement grid as used for the other tests. The tracer concentration was measured three times at each grid point, and each measurement was recorded. The measurements at each grid point were averaged to determine the average concentration at each grid point. The values for each grid point in the center twothirds of the stack were used to calculate the mean and standard deviation of concentration for the sampling location. The percent COV was calculated as 100 times the standard deviation divided by the mean. This value should be less than or equal to $20 \%$. The average concentration values for all grid points were also compared to the mean to determine if the concentration at any point deviates from the mean by more than $30 \%$.

The tracer-gas concentration was measured with an Innova AirTech Instruments A/S (Ballerup, Denmark) Model 1302 photoacoustic gas analyzer. The analyzer response was checked with calibration standards before and during the test series to verify that the instrument responds adequately to changes in concentration. If the indicated concentration was within $20 \%$ of the standard, the response was acceptable. A maximum bias of $4.8 \%$ was observed in these check readings. The effects of this bias on the COV were analyzed and found to be negligible. 
Figure 4.4 shows the tracer-gas sampling probe. Figure 4.5 shows the gas analyzer and Figure 4.6 the assembled sampling equipment. Procedure EMS-JAG-01, "Test to Determine Uniformity of a Tracer Gas at a Sampler Probe," was used for this test. The data sheets are given in Appendix E.

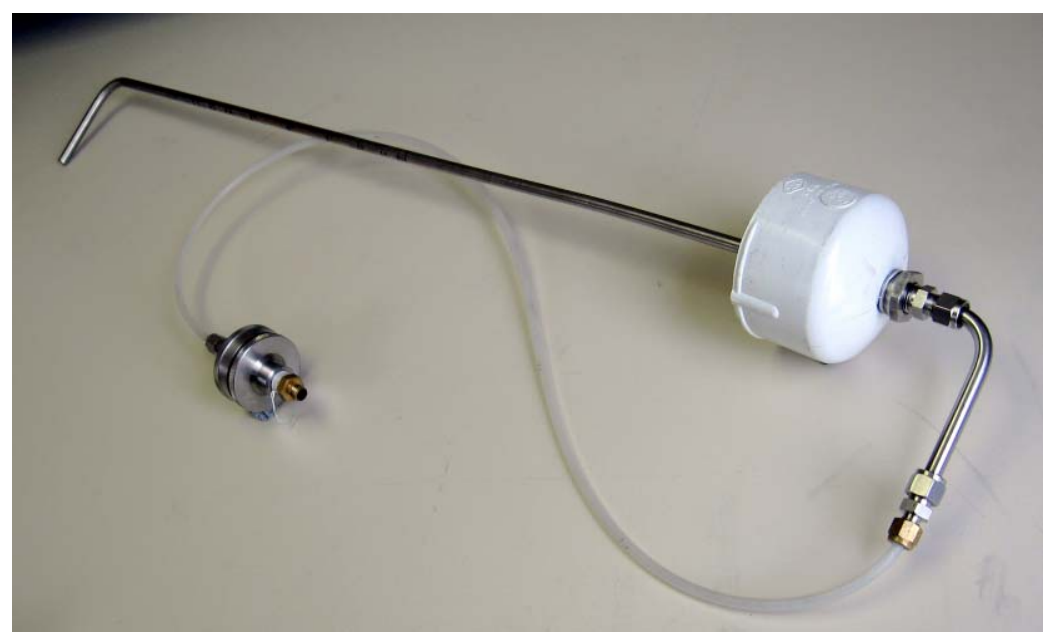

Figure 4.4. Tracer-Gas Sampling Assembly

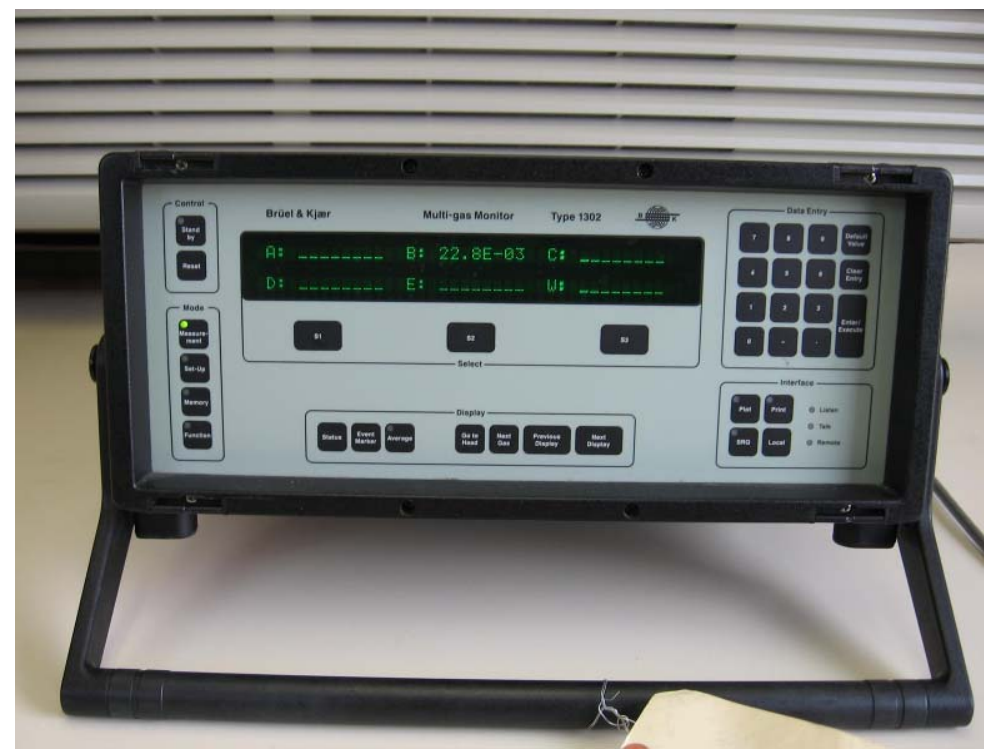

Figure 4.5. Tracer-Gas Analyzer 


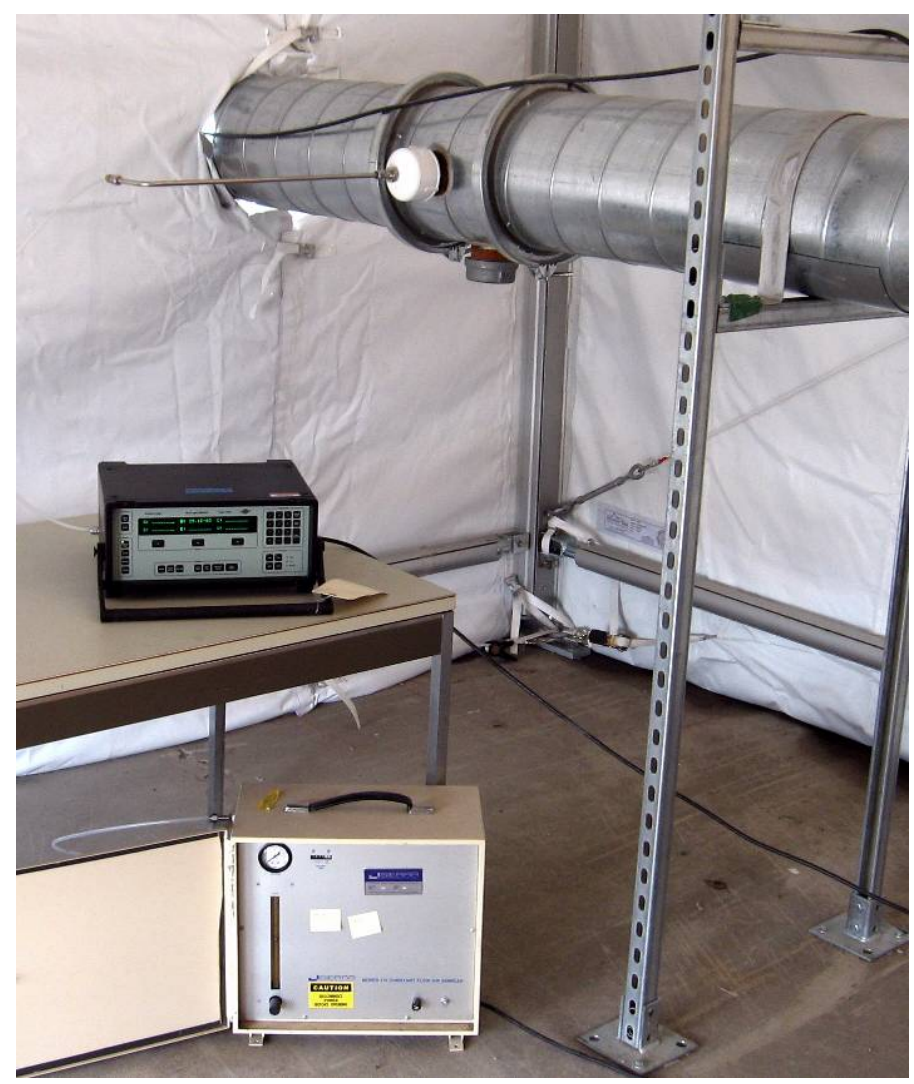

Figure 4.6. Tracer-Gas Test Setup

\subsection{Particle-Tracer Uniformity Test}

The test for uniformity of tracer particles is similar to the test for uniformity of tracer gases. The general approach is to inject a polydisperse aerosol into the test stack downstream of a fan. The concentration of the particles is then measured at the sampling grid points with a calibrated optical particle counter (OPC, Met-One Model A2408, Grants Pass, Oregon). A simple probe was used to extract the sample and deliver it to the OPC. Figure 4.7 shows the sampling probe and OPC located at a test port.

The tracer aerosol was vacuum pump oil (Fisherbrand 19 ${ }^{\mathrm{TM}}$ ). It is drawn into a compressed-air-driven spray nozzle and is housed in the chamber shown in Figure 4.8. The chamber provides a means for injecting the particles into the airflow through a probe.

The tracer injection ports were the same as for the gaseous tracer; however, only the centerline injection point was used as required in the standard. The layout of measurement points was the same as for all of the other tests.

The OPCs sort the number of particles into six size channels. Each concentration reading was the count of particles in the 9- to 11- $\mu \mathrm{m}$ channel. The readings were recorded on a data sheet. Three readings were taken at each point and averaged. The COV of the average concentration readings at all points was calculated, and the result was compared to the acceptance criterion for uniformity. The particle mixing 
was acceptable if the COV of the tracer particles of $10-\mu \mathrm{m}$ AD was less than or equal to $20 \%$ across the center two-thirds of the sampling plane.

Procedure EMS-JAG-02, “Test to Determine Uniformity of a Tracer Aerosol at a Sampler Probe," was used for this test. Appendix F contains the data sheets obtained.

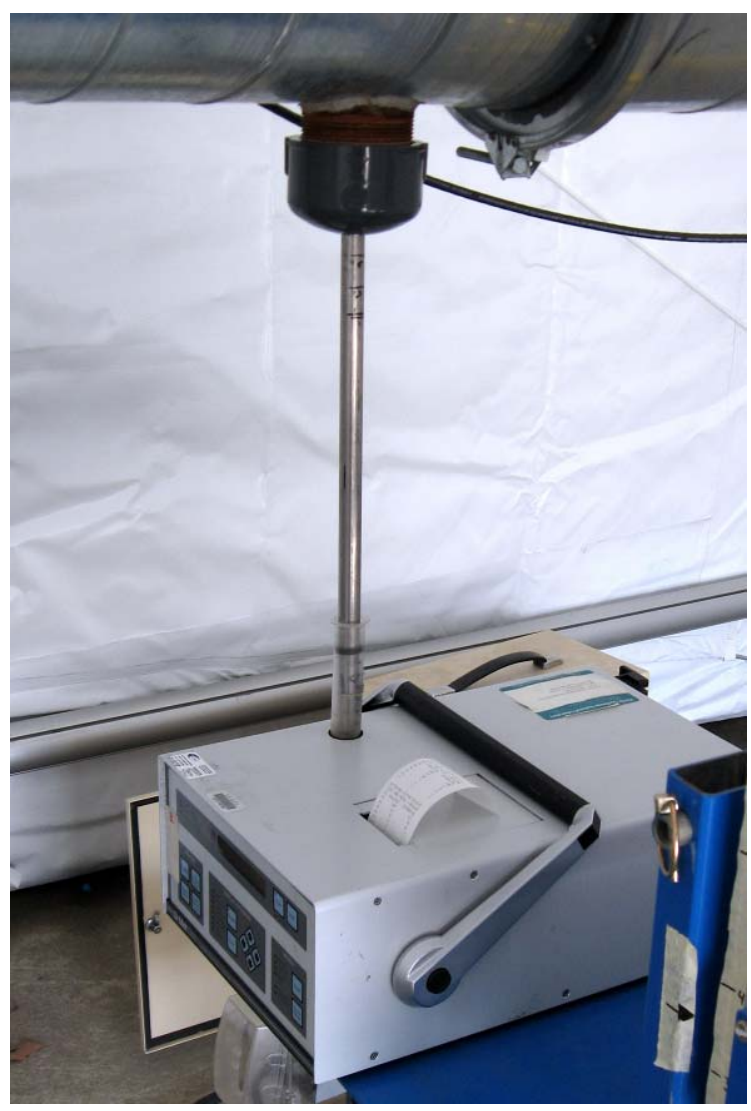

Figure 4.7. Particle Counter Connected to Probe 


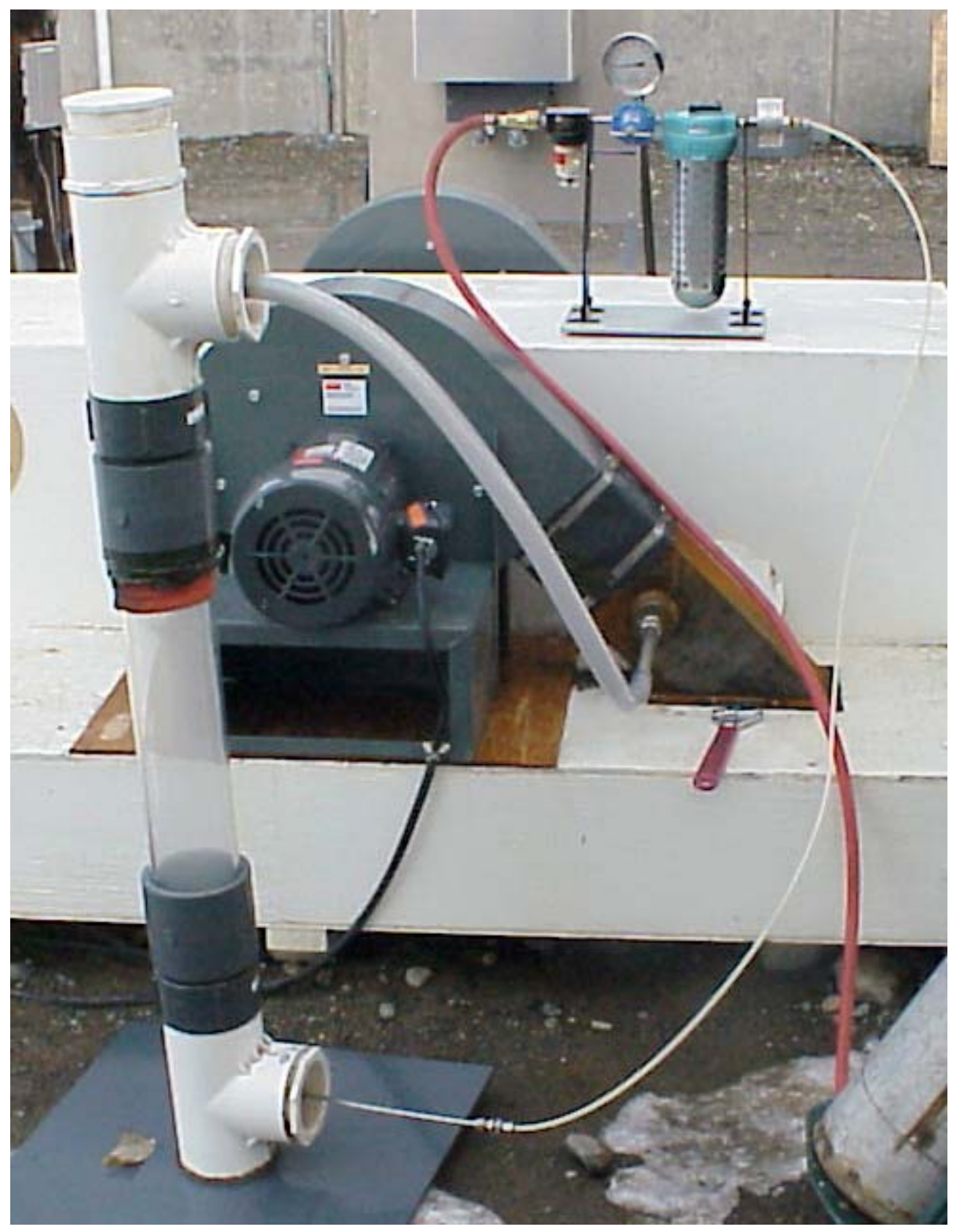

Figure 4.8. Particle Tracer Generator Apparatus (HVC2 not shown) 


\subsection{Quality Assurance Requirements}

\subsubsection{Application of RPP-WTP Quality Assurance Requirements}

Pacific Northwest National Laboratory (PNNL) is operated by Battelle for the U.S. Department of Energy. Its Pacific Northwest Division (PNWD) has a Quality Assurance Program that is based upon the requirements as defined in the United States Department of Energy (DOE) Order 414.1C, Quality Assurance, and 10 CFR 830, Energy/Nuclear Safety Management, Subpart A-Quality Assurance Requirements. PNWD chose to implement the requirements of DOE Order 414.1C and 10 CFR 830, Subpart A by integrating them into the laboratory's management systems and daily operating processes. The procedures necessary to implement the requirements are documented through the laboratory's Standards-Based Management System (SBMS).

PNNL implements the RPP-WTP quality requirements by performing work in accordance with the River Protection Project-Waste Treatment Plant Support Program (RPP-WTP) Quality Assurance Plan (RPP-WTP-QA-001, QAP). Work was performed to the quality requirements of NQA-1-1989, Part I, Basic and Supplementary Requirements, NQA-2a-1990, Part 2.7, and DOE/RW-0333P, Rev 13, Quality Assurance Requirements and Descriptions (QARD). These quality requirements are implemented through the River Protection Project-Waste Treatment Plant Support Program (RPP-WTP) Quality Assurance Manual (RPP-WTP-QA-003, QAM).

\subsubsection{Conduct of Experimental and Analytical Work}

Experiments that are not method-specific shall be performed in accordance with RPP-WTP's procedures QA-RPP-WTP-1101 "Scientific Investigations" and QA-RPP-WTP-1201 "Calibration Control System," making sure that sufficient data are taken with properly calibrated measuring and test equipment to obtain quality results. The instrumentation used for each test was recorded on each data sheet.

\subsubsection{Internal Data Verification and Validation}

RPP-WTP addresses internal verification and validation activities by conducting an independent technical review of the final data report in accordance with RPP-WTP's procedure QA-RPP-WTP-604. This review verifies that the reported results are traceable, inferences and conclusions are soundly based, and the reported work satisfies the Test Plan objectives. This review procedure is part of PNNL's RPP-WTP Quality Assurance Manual. 


\subsection{Test Runs}

A number of factors were considered in defining the appropriate set of test runs. In terms of defining the most limiting conditions, it is presumed that the least mixing would occur for tracer injected after Fan A and measured at Test Port 1 . Better mixing should be observed at the test ports more distant from the fans.

The current facility design shows that the planned air monitoring probe position will correspond to Test Port 2. The statement of work indicates that the qualification tests will be conducted in detail for the planned probe location, with less detailed testing at up to two other locations that cover the likely alternate positions. The tests should also be conducted at flow rates bracketing the range of expected flow. The different fan operating configurations should be addressed in the tests. And finally, the presence of dampers just upstream of the tracer injection ports was also addressed in this test plan because it is the first instance where the presence of dampers could be a factor.

To completely qualify a monitoring location for one set of operating conditions, the gas tracer test requires five runs (one for each injection area) and one run of each of the other tests. To qualify alternate test-port locations more distant from the fans or flow disturbance, only one of the set of gas tracer tests may be needed. Often the tests are more detailed for injection-port/test-port combinations in closest proximity to each other, with fewer test runs for injection-port/test-port combinations with a greater distance between them. Some repeat runs were added to the test matrix to obtain an indication of repeatability. Table 5.1 lists the number of test runs by operating configuration, injection and test-port combination, and test type.

The tests listed in the first three rows provided an approximate calibration of the fan-control settings. The tests in the next two rows allowed exploring the effect caused by the absence or presence of dampers just upstream of the injection ports. Test Port 1 and Injection Port A were used mostly because any damper effect would be most easily observed there. The series started with a group of tests with no dampers installed, and both series covered the major flow configurations. The rest of the tests were conducted with the dampers installed. Repeat tests were added to determine the repeatability of results.

The matrix of tests with dampers underwent some modifications based on results of initial tests without dampers to optimize the utility of the results. 
Table 5.1. Test Runs Performed for HV-C2 Configurations

\begin{tabular}{|c|c|c|c|c|c|}
\hline & & \multicolumn{4}{|c|}{ " Number of Test Runs } \\
\hline $\begin{array}{c}\text { Combination of } \\
\text { Injection/Test Port }\end{array}$ & $\begin{array}{l}\text { Target Scaled Stack } \\
\text { Flowrate, cfm }\end{array}$ & $\begin{array}{l}\text { Flow } \\
\text { Angle }\end{array}$ & Velocity & $\begin{array}{c}\text { Gas } \\
\text { Tracer }\end{array}$ & $\begin{array}{l}\text { Particle } \\
\text { Tracer }\end{array}$ \\
\hline N.A. & Both fans & - & 1 & - & - \\
\hline N.A. & Fan A & - & 1 & - & - \\
\hline N.A. & Fan B & - & 1 & - & - \\
\hline $\mathrm{A} / 1$ & $\begin{array}{c}\text { 909, Fan A only, no } \\
\text { dampers }\end{array}$ & 1 & 2 & 5 & 1 \\
\hline $\mathrm{B} / 1$ & $\begin{array}{c}\text { 909, Fan B only, no } \\
\text { dampers }\end{array}$ & 0 & 2 & 0 & 0 \\
\hline $\mathrm{A} / 1$ & \multirow{4}{*}{$\begin{array}{l}\text { 2091, both fans, no } \\
\text { dampers }\end{array}$} & 6 & 6 & 12 & 5 \\
\hline $\mathrm{A} / 2$ & & 0 & 0 & 1 & 0 \\
\hline $\mathrm{A} / 3$ & & 0 & 0 & 1 & 0 \\
\hline $\mathrm{B} / 1$ & & 0 & - & 1 & 0 \\
\hline $\mathrm{A} / 1$ & $\begin{array}{c}\text { Fan A only, dampers } \\
\text { partly closed }\end{array}$ & 1 & 1 & 5 & 1 \\
\hline $\mathrm{B} / 1$ & \multirow{3}{*}{$\begin{array}{c}\text { 2091, both fans, } \\
\text { dampers partly closed }\end{array}$} & 0 & 0 & 1 & 0 \\
\hline $\mathrm{B} / 2$ & & 0 & 0 & 1 & 0 \\
\hline $\mathrm{B} / 3$ & & 0 & 0 & 1 & 0 \\
\hline $\mathrm{A} / 1$ & \multirow{6}{*}{$\begin{array}{l}2091 \text { from both fans, } \\
\text { dampers full open }\end{array}$} & 1 & 1 & 5 & 1 \\
\hline $\mathrm{A} / 2$ & & 1 & 1 & 5 & 1 \\
\hline $\mathrm{A} / 3$ & & 1 & 1 & 5 & 1 \\
\hline $\mathrm{B} / 1$ & & 0 & - & 1 & 1 \\
\hline $\mathrm{B} / 2$ & & 0 & - & 1 & 1 \\
\hline $\mathrm{B} / 3$ & & 0 & - & 1 & 1 \\
\hline $\mathrm{A} / 1$ & \multirow{3}{*}{$\begin{array}{l}909 \text { Fan A only, } \\
\text { dampers full open }\end{array}$} & 1 & 1 & 1 & 1 \\
\hline $\mathrm{A} / 2$ & & 1 & 1 & 1 & 1 \\
\hline $\mathrm{A} / 3$ & & 1 & 1 & 1 & 1 \\
\hline $\mathrm{B} / 1$ & \multirow{3}{*}{$\begin{array}{l}909 \text { Fan B only, } \\
\text { dampers full open }\end{array}$} & 1 & 1 & 1 & 1 \\
\hline $\mathrm{B} / 2$ & & 1 & 1 & 1 & 1 \\
\hline $\mathrm{B} / 3$ & & 1 & 1 & 1 & 1 \\
\hline \multicolumn{2}{|c|}{ Total } & 17 & 6 & 52 & 111 \\
\hline
\end{tabular}




\subsection{Test Results}

The results will be presented in three sections as follows: (6.1) for fan-control correlation, (6.2) for tests without dampers installed, and (6.3) for tests with dampers installed.

\subsection{Fan-Control Correlation}

All of the measurements made for this test were done using Test Port 1. Flowrates were measured with the speed controls set at $30 \mathrm{~Hz}$ (half speed). The dampers were not installed. The flowrates were measured using the same procedure as for the velocity uniformity test. Table 6.1 lists the results, and the data sheets are in Appendix B. The first run was made with no prefilter on the high-efficiency particulate air (HEPA) filter inlets. Prefilters were used on the other three runs.

Table 6.1. Flowrates Measured at Test Port 1 During Control Correlation

\begin{tabular}{||c|c|c|c||}
\hline Operating Fans & Run No. & Flowrate cfm & \% COV with Center-Point \\
\hline A\&B-half speed, no prefilters & VT-1 & 2029 & 5.2 \\
\hline A\&B-half speed, prefilters & VT-2 & 1673 & 6.5 \\
\hline A- half speed, prefilters & VT-3 & 920 & 11.8 \\
\hline B half speed, prefilters & VT-4 & 913 & 6.3 \\
\hline
\end{tabular}

The measurement points were identified that best represented the average velocity. At those points, we measured velocity as a function of control setting, and the datasheets are given in Appendix B. Table 6.2 lists the control settings that would provide the minimum test flowrates. It was decided that for all of the remaining tests, the speed settings would be $40 \mathrm{~Hz}$ for two fans and $35 \mathrm{~Hz}$ for a single fan.

Table 6.2. Interpolated Fan-Control Settings

\begin{tabular}{|c|c|c|c||}
\hline Operating Fans & $\begin{array}{c}\text { Target Flow, } \\
\text { cfm }\end{array}$ & $\begin{array}{c}\text { Interpolated Control } \\
\text { Setting for Target, Hz }\end{array}$ & Run No. \\
\hline A \& B & 2091 & 37.4 & VF-1 \\
\hline A & 909 & 31.1 & VF-2 \\
\hline B & 909 & 30.5 & VF-3 \\
\hline A & 909 & 32.0 & VF-4 \\
\hline B & 909 & 30.4 & VF-5 \\
\hline
\end{tabular}

\subsection{Tests Without Dampers Installed}

\subsubsection{Flow-Angle Test}

Table 6.3 lists the results for the flow-angle tests performed on the scale model without the dampers installed. Tests were done for all fan combinations at Test Port 1. Tests with both fans were repeated five times. Run FA- 8 was also done with both fans operating, but with a contoured filler plug installed in 
the test port in an attempt to eliminate the possible effect of an eddy, caused by the empty space in the test port, on the readings nearest the test port. Figure 6.1 shows a chart of typical results from these tests. Appendix $\mathrm{C}$ provides the data sheets.

Table 6.3. Flow-Angle Tests on Scale Model Without Dampers

\begin{tabular}{|c|c|c|c||}
\hline Test Port & Fans & Run & $\begin{array}{c}\text { Average Absolute Angle } \\
\text { (degrees) }\end{array}$ \\
\hline 1 & A \& B & FA-1 & 4.59 \\
\hline 1 & A \& B & FA-2 & 5.02 \\
\hline 1 & A \& B & FA-3 & 4.96 \\
\hline 1 & A \& B & FA-4 & 4.30 \\
\hline 1 & A \& B & FA-7 & 4.44 \\
\hline 1 & A \& B & FA-8 & 3.75 \\
\hline 1 & A & FA-6 & 3.43 \\
\hline 1 & B & FA-5 & 5.46 \\
\hline
\end{tabular}
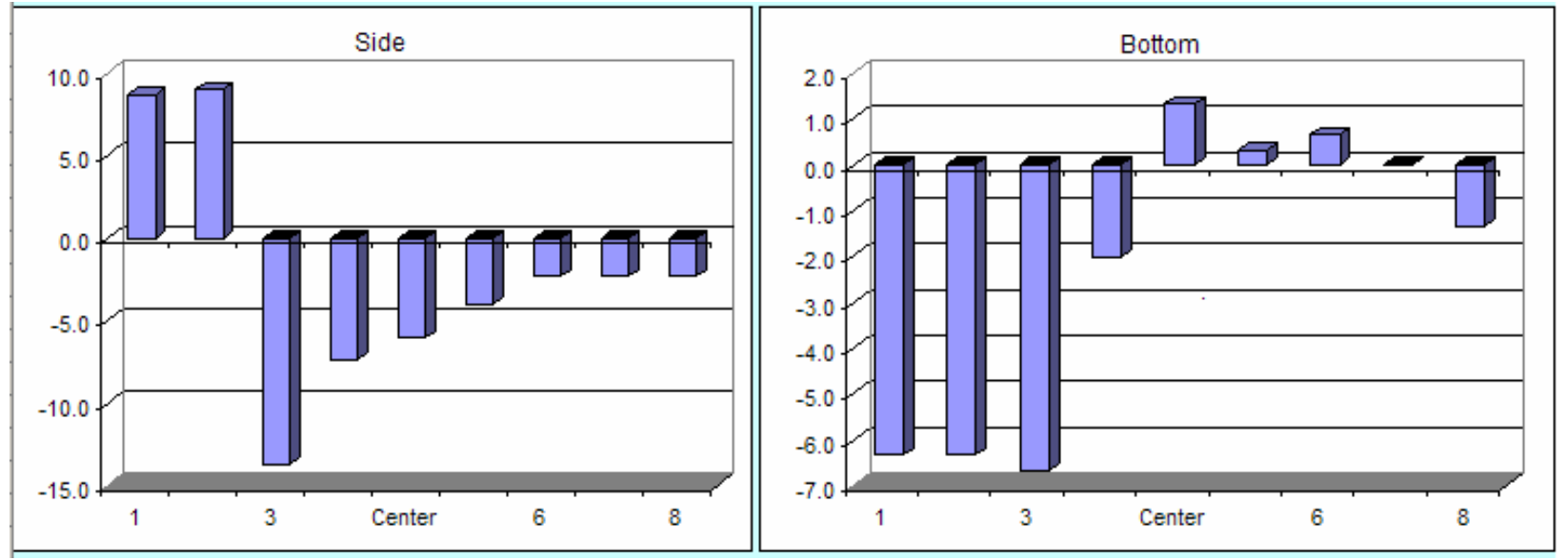

Figure 6.1. Plot of Scale-Model Flow-Angle Results, which are shown in degrees, with Both Fans Operating (Run FA-1)

\subsubsection{Velocity Uniformity Test}

\subsubsection{Scale-Model Tests}

Test runs were performed on the scale model using an 8-point traverse grid with the addition of a centerpoint. Generally, the addition of the centerpoint has little impact on the COV results and provides information on whether something interesting happens at the center. Appendix D provides the detailed results for each test. Table 6.4 summarizes the results for the various fan combinations. 
Table 6.4. Velocity Uniformity Tests on Scale Model at Test Port 1 Without Dampers

\begin{tabular}{|c|c|c|c|c|c||}
\hline Test Port & Operating Fans & Run No. & Flowrate cfm & \% COV & Meets COV Criterion \\
\hline 1 & A \& B & VT-1 & 2029 & 5.2 & Yes \\
\hline 1 & A \& B & VT-2 & 1673 & 6.5 & Yes \\
\hline 1 & A \& B & VT-5 & 2265 & 5.5 & Yes \\
\hline 1 & A \& B & VT-6 & 2244 & 5.8 & Yes \\
\hline 1 & A \& B & VT-7 & 2307 & 5.6 & Yes \\
\hline 1 & A \& B & VT-8 & 2282 & 7.0 & Yes \\
\hline 1 & A & VT-3 & 920 & 11.8 & Yes \\
\hline 1 & A & VT-9 & 1076 & 10.3 & Yes \\
\hline 1 & B & VT-4 & 913 & 6.3 & Yes \\
\hline 1 & B & VT-10 & 1049 & 5.9 & Yes \\
\hline
\end{tabular}

For the 8-point traverse grid, the center two-thirds area excludes the measurement points nearest the wall. The $\% \mathrm{COV}$ was calculated for the center two-thirds area, including centerpoint measurements. Runs VT-1 through VT-4 were originally done as part of the controls calibration, and the control setting was $30 \mathrm{~Hz}$. The other tests were done at $40 \mathrm{~Hz}$ for two fans and $35 \mathrm{~Hz}$ for single fans. Also, Run VT-1 was done without prefilters at the HEPA filter inlets and hence the somewhat higher flowrate. These slight variations in flowrate did not appear to have any impact on these results. With Fan A operated singly, the resulting COV was a little higher than that for the other fan configurations. The uniformity criterion was met in these tests. Figure 6.2 shows a plot of the velocity profile at Test Port 1 with Fans A and B operating.

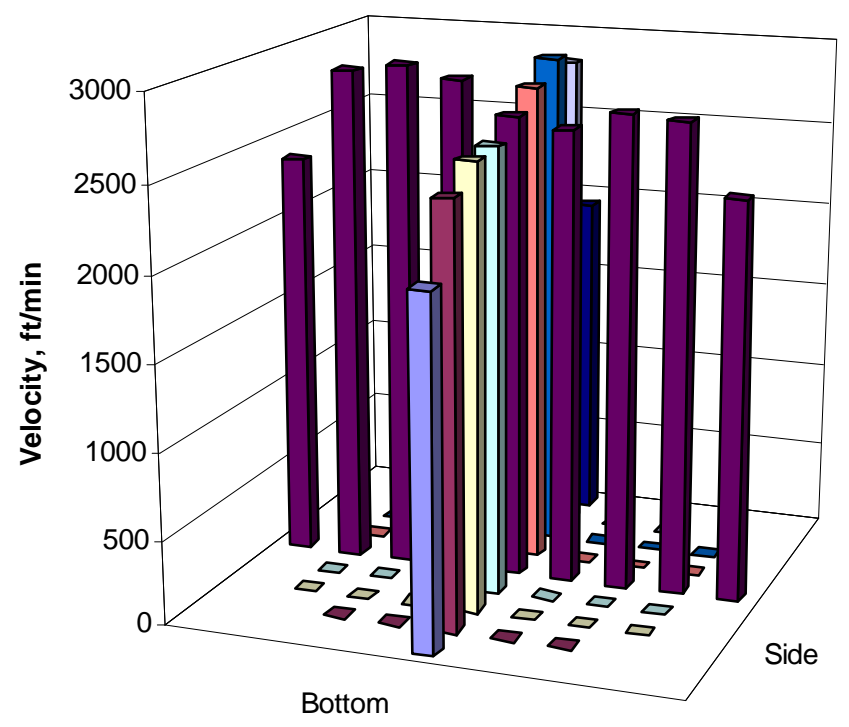

Figure 6.2. Scale-Model Velocity Profile with Fans A \& B Operating (Run VT-1) 


\subsubsection{Gas-Tracer Uniformity Test}

Twenty-one gas-tracer uniformity tests were performed on the scale model with no dampers installed. The gas-tracer injection ports are shown in Figure 3.1 and Figure 3.2. For a given test, the injection probe is positioned to inject the gas in one location within $25 \%$ of a hydraulic diameter from each of the corners of the rectangular cross section or at the center.

Appendix E provides the detailed data and results for each run, and the results are summarized in Table 6.5. The $\%$ COV was calculated for the measured gas concentration at the points in the center two-thirds area of the stack. The percent deviation from the mean concentration was also calculated for any point in the measurement grid. Figure 6.3 shows a sample plot of results from a gas-tracer test.

Table 6.5. Summarized Results of Gas-Tracer Uniformity Tests Without Dampers

\begin{tabular}{|c|c|c|c|c|c|c|}
\hline \hline \multicolumn{1}{|c|}{ Injection Port } & Operating Fans & Port & Run No. & Center ${ }^{2} \mathbf{3}$ \% COV & \% Deviation from Mean \\
\hline B & Center & A \& B & 1 & GT-21 & 24.7 & 43.3 \\
\hline A & Center & A \& B & 1 & GT-1 & 33.7 & 65.6 \\
\hline A & Center & A \& B & 1 & GT-8 & 9.3 & 27.8 \\
\hline A & Center & A \& B & 1 & GT-12 & 35.2 & 62.5 \\
\hline A & Far Left & A \& B & 1 & GT-5 & 29.8 & 60.8 \\
\hline A & Far Left & A \& B & 1 & GT-7 & 32.1 & 65.4 \\
\hline A & Far Right & A \& B & 1 & GT-4 & 37.5 & 60.7 \\
\hline A & Far Right & A \& B & 1 & GT-6 & 34.4 & 59.5 \\
\hline A & Far Right & A \& B & 1 & GT-10 & 37.6 & 59.6 \\
\hline A & Far Right & A \& B & 1 & GT-11 & 35.1 & 60.3 \\
\hline A & Far Right & A \& B & 2 & GT-19 & 14.6 & 34.8 \\
\hline A & Far Right & A \& B & 3 & GT-20 & 10.5 & 84.1 \\
\hline A & Near Left & A \& B & 1 & GT-2 & 30.3 & 78.0 \\
\hline A & Near Left & A \& B & 1 & GT-9 & 36.8 & 59.5 \\
\hline A & Near Right & A \& B & 1 & GT-3 & 33.8 & 6.4 \\
\hline A & Far Left & A & 1 & GT-16 & 9.1 & 11.0 \\
\hline A & Far Right & A & 1 & GT-17 & 2.9 & 5.6 \\
\hline A & Near Left & A & 1 & GT-15 & 5.8 & 4.3 \\
\hline A & Near Right & A & 1 & GT-14 & 2.6 & 6.0 \\
\hline A & Center & A & 1 & GT-13 & 2.9 & \\
\hline B & Center & B & 1 & GT-18 & 2.9 & \\
\hline \hline
\end{tabular}

With two streams coming together in this configuration, and with only one of the streams carrying the tracer, they did not mix together well until Test Port 3 was reached. With the flow only coming from one fan, the mixing of the tracer with the stream had occurred by the time Test Port 1 was reached. 


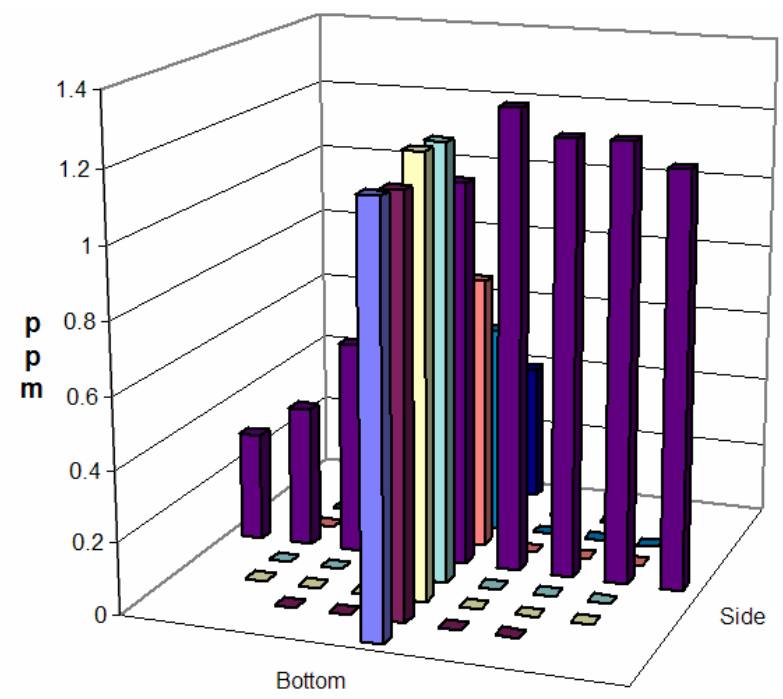

Figure 6.3. Plot of Gas-Tracer Results for Injection in Center at Port A with both Fans Operating (Run GT-1)

\subsubsection{Particle-Tracer Uniformity Test Without Dampers}

Nine runs of the particle-tracer uniformity test were performed before the dampers were installed. The tracer aerosol was injected along the duct centerlines downstream of the fans with the various combinations of operating fans. Table 6.6 summarizes the results for each test run. The $\%$ COV was calculated for the 9- to $11-\mu \mathrm{m}$-diameter particle concentration at the measurement points in the center two-thirds area of the stack.

During some runs, the output of the aerosol generator varied with time and could affect the test results. The concentration readings were normalized to equalize the centerpoint values and to remove that effect. Table 6.6 shows the COV values both with and without normalization. Cases where the time variation occurred are apparent when the COV values are compared. Where the temporal variation did not occur, both the normalized and un-normalized COVs were consistent.

With both fans operating, the particle tracer mixing was not satisfactory at Test Port 1, but was satisfactory at the other test ports. With only one fan operating, mixing was adequate even at Test Port 1.

Figure 6.4 shows the bar-chart particle concentration measurements for Run PT-1. The poor mixing is illustrated in the strong concentration gradient. 
Table 6.6. Particle-Tracer Uniformity Tests Without Dampers

\begin{tabular}{||c|c|c|c|c|c|}
\hline $\begin{array}{c}\text { Tracer } \\
\text { Injection Port }\end{array}$ & Operating Fans & Test Port & Run No. & $\begin{array}{c}\text { Normalized } \\
\text { \% COV }\end{array}$ & $\begin{array}{c}\text { Un-normalized } \\
\text { \% COV }\end{array}$ \\
\hline A & A \& B & 1 & PT-1 & 32.20 & 42.03 \\
\hline A & A \& B & 1 & PT-2 & 35.50 & 51.56 \\
\hline A & A \& B & 1 & PT-3 & 31.61 & 42.64 \\
\hline A & A \& B & 1 & PT-4 & 35.21 & 55.07 \\
\hline A & A \& B & 1 & PT-7 & 33.44 & 33.48 \\
\hline A & A \& B & 2 & PT-8 & 18.15 & 18.34 \\
\hline A & A \& B & 3 & PT-9 & 14.35 & 17.55 \\
\hline A & A & 1 & PT-5 & 9.81 & 27.05 \\
\hline B & B & 1 & PT-6 & 4.30 & 13.30 \\
\hline
\end{tabular}

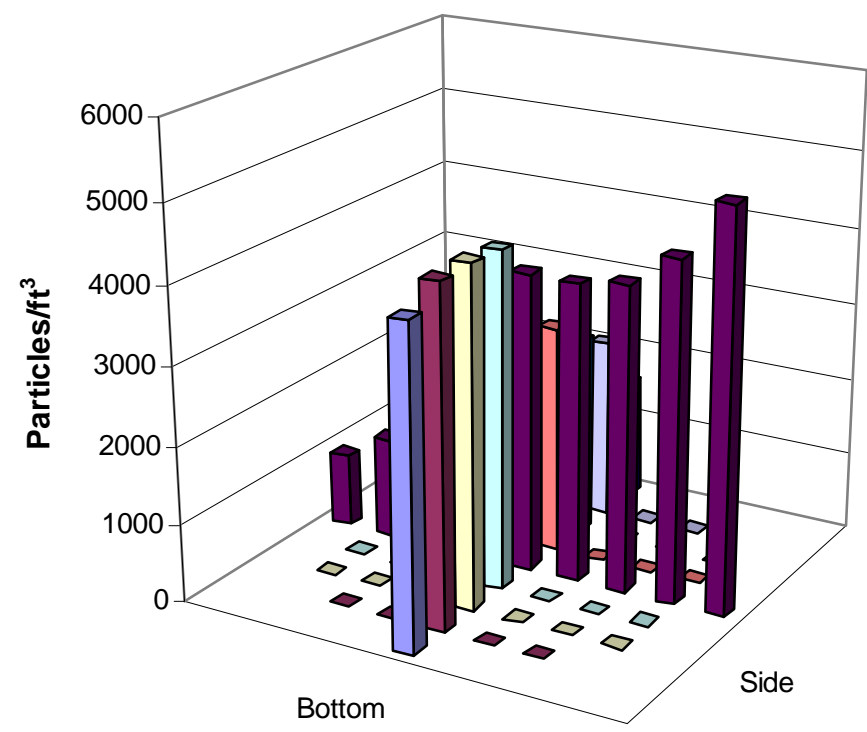

Figure 6.4. Plot of Particle-Tracer Concentration for Run PT-1

\subsection{Tests with Dampers Installed}

\subsubsection{Flow-Angle Test}

Table 6.7 lists the results for the flow-angle tests performed with the dampers installed. Except for Run FA-9, the contoured filler plug was used in all of these test runs. Figure 6.5 shows a chart of typical small-angle results from the scale model. Appendix $\mathrm{C}$ provides the data sheets. 
Table 6.7. Flow-Angle Results on Scale Model with Dampers Installed

\begin{tabular}{|c|c|c|c|c|c||}
\hline Run & Fans & Test Port & $\begin{array}{c}\text { Control Damper } \\
\text { Angle }\end{array}$ & $\begin{array}{c}\text { Backdraft Damper } \\
\text { Angle }\end{array}$ & $\begin{array}{c}\text { Average Absolute Angle } \\
\text { (degrees) }\end{array}$ \\
\hline FA-9 & A & 1 & 45 & 45 & 4.13 \\
\hline FA-10 & A & 1 & 90 & 70 & 3.44 \\
\hline FA-12 & A & 1 & 90 & 70 & 4.30 \\
\hline FA-13 & B & 1 & 90 & 70 & 6.15 \\
\hline FA-11 & A \& B & 1 & 90 & 70 & 4.31 \\
\hline FA-15 & A & 2 & 90 & 70 & 2.56 \\
\hline FA-16 & B & 2 & 90 & 70 & 5.04 \\
\hline FA-14 & A \& B & 2 & 90 & 70 & 3.98 \\
\hline FA-18 & A & 3 & 90 & 70 & 3.69 \\
\hline FA-17 & B & 3 & 90 & 70 & 4.54 \\
\hline FA-19 & A \& B & 3 & 90 & 70 & 4.78 \\
\hline
\end{tabular}
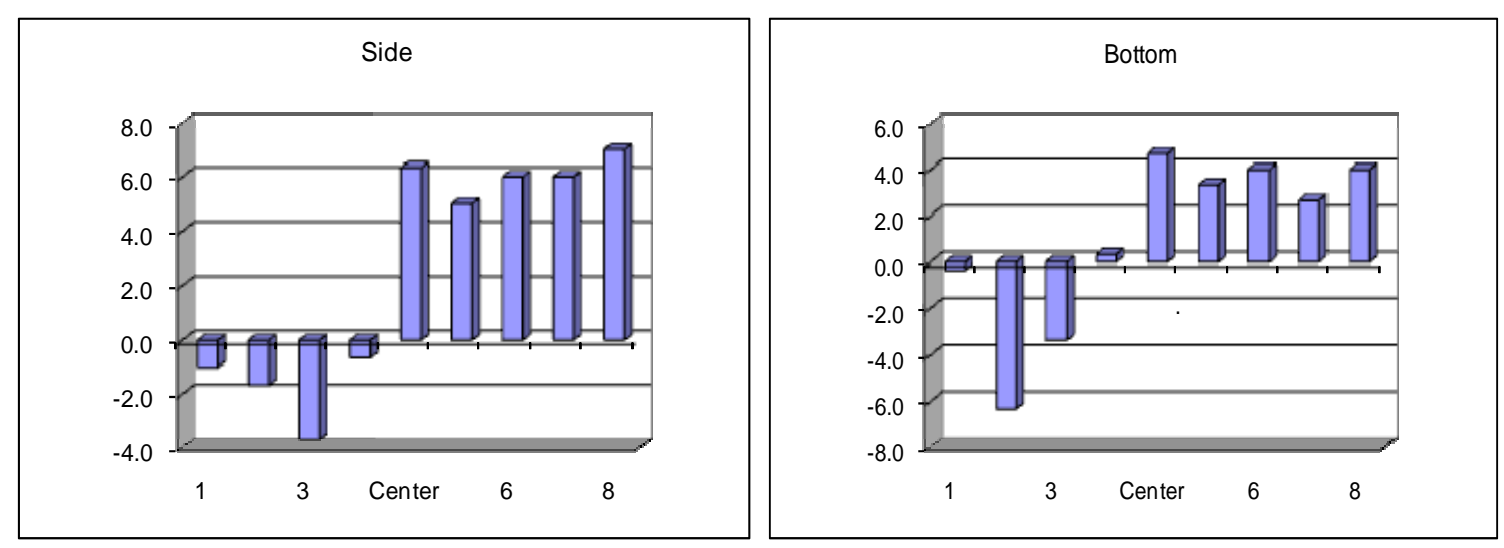

Figure 6.5. Plot of Flow-Angle Results, which are shown in degrees, with both Fans Operating (Run FA-14)

\subsubsection{Velocity Uniformity Test}

Fourteen velocity uniformity test runs were performed on the scale model with the dampers installed. Appendix D provides the detailed results for each test. Table 6.8 summarizes the results for the various fan combinations. The velocity uniformity criterion was met in all cases. 
Table 6.8. Velocity Uniformity Tests on Scale Model at Air-Sampling-Probe Location

\begin{tabular}{|c|c|c|c|c|c|c||}
\hline $\begin{array}{c}\text { Test } \\
\text { Port }\end{array}$ & $\begin{array}{c}\text { Operating } \\
\text { Fans }\end{array}$ & Run No. & $\begin{array}{c}\text { Control Damper } \\
\text { Setting (degrees) }\end{array}$ & $\begin{array}{c}\text { Back Flow Damper } \\
\text { Setting (degrees) }\end{array}$ & $\begin{array}{c}\text { Flowrate } \\
\text { cfm }\end{array}$ & \% COV \\
\hline 1 & A & VT-11 & 45 & 45 & 907 & 12.8 \\
\hline 1 & A \& B & VT-12 & 90 & 70 & 2128 & 7.8 \\
\hline 2 & A \& B & VT-13 & 90 & 70 & 2094 & 6.1 \\
\hline 3 & A \& B & VT-14 & 90 & 70 & 2117 & 4.4 \\
\hline 1 & A & VT-15 & 90 & 70 & 974 & 3.7 \\
\hline 2 & A & VT-16 & 90 & 70 & 973 & 3.6 \\
\hline 3 & A & VT-17 & 90 & 70 & 1002 & 3.4 \\
\hline 3 & B & VT-18 & 90 & 70 & 959 & 6.0 \\
\hline 2 & B & VT-19 & 90 & 70 & 977 & 6.0 \\
\hline 1 & B & VT-20 & 90 & 70 & 959 & 6.2 \\
\hline 3 & A \& B & VT-21 & 90 & 70 & 2136 & 5.1 \\
\hline 3 & A \& B & VT-22 & 90 & 70 & 2180 & 4.5 \\
\hline 2 & A \& B & VT-23 & 90 & 70 & 2132 & 5.1 \\
\hline 2 & A \& B & VT-24 & 90 & 70 & 2126 & 4.4 \\
\hline
\end{tabular}

With the dampers partly closed, the velocity uniformity result was definitely "less uniform" than the others, but within the criterion anyway. Figure 6.6 shows the plot of the velocity profile with the largest $\%$ COV (VT-11) at Test Port 1, with Fan A operating and the dampers set at 45 degrees.

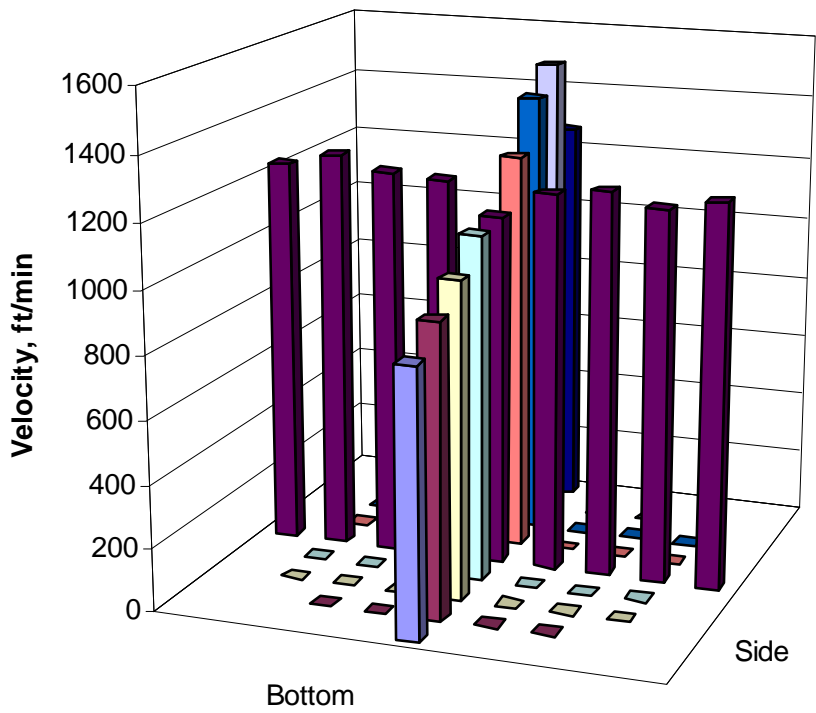

Figure 6.6. Scale-Model Velocity Profile with Fan A Operating and Dampers Set at 45 Degrees (Run VT-11) 


\subsubsection{Gas-Tracer Uniformity Test}

Thirty-three gas-tracer uniformity tests were performed on the scale model with the dampers installed. Table 6.9 summarizes these results for each run. The $\% \mathrm{COV}$ was calculated for the measured gas concentration at the points in the center two-thirds area of the stack. The percent deviation from the mean concentration was also calculated for any point in the measurement grid.

Table 6.9. Summarized Results of Gas-Tracer Uniformity Tests with Dampers

\begin{tabular}{|c|c|c|c|c|c|c|c|c|}
\hline \multicolumn{2}{|c|}{ Injection Port } & $\begin{array}{c}\text { Operating } \\
\text { Fans }\end{array}$ & $\begin{array}{l}\text { Test } \\
\text { Port }\end{array}$ & Run No. & $\begin{array}{c}\text { Control } \\
\text { Damper } \\
\text { Setting } \\
\text { (degrees) }\end{array}$ & $\begin{array}{c}\text { Back Flow } \\
\text { Damper } \\
\text { Setting } \\
\text { (degrees) }\end{array}$ & $\begin{array}{c}\text { Center } \\
2 / 3 \% \\
\text { COV }\end{array}$ & $\begin{array}{c}\% \\
\text { Deviation } \\
\text { from Mean }\end{array}$ \\
\hline $\bar{A}$ & Center & $\mathrm{A}$ & 1 & GT-40 & 45.0 & 70.0 & 1.4 & 3.5 \\
\hline $\mathrm{B}$ & Center & $A \& B$ & 1 & GT-50 & 45.0 & 45.0 & 2.7 & 5.1 \\
\hline B & Center & A \& B & 2 & GT-49 & 45.0 & 45.0 & 1.7 & 4.4 \\
\hline $\mathrm{B}$ & Center & $A \& B$ & 3 & GT-48 & 45.0 & 45.0 & 1.3 & 2.6 \\
\hline $\mathrm{A}$ & Far Left & $\mathrm{A}$ & 1 & GT-42 & 45.0 & 45.0 & 3.2 & 5.2 \\
\hline $\mathrm{A}$ & Far Right & $\mathrm{A}$ & 1 & GT-41 & 45.0 & 45.0 & 7.6 & 10.9 \\
\hline A & Near Left & $\mathrm{A}$ & 1 & GT-43 & 45.0 & 45.0 & 2.3 & 6.0 \\
\hline $\bar{A}$ & Near Right & $\mathrm{A}$ & 1 & GT-44 & 45.0 & 45.0 & 6.3 & 11.1 \\
\hline $\mathrm{A}$ & Center & $\mathrm{A}$ & 1 & GT-39 & 90.0 & 70.0 & 3.6 & 13.0 \\
\hline $\mathrm{A}$ & Center & $\mathrm{A}$ & 2 & GT-38 & 90.0 & 70.0 & 1.3 & 2.6 \\
\hline $\mathrm{A}$ & Center & $\mathrm{A}$ & 3 & GT-37 & 90.0 & 70.0 & 2.3 & 5.3 \\
\hline A & Center & $A \& B$ & 1 & GT-26 & 90.0 & 70.0 & 10.6 & 18.7 \\
\hline A & Center & $A \& B$ & 2 & GT-27 & 90.0 & 70.0 & 7.2 & 13.8 \\
\hline $\mathrm{A}$ & Center & $A \& B$ & 3 & GT-34 & 90.0 & 70.0 & 3.2 & 7.9 \\
\hline $\mathrm{B}$ & Center & $\mathrm{B}$ & 1 & GT-45 & 90.0 & 70.0 & 2.5 & 6.9 \\
\hline $\mathrm{B}$ & Center & $\mathrm{B}$ & 2 & GT-46 & 90.0 & 70.0 & 1.1 & 1.9 \\
\hline $\mathrm{B}$ & Center & $\mathrm{B}$ & 3 & GT-47 & 90.0 & 70.0 & 1.7 & 2.9 \\
\hline $\mathrm{B}$ & Center & $A \& B$ & 1 & GT-53 & 90.0 & 70.0 & 14.7 & 34.7 \\
\hline $\mathrm{B}$ & Center & $A \& B$ & 2 & GT-52 & 90.0 & 70.0 & 6.3 & 12.3 \\
\hline B & Center & $A \& B$ & 3 & GT-54 & 90.0 & 70.0 & 3.9 & 9.1 \\
\hline $\mathrm{A}$ & Far Left & $A \& B$ & 1 & GT-24 & 90.0 & 70.0 & 9.7 & 19.4 \\
\hline $\mathrm{A}$ & Far Left & $A \& B$ & 2 & GT-28 & 90.0 & 70.0 & 5.2 & 9.8 \\
\hline $\mathrm{A}$ & Far Left & $A \& B$ & 2 & GT-31 & 90.0 & 70.0 & 4.5 & 13.1 \\
\hline $\mathrm{A}$ & Far Left & $A \& B$ & 3 & GT-32 & 90.0 & 70.0 & 3.2 & 6.6 \\
\hline $\bar{A}$ & Far Right & $A \& B$ & 1 & GT-25 & 90.0 & 70.0 & 22.9 & 57.4 \\
\hline $\mathrm{A}$ & Far Right & $A \& B$ & 2 & GT-29 & 90.0 & 70.0 & 10.0 & 28.3 \\
\hline A & Far Right & $A \& B$ & 3 & GT-33 & 90.0 & 70.0 & 2.8 & 5.8 \\
\hline $\mathrm{A}$ & Near Left & $A \& B$ & 1 & GT-23 & 90.0 & 70.0 & 5.5 & 12.7 \\
\hline $\mathrm{A}$ & Near Left & $A \& B$ & 2 & GT-51 & 90.0 & 70.0 & 2.0 & 4.5 \\
\hline $\bar{A}$ & Near Left & $A \& B$ & 3 & GT-36 & 90.0 & 70.0 & 2.9 & 5.5 \\
\hline $\mathrm{A}$ & Near Right & $A \& B$ & 1 & GT-22 & 90.0 & 70.0 & 16.3 & 37.0 \\
\hline $\mathrm{A}$ & Near Right & A \& B & 2 & GT-30 & 90.0 & 70.0 & 5.7 & 9.6 \\
\hline $\bar{A}$ & Near Right & $A \& B$ & 3 & GT-35 & 90.0 & 70.0 & 3.5 & 7.9 \\
\hline
\end{tabular}


The dampers were partly closed for the first eight runs listed in Table 6.9. Both gas-mixing acceptance criteria were met for all test ports and for the fan configurations that were tested with the dampers partly closed to their $45^{\circ}$ positions.

The remaining 25 runs are listed in order of the injection position, fan configuration, and test port. Both acceptance criteria were met in all but three instances (GT-22, 25, and 53), and those were where Test Port 1 was used.

Figure 6.7 shows a sample plot of results from a gas-tracer test.

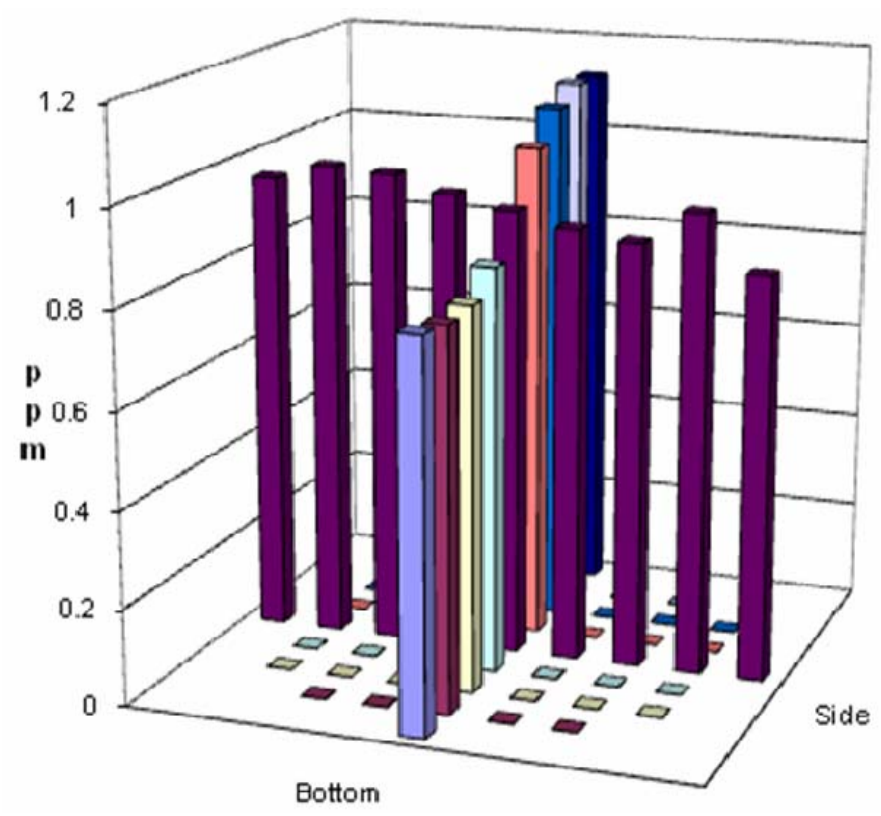

Figure 6.7. Plot of Gas-Tracer Results for Injection in Center at Port A with Fans A \& B Operating (Run GT-26)

\subsubsection{Particle-Tracer Uniformity Tests With Dampers}

Thirteen particle-tracer uniformity tests were performed with the dampers installed. The tracer aerosol was injected along the duct centerlines downstream of the fans with the various combinations of operating fans. Table 6.10 summarizes the results for each run. The $\%$ COV was calculated for the $9-$ to $11-\mu \mathrm{m}-$ diameter particle concentration at the measurement points in the center two-thirds area of the stack. Both the normalized and un-normalized \% COV results are shown.

With both fans operating, the dampers fully open, and with normalizing the measurements for time, satisfactory mixing occurred at Test Ports 2 and 3. With only one operating fan, the mixing was satisfactory for all three test ports.

Figure 6.8 shows the bar chart of normalized particle-concentration measurements for Run PT-22. The acceptance criterion was met in that the COV of the measured tracer particles was $13.5 \%$ across the center two-thirds of the sampling plane. 
Table 6.10. Particle-Tracer Uniformity Tests with Dampers

\begin{tabular}{||c|c|c|c|c|c|c|c||}
\hline $\begin{array}{c}\text { Injection } \\
\text { Port }\end{array}$ & $\begin{array}{c}\text { Operating } \\
\text { Fans }\end{array}$ & Test Port & Run No. & $\begin{array}{c}\text { Control Damper } \\
\text { Setting (degrees) }\end{array}$ & $\begin{array}{c}\text { Back Flow } \\
\text { Damper Setting } \\
\text { (degrees) }\end{array}$ & $\begin{array}{c}\text { Un- } \\
\text { Normalized } \\
\text { \% COV }\end{array}$ & $\begin{array}{c}\text { normalized } \\
\text { \% COV }\end{array}$ \\
\hline A & A \& B & 1 & PT-11 & 90 & 70 & 31.03 & 33.36 \\
\hline A & A \& B & 1 & PT-22 & 90 & 70 & 13.48 & 20.22 \\
\hline A & A \& B & 2 & PT-12 & 90 & 70 & 13.75 & 17.60 \\
\hline A & A \& B & 2 & PT-21 & 90 & 70 & 7.41 & 32.36 \\
\hline A & A \& B & 3 & PT-13 & 90 & 70 & 9.72 & 14.78 \\
\hline A & A \& B & 3 & PT-20 & 90 & 70 & 8.12 & 10.88 \\
\hline A & A & 1 & PT-10 & 45 & 45 & 3.47 & 10.71 \\
\hline A & A & 1 & PT-16 & 90 & 70 & 14.44 & 20.33 \\
\hline A & A & 2 & PT-15 & 90 & 70 & 2.46 & 4.79 \\
\hline A & A & 3 & PT-14 & 90 & 70 & 3.73 & 12.27 \\
\hline B & B & 1 & PT-17 & 90 & 70 & 2.03 & 6.82 \\
\hline B & B & 2 & PT-18 & 90 & 70 & 3.02 & 9.00 \\
\hline B & B & 3 & PT-19 & 90 & 70 & 3.61 & 5.42 \\
\hline
\end{tabular}

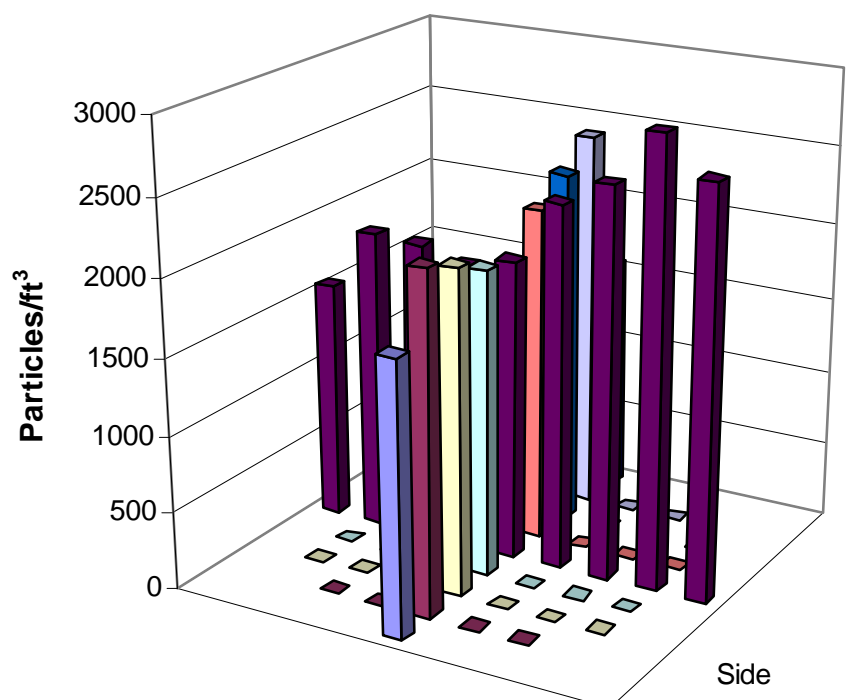

Bottom

Figure 6.8. Plot of Particle-Tracer Concentration for Run PT-22 


\subsection{Discussion of Results}

The following scatter charts reveal trends in the results obtained at the three test ports. They are based on the results in Table 6.1 to Table 6.10 .

Even though fewer tests were performed without the dampers installed, the scatter charts also provide some indication of the effect of the dampers on the results. This may provide some guidance for designing the tests to be performed for the other WTP stacks.

Figure 7.1 provides a scatter plot of the flow-angle tests. In all cases, the angles are small and have about the same magnitude with and without the dampers. The tests with dampers show a slight tendency for the flow angles to be lower at Test Ports 2 and 3 compared to Test Port 1 . The absence of the dampers seemed to have no effect on the flow angles measured at Test Port 1.
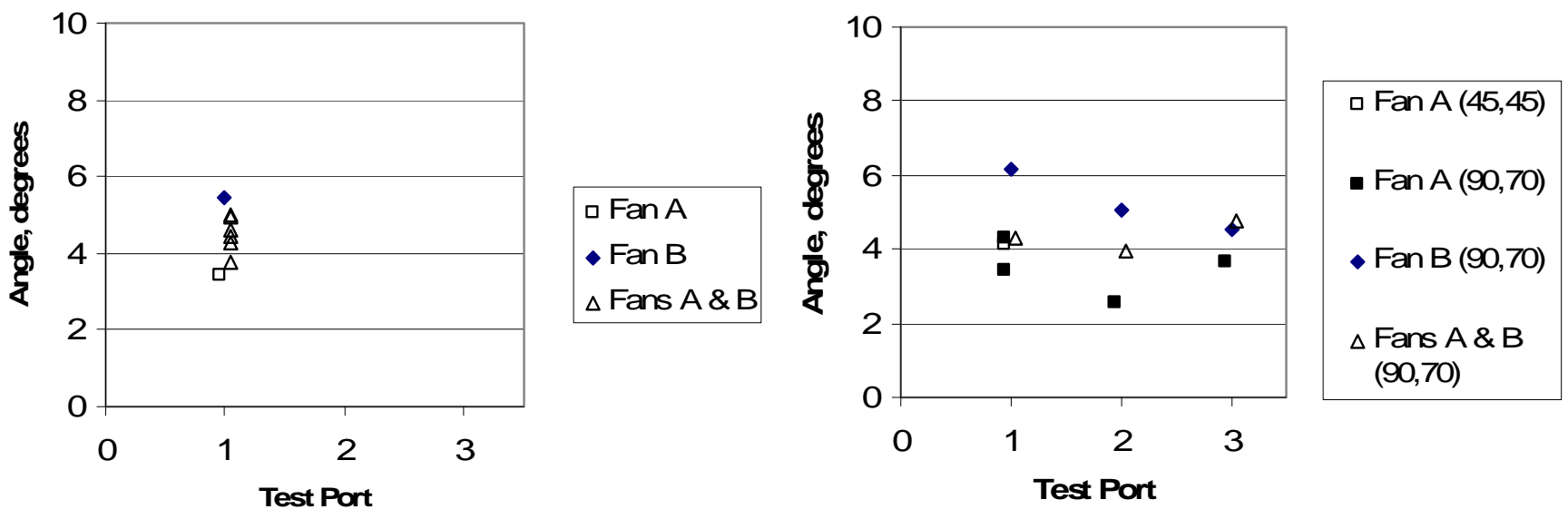

Figure 7.1. Flow-Angle Test Results Comparison (figure on left without dampers and figure on right with dampers)

With the dampers present, the scatter plots of the velocity uniformity results in Figure 7.2 show a slight improvement for test ports downstream of Test Port 1. At Test Port 1, the absence of the dampers seemed to have no effect on the results.

Figure 7.3 shows scatter plots of the gas-mixing \% COV results for each test port with and without the dampers installed. With both fans operating, which is the normal operating mode, note the trend toward greater uniformity with greater distance down the stack in the direction away from the fans. It can also be observed that the uniformity is somewhat more improved with the dampers installed than when they are absent. Similar trends do not appear when a single fan is operating. This is because the airstream with the tracer does not have to mix with another airstream without a tracer. The single 45-degree bend following either fan seems to be sufficient for mixing the tracer. In any case, the $\%$ COV values meet the criterion of $<20 \%$ at Test Ports 2 and 3. 

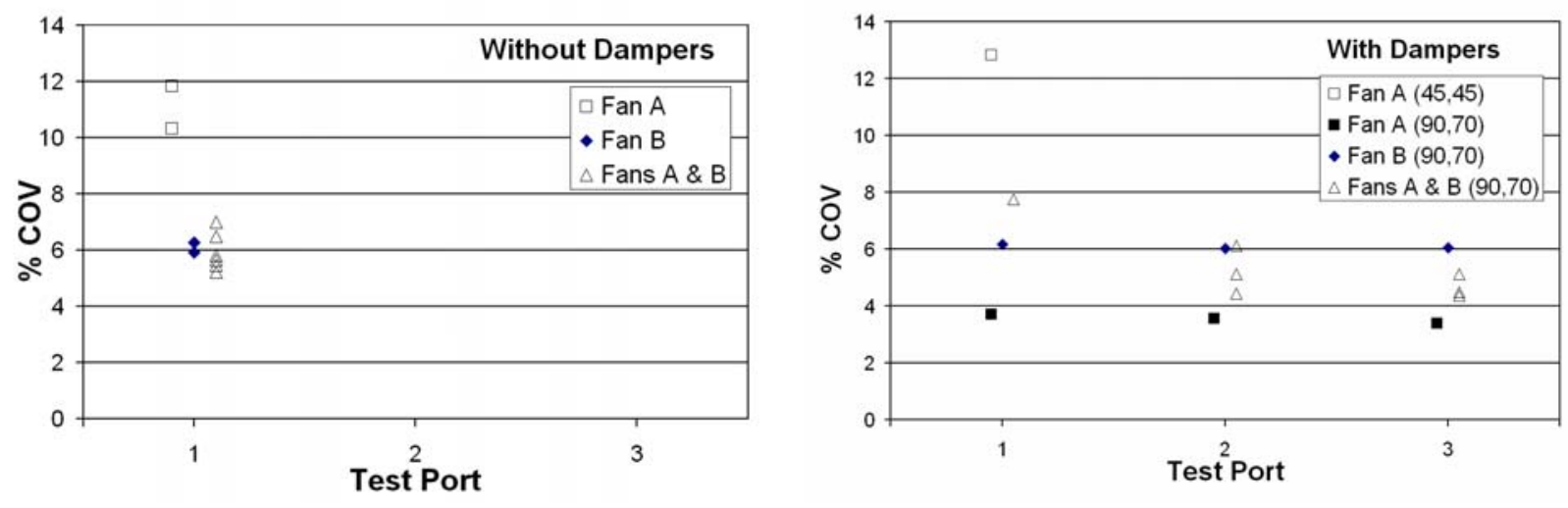

Figure 7.2. Velocity Uniformity Results (figure on left without dampers and figure on right with dampers)
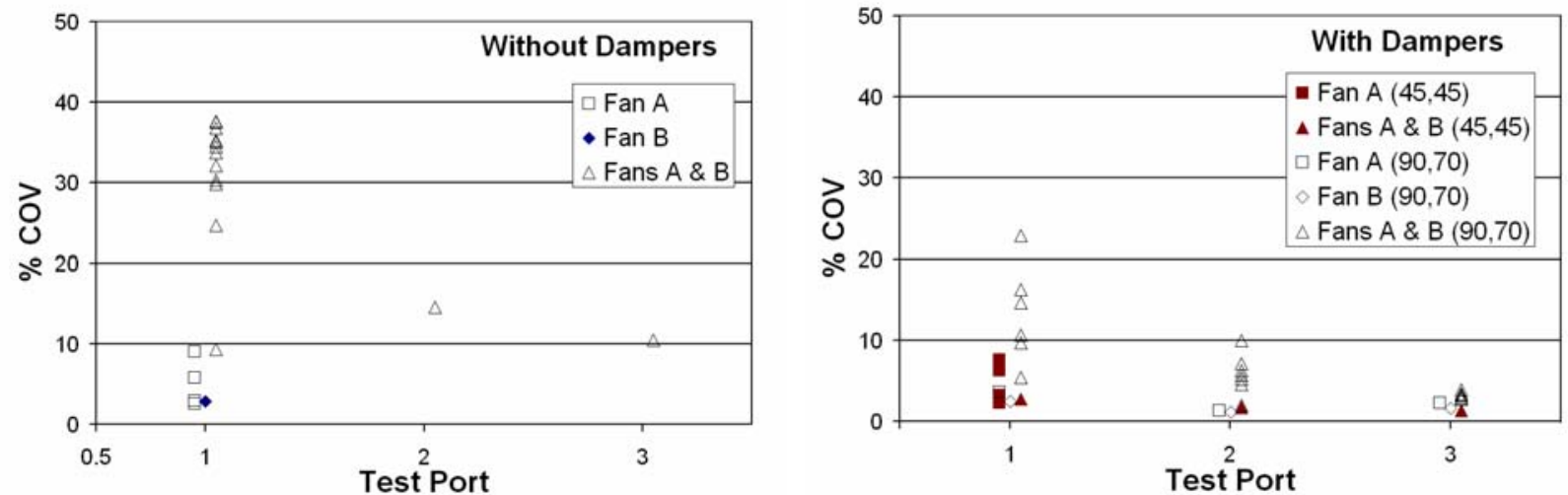

Figure 7.3. Gas Mixing \% COV Scatter Plot with and Without Dampers Installed

Figure 7.4 shows the scatter plots of the percent deviation from the mean of the gas-mixing tests with and without the dampers present. Similar to the \% COV results, and with both fans operating, there is a trend toward greater uniformity (less deviation) with distance down the stack in the direction away from the fans. It can also be observed that the deviation is somewhat more reduced with the dampers installed than when they are absent. With the dampers installed, the percent-deviation criterion is satisfied at both Test Port 2 and 3. Without the dampers, the criterion was only satisfied at Test Port 3. The same trends are not observable with only one fan operating, and for that operating mode, the criterion was met at all three test ports. 

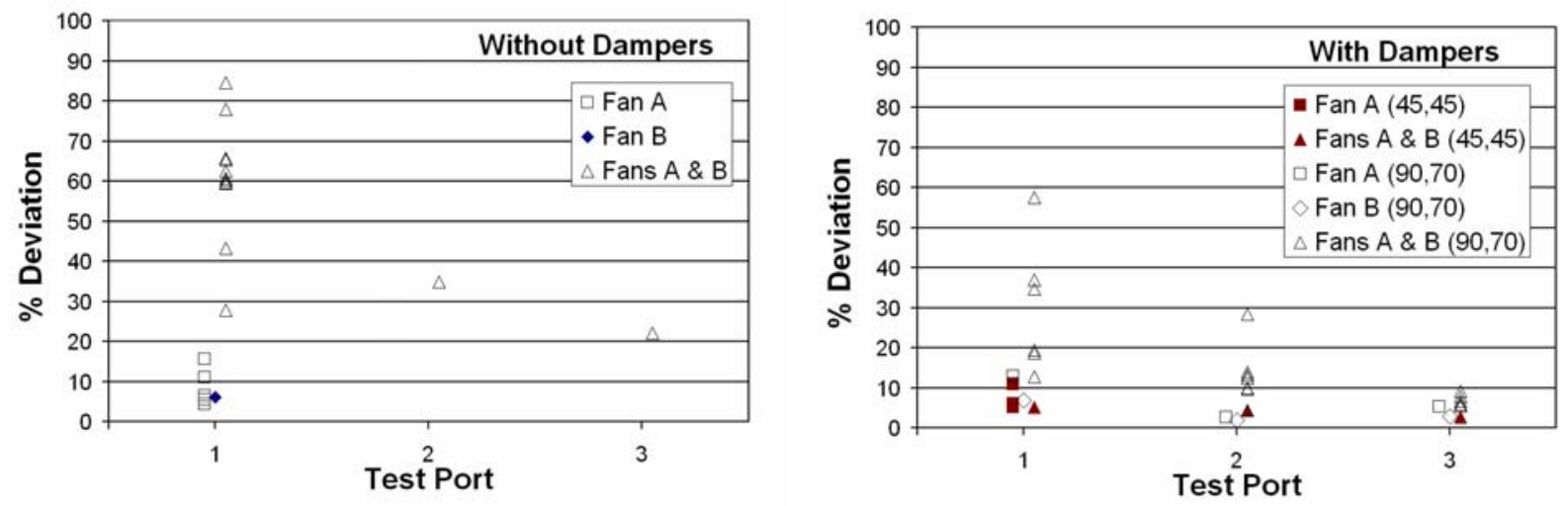

Figure 7.4. Gas Mixing \% Deviation Scatter Plot with and Without Dampers Installed

Figure 7.5 shows the scatter plots of the \% COV results of the particle mixing tests with and without the dampers present. Similar to the \% COV results for the gas-tracer mixing, and with both fans operating, there is a trend toward greater uniformity (lower \%COV) with distance down the stack in the direction away from the fans. It can also be observed that the $\% \mathrm{COV}$ is somewhat more reduced with the dampers installed than when they are absent. The percent-deviation criterion is satisfied at both Test Port 2 and 3. The same trends are not observable with only one fan operating, and for that operating mode, the criterion was met at all three test ports.

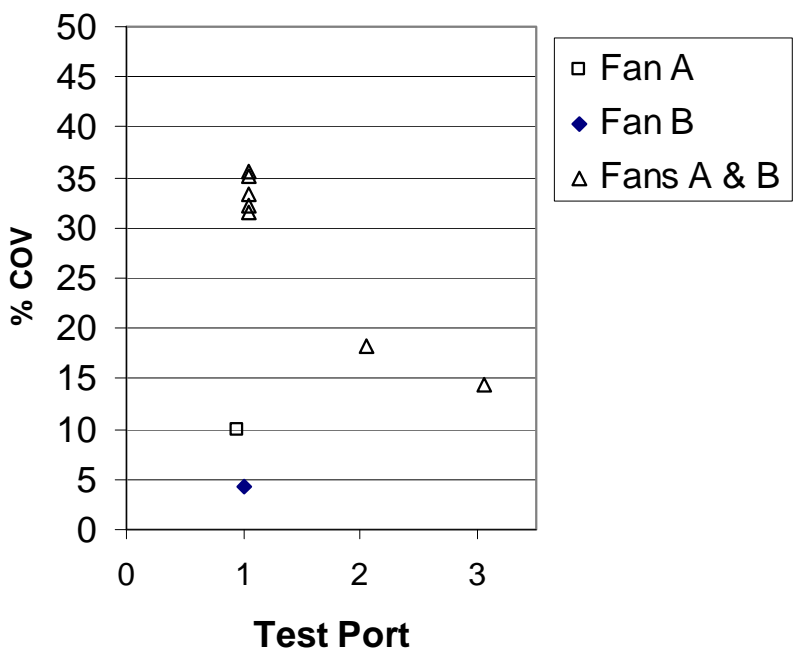

Normalized \% COV with No Dampers

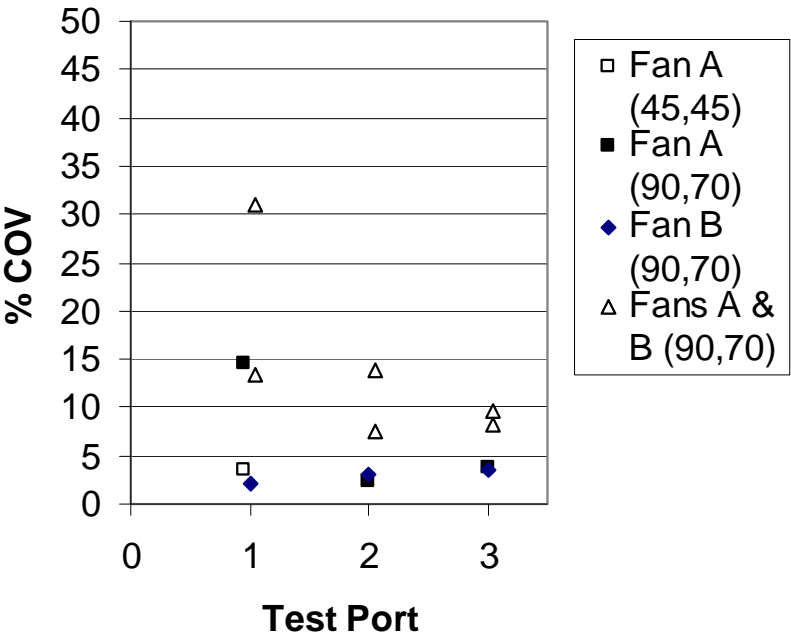

Normalized \% COV with Dampers

Figure 7.5. Particle Mixing \% COV Scatter Plot with and Without Dampers Installed 


\subsection{Conclusions}

Tests were performed to assess the suitability of the location of the air-sampling probe on the HV-C2 stack according to the criteria of ANSI N13.1-1999, Sampling and Monitoring Releases of Airborne Radioactive Substances from the Stacks and Ducts of Nuclear Facilities. A scale model of the stack was used for the tests. For each type of test, the following conclusions were reached.

1. Angular Flow-The purpose is to determine whether the velocity vector is aligned with the sampling nozzle. The average yaw angle relative to the nozzle axis should not be more than $20^{\circ}$. The measured average values ranged from 3.73 to 6.15 degrees, regardless of the test port and presence of the dampers. These same types of flow-angle measurements will have to be made on the constructed stack during cold startup to show that the mean flow angle is $<20^{\circ}$. There is no criterion for agreement with the scale-model flow-angle results.

2. Uniform Air Velocity-The acceptance criterion is that the COV of the air velocity must be $\leq 20 \%$ across the center two-thirds of the area of the stack. The maximum result was $12.8 \% \mathrm{COV}$, indicating that this criterion is met regardless of test port, fan configuration, and damper presence. The values declined with increasing distance from the duct junction. To confirm the validity of these scale-model tests, air-velocity uniformity measurements will have to be made on the constructed stack during cold startup to check for agreement with the velocity-uniformity results presented here for the same relative probe location. The agreement must be within 5\% COV.

3. Uniform Concentration of Tracer Gases-The two acceptance criteria are that 1) the COV of the measured tracer-gas concentration is $\leq 20 \%$ across the center two-thirds of the sampling plane and 2 ) at no point in the sampling plane does the concentration vary from the mean by $>30 \%$. With the dampers installed on the scale model, the results met the criteria at Test Ports 2 and 3. The maximum values observed were $10 \% \mathrm{COV}$ and $28.3 \%$ maximum deviation, both at Test Port 2. At Test Port 3, the maxima were $3.9 \% \mathrm{COV}$ and $9.1 \%$ maximum deviation. Without the dampers, the results were only acceptable at Test Port 3.

5. Uniform Concentration of Tracer Particles-The acceptance criterion is that the COV of particle concentration is $\leq 20 \%$ across the center two-thirds of the sampling plane. The data were normalized to account for drift in the particle generator. With the dampers installed on the scale model, the results met the criterion at Test Ports 2 and 3. The maximum values observed were $13.75 \%$ COV and 9.72\% COV at Test Port 2 and Test Port 3, respectively. Without the dampers, the results were still acceptable at both Test Ports 2 and 3.

6. The potential sampling-probe location represented by Test Port 1 did not satisfy all of the test criteria and would be unsuitable as a sampling probe location during normal operation. If only a single fan is operated at a time, then the Test Port 1 location would be satisfactory; however, that is not currently considered as a normal operating condition. 


\subsection{References}

40 CFR 60, Appendix A, Method 1. "Method 1 - Sample and Velocity Traverses for Stationary Sources." U.S. Environmental Protection Agency, Code of Federal Regulations,

40 CFR 60, Appendix A, Method 2, as amended. U.S. Environmental Protection Agency, Method 2 Determination of Stack Gas Velocity and Volumetric Flow Rate. U.S. Code of Federal Regulations.

American National Standards Institute (ANSI). 1999. Sampling and Monitoring Releases of Airborne Radioactive Substances From the Stacks and Ducts of Nuclear Facilities. ANSI/HPS N13.1 - 1999, American National Standards Institute, New York. 
Appendix A

\section{Dimensional Calculations for Scale Model WTP HV-C2 Stack}




\title{
Appendix A: Dimensional Calculations for Scale Model WTP HV-C2 Stack
}

\author{
John Glissmeyer \\ Revision 0 \\ February 7, 2006
}

\section{PURPOSE}

The purpose of this calculation is to provide key dimensions for a scale model of the WTP HVC2 air exhaust stack. Additional calculations will provide lower limit operating flowrates for the scale model.

\section{REFERENCES}

1. Attachment A -- The configuration of the scale model will mimic that of the attachments to this memorandum (CCN 111186) from BNI (Aaron Childers) to John Glissmeyer (January 19, 2005). The rendered model views and instrumentation diagrams are omitted here.

2. Attachment B -- Email from Dan Cragin (BNI) to John Glissmeyer (Battelle) of November 8, 2005, which refers to CCN 116112 containing the most current duct and flow parameters.

3. Attachment C -- BNI memorandum from Gerald M. Gaulden (BNI) to Jennifer SuCoker (BNI), Exhaust Stack Design Standardization Data, CCN 116112, March 23, 2005. Herein, the operating parameters for the $\mathrm{HV}-\mathrm{C} 2$ stack are enumerated: nominal stack height 71-ft, stack diameter 44-in, nominal airflow 40,000-cfm, discharge velocity 3500fpm, normal exhaust temperature $80^{\circ} \mathrm{F}$, discharge pressure 0.76 -in. $\mathrm{WG}$, and normal relative humidity $10-100 \%$. It was assumed that these parameters superseded those in the earlier CCN 111186.

4. Sampling and Monitoring Releases of Airborne Radioactive Substances From the Stacks and ducts of Nuclear Facilities. ANSI/HPS N13.1-1999. American National Standards Institute and the Health Physics Society, McLean, VA. Clause 5.2.2.2 of this standard provides guidance on the scaling parameters.

5. Attachment $\mathbf{D}$ - General arrangement of fan outlet and dampers as provided by Intermech (March 19, 2005), the ductwork subcontractor to BNI.

6. Attachment E-Catalog cut of Ruskin Control Damper. BNI will use the opposed blade style. Note that the frame is 8-in. deep.

7. Attachment $\mathbf{F}$ - Catalog cut of Ruskin Backdraft Damper. Note that the frame is 8-in. deep.

\section{ASSUMPTIONS}

1. The diameter of the actual stack will be 44 inches according to Reference 3, not 60 inches as shown in Attachment A. For the given flowrate, this gets the discharge air velocity up 
into the range where BNI wishes to standardize, i.e., $>3500 \mathrm{ft} / \mathrm{min}$. With the 60 inch duct, the velocity was only about $2000 \mathrm{ft} / \mathrm{min}$.

2. The dimensions of ducts feeding the stack will be unchanged from Attachment A.

3. The minimum distance from the last duct junction to the sampling probe location is assumed to remain at 9.5 diameters. As the duct gets smaller, this relative distance becomes smaller too. The bulk of the qualification tests will be done at this relative distance. However, it is noted that BNI may keep the distance from the fans to the sampling probe and the $90^{\circ}$ turn up to the vertical stack the same as in Attachment A for practical reasons. The qualification test results tend to improve at greater distances separating the last flow disturbance from the sampling location, so tests at 9.5 diameters will yield conservative results relative to those at longer distances. Some limited tests will be done at greater and lesser distances to verify that this is the case.

4. There are certain constraints on the scale model that must be satisfied. (These come from ANSI/HPS N13.1-1999.) A scale model used for qualifying the proposed sampling location must be geometrically similar (with proportional critical dimensions) to the actual stack. The sampling location of the model must be geometrically similar to the actual stack. The product of the mean air velocity times the hydraulic diameter of the actual stack must be within a factor of six of the scale model. The scale model hydraulic diameter must be at least $250 \mathrm{~mm}$. Finally, the Reynolds number of the scale model and the actual stack must be greater than 10,000 .

5. The size of the scaled stack diameter will be 12 inches.

6. The first item following the fan will be a rectangular cross section expansion from the fan outlet to the dampers, as shown in Attachment D.

7. The control damper will follow the expansion, see Attachment D.

8. The backdraft damper will follow the control damper, see Attachment D.

\section{CALCULATIONS}

1. The scaling factor for linear dimensions is

44 inches $/ 12$ inches $=3.667$

2. The key dimensions of the prototype stack were given in Reference 1 . However, the minimum distance from the duct junction to the sampling probe port was $9.5 \mathrm{D}$ (for diameters). Also, the minimum distance from the sampling probe port to the next flow disturbance was 5D. For the model, these distances become $9.5 \times 12$ and $5 \times 12$ inches, or 114 and 60 inches respectively.

3. Key dimensions are labeled in Attachment A with numeric labels. A spreadsheet (HVC2modelstacflo.xls, worksheet ScaledDims) was created to calculate the scaled down values for these key dimensions. The equation used was

$($ prototype dimension $) /($ scaling factor $)=$ scaled dimension

In this case, the scaling factor is 3.667. The results are included in Table 1.

For example, the spacing from the discharge of Fan $4 \mathrm{~B}$ to the start of the 44 inch diameter stack is given as $17-\mathrm{ft}, 10$-in. That is a total of

$(17-\mathrm{ft} \times 12-\mathrm{in} / \mathrm{ft})+10$-in $=214$ in. 
The scaled distance is

$214-$ in $/ 3.667=58.363$-in.

The scaled results are shown in Table 1 in decimal feet, decimal inches, and fractional inches.

4. The last sheet of Attachment A shows the presence of two dampers, a control damper and an isolation damper. Neither of these was shown in the other diagrams. Information was obtained from the ductwork subcontractor (Intermech) showing the general configuration of the fan discharge and dampers, as shown in Attachment D. Attachments E and F show Ruskin catalog cuts of the model of control and backdraft dampers that will be used.

Note that the depth of the dampers is 8 inches. This scales to a depth of

$8 / 3.667=2.182$ in

as listed in Table 1. Ruskin builds dampers to custom heights and widths. Therefore, it was assumed that the dampers would be the same size as the rectangular cross sections that they will mate with. The prototype and scaled dimensions are listed in Table 1.

5. The dampers will occupy space in the segment labeled as Fan 4B segment 1 in Table 1 and Attachment A. The balance of the space in that segment will be occupied by the rectangular expansion from the stack discharge to the damper cross section. The overall length of the expansion is

$7.636-2(2.182)=3.272$ in. 


\begin{tabular}{|c|c|c|c|c|c|c|c|c|c|c|c|}
\hline & $\mathrm{A}$ & $\mathrm{B}$ & $\mathrm{C}$ & $\mathrm{D}$ & \begin{tabular}{|l|l}
$\mathrm{E}$ & \\
\end{tabular} & $\mathrm{F}$ & $\mathrm{G}$ & $\mathrm{H}$ & $\mathrm{I}$ & $\mathrm{J}$ & \\
\hline 1 & \multicolumn{11}{|c|}{ Table 1 HV-C2 PRELIMINARY MODEL DIMENSIONS } \\
\hline 2 & \multicolumn{4}{|c|}{ 2/7/2006 Rev 0.} & \multicolumn{7}{|c|}{ Scaled } \\
\hline 3 & \multirow[b]{2}{*}{ Section } & \multirow[b]{2}{*}{ Orientation } & \multirow[b]{2}{*}{ Prototype ft } & \multirow[b]{2}{*}{ Prototype in } & \multicolumn{3}{|c|}{ Trial scaling, 1:x } & \multirow{2}{*}{\begin{tabular}{|r|}
\multicolumn{1}{|l|}{ Final ft } \\
3.67 \\
\end{tabular}} & \multirow{2}{*}{\begin{tabular}{|r|} 
Final in. \\
3.67 \\
\end{tabular}} & \multirow{2}{*}{\multicolumn{2}{|c|}{$\begin{array}{r}\text { Final in. } \\
3.67\end{array}$}} \\
\hline 4 & & & & & 1.83 & 2.45 & 3.67 & & & & \\
\hline 5 & \multirow[t]{2}{*}{ Stack 1} & \multirow[t]{2}{*}{$\mathrm{H}$} & \multirow[t]{2}{*}{17.833} & \multirow[t]{2}{*}{214.0} & \multirow[t]{2}{*}{9.727} & \multirow[t]{2}{*}{7.279} & \multirow[t]{2}{*}{4.864} & \multirow[t]{2}{*}{4.864} & \multirow[t]{2}{*}{58.363} & \multirow{2}{*}{\multicolumn{2}{|c|}{$58 \quad 6 / 16$}} \\
\hline 6 & & & & & & & & & & & \\
\hline 7 & \multirow{3}{*}{$\begin{array}{l}\text { Stack } 2 \\
\text { Stack } 3 \\
\text { Stack } 4\end{array}$} & $\mathrm{H}$ & 8.250 & 99.0 & 4.500 & 3.367 & 2.250 & 2.250 & 27.000 & 27 & \\
\hline 8 & & $\mathrm{H}$ min & 34.833 & 418.0 & 19.000 & 14.218 & 9.500 & 9.500 & 114.000 & 114 & \\
\hline 9 & & $\mathrm{H}$ min & 18.333 & 220.0 & 10.000 & 7.483 & 5.000 & 5.000 & 60.000 & 60 & \\
\hline 10 & Stack $2-4$ & $\mathrm{H} \min$ & 61.417 & 737.0 & 33.500 & 25.068 & 16.750 & 16.750 & 200.999 & 201 & \\
\hline 11 & & & & & & & & & & & \\
\hline 12 & Stack dia. & $\mathrm{H}$ & 3.667 & 44.0 & 2.000 & 1.497 & 1.000 & 1.000 & 12.000 & 12 & \\
\hline 13 & Stack 3 to probe tip & $\mathrm{H}$ min & 33.333 & 400.0 & 18.182 & 13.605 & 9.091 & 9.091 & 109.091 & 109 & $1 / 16$ \\
\hline 14 & & & & & & & & & & & \\
\hline 15 & Duct Width & $45 \mathrm{deg}$ & 2.500 & 30.0 & 1.364 & 1.020 & 0.682 & 0.682 & 8.182 & 8 & $3 / 16$ \\
\hline 16 & Duct Height & $45 \mathrm{deg}$ & 3.667 & 44.0 & 2.000 & 1.497 & 1.000 & 1.000 & 12.000 & 12 & \\
\hline 17 & & & & & & & & & & & \\
\hline 18 & Fan 4B-1 & $45 \mathrm{deg}$ & 2.333 & 28.0 & 1.273 & 0.952 & 0.636 & 0.636 & 7.636 & & $10 / 16$ \\
\hline 19 & Fan 4B-2 & $45 \mathrm{deg}$ & 3.797 & 45.6 & 2.071 & 1.550 & 1.036 & 1.036 & 12.427 & 12 & $7 / 16$ \\
\hline 20 & Fan 4B-3 & $45 \mathrm{deg}$ & 5.615 & 67.4 & 3.063 & 2.292 & 1.531 & 1.531 & 18.376 & 18 & $6 / 16$ \\
\hline 21 & Fan 4B-4 & $\mathrm{H}$ & 1.745 & 20.9 & 0.952 & 0.712 & 0.476 & 0.476 & 5.711 & & $11 / 16$ \\
\hline 22 & Fan 4B-5 & $\mathrm{H}$ & 2.797 & 33.6 & 1.526 & 1.142 & 0.763 & 0.763 & 9.154 & 9 & $2 / 16$ \\
\hline 23 & Fan 4B-6 & $\mathrm{H}$ & 2.276 & 27.3 & 1.241 & 0.929 & 0.621 & 0.621 & 7.449 & 7 & $7 / 16$ \\
\hline 24 & Fan 4B-RndDuct & $45 \mathrm{deg}$ & 3.333 & 40.0 & 1.818 & 1.361 & 0.909 & 0.909 & 10.909 & & $15 / 16$ \\
\hline 25 & & & & & & & & & & & \\
\hline 26 & Fan 4A-1 & $45 \mathrm{deg}$ & 12.688 & 152.3 & 6.920 & 5.179 & 3.460 & 3.460 & 41.523 & 41 & $8 / 16$ \\
\hline 27 & & & & & & & & & & & \\
\hline 28 & Control Damper & Width & 2.500 & 30.0 & 1.364 & 1.020 & 0.682 & 0.682 & 8.182 & 8 & $3 / 16$ \\
\hline 29 & & Height & 3.667 & 44.0 & 2.000 & 1.497 & 1.000 & 1.000 & 12.000 & 12 & \\
\hline 30 & & Length & 0.667 & 8.0 & 0.364 & 0.272 & 0.182 & 0.182 & 2.182 & 2 & $3 / 16$ \\
\hline 31 & & & & & & & & & & & \\
\hline 32 & Backdraft Damper & Width & 2.500 & 30.0 & 1.364 & 1.020 & 0.682 & 0.682 & 8.182 & 8 & $3 / 16$ \\
\hline 33 & & Height & 3.667 & 44.0 & 2.000 & 1.497 & 1.000 & 1.000 & 12.000 & 12 & \\
\hline 34 & & Length & 0.667 & 8.0 & 0.364 & 0.272 & 0.182 & 0.182 & 2.182 & 2 & $3 / 16$ \\
\hline 35 & & & & & & & & & & & \\
\hline 36 & Math Checks & Check & 1.000 & 12.0 & 0.545 & 0.408 & 0.273 & 0.273 & 3.273 & 3 & $4 / 16$ \\
\hline 37 & & Check & 5.000 & 60.0 & 2.727 & 2.041 & 1.364 & 1.364 & 16.364 & 16 & $6 / 16$ \\
\hline
\end{tabular}




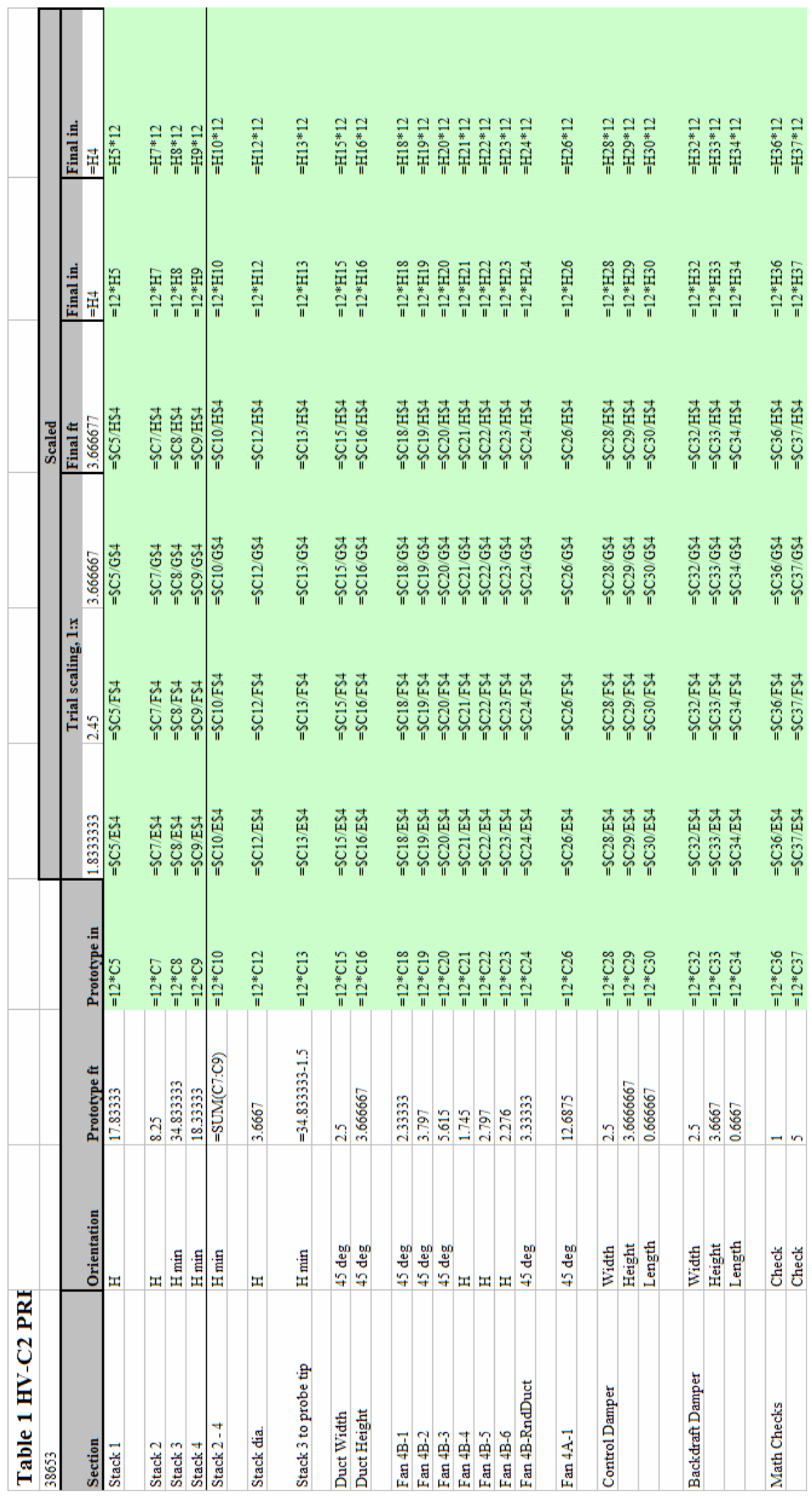




\section{ATTACHMENT A}

MEMORANDUM CCN 111186

January 19, 2005

All drawings are best-available copy in Attachment A. 


\section{Memorandum}

To: John Glissmeyer

From: Aaron Childers

Ext: (509) 371-3352
Date: January 19, 2005

$\mathrm{CCN}: 101967$

Subcontract 24590-101-TSA-W000-00004

\section{Subject: WTP/RPP STACK DISCHARGE SCALE MODEL SUPPORT FOR SUBCONTRACT 24590-TSA-W000-00004, FLOW ELEMENT PROCESS DATASHEETS FOR EACH FLUE EXHAUSTING FROM HLW, LAW, PTF, AND LAB FACILITIES}

John,

Please use the attached documents listed below for simulating the process conditions for each exhaust flue located in the HLW, LAW, PTF, and LAB facilities. In the attached documents, you will find anticipated process data conditions at the location of each inserted flow element for all exhaust flues in the HLW, LAW, PTF, and LAB facilities.

Regards,

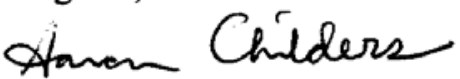

Aaron Childers

Controls and Instrument Engineer

High Level Waste Facility

Waste Treatment Project

Attachments: 1) High Level Waste Facility; Flow Process Data Sheet; SDJ-FE-3101/PD Rev 0; Decemeber 14, 2004

2) High Level Waste Facility; Flow Process Data Sheet; SDJ-FE-3104/PD Rev 0; Decemeber 14, 2004

3) High Level Waste Facility; Flow Process Data Sheet; SDJ-FE-3108/PD Rev 0; Decemeber 14, 2004 


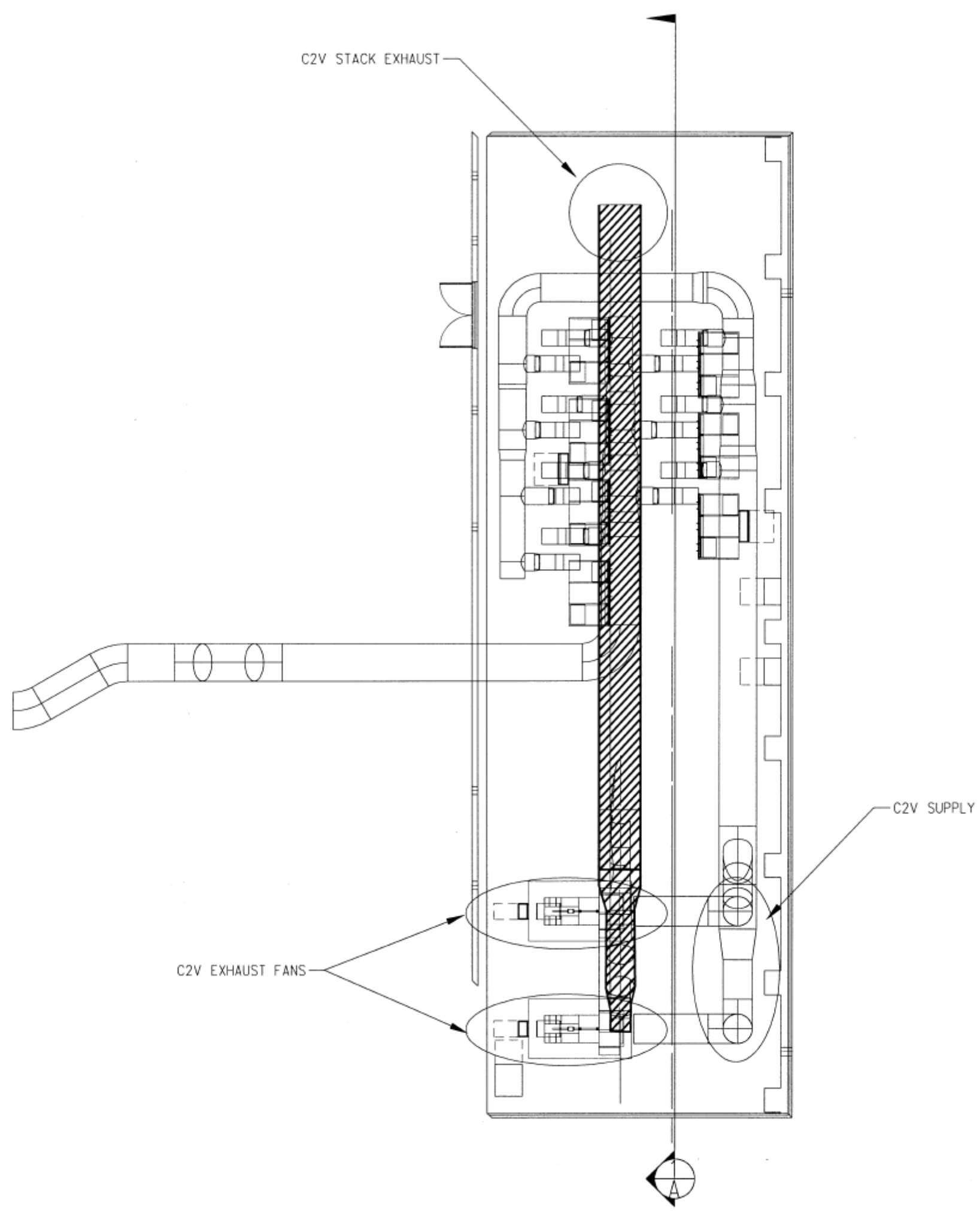




\section{Detail 1A}

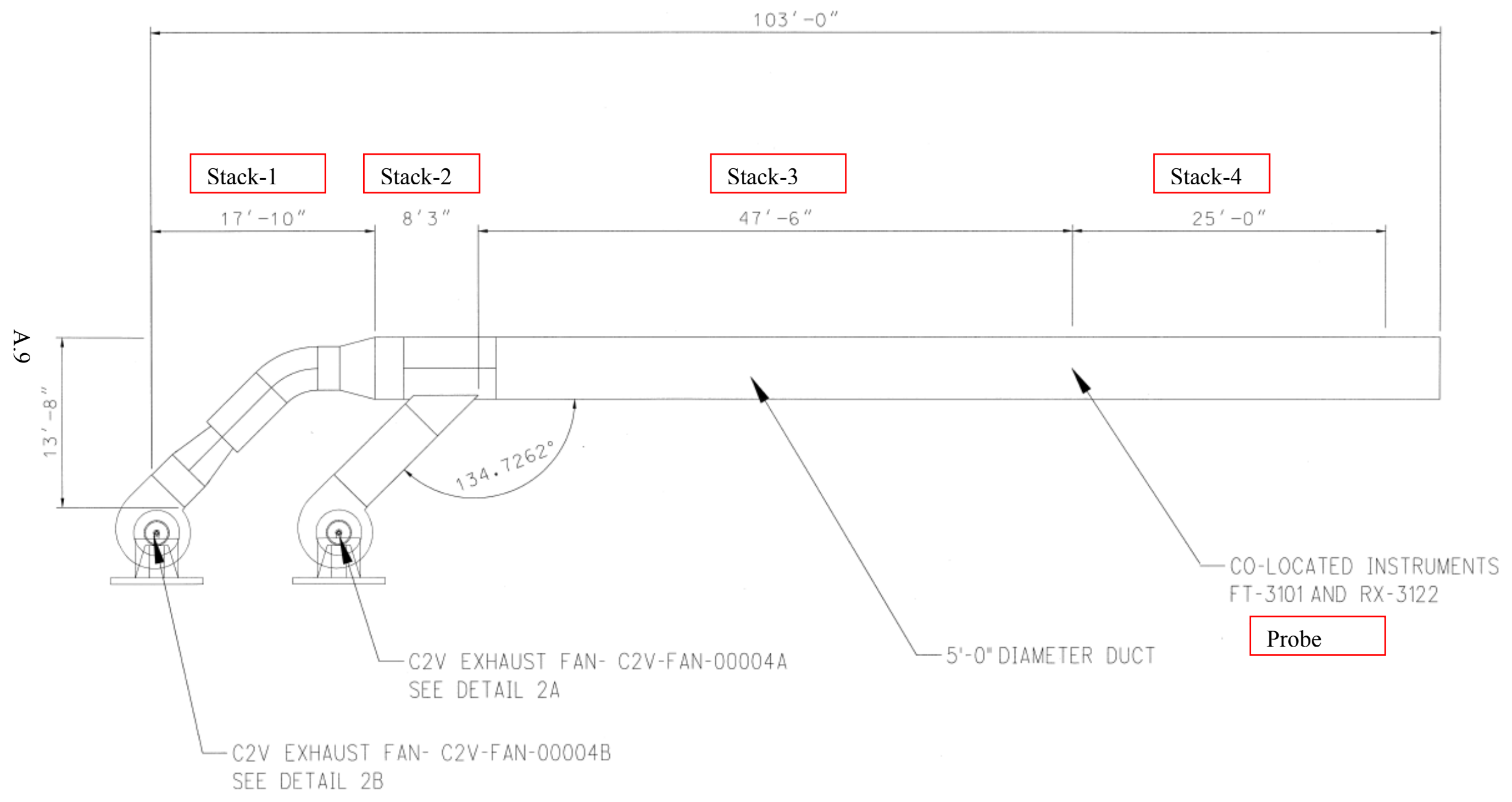




\section{DETAIL 2A: C2V-FAN-00004A}

Fan 4A dimensions

$\frac{p}{0}$

NOTES:

1. Between C2V-FAN-00004A to the main C2V exhaust duct header an on/off isolation damper, back draft damper, and an averaging pitot tube

is located in this duct

sitioning and

tails of the above elements are

unknown at this time.

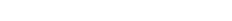




\section{DETAIL 2B: C2V-FAN-00004B}
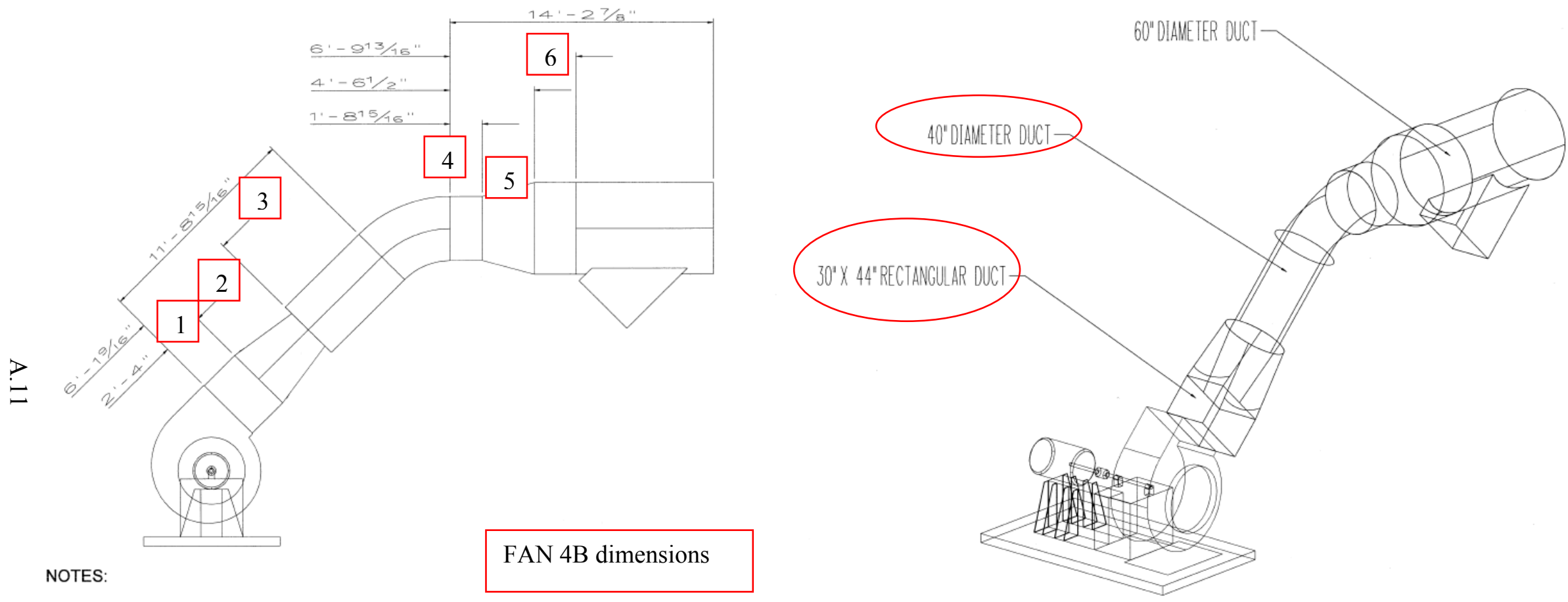

NOTES:

FAN 4B dimensions

1. Between C2V-FAN-00004B to the main C2V exhaust duct header an on/off isolation damper, back draft damper, and an averaging pitot tube resides in this duct run. Positioning and

installation details of the above elements are installation details of
unknown at this time.

2. The HVAC duct coming off of the C2V-FAN$00004 \mathrm{~B}$ is rectangular and transitions to a $40^{\prime \prime}$ round duct. The 40" round duct once again transitions into the 60 " main $\mathrm{C} 2 \mathrm{~V}$ exhaust header. 


\section{C2V STACK EXCERPT FROM V\&ID 24590-HLW-M8-SDJ-00002}

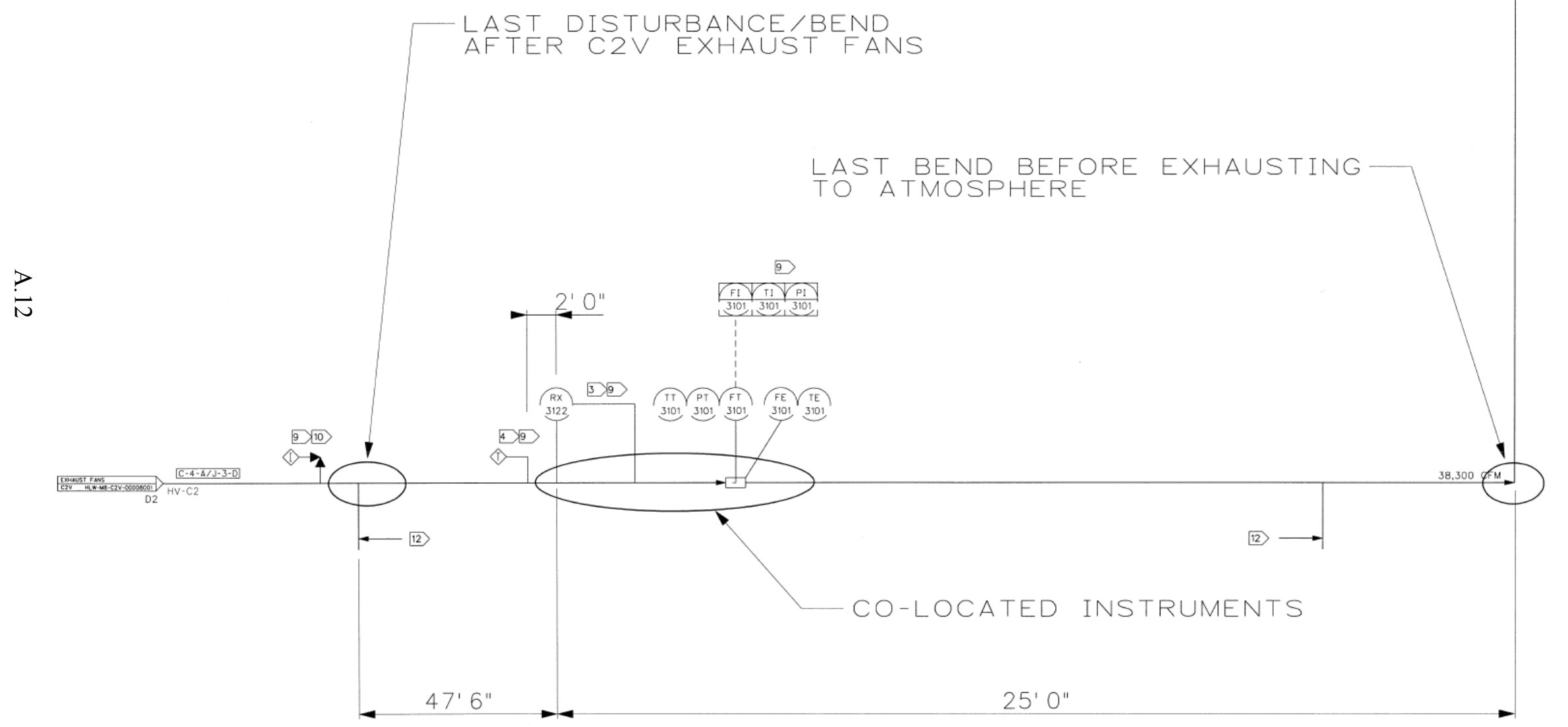



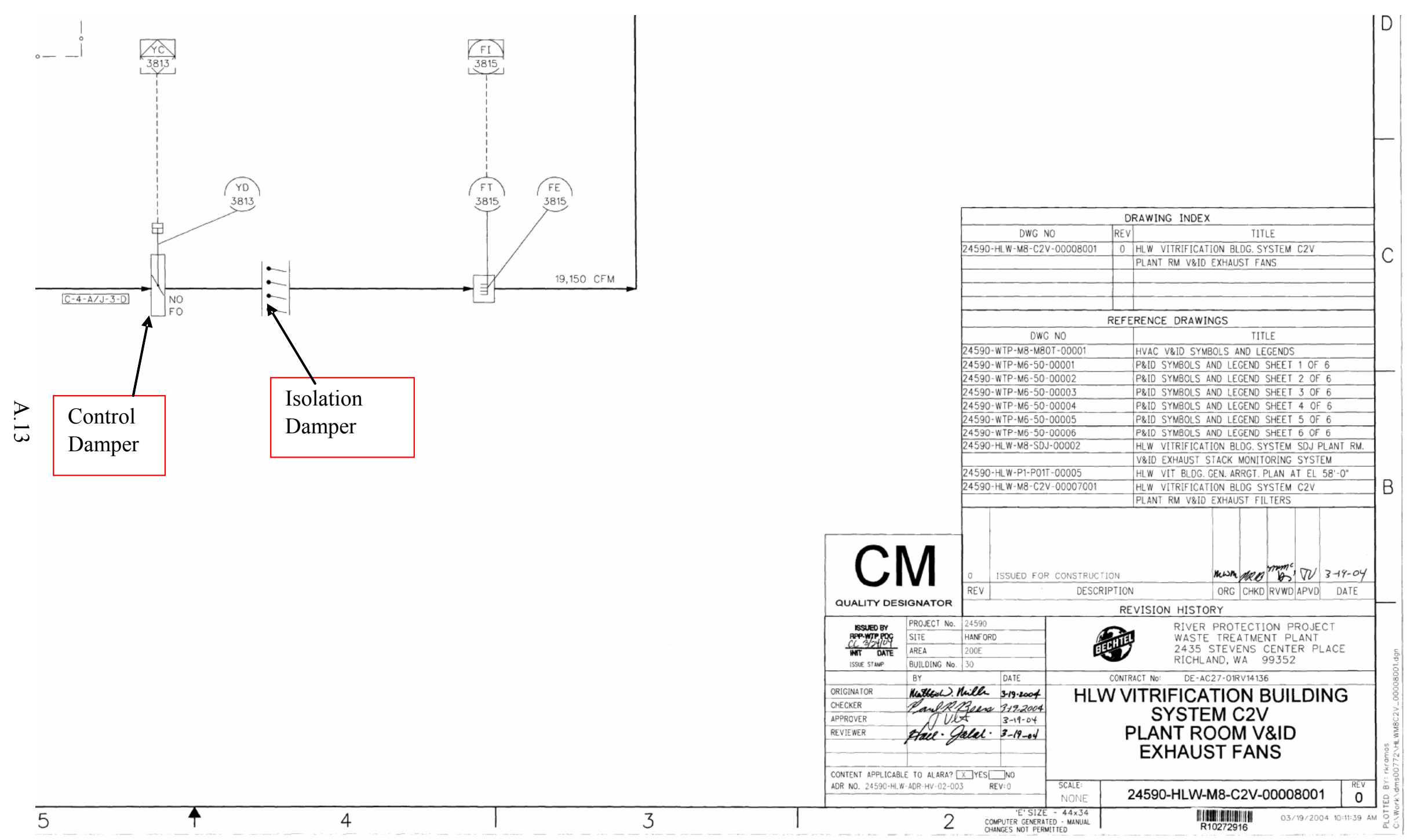


\section{ATTACHMENT B \\ MEMORANDUM CCN 111186 \\ January 19, 2005}

John,

In response to the e-mail below, please use the attached (CCN 116112 ) as a reference for your modelling efforts on the HV-C2 stack. This CCN represents the latest direction on WTP stack designs and target discharge velocities. If you have any questions regarding the use of this CCN please feel free to call either Jerry Gaulden (371-3598) or me.

Dan Cragin P.E.

WTP HVAC

(509) 371-8458

djcragin@bechtel.com < mailto:djcragin@bechtel.com>

-.---original Message-...-

From: Glissmeyer, John A [mailto:ja.glissmeyer@pnl.gov]

Sent: Thursday, November 03, 2005 5:25 PM

To: Weddle, Brian E; Garcia, Gerard

Cc: Icayan, Cesar

Subject: FW: Electronic Files- CCN 111186

Earlier this year, Aaron Childers submitted this package showing the conceptual design for the stack HV-C2. Shortly, we will be building a scale model of this stack to test the mixing at the proposed sampling location.

Brian - could you please check for design changes since January 2005 before I submit the scaled version of the design for fabrication?

I noticed that the airflow (38300) is considerably less than the value that was being used about a year ago and which appeared in my design review report $(58000 \mathrm{cfm})$; however the stack diameter had not changed. This would result in a discharge velocity much less than the standard range that Gerard Garcia proposed. Maybe Gerard's proposed range did not apply to C2 systems?

I was wondering if you two could set me straight.

Thanks,

John Glissmeyer 
ATTACHMENT C

MEMORANDUM CCN 116112

March 23, 2005

All drawings are best-available copy in Attachment C. 


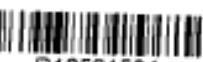

\section{Memorandum}

To: Jennifer Su-Coker - MS4-Cl

From: Gerald M. Gaulden, P.E.

Ext: $371-3582$

Fax: $371-3508$
Date: March 23, 2005

CCN: 116112

\section{Subject: VENTILATION EXHAUST STACK DESIGN DATA (AUGMENTED)}

Reference: $\quad$ CCN: 101188, Memorandum from G.M. Gaulden, "Exhaust Stack Design Standardization Data", March 4, 2005.

CCN: 094165, Meeting Minutes from G. Garcia, HVAC/FP Engineering Discipline Manager, October 11, 2004

The purpose of this memorandum is to supercede $\mathrm{CCN}$ : 101188 to augment the ventilation exhaust stack design data. The table has been augmented to include total pressure at stack discharge and nominal relative humidity. This current ventilation exhaust stack design data is being provided to RPP-WTP Environmental and Nuclear Safety (E\&NS) to support the Air Dispersion Model in the Environmental Risk Assessment for the Dangerous Waste Permit and the Radioactive Air Emissions Permit.

As engineering design on the RPP-WTP Project has progressed, the Central Engineering Groups have made a concerted effort with the Facility Design Teams to standardize the exhaust stack designs across all facilities. The most significant result of this effort has been the establishment of a nominal stack discharge velocity of 3,500 feet per minute $(\mathrm{fpm})$ within a range of $\pm 500 \mathrm{fpm}$. This velocity will maintain adequate exhaust plume rise above the stack discharge, thereby ensuring proper dispersion of the exhaust stream.

The reader is advised that the design data contained herein reflects the current engineering design of the RPP-WTP Facility. In some cases, further design evolution may require revision of this data.

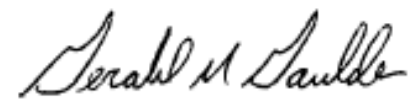

Gerald M. Gaulden, P.E.

Systems Lead

HVAC Central Engineering

GMG/gmg 
VENTILATION EXHAUST STACK DESIGN DATA

\begin{tabular}{|c|c|c|c|c|c|c|c|c|}
\hline Facility & $\begin{array}{c}\text { Emission } \\
\text { Unit }\end{array}$ & $\begin{array}{l}\text { Nominal } \\
\text { Stack } \\
\text { Height (ft) }\end{array}$ & $\begin{array}{c}\text { Stack } \\
\text { Diameter } \\
\text { (in) }\end{array}$ & $\begin{array}{l}\text { Nominal } \\
\text { Airflow } \\
(\mathrm{cfm})\end{array}$ & $\begin{array}{c}\text { Discharge } \\
\text { Velocity } \\
\text { (fpm) }\end{array}$ & $\begin{array}{c}\text { Normal } \\
\text { Exhaust } \\
\text { Temp (F) }\end{array}$ & $\begin{array}{l}\text { Discharge } \\
\text { Pressure } \\
(\text { in WG) }\end{array}$ & $\begin{array}{l}\text { Normal } \\
\text { Relative } \\
\text { Humidity }\end{array}$ \\
\hline \multirow{5}{*}{ PTF } & $\mathrm{C} 2 \mathrm{~V}$ & 200 & 54 & 63,400 & 4,000 & 80 & 1.00 & $10-80 \%$ \\
\hline & C3V & 200 & 62 & 72,500 & 3,500 & 95 & 0.76 & $10 \% \mathrm{~min}$ \\
\hline & $\mathrm{C} 5 \mathrm{~V}$ & 200 & 62 & 70,500 & 3,400 & 113 & 0.72 & $5 \%$ \\
\hline & PJV & \multicolumn{7}{|c|}{ Refer to CCN: 116807 for Process Exhaust Stack Design Data } \\
\hline & PVV & \multicolumn{7}{|c|}{ Refer to CCN: 116807 for Process Exhaust Stack Design Data } \\
\hline \multirow{4}{*}{ LAW } & $\mathrm{C} 2 \mathrm{~V}$ & 105 & 60 & 56,000 & 2,800 & 80 & 0.49 & $15 \%$ \\
\hline & $\mathrm{C} 3 \mathrm{~V}$ & 200 & 48 & 40,000 & 3,200 & 95 & 0.64 & $15 \%$ \\
\hline & C5V & 200 & 60 & 64,000 & 3,300 & 160 & 0.68 & $4 \%$ \\
\hline & LVP & \multicolumn{7}{|c|}{ Refer to CCN: 116807 for Proeess Exhaust Staek Design Bata } \\
\hline \multirow{7}{*}{ HLW } & $\mathrm{C} 2 \mathrm{~V}$ & 71 & 44 & 40,000 & 3,500 & 80 & 0.76 & $10-100 \%$ \\
\hline & C3V & 200 & 50 & $4 \mathbf{4 7 , 5 0 0}$ & 3,500 & 95 & 0.76 & $10 \% \mathrm{~min}$ \\
\hline & $\mathrm{C} 5 \mathrm{~V}$ & 200 & 52 & 51,000 & 3,400 & 113 & 0.72 & $5 \%$ \\
\hline & IHLW & 200 & 24 & 10,500 & 3,300 & 113 & 0.68 & $10 \% \min$ \\
\hline & PJV & \multicolumn{7}{|c|}{ Refer to CCN: 116807 for Process Exhaust Stack Design Data } \\
\hline & HOP A & \multicolumn{7}{|c|}{ Refer to CCN: 116807 for Process Exhaust Stack Design Data } \\
\hline & HOP B & \multicolumn{7}{|c|}{ Refer to CCN: 116807 for Process Exhaust Stack Design Data } \\
\hline \multirow{3}{*}{ LAB } & $\mathrm{C} 2 \mathrm{~V}$ & 118 & 48 & 42,000 & 3,300 & 80 & 0.68 & $10 \%$ \\
\hline & $\mathrm{C} 3 \mathrm{~V}$ & 118 & 60 & 69,000 & 3,500 & 95 & 0.76 & $30 \%$ \\
\hline & C5V & 118 & 28 & 14,500 & 3,400 & 95 & 0.72 & $10 \%$ \\
\hline
\end{tabular}

* Discharge Pressure is equal to Velocity Pressure since Static Pressure is negligible at point of discharge.

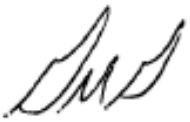

\section{Distribution}

\section{Addressee}

Braccia, Mark

Garcia, Gerard

Icayan, Cesar

Isern, Eric

Lawrence, Will

Merzouk, Karl

\section{MSIN}

MS4-B2

MS4-E2

MS5-K1

MS5-H

MS12-2B

MS12-2B

\section{Addressee}

Shea, Peter

Solis, Leo

Su-Coker, Jennifer

Sweeney, Sean

Thomas, John

PDC $\underline{\text { MSIN }}$

MS8-A

MS5-H

MS4-C1

MS12-2B

MS8-A

MS11-B 
The drawing is best-available copy in Attachment D. 


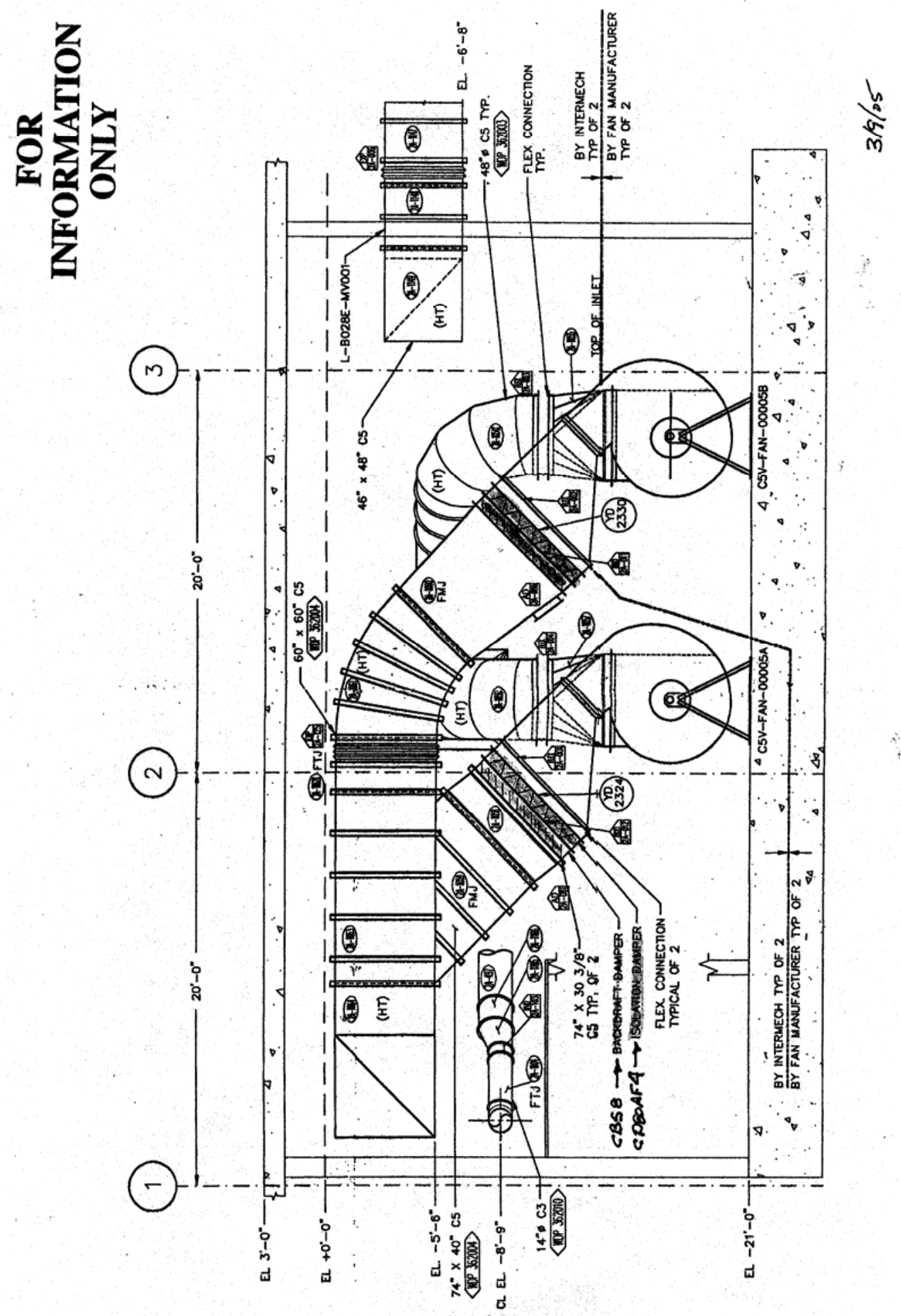




\section{ATTACHMENT E CATALOG CUT OF RUSKIN CONTROL DAMPER}

The drawing is best-available copy in Attachment E. 


\section{FOR \\ INFORMATION \\ ONLY}

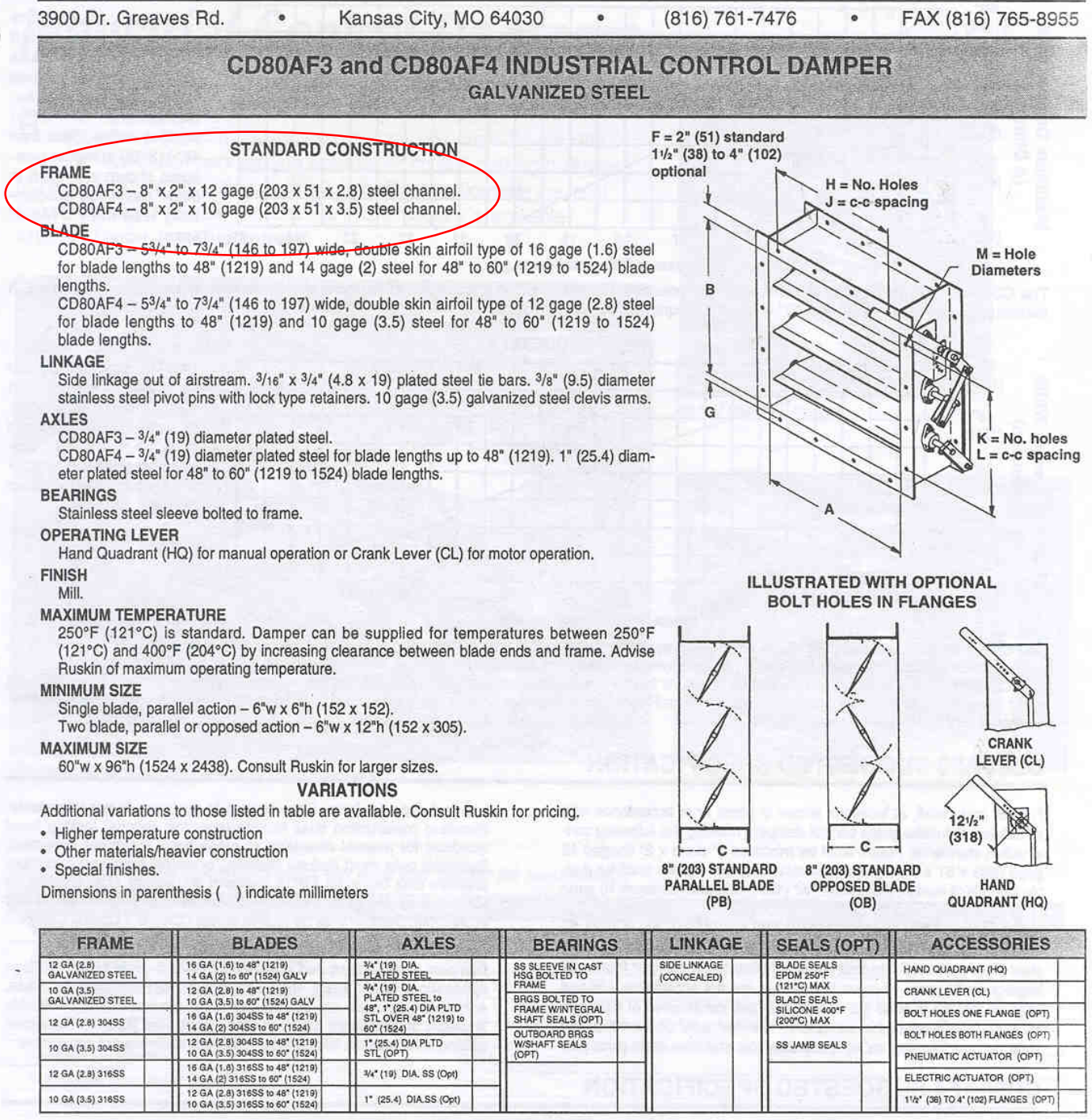

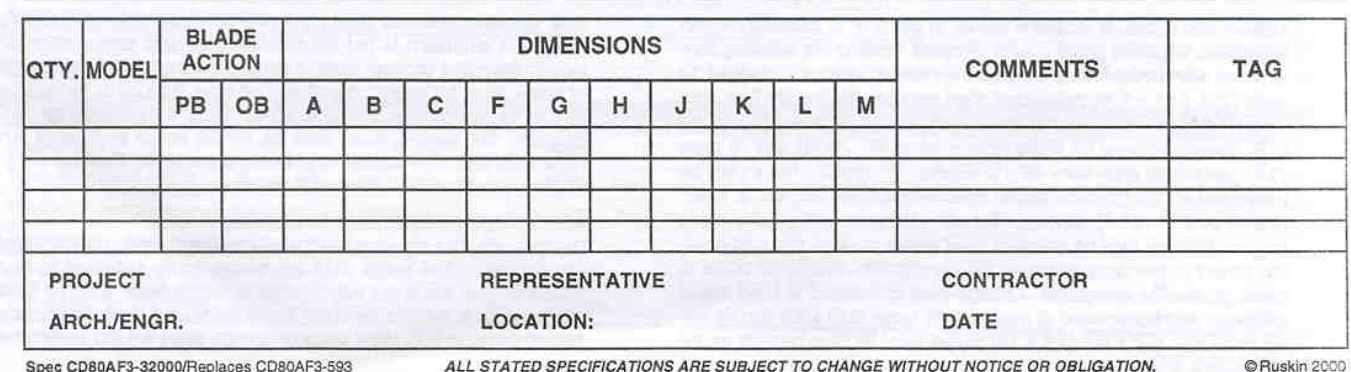

Spec CD80AF3-32000/Replaces CD80AF3-593

ALL STATED SPECIFICATIONS ARE SUBJECT TO CHANGE WITHOUT NOTICE OR OBLIGATION.

ORuskin 2000 


\section{ATTACHMENT F CATALOG CUT OF RUSKIN BACKDRAFT DAMPER}

The drawing is best-available copy in Attachment F. 


\section{RUSKIN ONLY

\begin{tabular}{rcccc}
\hline 3900 Dr. Greaves Rd. & Kansas City, MO 64030 & $(816) 761-7476$ & FAX (816) 765-8955 \\
CBS8 HEAVY DUTY & \\
& COUNTERBALANCED BACKDRAFT DAMPER & \\
\hline
\end{tabular}

FOR
INFORMATION

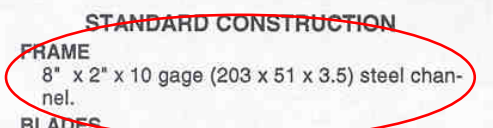

BLADES

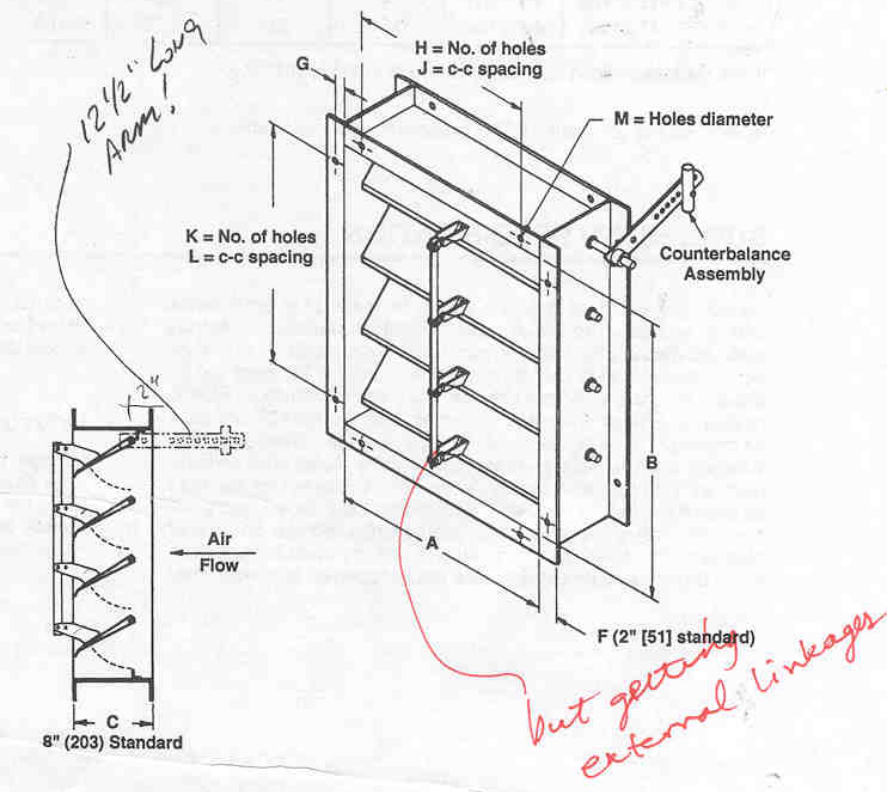

Double-skin, airfoil type of 18 (1.3) gage steel

with a $7^{*}$ (178) maximum width.

AXLES

$3 / 4$ " (19) diameter, plated steel.

BEARINGS

Ball bearings pressed to frame.

LINKAGE

$3 / 16^{\prime \prime}$ thick $\times 3 / 4^{\prime \prime}(5 \times 19)$ plated steel tie bar with

16 gage (1.6) linkage arms in airstream.

FINISH

Mill galvanized.

MINIMUM SIZE

6" w X 6"h $(152 \times 152)$

MAXIMUM SIZE

60 "w $\times 96$ "h (1524 × 2438).

MAXIMUM TEMPERATURE

$250^{\circ} \mathrm{F}\left(121^{\circ} \mathrm{C}\right)$.

\section{VARIATIONS}

Additional variations to those listed in table are available. Contact Ruskin for pricing.

- Heavier/Higher Temperature Construction

- Special Finishes

- Pressure Relief Applications

Dimensions shown in parentheses ( ) indicate millimeters.

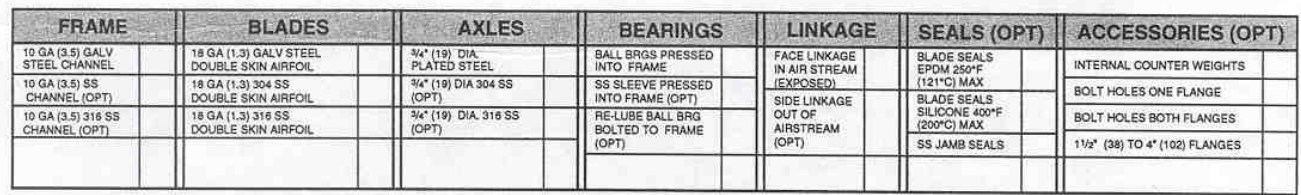

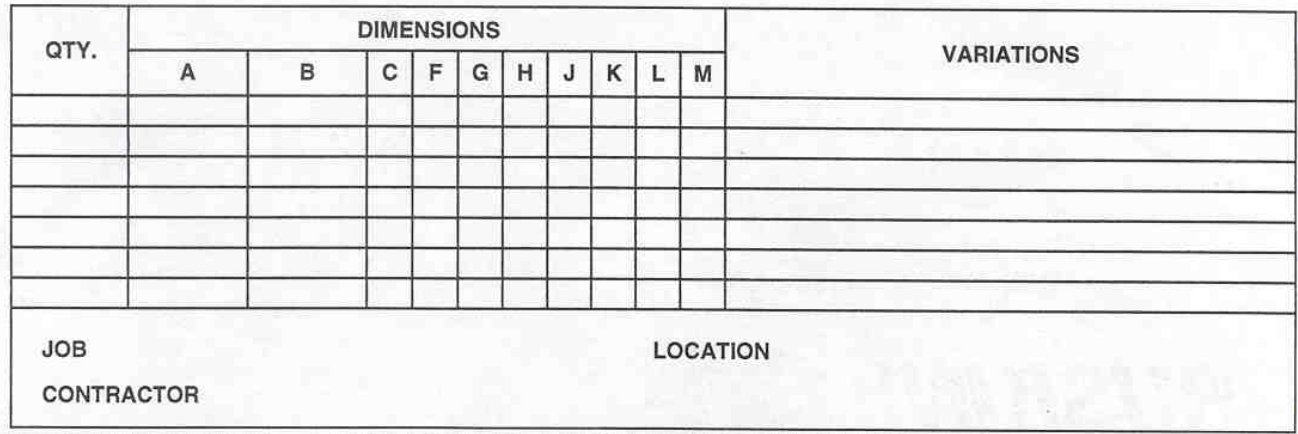


Appendix B

Flow-Calibration Data Sheets 


\section{Appendix B: Flow-Calibration Data Sheets}

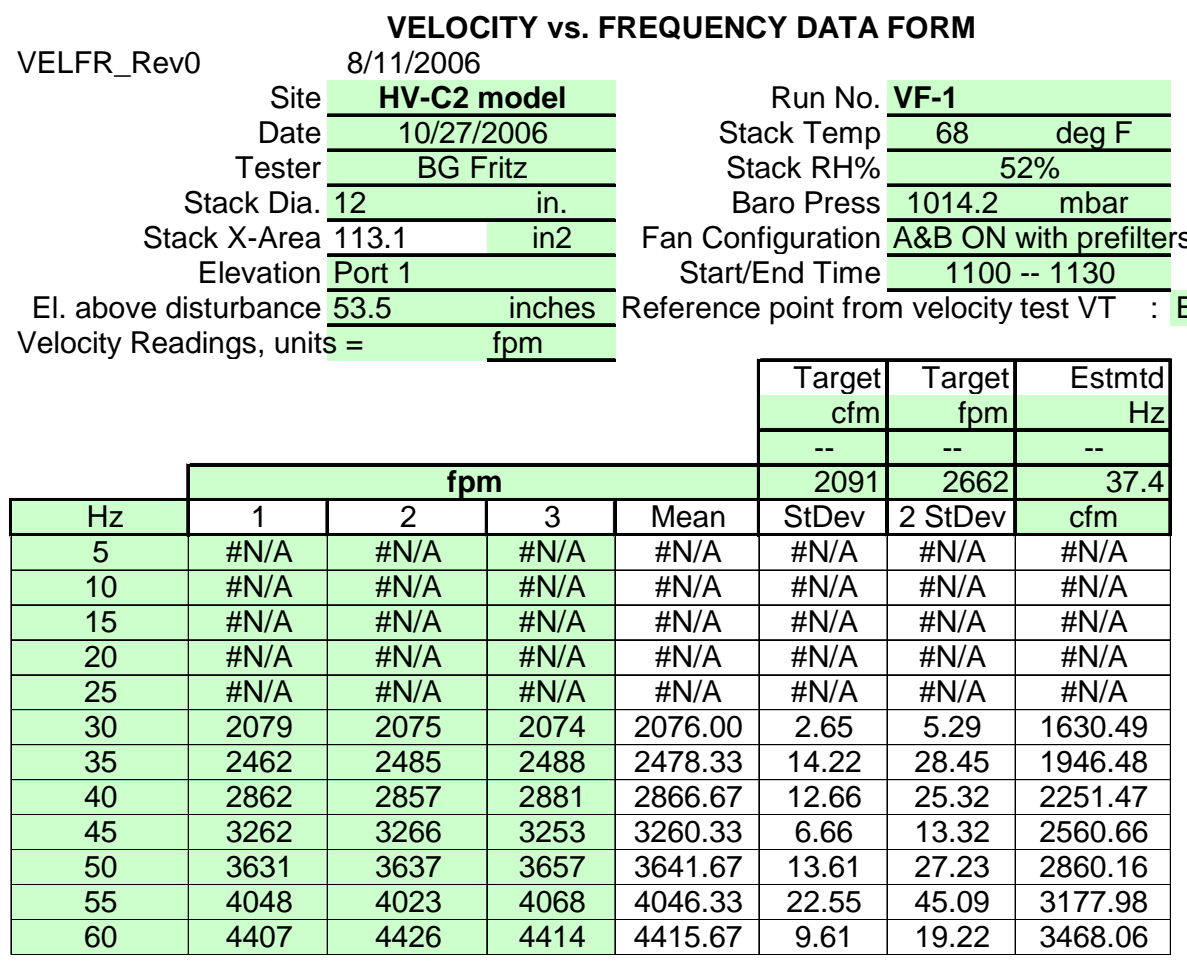

Instuments Used:

Solomat Zephyr SN 12951472

Stack pressure $=0.97 \mathrm{mbar}$

Total Stack Press = 1015 mbar

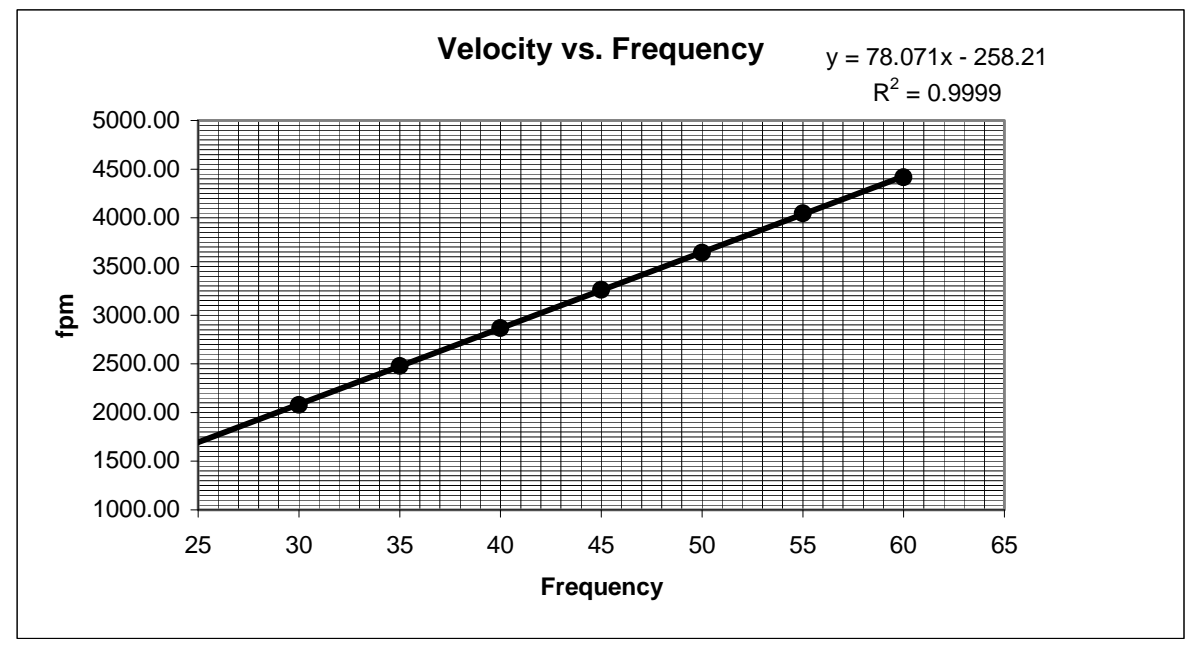

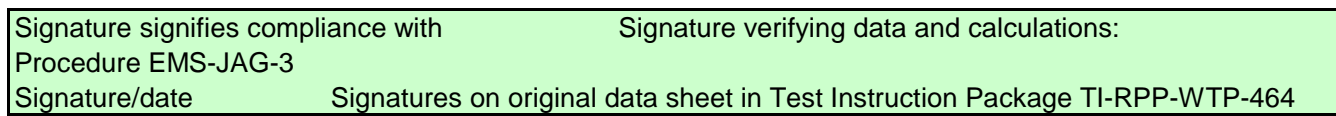




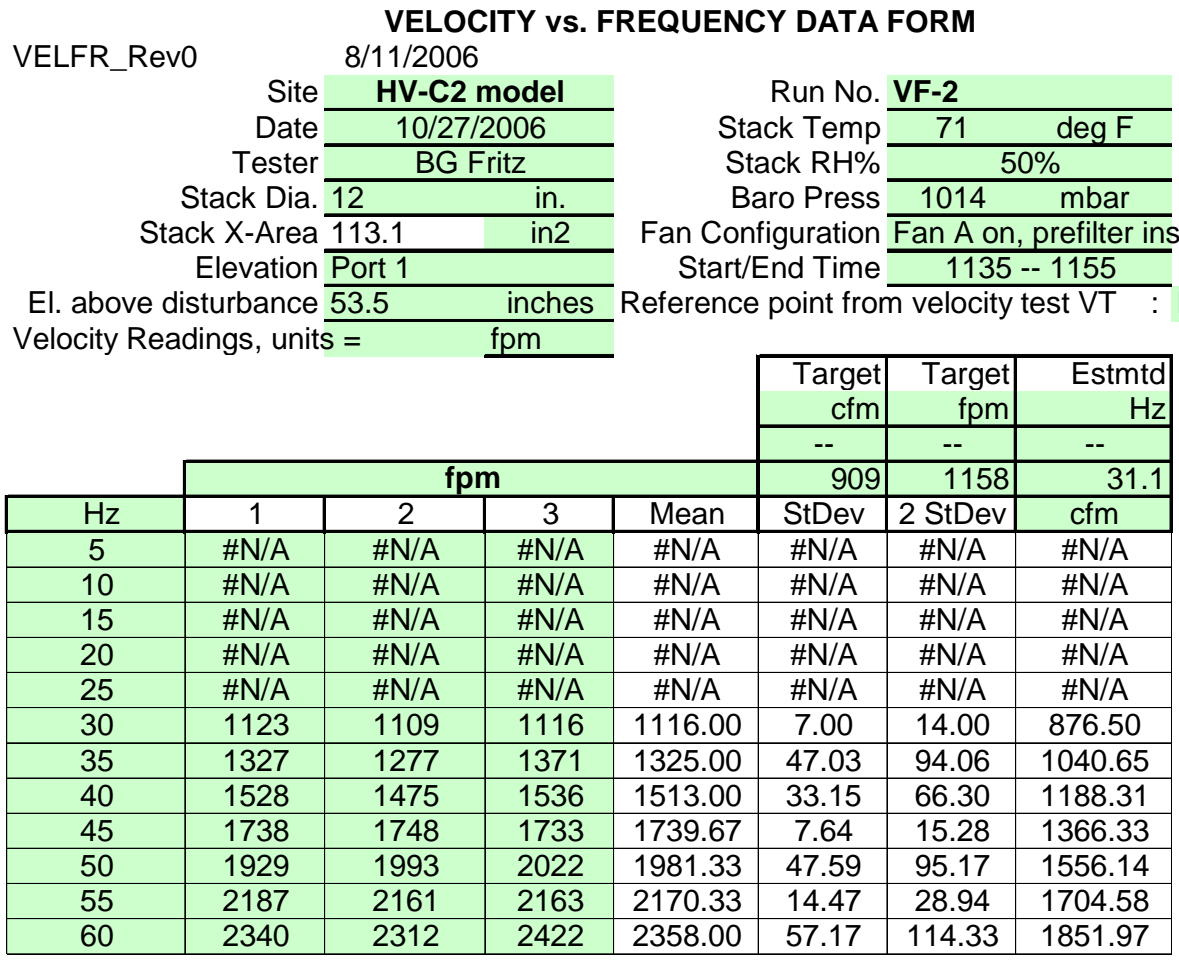

Instuments Used:

Solomat Zephyr SN 12951472

Stack static $=0.2 \mathrm{mbar}$

Total Stack Press $=1014$ mbar
Cal Exp. Date: $10 / 12 / 2007$

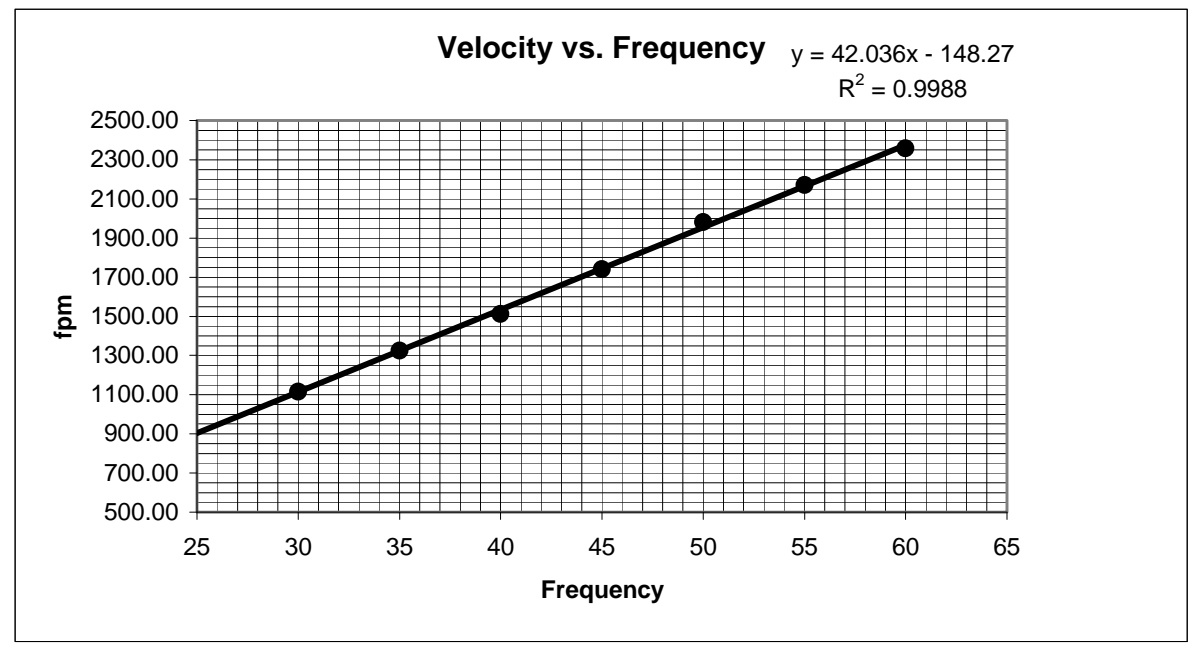

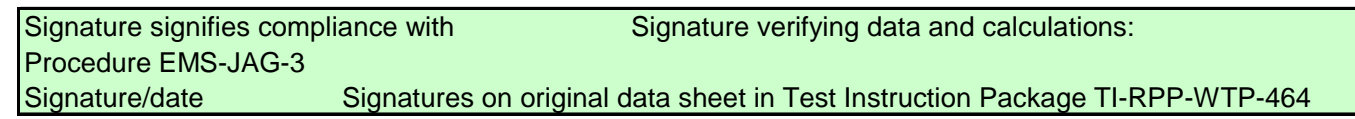

Reference: CCP-WTPSP-177

(HVC2_VELFR_Rev0).xls

VELFR Rev0.xls

VF2

3 August 2006

$4 / 9 / 2007$ 
VELFR_Rev0

\begin{tabular}{rlr} 
Site & \multicolumn{1}{c}{ HV-C2 model } \\
\cline { 2 - 2 } Date & \multicolumn{1}{c}{$10 / 27 / 2006$} \\
\cline { 2 - 2 } Tester & \multicolumn{2}{c}{ BG Fritz } \\
Stack Dia. & 12 & in. \\
Stack X-Area & 113.1 & in2 \\
Elevation & Port 1 &
\end{tabular}

El. above disturbance $\overline{53.5}$

Velocity Readings, units =

8/11/2006

\section{VELOCITY vS. FREQUENCY DATA FORM}

\begin{tabular}{|c|c|c|c|c|c|c|c|}
\hline & & & \multirow{2}{*}{$\begin{array}{c}\begin{array}{c}\text { Target } \\
\mathrm{cfm}\end{array} \\
-- \\
\end{array}$} & \multirow{2}{*}{$\begin{array}{r}\begin{array}{r}\text { Target } \\
\mathrm{fpm}\end{array} \\
--\end{array}$} & $\begin{array}{r}\text { Estmtd } \\
\mathrm{Hz} \\
\end{array}$ \\
\hline & & & & & & & -- \\
\hline & \multicolumn{4}{|c|}{ fpm } & 909 & 1158 & 30.5 \\
\hline $\mathrm{Hz}$ & 1 & 2 & 3 & Mean & StDev & $2 \mathrm{StDev}$ & cfm \\
\hline 5 & $\# \mathrm{~N} / \mathrm{A}$ & $\# \mathrm{~N} / \mathrm{A}$ & \#N/A & $\# \mathrm{~N} / \mathrm{A}$ & $\# \mathrm{~N} / \mathrm{A}$ & \#N/A & $\# \mathrm{~N} / \mathrm{A}$ \\
\hline 10 & $\# \mathrm{~N} / \mathrm{A}$ & $\# \mathrm{~N} / \mathrm{A}$ & $\# \mathrm{~N} / \mathrm{A}$ & $\# \mathrm{~N} / \mathrm{A}$ & $\# \mathrm{~N} / \mathrm{A}$ & \#N/A & $\# \mathrm{~N} / \mathrm{A}$ \\
\hline 15 & $\# \mathrm{~N} / \mathrm{A}$ & $\# \mathrm{~N} / \mathrm{A}$ & $\# \mathrm{~N} / \mathrm{A}$ & \#N/A & \#N/A & \#N/A & \#N/A \\
\hline 20 & $\# \mathrm{~N} / \mathrm{A}$ & $\# \mathrm{~N} / \mathrm{A}$ & $\# \mathrm{~N} / \mathrm{A}$ & $\# \mathrm{~N} / \mathrm{A}$ & $\# \mathrm{~N} / \mathrm{A}$ & $\# \mathrm{~N} / \mathrm{A}$ & $\# \mathrm{~N} / \mathrm{A}$ \\
\hline 25 & $\# \mathrm{~N} / \mathrm{A}$ & $\# \mathrm{~N} / \mathrm{A}$ & $\# \mathrm{~N} / \mathrm{A}$ & \#N/A & $\# \mathrm{~N} / \mathrm{A}$ & $\# \mathrm{~N} / \mathrm{A}$ & \#N/A \\
\hline 30 & 1153 & 1125 & 1168 & 1148.67 & 21.83 & 43.65 & 902.16 \\
\hline 35 & 1332 & 1323 & 1358 & 1337.67 & 18.18 & 36.35 & 1050.60 \\
\hline 40 & 1598 & 1528 & 1591 & 1572.33 & 38.55 & 77.11 & 1234.91 \\
\hline 45 & 1849 & 1777 & 1799 & 1808.33 & 36.90 & 73.79 & 1420.26 \\
\hline 50 & 1968 & 1964 & 2005 & 1979.00 & 22.61 & 45.21 & 1554.30 \\
\hline 55 & 2212 & 2181 & 2277 & 2223.33 & 48.99 & 97.99 & 1746.20 \\
\hline 60 & 2528 & 2398 & 2420 & 2448.67 & 69.58 & 139.16 & 1923.18 \\
\hline
\end{tabular}

Instuments Used:

Cal Exp. Date:

Solomat Zephyr SN 12951472 $10 / 12 / 2007$ For fun I looked at several averages at $45 \mathrm{~Hz}(30-35$ points). Typical Std. Dev. for each average was 120 (i.e., $1777+/-1$ S.D. $=1777+/$ - 120).

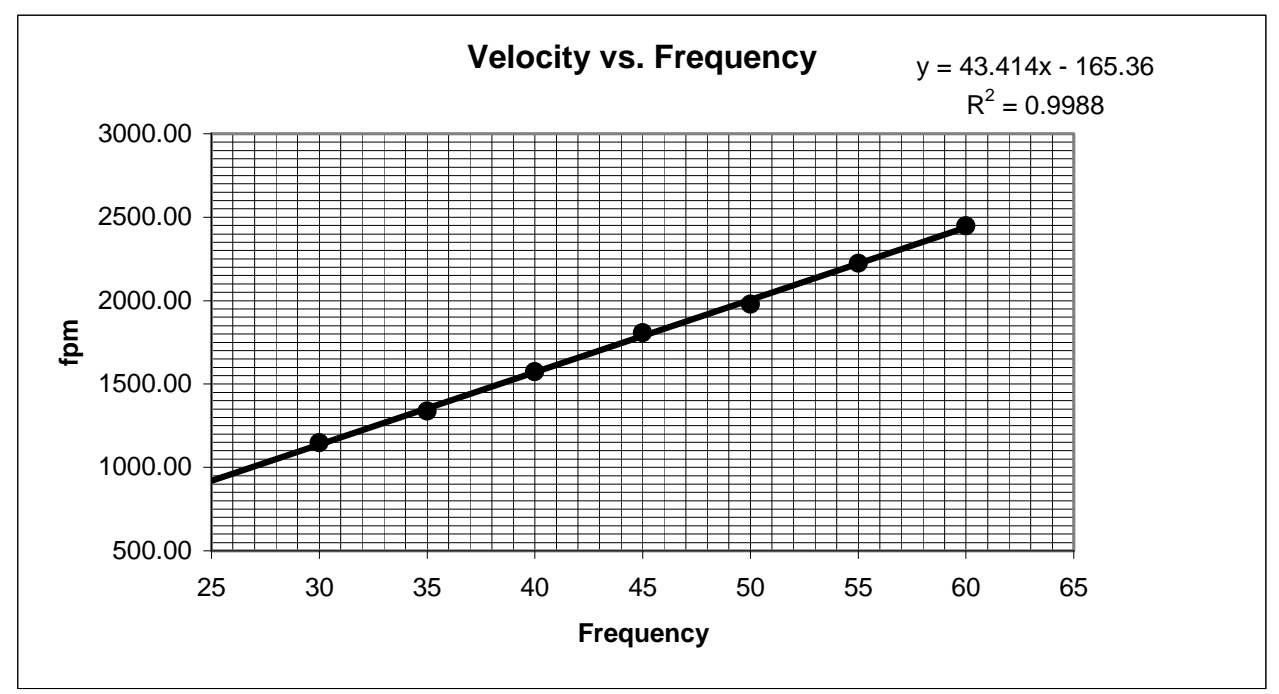

Signature signifies compliance with

Signature verifying data and calculations:

Procedure EMS-JAG-3

Signature/date

Signatures on original data sheet in Test Instruction Package TI-RPP-WTP-464

(HVC2_VELFR_Rev0).xls

Reference: CCP-WTPSP-177

VF3

VELFR_Rev0.xIs

$4 / 9 / 2007$

Run No. VF-3

Stack Temp 70

Baro Press $1014 \quad \mathrm{mbar}$

an Configuration Fan B, prefilter on, Fan A sealed Start/End Time 1300 -- 1325

Reference point from velocity test VT : Bottom \#4

d 
VELFR_Rev0

\begin{tabular}{|c|c|c|}
\hline ev0 & $8 / 11 / 2$ & \\
\hline Site & HV & nodel \\
\hline Date & & 006 \\
\hline Tester & & \\
\hline Stack Dia. & 12 & in. \\
\hline Stack X-Area & 113.1 & in2 \\
\hline Elevation & Port 1 & \\
\hline disturbance & 53.5 & inches \\
\hline adings, units & & $\mathrm{fpm}$ \\
\hline
\end{tabular}

Velocity Readings, units =

\section{VELOCITY vS. FREQUENCY DATA FORM}

Run No. VF-4

\begin{tabular}{rcc} 
Stack Temp & 72 & deg F \\
\cline { 2 - 3 } Stack RH\% & \multicolumn{2}{c}{$54 \%$} \\
\cline { 2 - 3 } Baro Press & 981 & mbar
\end{tabular}

Fan Configuration Fan A, prefilter on, Fan B sealed Start/End Time $1600-1640$ Reference point from velocity test VT : Side \#4

\begin{tabular}{|c|c|c|c|c|c|c|c|}
\hline & \multicolumn{3}{|c|}{\begin{tabular}{|r|r|r|} 
Target & Target & Estmtd \\
$\mathrm{cfm}$ & $\mathrm{fpm}$ & $\mathrm{Hz}$ \\
\end{tabular}} \\
\hline & & & & & -- & \begin{tabular}{|l|}
-- \\
\end{tabular} & -- \\
\hline & \multicolumn{4}{|c|}{ fpm } & 909 & 1158 & 32.0 \\
\hline$\overline{\mathrm{Hz}}$ & 1 & 2 & 3 & Mean & StDev & 2 StDev & cfm \\
\hline 5 & \#N/A & \#N/A & \#N/A & \#N/A & $\# N / A$ & $\# N / A$ & \#N/A \\
\hline 10 & \#N/A & \#N/A & \#N/A & \#N/A & \#N/A & \#N/A & \#N/A \\
\hline 15 & \#N/A & \#N/A & \#N/A & \#N/A & \#N/A & \#N/A & \#N/A \\
\hline 20 & \#N/A & \#N/A & \#N/A & \#N/A & \#N/A & \#N/A & \#N/A \\
\hline 25 & \#N/A & \#N/A & \#N/A & \#N/A & \#N/A & \#N/A & \#N/A \\
\hline 30 & 1044 & 1082 & 1109 & 1078.33 & 32.65 & 65.31 & 846.92 \\
\hline 35 & 1221 & 1302 & 1349 & 1290.67 & 64.75 & 129.50 & 1013.69 \\
\hline 40 & 1491 & 1568 & 1444 & 1501.00 & 62.60 & 125.20 & 1178.88 \\
\hline 45 & 1752 & 1797 & 1729 & 1759.33 & 34.59 & 69.18 & 1381.78 \\
\hline 50 & 1956 & 1952 & 1973 & 1960.33 & 11.15 & 22.30 & 1539.64 \\
\hline 55 & 2269 & 2157 & 2102 & 2176.00 & 85.11 & 170.21 & 1709.03 \\
\hline 60 & 2464 & 2495 & 2385 & 2448.00 & 56.72 & 113.44 & 1922.65 \\
\hline
\end{tabular}

Instuments Used:

Solomat Zephyr SN 12951472

1600 HMS Station $1154 \% \mathrm{RH}$ and 28.97 in. mercury barometric pressure.

Cal Exp. Date: $10 / 12 / 2007$

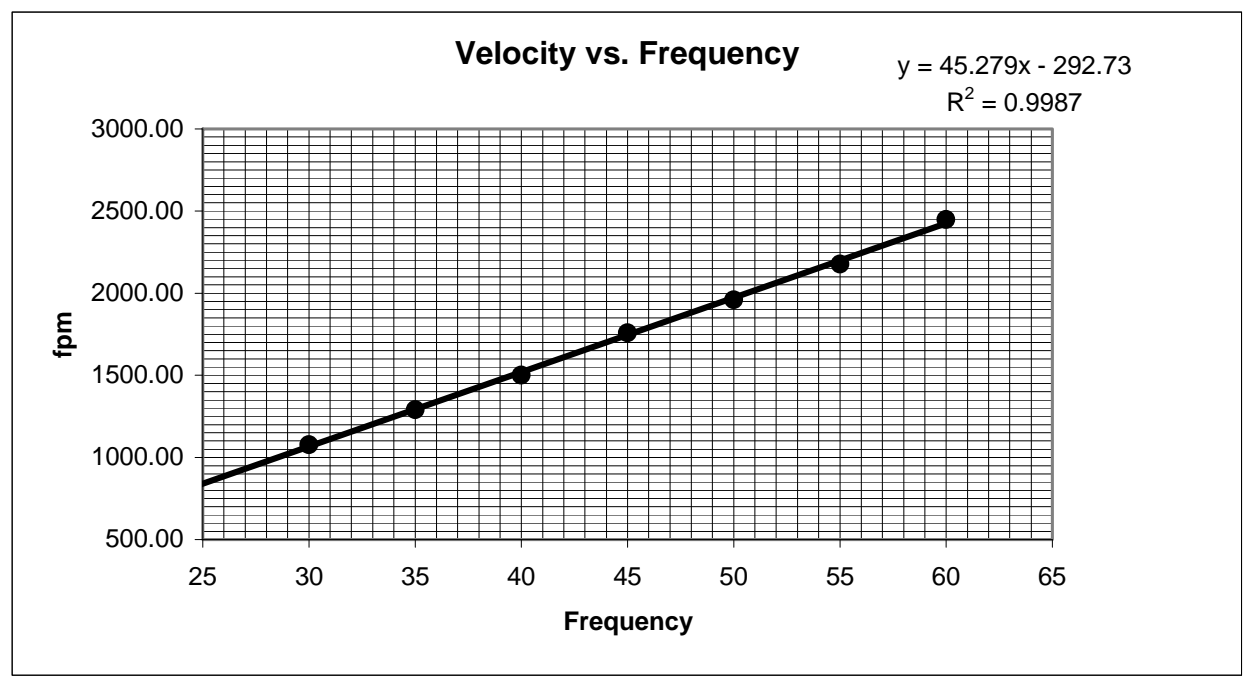

Signature signifies compliance with Procedure EMS-JAG-3 Signature/date Signature verifying data and calculations: 


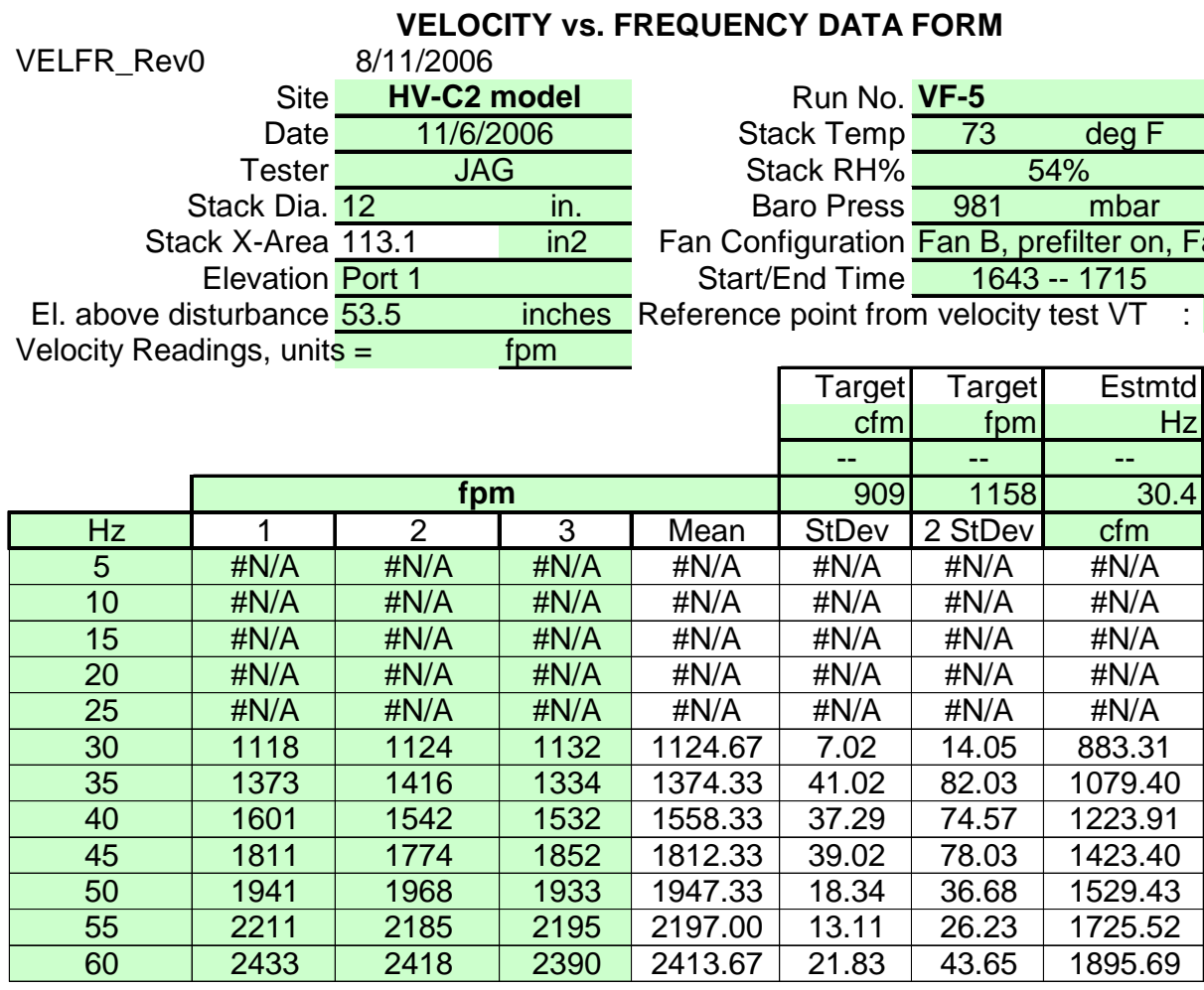

Instuments Used:

Cal Exp. Date:

Solomat Zephyr SN 12951472

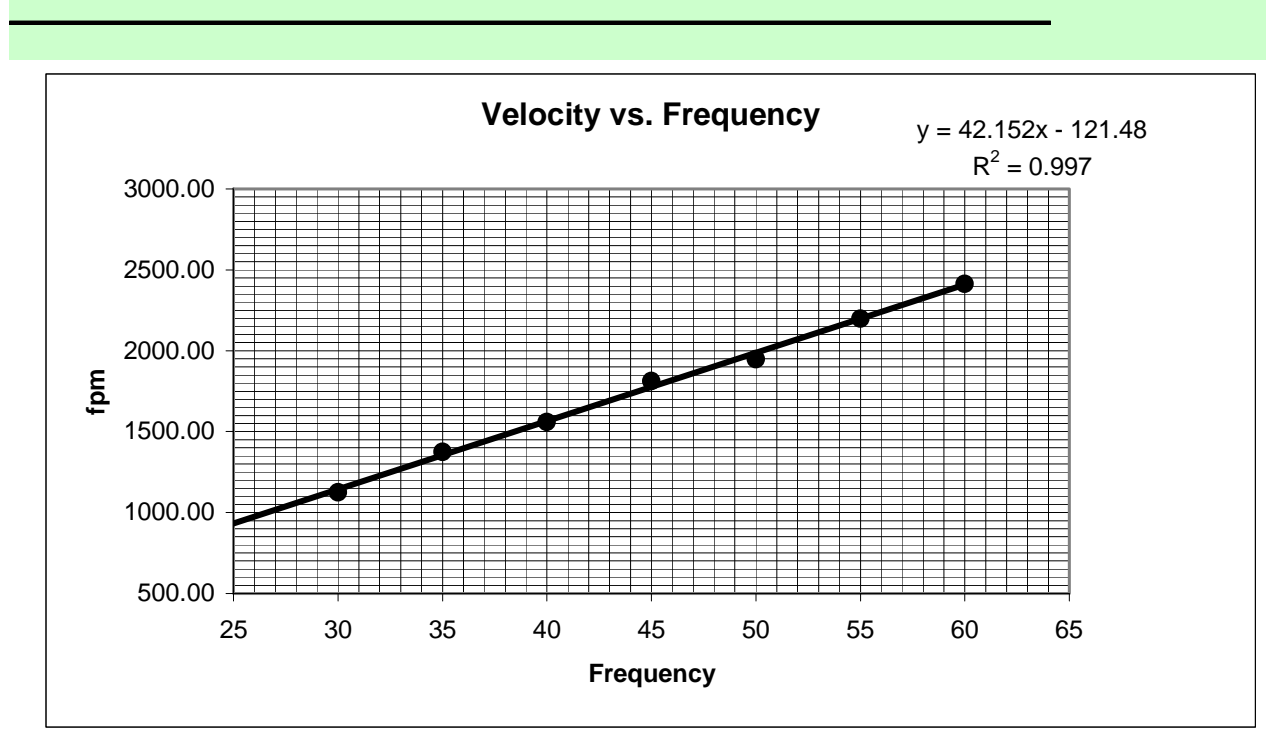

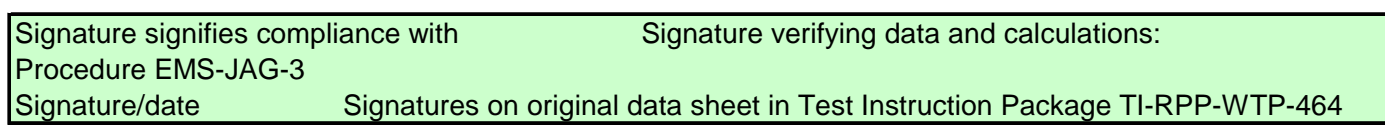




\section{Appendix C}

\section{Velocity Uniformity Data Sheets}




\section{Appendix C: Velocity Uniformity Data Sheets}

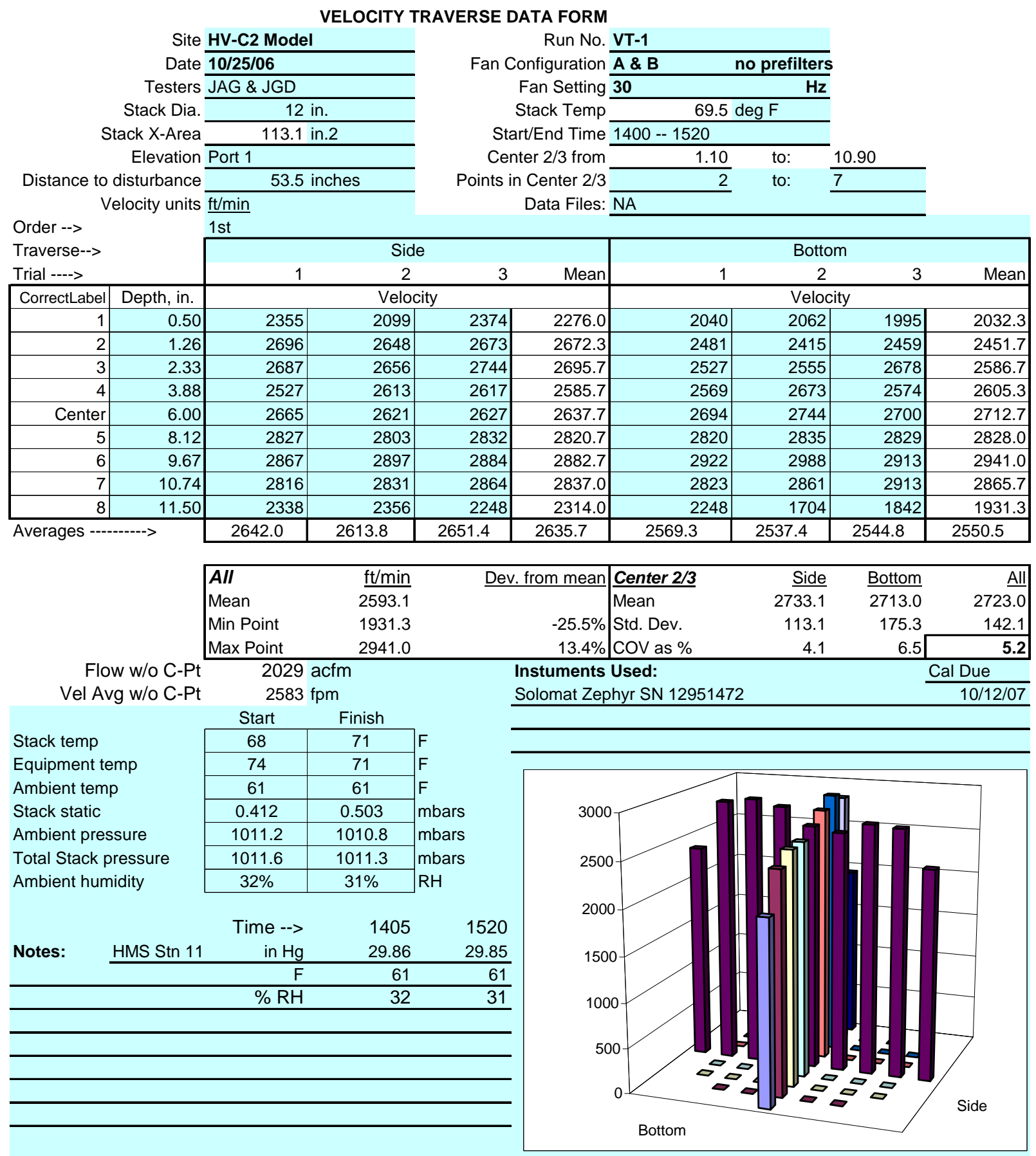

Signature signifies compliance with

Signature verifying data and calculations:

Procedure EMS-JAG-4

Signature/date

Signatures on original data sheet in Test Instruction Package TI-RPP-WTP-462

Reference: CCP-WTPSP-179

(HVC2_VEL-dataRev0 (1)).xIs/VT1

VEL-dataRev0.xls

$4 / 18 / 2007$

3 August 2006 


\section{VELOCITY TRAVERSE DATA FORM}

Site HV-C2 Model

Date 10/25/06

Testers JAG \& JGD

Stack Dia. Stack X-Area

Elevation Port 1

Distance to disturbance Velocity units $\underline{\mathrm{ft} / \mathrm{min}}$
Run No. VT-2

Fan Configuration A \& B

Fan Setting 30

Stack Temp

Start/End Time 1535 -- 1610

Center $2 / 3$ from

Points in Center $2 / 3$

Data Files: NA

\begin{tabular}{|c|c|c|c|c|c|c|c|c|c|}
\hline \multirow{3}{*}{\multicolumn{2}{|c|}{$\begin{array}{l}\text { Order --> } \\
\text { Traverse--> } \\
\text { Trial ----> }\end{array}$}} & & & & & & & & \\
\hline & & \multicolumn{4}{|c|}{ Side } & \multicolumn{4}{|c|}{ Bottom } \\
\hline & & 1 & 2 & \multirow[t]{2}{*}{3} & \multirow[t]{2}{*}{ Mean } & \multirow[t]{2}{*}{1} & 2 & 3 & Mean \\
\hline CorrectLabel & Depth, in. & \multicolumn{2}{|c|}{ Velocity } & & & & \multicolumn{3}{|c|}{ Velocity } \\
\hline 1 & 0.50 & 1867 & 1962 & 1724 & 1851.0 & 1733 & 1601 & 1608 & 1647.3 \\
\hline 2 & 1.26 & 2238 & 2242 & 2209 & 2229.7 & 1935 & 1980 & 1952 & 1955.7 \\
\hline 3 & 2.33 & 2350 & 2327 & 2275 & 2317.3 & 2079 & 2091 & 2101 & 2090.3 \\
\hline 4 & 3.88 & 2216 & 2256 & 2200 & 2224.0 & 2135 & 2056 & 2142 & 2111.0 \\
\hline Center & 6.00 & 2294 & 2256 & 2267 & 2272.3 & 2279 & 2302 & 2249 & 2276.7 \\
\hline 5 & 8.12 & 2376 & 2376 & 2334 & 2362.0 & 2407 & 2414 & 2393 & 2404.7 \\
\hline 6 & 9.67 & 2367 & 2442 & 2421 & 2410.0 & 2469 & 2451 & 2470 & 2463.3 \\
\hline 7 & 10.74 & 2434 & 2394 & 2390 & 2406.0 & 2410 & 2422 & 2430 & 2420.7 \\
\hline 8 & 11.50 & 1497 & 1543 & 1761 & 1600.3 & 1769 & 1339 & 1678 & 1595.3 \\
\hline Averages --- & $\cos _{-1}$ & 2182.1 & 2199.8 & 2175.7 & 2185.9 & 2135.1 & 2072.9 & 2113.7 & 2107.2 \\
\hline
\end{tabular}

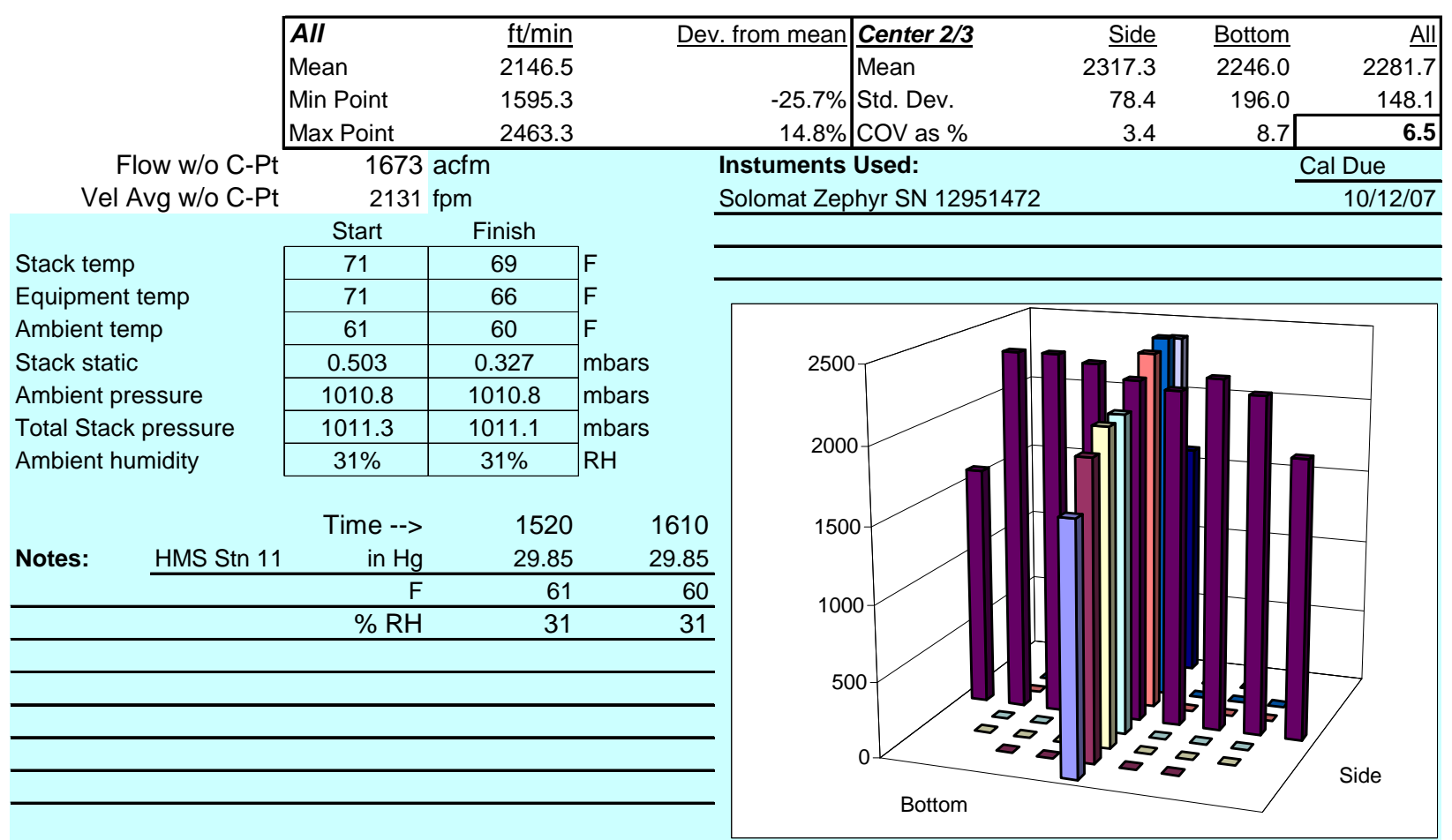

Signature signifies compliance with

Signature verifying data and calculations:

Procedure EMS-JAG-4

Signature/date

Signatures on original data sheet in Test Instruction Package TI-RPP-WTP-462

Reference: CCP-WTPSP-179

(HVC2_VEL-dataRev0 (1)).xIs/VT2

VEL-dataRev0.xls

$4 / 18 / 2007$

3 August 2006 


\section{VELOCITY TRAVERSE DATA FORM}

Site HV-C2 Model Date 10/26/06

Testers JAG \& BGF \& JGD

$$
\text { Stack Dia. }
$$

Stack X-Area

Elevation Port 1

Distance to disturbance Velocity units $\underline{\mathrm{ft} / \mathrm{min}}$
Run No. VT-3

Fan Configuration $\mathbf{A}$

Fan Setting $\mathbf{3 0 . 0 8}$

Stack Temp

Start/End Time 1410 -- 151

Center 2/3 from

Points in Center $2 / 3$

Data Files: NA

\begin{tabular}{|c|c|c|c|c|c|c|c|c|c|}
\hline \multirow{3}{*}{\multicolumn{2}{|c|}{$\begin{array}{l}\text { Order --> } \\
\text { Traverse--> } \\
\text { Trial ----> } \\
\end{array}$}} & $1 \mathrm{st}$ & & & & & & & \\
\hline & & \multicolumn{4}{|c|}{ Side } & \multicolumn{4}{|c|}{ Bottom } \\
\hline & & \multirow[t]{2}{*}{1} & \multicolumn{2}{|l|}{2} & \multirow[t]{2}{*}{ Mean } & 1 & & 3 & Mean \\
\hline CorrectLabel & Depth, in. & & \multicolumn{2}{|c|}{ Velocity } & & \multicolumn{4}{|c|}{ Velocity } \\
\hline 1 & 0.50 & 1062 & 914 & 1016 & 997.3 & \multirow{2}{*}{\multicolumn{2}{|c|}{922}} & 856 & 849.3 \\
\hline 2 & 1.26 & 1257 & 1168 & 1232 & 1219.0 & \multirow{2}{*}{\multicolumn{2}{|c|}{$\begin{array}{l}1046 \\
1094 \\
\end{array}$}} & 1069 & 1036.0 \\
\hline 3 & 2.33 & 1256 & 1299 & 1236 & 1263.7 & & & 1044 & 1083.7 \\
\hline 4 & 3.88 & 1100 & 1177 & 1176 & 1151.0 & \multicolumn{2}{|r|}{1034} & 1081 & 1059.3 \\
\hline Center & 6.00 & 1093 & 1100 & 1084 & 1092.3 & \multicolumn{2}{|r|}{1105} & 1117 & 1106.0 \\
\hline 5 & 8.12 & 1210 & 1098 & 1140 & 1149.3 & \multicolumn{2}{|r|}{1175} & 1206 & 1202.3 \\
\hline 6 & 9.67 & 1229 & 1172 & 1248 & 1216.3 & \multicolumn{2}{|r|}{1335} & 1451 & 1392.7 \\
\hline 7 & 10.74 & 1279 & 1331 & 1287 & 1299.0 & \multicolumn{2}{|l|}{1547} & 1606 & 1555.3 \\
\hline 8 & 11.50 & 1390 & 1235 & 1306 & 1310.3 & \multicolumn{2}{|r|}{1029} & 920 & 960.0 \\
\hline Averages --- & -----> & 1208.4 & 1166.0 & 1191.7 & 1188.7 & 1125.7 & 1139.2 & 1150.0 & 1138.3 \\
\hline
\end{tabular}

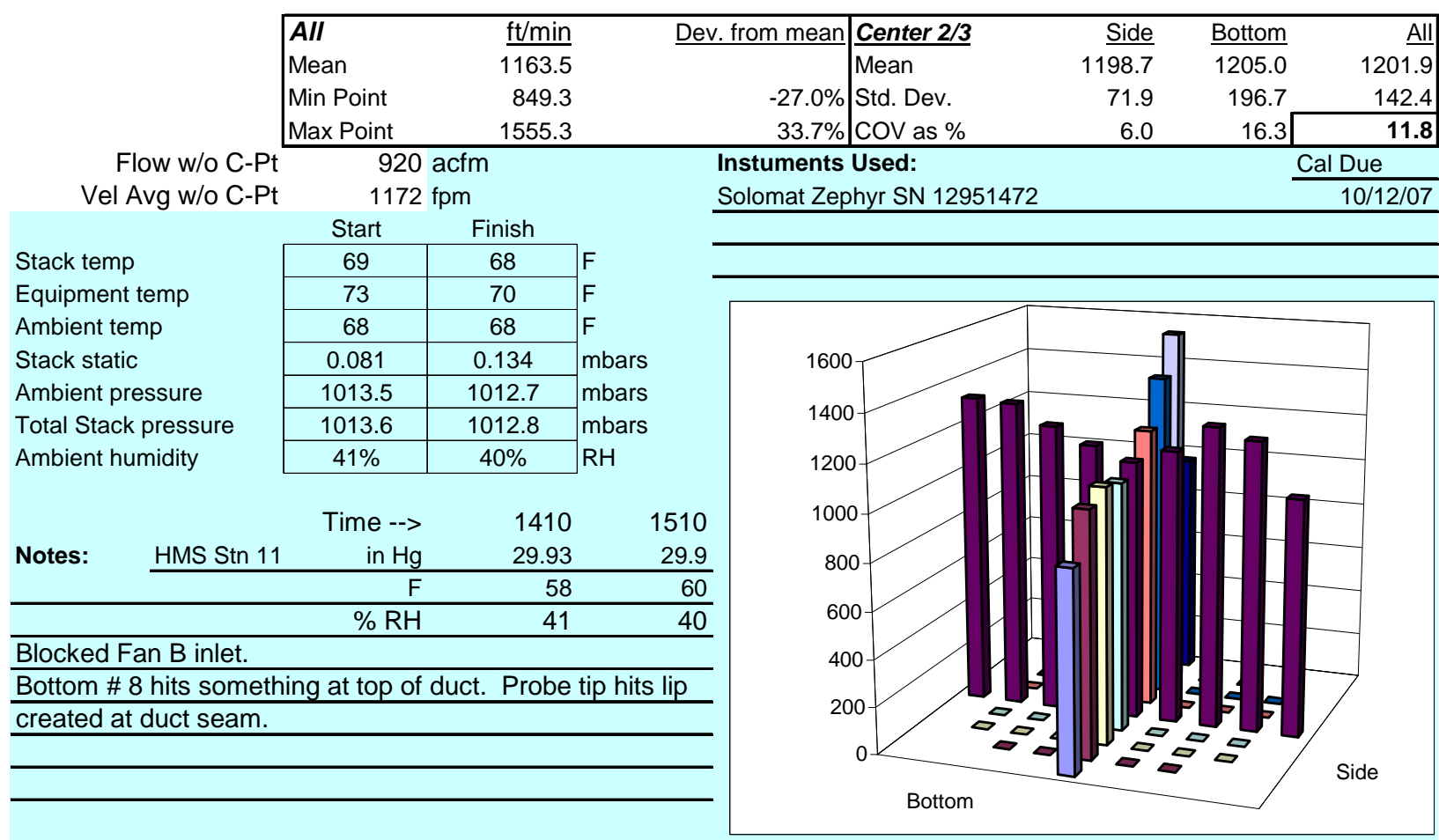

Signature signifies compliance with

Signature verifying data and calculations:

Procedure EMS-JAG-4

Signature/date

Signatures on original data sheet in Test Instruction Package TI-RPP-WTP-462

Reference: CCP-WTPSP-179

(HVC2_VEL-dataRev0 (1)).xIs/VT3

VEL-dataRev0.xls

$4 / 18 / 2007$

3 August 2006 


\section{VELOCITY TRAVERSE DATA FORM}

Site HV-C2 Model

Date 10/26/06

Testers BGF \& JGD

$$
\text { Stack Dia. }
$$

Stack X-Area

Elevation Port 1

Distance to disturbance

Velocity units $\underline{\mathrm{ft} / \mathrm{min}}$
Run No. VT-4

Fan Configuration $\mathbf{B}$

Fan Setting $\mathbf{3 0 . 0 8}$

Stack Temp

Start/End Time 1530 -- 1555

Center $2 / 3$ from

Points in Center 2/3

Data Files: NA

\begin{tabular}{|c|c|c|c|c|c|c|c|c|c|}
\hline \multirow{3}{*}{\multicolumn{2}{|c|}{$\begin{array}{l}\text { Order --> } \\
\text { Traverse--> } \\
\text { Trial ----> }\end{array}$}} & & & & & & & & \\
\hline & & \multicolumn{4}{|c|}{ Side } & \multicolumn{4}{|c|}{ Bottom } \\
\hline & & \multirow[t]{2}{*}{1} & 2 & \multirow[t]{2}{*}{3} & \multirow[t]{2}{*}{ Mean } & \multirow[t]{2}{*}{1} & 2 & 3 & Mean \\
\hline CorrectLabel & Depth, in. & & Velocity & & & & \multicolumn{3}{|c|}{ Velocity } \\
\hline 1 & 0.50 & 877 & 960 & 939 & 925.3 & 1039 & 946 & 920 & 968.3 \\
\hline 2 & 1.26 & 1074 & 1051 & 1022 & 1049.0 & 1208 & 1132 & 1105 & 1148.3 \\
\hline 3 & 2.33 & 1151 & 1147 & 1075 & 1124.3 & 1213 & 1194 & 1181 & 1196.0 \\
\hline 4 & 3.88 & 1177 & 1206 & 1132 & 1171.7 & 1241 & 1215 & 1252 & 1236.0 \\
\hline Center & 6.00 & 1207 & 1224 & 1216 & 1215.7 & 1250 & 1272 & 1276 & 1266.0 \\
\hline 5 & 8.12 & 1340 & 1297 & 1318 & 1318.3 & 1287 & 1256 & 1301 & 1281.3 \\
\hline 6 & 9.67 & 1308 & 1345 & 1311 & 1321.3 & 1284 & 1221 & 1256 & 1253.7 \\
\hline 7 & 10.74 & 1245 & 1262 & 1280 & 1262.3 & 1193 & 1222 & 1207 & 1207.3 \\
\hline 8 & 11.50 & 1219 & 1208 & 976 & 1134.3 & 912 & 1076 & 1000 & 996.0 \\
\hline Averages --- & ----> & 1177.6 & 1188.9 & 1141.0 & 1169.1 & 1180.8 & 1170.4 & 1166.4 & 1172.6 \\
\hline
\end{tabular}

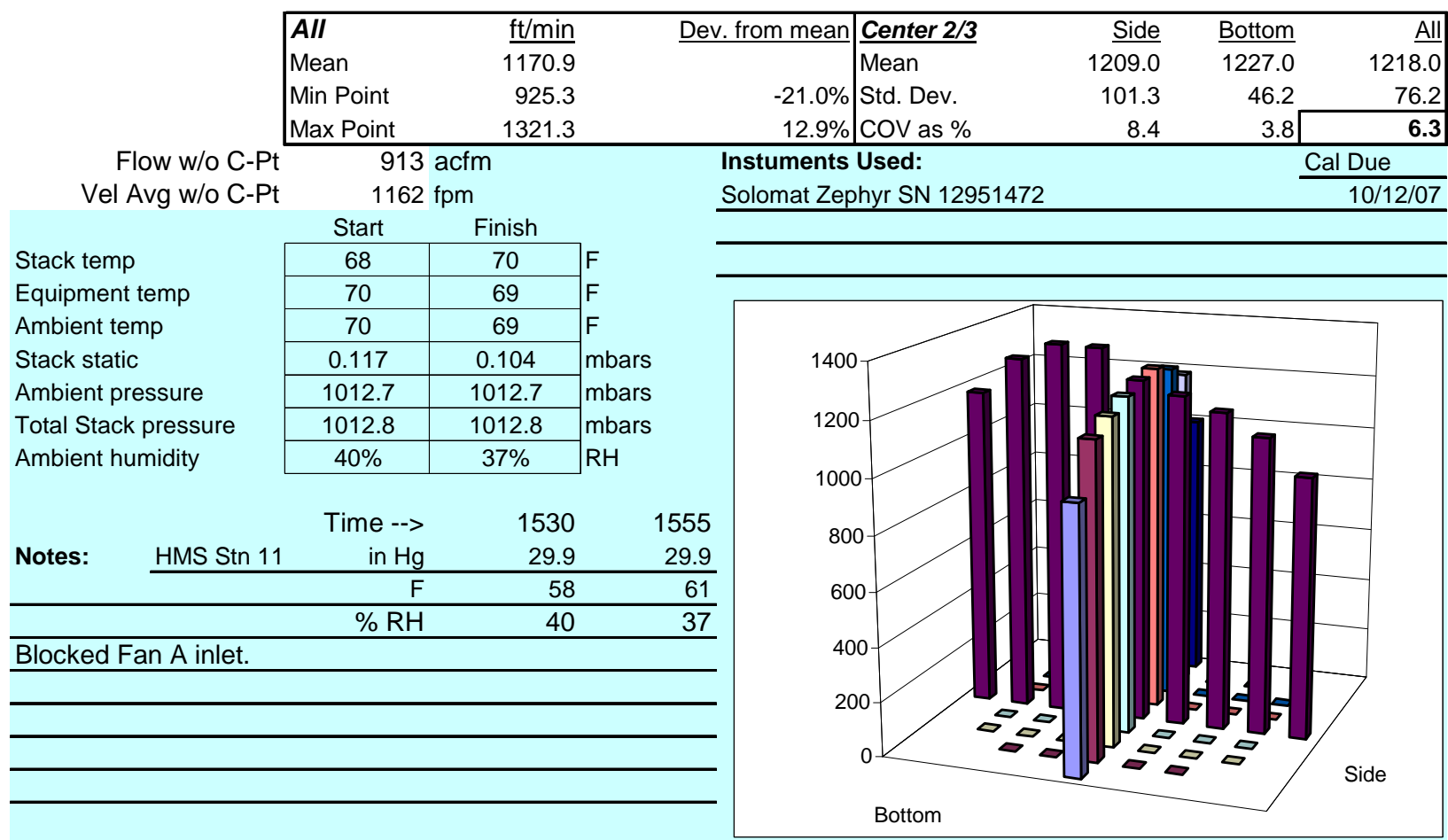

Signature signifies compliance with

Signature verifying data and calculations:

Procedure EMS-JAG-4

Signature/date

Signatures on original data sheet in Test Instruction Package TI-RPP-WTP-462

Reference: CCP-WTPSP-179

(HVC2_VEL-dataRev0 (1)).xIs/VT4

VEL-dataRev0.xls

$4 / 18 / 2007$

3 August 2006 


\section{VELOCITY TRAVERSE DATA FORM}

Site HV-C2 Model Date 11/7/06

Testers JAG \& JGD

$$
\text { Stack Dia. }
$$
Stack X-Area

Elevation Port

Distance to disturbance Velocity units $\overline{\mathrm{ft} / \mathrm{min}}$
Run No. VT-5

Fan Configuration $\mathbf{A}$ \& $\mathbf{B}$

Fan Setting 40

Stack Temp

Start/End Time 1445 -- 1530

Center $2 / 3$ from

Points in Center 2/3

Data Files: NA

\begin{tabular}{|c|c|c|c|c|c|c|c|c|c|}
\hline \multirow{3}{*}{\multicolumn{2}{|c|}{$\begin{array}{l}\text { Order --> } \\
\text { Traverse--> } \\
\text { Trial ----> }\end{array}$}} & \multicolumn{4}{|l|}{$1 \mathrm{st}$} & \multicolumn{4}{|l|}{ 2nd } \\
\hline & & \multicolumn{4}{|c|}{ Side } & \multicolumn{4}{|c|}{ Bottom } \\
\hline & & \multicolumn{2}{|l|}{1} & 3 & Mean & 1 & 2 & 3 & Mean \\
\hline CorrectLabel & Depth, in. & \multicolumn{4}{|c|}{ Velocity } & \multicolumn{4}{|c|}{ Velocity } \\
\hline 1 & 0.50 & 2695 & 2136 & 2546 & 2459.0 & 2110 & 1937 & 2328 & 2125.0 \\
\hline 2 & 1.26 & 3024 & 3078 & 3066 & 3056.0 & 2693 & 2736 & 2680 & 2703.0 \\
\hline 3 & 2.33 & 3030 & 3032 & 3139 & 3067.0 & 2808 & 2850 & 2860 & 2839.3 \\
\hline 4 & 3.88 & 3097 & 2991 & 3024 & 3037.3 & 2917 & 2936 & 2861 & 2904.7 \\
\hline Center & 6.00 & 2896 & 2966 & 3027 & 2963.0 & 3169 & 3082 & 3030 & 3093.7 \\
\hline 5 & 8.12 & 3065 & 3128 & 3071 & 3088.0 & 3256 & 3296 & 3282 & 3278.0 \\
\hline 6 & 9.67 & 3259 & 3252 & 3260 & 3257.0 & 3300 & 3312 & 3262 & 3291.3 \\
\hline 7 & 10.74 & 3190 & 3178 & 3058 & 3142.0 & 3125 & 3200 & 3074 & 3133.0 \\
\hline 8 & 11.50 & 2480 & 2450 & 2597 & 2509.0 & 2443 & 2103 & 2194 & 2246.7 \\
\hline Averages --- & & 2970.7 & 2912.3 & 2976.4 & 2953.1 & 2869.0 & 2828.0 & 2841.2 & 2846.1 \\
\hline
\end{tabular}

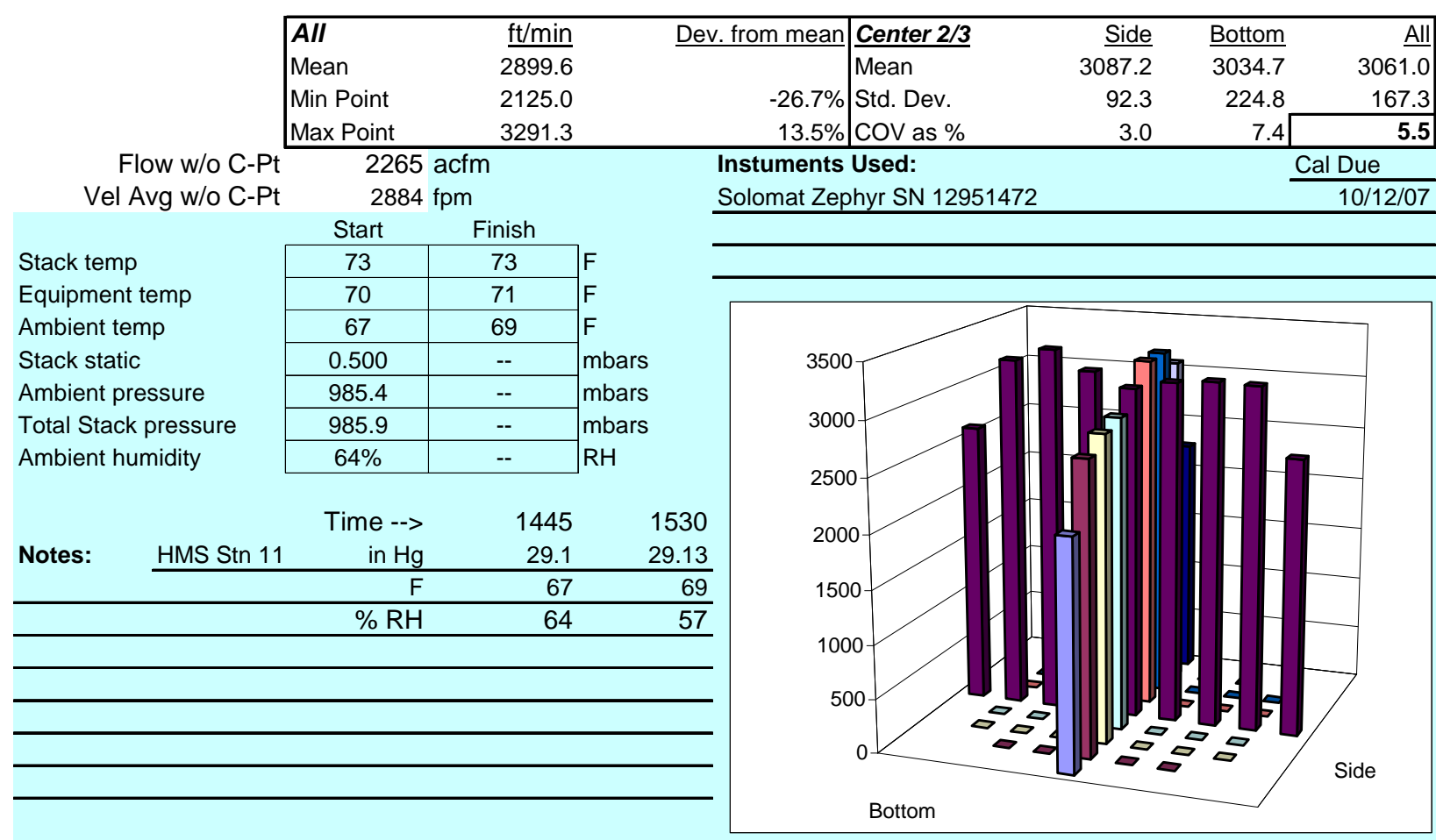

Signature signifies compliance with

Procedure EMS-JAG-4

Signature/date

Signature verifying data and calculations:

Signatures on original data sheet in Test Instruction Package TI-RPP-WTP-462

Reference: CCP-WTPSP-179

(HVC2_VEL-dataRev0 (1)).xIs/VT5

VEL-dataRev0.xls

$4 / 18 / 2007$

3 August 2006 


\section{VELOCITY TRAVERSE DATA FORM}

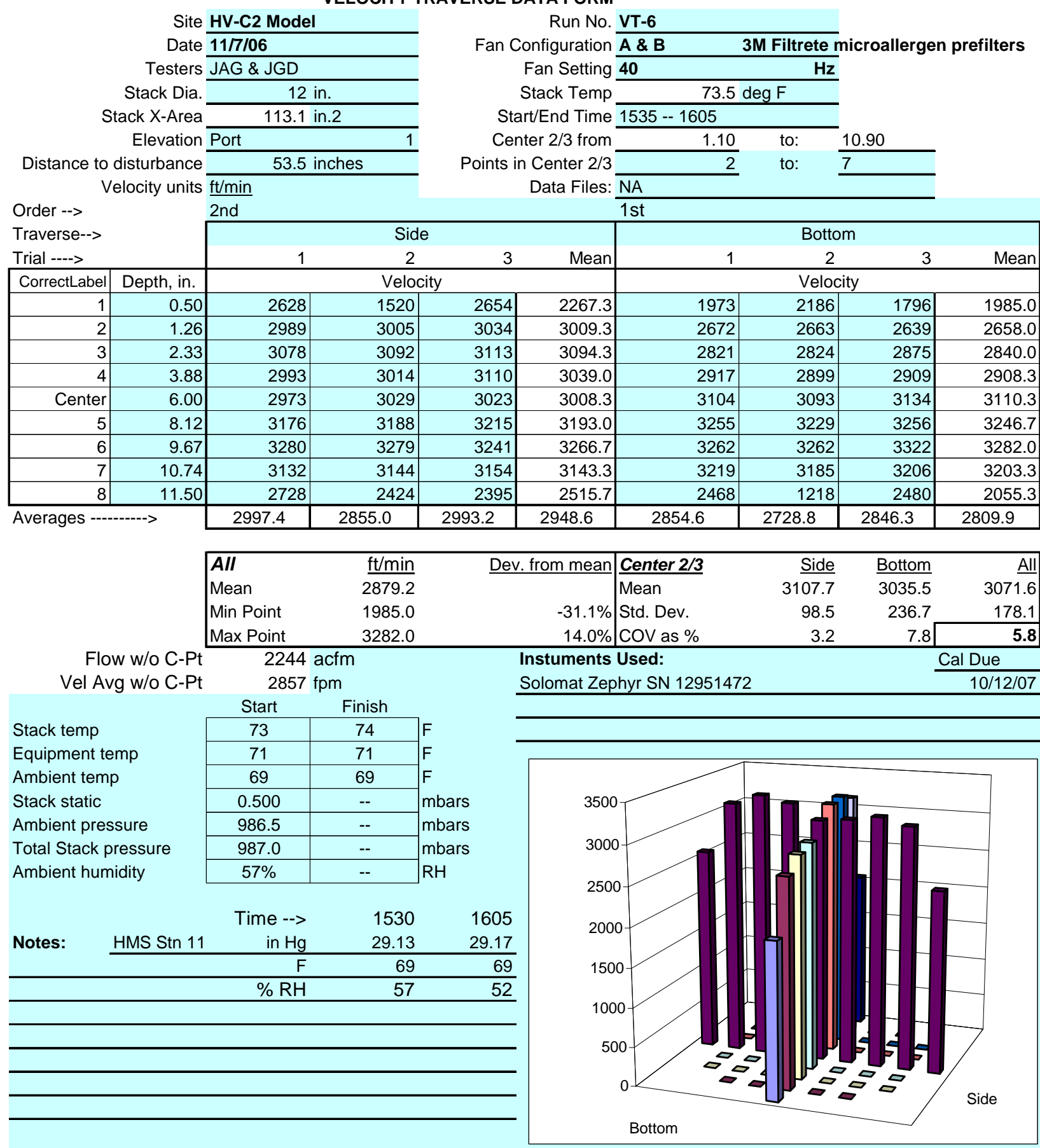

Signature signifies compliance with

Procedure EMS-JAG-4

Signature/date Signatures on original data sheet in Test Instruction Package TI-RPP-WTP-462

Reference: CCP-WTPSP-179

(HVC2_VEL-dataRev0 (1)).xIs/VT6

VEL-dataRev0.xls

$4 / 18 / 2007$

3 August 2006 


\section{VELOCITY TRAVERSE DATA FORM}

Site HV-C2 Model Date 11/7/06

Testers JAG \& JGD

$$
\text { Stack Dia. }
$$
Stack X-Area

Elevation Port

Distance to disturbance Velocity units $\overline{\mathrm{ft} / \mathrm{min}}$
Run No. VT-7

Fan Configuration $\mathbf{A}$ \& B

Fan Setting 40

Stack Temp

Start/End Time 1610 -- 1640

Center $2 / 3$ from

Points in Center 2/3

Data Files: NA

\begin{tabular}{|c|c|c|c|c|c|c|c|c|c|}
\hline \multirow{3}{*}{\multicolumn{2}{|c|}{$\begin{array}{l}\text { Order --> } \\
\text { Traverse--> } \\
\text { Trial ----> }\end{array}$}} & \multicolumn{4}{|l|}{$1 \mathrm{st}$} & \multicolumn{4}{|l|}{ 2nd } \\
\hline & & \multicolumn{4}{|c|}{ Side } & \multicolumn{4}{|c|}{ Bottom } \\
\hline & & \multicolumn{2}{|l|}{1} & 3 & Mean & 1 & 2 & 3 & Mean \\
\hline CorrectLabel & Depth, in. & \multicolumn{4}{|c|}{ Velocity } & \multicolumn{4}{|c|}{ Velocity } \\
\hline 1 & 0.50 & 2661 & 2645 & 2827 & 2711.0 & 2294 & 1940 & 1841 & 2025.0 \\
\hline 2 & 1.26 & 3078 & 3062 & 3110 & 3083.3 & 2723 & 2671 & 2779 & 2724.3 \\
\hline 3 & 2.33 & 3096 & 3094 & 3100 & 3096.7 & 2822 & 2965 & 2879 & 2888.7 \\
\hline 4 & 3.88 & 3096 & 3060 & 3021 & 3059.0 & 2881 & 2983 & 2917 & 2927.0 \\
\hline Center & 6.00 & 2995 & 3054 & 2960 & 3003.0 & 3154 & 3206 & 3201 & 3187.0 \\
\hline 5 & 8.12 & 3144 & 3203 & 3198 & 3181.7 & 3320 & 3295 & 3330 & 3315.0 \\
\hline 6 & 9.67 & 3265 & 3254 & 3283 & 3267.3 & 3339 & 3347 & 3355 & 3347.0 \\
\hline 7 & 10.74 & 3118 & 3144 & 3152 & 3138.0 & 3234 & 3225 & 3277 & 3245.3 \\
\hline 8 & 11.50 & 2736 & 2349 & 2393 & 2492.7 & 2446 & 2309 & 2709 & 2488.0 \\
\hline Averages --- & & 3021.0 & 2985.0 & 3004.9 & 3003.6 & 2912.6 & 2882.3 & 2920.9 & 2905.3 \\
\hline
\end{tabular}

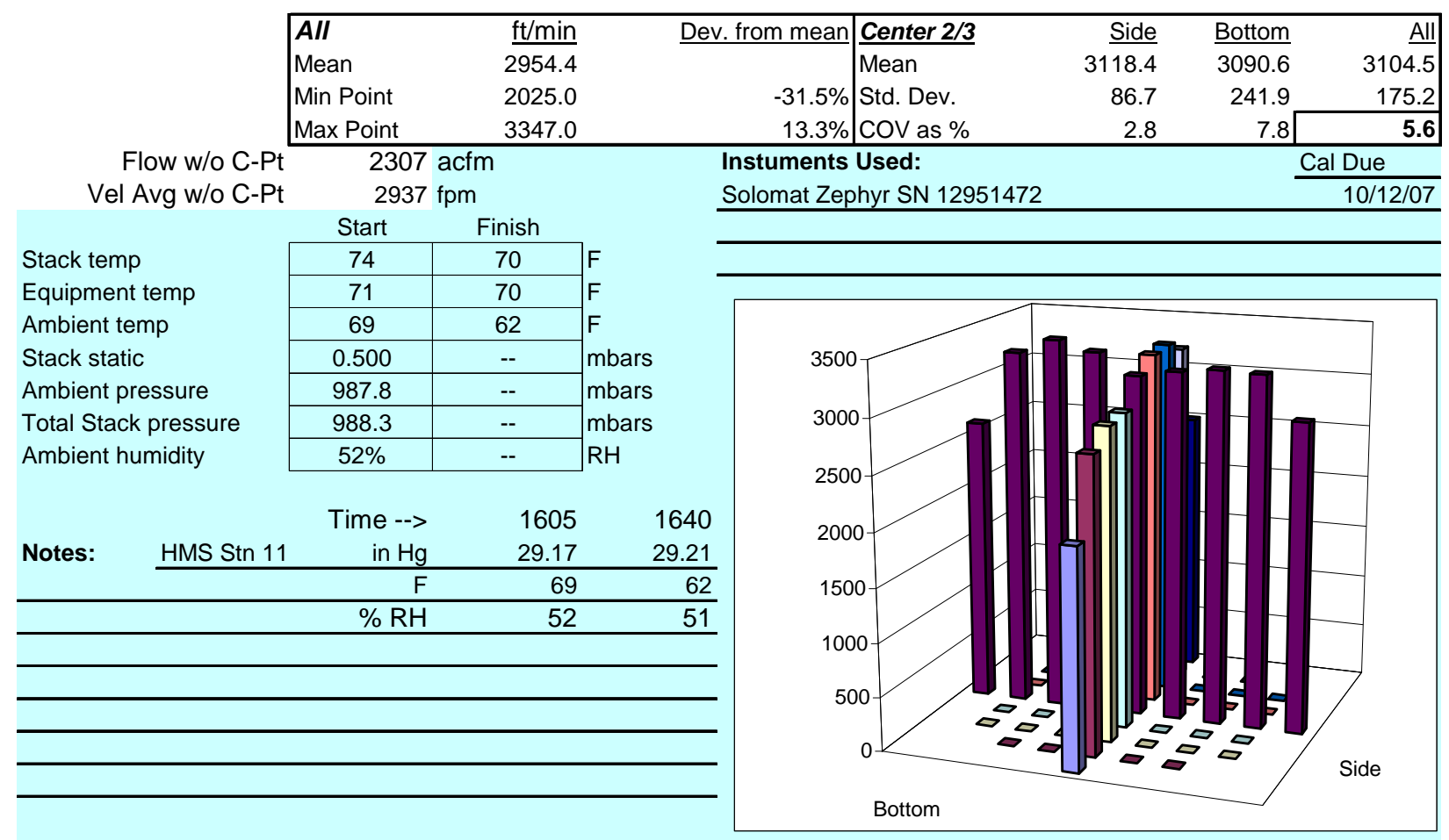

Signature signifies compliance with

Procedure EMS-JAG-4

Signature/date

Signature verifying data and calculations:

Signatures on original data sheet in Test Instruction Package TI-RPP-WTP-462

Reference: CCP-WTPSP-179

(HVC2_VEL-dataRev0 (1)).xIs/VT7

VEL-dataRev0.xls

$4 / 18 / 2007$

3 August 2006 


\section{VELOCITY TRAVERSE DATA FORM}

Site HV-C2 Model Date 11/9/06

Testers JAG

$$
\text { Stack Dia. }
$$
Stack X-Area

Elevation Port

Distance to disturbance Velocity units $\overline{\mathrm{ft} / \mathrm{min}}$
Run No. VT-8

Fan Configuration $\mathbf{A}$ \& $\mathbf{B}$

Fan Setting 40

Stack Temp

Start/End Time 1500 -- 1552

Center $2 / 3$ from

Points in Center 2/3

Data Files: NA

\begin{tabular}{|c|c|c|c|c|c|c|c|c|c|}
\hline \multirow{3}{*}{\multicolumn{2}{|c|}{$\begin{array}{l}\text { Order --> } \\
\text { Traverse--> } \\
\text { Trial ----> }\end{array}$}} & \multicolumn{4}{|l|}{$1 \mathrm{st}$} & \multicolumn{4}{|l|}{ 2nd } \\
\hline & & \multicolumn{4}{|c|}{ Side } & \multicolumn{4}{|c|}{ Bottom } \\
\hline & & \multicolumn{2}{|l|}{1} & 3 & Mean & 1 & 2 & 3 & Mean \\
\hline CorrectLabel & Depth, in. & \multicolumn{4}{|c|}{ Velocity } & \multicolumn{4}{|c|}{ Velocity } \\
\hline 1 & 0.50 & 1899 & 2827 & 2751 & 2492.3 & 1882 & 1632 & 1812 & 1775.3 \\
\hline 2 & 1.26 & 3005 & 3052 & 3060 & 3039.0 & 2662 & 2553 & 2634 & 2616.3 \\
\hline 3 & 2.33 & 3127 & 3144 & 3112 & 3127.7 & 2831 & 2845 & 2820 & 2832.0 \\
\hline 4 & 3.88 & 3009 & 2999 & 3090 & 3032.7 & 2896 & 2852 & 2881 & 2876.3 \\
\hline Center & 6.00 & 3049 & 2991 & 3085 & 3041.7 & 3124 & 3128 & 3089 & 3113.7 \\
\hline 5 & 8.12 & 3202 & 3246 & 3217 & 3221.7 & 3352 & 3330 & 3303 & 3328.3 \\
\hline 6 & 9.67 & 3336 & 3302 & 3302 & 3313.3 & 3366 & 3353 & 3385 & 3368.0 \\
\hline 7 & 10.74 & 3183 & 3121 & 3178 & 3160.7 & 3347 & 3355 & 3294 & 3332.0 \\
\hline 8 & 11.50 & 2778 & 2321 & 2487 & 2528.7 & 2275 & 2439 & 2649 & 2454.3 \\
\hline Averages --- & & 2954.2 & 3000.3 & 3031.3 & 2995.3 & 2859.4 & 2831.9 & 2874.1 & 2855.1 \\
\hline
\end{tabular}

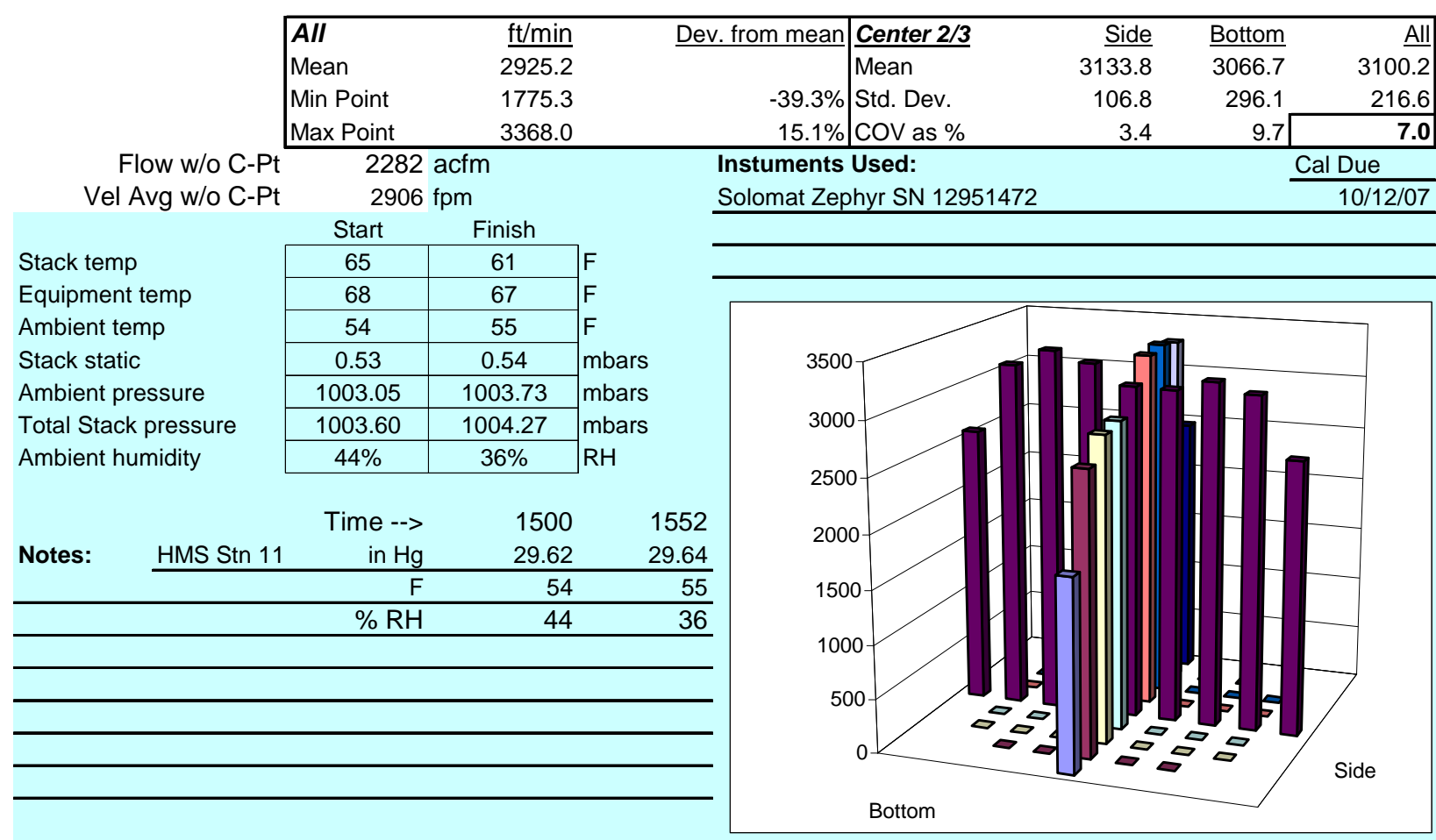

Signature signifies compliance with

Signature verifying data and calculations:

Procedure EMS-JAG-4

Signature/date

Signatures on original data sheet in Test Instruction Package TI-RPP-WTP-462

Reference: CCP-WTPSP-179

(HVC2_VEL-dataRev0 (1)).xIs/VT8

VEL-dataRev0.xls

$4 / 18 / 2007$

3 August 2006 


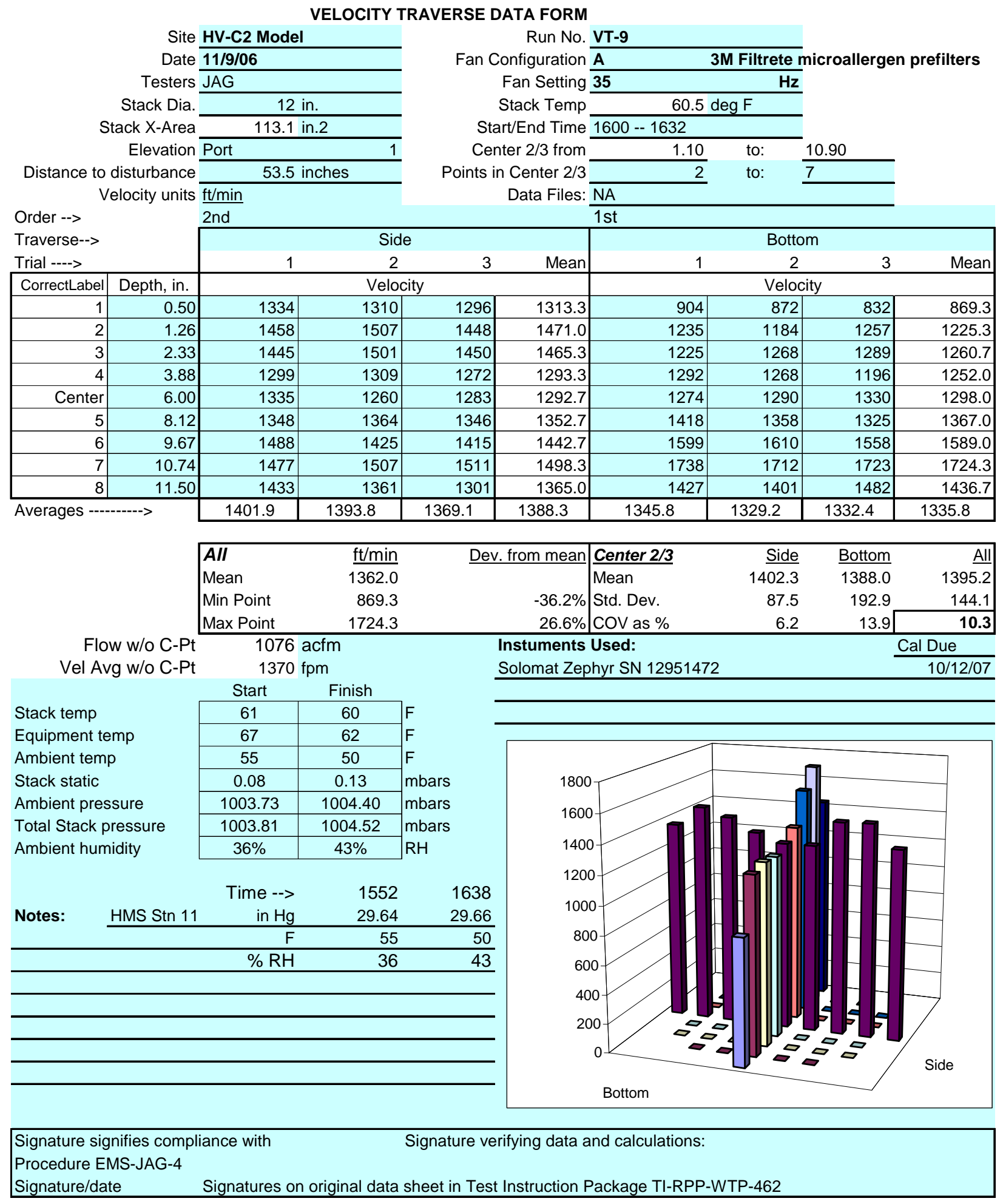

Reference: CCP-WTPSP-179

(HVC2_VEL-dataRev0 (1)).xls/VT9

VEL-dataRev0.xIs

$4 / 18 / 2007$

3 August 2006 


\section{VELOCITY TRAVERSE DATA FORM}

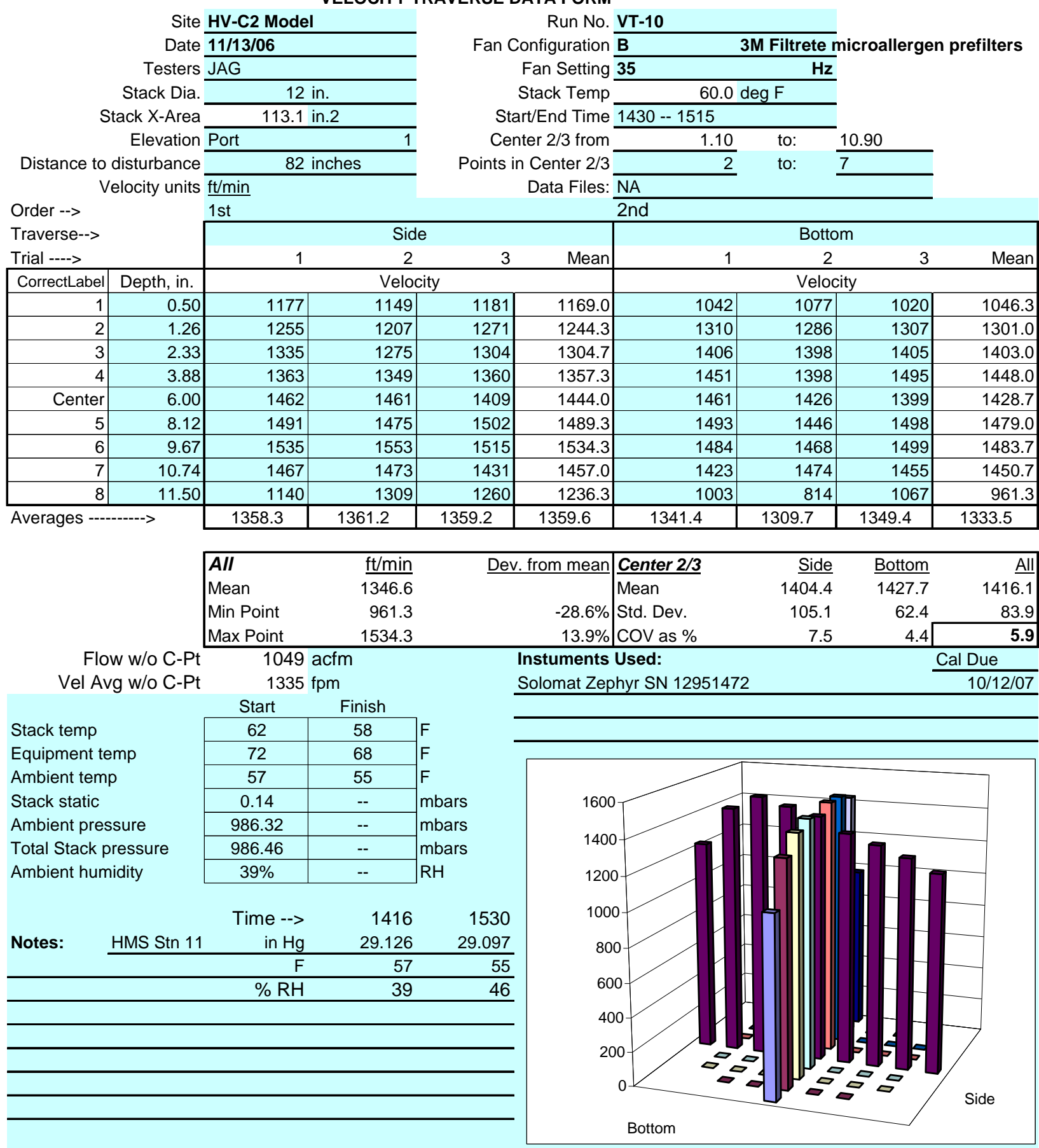

Signature signifies compliance with

Procedure EMS-JAG-4

Signature/date Signatures on original data sheet in Test Instruction Package TI-RPP-WTP-462

Reference: CCP-WTPSP-179

(HVC2_VEL-dataRev0 (1)).xIs/VT10

VEL-dataRev0.xIs

$4 / 18 / 2007$

3 August 2006 


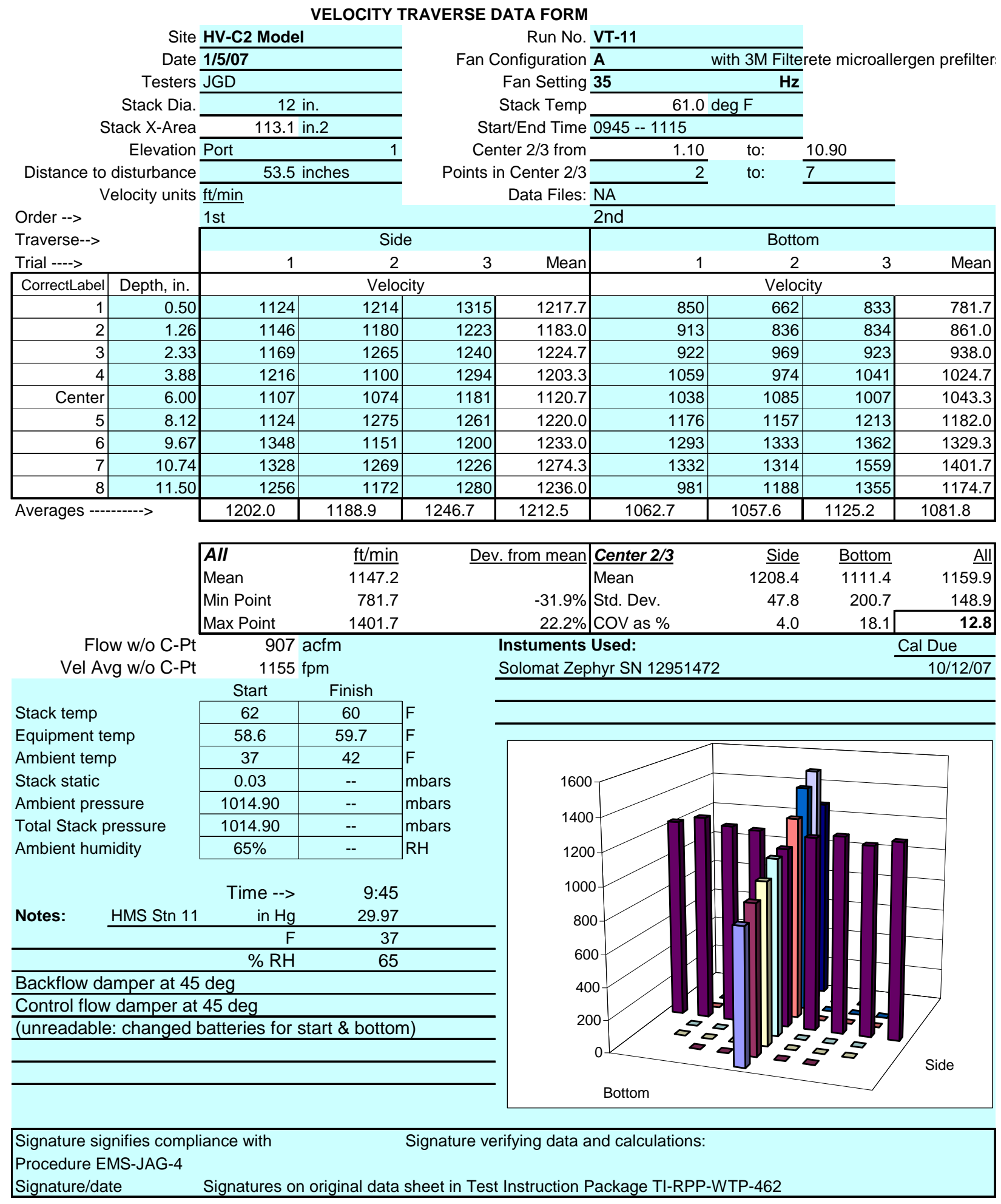

Reference: CCP-WTPSP-179

(HVC2_VEL-dataRev0 (1)).xIs/VT11

VEL-dataRev0.xIs

$4 / 18 / 2007$

3 August 2006 


\section{VELOCITY TRAVERSE DATA FORM}

Site HV-C2 Model

Date $\mathbf{1 / 5 / 0 7}$

Testers JGD \& MSP

Stack Dia. Stack X-Area

Elevation Port

Distance to disturbance Velocity units $\underline{\mathrm{ft} / \mathrm{min}}$
Run No. VT-12

Fan Configuration A \& B

Fan Setting 40

Stack Temp

Start/End Time 1315 -- 1430

Center $2 / 3$ from

Points in Center 2/3

Data Files: NA
Order -->
Traverse-->
Trial ---->
\begin{tabular}{|r|r|}
\hline CorrectLabel & Depth, in. \\
\hline 1 & 0.50 \\
\hline 2 & 1.26 \\
\hline 3 & 2.33 \\
\hline 4 & 3.88 \\
\hline Center & 6.00 \\
\hline 5 & 8.12 \\
\hline 6 & 9.67 \\
\hline 7 & 10.74 \\
\hline 8 & 11.50 \\
\hline
\end{tabular}

\begin{tabular}{|l|l|l|l|}
\hline Averages ---------> & 2808.7 & 2841.9 & 2855.6 \\
\cline { 2 - 4 }
\end{tabular} 2nd Velocity
Data Files: NA

\begin{tabular}{|r|r|r|r|r|}
\cline { 2 - 5 } & \multicolumn{5}{|c|}{ 1st } \\
\hline & \multicolumn{5}{|c|}{$\begin{array}{c}\text { Bottom } \\
2\end{array}$} \\
\hline 2589.0 & 1915 & 2241 & 2155 & 2103.7 \\
\hline 2852.3 & 2406 & 2495 & 2367 & 2422.7 \\
\hline 2980.3 & 2692 & 2599 & 2531 & 2607.3 \\
\hline 2799.3 & 2653 & 2650 & 2643 & 2648.7 \\
\hline 2657.0 & 2743 & 2722 & 2702 & 2722.3 \\
\hline 2760.3 & 3006 & 2977 & 2919 & 2967.3 \\
\hline 3031.7 & 3219 & 3211 & 3113 & 3181.0 \\
\hline 3063.0 & 3147 & 3076 & 3110 & 3111.0 \\
\hline 2785.3 & 1804 & 1178 & 1370 & 1450.7 \\
\hline 2835.4 & 2620.6 & 2572.1 & 2545.6 & 2579.4 \\
\hline
\end{tabular}

\begin{tabular}{|ll}
\hline All & $\underline{\mathrm{ft} / \mathrm{min}}$ \\
Mean & 2707.4 \\
Min Point & 1450.7 \\
Max Point & 3181.0 \\
\hline
\end{tabular}

Flow w/o C-Pt Side

\begin{tabular}{|l|l|r|r|r|}
\hline 50 & 2733 & 2660 & 2374 & 2589.0 \\
\hline 33 & 2888 & 2875 & 2794 & 2852.3 \\
\hline 6.00 & 2939 & 2975 & 3027 & 2980.3 \\
\hline 12 & 2628 & 2799 & 2871 & 2799.3 \\
\hline .67 & 2723 & 2622 & 2725 & 2657.0 \\
\hline .74 & 3027 & 3085 & 3075 & 3031.7 \\
\hline & 2681 & 2762 & 2913 & 3063.0 \\
\hline
\end{tabular}

$2128 \mathrm{acfm}$
Vel Avg w/o C-Pt $2710 \mathrm{fpm}$

\begin{tabular}{|c|c|l|}
\multicolumn{1}{c|}{ Start } & Finish & \\
\hline $62(58-65)$ & 62 & $F$ \\
\hline 57 & 60.6 & F \\
\hline $64(41)$ & 61 & F \\
\hline 0.40 & -- & mbars \\
\hline 1012.00 & -- & mbars \\
\hline 1012.00 & -- & mbars \\
\hline $56(25) \%$ & -- & RH \\
\hline
\end{tabular}

Stack temp Equipment temp Ambient temp Stack static Ambient pressure Total Stack pressure Ambient humidity

$$
\begin{array}{|l}
\hline 62 \\
\hline \\
\hline \\
\hline \\
\hline \\
\hline 5 \\
\hline 56 \\
\hline
\end{array}
$$

\begin{tabular}{lrrr} 
& & Time --> & $13: 15$ \\
Notes: & HMS Stn 11 & in Hg & 29.88 \\
\cline { 2 - 4 } & \% RH & 41 \\
\hline \multicolumn{3}{c}{ \% } \\
\hline Control dampers: Full Open \\
\hline Backflow: 70 deg.
\end{tabular}

\begin{tabular}{r|l} 
Dev. from mean & Center $2 / 3$ \\
& Mean \\
$-46.4 \%$ & Std. Dev. \\
$17.5 \%$ & COV as \% \\
\hline
\end{tabular}

\begin{tabular}{|c|c|c|}
\hline Side & Bottom & All \\
\hline 2877.7 & 2808.6 & 2843.2 \\
\hline 151.6 & 282.2 & 220.6 \\
\hline 5.3 & 10.0 & 7.8 \\
\hline
\end{tabular}

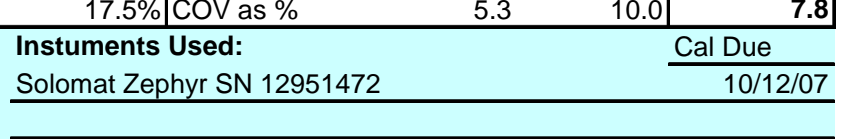

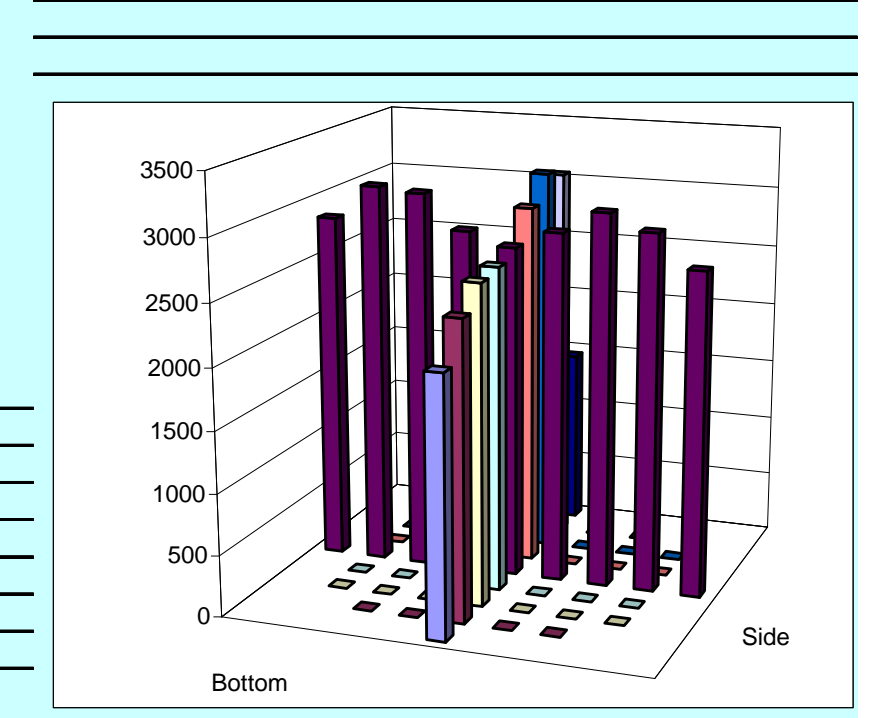

Signature signifies compliance with Procedure EMS-JAG-4

Signature/date Signatures on original data sheet in Test Instruction Package TI-RPP-WTP-462

Signature verifying data and calculations:

Reference: CCP-WTPSP-179

(HVC2_VEL-dataRev0 (1)).xIs/VT12 VEL-dataRev0.xls $4 / 18 / 2007$ 


\section{VELOCITY TRAVERSE DATA FORM}

Site HV-C2 Model

Date 1/5/07

Testers JGD \& MSP

$$
\text { Stack Dia. }
$$

Stack X-Area

Elevation Port

Distance to disturbance

Velocity units $\underline{\mathrm{ft} / \mathrm{min}}$
Run No. VT-13

Fan Configuration $\mathbf{A}$ \& $\mathbf{B}$

Fan Setting $\mathbf{4 0}$

Stack Temp

Start/End Time $1430-1510$

Center $2 / 3$ from

Points in Center $2 / 3$

Data Files: NA
Order -->
Traverse-->
Trial ---->
\begin{tabular}{|r|r|}
\hline CorrectLabel & Depth, in. \\
\hline 1 & 0.50 \\
\hline 2 & 1.26 \\
\hline 3 & 2.33 \\
\hline 4 & 3.88 \\
\hline Center & 6.00 \\
\hline 5 & 8.12 \\
\hline 6 & 9.67 \\
\hline 7 & 10.74 \\
\hline 8 & 11.50 \\
\hline
\end{tabular}

\begin{tabular}{l|l}
\hline Averages ---------> & 2738.9 \\
\hline
\end{tabular}

\section{2nd}

13.75 inches

\begin{tabular}{|c|c|}
\hline \multicolumn{2}{|r|}{ Side } \\
\hline 1 & 2 \\
\hline & Velocit \\
\hline 2282 & 2538 \\
\hline 2586 & 2663 \\
\hline 2793 & 2785 \\
\hline 2832 & 2881 \\
\hline 2806 & 2827 \\
\hline 2871 & 2900 \\
\hline 2899 & 2819 \\
\hline 2819 & 2871 \\
\hline 2762 & 2739 \\
\hline 2738.9 & 2780.3 \\
\hline$A I I$ & $\underline{\mathrm{ft} / \mathrm{min}}$ \\
\hline Mean & $\overline{2681.7}$ \\
\hline Min Point & 2237.0 \\
\hline Max Point & 2889.3 \\
\hline
\end{tabular}

Flow w/o C-Pt Vel Avg w/o C-Pt

Stack temp

Equipment temp

Ambient temp

Stack static

Ambient pressure

Total Stack pressure

Ambient humidity

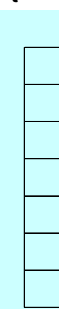

\begin{tabular}{|c|c|l}
\multicolumn{1}{c|}{ Start } & Finish & \\
\hline 62 & 61 & $F$ \\
\hline 60.6 & 63.3 & $F$ \\
\hline 60 & 60 & F \\
\hline 0.14 & -- & mbars \\
\hline 1012.00 & -- & mbars \\
\hline 1012.00 & -- & mbars \\
\hline $26 \%$ & -- & RH \\
\hline
\end{tabular}

\begin{tabular}{|c|c|c|c|}
\hline \multirow[t]{2}{*}{ Notes: } & \multirow[t]{2}{*}{ HMS Stn 11} & Time --> & $\begin{array}{r}14: 30 \\
29.82\end{array}$ \\
\hline & & $\mathrm{F}$ & 41 \\
\hline & & $\% \mathrm{RH}$ & 55 \\
\hline \multicolumn{4}{|c|}{ Control dampers: full open } \\
\hline Backflc & 0 deg. & & \\
\hline
\end{tabular}

Backflow: 70 deg.

\begin{tabular}{|c|c|c|c|c|c|}
\hline \multicolumn{6}{|c|}{$1 s t$} \\
\hline \multirow{3}{*}{3} & \multirow{3}{*}{ Mean } & \multicolumn{4}{|c|}{ Bottom } \\
\hline & & \multirow[t]{2}{*}{1} & 2 & 3 & Mean \\
\hline & & & \multicolumn{2}{|c|}{ Velocity } & \\
\hline 2190 & 2336.7 & 2308 & 2240 & 2163 & 2237.0 \\
\hline 2516 & 2588.3 & 2337 & 2331 & 2370 & 2346.0 \\
\hline 2757 & 2778.3 & 2501 & 2496 & 2437 & 2478.0 \\
\hline 2871 & 2861.3 & 2693 & 2654 & 2616 & 2654.3 \\
\hline 2819 & 2817.3 & 2772 & 2827 & 2805 & 2801.3 \\
\hline 2852 & 2874.3 & 2863 & 2857 & 2866 & 2862.0 \\
\hline 2915 & 2877.7 & 2913 & 2884 & 2871 & 2889.3 \\
\hline 2836 & 2842.0 & 2792 & 2775 & 2830 & 2799.0 \\
\hline 2633 & 2711.3 & 2547 & 2540 & 2460 & 2515.7 \\
\hline 09.9 & 2743.0 & 2636.2 & 2622.7 & 2602.0 & 2620.3 \\
\hline
\end{tabular}

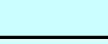

with 3M Filterete microallergen prefilter:

$61.5 \mathrm{~Hz}$

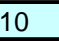
10 to: 10.90

to: 


\section{VELOCITY TRAVERSE DATA FORM}

Site HV-C2 Model

Date 1/5/07

Testers JGD \& MSP

\section{Stack Dia.} Stack X-Area

Elevation Port

Distance to disturbance Velocity units $\underline{\mathrm{ft} / \mathrm{min}}$
Run No. VT-14

Fan Configuration $\mathbf{A}$ \& $\mathbf{B}$

Fan Setting 40

Stack Temp

Start/End Time 1510 -- 1540

Center $2 / 3$ from

Points in Center $2 / 3$

Data Files: NA

\begin{tabular}{|c|c|c|c|c|c|c|c|c|c|}
\hline \multirow{3}{*}{\multicolumn{2}{|c|}{$\begin{array}{l}\text { Order --> } \\
\text { Traverse--> } \\
\text { Trial ----> }\end{array}$}} & \multicolumn{8}{|c|}{ 2nd } \\
\hline & & \multicolumn{4}{|c|}{ Side } & \multicolumn{4}{|c|}{ Bottom } \\
\hline & & 1 & 2 & 3 & Mean & 1 & 2 & 3 & Mean \\
\hline CorrectLabel & Depth, in. & \multicolumn{4}{|c|}{ Velocity } & \multicolumn{4}{|c|}{ Velocity } \\
\hline 1 & 0.50 & 2364 & 2482 & 2270 & 2372.0 & 2356 & 2298 & 2321 & 2325.0 \\
\hline 2 & 1.26 & 2697 & 2685 & 2665 & 2682.3 & 2620 & 2480 & 2537 & 2545.7 \\
\hline 3 & 2.33 & 2893 & 2850 & 2813 & 2852.0 & 2641 & 2624 & 2644 & 2636.3 \\
\hline 4 & 3.88 & 2909 & 2878 & 2922 & 2903.0 & 2809 & 2798 & 2798 & 2801.7 \\
\hline Center & 6.00 & 2893 & 2848 & 2857 & 2866.0 & 2856 & 2883 & 2864 & 2867.7 \\
\hline 5 & 8.12 & 2874 & 2835 & 2917 & 2875.3 & 2996 & 2967 & 2946 & 2969.7 \\
\hline 6 & 9.67 & 2800 & 2847 & 2900 & 2849.0 & 2932 & 2964 & 2986 & 2960.7 \\
\hline 7 & 10.74 & 2735 & 2704 & 2772 & 2737.0 & 2763 & 2767 & 2742 & 2757.3 \\
\hline 8 & 11.50 & 2530 & 2526 & 2422 & 2492.7 & 2502 & 2310 & 2316 & 2376.0 \\
\hline Averages --- & -----> & 2743.9 & 2739.4 & 2726.4 & 2736.6 & 2719.4 & 2676.8 & 2683.8 & 2693.3 \\
\hline
\end{tabular}

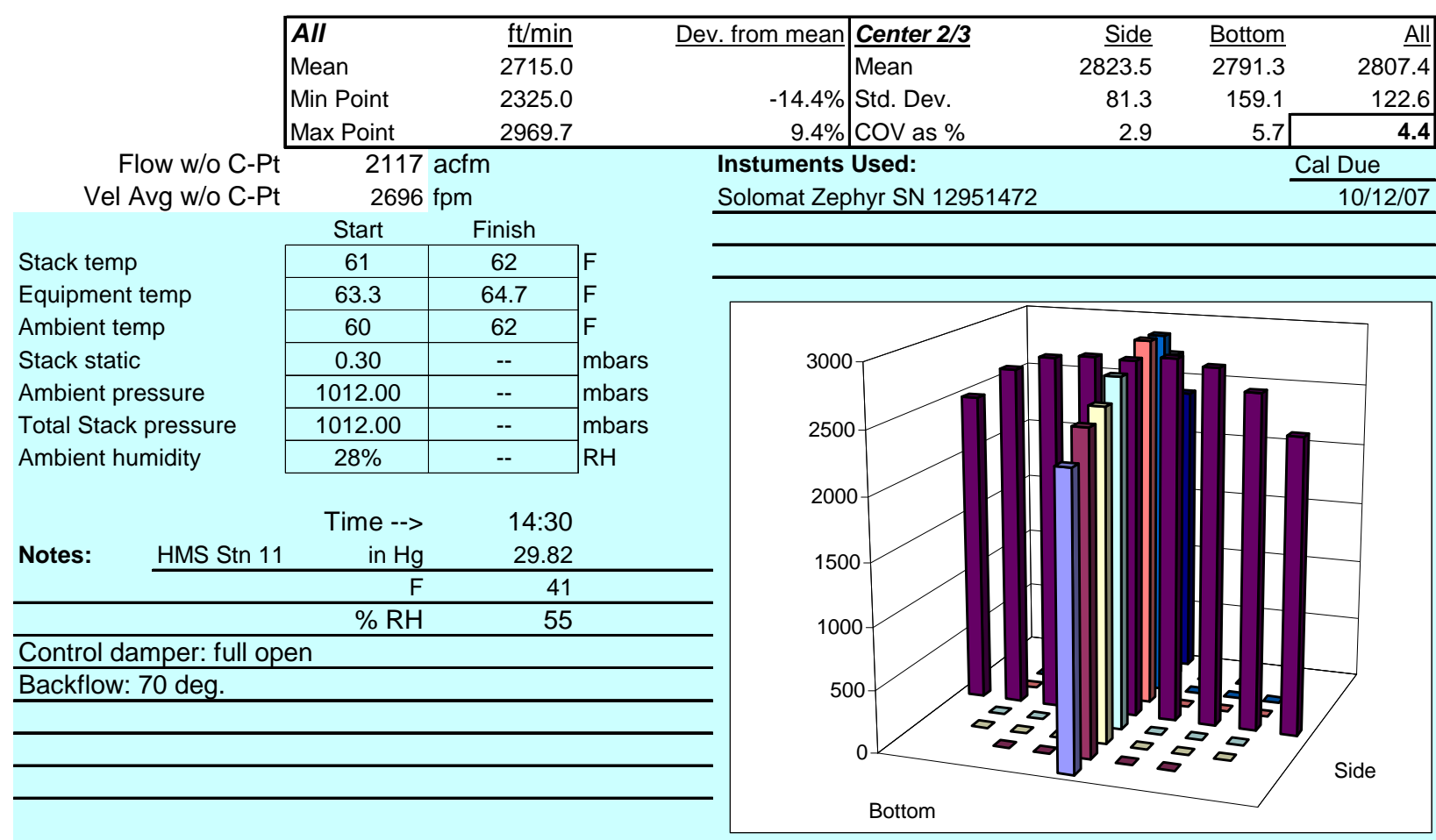

Signature signifies compliance with

Signature verifying data and calculations:

Procedure EMS-JAG-4

Signature/date

Signatures on original data sheet in Test Instruction Package TI-RPP-WTP-462

Reference: CCP-WTPSP-179

(HVC2_VEL-dataRev0 (1)).xIs/VT14

VEL-dataRev0.xls

$4 / 18 / 2007$

3 August 2006 


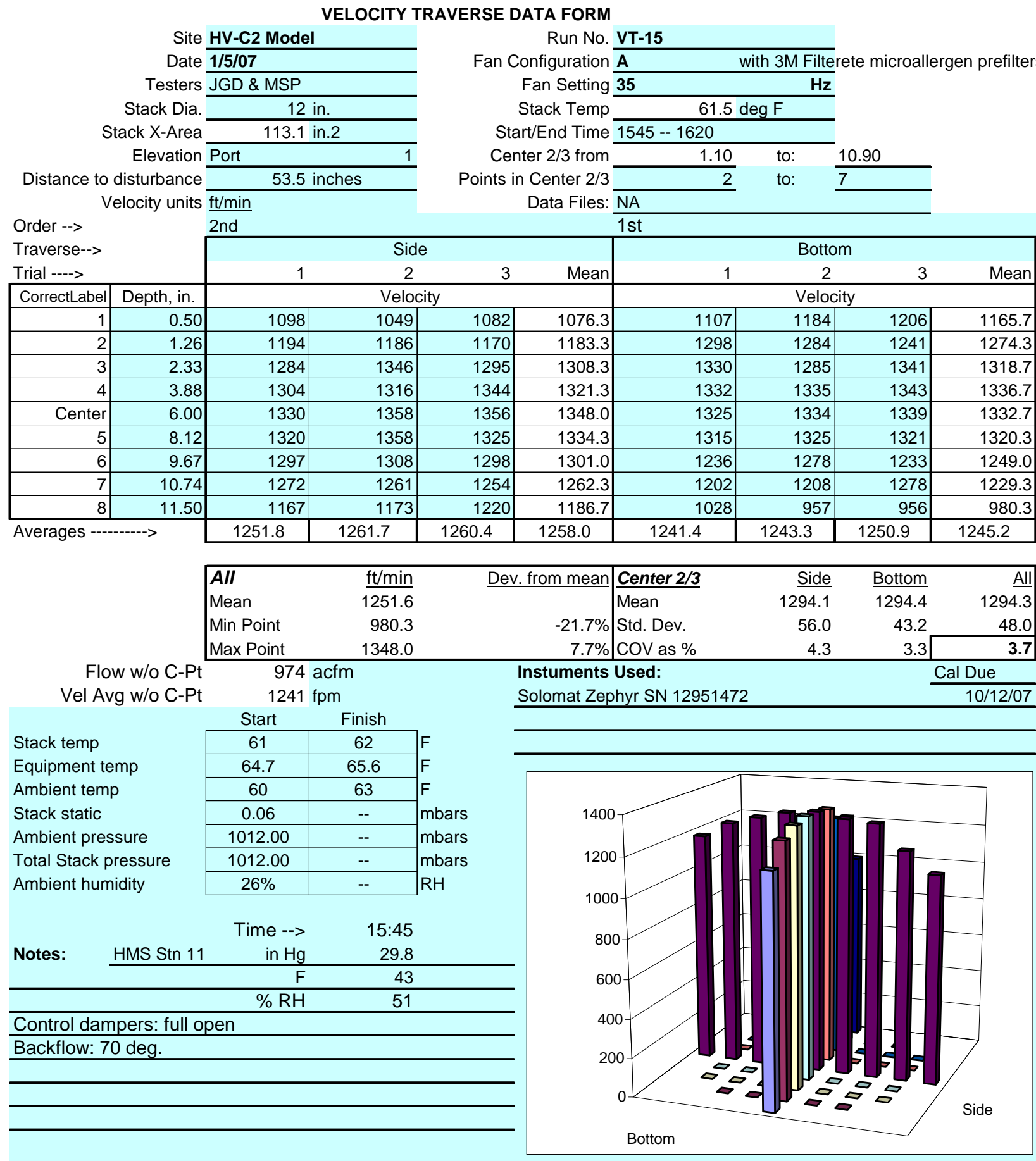

Signature signifies compliance with

Procedure EMS-JAG-4

Signature/date Signatures on original data sheet in Test Instruction Package TI-RPP-WTP-462

Reference: CCP-WTPSP-179

(HVC2_VEL-dataRev0 (1)).xIs/VT15

VEL-dataRev0.xIs

$4 / 18 / 2007$

3 August 2006 


\section{VELOCITY TRAVERSE DATA FORM}

Site HV-C2 Model Date 1/5/07

Testers JGD \& MSP

\section{Stack Dia.} Stack X-Area

Elevation Port

Distance to disturbance Velocity units $\mathrm{ft} / \mathrm{min}$
Run No. VT-16

Fan Configuration $\mathbf{A}$

Fan Setting 35

Start/End Time 1620 -- 1645

Center $2 / 3$ from

Points in Center $2 / 3$
Stack Temp
Order -->
Traverse-->
Trial ---->
\begin{tabular}{|r|r|}
\hline CorrectLabel & Depth, in. \\
\hline 1 & 0.50 \\
\hline 2 & 1.26 \\
\hline 3 & 2.33 \\
\hline 4 & 3.88 \\
\hline Center & 6.00 \\
\hline 5 & 8.12 \\
\hline 6 & 9.67 \\
\hline 7 & 10.74 \\
\hline 8 & 11.50 \\
\hline
\end{tabular}

\begin{tabular}{l|l} 
& \\
Averages ---------> & 1225.8
\end{tabular}

\section{2nd}

113.75 inches

\begin{tabular}{|c|c|}
\hline \multirow[b]{2}{*}{1} & Side \\
\hline & 2 \\
\hline & Velocit \\
\hline 1018 & 1059 \\
\hline 1127 & 1220 \\
\hline 1328 & 1310 \\
\hline 1338 & 1368 \\
\hline 1326 & 1324 \\
\hline 1289 & 1301 \\
\hline 1256 & 1259 \\
\hline 1220 & 1194 \\
\hline 1130 & 1194 \\
\hline 1225.8 & 1247.7 \\
\hline$A I I$ & $\underline{\mathrm{ft} / \mathrm{min}}$ \\
\hline Mean & $\overline{1247.9}$ \\
\hline Min Point & 1071.7 \\
\hline Max Point & 1335.0 \\
\hline
\end{tabular}

Flow w/o C-Pt Vel Avg w/o C-Pt

Stack temp Equipment temp Ambient temp Stack static Ambient pressure Total Stack pressure Ambient humidity

$$
\begin{array}{|l}
\hline \\
\hline \\
\hline \\
\hline 1 \\
\hline 101 \\
\hline
\end{array}
$$

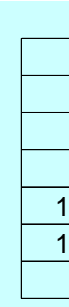
$973 \mathrm{acfm}$ $1239 \mathrm{fpm}$ Start Finish

\begin{tabular}{|c|c|}
\hline 62 & 62 \\
\hline 65.6 & 66.7 \\
\hline 63 & 61 \\
\hline 0.09 & -- \\
\hline 1012.00 & -- \\
\hline 1012.00 & -- \\
\hline $25 \%$ & -- \\
\hline
\end{tabular}

Time --> 15:43

\begin{tabular}{lrrr} 
Notes: & HMS Stn 11 & in $\mathrm{Hg}$ & 29.8 \\
\cline { 2 - 4 } & $\mathrm{F}$ & 43 \\
\hline & $\% \mathrm{RH}$ & 51 \\
\hline
\end{tabular}

Data Files: $\overline{\text { NA }}$

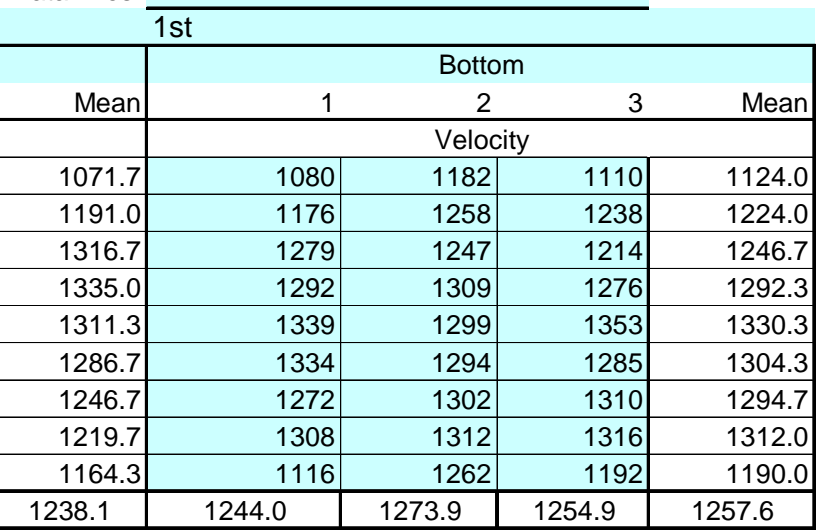

with 3M Filterete microallergen prefilter

$62.0 \mathrm{~Hz}$

5

(n)

\begin{tabular}{|c|c|c|c|c|}
\hline Dev. from mean & Center $2 / 3$ & Side & Bottom & All \\
\hline & Mean & 1272.4 & 1286.3 & 1279.4 \\
\hline$-14.1 \%$ & Std. Dev. & 54.3 & 37.6 & 45.4 \\
\hline $7.0 \%$ & cov as $\%$ & 4.3 & 2.9 & 3.6 \\
\hline
\end{tabular}

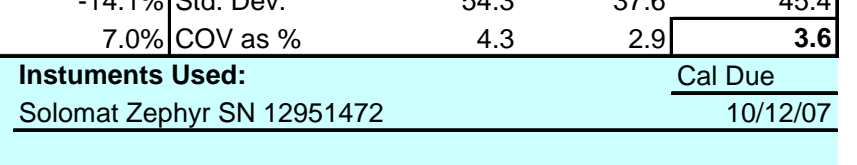
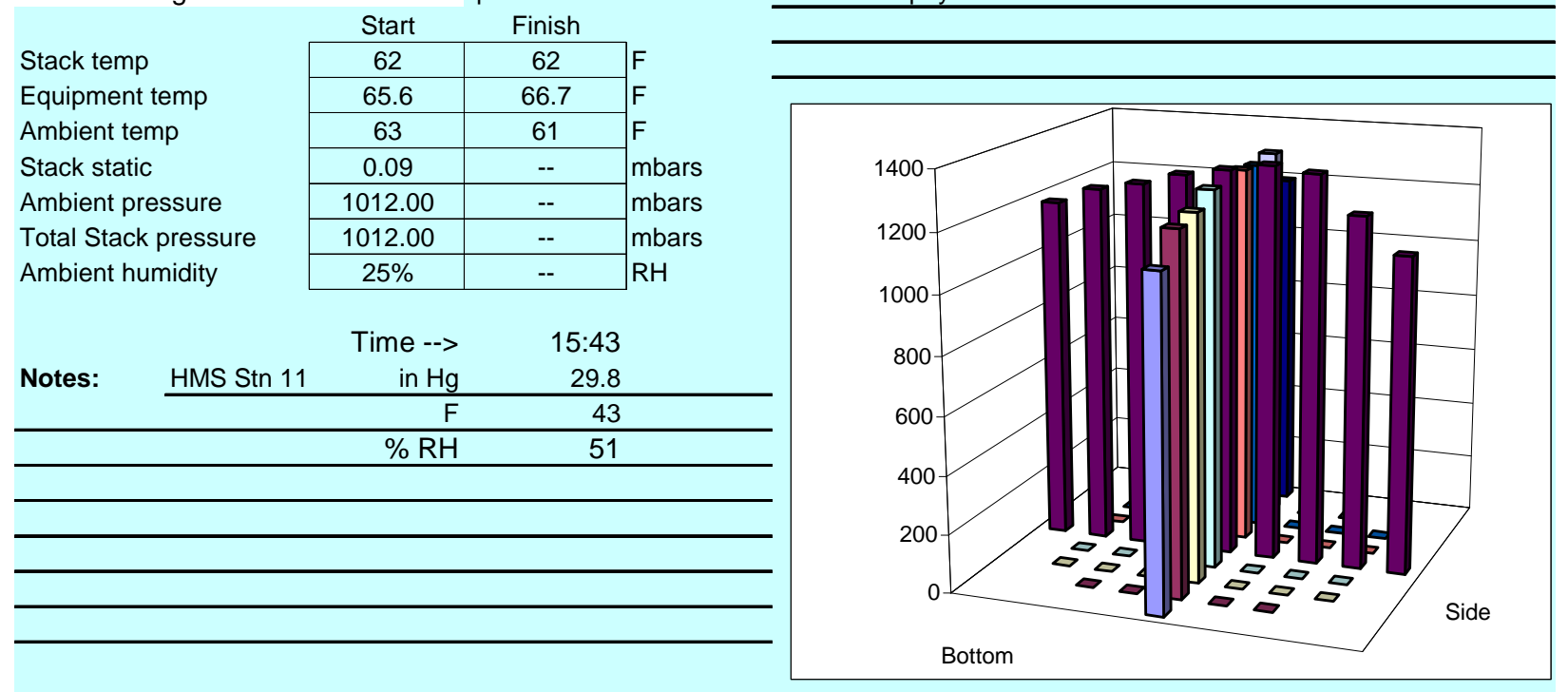

Signature signifies compliance with Procedure EMS-JAG-4

Signature/date

Signature verifying data and calculations:

Reference: CCP-WTPSP-179

VEL-dataRev0.xls

3 August 2006
Signatures on original data sheet in Test Instruction Package TI-RPP-WTP-462

(HVC2_VEL-dataRev0 (1)).xIs/VT16 $4 / 18 / 2007$ 


\title{
VELOCITY TRAVERSE DATA FORM
}

Site HV-C2 Model

Date 1/8/07

Testers JGD \& MSP

Stack Dia. Stack X-Area

Elevation Port

Distance to disturbance Velocity units $\underline{\mathrm{ft} / \mathrm{min}}$
Run No. VT-17

Fan Configuration $\mathbf{A}$

Fan Setting 35

Stack Temp

Start/End Time 1115 -- 114

Center $2 / 3$ from

Points in Center $2 / 3$

Data Files: NA
Order -->
Traverse-->
Trial ---->
\begin{tabular}{|r|r|}
\hline CorrectLabel & Depth, in. \\
\hline 1 & 0.50 \\
\hline 2 & 1.2 \\
\hline 3 & 2.33 \\
\hline 4 & 3.88 \\
\hline Center & 6.00 \\
\hline 5 & 8.12 \\
\hline 6 & 9.67 \\
\hline 7 & 10.74 \\
\hline 8 & 11.50 \\
\hline
\end{tabular}

\begin{tabular}{l|l}
\hline Averages ---------> & 1279.9 \\
\cline { 2 - 2 }
\end{tabular} 2nd

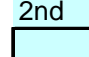

74.25 inches

Side

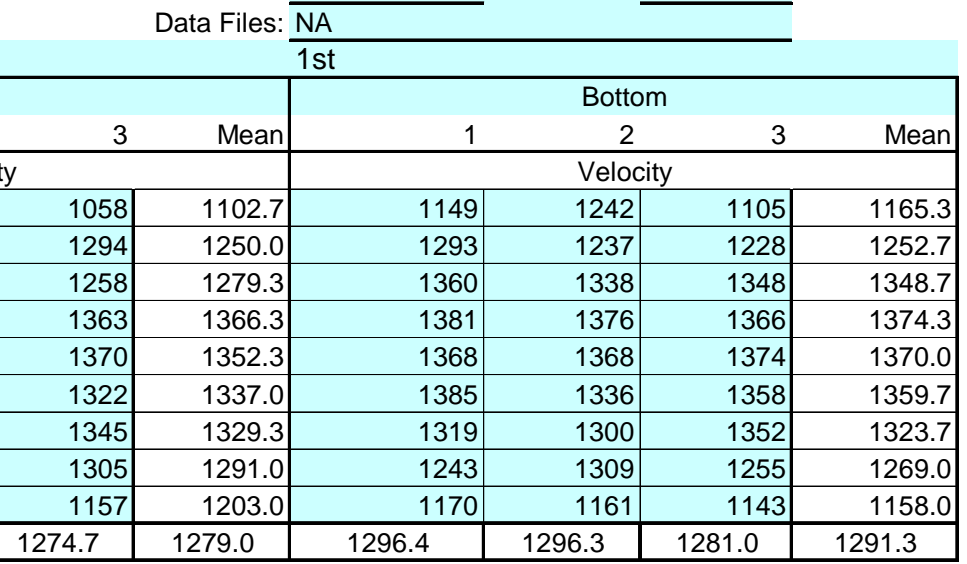

with 3M Filterete microallergen prefilter:

$63.0 \mathrm{~Hz}$

\author{
45
}

10.90

\begin{tabular}{|c|c|c|}
\hline \multicolumn{3}{|c|}{ Velocity } \\
\hline 1124 & 1126 & 105 \\
\hline 1235 & 1221 & $12 \mathrm{~s}$ \\
\hline 1287 & 1293 & 125 \\
\hline 1390 & 1346 & 13 \\
\hline 1319 & 1368 & 13 \\
\hline 1326 & 1363 & 132 \\
\hline 1306 & 1337 & 132 \\
\hline 1290 & 1278 & 13 \\
\hline 1242 & 1210 & 115 \\
\hline 1279.9 & 1282.4 & 1274.7 \\
\hline
\end{tabular}

\begin{tabular}{|ll}
\hline AII & $\underline{\mathrm{ft} / \mathrm{min}}$ \\
Mean & 1285.1 \\
Min Point & 1102.7 \\
Max Point & 1374.3 \\
\hline
\end{tabular}

Flow w/o C-Pt 1002 acfm Vel Avg w/o C-Pt

$1276 \mathrm{fpm}$

\begin{tabular}{|c|c|l}
\multicolumn{1}{c|}{ Start } & \multicolumn{1}{c|}{ Finish } & \\
\hline 64 & 62 & $\mathrm{~F}$ \\
\hline 66.5 & 69.2 & $\mathrm{~F}$ \\
\hline 67 & 64 & $\mathrm{~F}$ \\
\hline 0.04 & -- & mbars \\
\hline 1022.00 & -- & mbars \\
\hline 1022.00 & -- & mbars \\
\hline $31 \%$ & -- & $\mathrm{RH}$ \\
\hline
\end{tabular}

Stack temp

Equipment temp

Ambient temp

Stack static

Ambient pressure

Total Stack pressure

Ambient humidity

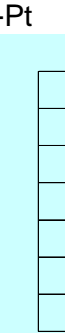

\begin{tabular}{|c|c|c|c|c|}
\hline \multirow{3}{*}{ Notes: } & \multirow{3}{*}{ HMS Stn 11} & \multirow{2}{*}{$\begin{array}{r}\text { Time --> } \\
\text { in } \mathrm{Hg}\end{array}$} & \multirow{2}{*}{$\begin{array}{r}11: 15 \\
30.19\end{array}$} & \multirow{2}{*}{$\begin{array}{r}11: 45 \\
30.18 \\
\end{array}$} \\
\hline & & & & \\
\hline & & $\mathrm{F}$ & 48 & 49 \\
\hline & & $\% \mathrm{RH}$ & 57 & 58 \\
\hline \multicolumn{5}{|c|}{ Control dampers: full open } \\
\hline Backflo & 70 deg. & & & \\
\hline
\end{tabular}

Dev. from mean Center 2/3

Mean

$-14.2 \%$ Std. Dev.

$6.9 \%$ COV as $\%$

\begin{tabular}{rrr} 
Side & Bottom & All \\
\cline { 3 - 3 } 1315.0 & 1328.3 & 1321.7 \\
42.4 & 49.2 & 44.7 \\
& 3.7 & 3.4 \\
\hline
\end{tabular}

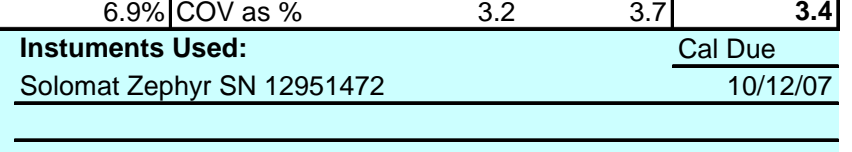
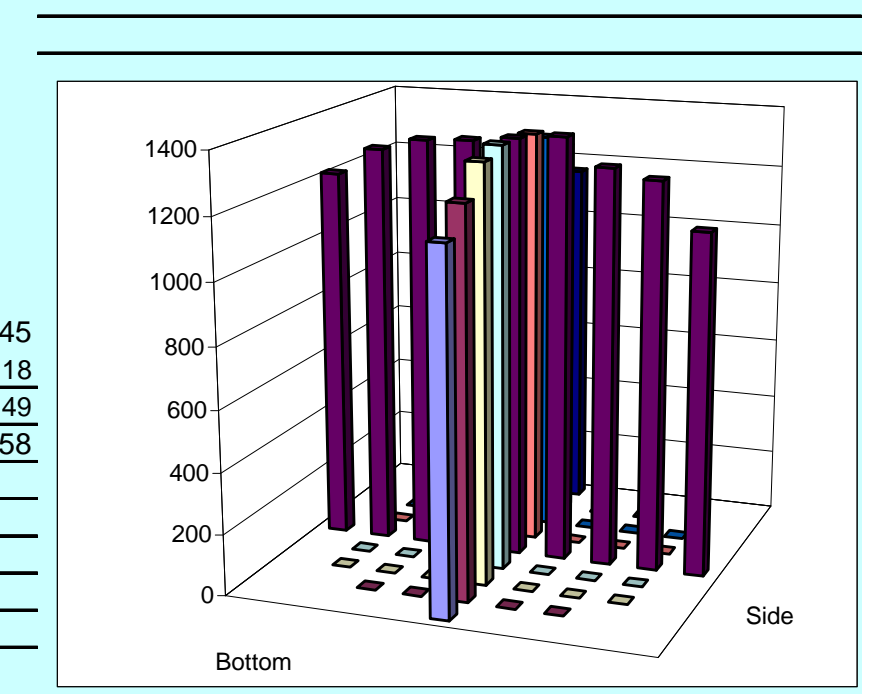

Signature signifies compliance with

Procedure EMS-JAG-4

Signature/date

Signatures on original data sheet in Test Instruction Package TI-RPP-WTP-462

Reference: CCP-WTPSP-179

(HVC2_VEL-dataRev0 (1)).xIs/VT17

VEL-dataRev0.xls

$4 / 18 / 2007$

3 August 2006 


\section{VELOCITY TRAVERSE DATA FORM}

Site HV-C2 Model

Date 1/8/07

Testers JGD \& MSP

Stack Dia. Stack X-Area

Elevation Port

Distance to disturbance Velocity units $\mathrm{ft} / \mathrm{min}$
Run No. VT-18

Fan Configuration $\mathbf{B}$

Fan Setting 35

Stack Temp

Start/End Time 1145 -- 1220

Center $2 / 3$ from

Points in Center 2/3

Data Files: NA

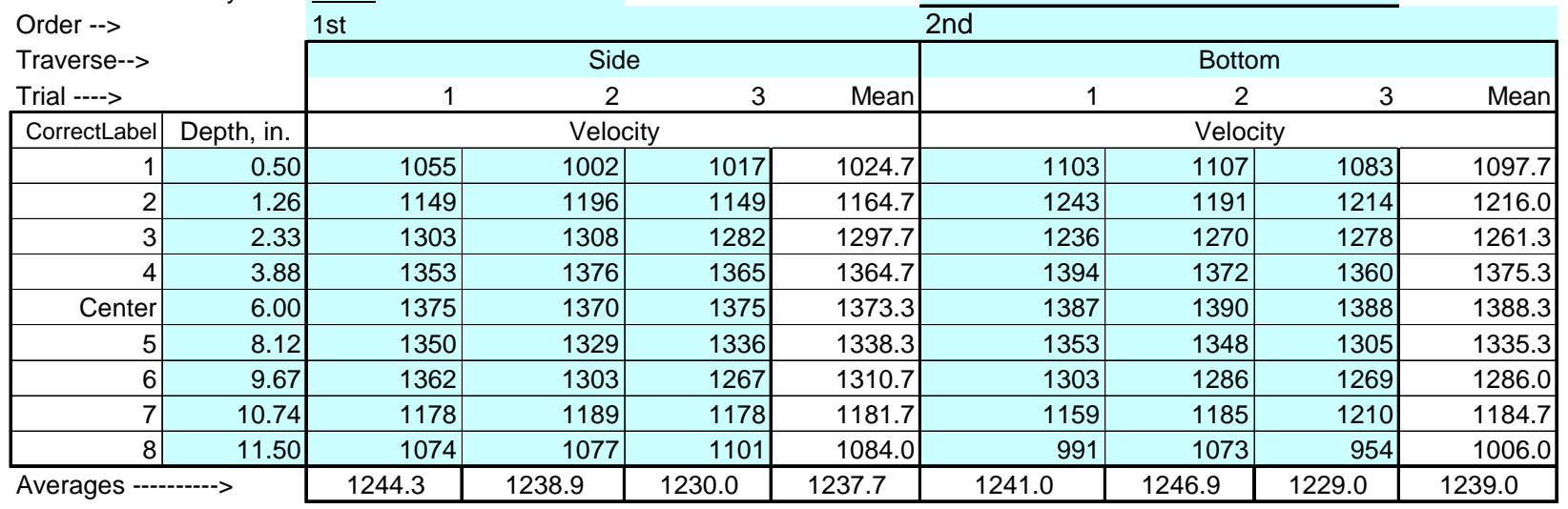

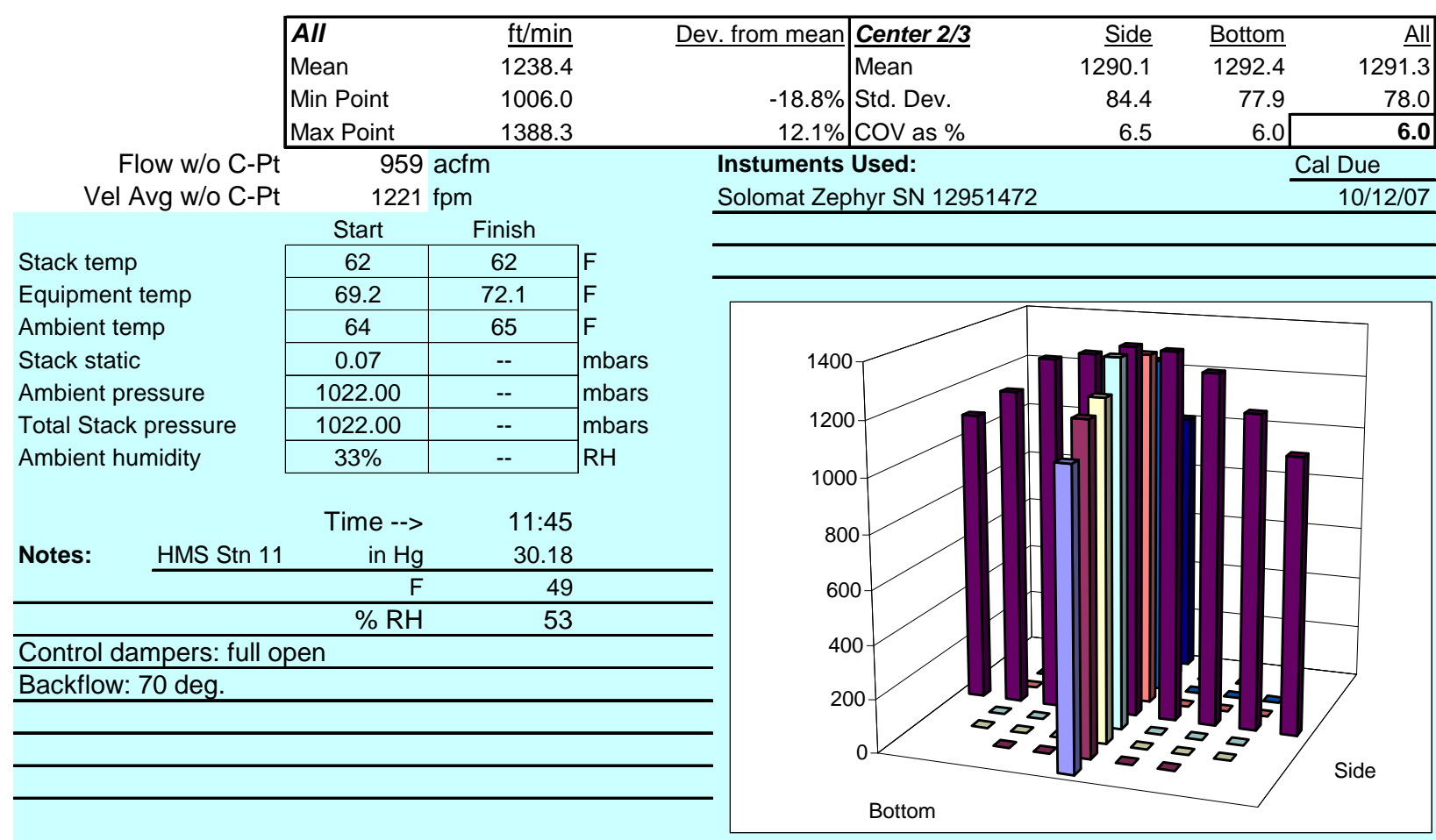

Signature signifies compliance with

Procedure EMS-JAG-4

Signature/date

Signature verifying data and calculations:

with 3M Filterete microallergen prefilter

$\mathrm{Hz}$

\begin{tabular}{llll} 
& 1.10 & to: & 10.90 \\
\hline & 2 & to: &
\end{tabular}

o: $\quad 10.90$

202.75 inches 2nd

Reference: CCP-WTPSP-179

Signatures on original data sheet in Test Instruction Package TI-RPP-WTP-462

VEL-dataRev0.xIs

(HVC2_VEL-dataRev0 (1)).xIs/VT18

3 August 2006

$4 / 18 / 2007$ 


\section{VELOCITY TRAVERSE DATA FORM}

Site HV-C2 Model

Date $1 / 8 / 07$

Testers JGD \& MSP

Stack Dia. Stack X-Area

Elevation Port

Distance to disturbance Velocity units $\underline{\mathrm{ft} / \mathrm{min}}$
Run No. VT-19

Fan Configuration $\mathbf{B}$

Fan Setting 35

Stack Temp

Start/End Time $1220--1250$

Center $2 / 3$ from

Points in Center 2/3

Data Files: NA

\begin{tabular}{|c|c|c|c|c|c|c|c|c|c|}
\hline \multirow{3}{*}{\multicolumn{2}{|c|}{$\begin{array}{l}\text { Order --> } \\
\text { Traverse--> } \\
\text { Trial ----> }\end{array}$}} & \multicolumn{4}{|l|}{ 2nd } & \multicolumn{4}{|l|}{ 1nd } \\
\hline & & \multicolumn{4}{|c|}{ Side } & \multicolumn{4}{|c|}{ Bottom } \\
\hline & & 1 & 2 & 3 & Mean & 1 & 2 & 3 & Mean \\
\hline CorrectLabel & Depth, in. & \multicolumn{4}{|c|}{ Velocity } & \multicolumn{4}{|c|}{ Velocity } \\
\hline 1 & 0.50 & 1026 & 920 & 1050 & 998.7 & 1028 & 1105 & 1088 & 1073.7 \\
\hline 2 & 1.26 & 1127 & 1111 & 1112 & 1116.7 & 1174 & 1195 & 1212 & 1193.7 \\
\hline 3 & 2.33 & 1224 & 1237 & 1268 & 1243.0 & 1300 & 1283 & 1264 & 1282.3 \\
\hline 4 & 3.88 & 1321 & 1301 & 1305 & 1309.0 & 1403 & 1352 & 1371 & 1375.3 \\
\hline Center & 6.00 & 1379 & 1345 & 1373 & 1365.7 & 1381 & 1338 & 1362 & 1360.3 \\
\hline 5 & 8.12 & 1360 & 1364 & 1377 & 1367.0 & 1413 & 1362 & 1379 & 1384.7 \\
\hline 6 & 9.67 & 1337 & 1350 & 1340 & 1342.3 & 1323 & 1344 & 1349 & 1338.7 \\
\hline 7 & 10.74 & 1240 & 1261 & 1278 & 1259.7 & 1249 & 1250 & 1296 & 1265.0 \\
\hline 8 & 11.50 & 1155 & 1244 & 1148 & 1182.3 & 1195 & 1168 & 1140 & 1167.7 \\
\hline Averages --- & $---->$ & 1241.0 & 1237.0 & 1250.1 & 1242.7 & \begin{tabular}{l|l}
1274.0 \\
\end{tabular} & 1266.3 & 1273.4 & 1271.3 \\
\hline
\end{tabular}

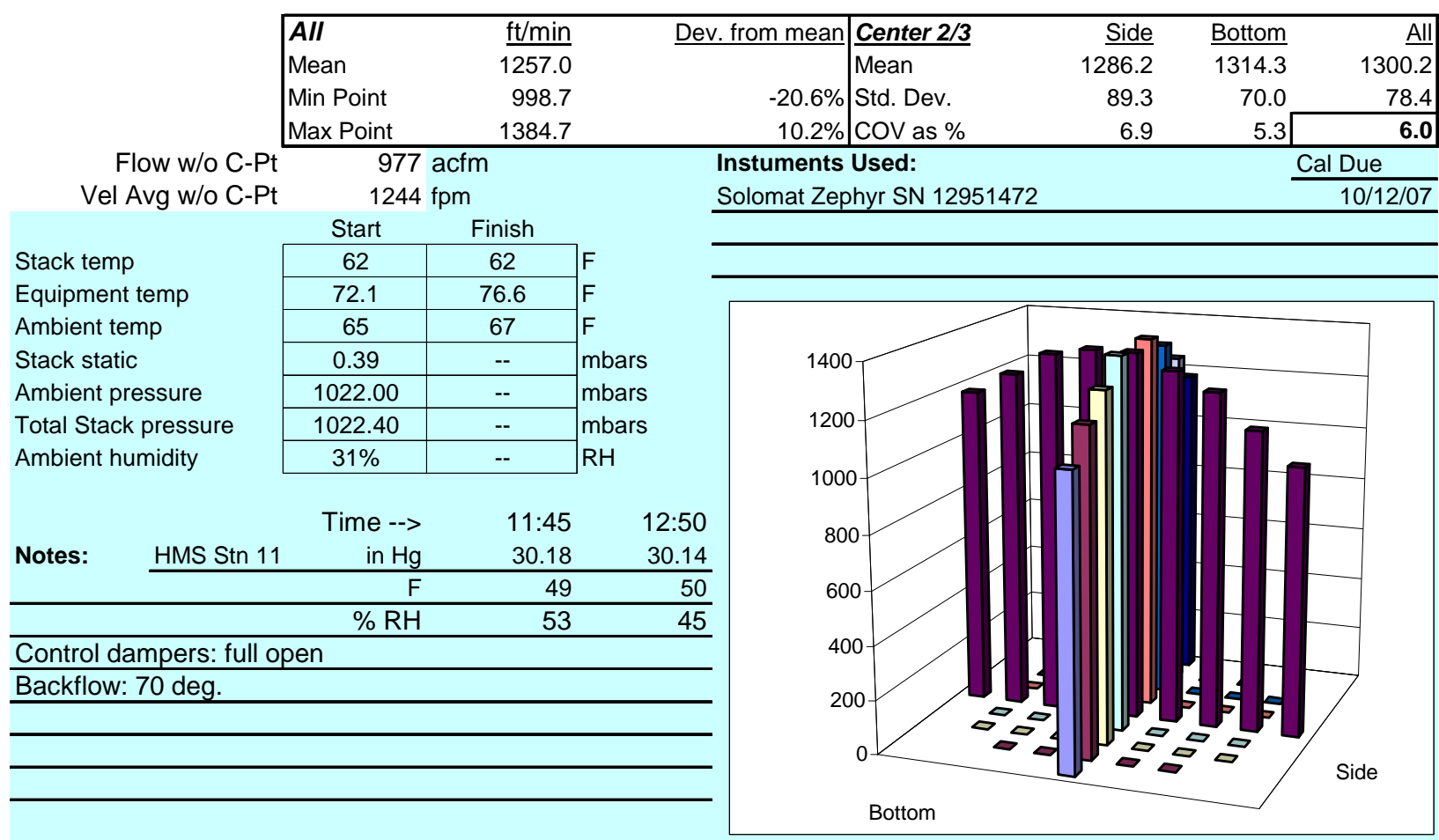

Signature signifies compliance with

Signature verifying data and calculations:

Procedure EMS-JAG-4

Signature/date

Signatures on original data sheet in Test Instruction Package TI-RPP-WTP-462

Reference: CCP-WTPSP-179

(HVC2_VEL-dataRev0 (1)).xIs/VT19

VEL-dataRev0.xls

$4 / 18 / 2007$

3 August 2006 


\section{VELOCITY TRAVERSE DATA FORM}

Site HV-C2 Model

Date $1 / 8 / 07$

Testers JGD \& MSP

Stack Dia. Stack X-Area

Elevation Port

Distance to disturbance Velocity units $\underline{\mathrm{ft} / \mathrm{min}}$
Run No. VT-20

Fan Configuration $\mathbf{B}$

Fan Setting 35

Stack Temp

Start/End Time $1250--1330$

Center $2 / 3$ from

Points in Center 2/3

Data Files: NA

\begin{tabular}{|c|c|c|c|c|c|c|c|c|c|}
\hline \multirow{3}{*}{\multicolumn{2}{|c|}{$\begin{array}{l}\text { Order --> } \\
\text { Traverse--> } \\
\text { Trial ----> } \\
\end{array}$}} & \multicolumn{4}{|l|}{$1 \mathrm{st}$} & \multicolumn{4}{|l|}{ 2nd } \\
\hline & & \multicolumn{4}{|c|}{ Side } & \multicolumn{4}{|c|}{ Bottom } \\
\hline & & \multirow[t]{2}{*}{1} & 2 & \multirow[t]{2}{*}{3} & \multirow[t]{2}{*}{ Mean } & \multirow[t]{2}{*}{1} & 2 & 3 & \multirow[t]{2}{*}{ Mean } \\
\hline CorrectLabel & Depth, in. & & & & & & \multicolumn{2}{|c|}{ Velocity } & \\
\hline 1 & 0.50 & \multicolumn{4}{|c|}{\begin{tabular}{l}
\multicolumn{2}{|c|}{ Velocity } \\
794
\end{tabular}} & 1008 & 1041 & 1046 & 1031.7 \\
\hline 2 & 1.26 & 1200 & 1210 & 1158 & 1189.3 & 1167 & 1118 & 1209 & 1164.7 \\
\hline 3 & 2.33 & 1237 & 1244 & 1223 & 1234.7 & 1265 & 1236 & 1218 & 1239.7 \\
\hline 4 & 3.88 & 1265 & 1231 & 1285 & 1260.3 & 1296 & 1306 & 1316 & 1306.0 \\
\hline Center & 6.00 & 1314 & 1342 & 1303 & 1319.7 & 1323 & 1335 & 1355 & 1337.7 \\
\hline 5 & 8.12 & 1387 & 1375 & 1302 & 1354.7 & 1431 & 1413 & 1425 & 1423.0 \\
\hline 6 & 9.67 & 1322 & 1381 & 1372 & 1358.3 & 1399 & 1423 & 1470 & 1430.7 \\
\hline 7 & 10.74 & 1308 & 1289 & 1276 & 1291.0 & 1293 & 1395 & 1402 & 1363.3 \\
\hline 8 & 11.50 & 994 & 1164 & 1054 & 1070.7 & 863 & 1294 & 753 & 970.0 \\
\hline Averages ---- & 7 & 1198.8 & 1225.6 & 1220.3 & 1214.9 & 1227.2 & 1284.6 & 1243.8 & 1251.9 \\
\hline
\end{tabular}

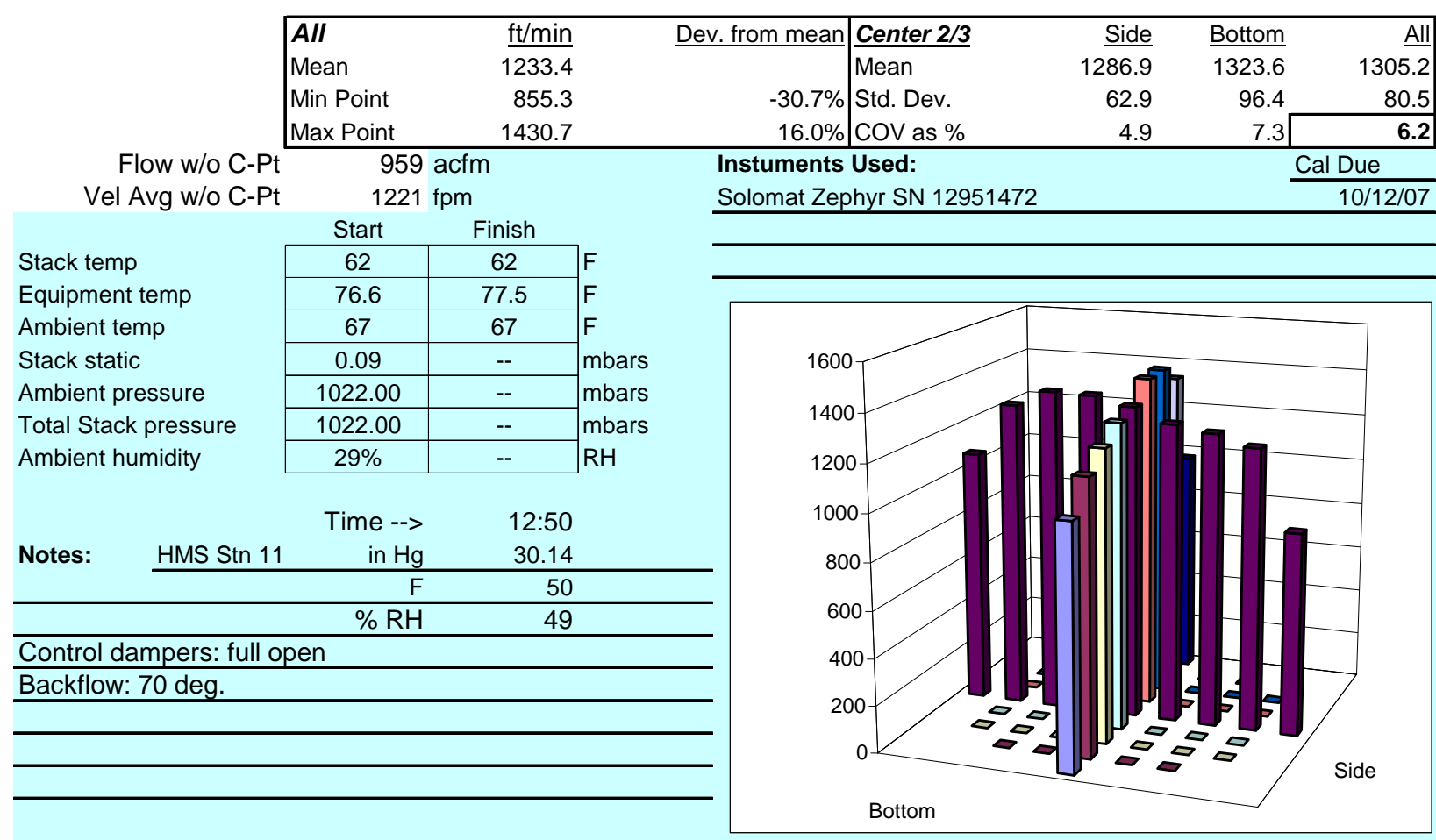

Signature signifies compliance with

Procedure EMS-JAG-4

Signature/date

Signature verifying data and calculations:

Signatures on original data sheet in Test Instruction Package TI-RPP-WTP-462

Reference: CCP-WTPSP-179

(HVC2_VEL-dataRev0 (1)).xIs/VT20

VEL-dataRev0.xls

$4 / 18 / 2007$

3 August 2006 


\section{VELOCITY TRAVERSE DATA FORM}

Site HV-C2 Model Date 2/5/07

Testers BGF

\begin{tabular}{rr} 
Stack Dia. & \multicolumn{1}{c}{12 in. } \\
\cline { 2 - 2 } Stack X-Area \\
\cline { 2 - 2 } Elevation & 113.1 in.2 \\
\cline { 2 - 2 }
\end{tabular}

Distance to disturbance Velocity units $\underline{\mathrm{ft} / \mathrm{min}}$
Run No. VT-21

Fan Configuration A \& B with 3M Filterete microallergen prefilter: Fan Setting 40

Stack Temp

Start/End Time $0940--1015$

Center $2 / 3$ from Points in Center $2 / 3$ Data Files: NA

\begin{tabular}{|c|c|c|c|c|c|c|c|c|c|}
\hline \multirow{3}{*}{\multicolumn{2}{|c|}{$\begin{array}{l}\text { Order --> } \\
\text { Traverse--> } \\
\text { Trial ----> }\end{array}$}} & \multicolumn{4}{|l|}{ 1st } & \multicolumn{4}{|l|}{ 2nd } \\
\hline & & \multicolumn{4}{|c|}{ Side } & \multicolumn{4}{|c|}{ Bottom } \\
\hline & & 1 & 2 & 3 & Mean & 1 & 2 & 3 & Mean \\
\hline CorrectLabel & Depth, in. & \multicolumn{4}{|c|}{ Velocity } & \multicolumn{4}{|c|}{ Velocity } \\
\hline 1 & 0.50 & 2474 & 2388 & 2422 & 2428.0 & 2490 & 2496 & 2499 & 2495.0 \\
\hline 2 & 1.26 & 2680 & 2595 & 2625 & 2633.3 & 2628 & 2674 & 2700 & 2667.3 \\
\hline 3 & 2.33 & 2807 & 2849 & 2934 & 2863.3 & 2780 & 2849 & 2878 & 2835.7 \\
\hline 4 & 3.88 & 3021 & 3017 & 2991 & 3009.7 & 2905 & 2947 & 2950 & 2934.0 \\
\hline Center & 6.00 & 3006 & 2987 & 2992 & 2995.0 & 3013 & 3108 & 2956 & 3025.7 \\
\hline 5 & 8.12 & 2967 & 2966 & 2902 & 2945.0 & 2960 & 3024 & 2995 & 2993.0 \\
\hline 6 & 9.67 & 2831 & 2816 & 2835 & 2827.3 & 2883 & 2883 & 2899 & 2888.3 \\
\hline 7 & 10.74 & 2647 & 2591 & 2602 & 2613.3 & 2671 & 2688 & 2724 & 2694.3 \\
\hline 8 & 11.50 & 2354 & 2372 & 2418 & 2381.3 & 2372 & 2315 & 2241 & 2309.3 \\
\hline Averages --- & $---->$ & 2754.1 & 2731.2 & 2746.8 & 2744.0 & 2744.7 & 2776.0 & 2760.2 & 2760.3 \\
\hline
\end{tabular}

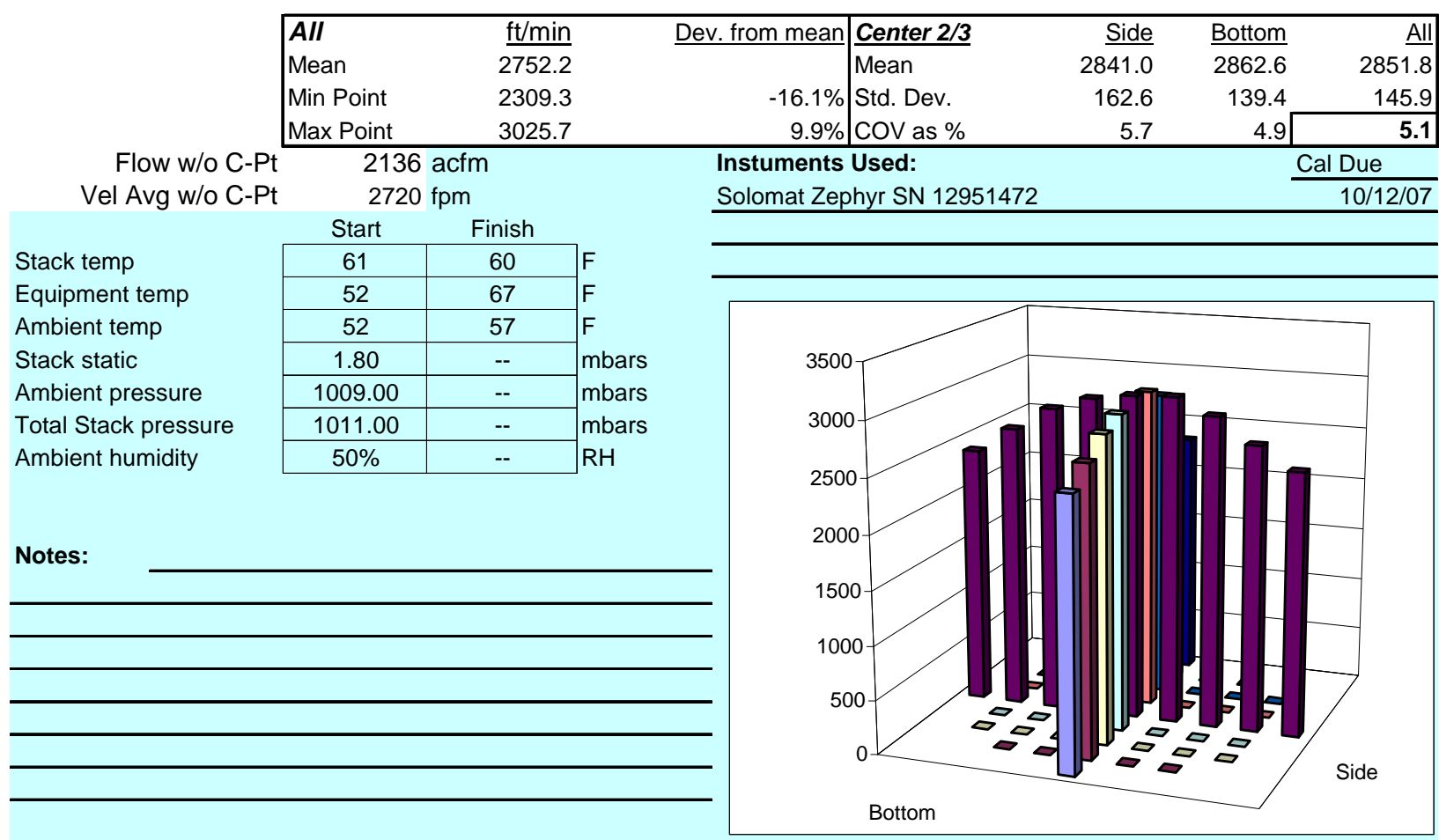

Signature signifies compliance with Signature verifying data and calculations:

Procedure EMS-JAG-4

Signature/date

Signatures on original data sheet in Test Instruction Package TI-RPP-WTP-462

Reference: CCP-WTPSP-179

(HVC2_VEL-dataRev0 (1)).xIs/VT21

VEL-dataRev0.xIs

$4 / 18 / 2007$

3 August 2006 


\section{VELOCITY TRAVERSE DATA FORM}

Site HV-C2 Model

Date 2/5/07

Testers BGF \& MSP

$$
\text { Stack Dia. }
$$

Stack X-Area

Elevation Port

Distance to disturbance

Velocity units $\underline{\mathrm{ft} / \mathrm{min}}$
Run No. VT-22

Fan Configuration $\mathbf{A}$ \& $\mathbf{B}$

Fan Setting 40

Stack Temp

Start/End Time 1020 -- 1045

Center $2 / 3$ from

Points in Center $2 / 3$

Data Files: NA
Order -->

Traverse-->

Trial ---->

\begin{tabular}{|r|r|}
\hline CorrectLabel & Depth, in. \\
\hline 1 & 0.50 \\
\hline 2 & 1.2 \\
\hline 3 & 2.33 \\
\hline 4 & 3.88 \\
\hline Center & 6.00 \\
\hline 5 & 8.12 \\
\hline 6 & 9.6 \\
\hline 7 & 10.7 \\
\hline 8 & 11.50 \\
\hline
\end{tabular}

\begin{tabular}{l|l}
\hline Averages ---------> & 2797.8 \\
\hline
\end{tabular}

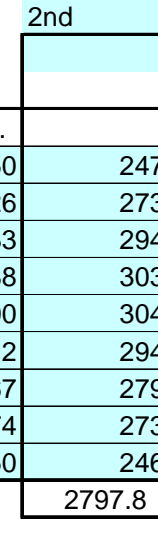

3

\begin{tabular}{|lr}
\hline All & $\underline{\mathrm{ft} / \mathrm{min}}$ \\
Mean & 2805.5 \\
Min Point & 2443.7 \\
Max Point & 3051.3 \\
\hline
\end{tabular}

Flow w/o C-Pt Vel Avg w/o C-Pt

Stack temp

Equipment temp

Ambient temp

Stack static

Ambient pressure

Total Stack pressure

Ambient humidity

\begin{tabular}{|c|c|l}
\multicolumn{1}{c|}{ Start } & Finish & \\
\hline 60 & 66 & $F$ \\
\hline 52 & 69 & $F$ \\
\hline 52 & 60 & F \\
\hline 1.80 & -- & mbars \\
\hline 1009.00 & -- & mbars \\
\hline 1011.00 & -- & mbars \\
\hline $46 \%$ & -- & RH \\
\hline
\end{tabular}

Notes:

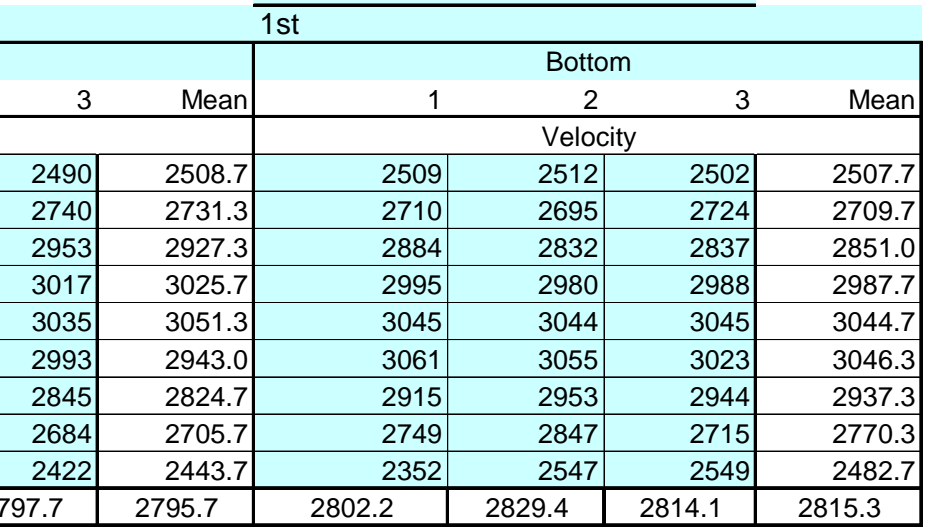

with 3M Filterete microallergen prefilter:

Hz

1.10

1.10

to: 10.90
Dev. from mean Center 2/3
Mean
$-12.9 \%$ Std. Dev.
$8.8 \%$ COV as $\%$

Side
2887.0
136.6
4.7

\begin{tabular}{rr}
$\frac{\text { Bottom }}{2906.7}$ & All \\
133.3 & 2896.9 \\
4.6 & 130.1 \\
\hline
\end{tabular}

\begin{tabular}{lcc} 
Instuments Used: & Cal Due \\
Solomat Zephyr SN 12951472 & 10/12/07 \\
\hline
\end{tabular}
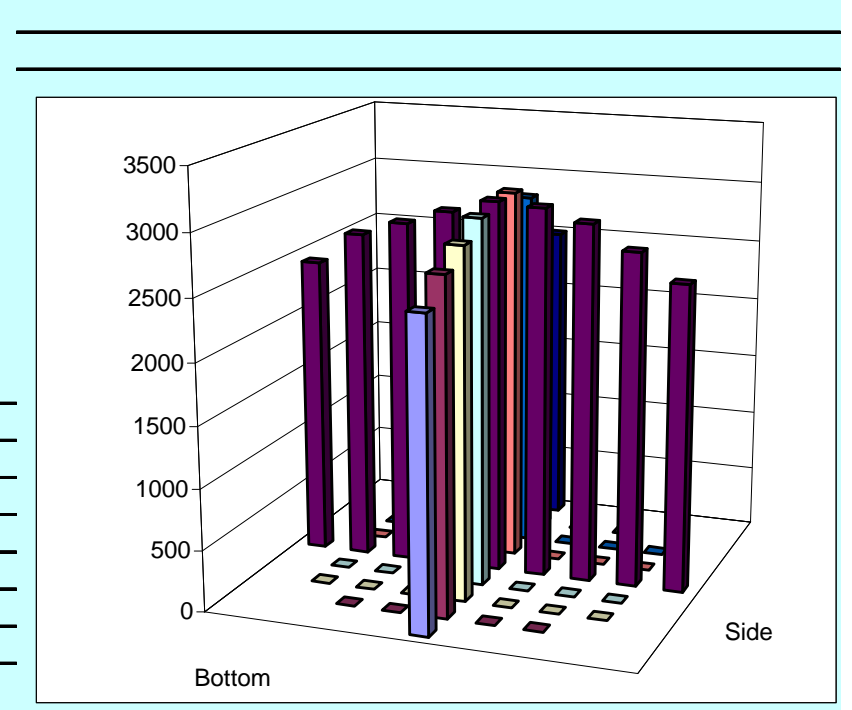

Signature signifies compliance with

Procedure EMS-JAG-4

Signature/date

Signatures on original data sheet in Test Instruction Package TI-RPP-WTP-462

Reference: CCP-WTPSP-179

(HVC2_VEL-dataRev0 (1)).xls/VT22

VEL-dataRev0.xIs

$4 / 18 / 2007$

3 August 2006 


\section{VELOCITY TRAVERSE DATA FORM}

Site HV-C2 Model Date 2/5/07

Testers MSP

\begin{tabular}{rr} 
Stack Dia. & \multicolumn{1}{c}{12 in. } \\
\cline { 2 - 2 } Stack X-Area \\
\cline { 2 - 2 } Elevation & 113.1 in.2 \\
\cline { 2 - 2 }
\end{tabular}

Distance to disturbance Velocity units $\mathrm{ft} / \mathrm{min}$
Run No. VT-23

Fan Configuration A \& B with 3M Filterete microallergen prefilter: Fan Setting 40

Stack Temp

Start/End Time 1055 -- 1140

Center $2 / 3$ from Points in Center $2 / 3$ Data Files: NA

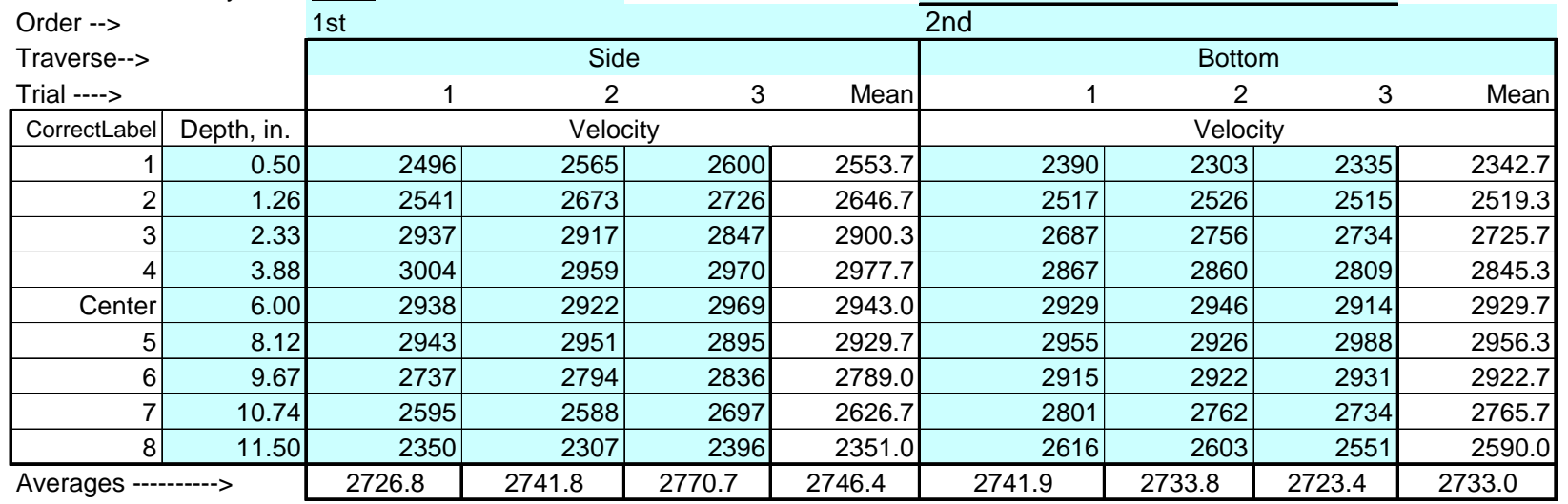

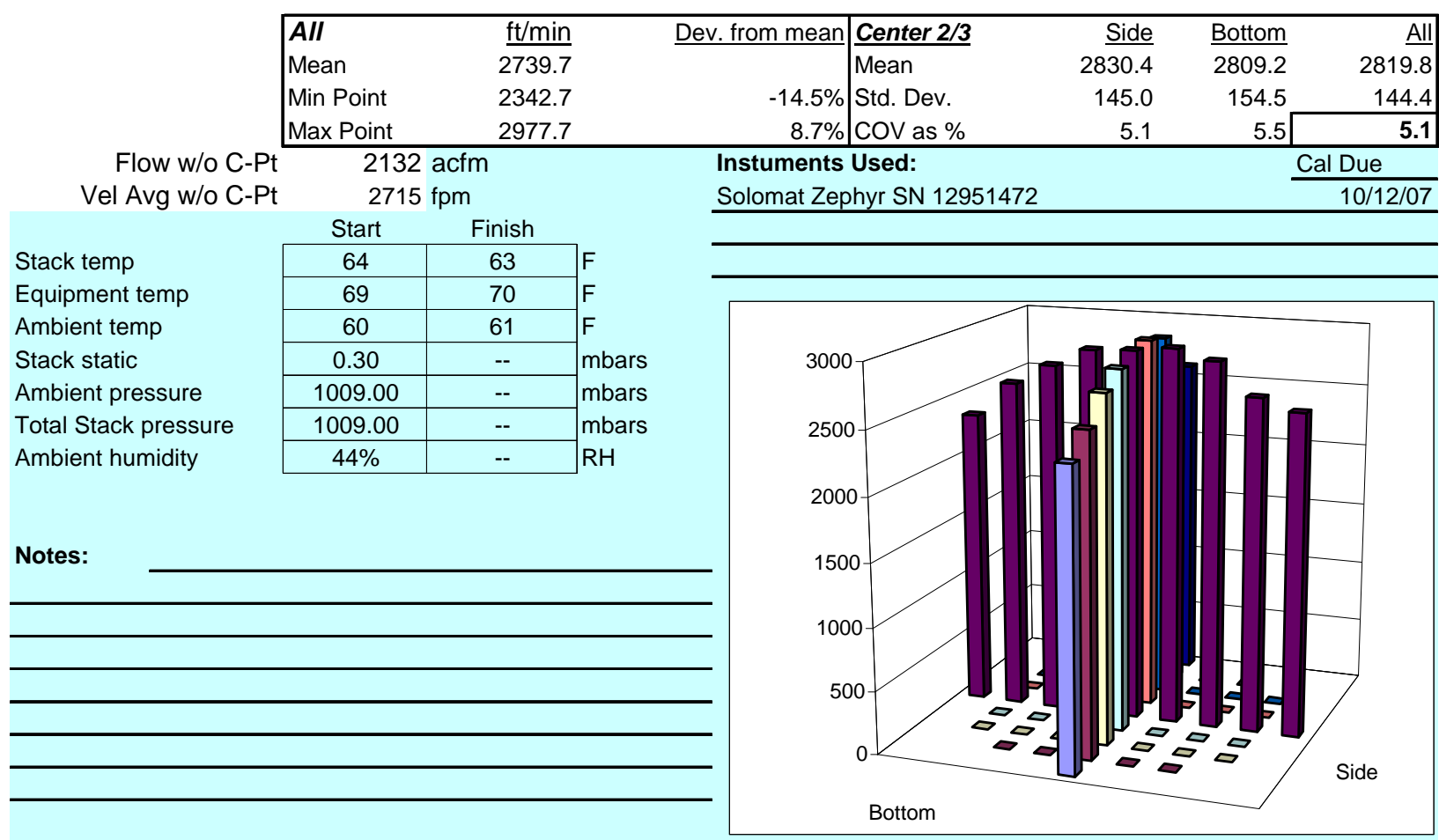

Signature signifies compliance with Signature verifying data and calculations:

Procedure EMS-JAG-4

Signature/date

Signatures on original data sheet in Test Instruction Package TI-RPP-WTP-462

Reference: CCP-WTPSP-179

(HVC2_VEL-dataRev0 (1)).xIs/VT23

VEL-dataRev0.xIs

$4 / 18 / 2007$

3 August 2006 


\section{VELOCITY TRAVERSE DATA FORM}

Site HV-C2 Model Date 2/5/07

Testers MSP

\begin{tabular}{rr} 
Stack Dia. & \multicolumn{1}{c}{12 in. } \\
\cline { 2 - 2 } Stack X-Area \\
\cline { 2 - 2 } Elevation & 113.1 in.2 \\
\cline { 2 - 2 }
\end{tabular}

Distance to disturbance Velocity units $\underline{\mathrm{ft} / \mathrm{min}}$
Run No. VT-24

Fan Configuration A \& B

Fan Setting 40

Stack Temp

Start/End Time 1145 -- 1220

Center $2 / 3$ from

Points in Center $2 / 3$

Order -->

Traverse-->

Trial ---->

\begin{tabular}{|r|r|}
\hline CorrectLabel & Depth, in. \\
\hline 1 & 0.50 \\
\hline 2 & 1.2 \\
\hline 3 & 2.33 \\
\hline 4 & 3.88 \\
\hline Center & 6.00 \\
\hline 5 & 8.12 \\
\hline 6 & 9.6 \\
\hline 7 & 10.7 \\
\hline 8 & 11.50 \\
\hline
\end{tabular}

\begin{tabular}{|l|l}
\hline Averages ---------> & 2735.3 \\
\hline
\end{tabular}

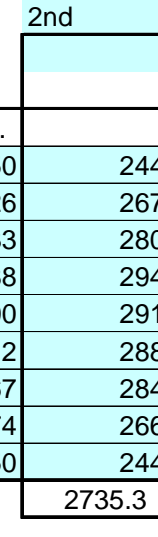

13.75 inches

Side
Data Files: $\frac{\text { NA }}{1 \mathrm{st}}$

\begin{tabular}{|c|c|c|c|c|c|}
\hline & \multicolumn{4}{|c|}{ st } \\
\hline \multirow[b]{2}{*}{3} & \multirow[b]{2}{*}{ Mean } & \multicolumn{4}{|c|}{ Bottom } \\
\hline & & 1 & 2 & 3 & Mean \\
\hline & & \multicolumn{4}{|c|}{ Velocity } \\
\hline 2399 & 2369.0 & 2355 & 2224 & 2366 & 2315.0 \\
\hline 2625 & 2628.3 & 2538 & 2462 & 2741 & 2580.3 \\
\hline 2875 & 2829.0 & 2644 & 2709 & 2876 & 2743.0 \\
\hline 2933 & 2949.3 & 2835 & 2843 & 2941 & 2873.0 \\
\hline 2937 & 2942.7 & 2939 & 2960 & 2965 & 2954.7 \\
\hline 2913 & 2905.7 & 2964 & 2949 & 2939 & 2950.7 \\
\hline 2776 & 2816.3 & 2884 & 2893 & 2903 & 2893.3 \\
\hline 2697 & 2686.7 & 2754 & 2782 & 2763 & 2766.3 \\
\hline 2498 & 2457.0 & 2522 & 2570 & 2522 & 2538.0 \\
\hline 739.2 & 2731.6 & 2715.0 & 2710.2 & 2779.6 & 2734.9 \\
\hline
\end{tabular}

\begin{tabular}{r|l} 
Dev. from mean & Center $\mathbf{2 / 3}$ \\
$-15.3 \%$ & Mean \\
Std. Dev. \\
$8.1 \%$ & COV as $\%$ \\
\hline
\end{tabular}

with 3M Filterete microallergen prefilter:

$63.0 \operatorname{deg} \mathrm{F}$

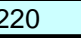

1220

to:

to: 10.90

\begin{tabular}{|c|c|c|}
\hline Side & Bottom & All \\
\hline 2822.6 & 2823.0 & 2822.8 \\
\hline 125.0 & 135.1 & 125.0 \\
\hline 4.4 & 4.8 & 4.4 \\
\hline
\end{tabular}

Flow w/o C-Pt Vel Avg w/o C-Pt

2954.7

$2126 \mathrm{acfm}$ $2706 \mathrm{fpm}$

\begin{tabular}{|c|c|}
\hline \multicolumn{2}{|c|}{$2706 \mathrm{fpm}$} \\
\hline Start & Finish \\
\hline 63 & 63 \\
\hline 70 & 70 \\
\hline 60 & 62 \\
\hline 0.30 & -- \\
\hline 1008.00 & -- \\
\hline 1008.00 & -- \\
\hline $44 \%$ & -- \\
\hline
\end{tabular}

Stack temp

Equipment temp

Ambient temp

Stack static

Ambient pressure

Total Stack pressure

Ambient humidity

\section{Notes:}
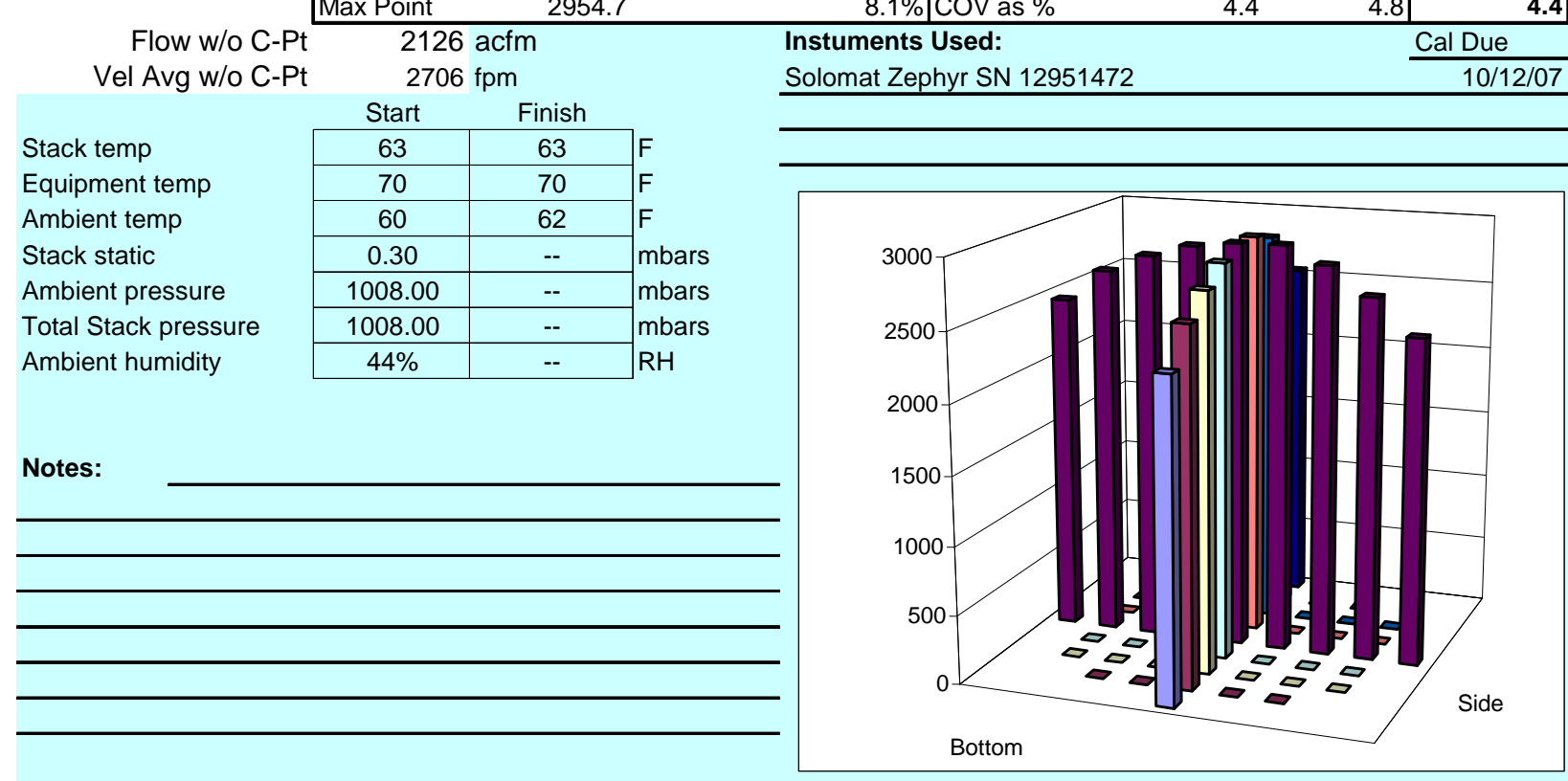

Signature signifies compliance with

Procedure EMS-JAG-4

Signature/date

Signatures on original data sheet in Test Instruction Package TI-RPP-WTP-462

Reference: CCP-WTPSP-179

(HVC2_VEL-dataRev0 (1)).xIs/VT24

VEL-dataRev0.xls

3 August 2006 
Appendix D

\section{Flow-Angle Data Sheets}




\section{Appendix D: Flow-Angle Data Sheets}

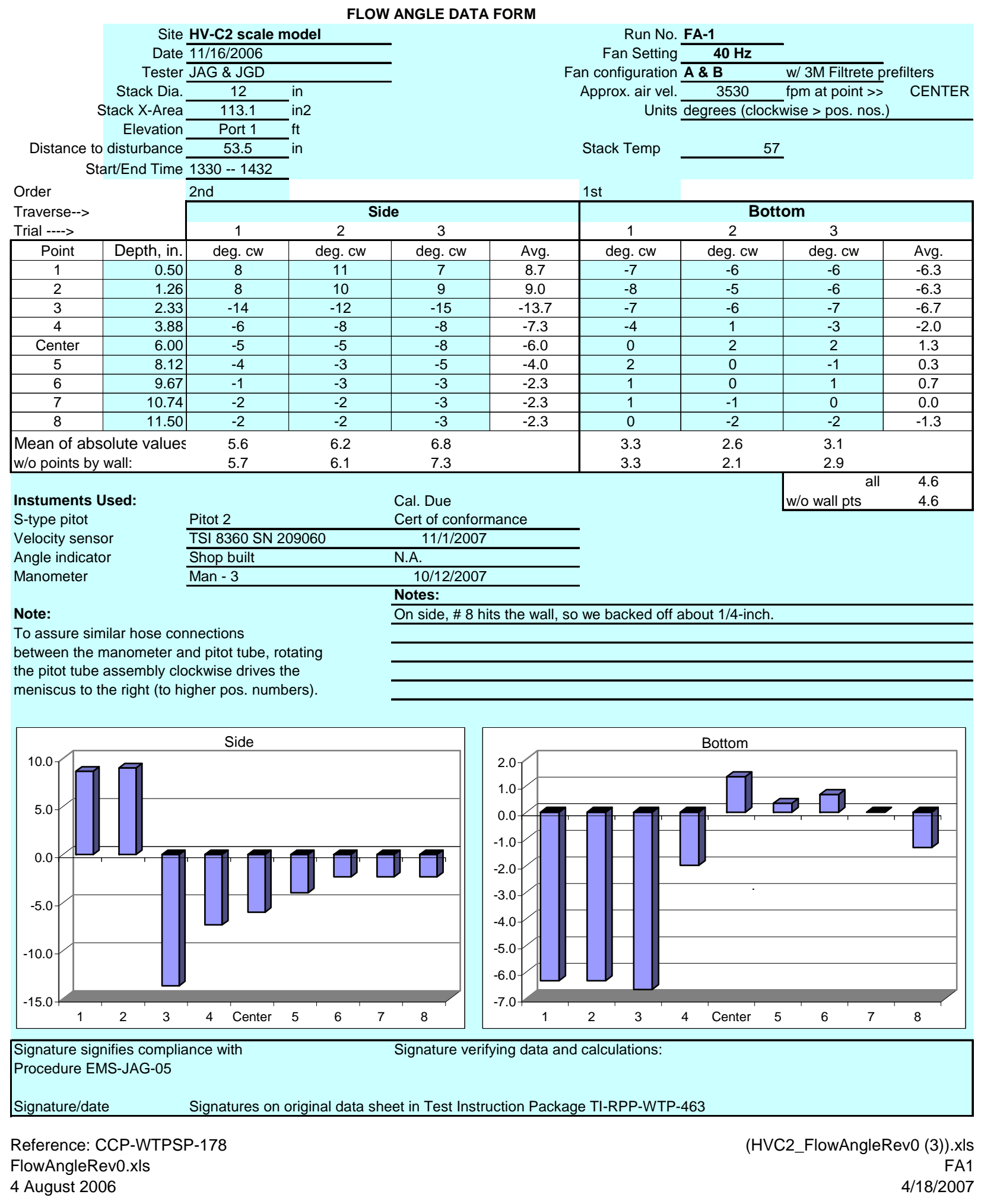

D. 1 


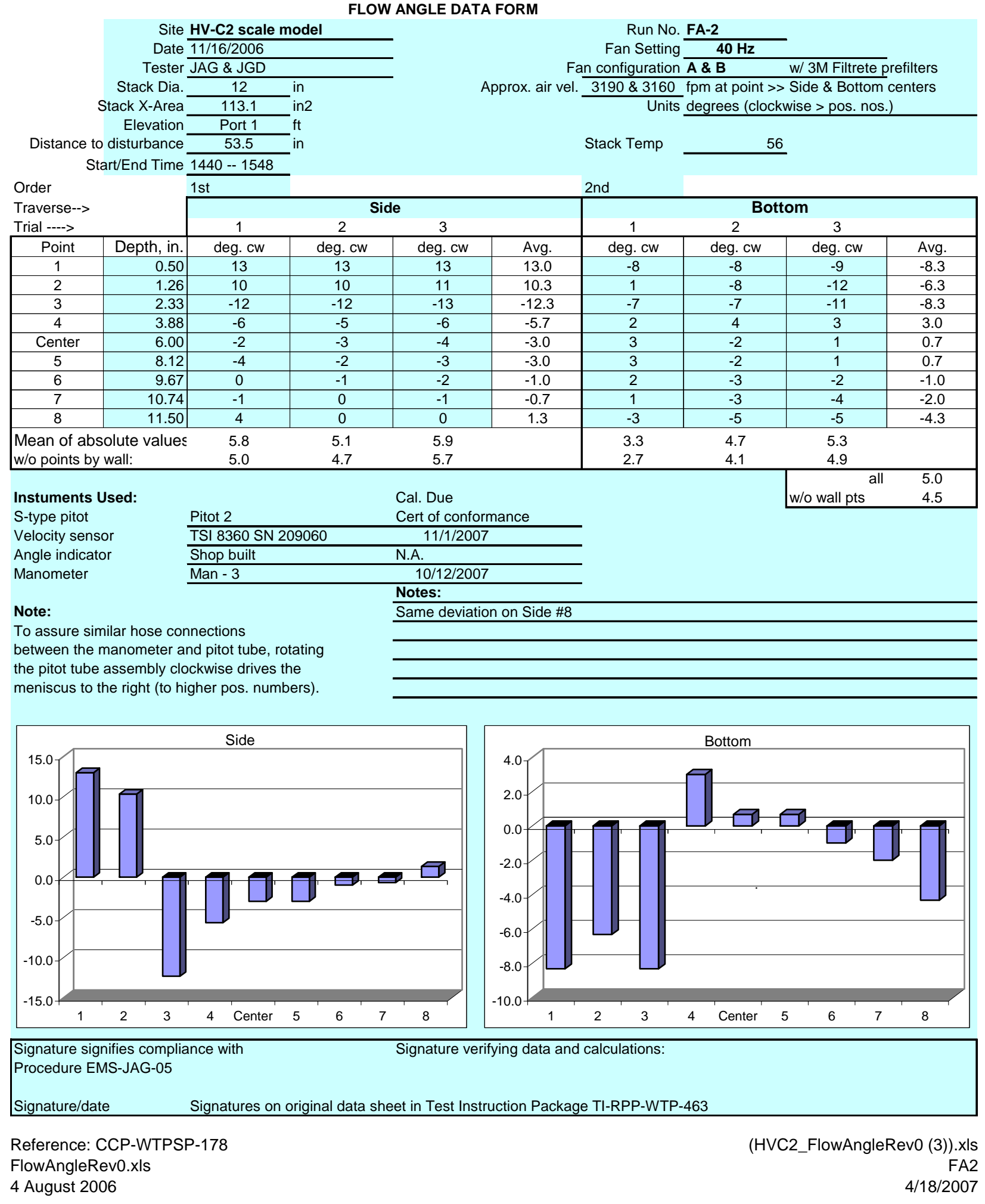




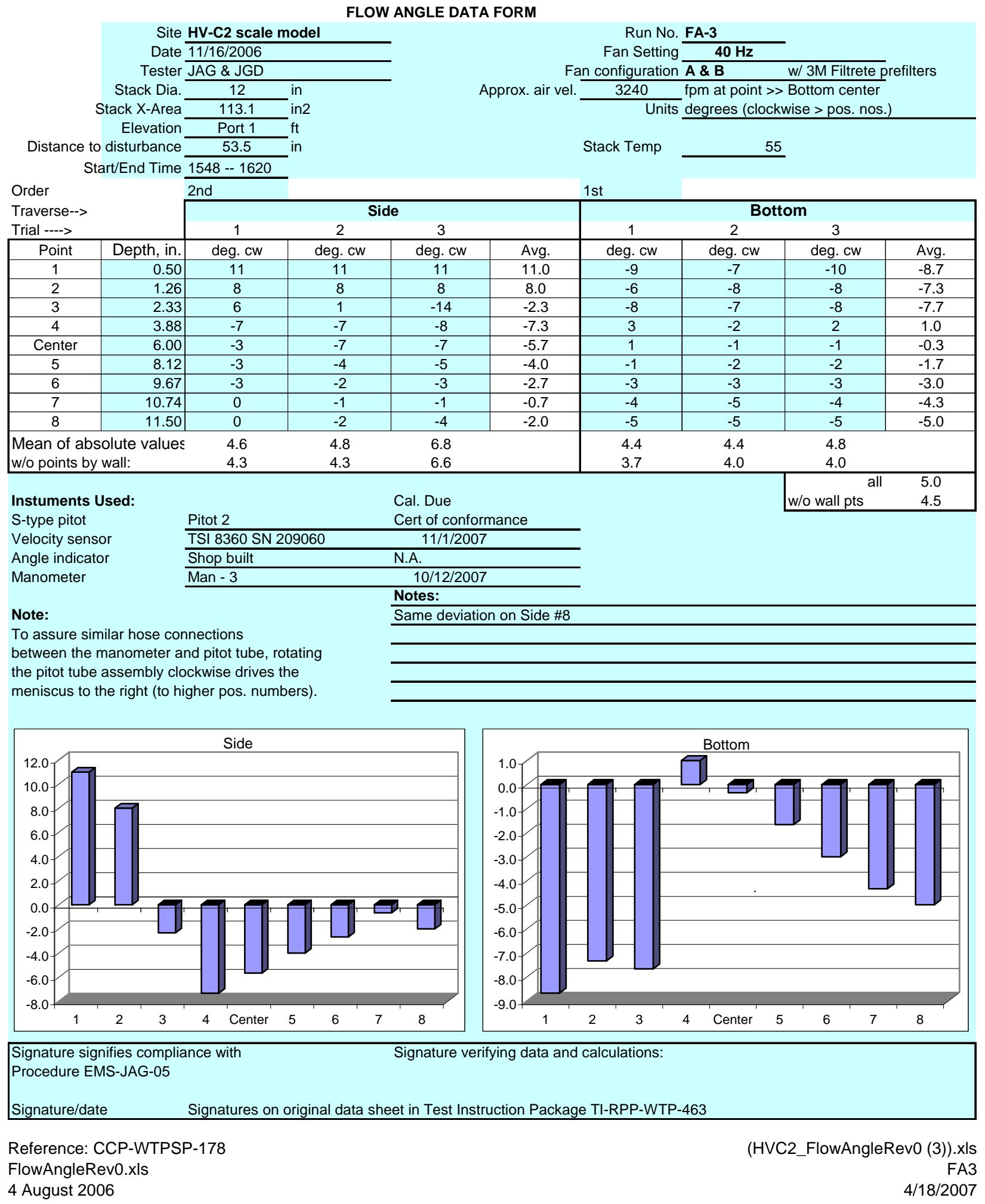




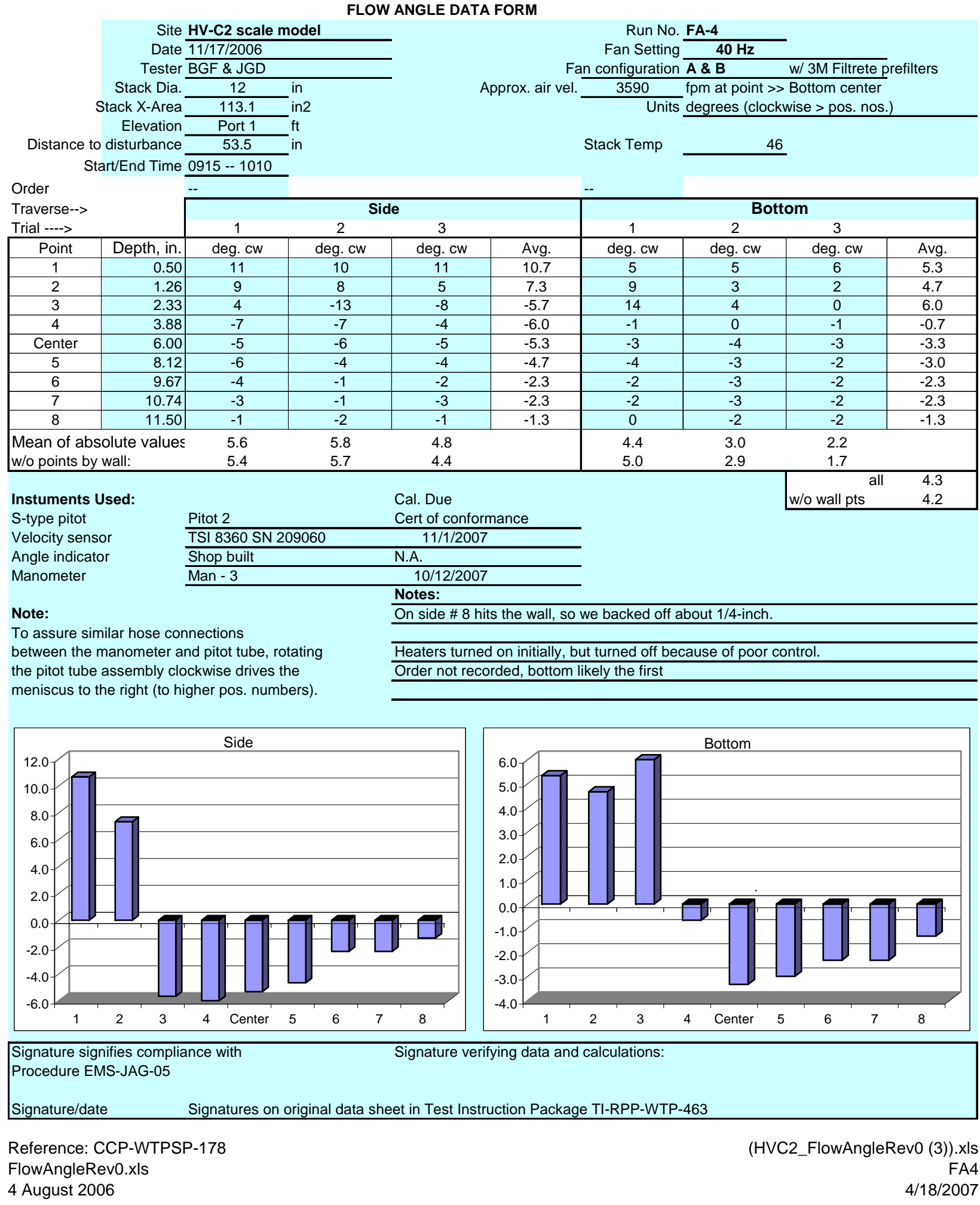




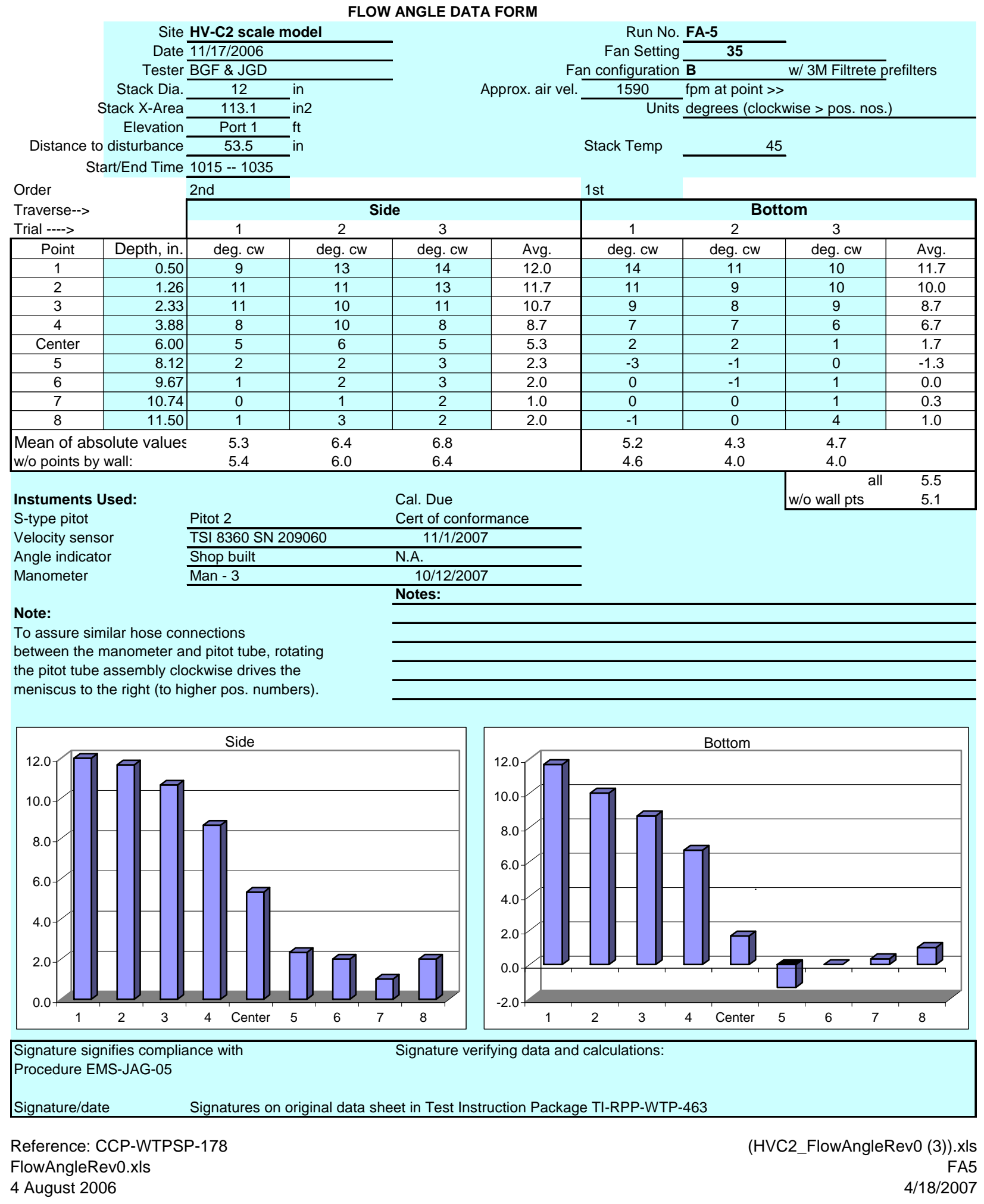




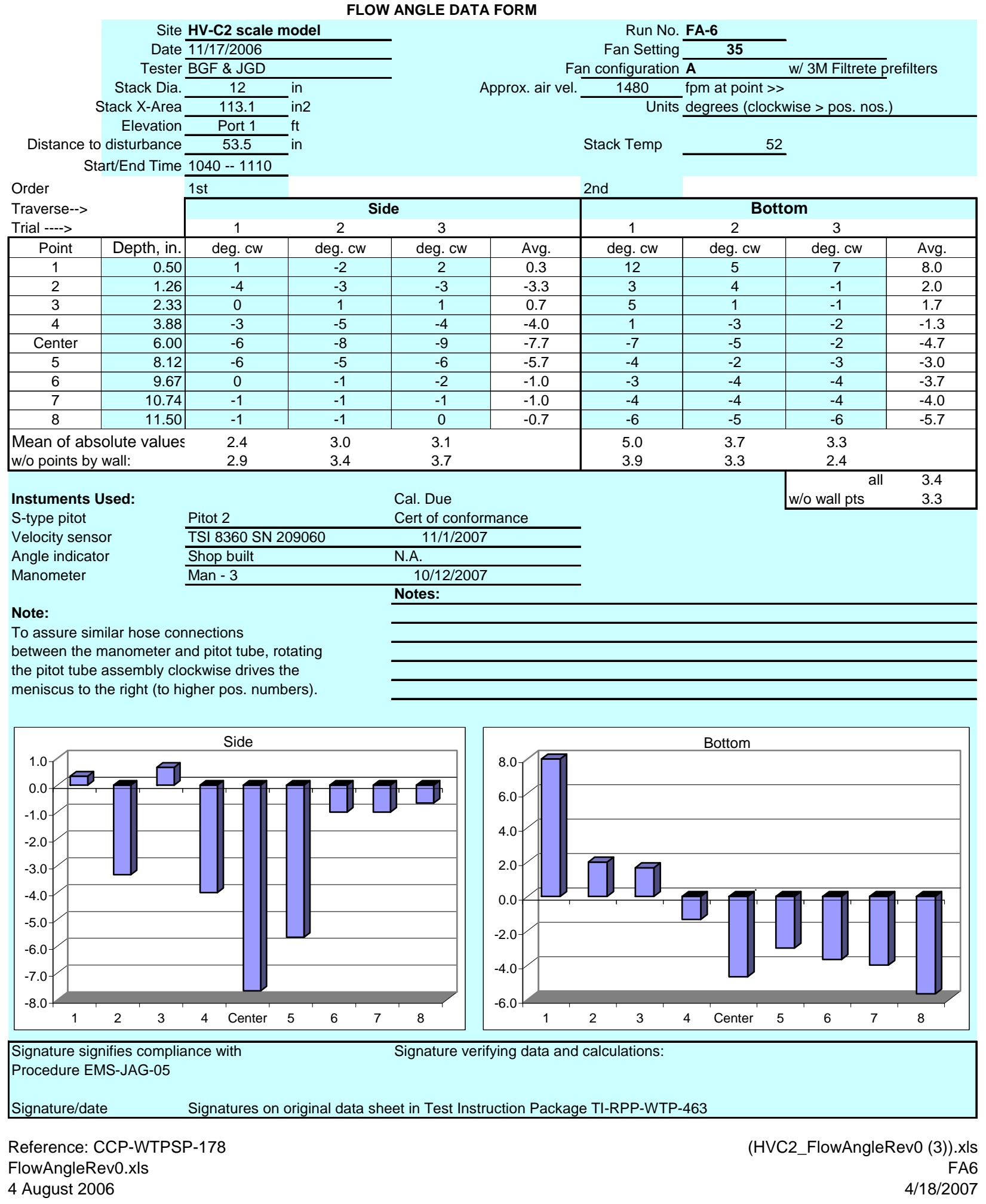




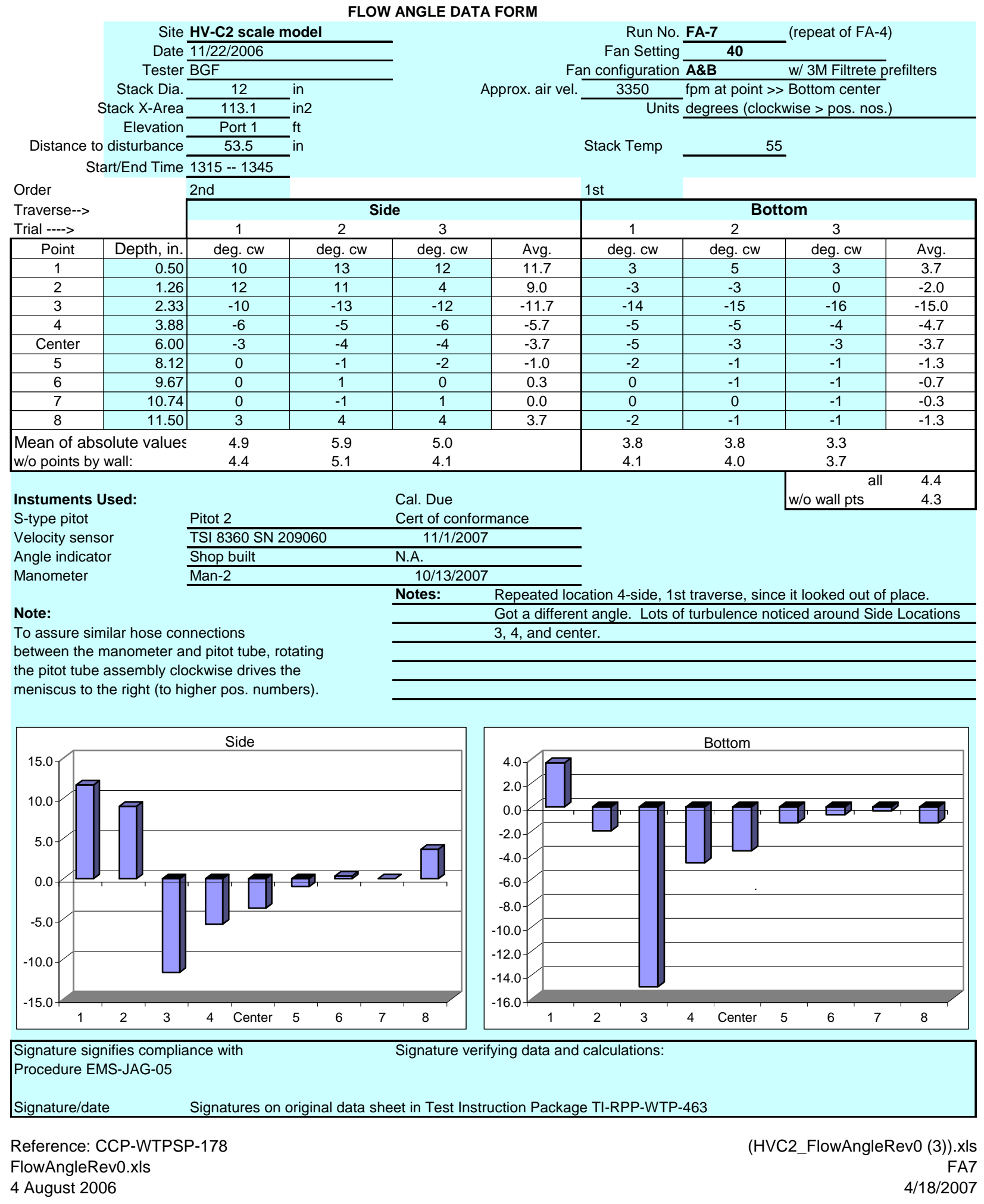




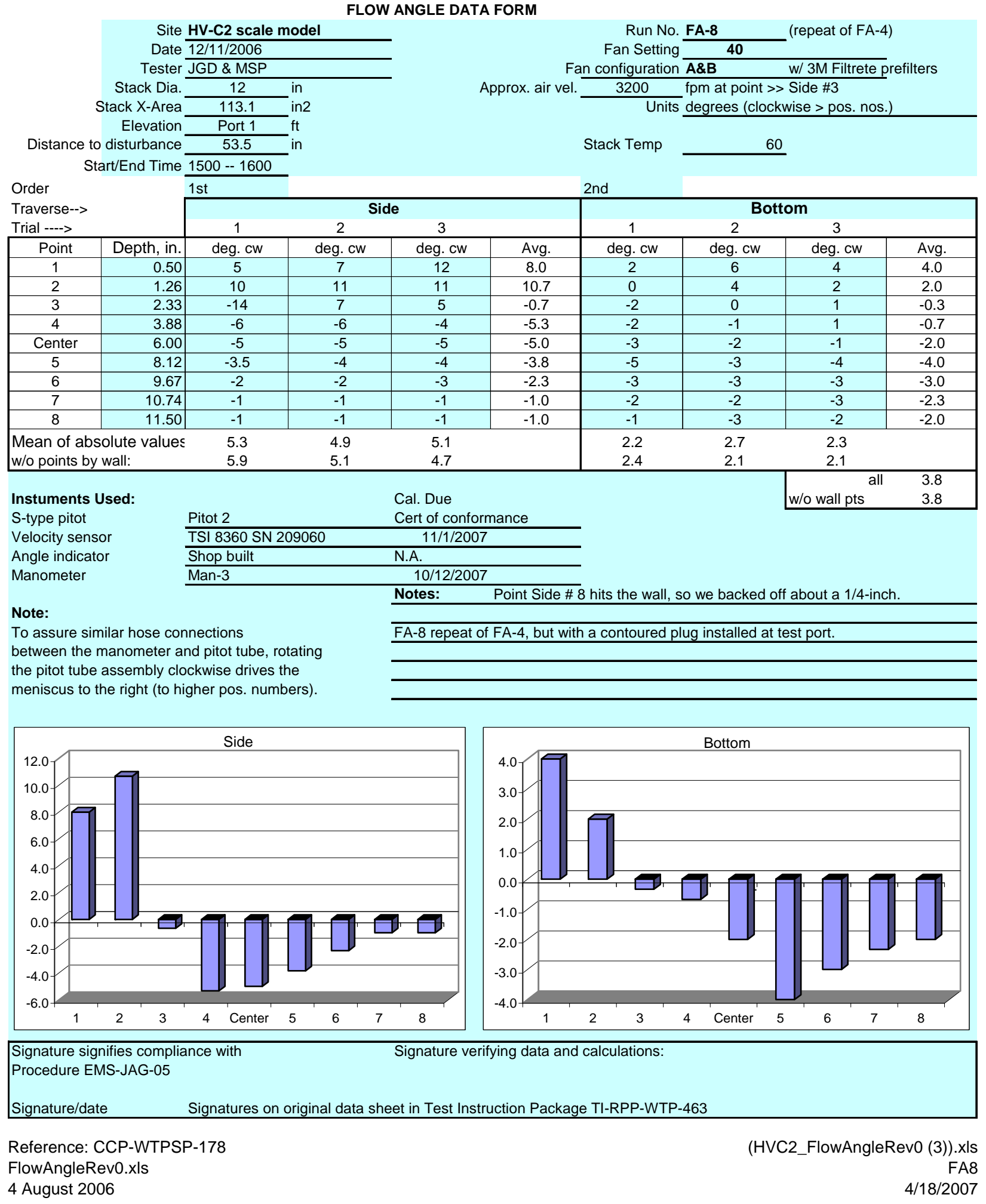




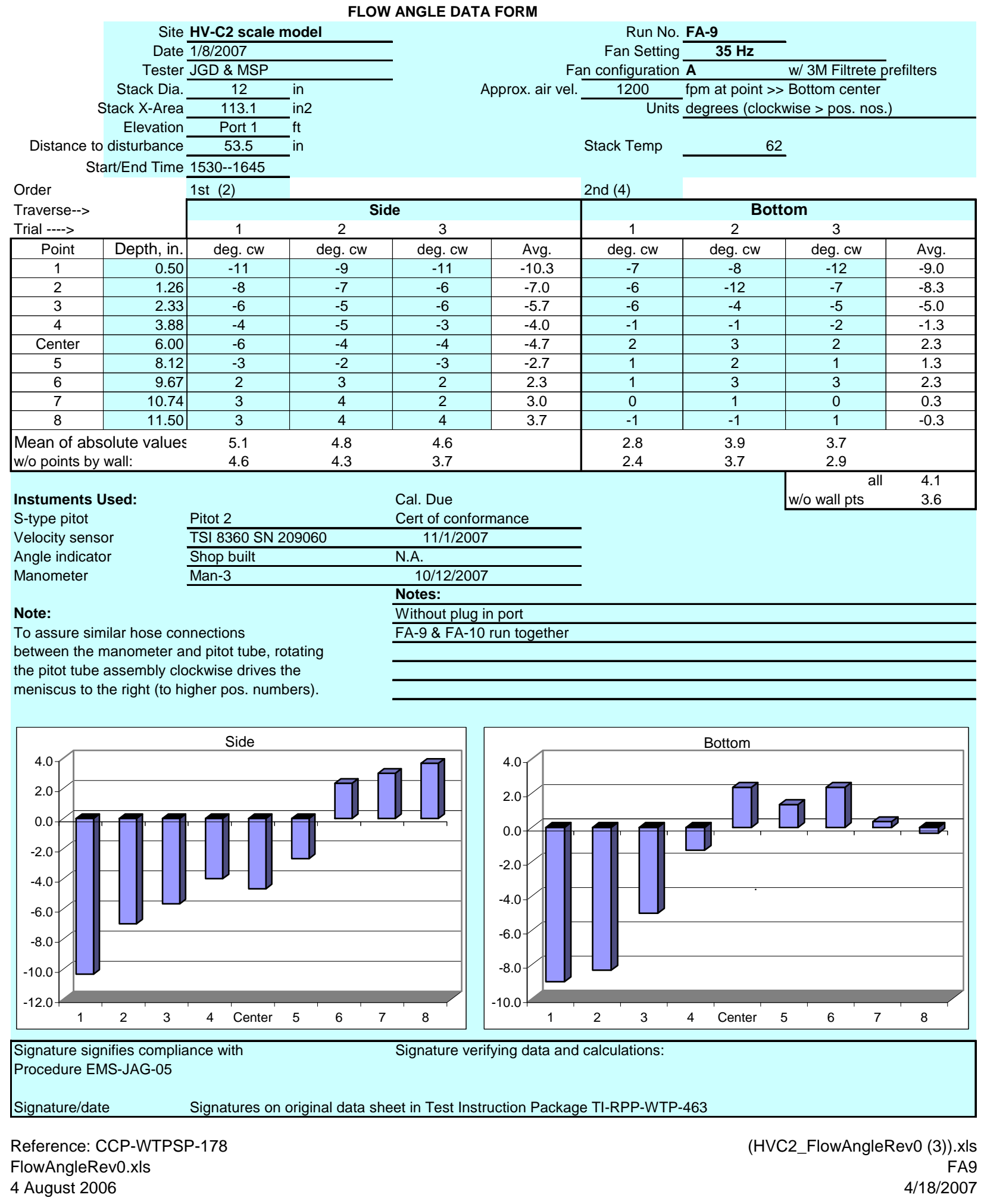




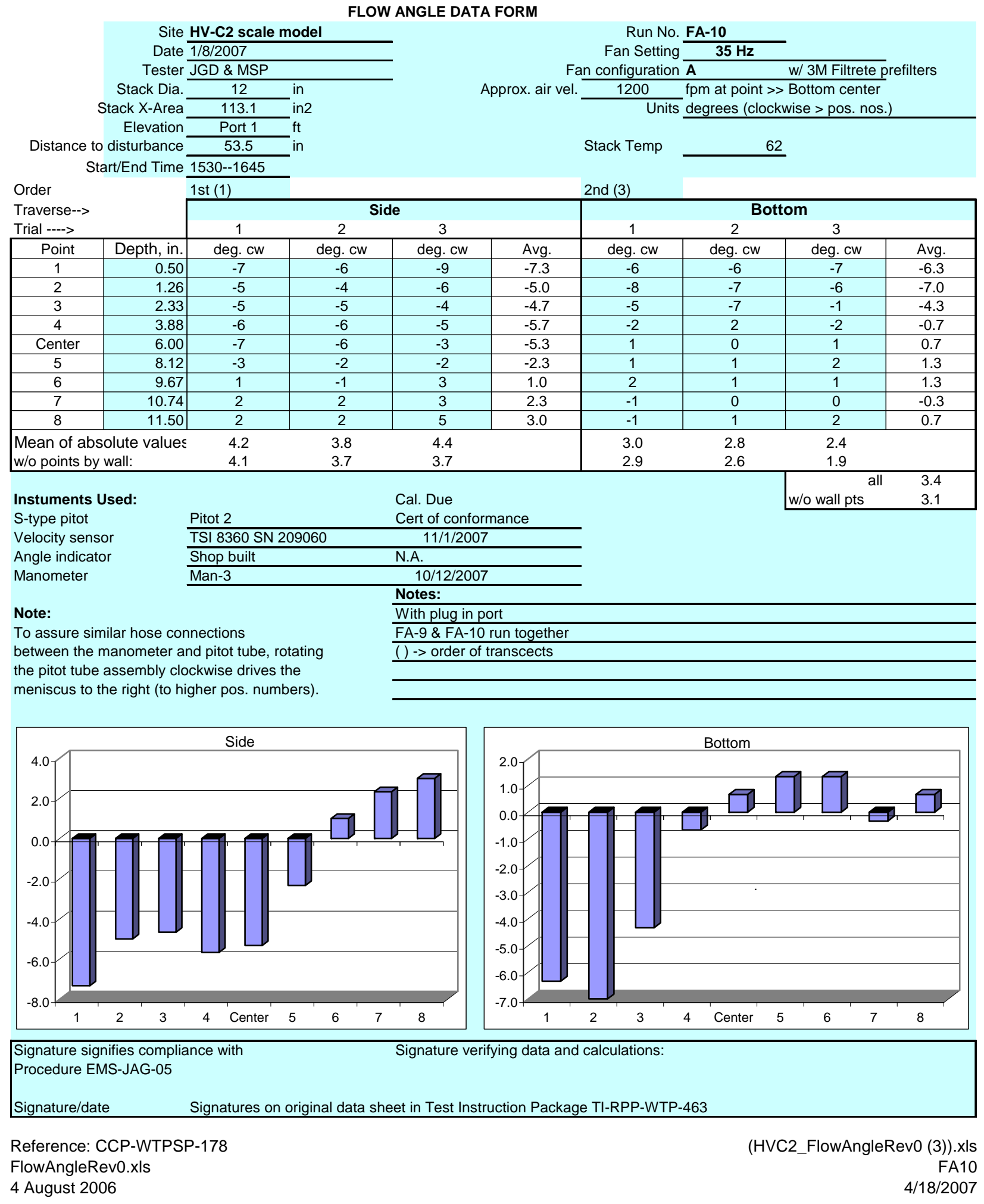




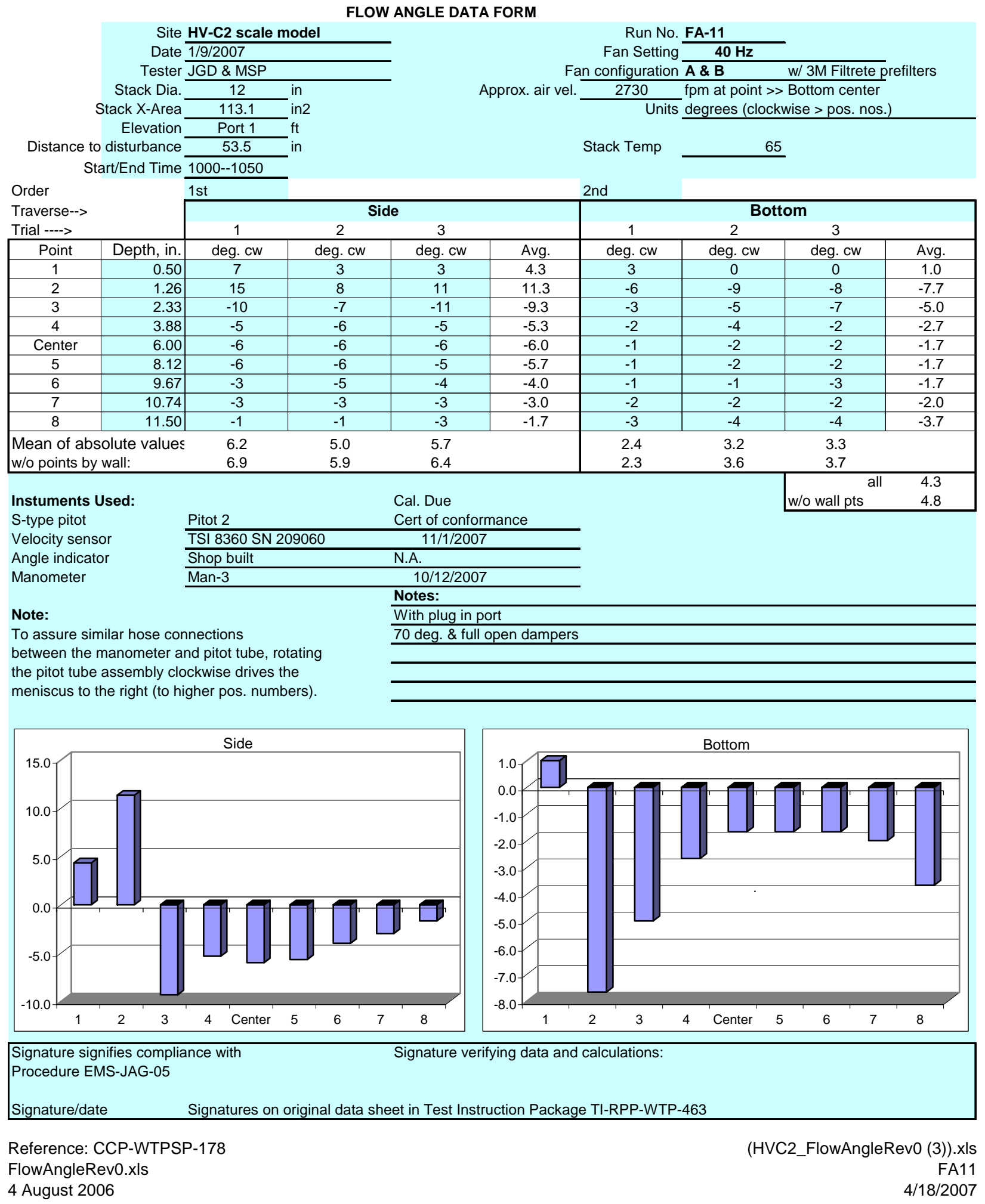




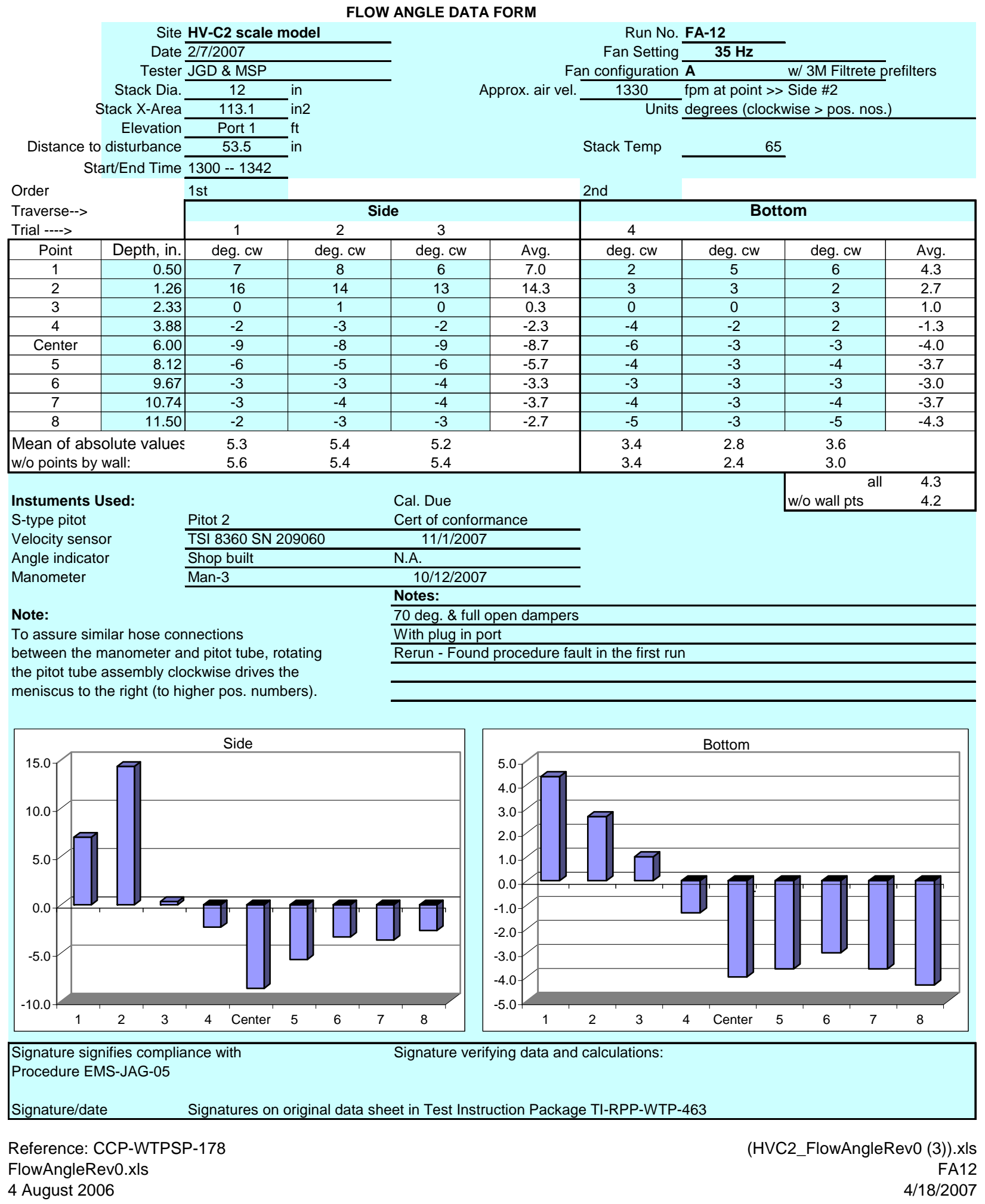




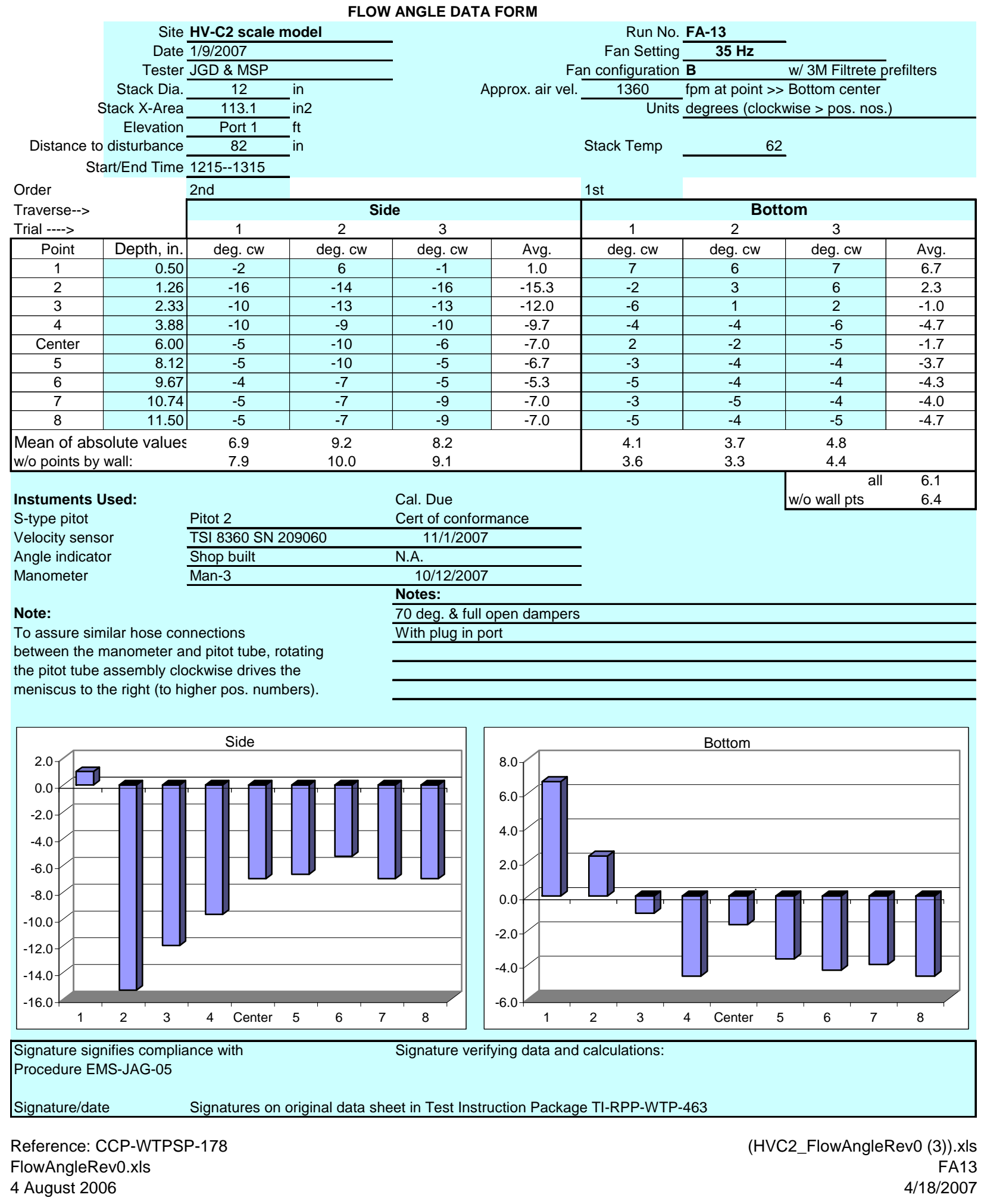




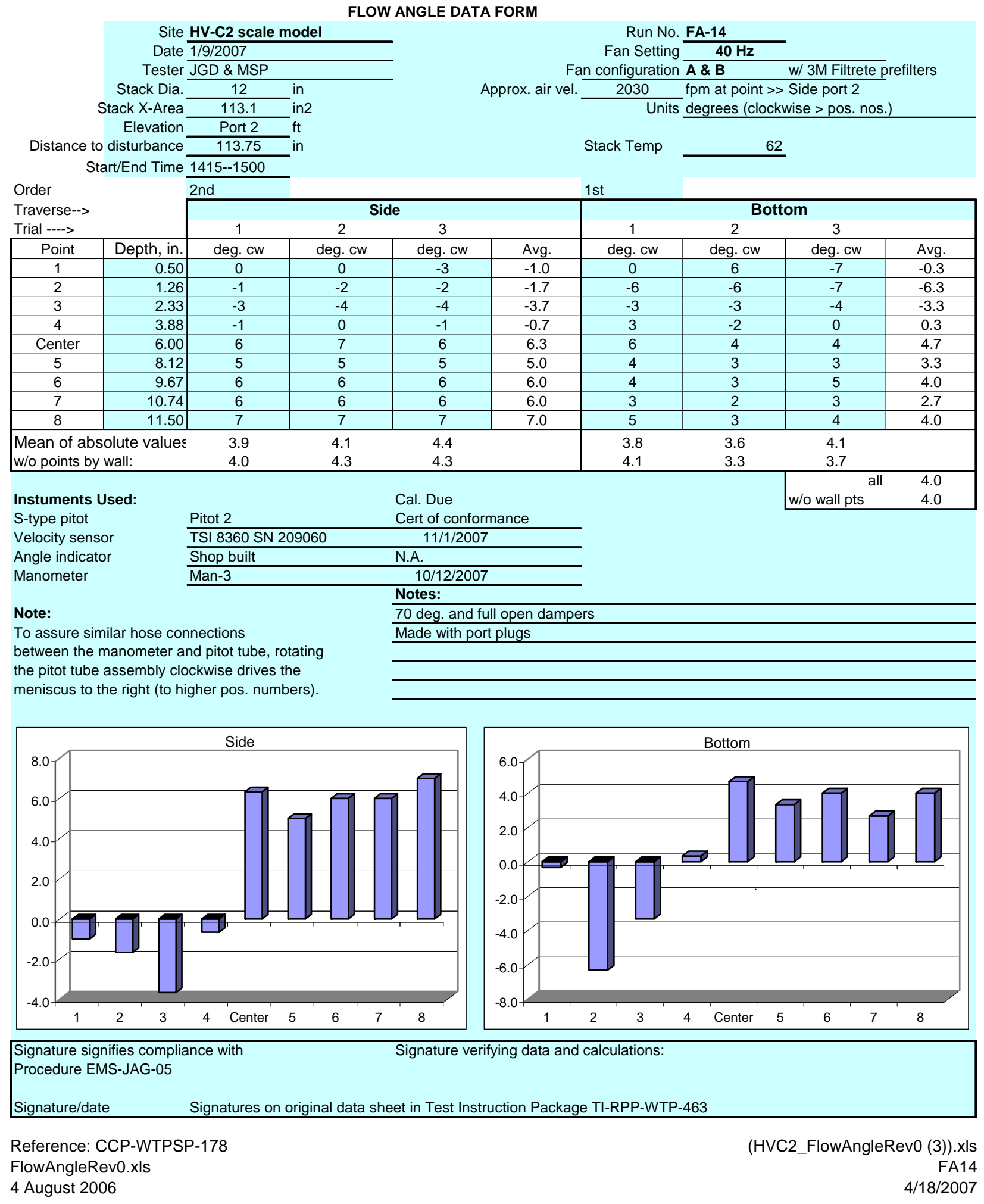




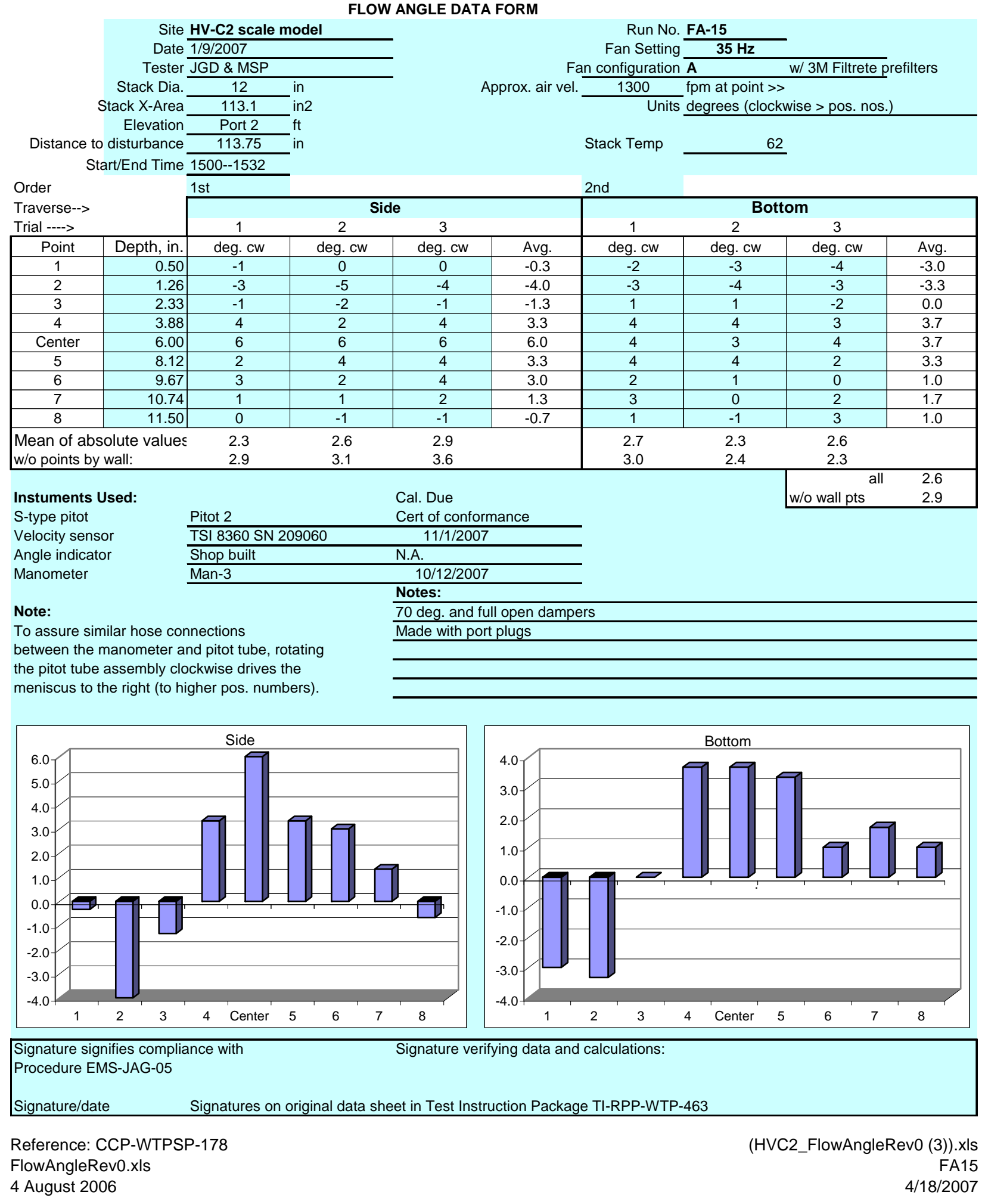




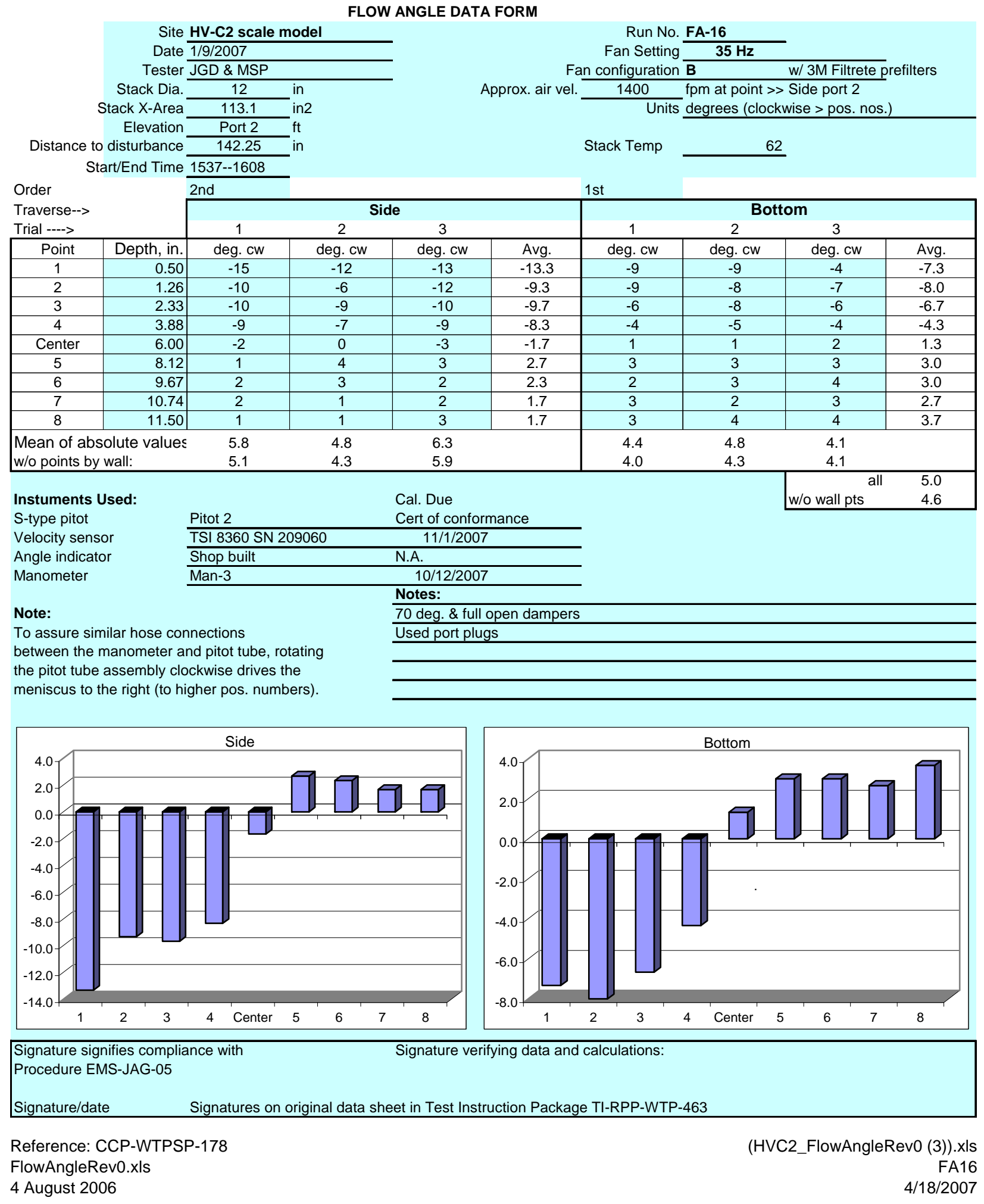




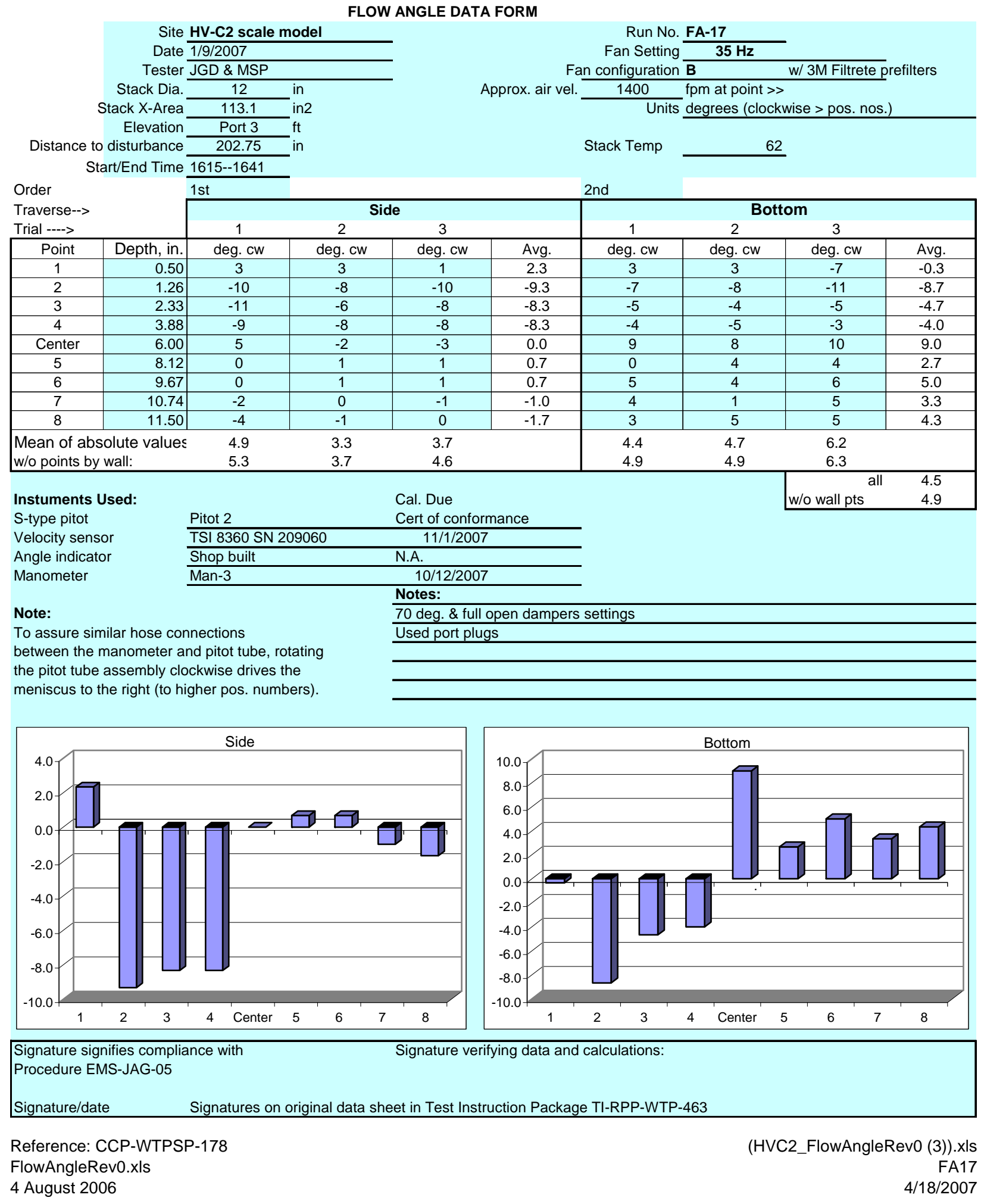




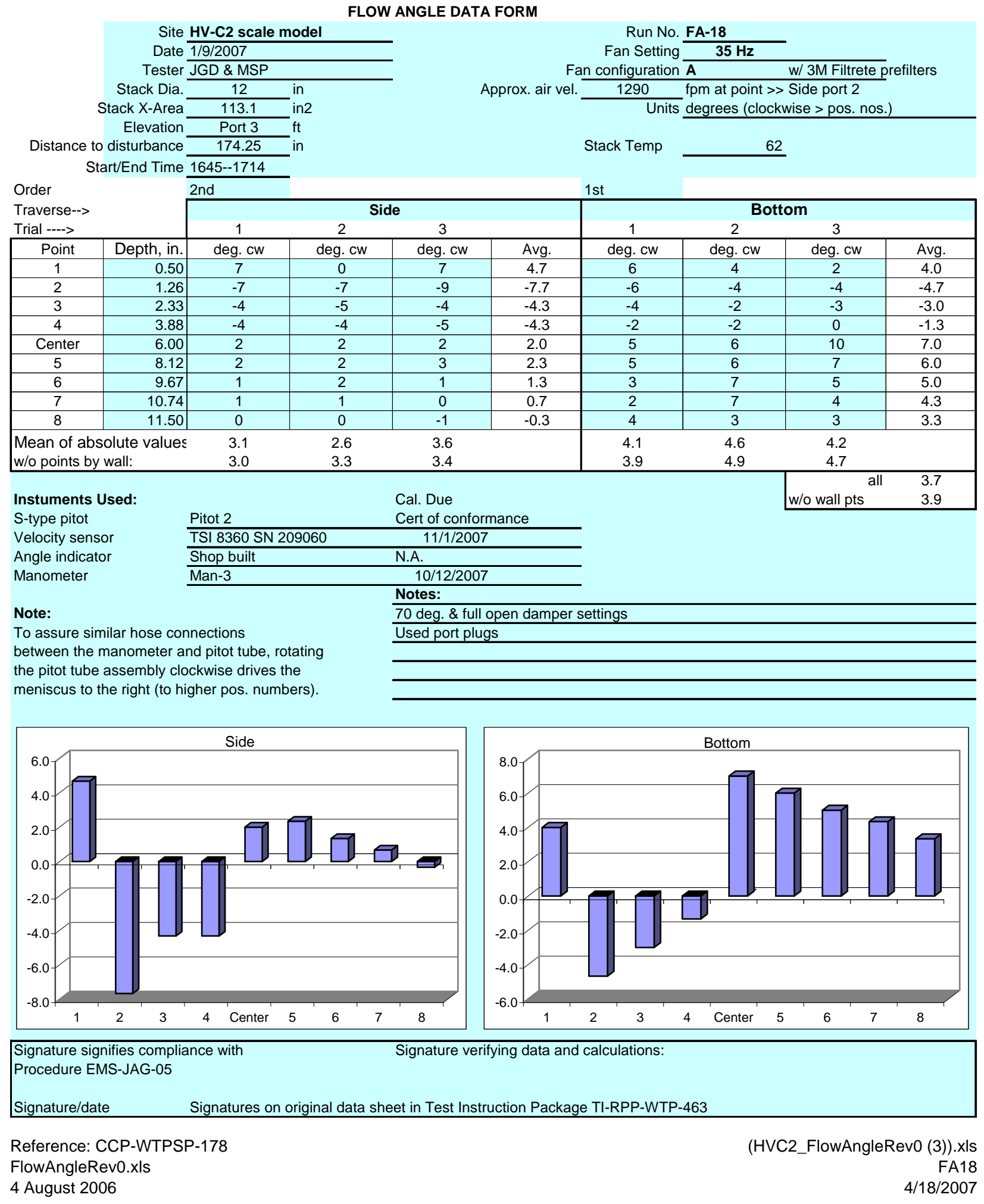




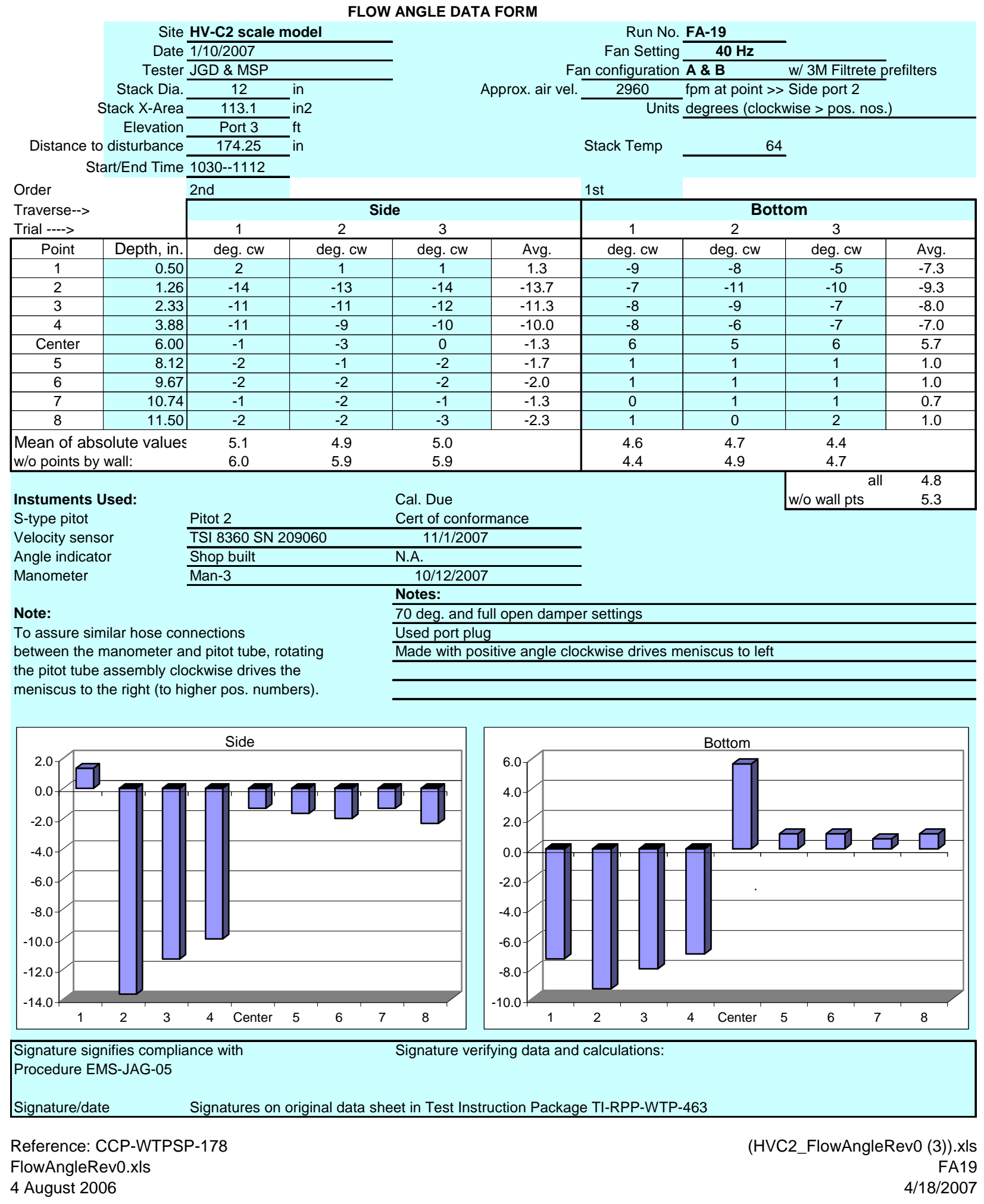


Appendix E

\section{Tracer-Gas Uniformity Data Sheets}




\section{Appendix E: Tracer-Gas Uniformity Data Sheets}

Rev. 0

31-Jul-06

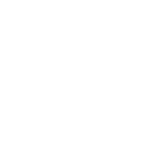

Distance to disturt

disturbance

Measurement units ppm SF6

\begin{tabular}{|c|c|}
\hline $\begin{array}{l}\text { Traverse--> } \\
\text { Trial ----> }\end{array}$ & \\
\hline Point & Depth, in. \\
\hline 1 & 0.5 \\
\hline 2 & 1.2 \\
\hline 3 & 2.3 \\
\hline 4 & 3.8 \\
\hline Center & 6.0 \\
\hline 5 & 8.1 \\
\hline 6 & 9.6 \\
\hline 7 & 10.7 \\
\hline 8 & 11.5 \\
\hline
\end{tabular}

2nd

\section{TRACER GAS TRAVERSE DATA FORM}

Run No. GT-1

Fan Configuration A \& B

Fan Setting 40

Stack Temp

Start/End Time 1115 -- 1245

Center $2 / 3$ from

Points in Center $2 / 3$

Injection Point A -- Center w/ 3M filterete prefilters

$\mathrm{Hz}$

$61 \operatorname{deg} \mathrm{F}$

1.10 to: 10.90

$1 \mathrm{st}$

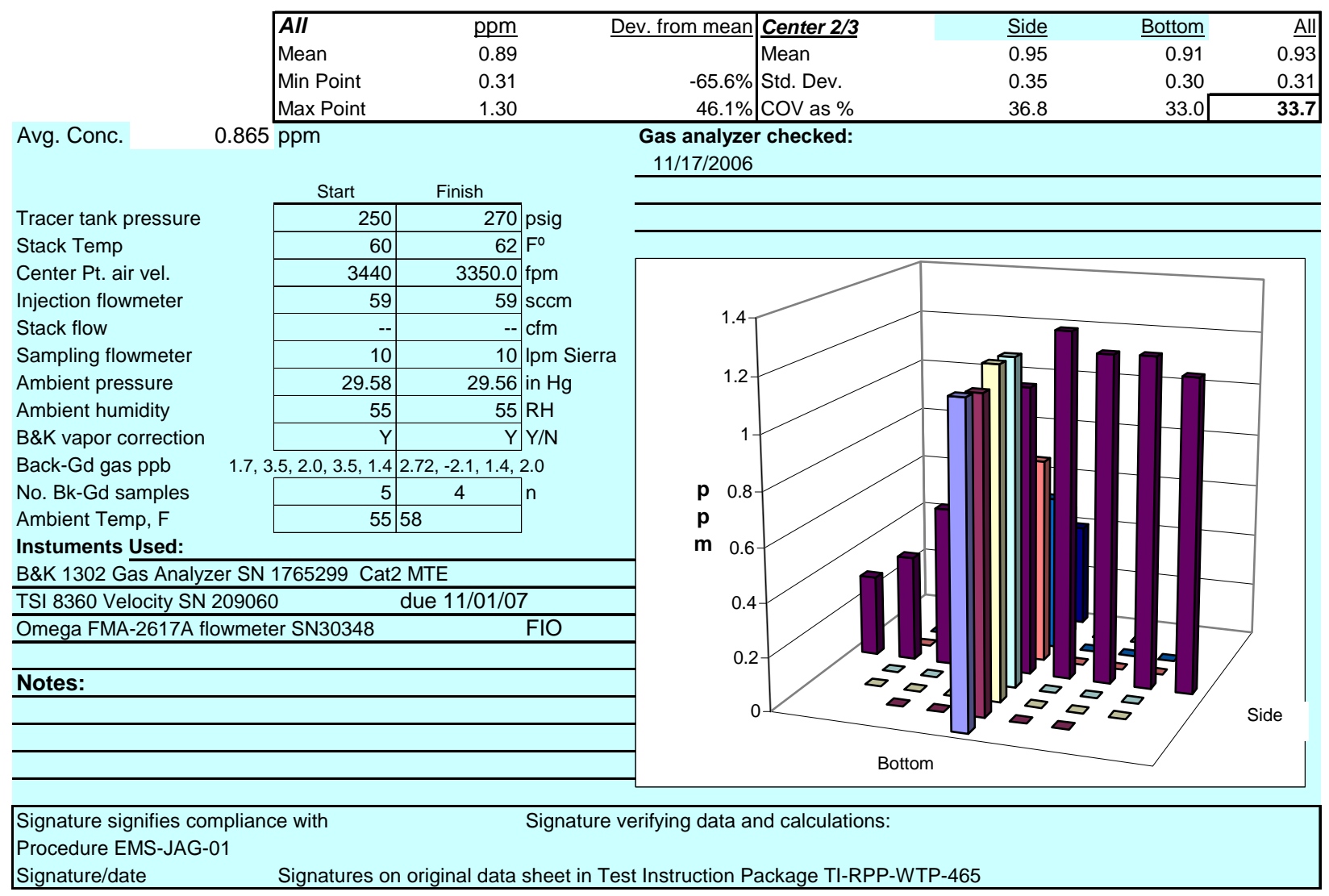

Reference: CCP-WTPSP-176

(HVC2_gas-dataRev0 (8)).xls

gas-dataRev0.xls

GT1

31 July 2006

4/18/2007 
Rev. 0

31-Jul-06

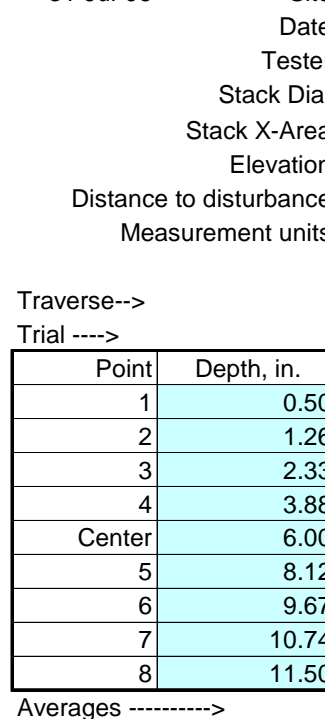

TRACER GAS TRAVERSE DATA FORM

Site HV-C2 Model

Date 11/20/2006

Tester BG Fritz

ack Dia.

$\frac{12 \text { in. }}{\frac{113.1 \text { in. }^{2}}{\text { Port } 1}}$

53.5 inches

ppm SF6

Fan Setting 40

Stack Temp

Center $2 / 3$ from

Points in Center $2 / 3$

Run No. GT-2

Fan Configuration $\mathrm{A}$ \& B on

Start/End Time $2 \mathrm{pm} / 3: 30$

Injection Point $\overline{A \text { near port, left side }}$

w/ 3M filterete prefilters

$\mathrm{Hz}$

$60.5 \operatorname{deg} F$ 1st 2nd

Avg. Conc.

$$
0.819 \mathrm{ppm}
$$

$0.819 \mathrm{ppm}$

\begin{tabular}{|lr}
\hline All & ppm \\
Mean & 0.82 \\
Min Point & 0.38 \\
Max Point & 1.51 \\
\hline
\end{tabular}

Tracer tank pressure

Stack Temp

Center Pt. air vel.

Injection flowmeter

Stack flow

Sampling flowmeter

Ambient pressure

Ambient humidity

B\&K vapor correction

Back-Gd gas ppb

No. Bk-Gd samples

Ambient Temp, $F$

Instuments Used:

B\&K 1302 Gas Analyzer SN 1765299 Cat2 MTE

TSI 8360 Velocity SN 209060

Omega FMA-2617A flowmeter SN30348

due $11 / 01 / 07$

\begin{tabular}{|c|c|}
\hline Start & Finish \\
\hline 300 & 300 \\
\hline 61 & 60 \\
\hline 3300 & 3240.0 \\
\hline 59 & 59 \\
\hline-- & -- \\
\hline 10 & 10 \\
\hline 29.53 & 29.52 \\
\hline 47 & -- \\
\hline yes & yes \\
\hline
\end{tabular}

\begin{tabular}{rl|r}
\hline $5.5,5.5,7.3,5.4$ & $7.3,4.7,8.0,8.1$ \\
\hline
\end{tabular}

$$
\begin{array}{|r|l|l|}
\hline 4 & 4 \\
\hline 58 & 58 \\
\end{array}
$$

due $11 / 01 / 07$

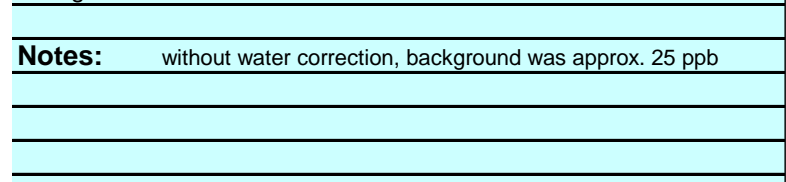

\begin{tabular}{r|l} 
Dev. from mean & Center 2/3 \\
$-53.4 \%$ & Mean \\
$84.6 \%$ & Ctd. Dev. \\
COV as \%
\end{tabular}

$11 / 17 / 2006$

Signature signifies compliance with

Signature verifying data and calculations:

Procedure EMS-JAG-01

Signature/date

Signatures on original data sheet in Test Instruction Package TI-RPP-WTP-465

Reference: CCP-WTPSP-176

(HVC2_gas-dataRev0 (8)).xls

gas-dataRev0.xls

GT2

31 July 2006

$4 / 18 / 2007$ 
Rev. 0

31-Jul-06

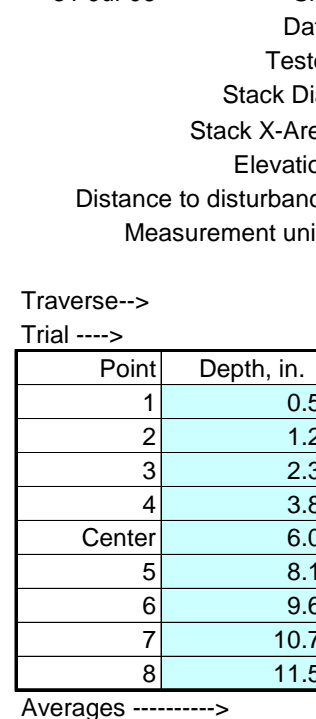

TRACER GAS TRAVERSE DATA FORM

Site HV-C2 Model

Date 11/21/2006

Tester BG Fritz

Stack Dia.

$\frac{12 \text { in. }}{\frac{113.1 \text { in. }^{2}}{\text { Port } 1}}$

53.5 inches

ppm SF6

Fan Setting 40

Stack Temp

Center $2 / 3$ from

Points in Center $2 / 3$

Run No. GT-3

Fan Configuration $\mathrm{A}$ \& $\mathrm{B}$ on

Start/End Time 11:15/1:30

Injection Point A near port, right side

w/ 3M filterete prefilters

$\mathrm{Hz}$

$56 \operatorname{deg} F$

1.10 to: 10.90

1st

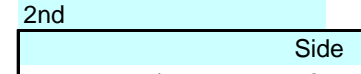

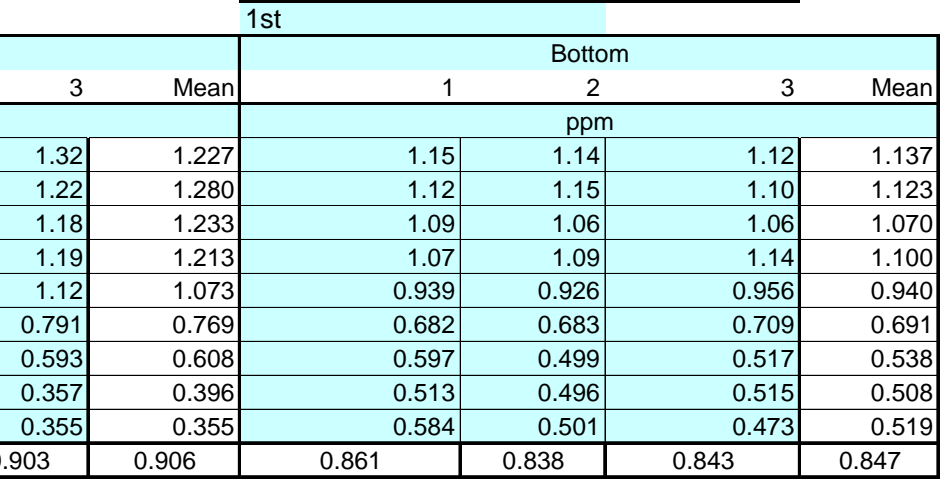

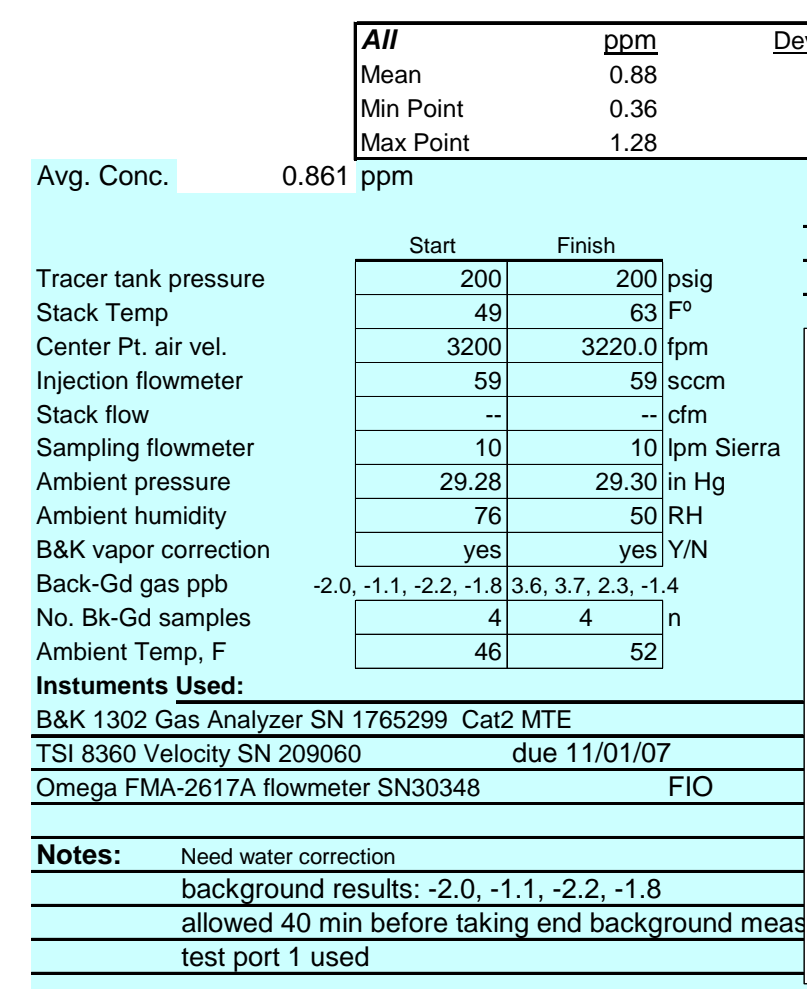

\begin{tabular}{r|l} 
Dev. from mean & $\frac{\text { Center } 2 / 3}{\text { Mean }}$ \\
$-59.5 \%$ & Std. Dev. \\
$46.0 \%$ & COV as \% \\
\hline
\end{tabular}

\section{Gas analyzer checked:}

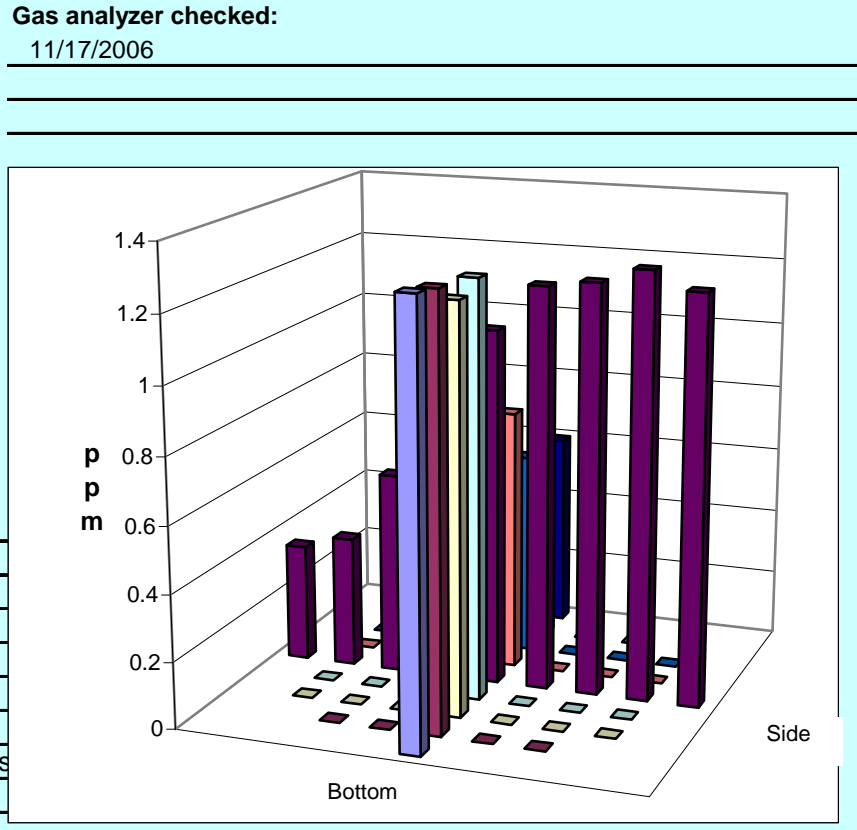

Signature signifies compliance with

Signature verifying data and calculations:

Procedure EMS-JAG-01

Signature/date

Signatures on original data sheet in Test Instruction Package TI-RPP-WTP-465

Reference: CCP-WTPSP-176

(HVC2_gas-dataRev0 (8)).xls

gas-dataRev0.xls

GT3

31 July 2006

$4 / 18 / 2007$ 
Rev. 0

31-Jul-06

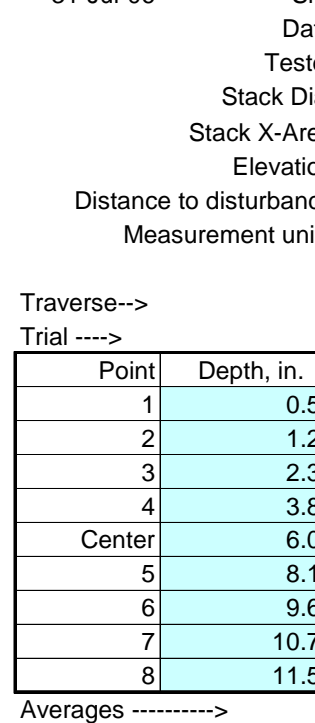

TRACER GAS TRAVERSE DATA FORM

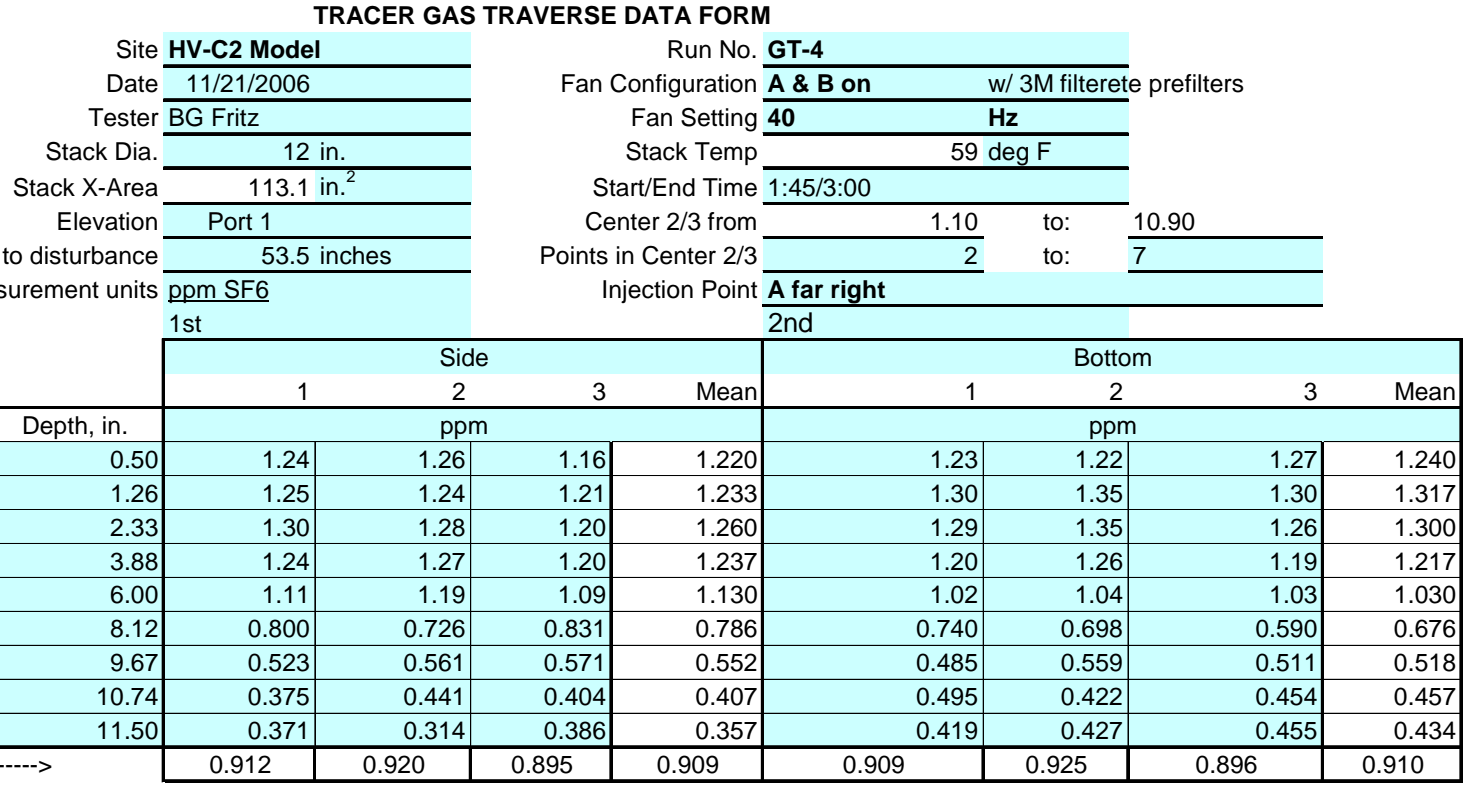

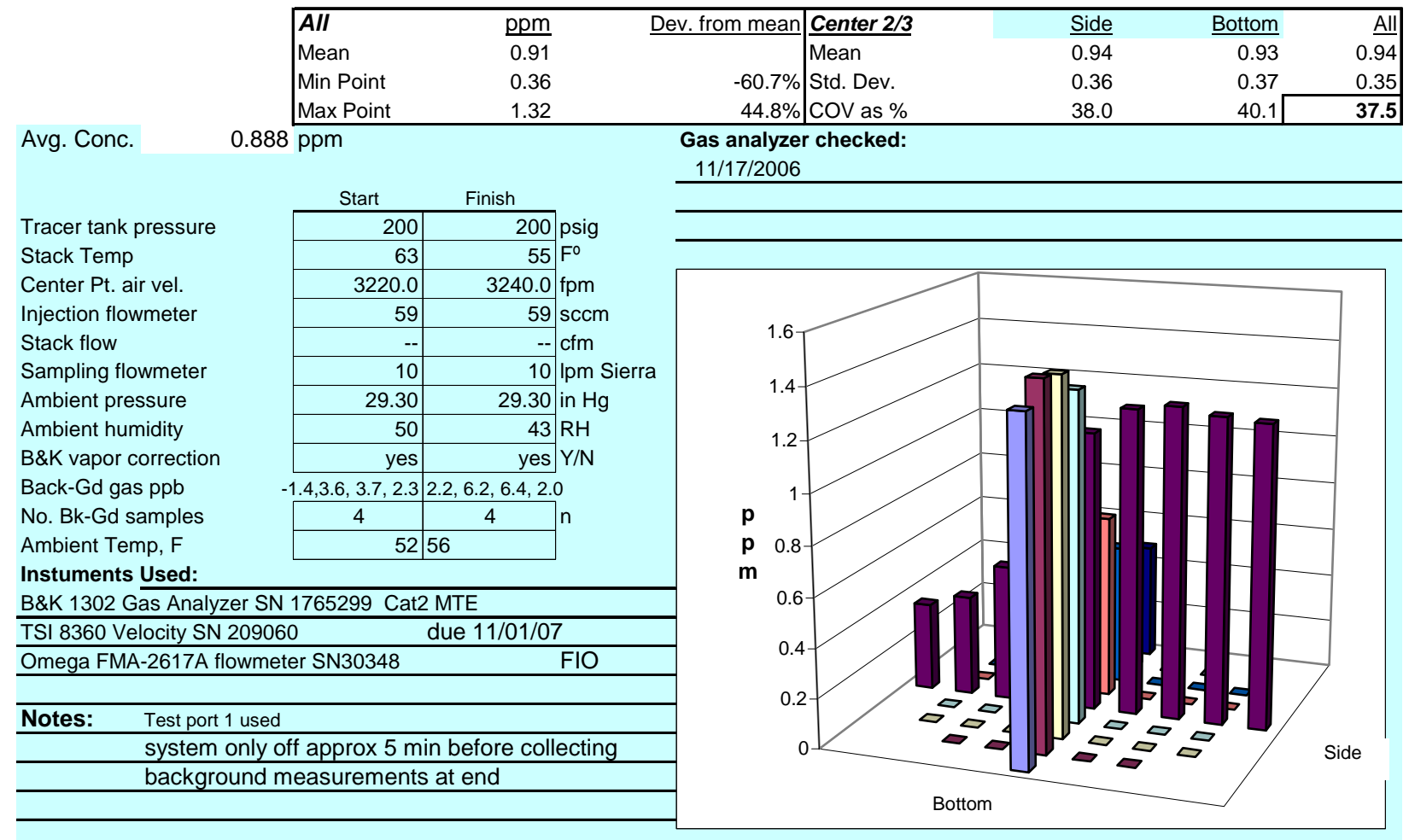

Signature signifies compliance with

Signature verifying data and calculations:

Procedure EMS-JAG-01

Signature/date

Signatures on original data sheet in Test Instruction Package TI-RPP-WTP-465

Reference: CCP-WTPSP-176

(HVC2_gas-dataRev0 (8)).xls

gas-dataRev0.xls

GT4

31 July 2006

$4 / 18 / 2007$ 
Rev. 0

31-Jul-06

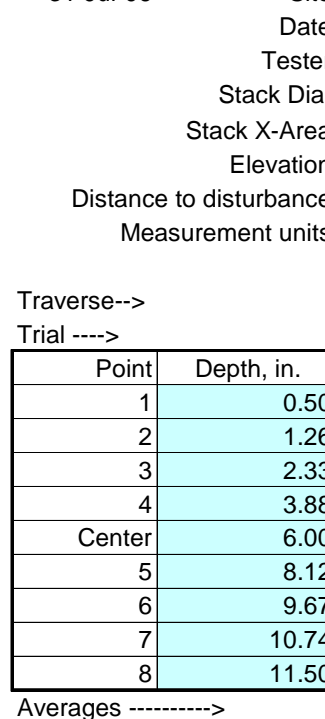

TRACER GAS TRAVERSE DATA FORM

Site HV-C2 Model

Date 11/22/2006

Tester BG Fritz

ack Dia.

$\frac{12 \text { in. }}{\frac{113.1 \text { in. }^{2}}{\text { Port } 1}}$

53.5 inches

ppm SF6

Fan Setting $\mathbf{4 0}$

Stack Temp

Center $2 / 3$ from

Points in Center $2 / 3$

Run No. GT-5

Fan Configuration $\mathbf{A}$ \& $\mathbf{B}$ on $\quad$ w/ 3M filterete prefilters

Start/End Time 12:00/1:00

Injection Point $\mathbf{A}$ far left

$\mathrm{Hz}$

$55 \operatorname{deg} F$

1.10 to: $\frac{10.90}{7}$

2 to: $\frac{10}{7}$ 1st

2nd

\begin{tabular}{|c|c|c|c|c|c|c|c|}
\hline 1 & 2 & 3 & Mean & 1 & 2 & 3 & Mean \\
\hline \multicolumn{4}{|c|}{ ppm } & \multicolumn{4}{|c|}{ ppm } \\
\hline 0.672 & 0.642 & 0.663 & 0.659 & 1.50 & 1.60 & 1.58 & 1.560 \\
\hline 0.774 & 0.775 & 0.715 & 0.755 & 1.53 & 1.51 & 1.52 & 1.520 \\
\hline 0.847 & 0.843 & 0.829 & 0.840 & 1.47 & 1.50 & 1.48 & 1.483 \\
\hline 1.01 & 1.01 & 0.998 & 1.006 & 1.42 & 1.31 & 1.31 & 1.347 \\
\hline 1.17 & 1.16 & 1.19 & 1.173 & 1.150 & 1.16 & 1.09 & 1.133 \\
\hline 1.050 & 1.080 & 1.030 & 1.053 & 0.845 & 0.812 & 0.850 & 0.836 \\
\hline 0.923 & 0.875 & 0.909 & 0.902 & 0.654 & 0.660 & 0.686 & 0.667 \\
\hline 0.795 & 0.756 & 0.712 & 0.754 & 0.619 & 0.535 & 0.536 & 0.563 \\
\hline 0.735 & 0.721 & 0.668 & 0.708 & 0.518 & 0.503 & 0.482 & 0.501 \\
\hline 0.886 & 0.874 & 0.857 & 0.872 & 1.078 & 1.066 & 1.059 & 1.068 \\
\hline
\end{tabular}

Avg. Conc.

\begin{tabular}{|lr}
\hline All & ppm \\
Mean & 0.97 \\
Min Point & 0.50 \\
Max Point & 1.56 \\
\hline
\end{tabular}

$0.947 \mathrm{ppm}$

Start

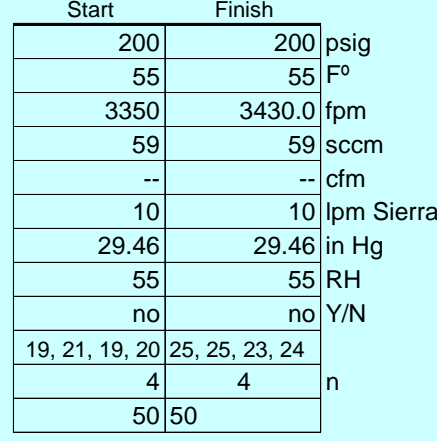

Tracer tank pressure

Stack Temp

Center Pt. air vel.

Injection flowmeter

Stack flow

Sampling flowmeter

Ambient pressure

Ambient humidity

B\&K vapor correction

Back-Gd gas ppb

No. Bk-Gd samples

Ambient Temp, $F$

Instuments Used:

B\&K 1302 Gas Analyzer SN 1765299 Cat2 MTE

TSI 8360 Velocity SN 209060

Omega FMA-2617A flowmeter SN30348

due $11 / 01 / 07$

Notes: $\quad$ with water correction, background around 7 or $8 \mathrm{ppb}$ \begin{tabular}{r|l} 
Dev. from mean & Center $\mathbf{2 / 3}$ \\
$-48.4 \%$ & Mean \\
$60.8 \%$ & Ctd. Dev. \\
CoV as \%
\end{tabular}

Gas analyzer checked:

$11 / 17 / 2006$

Signature signifies compliance with

Signature verifying data and calculations:

Procedure EMS-JAG-01

Signature/date

Signatures on original data sheet in Test Instruction Package TI-RPP-WTP-465

Reference: CCP-WTPSP-176

(HVC2_gas-dataRev0 (8)).xls

gas-dataRev0.xls

GT5

31 July 2006

$4 / 18 / 2007$ 
Rev. 0

31-Jul-06

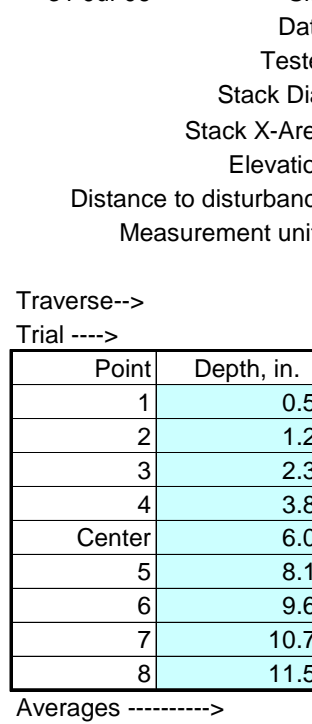

TRACER GAS TRAVERSE DATA FORM

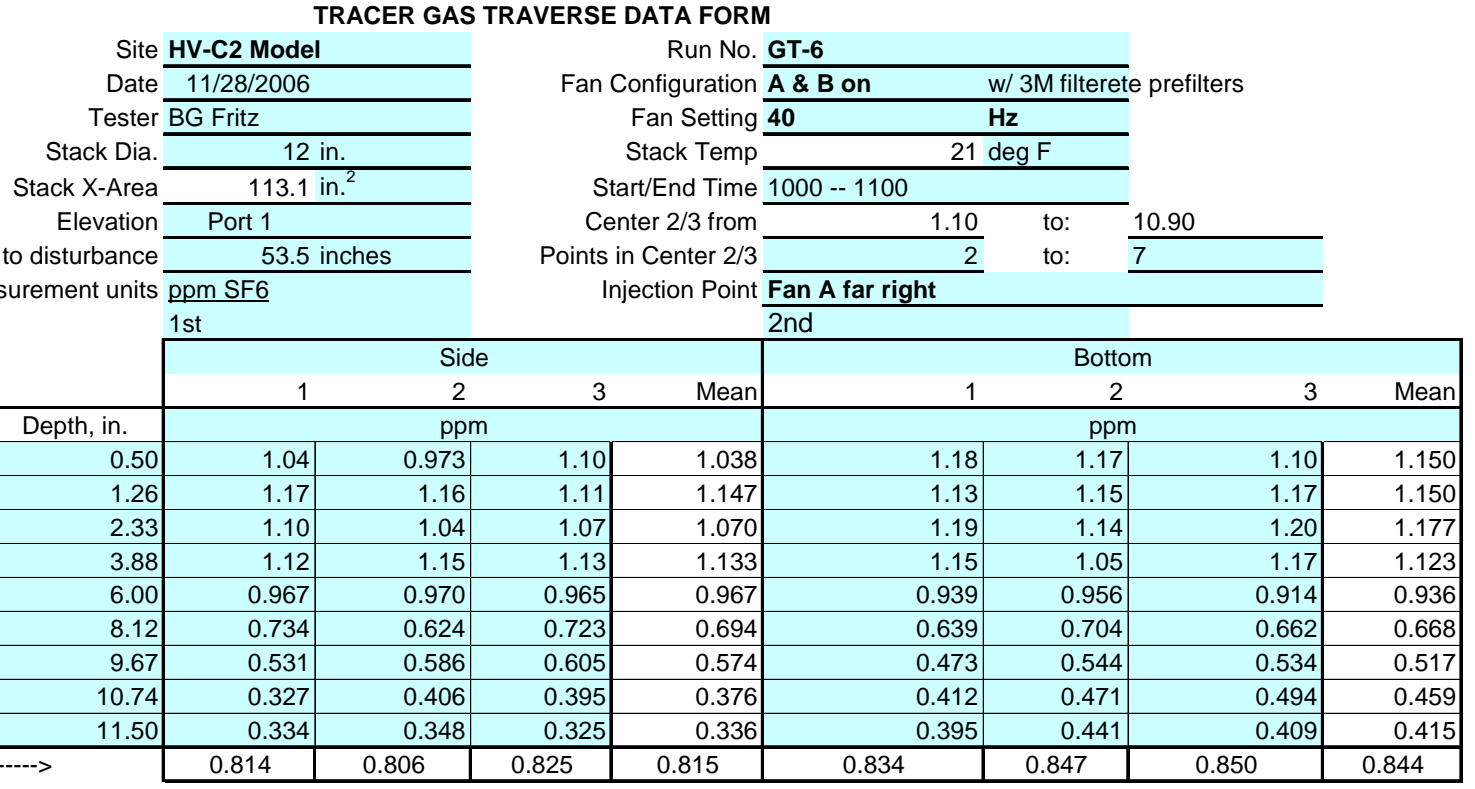

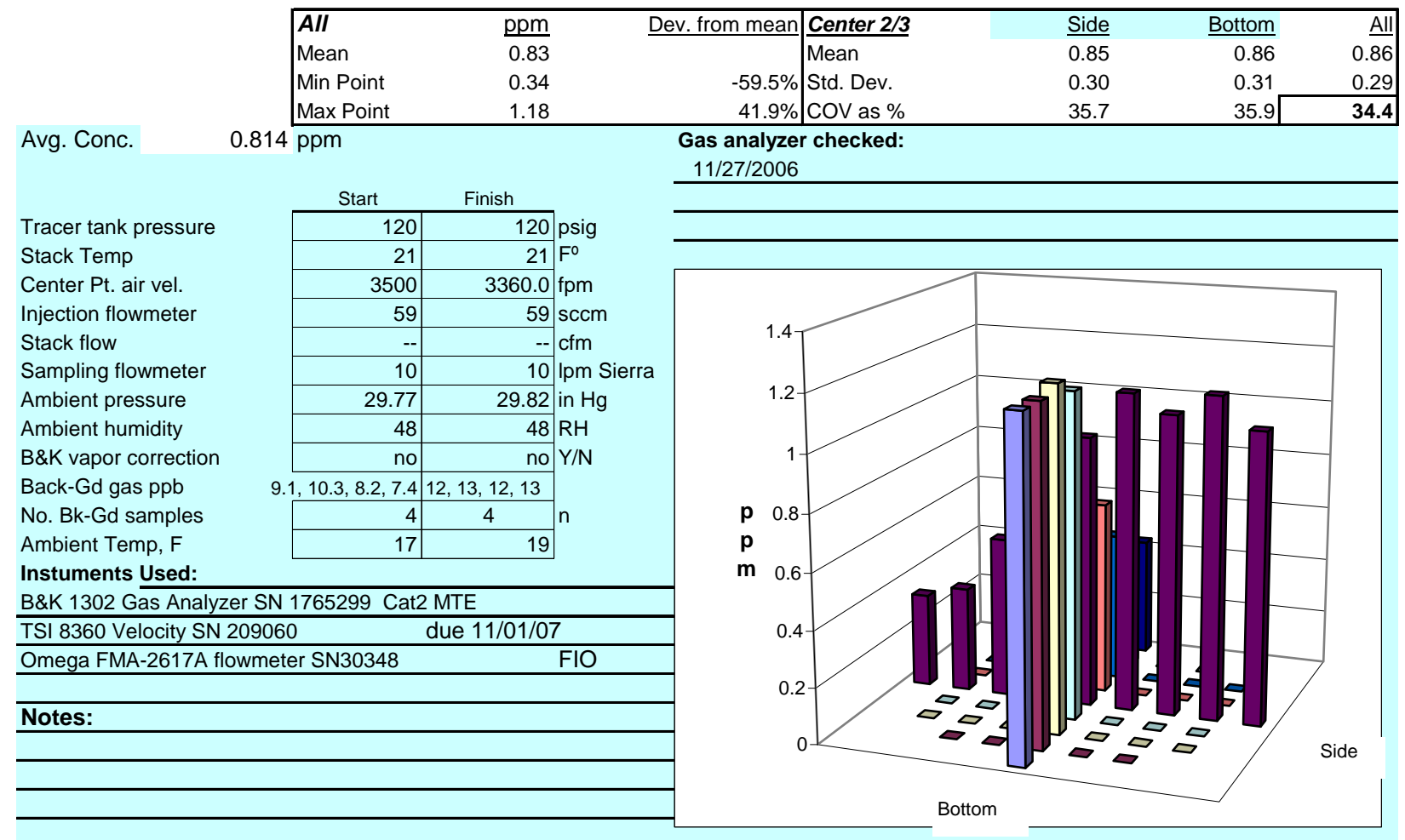

Signature signifies compliance with

Signature verifying data and calculations:

Procedure EMS-JAG-01

Signature/date

Signatures on original data sheet in Test Instruction Package TI-RPP-WTP-465

Reference: CCP-WTPSP-176

(HVC2_gas-dataRev0 (8)).xls

gas-dataRev0.xls

GT6

31 July 2006

$4 / 18 / 2007$ 
Rev. 0

31-Jul-06

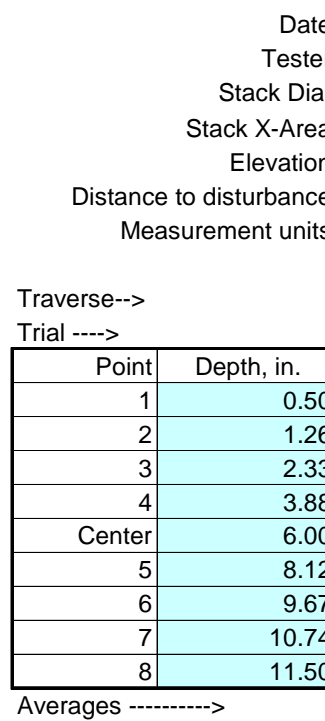

TRACER GAS TRAVERSE DATA FORM

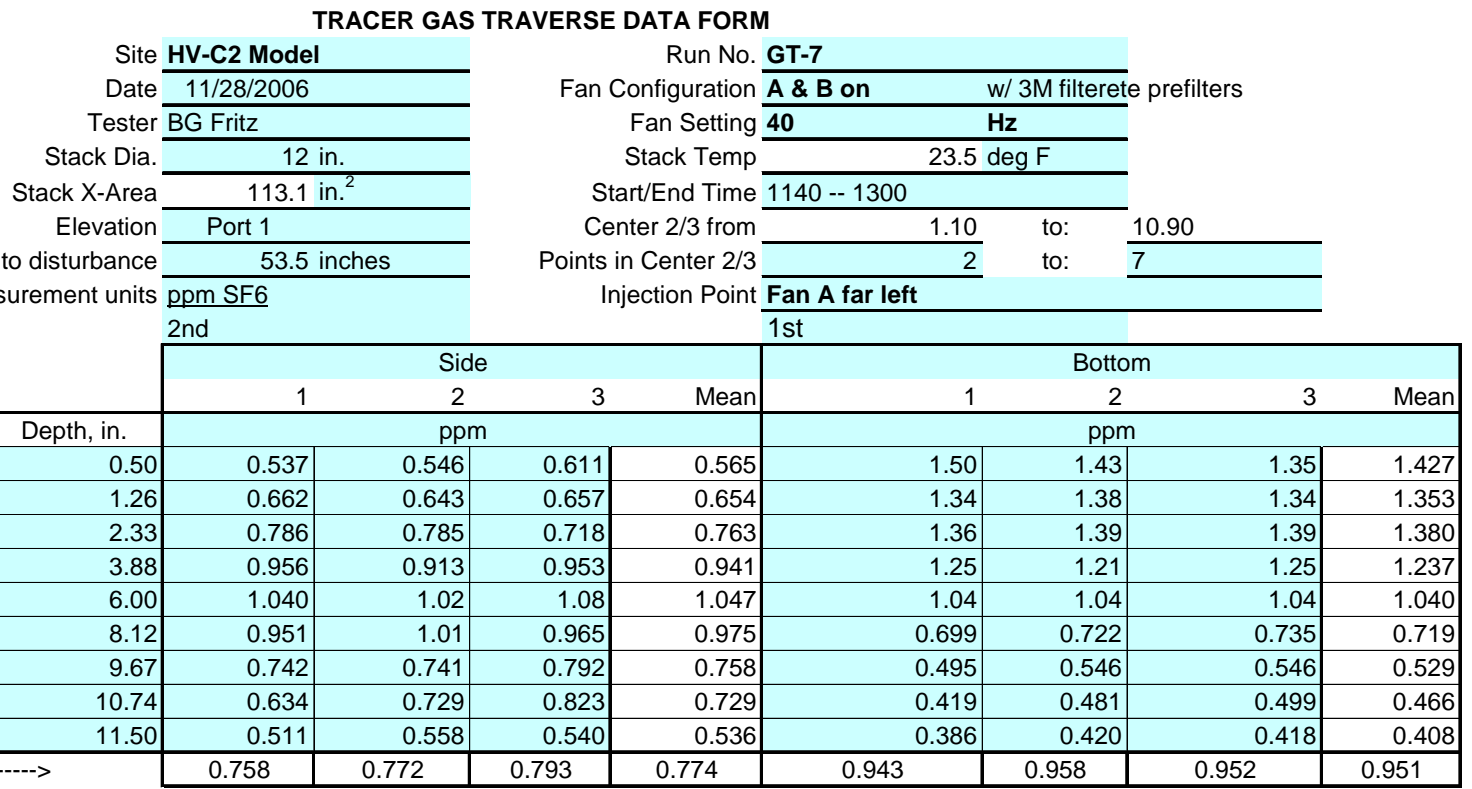

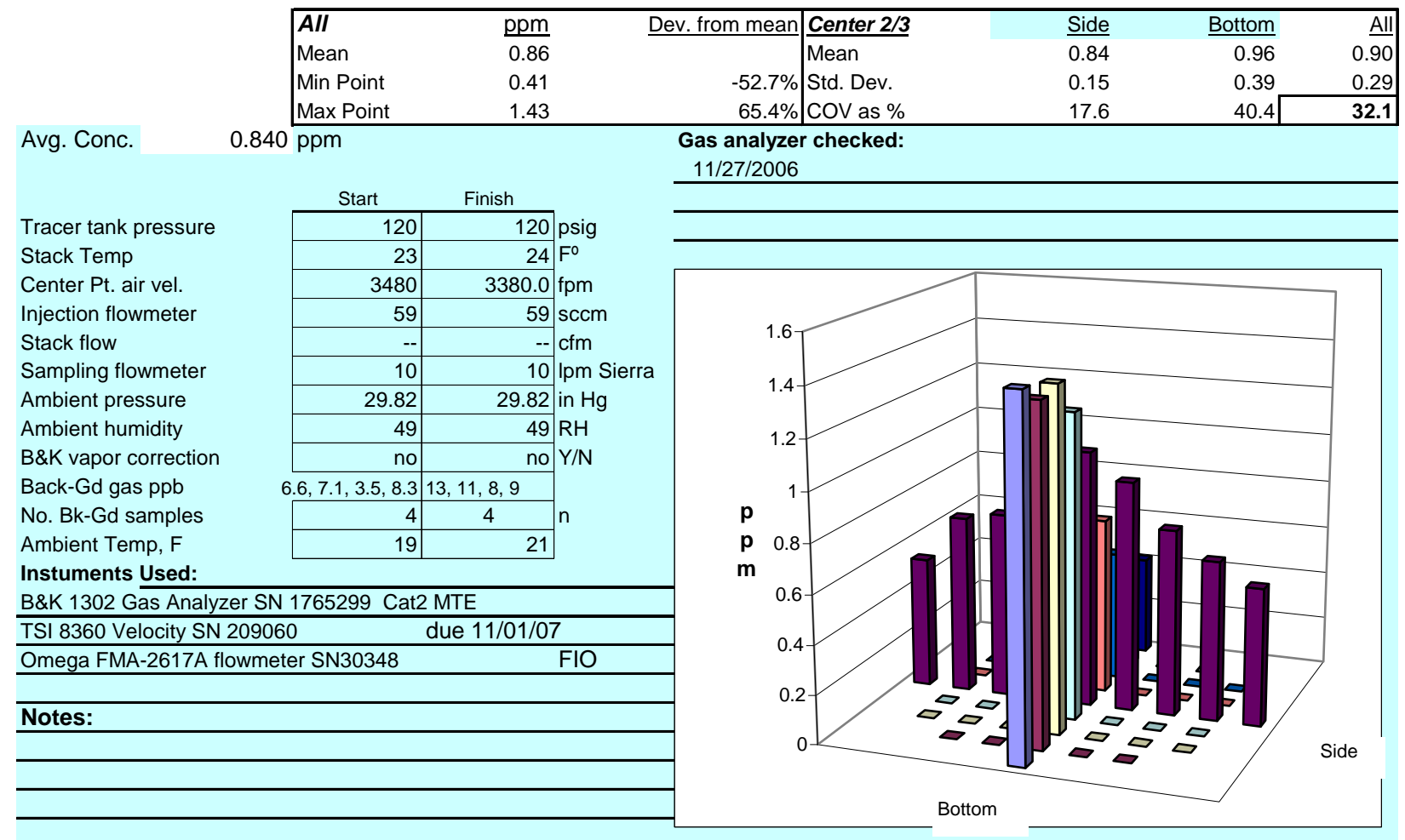

Signature signifies compliance with

Signature verifying data and calculations:

Procedure EMS-JAG-01

Signature/date

Signatures on original data sheet in Test Instruction Package TI-RPP-WTP-465

Reference: CCP-WTPSP-176

(HVC2_gas-dataRev0 (8)).xls

gas-dataRev0.xls

GT7

31 July 2006

$4 / 18 / 2007$ 
Rev. 0

31-Jul-06

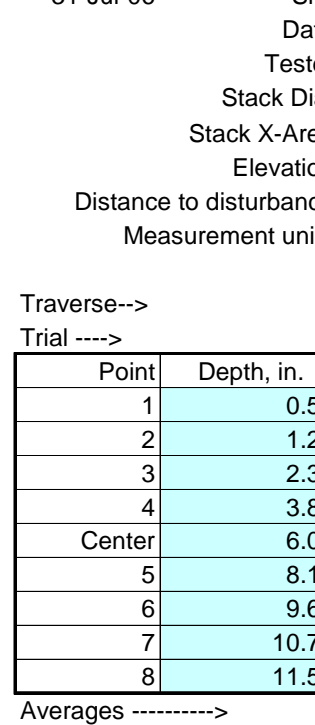

TRACER GAS TRAVERSE DATA FORM

Site HV-C2 Model

Date 11/28/2006

Tester BG Fritz \& JGD

Stack Dia.

$\frac{12 \text { in. }^{2}}{113.1 \text { in. }^{2}}$

53.5 inches

ppm SF6

Fan Setting 40

Stack Temp

Center $2 / 3$ from

Points in Center 2/3

Run No. GT-8

Fan Configuration $\mathrm{A}$ \& B on

Start/End Time 1315 -- 1343

Injection Point Fan A center

w/ 3M filterete prefilters

$\mathrm{Hz}$

$24 \operatorname{deg} F$

433

1.10 to: 10.90

2 to: $\frac{10}{7}$

2nd

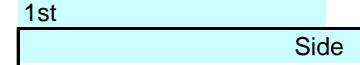

\begin{tabular}{|r|r|r|r|r|r|}
\multicolumn{2}{|c|}{} & \multicolumn{5}{|c|}{$\begin{array}{c}\text { Bottom } \\
2\end{array}$} \\
\hline \multicolumn{2}{|c|}{ Mean } & 1 & \multicolumn{5}{c|}{$\mathrm{ppm}$} \\
\hline 0.768 & 0.761 & 0.900 & 0.891 & 0.928 & 0.906 \\
\hline 0.812 & 0.808 & 0.911 & 0.893 & 0.976 & 0.927 \\
\hline 0.822 & 0.825 & 0.919 & 0.921 & 0.916 & 0.919 \\
\hline 0.882 & 0.867 & 0.949 & 0.961 & 0.902 & 0.937 \\
\hline 0.968 & 0.955 & 0.970 & 0.927 & 0.949 & 0.949 \\
\hline 0.937 & 0.932 & 0.881 & 0.860 & 0.938 & 0.893 \\
\hline 0.866 & 0.844 & 0.754 & 0.822 & 0.840 & 0.805 \\
\hline 0.677 & 0.711 & 0.668 & 0.767 & 0.744 & 0.726 \\
\hline 0.586 & 0.607 & 0.680 & 0.795 & 0.797 & 0.757 \\
\hline 0.813 & 0.812 & 0.848 & 0.871 & 0.888 & 0.869 \\
\hline
\end{tabular}

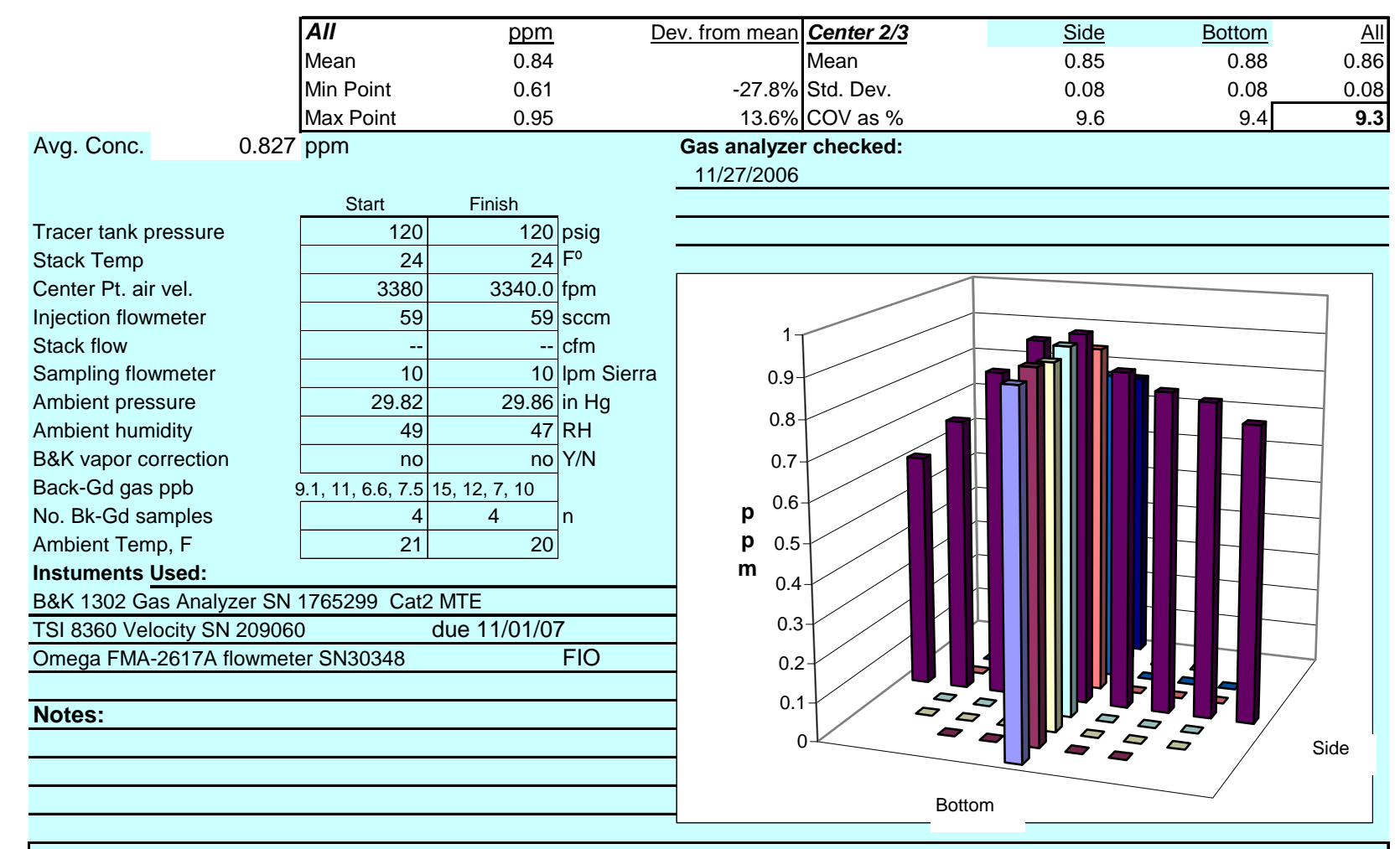

Signature signifies compliance with

Signature verifying data and calculations:

Procedure EMS-JAG-01

Signature/date

Signatures on original data sheet in Test Instruction Package TI-RPP-WTP-465

Reference: CCP-WTPSP-176

(HVC2_gas-dataRev0 (8)).xls

gas-dataRev0.xls

GT8

31 July 2006

4/18/2007 
Rev. 0

31-Jul-06

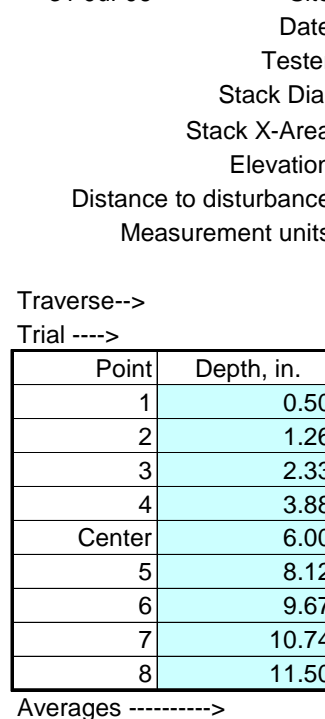

TRACER GAS TRAVERSE DATA FORM

Site HV-C2 Model

Date 11/28/2006

Tester JGD \& JAG

Stack Dia

$\frac{12 \text { in. }^{2}}{\frac{113.1 \text { in. }^{2}}{\text { Port } 1}}$

53.5 inches

ppm SF6

Fan Setting 40

Stack Temp

Center $2 / 3$ from

Points in Center 2/3

Run No. GT-9

Fan Configuration $\mathbf{A}$ \& B on

Start/End Time 1345 -- 1450 Injection Point Fan A near left

w/ 3M filterete prefilters

$\mathrm{Hz}$

$24 \operatorname{deg} F$

$\begin{array}{rll}1.10 & & \\ 2 & \text { to: } & 10.90 \\ 7 & \text { to: }\end{array}$
1st

2nd

(15t

\begin{tabular}{r|rr} 
Mean & 1 & 2 \\
\hline & &
\end{tabular}

$\begin{array}{cc}1 & 2 \\ \mathrm{ppm}\end{array}$

\begin{tabular}{|r|r|r|r|}
\hline 1.39 & 1.50 & 1.42 & 1.43 \\
\hline 1.35 & 1.45 & 1.31 & 1.37 \\
\hline 1.31 & 1.29 & 1.29 & 1.297 \\
1.14 & 1.10 & 1.10 & 1.113 \\
\hline 0.819 & 0.900 & 0.811 & 0.843 \\
\hline 0.558 & 0.565 & 0.641 & 0.588 \\
\hline 0.440 & 0.382 & 0.414 & 0.41 \\
\hline 0.316 & 0.289 & 0.301 & 0.302 \\
\hline 0.260 & 0.261 & 0.252 & 0.258 \\
\hline 843 & 0.860 & 0.838 & 0.847 \\
\hline
\end{tabular}

\begin{tabular}{r|r} 
& \\
0.734 & 0.8 \\
\hline 0.772 & 0.7 \\
\hline 0.847 & 0.7 \\
\hline 0.920 & 0.8 \\
0.797 & 0.8 \\
0.723 & 0.7 \\
\hline 0.708 & 0.697 \\
\hline 0.693 & 0.7 \\
\hline 0.703 & 0.7 \\
\hline 66 & 0.776 \\
\hline
\end{tabular}

\begin{tabular}{|r|r|r|}
\hline \multicolumn{2}{|c|}{ p } & Mean \\
\hline 0.853 & 0.706 & 0.764 \\
\hline 0.798 & 0.796 & 0.789 \\
\hline 0.742 & 0.868 & 0.819 \\
\hline 0.835 & 0.880 & 0.878 \\
\hline 0.866 & 0.712 & 0.792 \\
\hline 0.718 & 0.731 & 0.724 \\
0.697 & 0.692 & 0.699 \\
\hline 0.744 & 0.641 & 0.693 \\
\hline 0.734 & 0.823 & 0.753 \\
\hline 76 & 0.761 & 0.768 \\
\hline
\end{tabular}

\begin{tabular}{|ll}
\hline All & $\mathrm{ppm}$ \\
Mean & 0.81 \\
Min Point & 0.26 \\
Max Point & 1.44 \\
\hline
\end{tabular}

Avg. Conc.

0.806 ppm

Start

\begin{tabular}{|c|c|c|}
\hline Stait & IIIISII & \\
\hline 120 & 120 & psig \\
\hline 24 & 24 & $F^{\circ}$ \\
\hline 3340 & 3310 & fpm \\
\hline 59 & 59 & $\mathrm{sccm}$ \\
\hline- & -- & $\mathrm{cfm}$ \\
\hline 10 & 10 & Ipm Sierra \\
\hline 29.86 & 29.90 & in $\mathrm{Hg}$ \\
\hline 47 & 48 & $\mathrm{RH}$ \\
\hline no & no & $\mathrm{Y} / \mathrm{N}$ \\
\hline $12,7,10,9$ & $10,11.6,4.8,6$ & 3 \\
\hline 4 & 4 & n \\
\hline 20 & 20 & \\
\hline
\end{tabular}

Tracer tank pressure

Stack Temp

Center Pt. air vel.

Injection flowmeter

Stack flow

Sampling flowmeter

Ambient pressure

Ambient humidity

B\&K vapor correction

Back-Gd gas ppb

No. Bk-Gd samples

Ambient Temp, F

Instuments Used:

B\&K 1302 Gas Analyzer SN 1765299 Cat2 MTE

TSI 8360 Velocity SN 209060

Omega FMA-2617A flowmeter SN30348

due $11 / 01 / 07$

FIO

\section{Notes:}

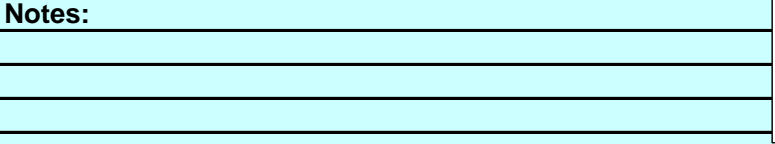

\begin{tabular}{|c|c|c|c|c|}
\hline Dev. from mean & Center $2 / 3$ & Side & Bottom & All \\
\hline & Mean & $\overline{0.85}$ & 0.77 & 0.81 \\
\hline$-68.1 \%$ & Std. Dev. & 0.43 & 0.07 & 0.30 \\
\hline $78.0 \%$ & COV as $\%$ & 50.6 & 8.9 & 36.8 \\
\hline
\end{tabular}

Gas analyzer checked:

$11 / 27 / 2006$

Signature signifies compliance with

Signature verifying data and calculations:

Procedure EMS-JAG-01

Signature/date

Signatures on original data sheet in Test Instruction Package TI-RPP-WTP-465

Reference: CCP-WTPSP-176

(HVC2_gas-dataRev0 (8)).xls

gas-dataRev0.xls

GT9

31 July 2006

4/18/2007 
Rev. 0

31-Jul-06

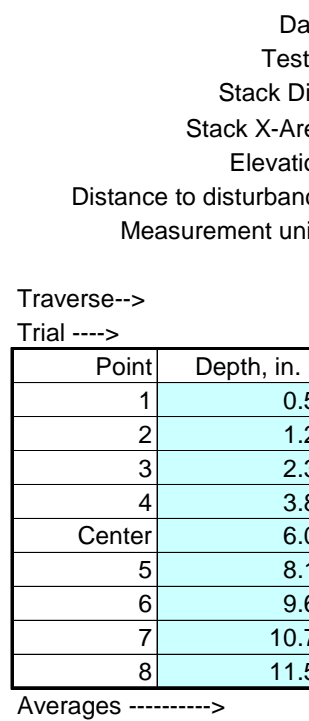

TRACER GAS TRAVERSE DATA FORM

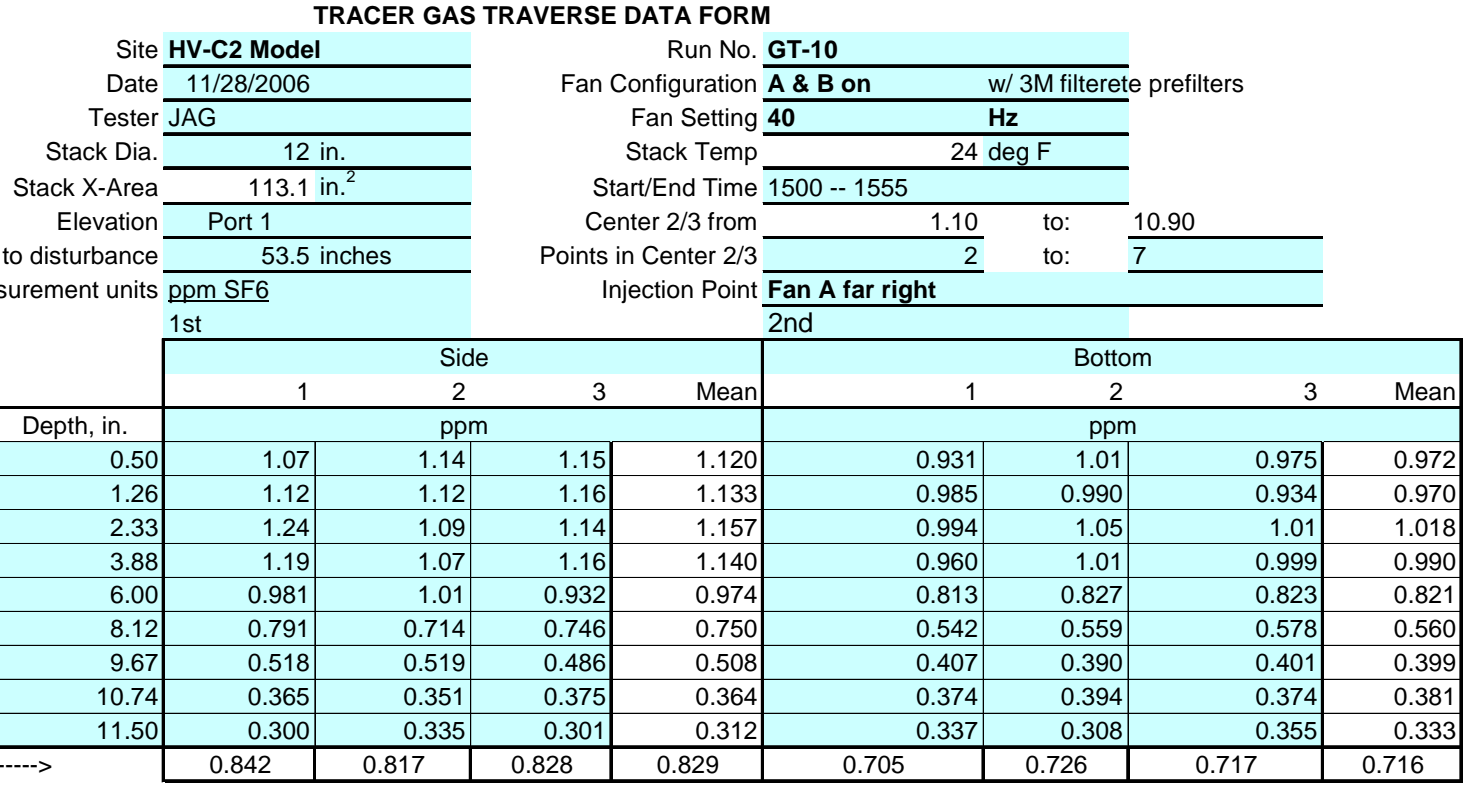

Avg. Conc. $0.757 \mathrm{ppm}$

\begin{tabular}{|lr}
\hline All & ppm \\
Mean & 0.77 \\
Min Point & 0.31 \\
Max Point & 1.16 \\
\hline
\end{tabular}

Tracer tank pressure Stack Temp Center Pt. air vel. Injection flowmeter Stack flow

Sampling flowmeter Ambient pressure Ambient humidity B\&K vapor correction Back-Gd gas ppb No. Bk-Gd samples Ambient Temp, $F$ Instuments Used: B\&K 1302 Gas Analyzer SN 1765299 Cat2 MTE TSI 8360 Velocity SN 209060 Omega FMA-2617A flowmeter SN30348 due $11 / 01 / 07$

\begin{tabular}{|c|c|}
\hline Start & Finish \\
\hline 120 & 150 \\
\hline 24 & 24 \\
\hline 3310 & 3360 \\
\hline 59 & 59 \\
\hline-- & - \\
\hline 10 & 10 \\
\hline 29.90 & 29.92 \\
\hline 48 & 51 \\
\hline no & no \\
\hline
\end{tabular}

\begin{tabular}{r|r}
\hline $10,11.6,4.8,6.3$ & $20.1,15.5,16.7,18.5$ \\
\hline
\end{tabular} \begin{tabular}{|r|r|}
\hline 4 & 4 \\
\hline 20 & \\
\hline
\end{tabular} \begin{tabular}{r|l} 
Dev. from mean & Center 2/3 \\
\hline$-59.6 \%$ & Mean \\
$49.8 \%$ & CoV. Dev. \\
Cov \%
\end{tabular} Gas analyzer checked:

$11 / 27 / 2006$

\section{Notes:}
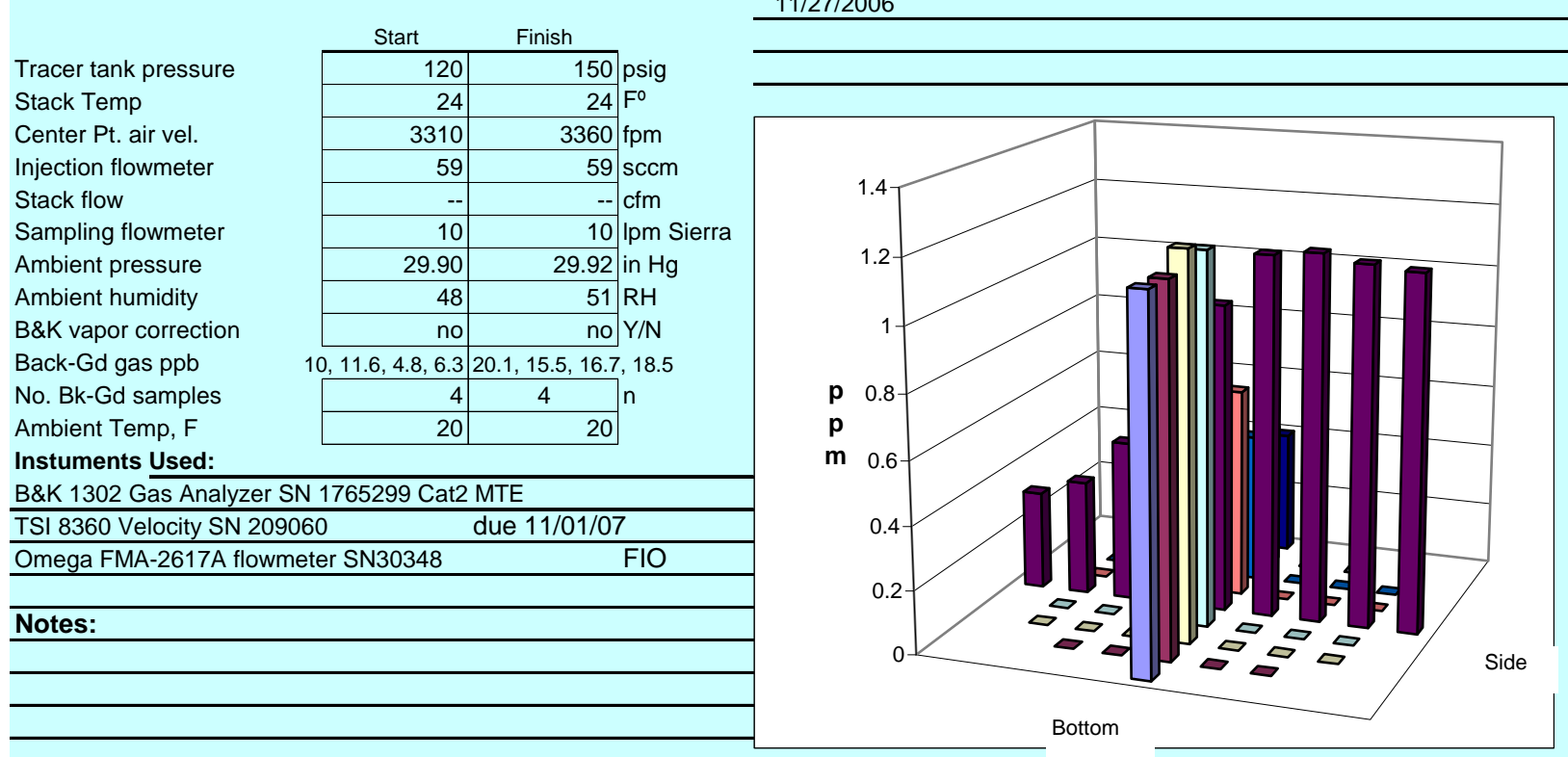

Signature signifies compliance with Signature verifying data and calculations:

Procedure EMS-JAG-01

Signature/date Signatures on original data sheet in Test Instruction Package TI-RPP-WTP-465

Reference: CCP-WTPSP-176

(HVC2_gas-dataRev0 (8)).xls gas-dataRev0.xls

GT10 31 July 2006

$4 / 18 / 2007$ 
Rev. 0

31-Jul-06

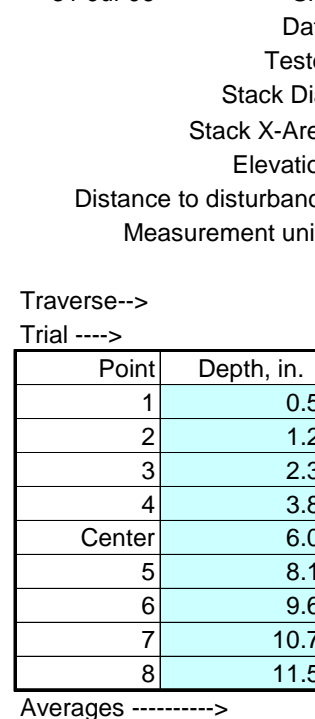

TRACER GAS TRAVERSE DATA FORM

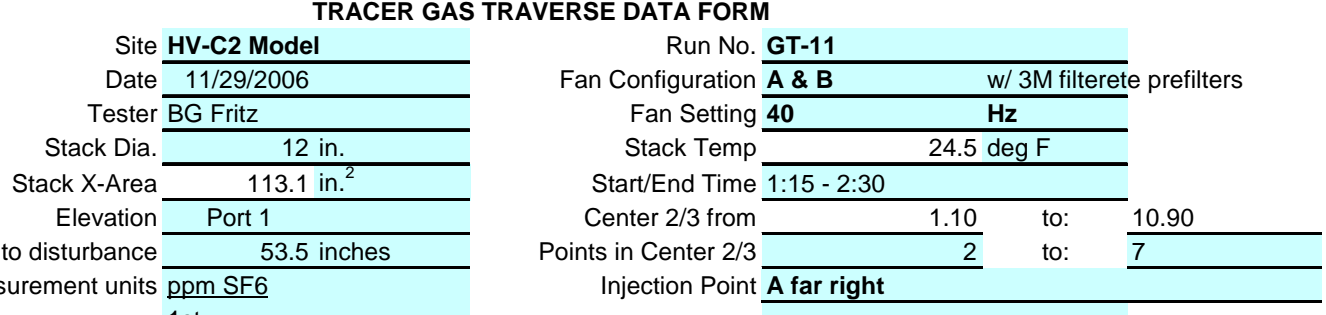

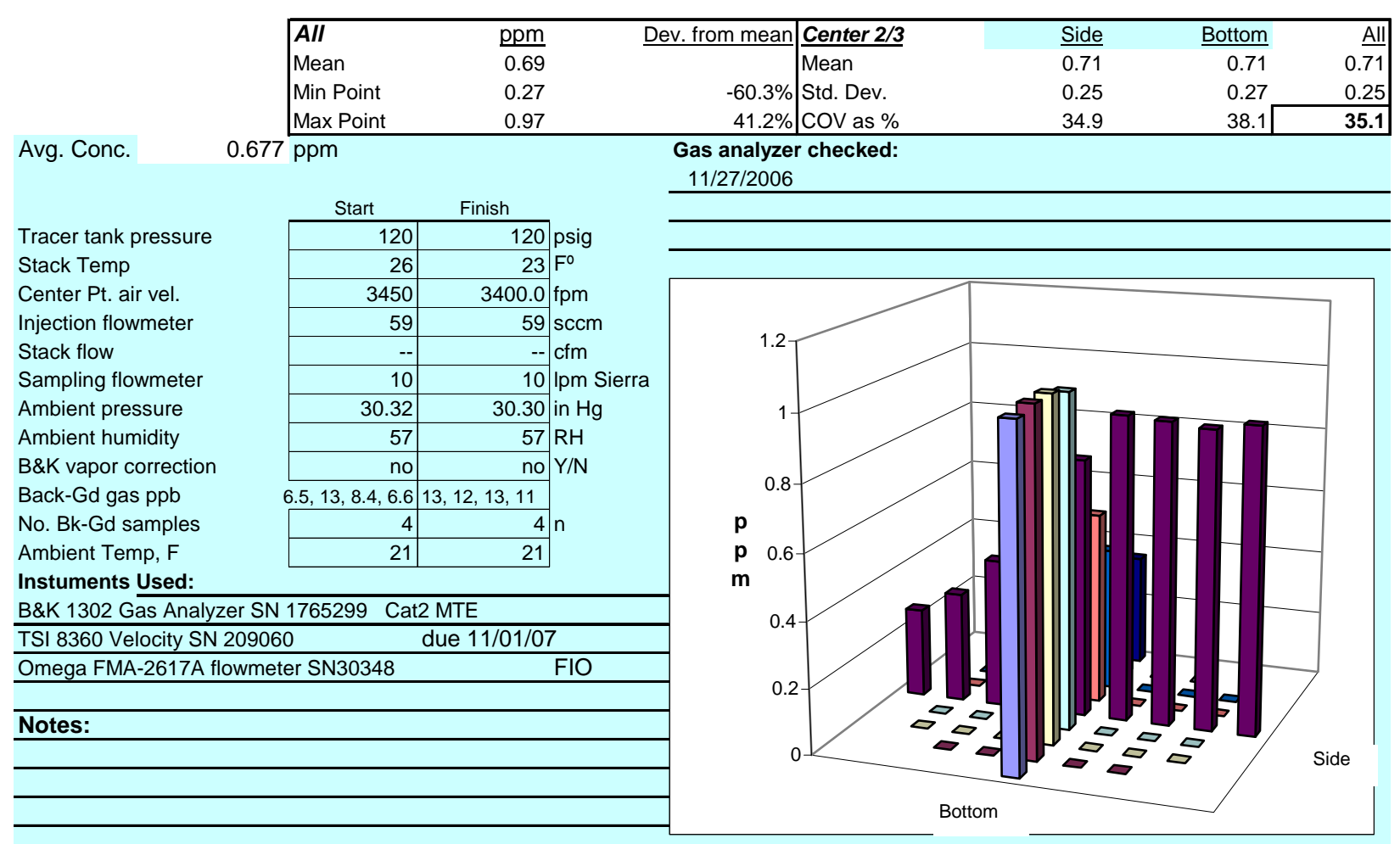

Signature signifies compliance with

Signature verifying data and calculations:

Procedure EMS-JAG-01

Signature/date

Signatures on original data sheet in Test Instruction Package TI-RPP-WTP-465

Reference: CCP-WTPSP-176

(HVC2_gas-dataRev0 (8)).xls

gas-dataRev0.xls

GT11

31 July 2006

$4 / 18 / 2007$ 
Rev. 0

31-Jul-06

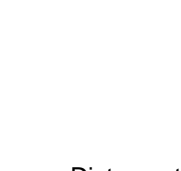

\begin{aligned} & TRACER GAS \\ & Site \multicolumn{1}{c}{ HV-C2 Model } \\ & Date $\frac{11 / 29 / 2006}{} \\ &$ Tester BG Fritz \\ & Stack Dia. 12 in. \\ & Stack X-Area 113.1 in. $^{2} \\ &$ Elevation Port 1 \\ & \hline\end{aligned}

istance to disturbance Measurement units ppm SF6
53.5 inches

and

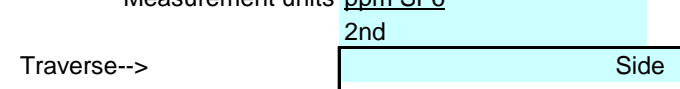

\begin{tabular}{l|cc}
\cline { 2 - 2 } Traverse--> & \multicolumn{2}{c}{ Side } \\
Trial ----> & 1 & 2 \\
\hline
\end{tabular}

\begin{tabular}{|c|c|c|c|c|c|c|c|c|c|}
\hline \multirow{3}{*}{$\begin{array}{l}\text { Traverse--> } \\
\text { Trial ----> }\end{array}$} & & \multicolumn{4}{|l|}{ 2nd } & \multicolumn{4}{|l|}{ 1st } \\
\hline & & \multicolumn{4}{|c|}{ Side } & \multicolumn{4}{|c|}{ Bottom } \\
\hline & & 1 & 2 & 3 & Mean & 1 & 2 & 3 & Mean \\
\hline Point & Depth, in. & \multicolumn{4}{|c|}{ ppm } & \multicolumn{4}{|c|}{ ppm } \\
\hline 1 & 0.50 & 0.859 & 0.888 & 0.902 & 0.883 & 0.957 & 0.943 & 0.983 & 0.961 \\
\hline 2 & 1.26 & 0.888 & 0.823 & 0.851 & 0.854 & 1.04 & 0.932 & 1 & 0.991 \\
\hline 3 & 2.33 & 0.918 & 0.931 & 1.01 & 0.953 & 0.993 & 1.02 & 0.999 & 1.004 \\
\hline 4 & 3.88 & 0.893 & 0.969 & 1.03 & 0.964 & 1.05 & 1.01 & 0.991 & 1.017 \\
\hline Center & 6.00 & 0.916 & 0.881 & 0.832 & 0.876 & 0.827 & 0.821 & 0.835 & 0.828 \\
\hline 5 & 8.12 & 0.639 & 0.650 & 0.629 & 0.639 & 0.608 & 0.584 & 0.612 & 0.601 \\
\hline 6 & 9.67 & 0.474 & 0.446 & 0.475 & 0.465 & 0.446 & 0.458 & 0.461 & 0.455 \\
\hline 7 & 10.74 & 0.300 & 0.314 & 0.285 & 0.300 & 0.347 & 0.360 & 0.359 & 0.355 \\
\hline 8 & 11.50 & 0.293 & 0.242 & 0.263 & 0.266 & 0.345 & 0.351 & 0.340 & 0.345 \\
\hline Averages ---- & $-->$ & 0.687 & 0.683 & 0.697 & 0.689 & 0.735 & 0.720 & 0.731 & 0.729 \\
\hline
\end{tabular}

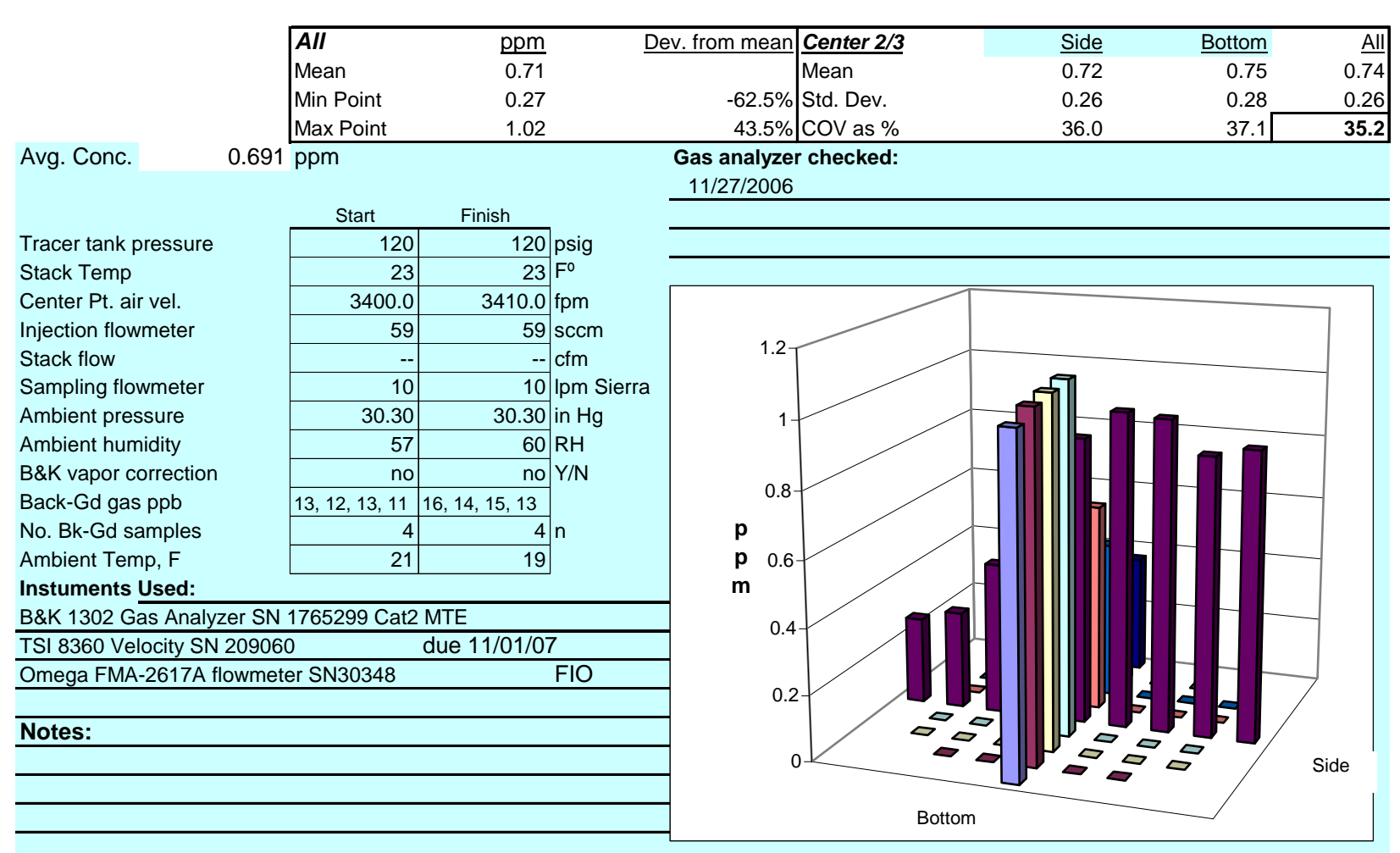

Signature signifies compliance with

Signature verifying data and calculations:

Procedure EMS-JAG-01

Signature/date

Signatures on original data sheet in Test Instruction Package TI-RPP-WTP-465

Reference: CCP-WTPSP-176

gas-dataRev0.xls

(HVC2_gas-dataRev0 (8)).xls

31 July 2006

GT12

$4 / 18 / 2007$ 
Rev. 0

31-Jul-06

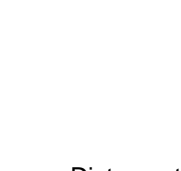

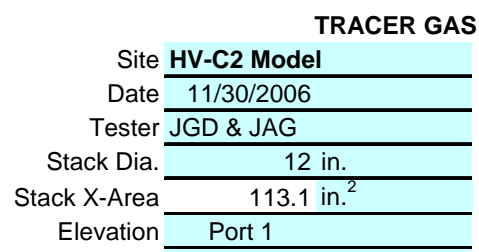

Distance to disturbance Measurement units ppm SF6
53.5 inches

st

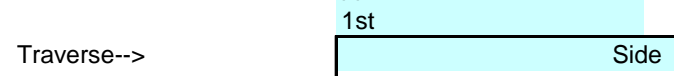

Trial ---->

\begin{tabular}{|c|c|c|c|c|c|c|c|c|c|}
\hline \multirow{3}{*}{$\begin{array}{l}\text { Traverse--> } \\
\text { Trial ----> }\end{array}$} & & $1 \mathrm{st}$ & & & & 2nd & & & \\
\hline & & \multicolumn{4}{|c|}{ Side } & \multicolumn{4}{|c|}{ Bottom } \\
\hline & & 1 & 2 & 3 & Mean & 1 & 2 & 3 & Mean \\
\hline \begin{tabular}{r|} 
Point \\
\end{tabular} & Depth, in. & \multicolumn{4}{|c|}{$\mathrm{ppm}$} & \multicolumn{4}{|c|}{$\mathrm{ppm}$} \\
\hline 1 & 0.50 & 1.64 & 1.59 & 1.60 & 1.610 & 1.58 & 1.61 & 1.58 & 1.590 \\
\hline 2 & 1.26 & 1.73 & 1.62 & 1.64 & 1.663 & 1.55 & 1.59 & 1.62 & 1.587 \\
\hline 3 & 2.33 & 1.70 & 1.70 & 1.62 & 1.673 & 1.66 & 1.61 & 1.70 & 1.657 \\
\hline 4 & 3.88 & 1.64 & 1.66 & 1.71 & 1.670 & 1.64 & 1.67 & 1.58 & 1.630 \\
\hline Center & 6.00 & 1.71 & 1.57 & 1.57 & 1.617 & 1.62 & 1.55 & 1.52 & 1.563 \\
\hline 5 & 8.12 & 1.65 & 1.66 & 1.50 & 1.603 & 1.54 & 1.55 & 1.55 & 1.547 \\
\hline 6 & 9.67 & 1.56 & 1.56 & 1.50 & 1.540 & 1.57 & 1.57 & 1.70 & 1.613 \\
\hline 7 & 10.74 & 1.56 & 1.69 & 1.70 & 1.650 & 1.50 & 1.54 & 1.64 & 1.560 \\
\hline 8 & 11.50 & 1.57 & 1.56 & 1.73 & 1.620 & 1.66 & 1.54 & 1.53 & 1.577 \\
\hline Averages ---- & $\cdots>$ & 1.640 & 1.623 & 1.619 & 1.627 & 1.59 & 1.58 & 1.60 & 1.591 \\
\hline
\end{tabular}

\begin{tabular}{|lr}
\hline All & $\mathrm{ppm}$ \\
Mean & 1.61 \\
Min Point & 1.54 \\
Max Point & 1.67
\end{tabular}

Avg. Conc.

$1.612 \mathrm{ppm}$

Start

\begin{tabular}{|c|c|c|}
\hline Start & Finisn & \\
\hline 120 & 180 & psig \\
\hline 32.5 & 30 & \multirow{4}{*}{$\begin{array}{l}\mathrm{fpm} \\
\mathrm{sccm} \\
\mathrm{cfm}\end{array}$} \\
\hline 1570.0 & 1560.0 & \\
\hline 59 & 59 & \\
\hline-- & -- & \\
\hline 10 & 10 & \multirow{2}{*}{$\begin{array}{l}\text { Ipm Sierra } \\
\text { in } \mathrm{Hg}\end{array}$} \\
\hline 29.968 & 29.97 & \\
\hline 67 & 67 & \multirow{3}{*}{$\begin{array}{l}\mathrm{RH} \\
\mathrm{Y} / \mathrm{N}\end{array}$} \\
\hline no & no & \\
\hline $16,17,17,17$ & $13,12,13,11$ & \\
\hline 4 & 4 & \multirow{2}{*}{$n$} \\
\hline 28 & 27 & \\
\hline
\end{tabular}

Tracer tank pressure

Stack Temp

Center Pt. air vel.

Injection flowmeter

Stack flow

Sampling flowmeter

Ambient pressure

Ambient humidity

B\&K vapor correction

Back-Gd gas ppb

No. Bk-Gd samples

Ambient Temp, F

Instuments Used:

B\&K 1302 Gas Analyzer SN 1765299 Cat2 MTE

TSI 8360 Velocity SN 209060

Omega FMA-2617A flowmeter SN30348

due $11 / 01 / 07$

Notes: $1015 \mathrm{mbar}$
Run No. GT-13

Fan Configuration $\mathbf{A}$ Fan Setting 35

Stack Temp

Start/End Time

Center $2 / 3$ from

Points in Center 2/3

Injection Point A center w/ 3M filterete prefilters

$\mathrm{Hz}$

$31.25 \operatorname{deg} F$

$\begin{array}{rll}1.10 & \text { to: } & 10.90 \\ 2 & \text { to: } & \end{array}$

7

Bottom

\begin{tabular}{|c|c|c|c|c|}
\hline Dev. from mean & Center 2/3 & Side & Bottom & All \\
\hline & Mean & 1.63 & 1.59 & 1.61 \\
\hline$-4.3 \%$ & Std. Dev. & 0.05 & 0.04 & 0.05 \\
\hline $4.0 \%$ & Cov as $\%$ & 3.0 & 2.6 & 2.9 \\
\hline
\end{tabular}

Gas analyzer checked:

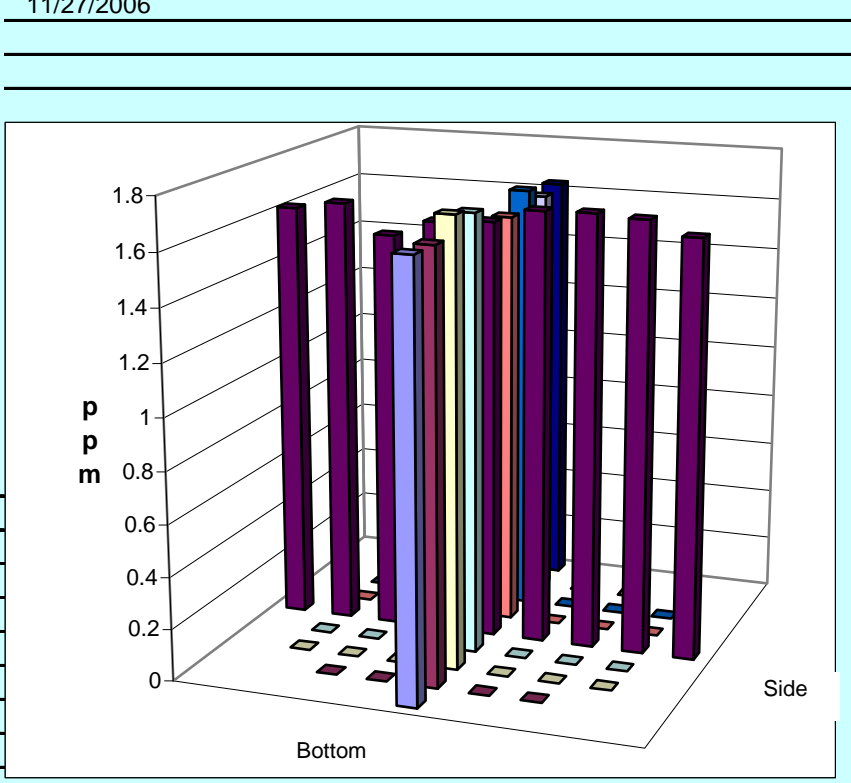

Signature signifies compliance with

Signature verifying data and calculations:

Procedure EMS-JAG-01

Signature/date

Signatures on original data sheet in Test Instruction Package TI-RPP-WTP-465

Reference: CCP-WTPSP-176

(HVC2_gas-dataRev0 (8)).xls

gas-dataRev0.xls

GT13

31 July 2006

$4 / 18 / 2007$ 
Rev. 0

31-Jul-06

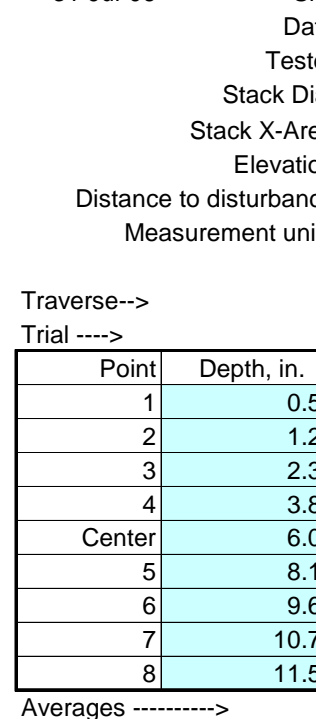

TRACER GAS TRAVERSE DATA FORM

\begin{tabular}{|c|c|}
\hline \multicolumn{2}{|c|}{ Site HV-C2 Model } \\
\hline Date & $12 / 1 / 2006$ \\
\hline Tester & BGF \\
\hline Stack Dia. & 12 in. \\
\hline Stack X-Area & 113.1 in. $^{2}$ \\
\hline Elevation & Port 1 \\
\hline o disturbance & 53.5 inches \\
\hline
\end{tabular}

\section{Run No. GT-14}

Fan Configuration $\begin{array}{ll}\mathbf{A} & \mathrm{W} / 3 \mathrm{M} \text { filterete } \\ & \end{array}$

Fan Setting 37

Stack Temp

Start/End Time 0845 -- 0940

Center $2 / 3$ from

Points in Center $2 / 3$

Injection Point A near right

$\mathrm{W} / 3 \mathrm{M}$ filterete prefilters

$24 \operatorname{deg} \mathrm{F}$

1st 2 nd

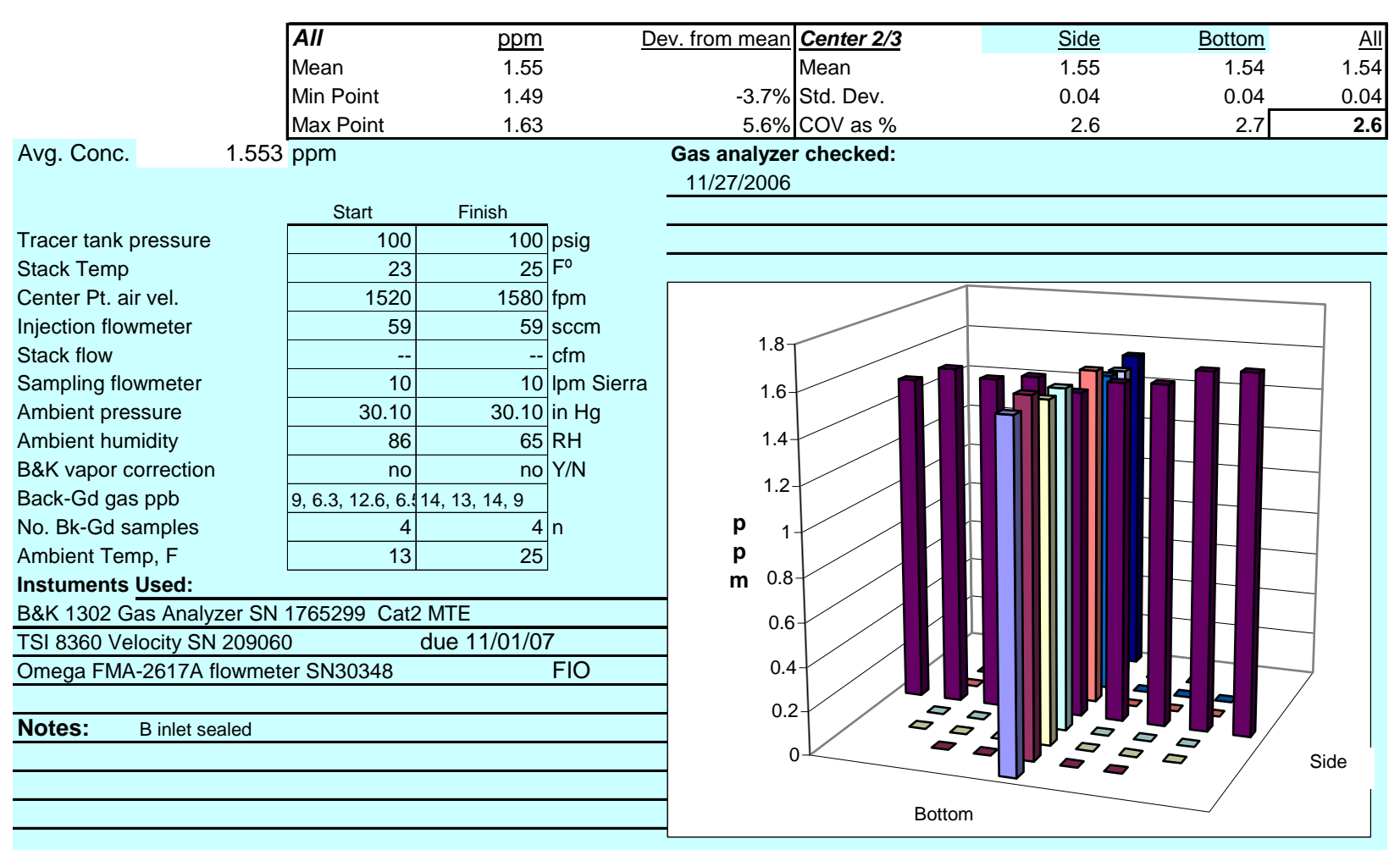

Signature signifies compliance with

Signature verifying data and calculations:

Procedure EMS-JAG-01

Signature/date

Signatures on original data sheet in Test Instruction Package TI-RPP-WTP-465

Reference: CCP-WTPSP-176

gas-dataRev0.xls

(HVC2_gas-dataRev0 (8)).xls

31 July 2006

GT14

$4 / 18 / 2007$ 
Rev. 0

31-Jul-06

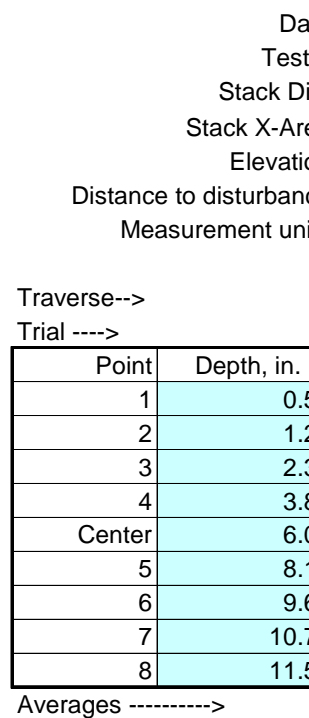

TRACER GAS TRAVERSE DATA FORM

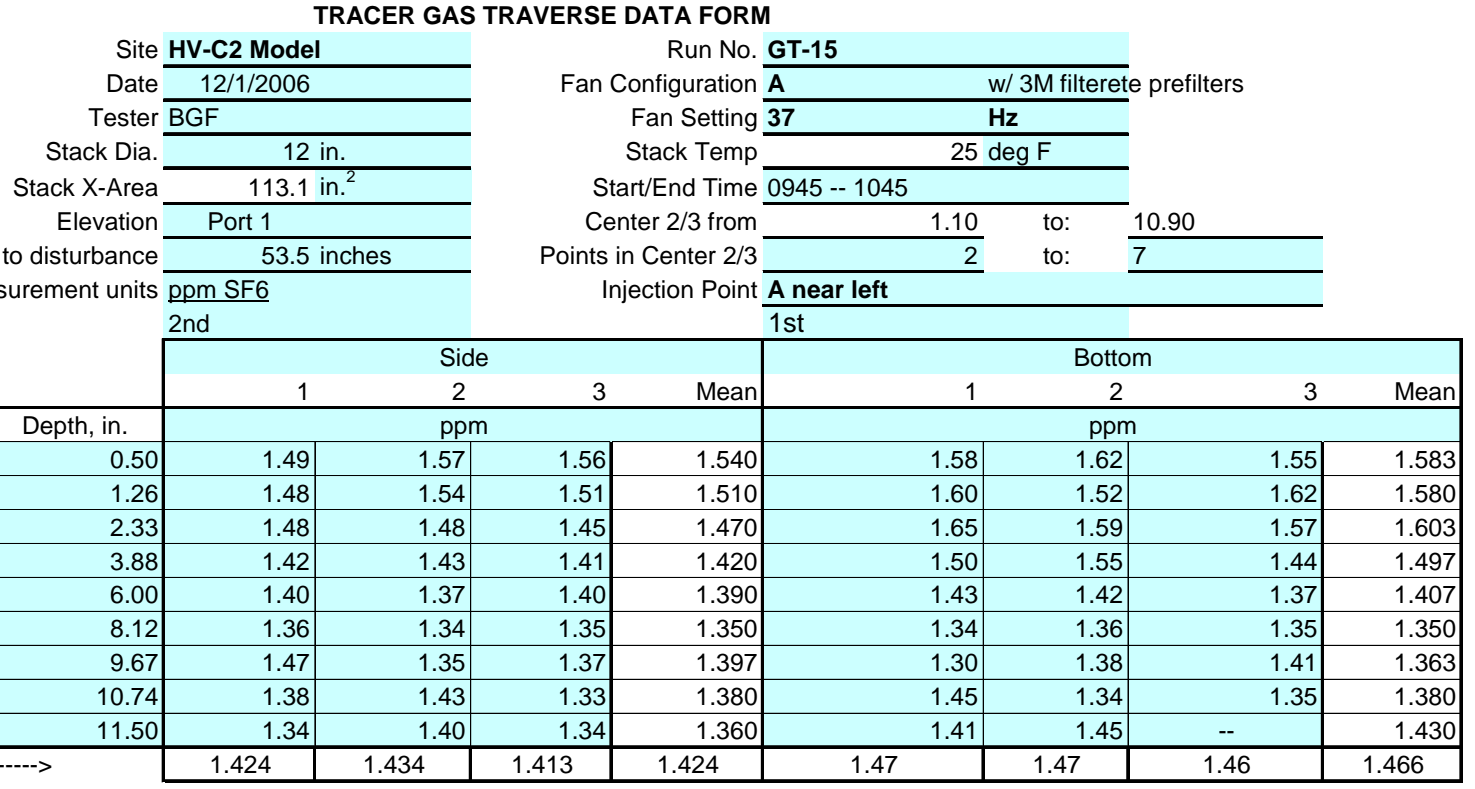

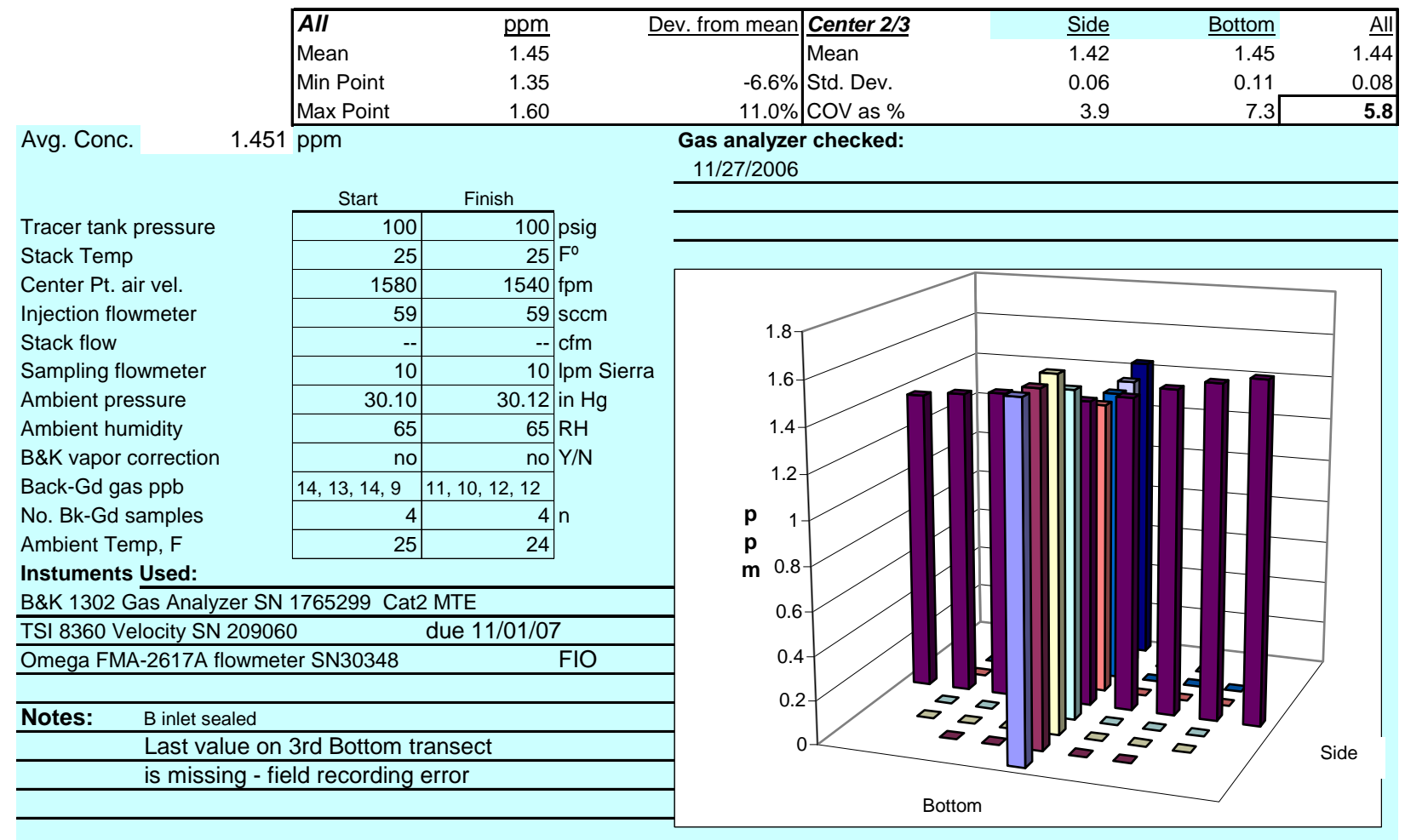

Signature signifies compliance with

Signature verifying data and calculations:

Procedure EMS-JAG-01

Signature/date

Signatures on original data sheet in Test Instruction Package TI-RPP-WTP-465

Reference: CCP-WTPSP-176

(HVC2_gas-dataRev0 (8)).xIs

gas-dataRev0.xls

GT15

31 July 2006

$4 / 18 / 2007$ 
Rev. 0

31-Jul-06

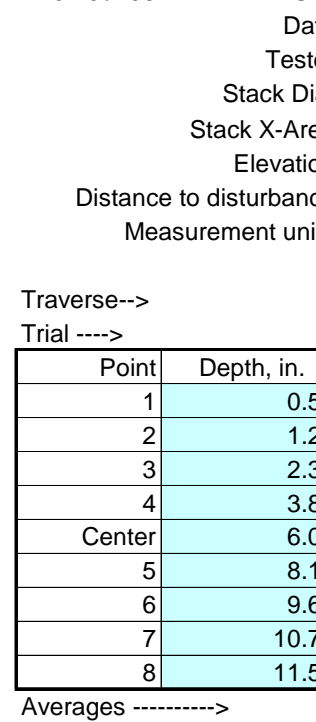

TRACER GAS TRAVERSE DATA FORM

Site HV-C2 Model

Date 12/1/2006

Tester BGF

Stack Dia.

\begin{tabular}{|c|}
\hline 12 in. \\
\hline 113.1 in. $^{2}$ \\
\hline Port 1 \\
\hline
\end{tabular}

53.5 inches

ppm SF6
Run No. GT-16

Fan Configuration $\mathbf{A}$ Fan Setting 35

Stack Temp

Start/End Time 1200 -- 123

Center $2 / 3$ from Points in Center $2 / 3$ Injection Point $\mathbf{A}$ far left w/ 3M filterete prefilters

$\mathrm{Hz}$

$34 \operatorname{deg} F$

5

1.10 to: 10.90

$\frac{1.10}{2}$ to: $\frac{10}{7}$ 2nd

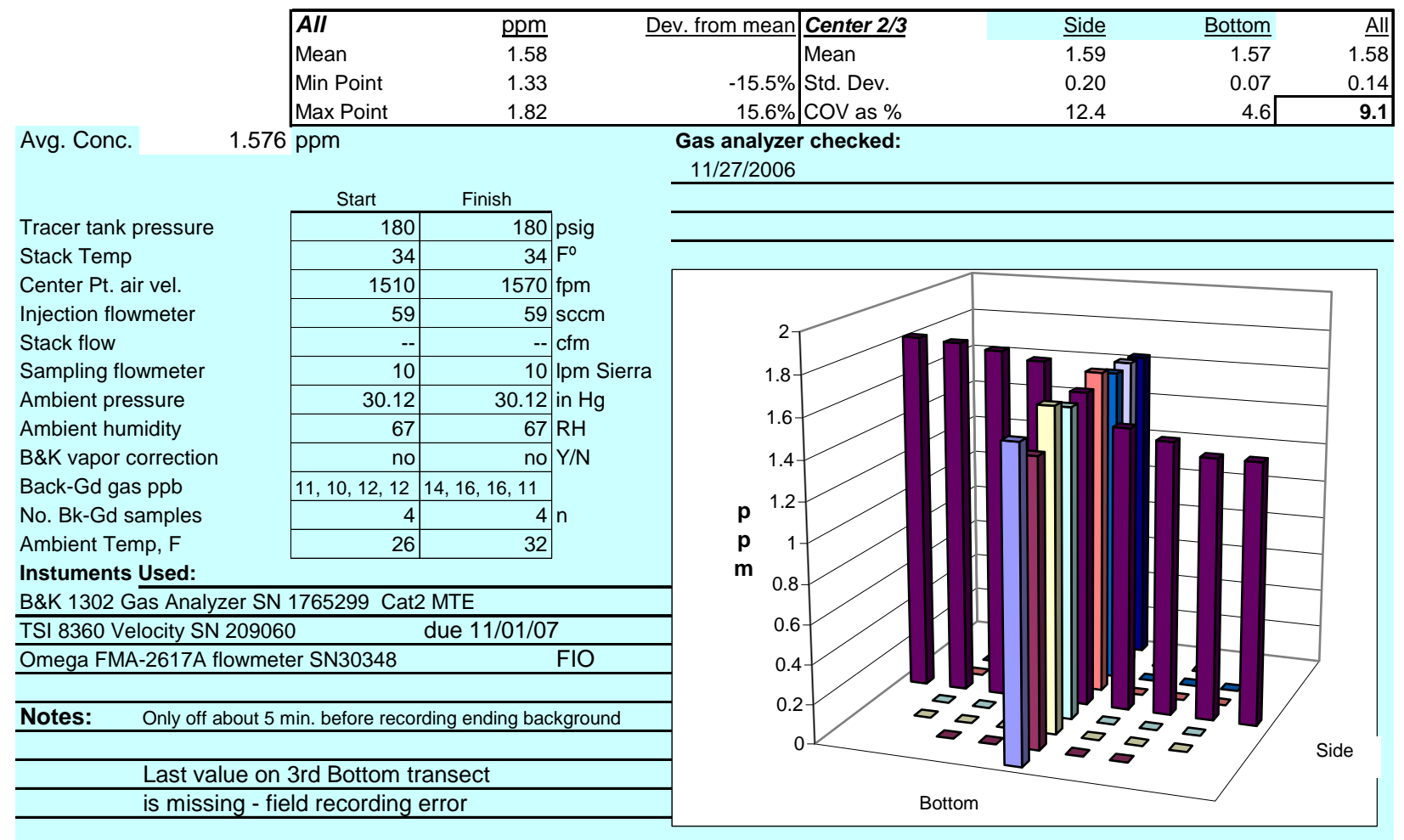

Signature signifies compliance with

Signature verifying data and calculations:

Procedure EMS-JAG-01

Signature/date

Signatures on original data sheet in Test Instruction Package TI-RPP-WTP-465

Reference: CCP-WTPSP-176

(HVC2_gas-dataRev0 (8)).xls

gas-dataRev0.xls

GT16

31 July 2006

4/18/2007 
Rev. 0

31-Jul-06

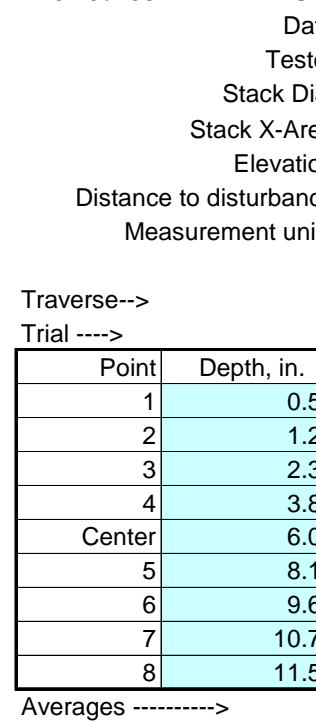

TRACER GAS TRAVERSE DATA FORM

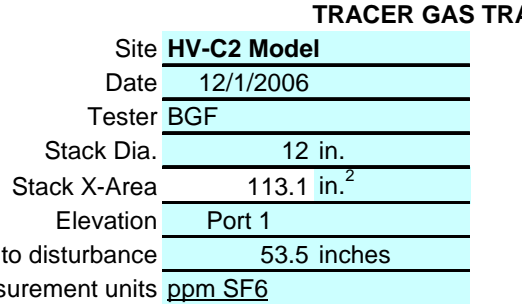

\section{Run No. GT-17}

Fan Configuration $\mathbf{A}$

Fan Setting 35

Stack Temp

Start/End Time

Center $2 / 3$ from

Points in Center $2 / 3$ Injection Point $\mathrm{A}$ far right

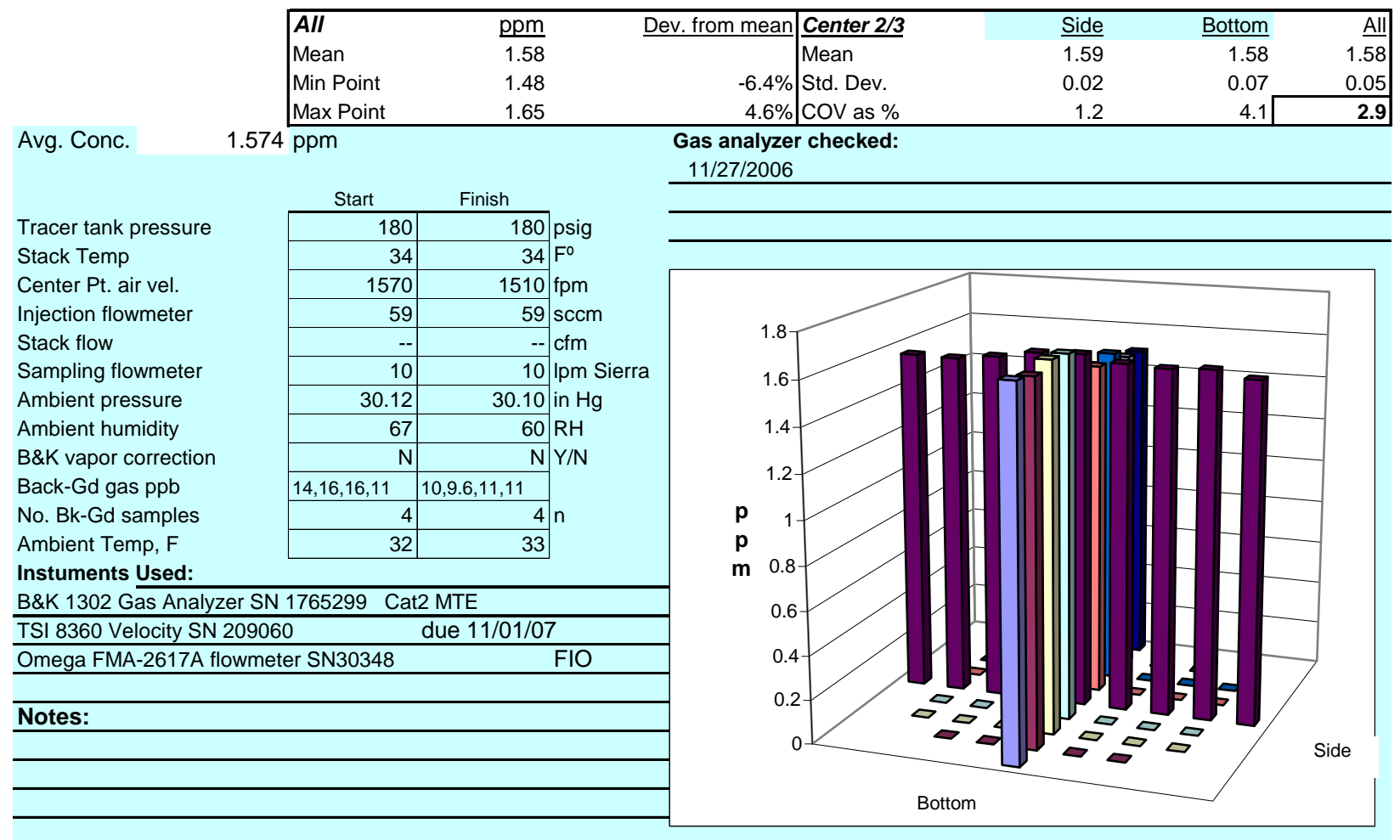

Signature signifies compliance with Signature verifying data and calculations:

Procedure EMS-JAG-01

Signature/date

Signatures on original data sheet in Test Instruction Package TI-RPP-WTP-465

Reference: CCP-WTPSP-176

gas-dataRev0.xls

(HVC2_gas-dataRev0 (8)).xls

31 July 2006

GT17

$4 / 18 / 2007$ 
Rev. 0

31-Jul-06

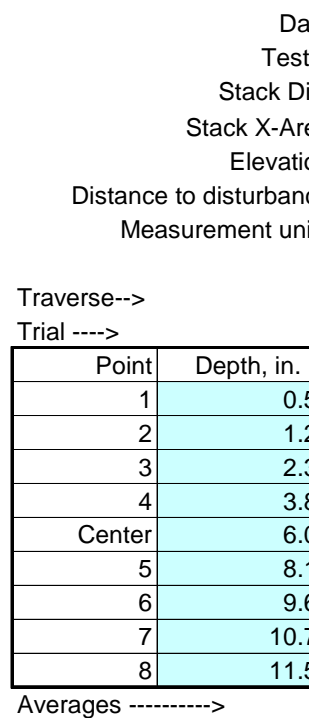

TRACER GAS TRAVERSE DATA FORM

Site HV-C2 Model

Date 12/1/2006

Tester BGF

Stack Dia.

$\frac{12 \text { in. }}{113.1 \text { in. }^{2}}$

82 inches

ppm SF6

Run No. GT-18

Fan Configuration $\begin{array}{lll}\mathbf{B} \text { on } \quad \mathbf{A} \text { off } & \text { w/ } 3 \mathrm{M} \text { filterete } & \text { prefilters }\end{array}$

Fan Setting 35

Stack Temp

Start/End Time 1315 -- 1400

Center $2 / 3$ from

Points in Center $2 / 3$ Injection Point $\mathbf{B}$ center

$\mathrm{Hz}$

1400

$34.5 \operatorname{deg} F$

1.10 to: 10.90

1st 2nd

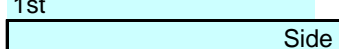

Bottom

\begin{tabular}{llll}
$3-$ Mean & 1 & 2 \\
\hline
\end{tabular}

$2 \quad 3 \quad$ Mean

\begin{tabular}{l|r|r|}
\hline & & ppm \\
\hline .50 & 1.62 & 1.64 \\
\hline .26 & 1.58 & 1.61 \\
\hline .33 & 1.65 & 1.64 \\
\hline .88 & 1.61 & 1.64 \\
\hline .00 & 1.66 & 1.61 \\
\hline 8.12 & 1.57 & 1.55 \\
\hline 9.67 & 1.56 & 1.54 \\
\hline 0.74 & 1.49 & 1.50 \\
\hline 1.50 & 1.51 & 1.53 \\
\hline \multicolumn{2}{|c|}{1.583} & 1.584 \\
\cline { 2 - 3 } & AlI & \\
Mean & 1.58 \\
Min Point & 1.48 \\
Max Point & 1.64 \\
\hline
\end{tabular}

Avg. Conc.

1.64

\begin{tabular}{|l|r|r|r|r|r|}
\hline \multicolumn{2}{|c|}{} & \multicolumn{5}{|c|}{$\mathrm{ppm}$} \\
\hline 1.61 & 1.623 & 1.54 & 1.53 & 1.54 & 1.537 \\
\hline 1.62 & 1.603 & 1.51 & 1.54 & 1.54 & 1.530 \\
\hline 1.63 & 1.640 & 1.55 & 1.60 & 1.57 & 1.573 \\
\hline 1.63 & 1.627 & 1.55 & 1.56 & 1.55 & 1.553 \\
\hline 1.58 & 1.613 & 1.60 & 1.63 & 1.62 & 1.617 \\
\hline 1.55 & 1.567 & 1.60 & 1.68 & 1.64 & 1.640 \\
\hline 1.46 & 1.550 & 1.64 & 1.60 & 1.60 & 1.613 \\
\hline 1.44 & 1.493 & 1.62 & 1.59 & 1.57 & 1.593 \\
\hline 566 & 1.578 & 1.56 & 1.55 & 1.54 & 1.550 \\
\hline
\end{tabular}

Tracer tank pressure

Stack Temp

Center Pt. air vel.

Injection flowmeter

Stack flow

Sampling flowmeter

Ambient pressure

Ambient humidity

B\&K vapor correction

Back-Gd gas ppb

No. Bk-Gd samples

Ambient Temp, F

Instuments Used:

\begin{tabular}{|c|c|c|}
\hline Start & Finish & \\
\hline 180 & 200 & psig \\
\hline 34 & 35 & $F^{\circ}$ \\
\hline 1660 & 1610 & $\mathrm{fpm}$ \\
\hline 59 & 59 & $\mathrm{sccm}$ \\
\hline- & -- & $\mathrm{cfm}$ \\
\hline 10 & 10 & Ipm Sierra \\
\hline 30.1 & 30.1 & in $\mathrm{Hg}$ \\
\hline 60 & 60 & $\mathrm{RH}$ \\
\hline no & no & $Y / N$ \\
\hline $10,9.6,11,11$ & $14,13,13,14$ & \\
\hline 4 & 4 & $n$ \\
\hline 33 & 33 & \\
\hline
\end{tabular}

B\&K 1302 Gas Analyzer SN 1765299 Cat2 MTE

TSI 8360 Velocity SN $209060 \quad$ due 11/01/07

Omega FMA-2617A flowmeter SN30348

$\mathrm{FIO}$

\section{Notes:}

\begin{tabular}{|c|c|c|c|c|}
\hline Dev. from mean & Center 2/3 & Side & Bottom & All \\
\hline & Mean & 1.58 & 1.59 & 1.59 \\
\hline$-6.0 \%$ & Std. Dev. & 0.05 & 0.04 & 0.05 \\
\hline $3.9 \%$ & COV as $\%$ & 3.4 & 2.4 & 2.9 \\
\hline
\end{tabular}

Gas analyzer checked:

$11 / 27 / 2006$

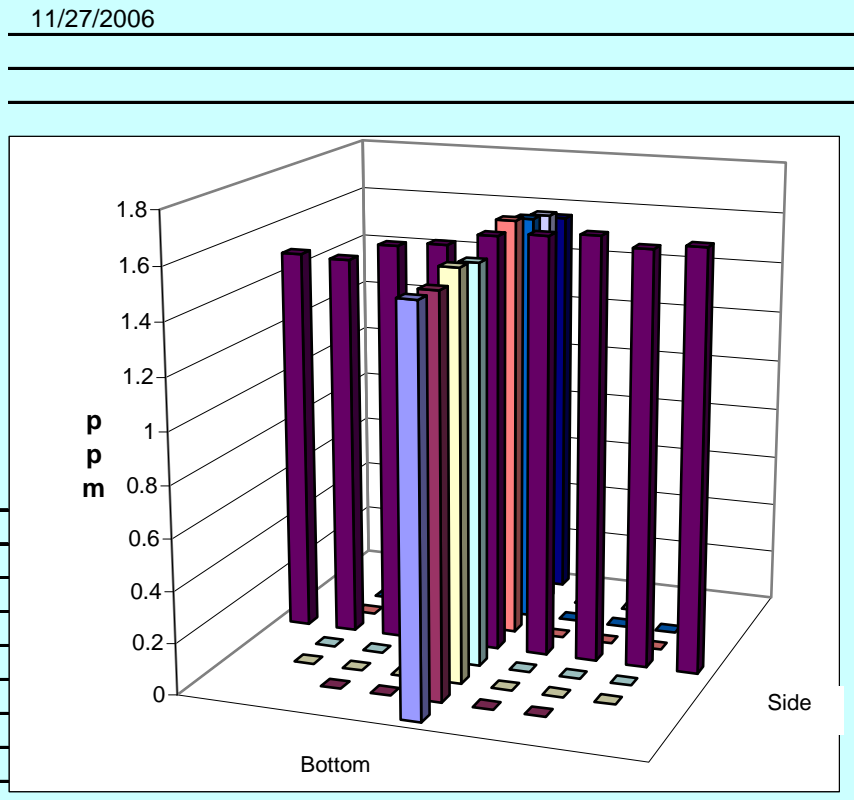

Signature signifies compliance with Procedure EMS-JAG-01

Signature/date

Signatures on original data sheet in Test Instruction Package TI-RPP-WTP-465

Reference: CCP-WTPSP-176

(HVC2_gas-dataRev0 (8)).xIs

gas-dataRev0.xls

GT18

31 July 2006

$4 / 18 / 2007$ 
Rev. 0

31-Jul-06

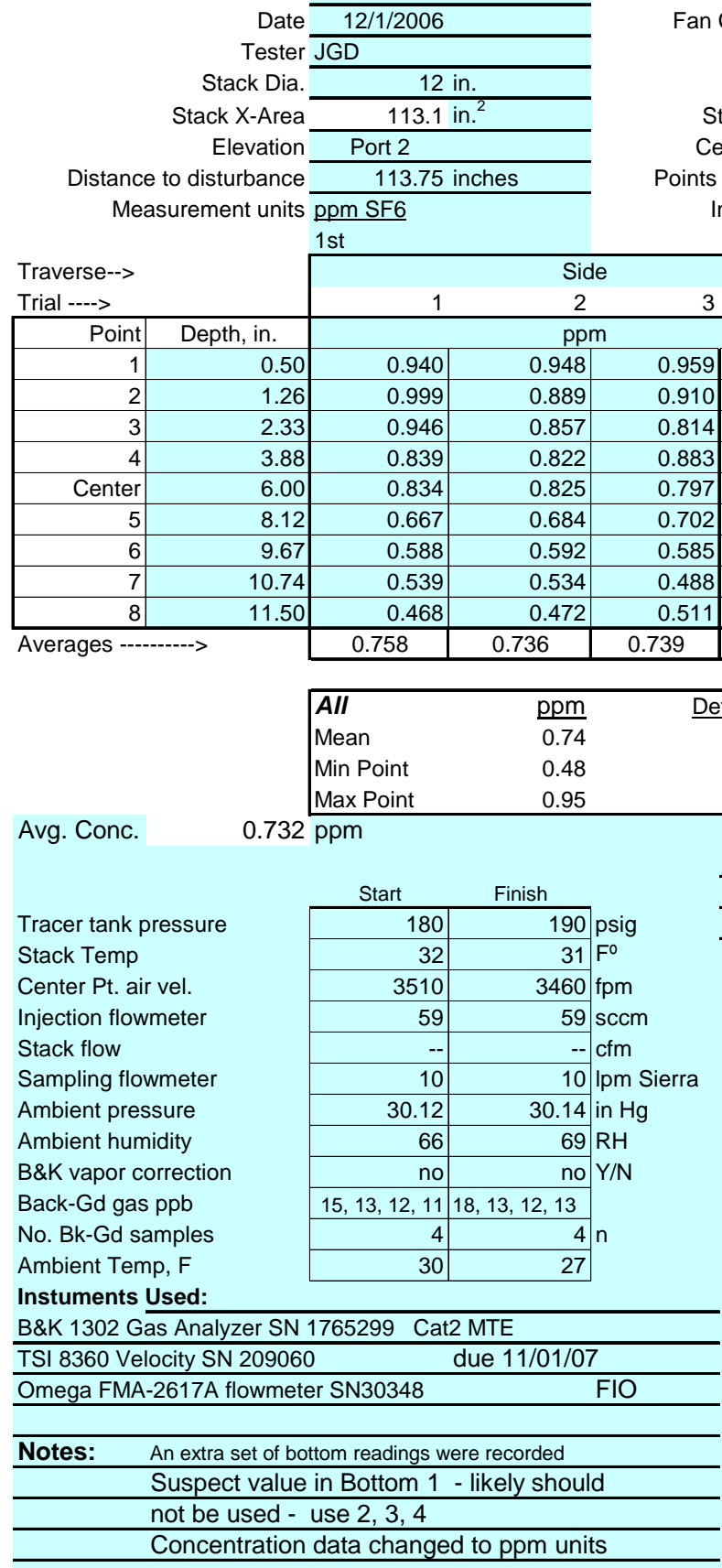

\section{TRACER GAS TRAVERSE DATA FORM}

Run No. GT-19

Fan Configuration A \& B Fan Setting 40

Stack Temp

Start/End Time 1445 -- 1600

Center $2 / 3$ from

oints in Center $2 / 3$ Injection Point $\mathbf{A}$ far right w/ 3M filterete prefilters

$\mathrm{Hz}$

$31.5 \operatorname{deg} \mathrm{F}$

1.10 to: 10.90

2 to: $\frac{10}{7}$ 2nd |

Mean $\quad 4 \quad 2$

$2 \quad 3 \quad$ Mean

\begin{tabular}{|r|r|r|r|r|}
\hline & \multicolumn{4}{|c|}{$\mathrm{ppm}$} \\
\hline 0.949 & 0.775 & 0.681 & 0.725 & 0.727 \\
\hline 0.933 & 0.714 & 0.810 & 0.710 & 0.745 \\
\hline 0.872 & 0.742 & 0.775 & 0.791 & 0.769 \\
\hline 0.848 & 0.807 & 0.777 & 0.803 & 0.796 \\
\hline 0.684 & 0.769 & 0.856 & 0.810 & 0.812 \\
\hline 0.588 & 0.713 & 0.761 & 0.749 & 0.741 \\
\hline 0.520 & 0.700 & 0.675 & 0.721 & 0.699 \\
\hline 0.484 & 0.654 & 0.730 & 0.744 & 0.709 \\
\hline 0.744 & 0.655 & 0.644 & 0.664 & 0.654 \\
\hline
\end{tabular}

\begin{tabular}{|c|c|c|c|c|}
\hline from mean & Center $2 / 3$ & Side & Bottom & All \\
\hline & Mean & 0.75 & 0.75 & 0.75 \\
\hline$-34.8 \%$ & Std. Dev. & 0.16 & 0.04 & 0.11 \\
\hline $28.0 \%$ & cov as $\%$ & 20.7 & 5.6 & 14.6 \\
\hline
\end{tabular}
Gas analyzer checked:

$11 / 27 / 2006$

Signature signifies compliance with

Signature verifying data and calculations:

Procedure EMS-JAG-01

Signature/date

Signatures on original data sheet in Test Instruction Package TI-RPP-WTP-465

Reference: CCP-WTPSP-176

(HVC2_gas-dataRev0 (8)).xls

gas-dataRev0.xls

GT19

31 July 2006

$4 / 18 / 2007$ 
Rev. 0

31-Jul-06

\begin{tabular}{|c|c|}
\hline \multicolumn{2}{|c|}{ Site HV-C2 Model } \\
\hline Date & $12 / 4 / 2006$ \\
\hline Tester & 3G Fritz \\
\hline Stack Dia. & 12 in. \\
\hline Stack X-Area & 113.1 in. $^{2}$ \\
\hline Elevation & Port 3 \\
\hline
\end{tabular}

Distance to disturbance Measurement units ppm SF6
TRACER GAS TRAVERSE DATA FORM

Run No. GT-20

Fan Configuration A\&B ON

Fan Setting 40

Stack Temp

Start/End Time 13:00-14:00

Center $2 / 3$ from

Points in Center 2/3 Injection Point $\mathbf{A}$ far right w/ 3M filterete prefilters

$\mathrm{Hz}$

$62 \operatorname{deg} F$

1.10 to: $\frac{10.90}{7}$

2 to: $\frac{1}{7}$

$1 \mathrm{st}$

\begin{tabular}{|c|c|c|c|c|c|c|c|c|c|}
\hline \multirow{3}{*}{$\begin{array}{l}\text { Traverse--> } \\
\text { Trial ----> }\end{array}$} & & \multicolumn{8}{|c|}{ 1st } \\
\hline & & \multicolumn{4}{|c|}{ Side } & \multicolumn{4}{|c|}{ Bottom } \\
\hline & & 1 & 2 & 3 & Mean & 1 & 2 & 3 & Mean \\
\hline Point & Depth, in. & \multicolumn{4}{|c|}{ ppm } & \multicolumn{4}{|c|}{ ppm } \\
\hline 1 & 0.50 & 0.879 & 0.911 & 1.05 & 0.947 & 0.706 & 0.678 & 0.705 & 0.696 \\
\hline 2 & 1.26 & 0.932 & 0.92 & 0.884 & 0.912 & 0.729 & 0.689 & 0.767 & 0.728 \\
\hline 3 & 2.33 & 0.886 & 0.908 & 0.905 & 0.900 & 0.712 & 0.806 & 0.792 & 0.770 \\
\hline 4 & 3.88 & 0.865 & 0.898 & 0.877 & 0.880 & 0.853 & 0.822 & 0.852 & 0.842 \\
\hline Center & 6.00 & 0.795 & 0.858 & 0.847 & 0.833 & 0.857 & 0.83 & 0.875 & 0.854 \\
\hline 5 & 8.12 & 0.724 & 0.750 & 0.720 & 0.731 & 0.904 & 0.807 & 0.816 & 0.842 \\
\hline 6 & 9.67 & 0.702 & 0.704 & 0.658 & 0.688 & 0.816 & 0.845 & 0.838 & 0.833 \\
\hline 7 & 10.74 & 0.665 & 0.653 & 0.562 & 0.627 & 0.921 & 0.871 & 0.757 & 0.850 \\
\hline 8 & 11.50 & 0.732 & 0.610 & 0.606 & 0.649 & 0.924 & 0.864 & 0.885 & 0.891 \\
\hline Averages --- & $--->$ & 0.798 & 0.801 & 0.790 & 0.796 & 0.825 & 0.801 & 0.810 & 0.812 \\
\hline
\end{tabular}

Avg. Conc.

$0.799 \mathrm{ppm}$

\begin{tabular}{|lr}
\hline All & ppm \\
Mean & 0.80 \\
Min Point & 0.63 \\
Max Point & 0.95 \\
\hline
\end{tabular}

Tracer tank pressure

Stack Temp

Center Pt. air vel.

Injection flowmeter

Stack flow

Sampling flowmeter

Ambient pressure

Ambient humidity

B\&K vapor correction

Back-Gd gas ppb

No. Bk-Gd samples

Ambient Temp, $\mathrm{F}$

Instuments Used:

B\&K 1302 Gas Analyzer SN 1765299 Cat2 MTE

TSI 8360 Velocity SN 209060

Omega FMA-2617A flowmeter SN30348

due $11 / 01 / 07$

\begin{tabular}{|c|c|c|}
\hline Start & Finish & \\
\hline 180 & 180 & psig \\
\hline 62 & 62 & $F^{\circ}$ \\
\hline 3200 & 3260.0 & $\mathrm{fpm}$ \\
\hline 59 & 59 & $\mathrm{sccm}$ \\
\hline-- & -- & $\mathrm{cfm}$ \\
\hline 10 & 10 & Ipm Sierra \\
\hline 30.03 & 30.03 & in $\mathrm{Hg}$ \\
\hline 79 & 79 & $\mathrm{RH}$ \\
\hline no & no & $Y / N$ \\
\hline $14,10,11,12$ & $17,15,15,12$ & \\
\hline 4 & & $n$ \\
\hline 25 & 25 & \\
\hline
\end{tabular}

Notes: Duct Heaters ON

\begin{tabular}{|c|c|c|c|c|}
\hline Dev. from mear & Center 2/3 & Side & Bottom & All \\
\hline & Mean & 0.80 & 0.82 & 0.81 \\
\hline$-22.1 \%$ & Std. Dev. & 0.11 & 0.05 & 0.08 \\
\hline $17.7 \%$ & cov as $\%$ & 14.3 & 5.9 & 10.5 \\
\hline
\end{tabular}

Gas analyzer checked:

$12 / 4 / 2006$

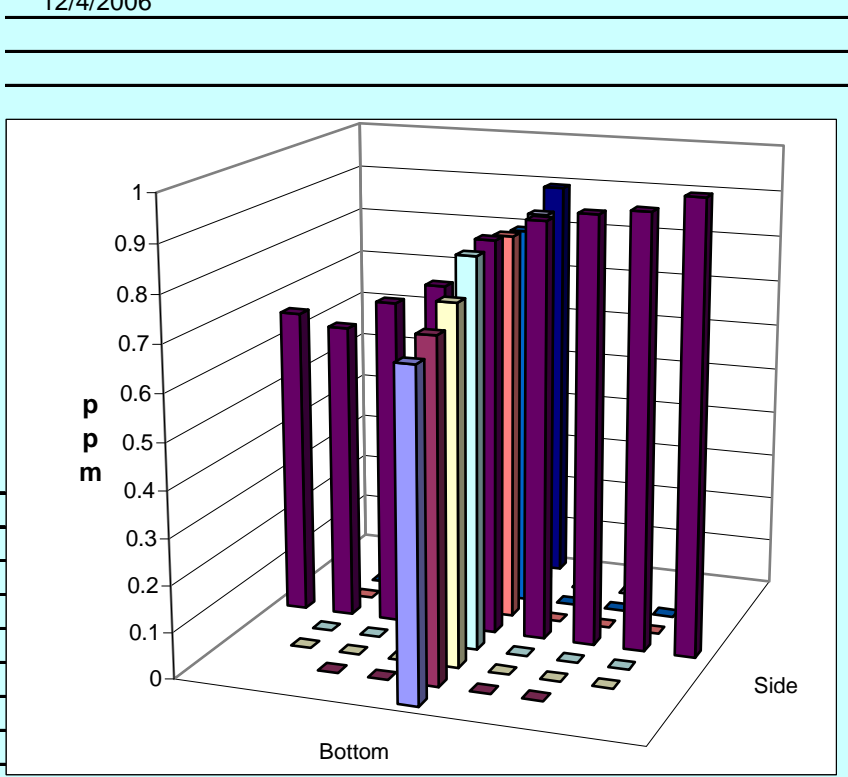

Signature signifies compliance with Procedure EMS-JAG-01

Signature/date

Signatures on original data sheet in Test Instruction Package TI-RPP-WTP-465

Reference: CCP-WTPSP-176

(HVC2_gas-dataRev0 (8)).xls

gas-dataRev0.xls

GT20

31 July 2006

$4 / 18 / 2007$ 
Rev. 0

31-Jul-06

$\begin{aligned} \text { Site } & \text { HV-C2 Model } \\ \text { Date } & \frac{12 / 4 / 2006}{12 \text { in. }} \\ \text { Tester } & \text { BG Fritz } \\ \text { Stack Dia. } & \frac{113.1 \text { in. }^{2}}{} \\ \text { Stack X-Area } & \text { Port 1 }\end{aligned}$

Distance to disturbance Measurement units ppm SF6
TRACER GAS TRAVERSE DATA FORM

Run No. GT-21

Fan Configuration $\mathbf{A \& B}$ on

Fan Setting 40

Stack Temp

Start/End Time

Center $2 / 3$ from

Points in Center $2 / 3$ Injection Point $\mathbf{B}$ center

\begin{tabular}{l} 
Measurement units ppm SF6 \\
\cline { 4 - 10 }
\end{tabular}

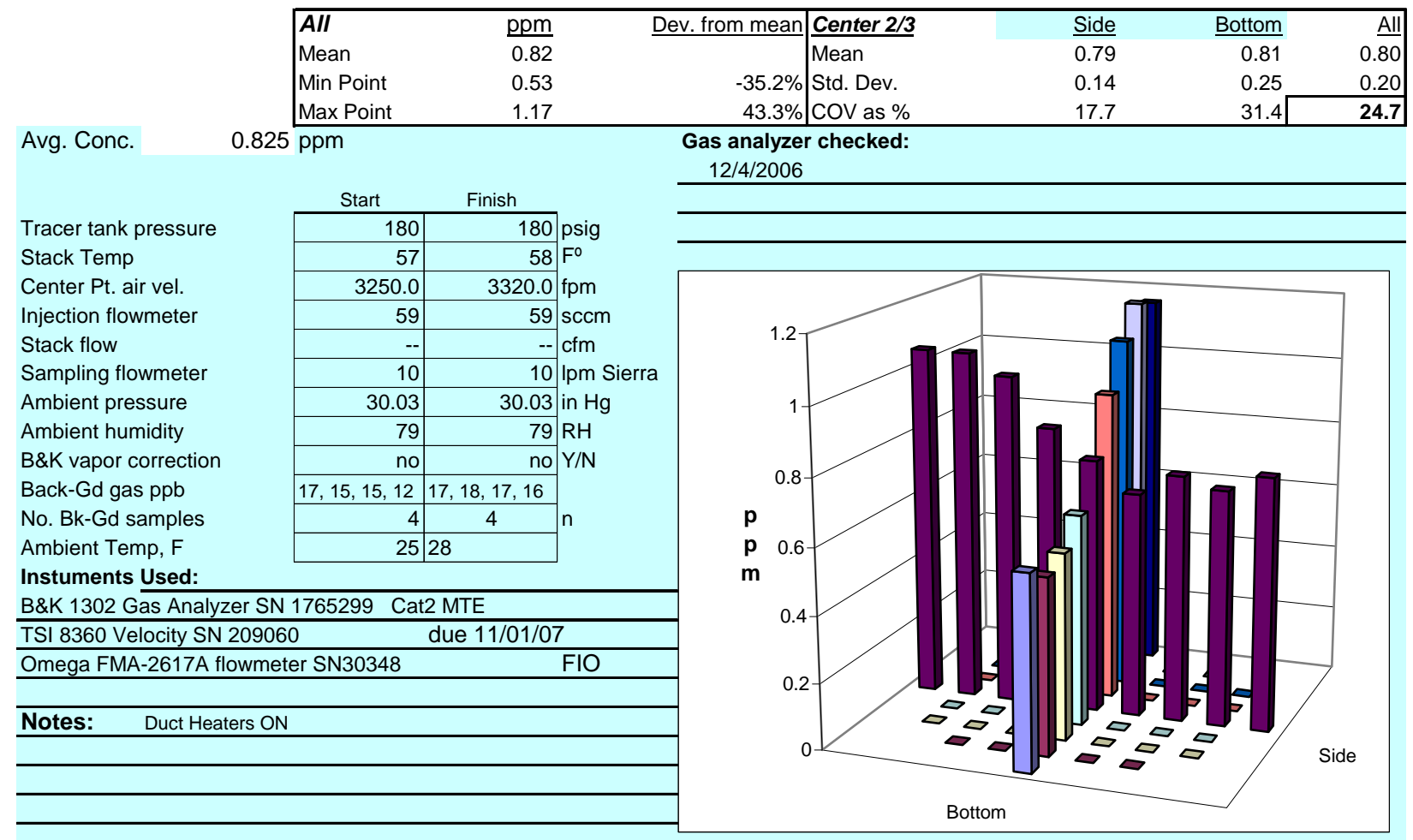

Signature signifies compliance with

Signature verifying data and calculations:

Procedure EMS-JAG-01

Signature/date

Signatures on original data sheet in Test Instruction Package TI-RPP-WTP-465

Reference: CCP-WTPSP-176

(HVC2_gas-dataRev0 (8)).xls

gas-dataRev0.xls

GT21

31 July 2006

$4 / 18 / 2007$ 
Rev. 0

31-Jul-06

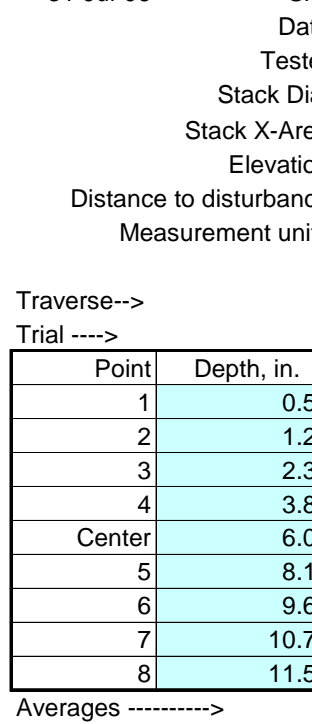

TRACER GAS TRAVERSE DATA FORM

Site HV-C2 Model

Date 1/17/2007

Tester BGF, MSP

Stack Dia.

ck X-Area

Elevation

$\frac{12 \text { in. }}{\frac{113.1 \text { in. }^{2}}{\text { Port } 1}}$

53.5 inches

ppm SF6
Run No. GT-22

Fan Configuration A \& B

Fan Setting 40

Stack Temp

Start/End Time 1340 -- 1501

Center $2 / 3$ from

Points in Center $2 / 3$ Injection Point $\mathrm{A}$ near right w/ 3M filterete prefilters

$\mathrm{Hz}$

$50 \operatorname{deg} F$

01

1.10 to: 10.90

$\frac{10}{2}$ to: $\frac{10}{7}$

\begin{tabular}{l|ll}
\cline { 3 - 3 } 2 2nd & \\
\hline & & Bottom
\end{tabular}

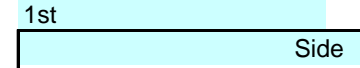

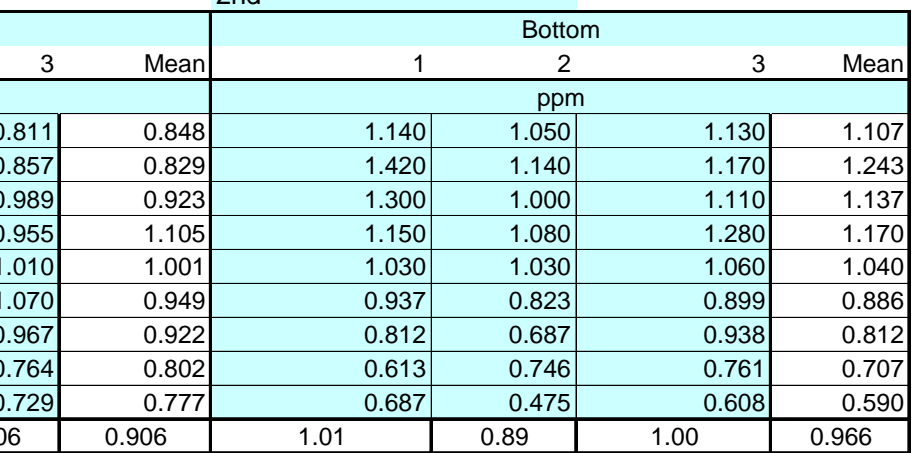

Avg. Conc. 0.925 ppm

Tracer tank pressure Stack Temp Center Pt. air vel. Injection flowmeter Stack flow

Sampling flowmeter Ambient pressure Ambient humidity B\&K vapor correction Back-Gd gas ppb No. Bk-Gd samples Ambient Temp, F Instuments Used: B\&K 1302 Gas Analyzer SN 1788615 Cat2 MTE TSI 8360 Velocity SN 209060 Omega FMA-2617A flowmeter SN30348 due $11 / 01 / 07$

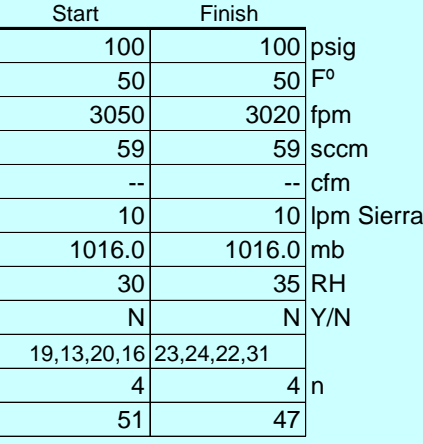

Notes: Ambient pressure in $\mathrm{hPa}$

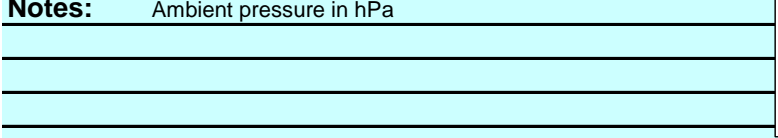

\begin{tabular}{r|l} 
Dev. from mean & Center $2 / 3$ \\
\hline$-37.0 \%$ & Mean \\
$32.8 \%$ & Std. Dev. \\
COV as \%
\end{tabular}
Gas analyzer checked:

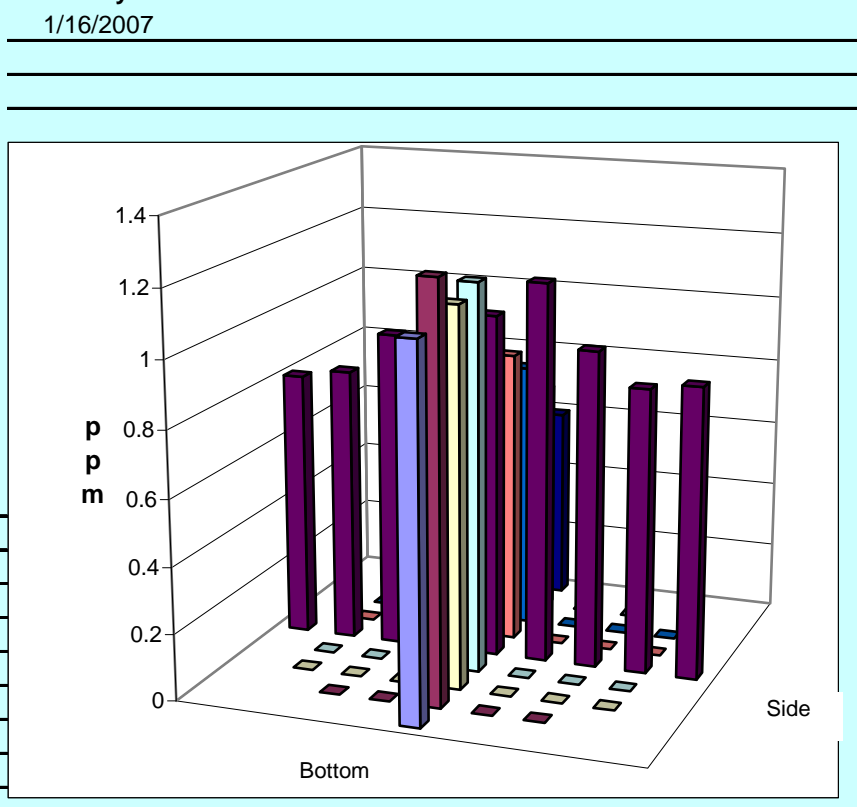

Signature signifies compliance with Signature verifying data and calculations:

Procedure EMS-JAG-01

Signature/date Signatures on original data sheet in Test Instruction Package TI-RPP-WTP-465

Reference: CCP-WTPSP-176

(HVC2_gas-dataRev0 (8)).xls gas-dataRev0.xls

GT22 31 July 2006

4/18/2007 
Rev. 0

31-Jul-06

$\begin{aligned} \text { Site } & \text { HV-C2 Model } \\ \text { Date } & \frac{1 / 17 / 2007}{\text { MSP }} \\ \text { Tester } & \\ \text { Stack Dia. } & 12 \text { in. } \\ \text { Stack X-Area } & 113.1 \text { in. }^{2} \\ \text { Elevation } & \text { Port 1 }\end{aligned}$

Distance to disturbance Measurement units ppm SF6
TRACER GAS TRAVERSE DATA FORM

Run No. GT-23

Fan Configuration $\mathbf{A}$ \& $\mathbf{B}$ Fan Setting 40

Stack Temp

Start/End Time 1505 -- 155

Center $2 / 3$ from

Points in Center $2 / 3$ Injection Point $\mathrm{A}$ near left w/ 3M filterete prefilters

$\mathrm{Hz}$

$50.5 \operatorname{deg} \mathrm{F}$

1.10 to: 10.90

2 to: $\frac{1}{7}$

$1 \mathrm{st}$

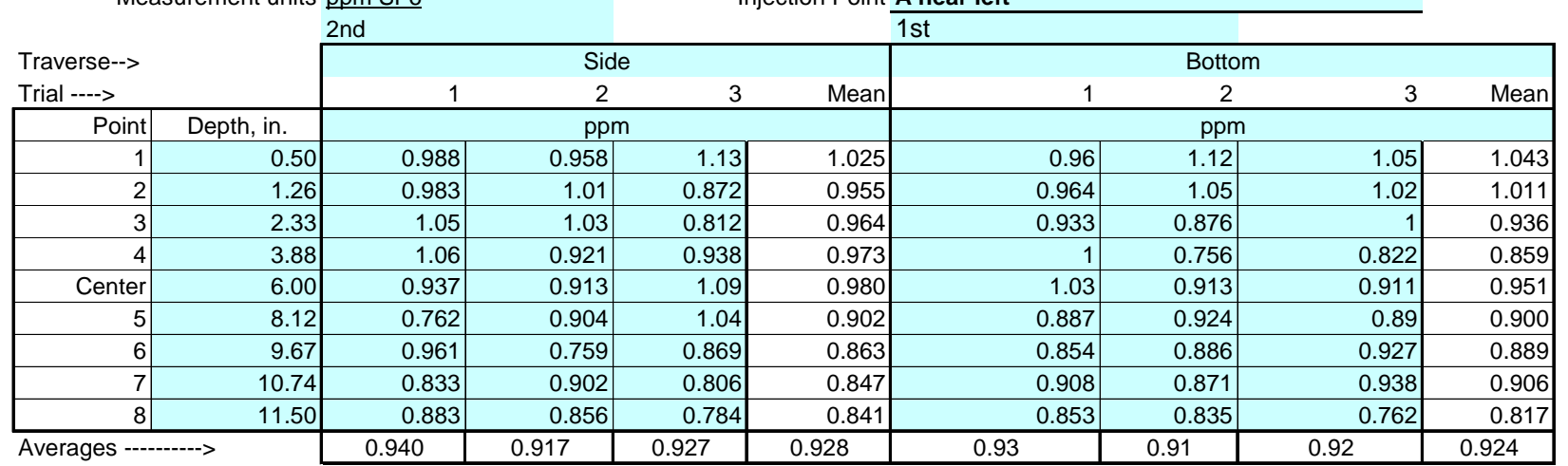

Avg. Conc. $0.921 \mathrm{ppm}$

Tracer tank pressure Stack Temp Center Pt. air vel. Injection flowmeter Stack flow Sampling flowmeter Ambient pressure Ambient humidity B\&K vapor correction Back-Gd gas ppb No. Bk-Gd samples Ambient Temp, $\mathrm{F}$ Instuments Used: B\&K 1302 Gas Analyzer SN 1788615 Cat2 MTE TSI 8360 Velocity SN 209060 Omega FMA-2617A flowmeter SN30348 due $11 / 01 / 07$

\begin{tabular}{|c|c|c|}
\hline Start & Finish & \\
\hline 100 & 100 & psig \\
\hline 50 & 51 & $F^{0}$ \\
\hline 3020 & 3020 & $\mathrm{fpm}$ \\
\hline 59 & 59 & $\mathrm{sccm}$ \\
\hline-- & -- & $\mathrm{cfm}$ \\
\hline 10 & 10 & Ipm Sierra \\
\hline 1016.0 & 1017.0 & $\mathrm{mb}$ \\
\hline 35 & 34 & $\mathrm{RH}$ \\
\hline $\mathrm{N}$ & $\mathrm{N}$ & $\mathrm{Y} / \mathrm{N}$ \\
\hline $21,23,20,19$ & $24,25,31,26$ & \\
\hline 4 & & $n$ \\
\hline 47 & 48 & \\
\hline
\end{tabular}

Notes: Ambient pressure in $\mathrm{hPa}$

A

\begin{tabular}{r|l} 
Dev. from mean & Center 2/3 \\
\hline$-11.8 \%$ & Mean \\
$12.7 \%$ & Std. Dev. \\
COV as \%
\end{tabular}
Gas analyzer checked: $1 / 16 / 2007$

Signature signifies compliance with Signature verifying data and calculations: Procedure EMS-JAG-01 Signature/date Signatures on original data sheet in Test Instruction Package TI-RPP-WTP-465

Reference: CCP-WTPSP-176

(HVC2_gas-dataRev0 (8)).xls gas-dataRev0.xls

GT23 31 July 2006

$4 / 18 / 2007$ 
Rev. 0

31-Jul-06

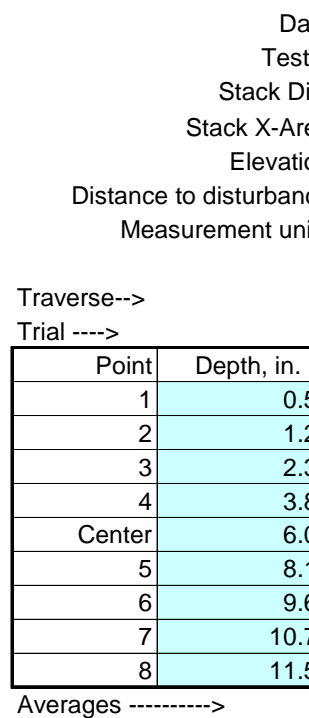

TRACER GAS TRAVERSE DATA FORM

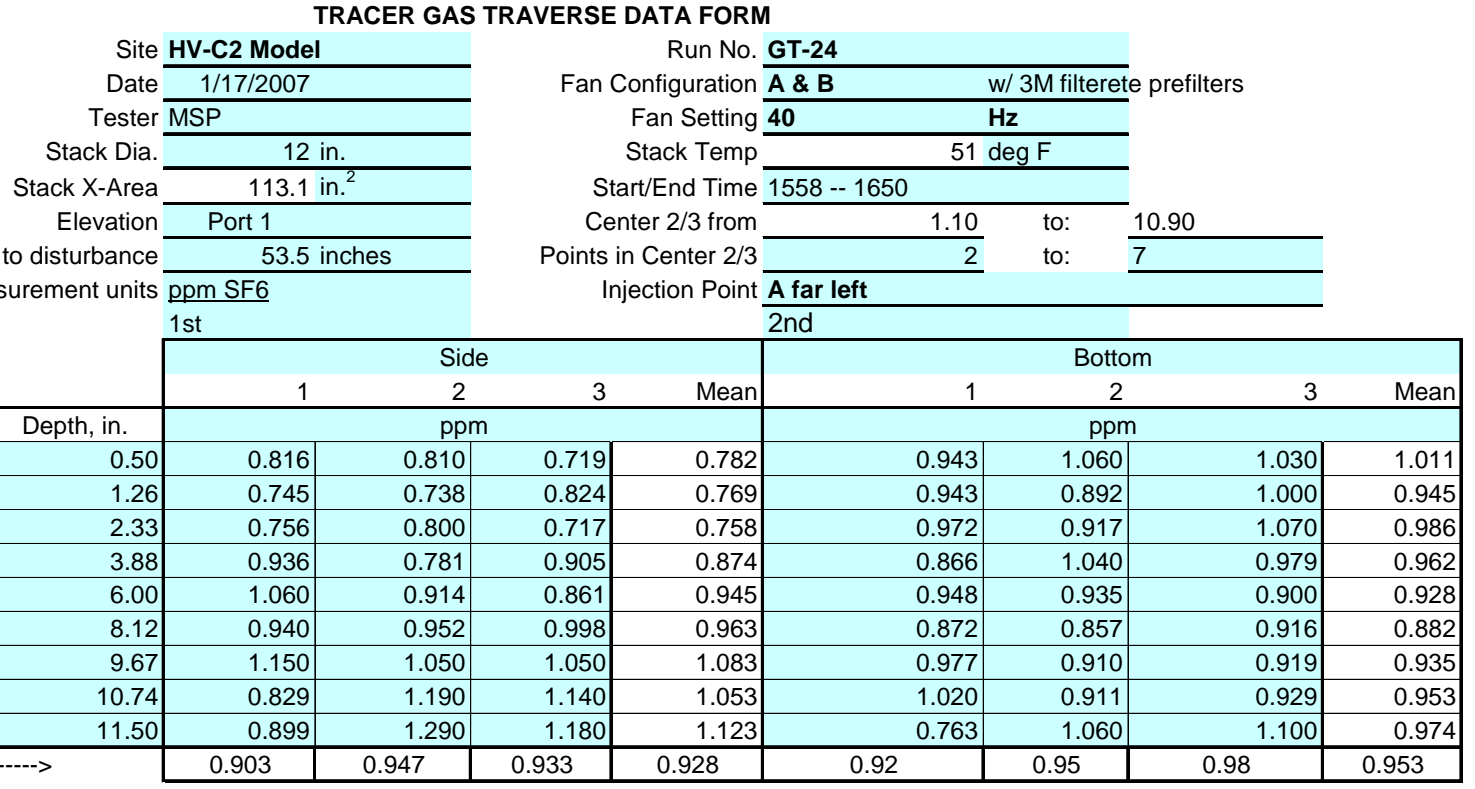

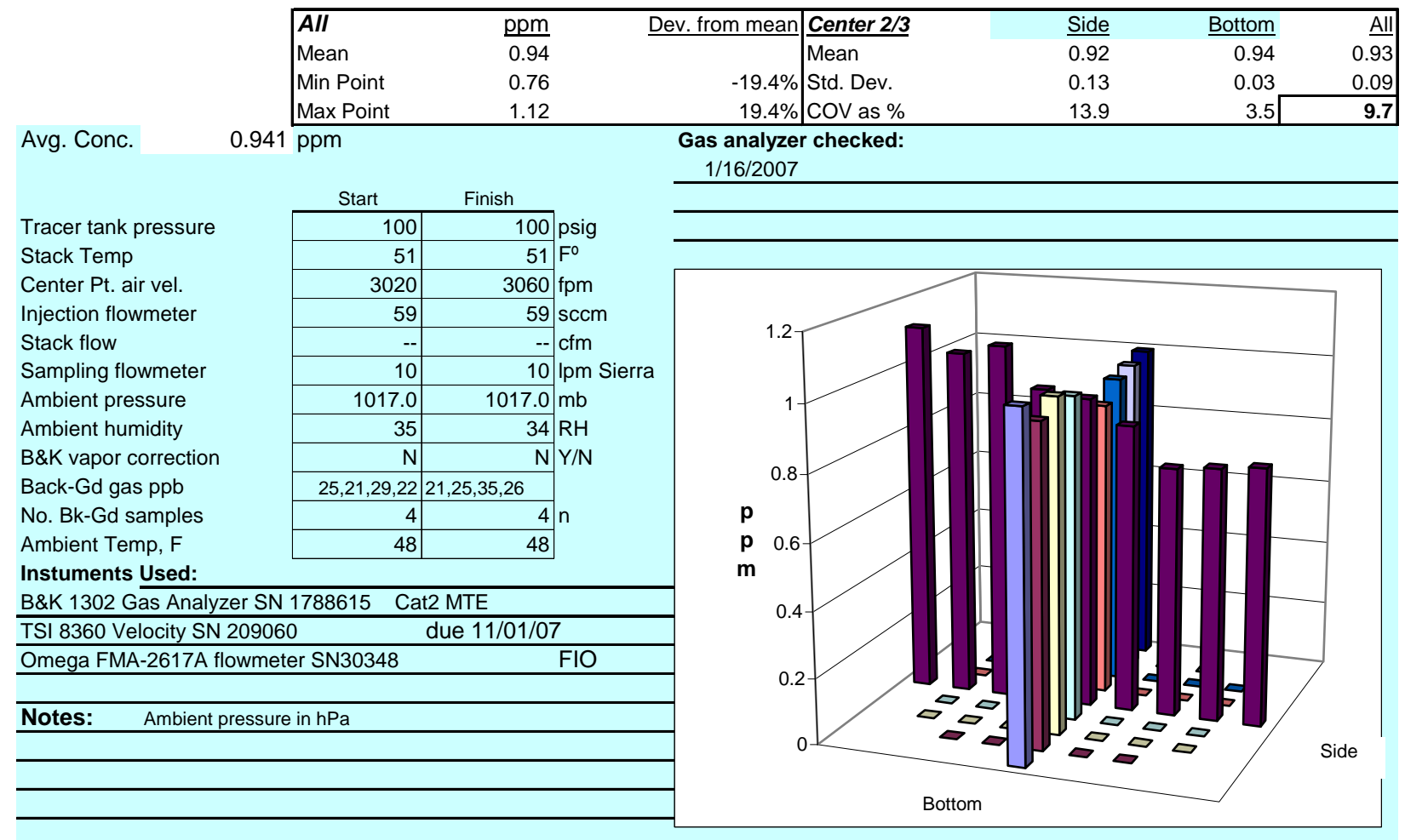

Signature signifies compliance with

Signature verifying data and calculations:

Procedure EMS-JAG-01

Signature/date

Signatures on original data sheet in Test Instruction Package TI-RPP-WTP-465

Reference: CCP-WTPSP-176

(HVC2_gas-dataRev0 (8)).xls

gas-dataRev0.xls

GT24

31 July 2006

$4 / 18 / 2007$ 
Rev. 0

31-Jul-06

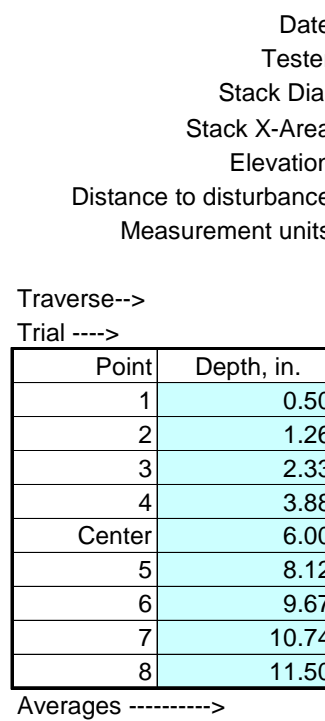

TRACER GAS TRAVERSE DATA FORM

\begin{tabular}{|c|c|c|c|c|c|c|c|c|}
\hline \multicolumn{7}{|c|}{ TRACER GAS TRAVERSE DATA FORM } & & \\
\hline \multicolumn{3}{|c|}{ Site HV-C2 Model } & \multicolumn{4}{|c|}{ Run No. GT-25 } & \multirow[b]{2}{*}{ te prefilters } & \\
\hline Date & $1 / 18 / 2007$ & & \multicolumn{3}{|c|}{ Fan Configuration A \& B } & \multirow{2}{*}{$\begin{array}{l}\mathrm{w} / 3 \mathrm{M} \text { filteret } \\
\mathrm{Hz}\end{array}$} & & \\
\hline \multicolumn{3}{|c|}{ Tester $\frac{1 / 10 / \angle \mathrm{VO} /}{\mathrm{MSP}}$} & \multirow{2}{*}{\multicolumn{2}{|c|}{ Fan Setting }} & 40 & & & \\
\hline Stack Dia. & \multicolumn{2}{|c|}{12 in. } & \multirow{2}{*}{\multicolumn{2}{|c|}{$\begin{array}{r}\text { Stack Temp } \\
\text { Start/End Time }\end{array}$}} & & $54.5 \operatorname{deg} F$ & & \\
\hline Stack X-Area & \multicolumn{2}{|c|}{113.1 in. $^{2}$} & \multicolumn{3}{|c|}{ Start/End Time 1239 -- 1345} & & & \\
\hline Elevation & \multicolumn{2}{|c|}{ Port 1} & \multicolumn{2}{|c|}{ Center $2 / 3$ from } & 1.10 & to: & 10.90 & \\
\hline to disturbance. & \multicolumn{2}{|c|}{53.5 inches } & \multirow{2}{*}{\multicolumn{2}{|c|}{ Points in Center $2 / 3$}} & 2 & to: & 7 & \\
\hline \multirow{4}{*}{ urement units } & \multicolumn{2}{|c|}{ Injection Point } & & & \multicolumn{3}{|l|}{ A far right } & \\
\hline & \multicolumn{4}{|l|}{ 2nd } & \multicolumn{4}{|l|}{$1 \mathrm{st}$} \\
\hline & \multicolumn{4}{|c|}{ Side } & \multicolumn{4}{|c|}{ Bottom } \\
\hline & 1 & 2 & 3 & Mean & 1 & 2 & 3 & Mean \\
\hline Depth, in. & \multicolumn{4}{|c|}{$\mathrm{ppm}$} & \multicolumn{4}{|c|}{$\mathrm{ppm}$} \\
\hline 0.50 & 0.538 & 0.711 & 0.637 & 0.629 & 1.100 & 1.060 & 0.804 & 0.988 \\
\hline 1.26 & 0.555 & 0.759 & 0.868 & 0.727 & 1.300 & 1.180 & 1.130 & 1.203 \\
\hline 2.33 & 0.816 & 0.805 & 0.658 & 0.760 & 1.420 & 1.300 & 1.580 & 1.433 \\
\hline 3.88 & 0.995 & 0.954 & 1.160 & 1.036 & 1.090 & 1.410 & 1.110 & 1.203 \\
\hline 6.00 & 1.180 & 1.210 & 1.090 & 1.160 & 1.080 & 1.070 & 1.110 & 1.087 \\
\hline 8.12 & 1.080 & 1.170 & 1.230 & 1.160 & 0.997 & 1.070 & 0.789 & 0.952 \\
\hline 9.67 & 0.962 & 1.030 & 1.010 & 1.001 & 0.725 & 0.881 & 0.788 & 0.798 \\
\hline 10.74 & 0.971 & 0.925 & 1.000 & 0.965 & 0.470 & 0.722 & 0.533 & 0.575 \\
\hline 11.50 & 0.745 & 0.837 & 0.812 & 0.798 & 0.503 & 0.345 & 0.349 & 0.399 \\
\hline$->$ & 0.871 & 0.933 & 0.941 & 0.915 & 0.97 & 1.00 & 0.91 & 0.960 \\
\hline
\end{tabular}

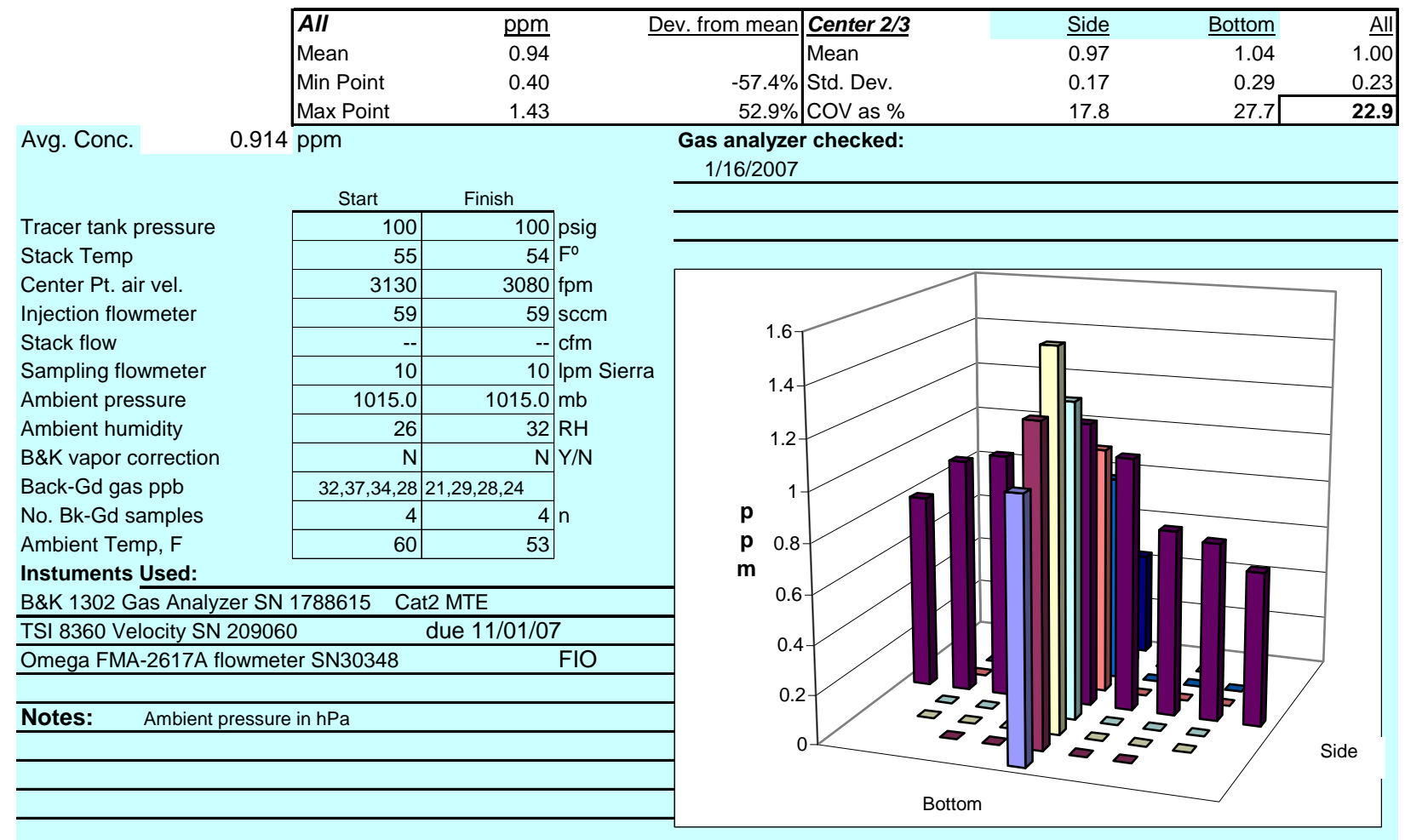

Signature signifies compliance with

Signature verifying data and calculations:

Procedure EMS-JAG-01

Signature/date

Signatures on original data sheet in Test Instruction Package TI-RPP-WTP-465

Reference: CCP-WTPSP-176

(HVC2_gas-dataRev0 (8)).xls

gas-dataRev0.xls

GT25

31 July 2006

$4 / 18 / 2007$ 
Rev. 0

31-Jul-06

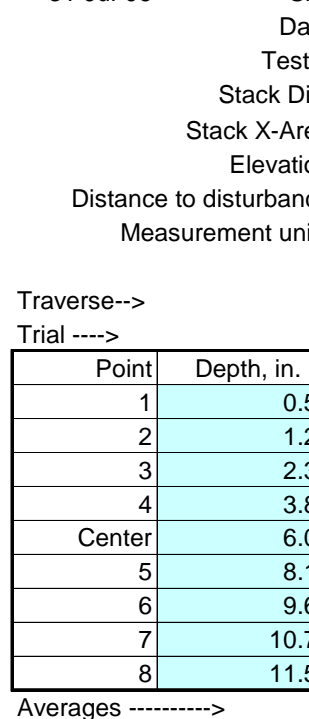

TRACER GAS TRAVERSE DATA FORM

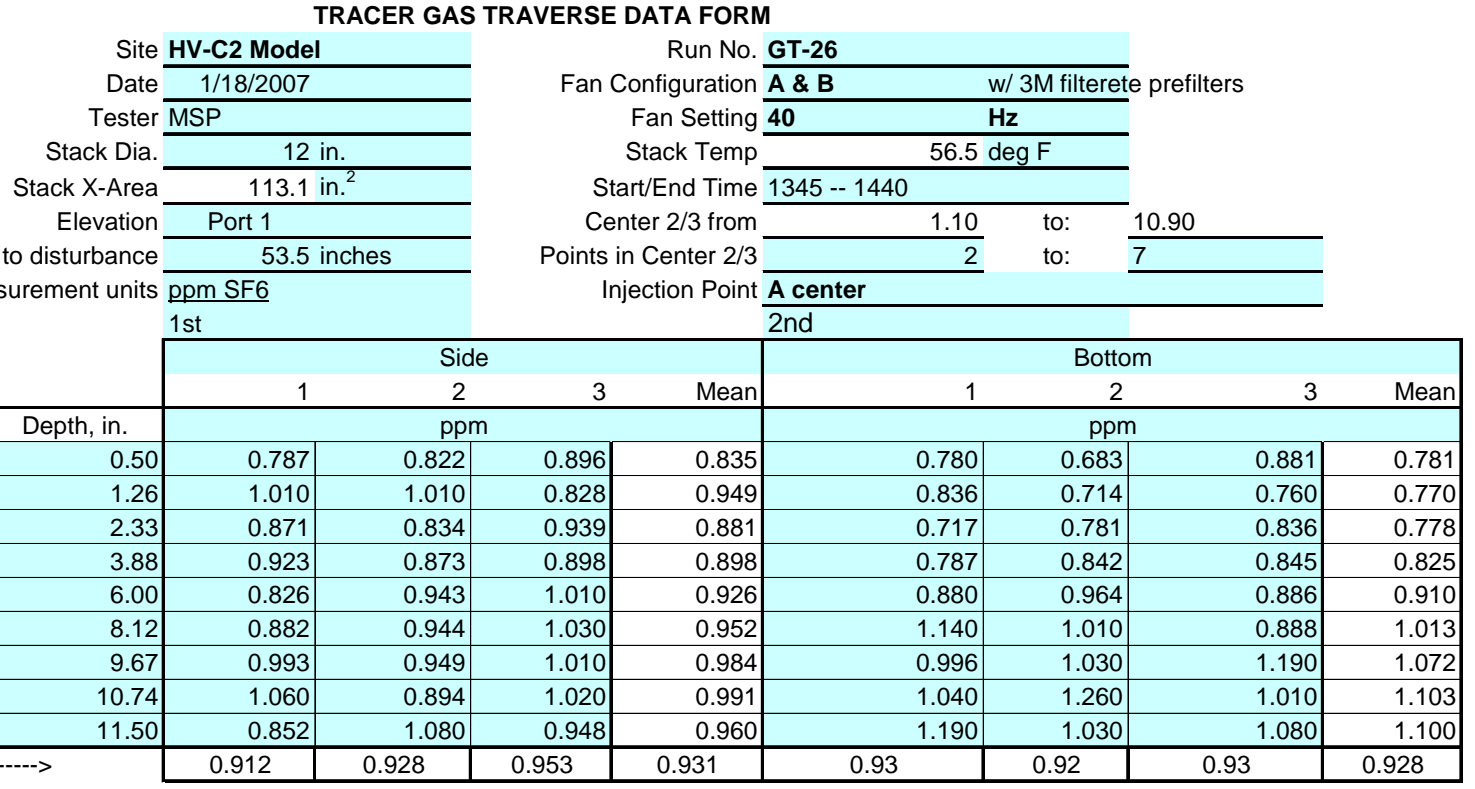

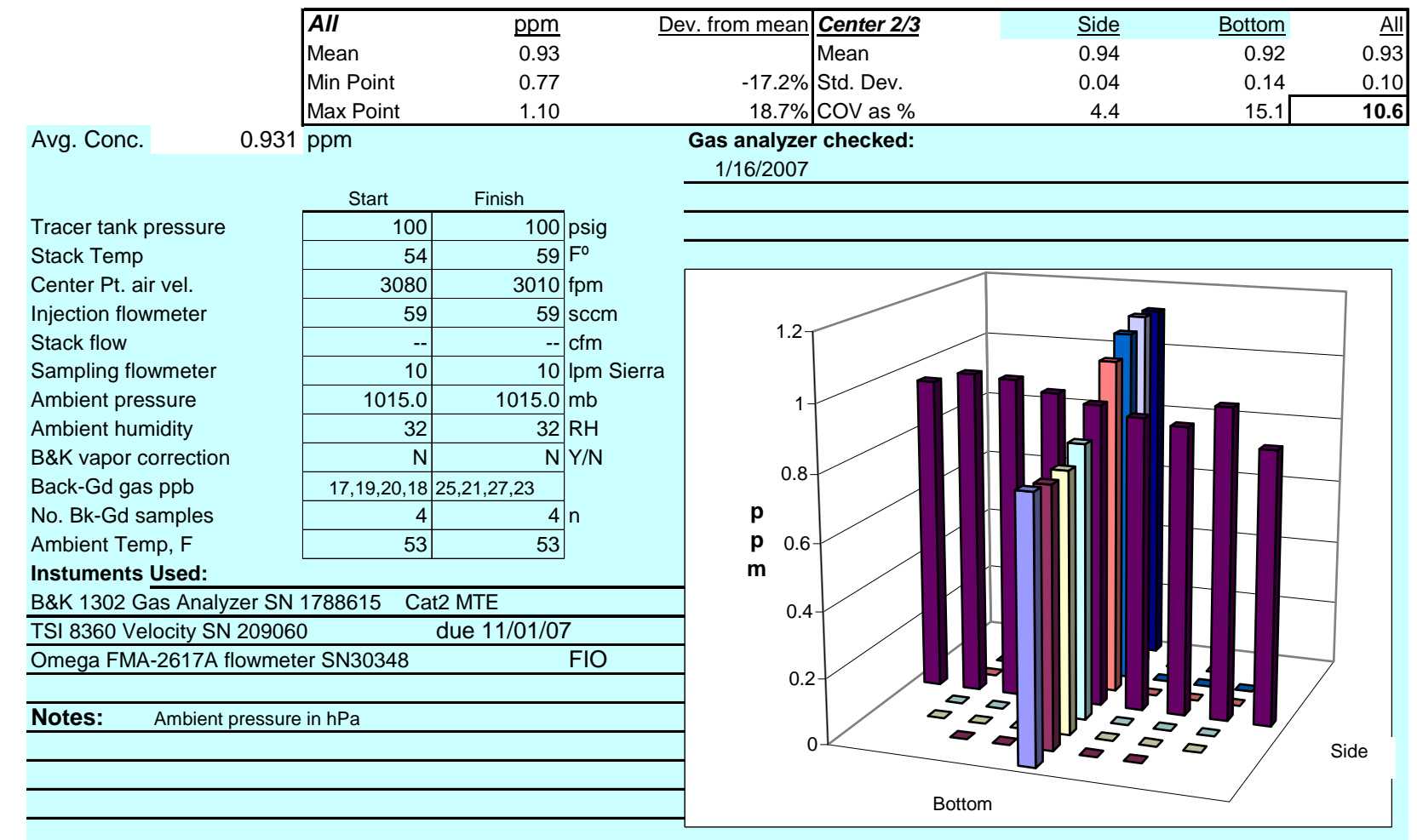

Signature signifies compliance with Signature verifying data and calculations:

Procedure EMS-JAG-01

Signature/date

Signatures on original data sheet in Test Instruction Package TI-RPP-WTP-465

Reference: CCP-WTPSP-176

(HVC2_gas-dataRev0 (8)).xls

gas-dataRev0.xls

GT26

31 July 2006

$4 / 18 / 2007$ 
Rev. 0

31-Jul-06

$\begin{aligned} \text { Site } & \text { HV-C2 Model } \\ \text { Date } & \frac{1 / 18 / 2007}{M} \\ \text { Tester } & \text { MSP } \\ \text { Stack Dia. } & 12 \text { in. } \\ \text { Stack X-Area } & 113.1 \text { in. }^{2} \\ \text { Elevation } & \text { Port } 2\end{aligned}$

Distance to disturbance Measurement units ppm SF6
TRACER GAS TRAVERSE DATA FORM

Run No. GT-27

Fan Configuration A \& B Fan Setting 40

Stack Temp

Start/End Time

Center $2 / 3$ from

Points in Center $2 / 3$ Injection Point $\mathrm{A}$ center w/ 3M filterete prefilters

$\mathrm{Hz}$

$56 \operatorname{deg} \mathrm{F}$

1.10 to: $\frac{10.90}{7}$

2 to: $\frac{10}{7}$

$1 \mathrm{st}$

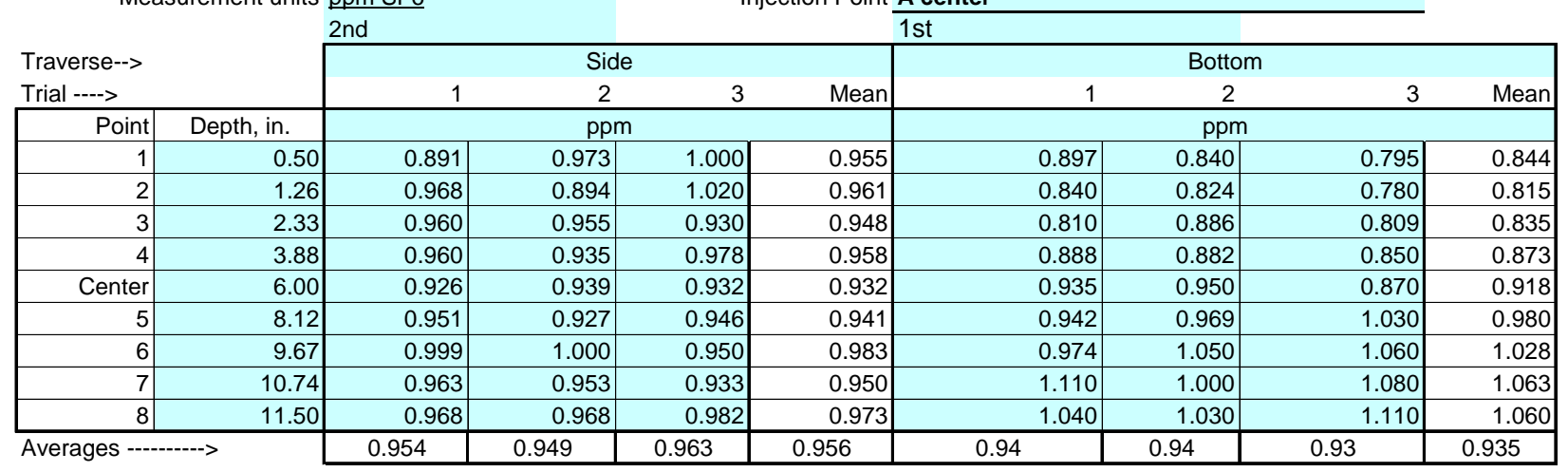

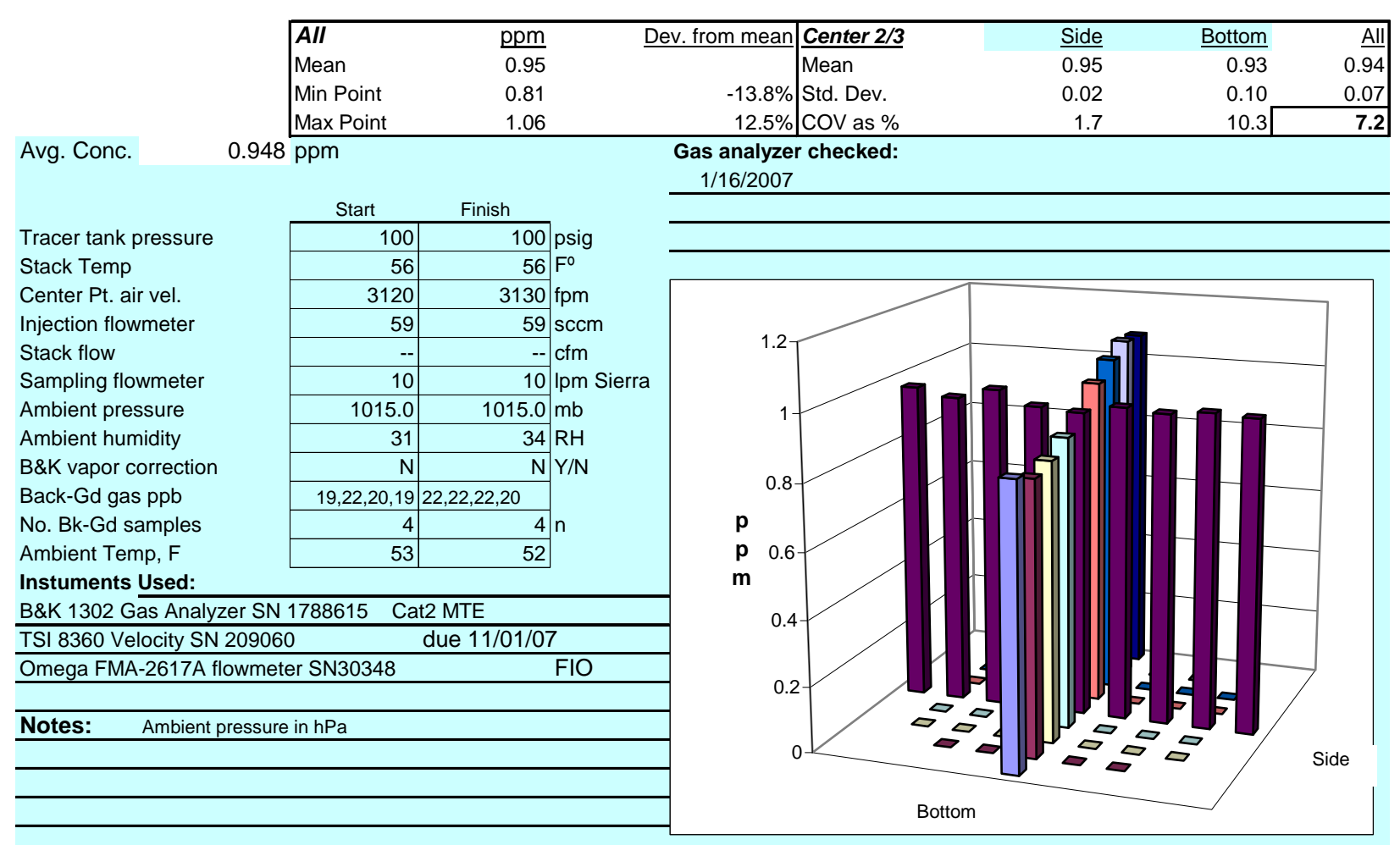

Signature signifies compliance with Signature verifying data and calculations:

Procedure EMS-JAG-01

Signature/date

Signatures on original data sheet in Test Instruction Package TI-RPP-WTP-465

Reference: CCP-WTPSP-176

(HVC2_gas-dataRev0 (8)).xls

gas-dataRev0.xls

GT27

31 July 2006

4/18/2007 
Rev. 0

31-Jul-06

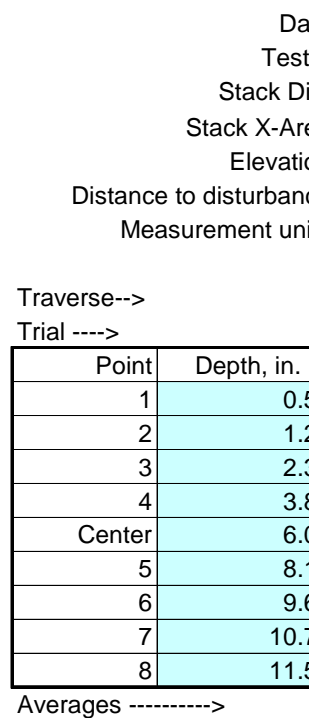

TRACER GAS TRAVERSE DATA FORM

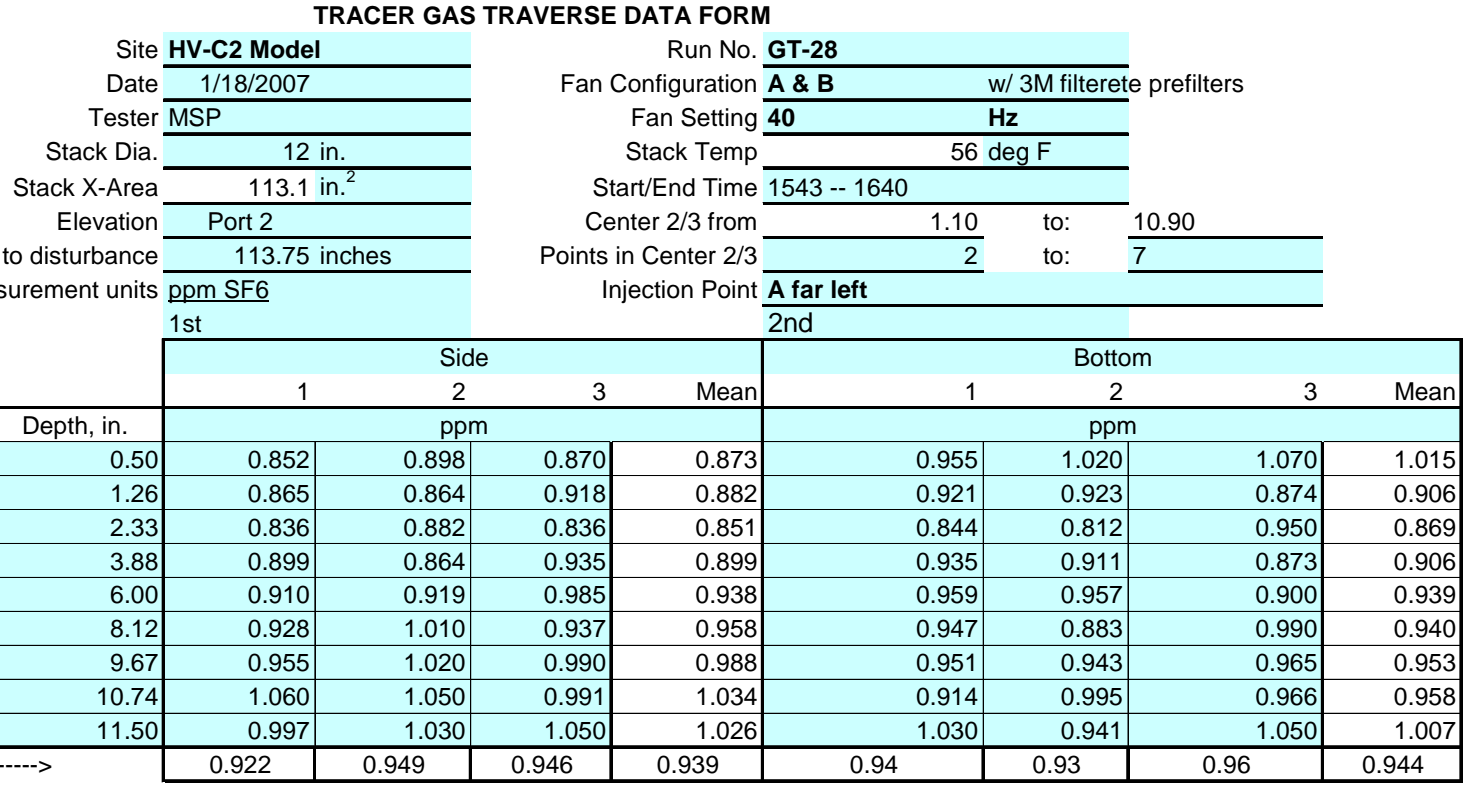

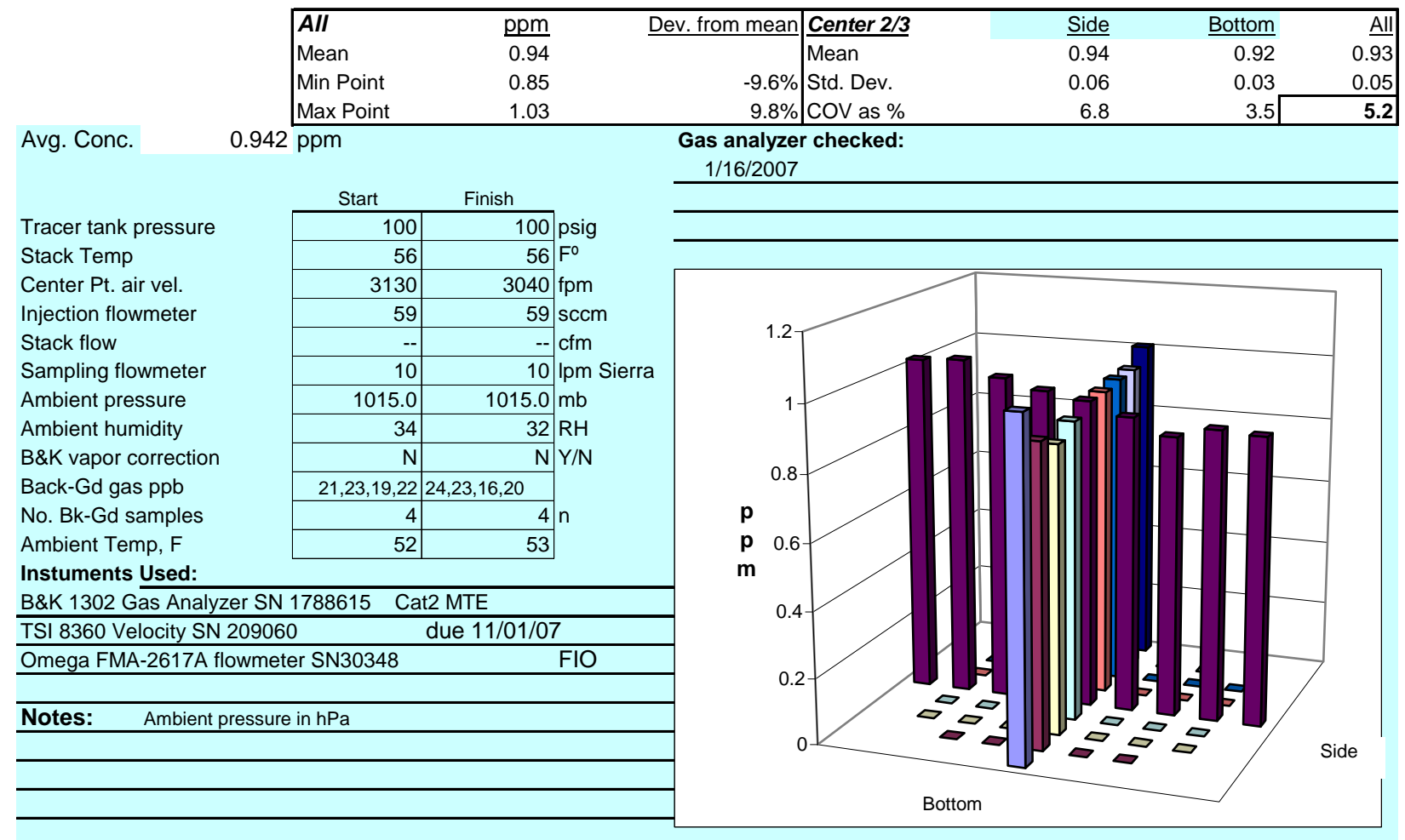

Signature signifies compliance with

Signature verifying data and calculations:

Procedure EMS-JAG-01

Signature/date

Signatures on original data sheet in Test Instruction Package TI-RPP-WTP-465

Reference: CCP-WTPSP-176

(HVC2_gas-dataRev0 (8)).xls

gas-dataRev0.xls

GT28

31 July 2006

$4 / 18 / 2007$ 
Rev. 0

31-Jul-06

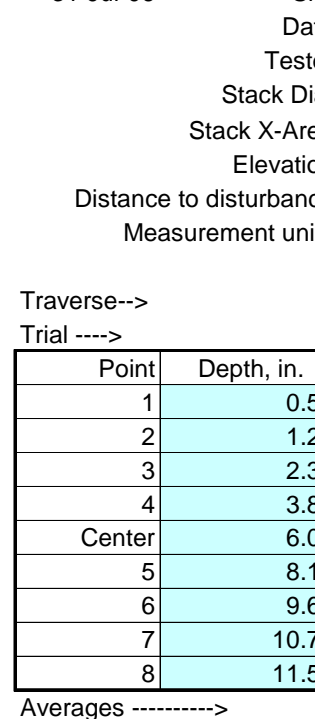

TRACER GAS TRAVERSE DATA FORM

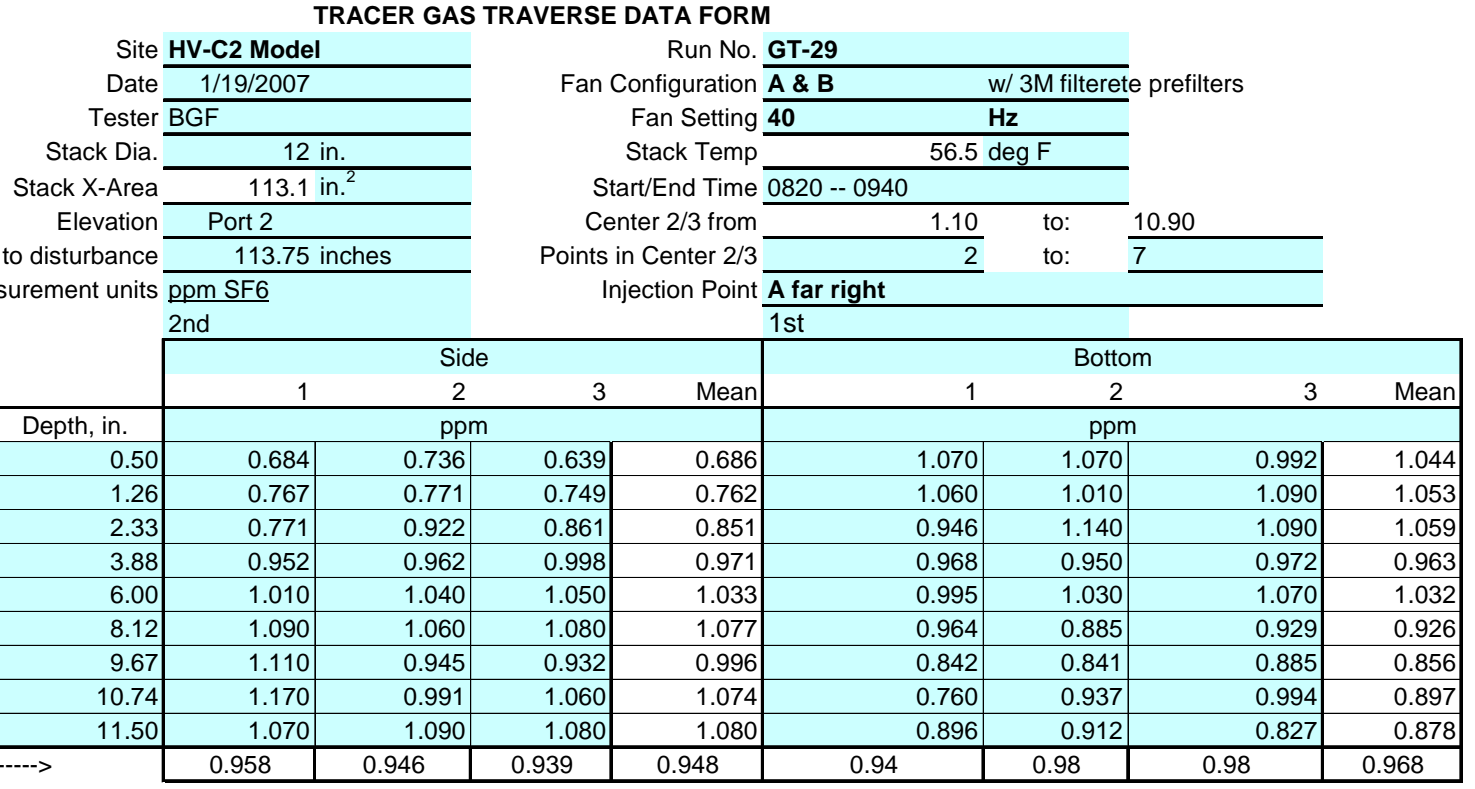

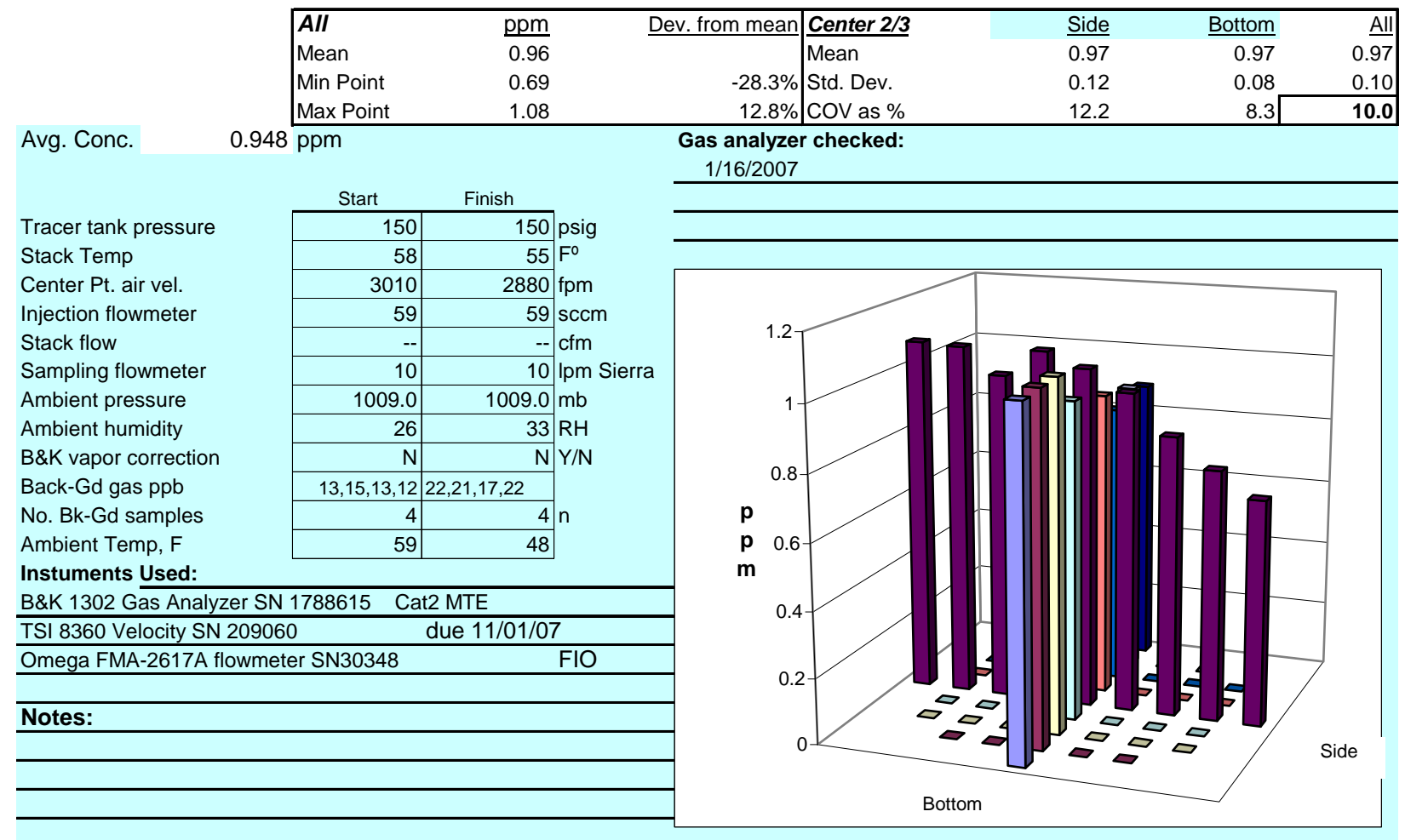

Signature signifies compliance with Signature verifying data and calculations:

Procedure EMS-JAG-01

Signature/date

Signatures on original data sheet in Test Instruction Package TI-RPP-WTP-465

Reference: CCP-WTPSP-176

(HVC2_gas-dataRev0 (8)).xls

gas-dataRev0.xls

GT29

31 July 2006

$4 / 18 / 2007$ 
Rev. 0

31-Jul-06

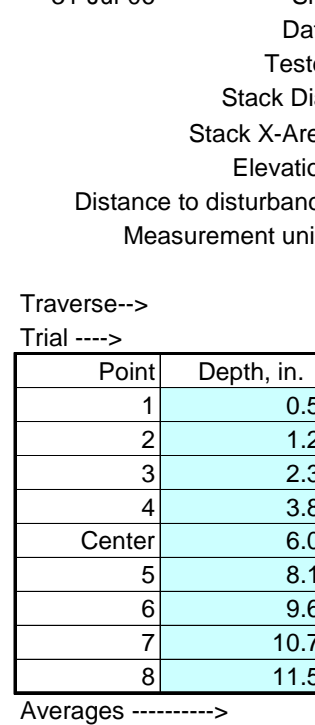

TRACER GAS TRAVERSE DATA FORM

Site HV-C2 Model

Date 1/19/2007

Tester BGF, JGD

Stack Dia.

evation

\begin{tabular}{c}
\hline 12 in. \\
\hline 113.1 in. $^{2}$ \\
\hline Port 2 \\
\hline 113.75 inches
\end{tabular}

113.75 inches

ppm SF6
Run No. GT-30

Fan Configuration A \& B Fan Setting 40

Stack Temp

Start/End Time 0950 -- 1030

Center $2 / 3$ from

Points in Center $2 / 3$ Injection Point $\mathrm{A}$ near right w/ 3M filterete prefilters

$\mathrm{Hz}$

$55 \operatorname{deg} F$

10 to: 10.90

2 to: $\frac{10}{7}$

2nd

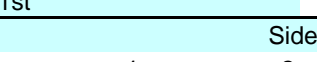

| Bottom

Bottom

\begin{tabular}{|r|r|r|r|r|r|}
\hline 3 & Mean & 1 & 2 & 3 & Mean \\
\hline & & \multicolumn{4}{|c|}{$\mathrm{ppm}$} \\
\hline 35 & 0.944 & 0.997 & 0.844 & 0.903 & 0.915 \\
\hline 49 & 0.920 & 1.030 & 1.140 & 0.968 & 1.046 \\
\hline 42 & 1.034 & 1.010 & 0.867 & 0.967 & 0.948 \\
\hline 78 & 1.004 & 0.996 & 0.998 & 1.020 & 1.005 \\
\hline 70 & 0.928 & 0.962 & 1.010 & 1.090 & 1.021 \\
\hline 13 & 0.970 & 0.967 & 1.020 & 1.000 & 0.996 \\
\hline 81 & 0.907 & 0.929 & 1.020 & 0.992 & 0.980 \\
\hline 76 & 0.877 & 0.880 & 0.912 & 0.866 & 0.886 \\
\hline & 0.876 & 0.934 & 0.952 & 0.863 & 0.916 \\
\hline
\end{tabular}

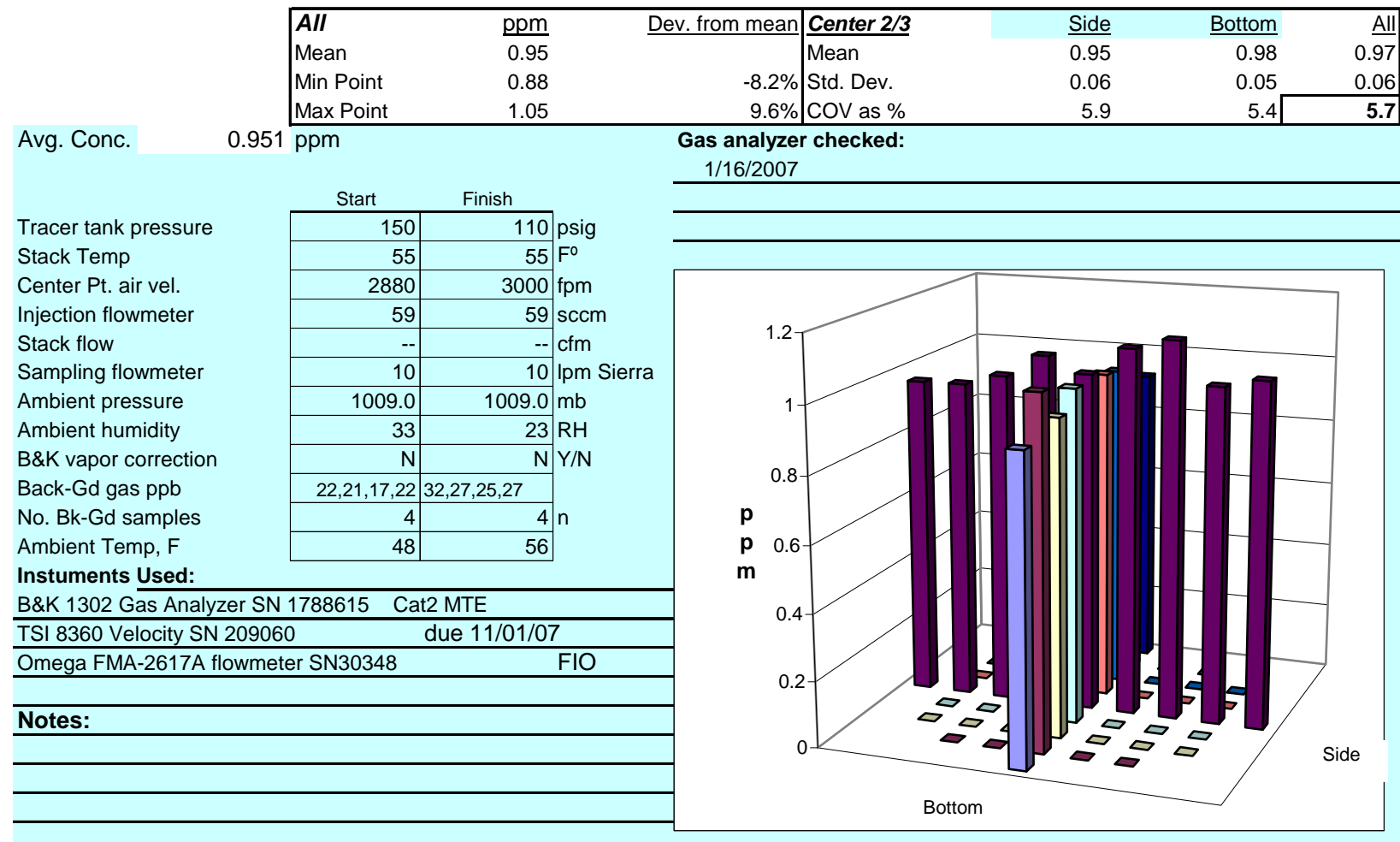

Signature signifies compliance with

Signature verifying data and calculations:

Procedure EMS-JAG-01

Signature/date

Signatures on original data sheet in Test Instruction Package TI-RPP-WTP-465

Reference: CCP-WTPSP-176

(HVC2_gas-dataRev0 (8)).xls

gas-dataRev0.xls

GT30

31 July 2006

4/18/2007 
Rev. 0

31-Jul-06

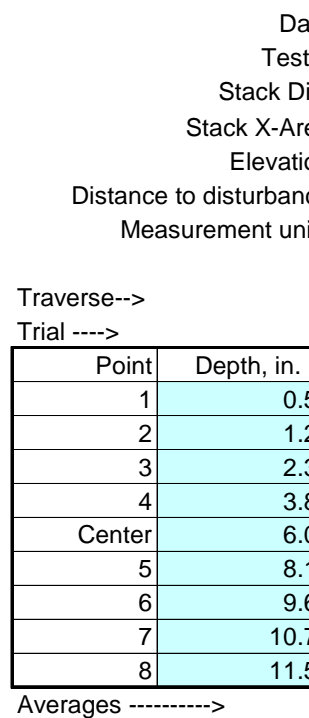

TRACER GAS TRAVERSE DATA FORM

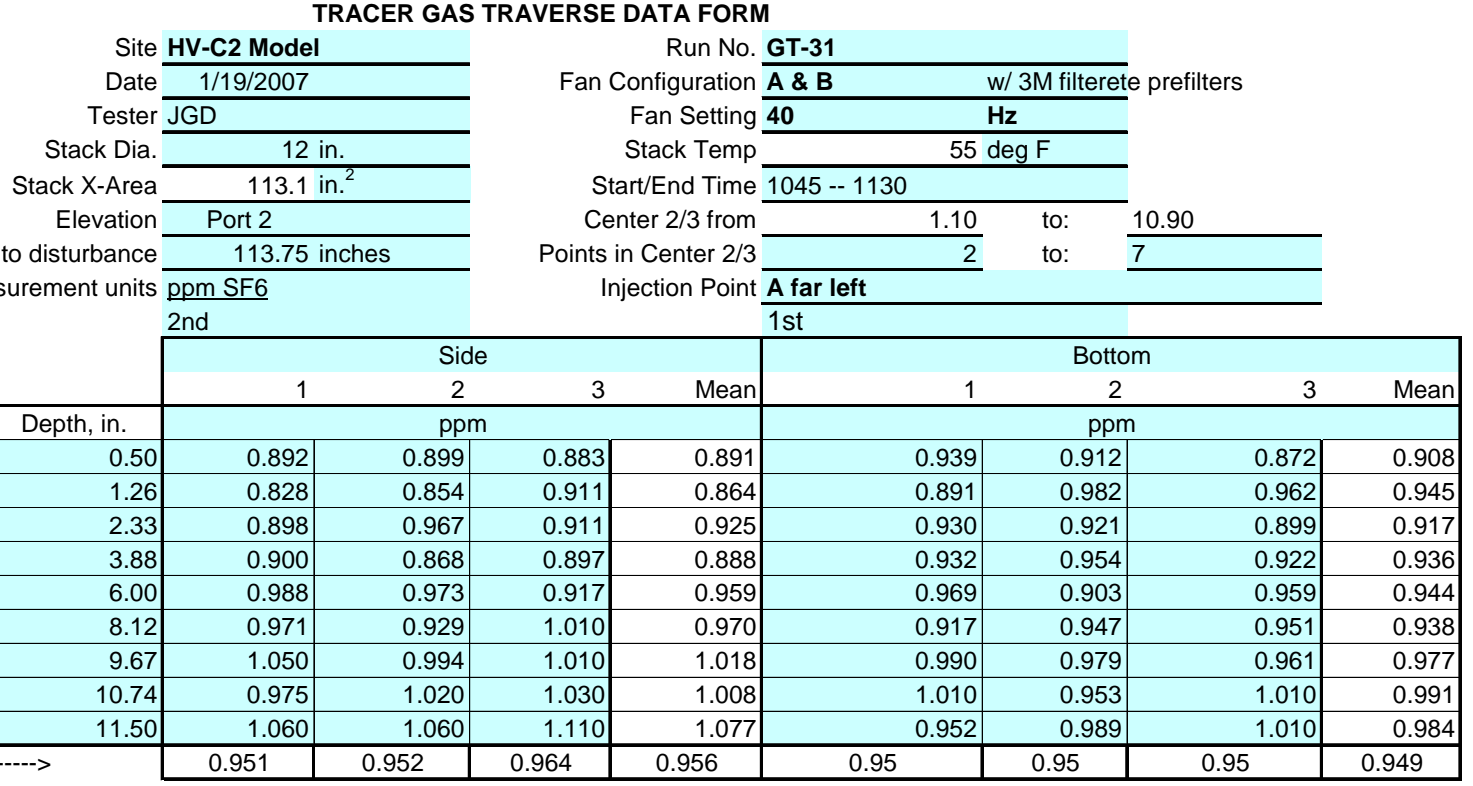

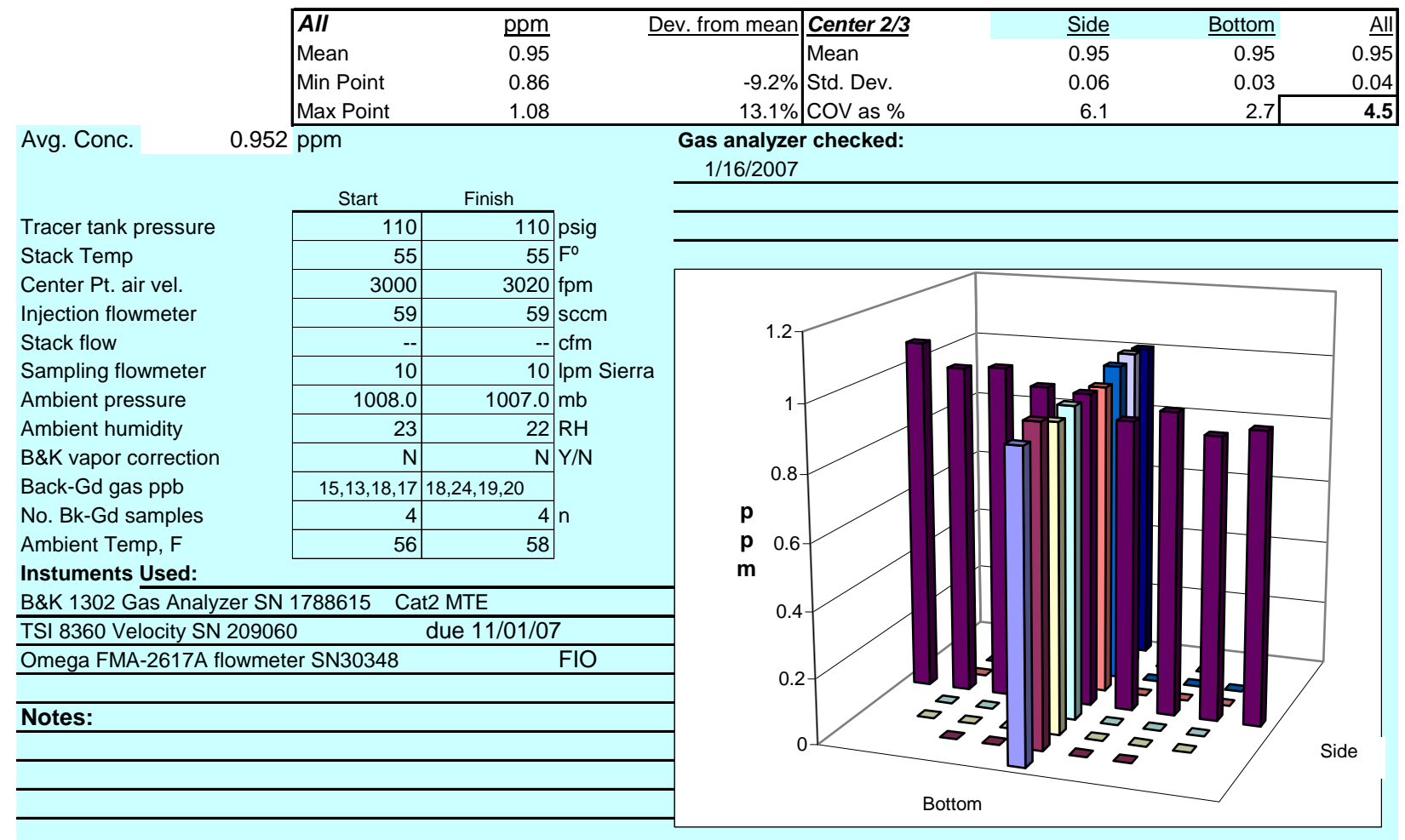

Signature signifies compliance with Signature verifying data and calculations:

Procedure EMS-JAG-01

Signature/date

Signatures on original data sheet in Test Instruction Package TI-RPP-WTP-465

Reference: CCP-WTPSP-176

(HVC2_gas-dataRev0 (8)).xls

gas-dataRev0.xls

GT31

31 July 2006

$4 / 18 / 2007$ 
Rev. 0

31-Jul-06

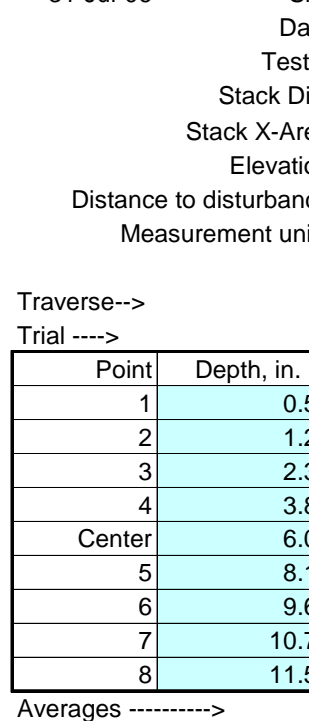

TRACER GAS TRAVERSE DATA FORM

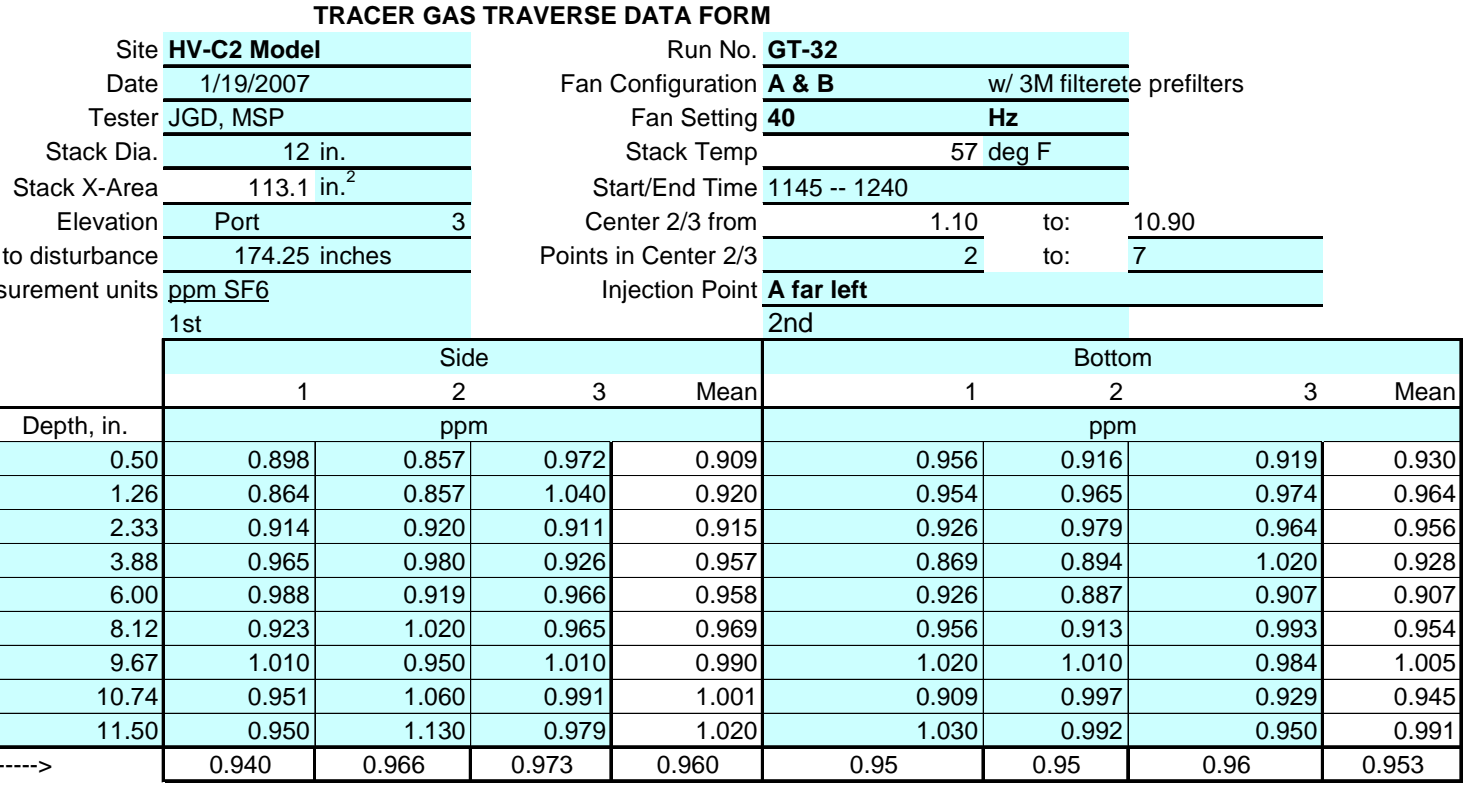

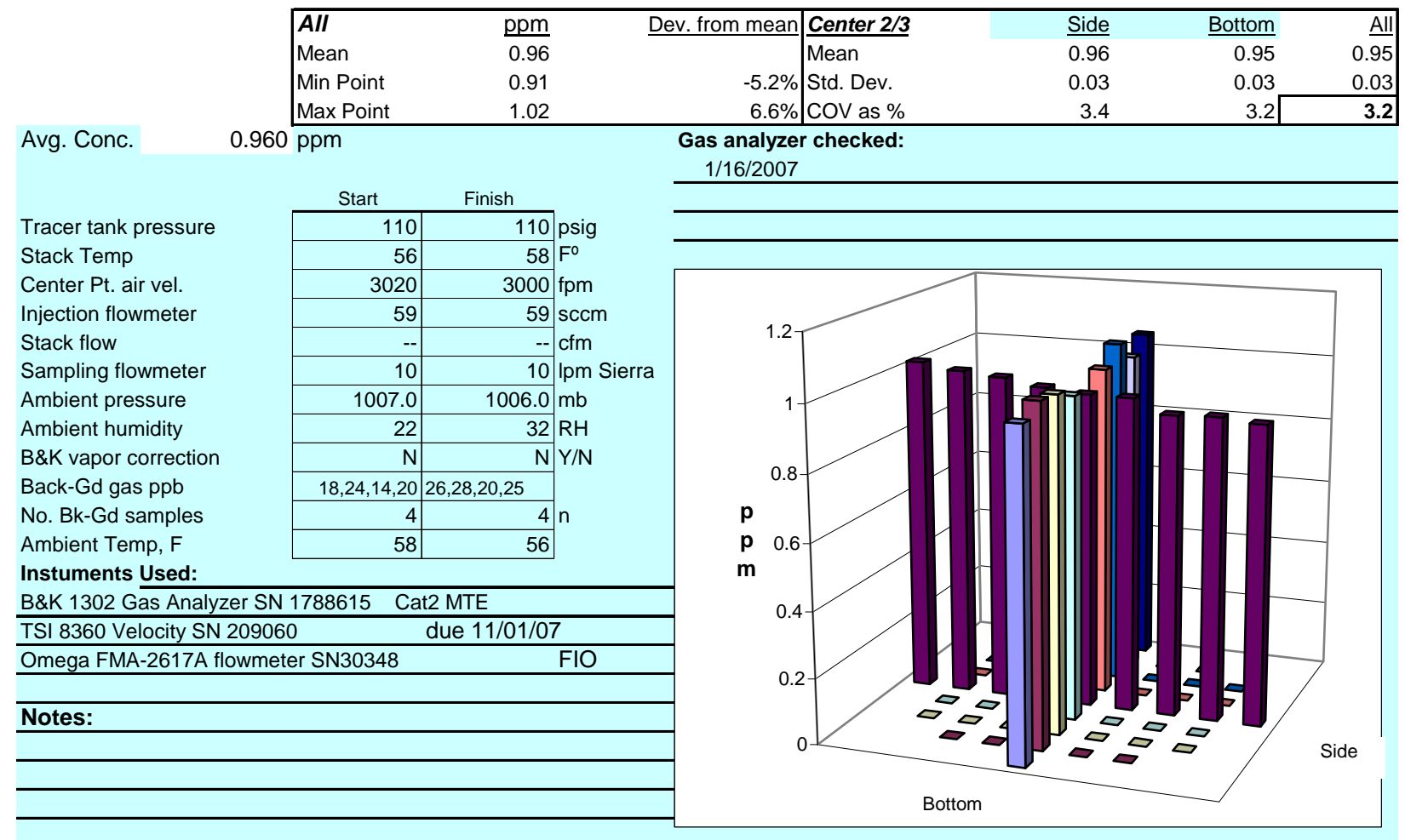

Signature signifies compliance with Signature verifying data and calculations:

Procedure EMS-JAG-01

Signature/date

Signatures on original data sheet in Test Instruction Package TI-RPP-WTP-465

Reference: CCP-WTPSP-176

(HVC2_gas-dataRev0 (8)).xls

gas-dataRev0.xls

GT32

31 July 2006

$4 / 18 / 2007$ 
TRACER GAS TRAVERSE DATA FORM

Rev. 0

31-Jul-06

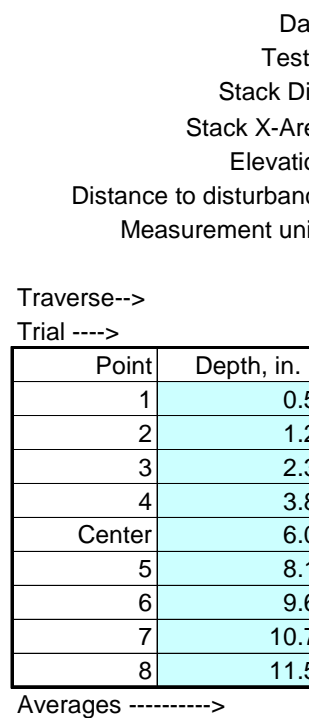

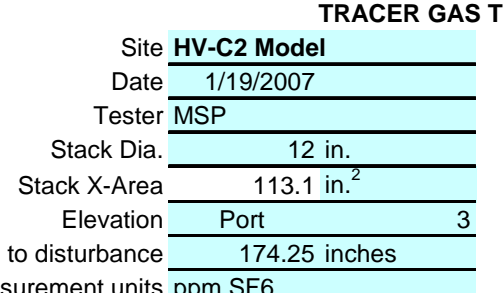

ppm SF6

2nd

2nd

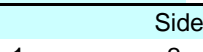

\begin{tabular}{ccc|ccc}
\cline { 4 - 5 } & \multicolumn{1}{l|}{ 1st } & & \\
\hline Side & 3 & Mean & & Bottom \\
2 & 3 & & 1 & 2 \\
\hline
\end{tabular}

Fan Configuration $\mathrm{A} \& \mathrm{~B}$

Fan Setting $\mathbf{4 0}$

Stack Temp

Start/End Time

Center $2 / 3$ from

Points in Center 2/3

Injection Point $\mathbf{A}$ far right

$w / 3 M$ filterete prefilters

$\mathrm{Hz} / 3 \mathrm{M}$ filterete

$57.5 \operatorname{deg} \mathrm{F}$

1.10 to: 10.90

2 to: $\underline{7}$

\begin{tabular}{|c|c|c|c|c|c|c|c|}
\hline 1 & 2 & 3 & Mean & 1 & 2 & 3 & Mean \\
\hline \multicolumn{4}{|c|}{$\mathrm{ppm}$} & \multicolumn{4}{|c|}{$\mathrm{ppm}$} \\
\hline 0.903 & \begin{tabular}{|l|}
0.928 \\
\end{tabular} & 0.935 & 0.922 & 0.878 & 0.928 & 0.897 & 0.901 \\
\hline 0.991 & 0.888 & 0.929 & 0.936 & 0.903 & 0.905 & 0.925 & 0.911 \\
\hline 0.868 & 0.976 & 0.934 & 0.926 & 0.976 & 0.932 & 0.995 & 0.968 \\
\hline 0.877 & 0.867 & 0.971 & 0.905 & 0.889 & 0.910 & 1.030 & 0.943 \\
\hline 0.873 & 0.959 & 0.937 & 0.923 & 0.904 & 1.080 & 0.902 & 0.962 \\
\hline 0.931 & 1.000 & 0.997 & 0.976 & 0.935 & 0.937 & 0.931 & 0.934 \\
\hline 0.922 & 1.030 & 0.948 & 0.967 & 0.929 & 1.080 & 0.931 & 0.980 \\
\hline 0.949 & 0.948 & 0.943 & 0.947 & 0.874 & 0.894 & 0.942 & 0.903 \\
\hline 0.965 & 0.946 & 1.070 & 0.994 & 0.908 & 0.896 & 0.901 & 0.902 \\
\hline 0.920 & 0.949 & 0.963 & 0.944 & 0.91 & \begin{tabular}{|l|l|}
0.95 \\
\end{tabular} & 0.94 & 0.934 \\
\hline
\end{tabular}

Avg. Conc.

\begin{tabular}{|lr}
\hline All & ppm \\
Mean & 0.94 \\
Min Point & 0.90 \\
Max Point & 0.99 \\
\hline
\end{tabular}

$0.938 \mathrm{ppm}$

0.99

\begin{tabular}{r|l} 
Dev. from mean & Center $2 / 3$ \\
$-4.0 \%$ & Mean \\
$5.8 \%$ & Std. Dev. \\
COV as \%
\end{tabular}

Gas analyzer checked:

Start Finish

Tracer tank pressure

Stack Temp

Center Pt. air vel.

Injection flowmeter

Stack flow

Sampling flowmeter

Ambient pressure

Ambient humidity

B\&K vapor correction

Back-Gd gas ppb

No. Bk-Gd samples

Ambient Temp, $F$

Instuments Used:

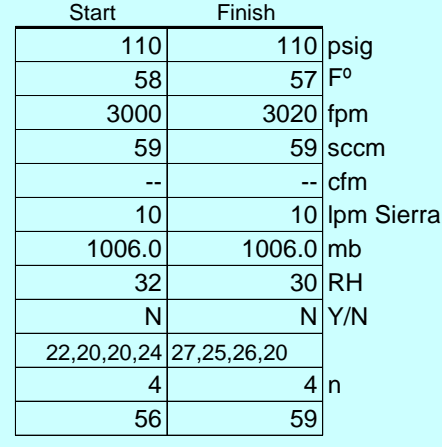

B\&K 1302 Gas Analyzer SN 1788615 Cat2 MTE

\begin{tabular}{ll}
\hline TSI 8360 Velocity SN 209060 & due 11/01/07 \\
\hline
\end{tabular}

Omega FMA-2617A flowmeter SN30348 FIO

Notes:

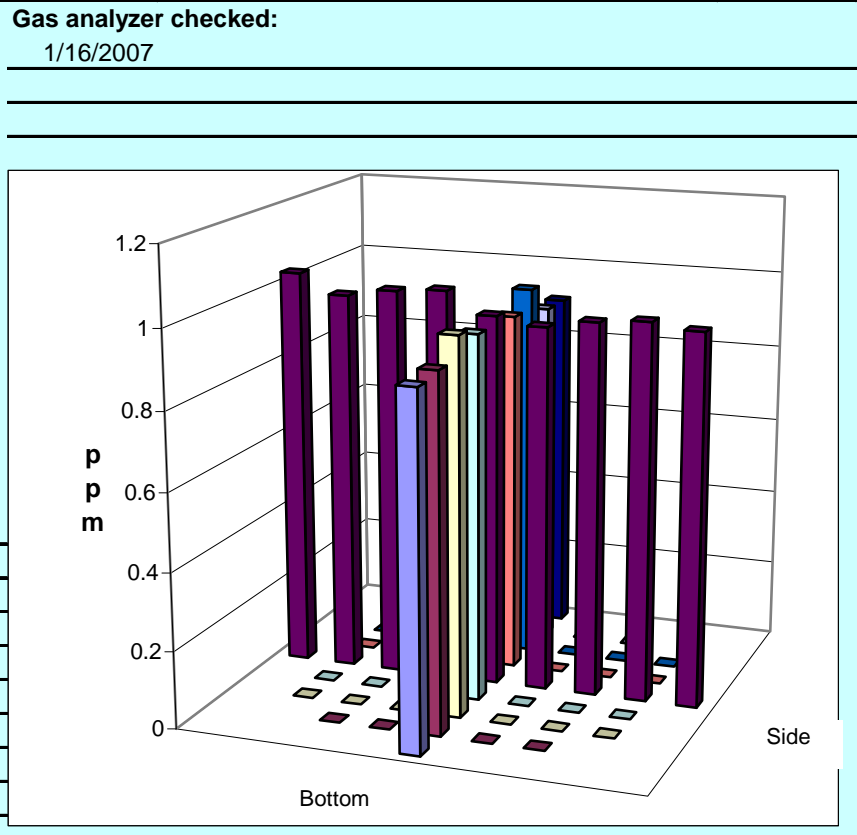

Signature signifies compliance with

Signature verifying data and calculations:

Procedure EMS-JAG-01

Signature/date

Signatures on original data sheet in Test Instruction Package TI-RPP-WTP-465

Reference: CCP-WTPSP-176

(HVC2_gas-dataRev0 (8)).xls

gas-dataRev0.xls

GT33

31 July 2006

$4 / 18 / 2007$ 
Rev. 0

31-Jul-06

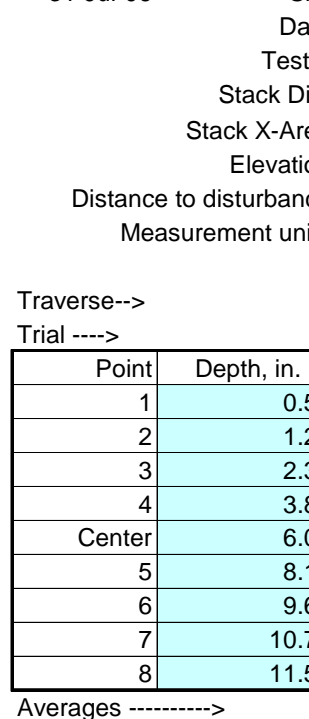

TRACER GAS TRAVERSE DATA FORM

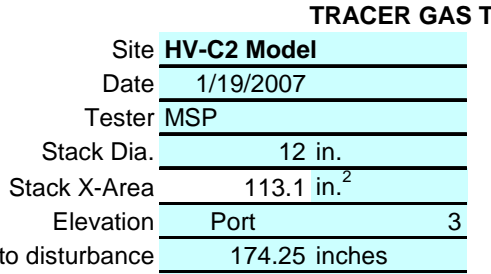

174.25 inches

ppm SF6

1 st

$\begin{array}{cc} & \text { Side } \\ 1 & 2\end{array}$

\begin{tabular}{lll|cc} 
& & & & \\
2 & 3 & Mettom
\end{tabular}

Fan Configuration $\mathbf{A} \& \mathbf{B}$

Fan Setting $\mathbf{4 0}$

w/ 3M filterete prefilters

Stack Temp

Start/End Time 1335 -- 1415

Center $2 / 3$ from

Points in Center $2 / 3$

Injection Point $\mathbf{A}$ center

$\mathrm{Hz}$

$57 \operatorname{deg} F$

15

1.10 to: 10.90

2 to: 7

Bottom

\begin{tabular}{r|r|r|r|r|r|r|}
\multicolumn{2}{c|}{$\mathrm{ppm}$} & \multicolumn{4}{|c|}{$\mathrm{ppm}$} \\
\hline 0.943 & 0.936 & 0.950 & 0.902 & 0.856 & 0.912 & 0.890 \\
\hline 0.953 & 0.935 & 0.962 & 0.918 & 0.956 & 0.899 & 0.924 \\
\hline
\end{tabular}

\begin{tabular}{|c|c|c|r|r|r|r|}
0.953 & 0.935 & 0.962 & 0.918 & 0.956 & 0.899 & 0.924 \\
\hline 0.966 & 0.927 & 0.954 & 0.903 & 0.891 & 0.982 & 0.925 \\
\hline 0.954 & 0.938 & 0.949 & 0.932 & 0.939 & 0.910 & 0.927 \\
\hline 0.964 & 0.980 & 0.980 & 0.965 & 0.965 & 0.957 & 0.962 \\
\hline 0.936 & 0.931 & 0.936 & 0.994 & 0.976 & 0.995 & 0.988 \\
\hline 0.938 & 0.952 & 0.934 & 0.952 & 1.020 & 1.020 & 0.997 \\
\hline 0.933 & 0.939 & 0.941 & 1.020 & 1.010 & 1.040 & 1.023 \\
\hline 0.945 & 0.934 & 0.942 & 1.070 & 1.040 & 0.985 & 1.032 \\
\hline 948 & 0.941 & 0.950 & 0.96 & 0.96 & 0.97 & 0.963 \\
\hline
\end{tabular}

Avg. Conc.

\begin{tabular}{|ll}
\hline AII & $\mathrm{ppm}$ \\
Mean & 0.96 \\
Min Point & 0.89 \\
Max Point & 1.03 \\
\hline
\end{tabular}

\begin{tabular}{r|l} 
Dev. from mean & Center $2 / 3$ \\
\hline$-6.9 \%$ & Mean \\
$7.9 \%$ & Ctd. Dev. \\
COV as \%
\end{tabular}

\begin{tabular}{|rrr|}
\hline Side & Bottom & All \\
0.95 & 0.96 & 0.96 \\
0.02 & 0.04 & 0.03 \\
1.7 & 4.2 & $\mathbf{3 . 2}$ \\
\hline
\end{tabular}

Tracer tank pressure

Stack Temp

Center Pt. air vel.

Injection flowmeter

Stack flow

Sampling flowmeter Ambient pressure

Ambient humidity

B\&K vapor correction

Back-Gd gas ppb

No. Bk-Gd samples

Ambient Temp, F

Instuments Used:

\begin{tabular}{|c|c|c|}
\hline Start & Finish & \\
\hline 110 & 110 & psig \\
\hline 57 & 57 & $\mathrm{~F}^{\circ}$ \\
\hline 3020 & 2980 & $\mathrm{fpm}$ \\
\hline 59 & 59 & $\mathrm{sccm}$ \\
\hline-- & -- & $\mathrm{cfm}$ \\
\hline 10 & 10 & Ipm Sierra \\
\hline 1006.0 & 1005.0 & $\mathrm{mb}$ \\
\hline 30 & 30 & $\mathrm{RH}$ \\
\hline $\mathrm{N}$ & $\mathrm{N}$ & $\mathrm{Y} / \mathrm{N}$ \\
\hline $17,15,19,16$ & $20,22,21,20$ & \\
\hline 4 & 4 & $n$ \\
\hline 59 & 59 & \\
\hline
\end{tabular}

B\&K 1302 Gas Analyzer SN 1788615 Cat2 MTE

TSI 8360 Velocity SN 209060 due 11/01/07

Omega FMA-2617A flowmeter SN30348

$\mathrm{FIO}$

\section{Notes:}

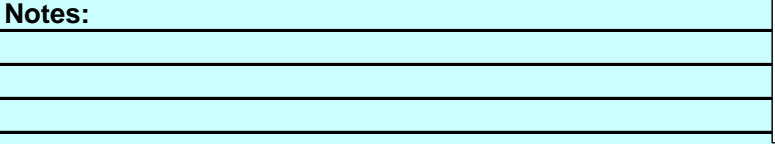

Gas analyzer checked:

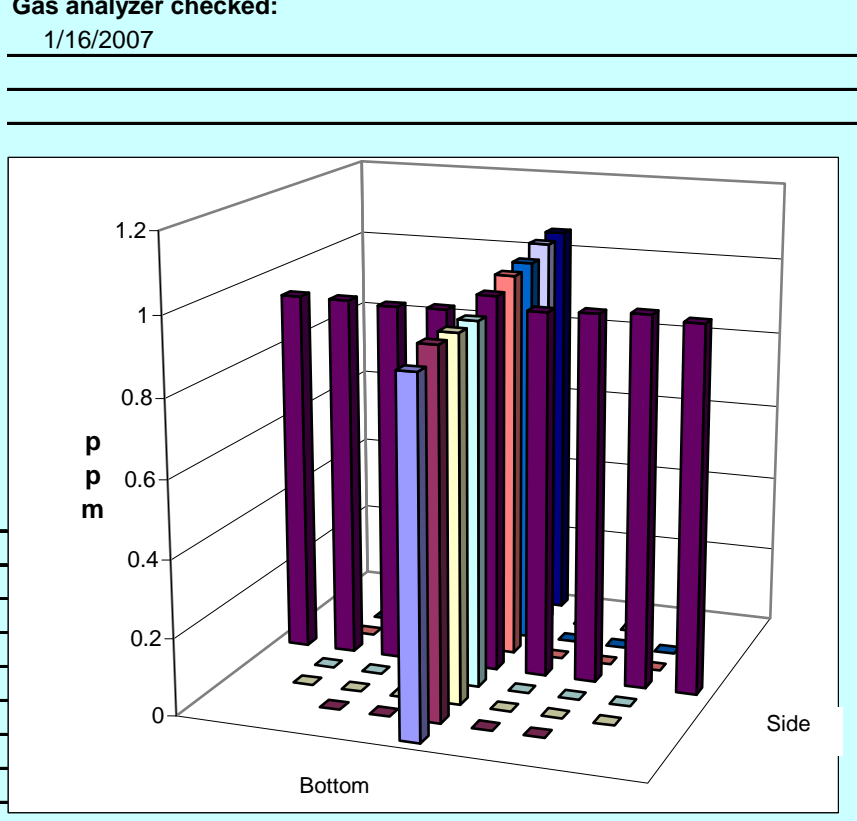

Signature signifies compliance with

Signature verifying data and calculations:

Procedure EMS-JAG-01

Signature/date

Signatures on original data sheet in Test Instruction Package TI-RPP-WTP-465

Reference: CCP-WTPSP-176

gas-dataRev0.xls

(HVC2_gas-dataRev0 (8)).xls

31 July 2006

GT34

$4 / 18 / 2007$ 
Rev. 0

31-Jul-06

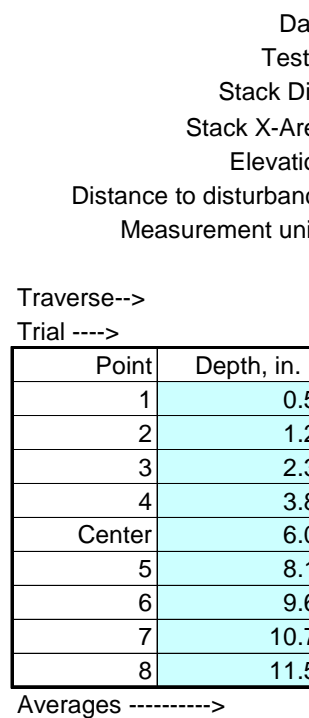

TRACER GAS TRAVERSE DATA FORM

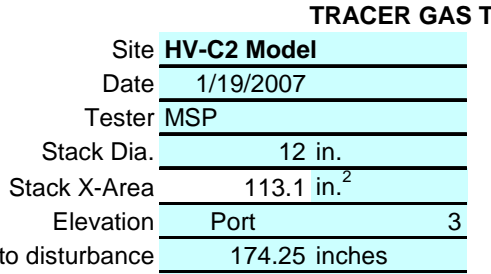

174.25 inches

ppm SF6

2nd

\section{Run No. GT-35}

Fan Configuration $\mathbf{A}$ \& B

Fan Setting 40

Stack Temp

Start/End Time

Center $2 / 3$ from

Points in Center $2 / 3$ Injection Point A near right $w / 3 M$ filterete prefilters

$\mathrm{Hz}$

$56.5 \operatorname{deg} \mathrm{F}$

510

1.10 to: 10.90

$\frac{1.10}{2}$ to: $\frac{10.90}{7}$

2

\begin{tabular}{|c|c|c|c|c|c|}
\hline \multicolumn{2}{|c|}{1} & \multicolumn{4}{|l|}{$1 \mathrm{st}$} \\
\hline \multirow[b]{2}{*}{3} & & \multicolumn{4}{|c|}{ Bottom } \\
\hline & Mean & 1 & 2 & 3 & Mean \\
\hline & & \multicolumn{4}{|c|}{ ppm } \\
\hline 0.899 & 0.945 & 1.010 & 0.949 & 0.989 & 0.983 \\
\hline 0.914 & 0.953 & 0.975 & 0.934 & 0.962 & 0.957 \\
\hline 1.040 & 1.005 & 1.090 & 0.972 & 0.932 & 0.998 \\
\hline 1.010 & 0.984 & 0.973 & 1.030 & 0.966 & 0.990 \\
\hline 0.991 & 0.979 & 0.986 & 0.980 & 0.994 & 0.987 \\
\hline 0.924 & 0.951 & 0.980 & 0.936 & 0.944 & 0.953 \\
\hline 0.945 & 0.932 & 0.970 & 0.950 & 0.951 & 0.957 \\
\hline 0.876 & 0.882 & 0.961 & 0.910 & 0.895 & 0.922 \\
\hline 0.845 & 0.912 & 0.977 & 0.967 & 0.896 & 0.947 \\
\hline 0.938 & 0.949 & 0.99 & 0.96 & 0.95 & 0.966 \\
\hline
\end{tabular}

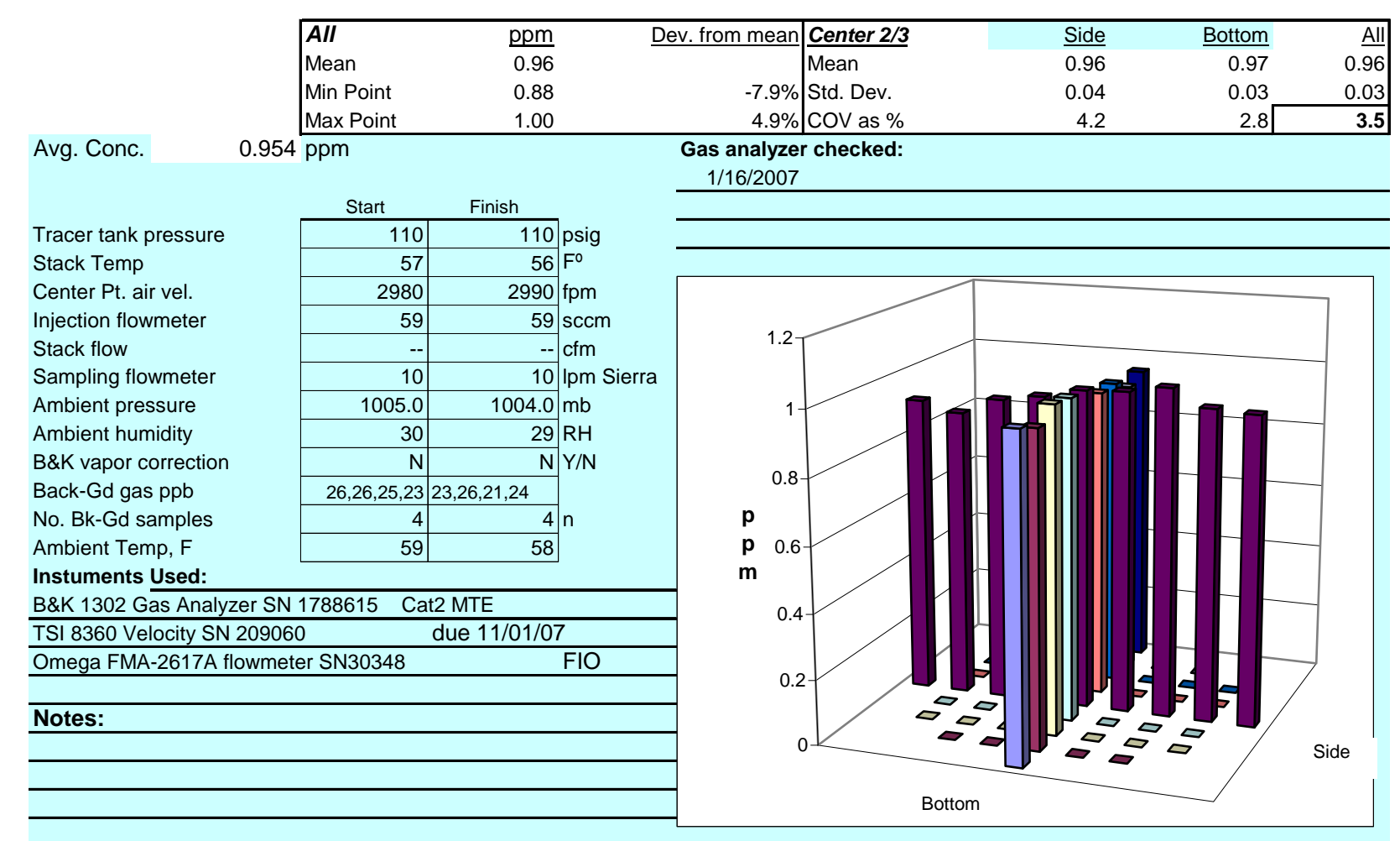

Signature signifies compliance with Signature verifying data and calculations:

Procedure EMS-JAG-01

Signature/date

Signatures on original data sheet in Test Instruction Package TI-RPP-WTP-465

Reference: CCP-WTPSP-176

gas-dataRev0.xls

(HVC2_gas-dataRev0 (8)).xls

31 July 2006

GT35

4/18/2007 
Rev. 0

31-Jul-06

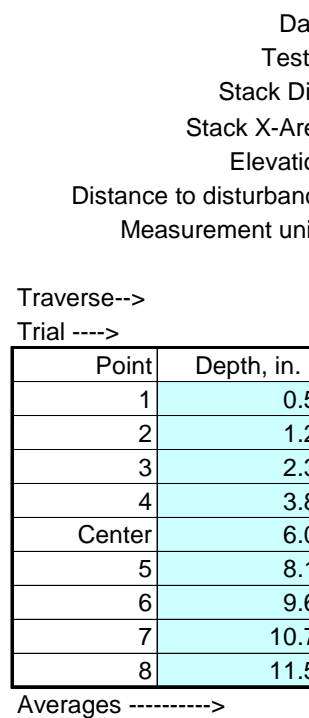

TRACER GAS TRAVERSE DATA FORM

\begin{aligned} & \multicolumn{1}{c}{ TRACER GAS TRA } \\ & Site HV-C2 Model \\ & Date $1 / 19 / 2007 \\ &$ Tester MSP \\ & Stack Dia. 12 in. \\ & Stack X-Area 113.1 in. $^{2} \\ &$ Elevation Port \\ & to disturbance 174.25 inches \\ & spm SF6 \end{aligned}

Run No. GT-36

Fan Configuration $A \& B$

Fan Setting 40

w/ 3M filterete prefilters

Stack Temp

Start/End Time 1512 -- 1600

Center $2 / 3$ from

Points in Center $2 / 3$

Injection Point $\mathrm{A}$ near left

$\mathrm{Hz}$

Hz

2nd

Bottom

\begin{tabular}{|c|c|c|c|c|c|c|}
\hline \multicolumn{3}{|c|}{ Side } & \multicolumn{4}{|c|}{ Bottom } \\
\hline 2 & 3 & Mean & 1 & 2 & 3 & Mean \\
\hline \multicolumn{2}{|c|}{ ppm } & & \multicolumn{4}{|c|}{ ppm } \\
\hline 0.888 & 0.958 & 0.959 & 0.963 & 0.965 & 0.955 & 0.961 \\
\hline 1.070 & 0.984 & 0.996 & 0.930 & 0.919 & 0.845 & 0.898 \\
\hline 0.976 & 0.919 & 0.947 & 0.950 & 0.906 & 0.959 & 0.938 \\
\hline 0.972 & 0.960 & 0.970 & 0.956 & 0.973 & 0.909 & 0.946 \\
\hline 0.884 & 0.913 & 0.913 & 1.000 & 0.860 & 0.931 & 0.930 \\
\hline 0.930 & 0.972 & 0.954 & 1.030 & 0.983 & 0.908 & 0.974 \\
\hline 0.917 & 0.936 & 0.905 & 0.944 & 0.960 & 0.991 & 0.965 \\
\hline 0.930 & 0.926 & 0.948 & 0.946 & 0.917 & 0.937 & 0.933 \\
\hline 0.883 & 0.905 & 0.895 & 0.978 & 0.921 & 0.974 & 0.958 \\
\hline 39 & 0.941 & 0.943 & 0.97 & 0.93 & 0.93 & 0.945 \\
\hline
\end{tabular}

Avg. Conc.

0.947 Mpm Point

$\mathrm{ppm}$
0.94
0.90
1.00

\begin{tabular}{r|l} 
Dev. from mean & Center 2/3 \\
$-5.2 \%$ & Mean \\
$5.5 \%$ & Ctd. Dev. \\
COV as \%
\end{tabular}

\begin{tabular}{rrr|} 
Side & Bottom & All \\
\hline 0.95 & 0.94 & 0.94 \\
0.03 & 0.02 & 0.03 \\
3.3 & 2.6 & $\mathbf{2 . 9}$ \\
\hline
\end{tabular}

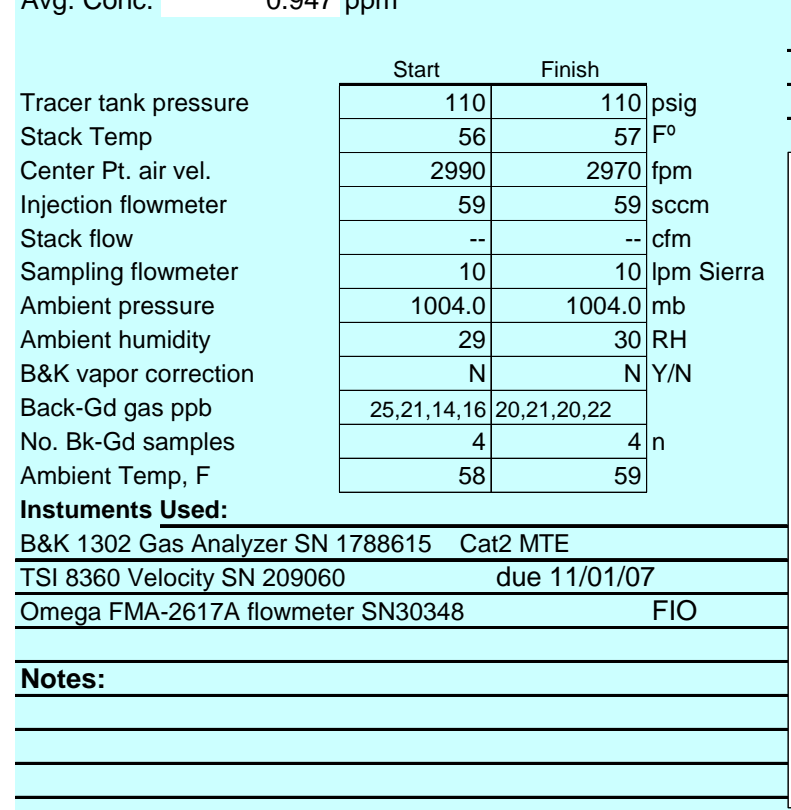

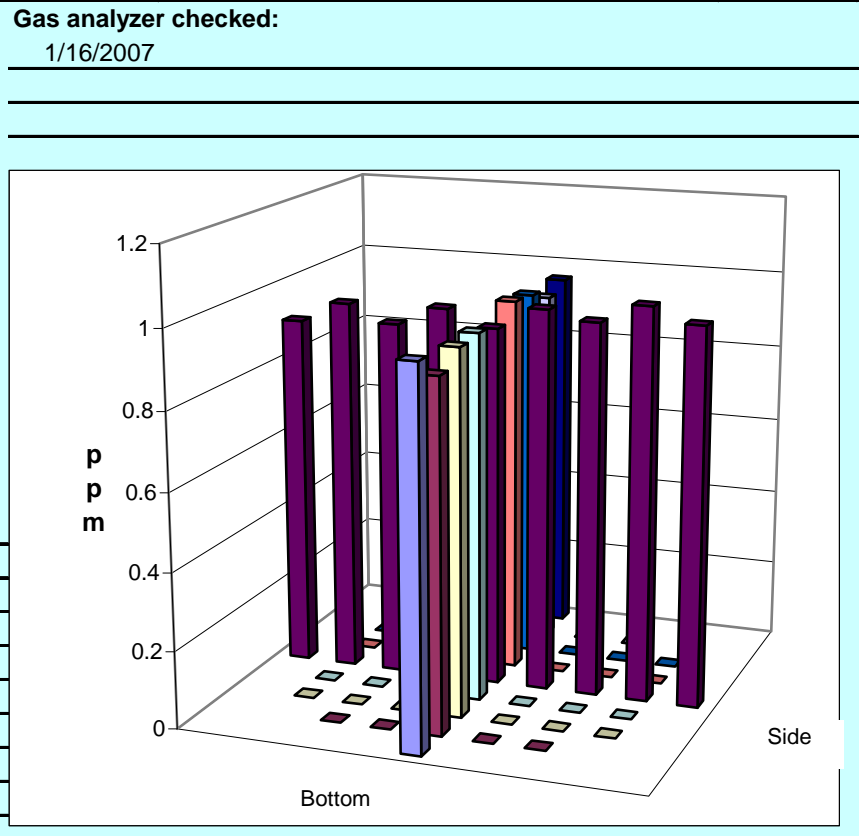

Signature signifies compliance with

Signature verifying data and calculations:

Procedure EMS-JAG-01

Signature/date

Signatures on original data sheet in Test Instruction Package TI-RPP-WTP-465

Reference: CCP-WTPSP-176

gas-dataRev0.xls

(HVC2_gas-dataRev0 (8)).xls

31 July 2006

GT36

4/18/2007 
Rev. 0

31-Jul-06

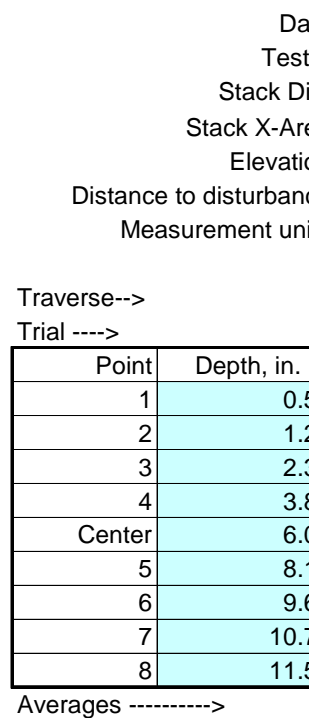

TRACER GAS TRAVERSE DATA FORM

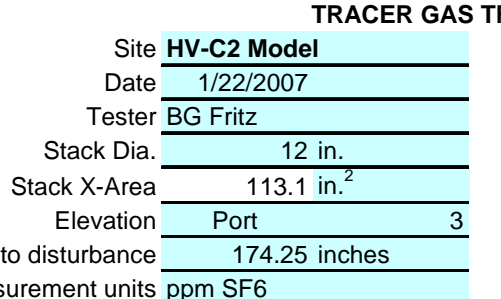

\section{Run No. GT-37}

Fan Configuration $\mathbf{A}$ Fan Setting 35

Stack Temp

Start/End Time 10:00/11:00

Center $2 / 3$ from

Points in Center $2 / 3$

Injection Point A center

$\mathrm{w} / 3 \mathrm{M}$ filterete prefilters

\begin{tabular}{cc}
\hline & $\mathbf{H z}$ \\
$61.5 \mathrm{deg} F$ \\
\end{tabular}

2nd $\quad$ Side

Ist

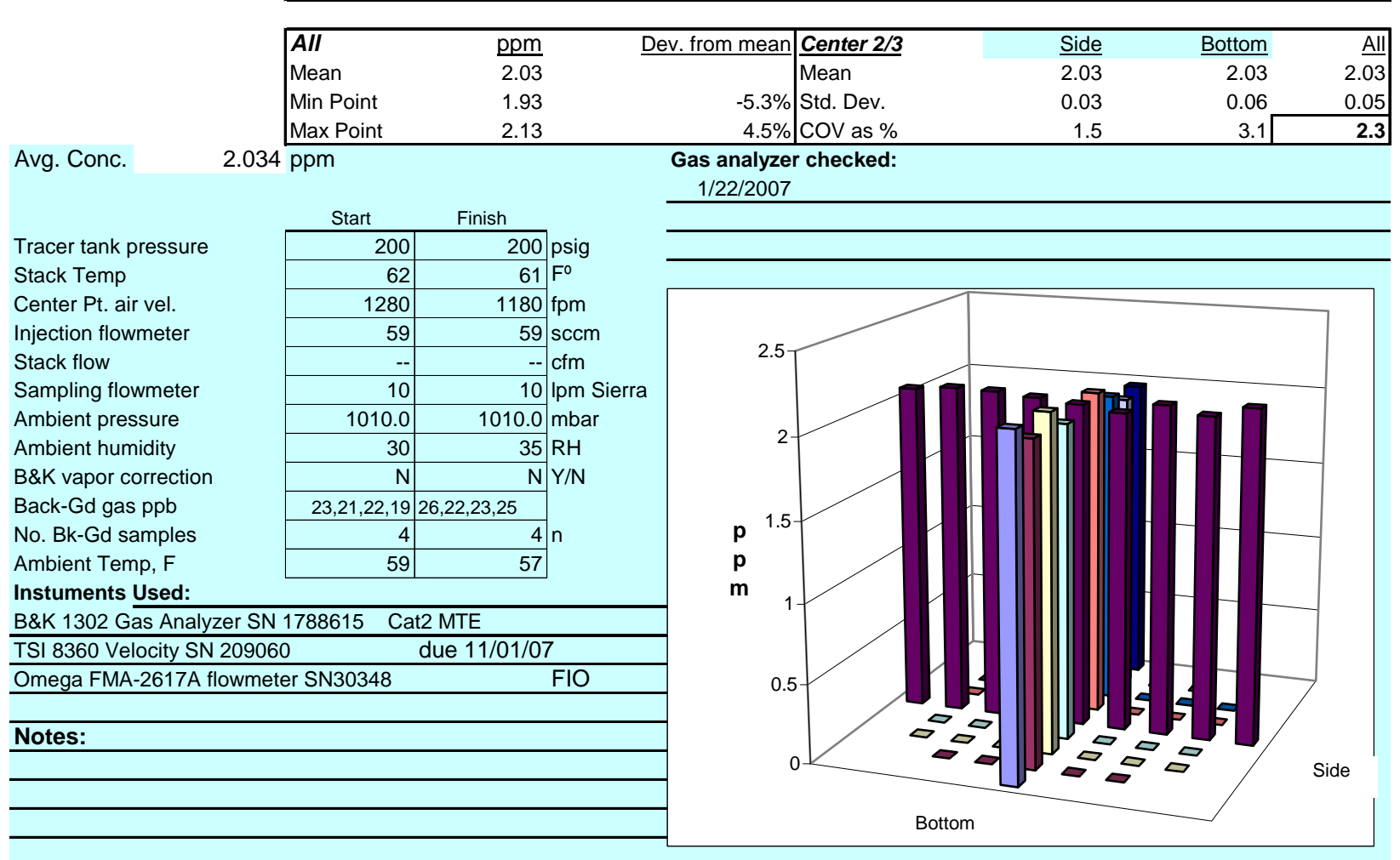

Signature signifies compliance with

Signature verifying data and calculations:

Procedure EMS-JAG-01

Signature/date

Signatures on original data sheet in Test Instruction Package TI-RPP-WTP-465

Reference: CCP-WTPSP-176

gas-dataRev0.xls

(HVC2_gas-dataRev0 (8)).xls

31 July 2006

GT37

$4 / 18 / 2007$ 
Rev. 0

31-Jul-06

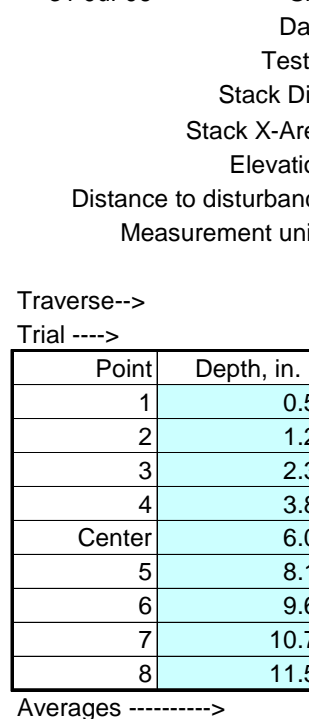

TRACER GAS TRAVERSE DATA FORM

\begin{tabular}{|c|c|c|}
\hline \multicolumn{3}{|c|}{ Site HV-C2 Model } \\
\hline Date & $1 / 22 / 2007$ & \\
\hline Tester & Fritz & \\
\hline Stack Dia. & 12 in. & \\
\hline Stack X-Area & 113.1 in. $^{2}$ & \\
\hline Elevation & Port & 2 \\
\hline
\end{tabular}

113.75 inches

ppm SF6

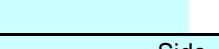

Side

Run No. GT-38

Fan Configuration $\mathbf{A}$

Fan Setting 35

Stack Temp

Start/End Time 12:30/13:1

Center $2 / 3$ from

Points in Center $2 / 3$

Injection Point A center

w/ 3M filterete prefilters

$\mathrm{Hz}$

$64 \operatorname{deg} F$

2nd

Bottom

$\begin{array}{rrrr}3 & \text { Mean } & 1 & 2\end{array}$

$1 \quad 2$

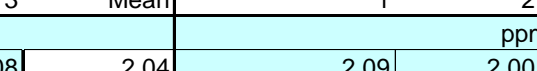

\begin{tabular}{|l|l|l|l|r|r|r|}
\hline 2.06 & 2.08 & 2.04 & 2.09 & 2.00 & 2.03 & 2.04 \\
\hline 2.03 & 2.06 & 2.04 & 2.09 & 2.10 & 1.98 & 2.06 \\
\hline 2.01 & 2.05 & 2.02 & 2.02 & 2.07 & 2.02 & 2.04 \\
\hline 1.98 & 2.08 & 2.02 & 2.02 & 1.98 & 2.07 & 2.02 \\
\hline 2.03 & 1.99 & 2.02 & 2.07 & 2.11 & 2.04 & 2.07 \\
\hline 2.04 & 2.07 & 2.04 & 2.06 & 2.09 & 2.08 & 2.08 \\
\hline 2.02 & 1.98 & 1.98 & 2.10 & 2.07 & 2.05 & 2.07 \\
\hline 2.00 & 2.01 & 2.01 & 2.04 & 1.99 & 2.02 & 2.02 \\
\hline 2.02 & 2.02 & 2.01 & 2.08 & 2.06 & 2.12 & 2.09 \\
\hline
\end{tabular}

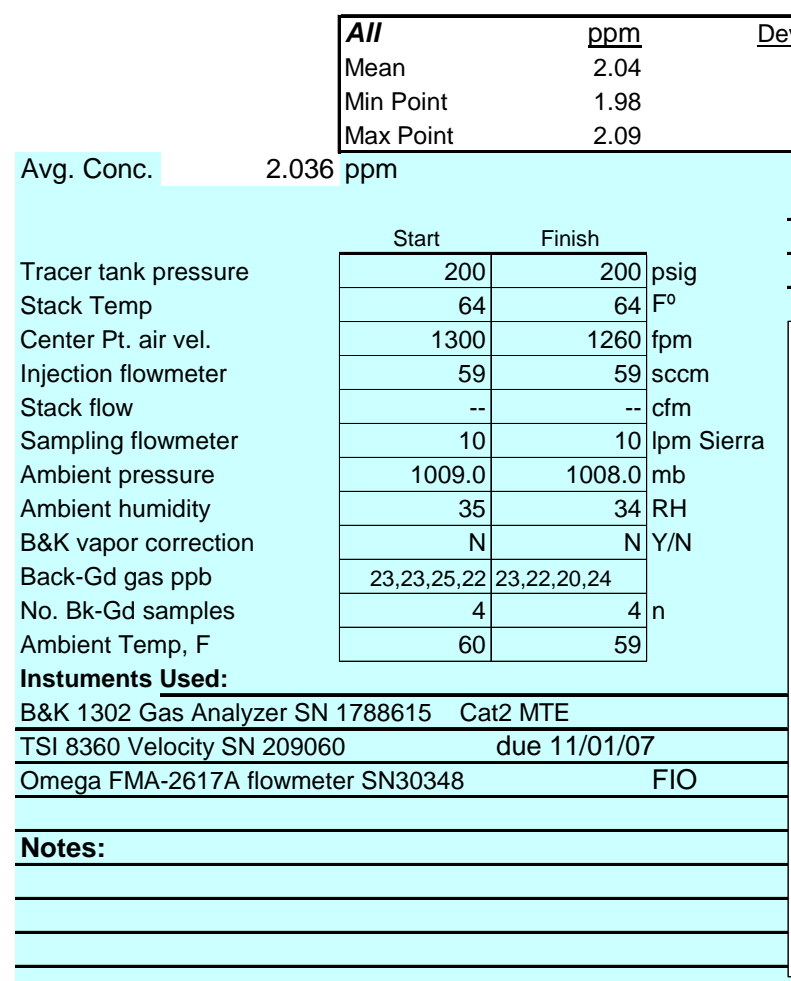

\begin{tabular}{r|l} 
Dev. from mean & Center 2/3 \\
\hline Mean \\
$-2.6 \%$ & Std. Dev. \\
$2.4 \%$ & COV as \% \\
\hline
\end{tabular}

Gas analyzer checked:

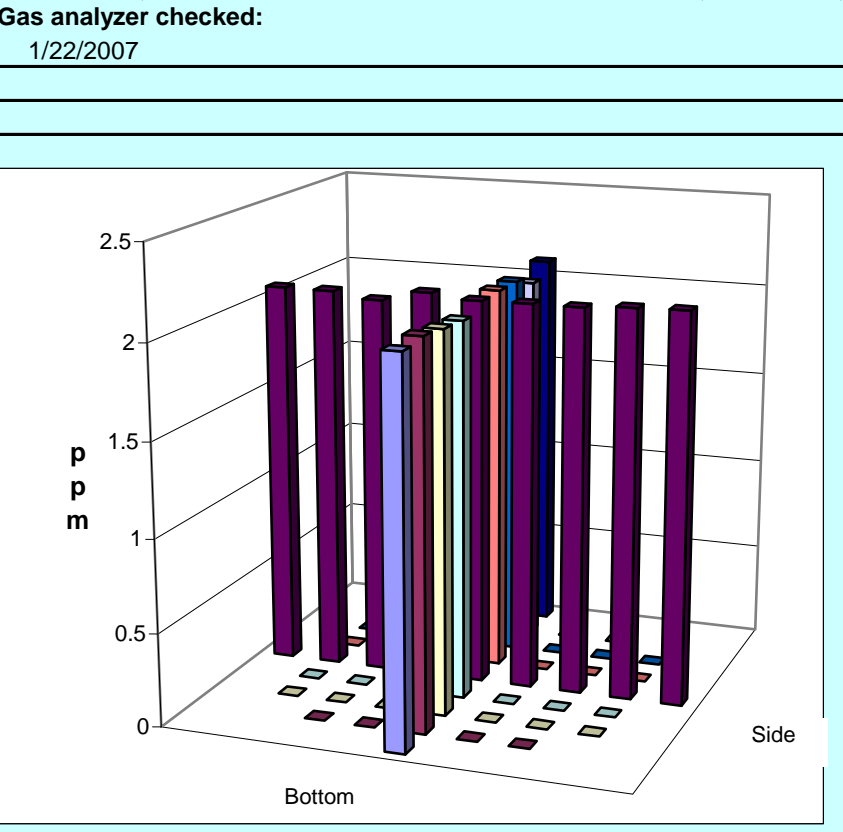

Signature signifies compliance with

Signature verifying data and calculations:

Procedure EMS-JAG-01

Signature/date

Signatures on original data sheet in Test Instruction Package TI-RPP-WTP-465

Reference: CCP-WTPSP-176

(HVC2_gas-dataRev0 (8)).xIs

gas-dataRev0.xls

GT38

31 July 2006

$4 / 18 / 2007$ 
Rev. 0

31-Jul-06

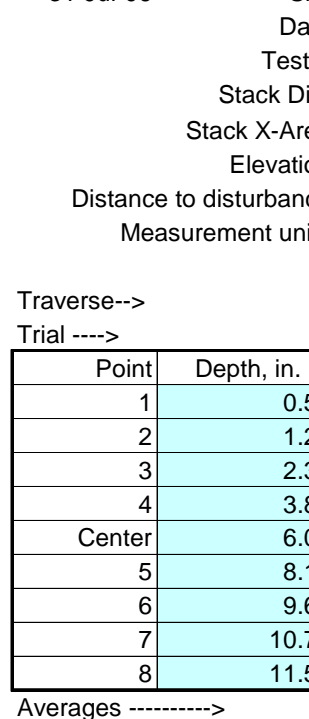

TRACER GAS TRAVERSE DATA FORM

Site HV-C2 Model

Date 1/22/2007

Tester BG Fritz

Stack Dia.

\begin{tabular}{l}
$\frac{12 \text { in. }}{2}$ \\
\cline { 2 - 2 }
\end{tabular}

ppm SF6
Run No. GT-39

Fan Configuration $\mathbf{A}$ Fan Setting 35

Stack Temp

Start/End Time 13:20/14:10

Center $2 / 3$ from Points in Center 2/3 Injection Point $\mathbf{A}$ center w/ 3M filterete prefilters

$\mathrm{Hz}$

$63 \mathrm{deg} F$

10

1.10 to: 10.90

$\frac{1.10}{2}$ to: $\frac{10.90}{7}$ 2nd

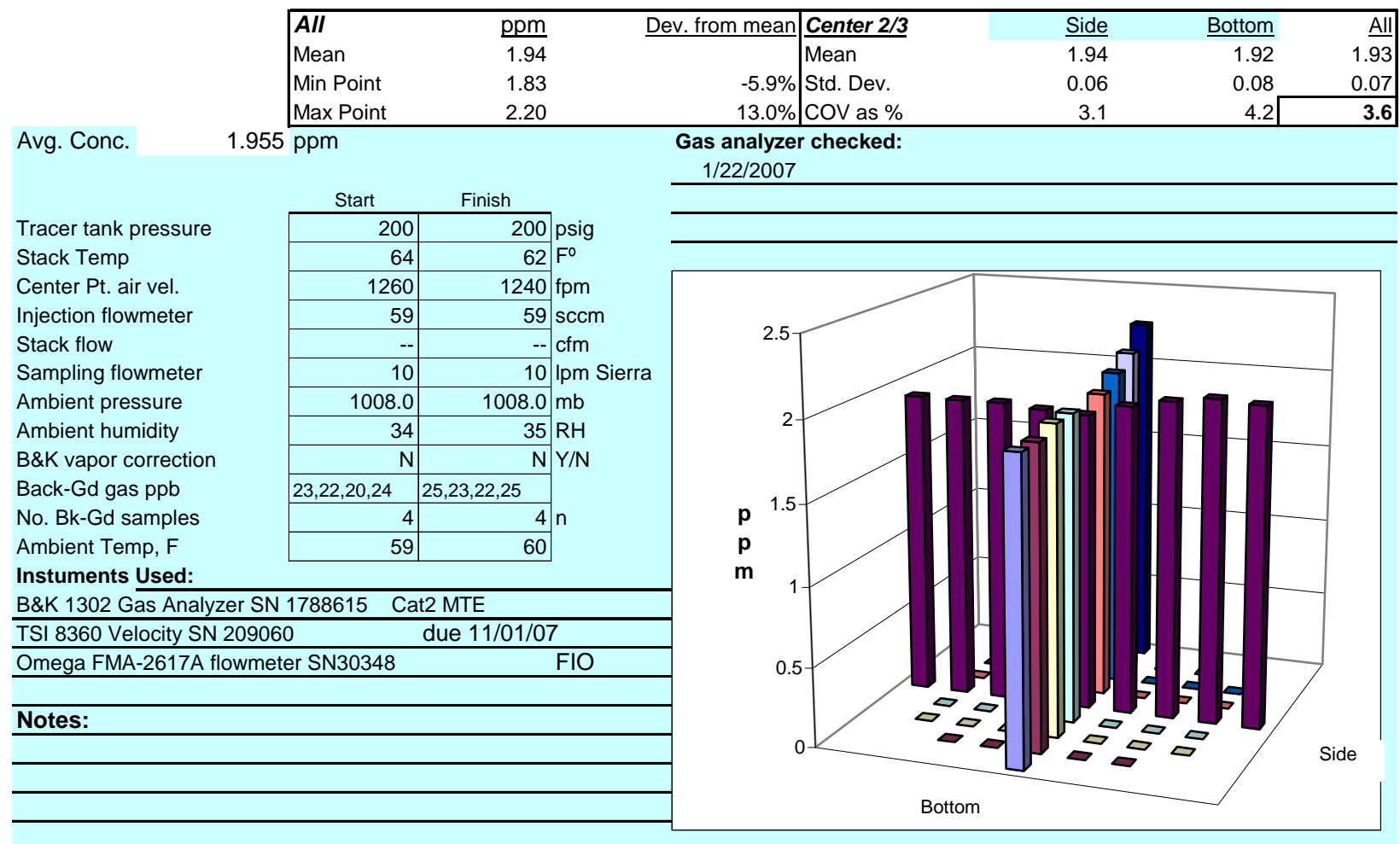

Signature signifies compliance with Signature verifying data and calculations:

Procedure EMS-JAG-01

Signature/date

Signatures on original data sheet in Test Instruction Package TI-RPP-WTP-465

Reference: CCP-WTPSP-176

(HVC2_gas-dataRev0 (8)).xls gas-dataRev0.xls

GT39

31 July 2006

$4 / 18 / 2007$ 
Rev. 0

31-Jul-06

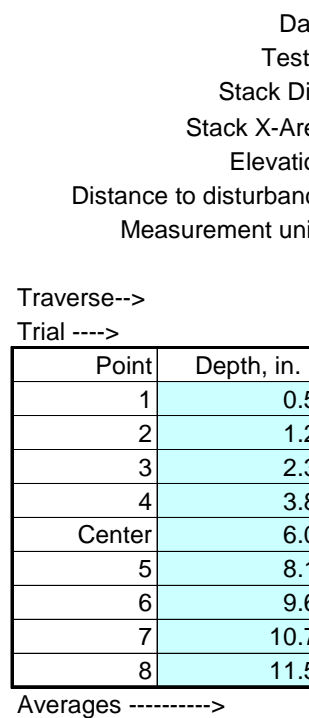

TRACER GAS TRAVERSE DATA FORM

Site HV-C2 Model

Date 1/23/2007

Tester BGF

Stack Dia.

\begin{tabular}{c}
\hline \multicolumn{1}{c}{12 in. } \\
\hline 113.1 in. $^{2}$ \\
\hline Port \\
\hline 53.5 inches
\end{tabular}

ppm SF6

\section{Run No. GT-40}

Fan Configuration $\mathbf{A}$ Fan Setting 35

Stack Temp

Start/End Time

Center $2 / 3$ from

Points in Center $2 / 3$ Injection Point $\mathrm{A}$ Center w/ 3M filterete prefilters

$\mathrm{Hz}$

$61.5 \operatorname{deg} \mathrm{F}$

0

1.10 to: 10.90

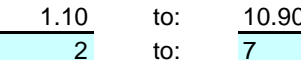

-

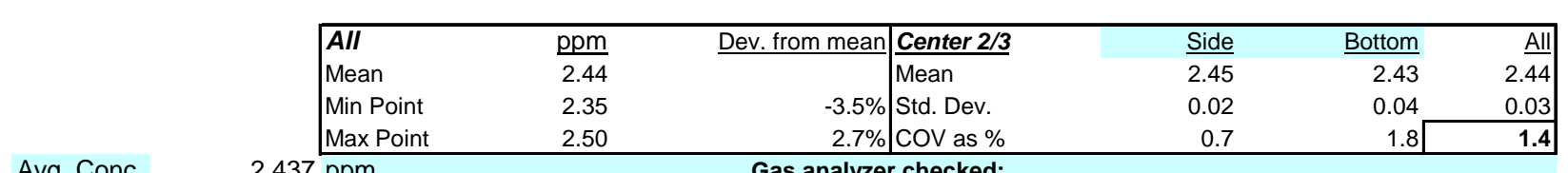

Avg. Conc.

$2.437 \mathrm{ppm}$

Tracer tank pressure

Stack Temp

Center Pt. air vel.

Injection flowmeter

Stack flow

Sampling flowmeter

Ambient pressure

Ambient humidity

$B \& K$ vapor correction

Back-Gd gas ppb

No. Bk-Gd samples

Ambient Temp, $\mathrm{F}$

Instuments Used:

B\&K 1302 Gas Analyzer SN 1788615 Cat2 MTE

TSI 8360 Velocity SN 209060

Omega FMA-2617A flowmeter SN30348 FIO

due $11 / 01 / 07$

\section{Notes:}

\begin{tabular}{|c|c|c|}
\hline Start & Finish & \\
\hline 200 & 200 & psig \\
\hline 61 & 62 & $F^{\circ}$ \\
\hline 980 & 1070 & $\mathrm{fpm}$ \\
\hline 59 & 59 & $\mathrm{sccm}$ \\
\hline-- & -- & $\mathrm{cfm}$ \\
\hline 10 & 10 & Ipm Sierra \\
\hline 1009.0 & 1010.0 & $\mathrm{mb}$ \\
\hline 32 & 39 & $\mathrm{RH}$ \\
\hline $\mathrm{N}$ & $\mathrm{N}$ & $\mathrm{Y} / \mathrm{N}$ \\
\hline $30,25,25,27$ & $22,25,27,25$ & \\
\hline 4 & & $\mathrm{n}$ \\
\hline 53 & 55 & \\
\hline
\end{tabular}

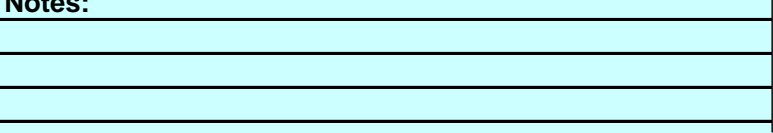

Signature signifies compliance with Procedure EMS-JAG-01

Signature/date

Signatures on original data sheet in Test Instruction Package TI-RPP-WTP-465

Reference: CCP-WTPSP-176

gas-dataRev0.xls

(HVC2_gas-dataRev0 (8)).xls

31 July 2006

Gas analyzer checked:

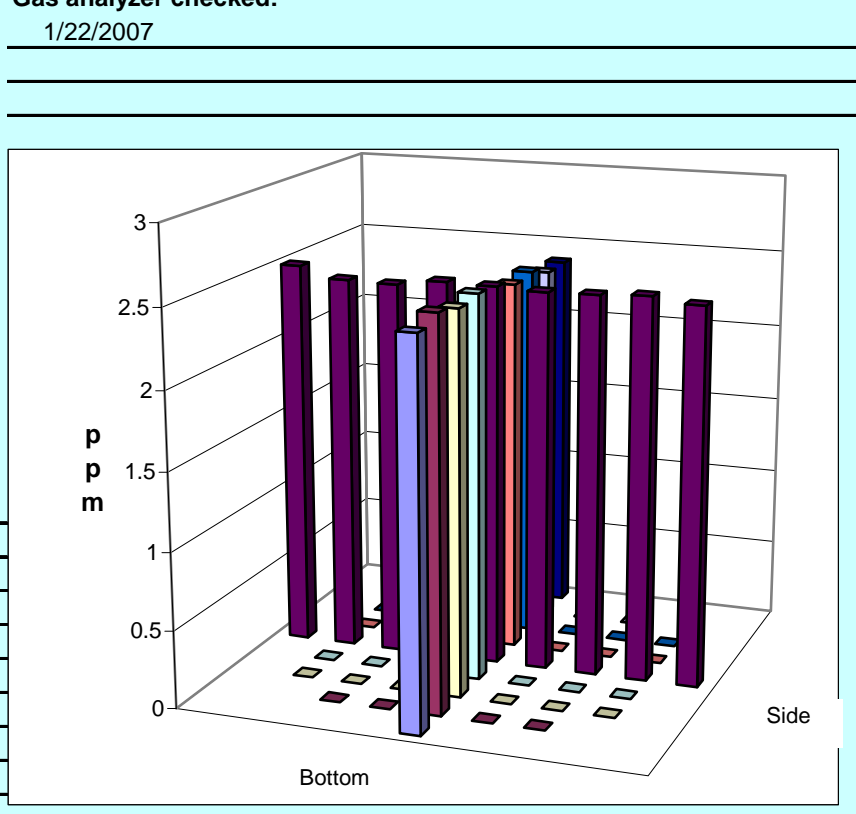

Signature verifying data and calculations: 
Rev. 0

31-Jul-06

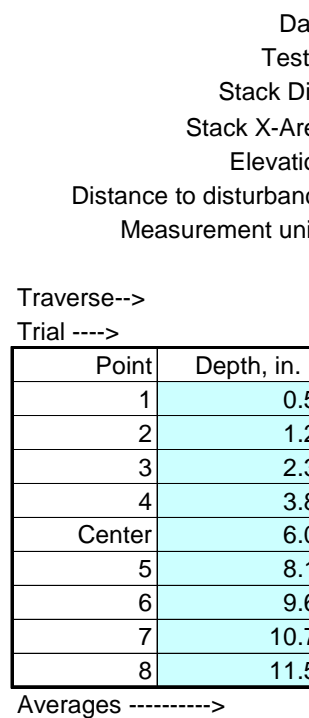

Avg. Conc.

$2.495 \mathrm{ppm}$

Tracer tank pressure

Stack Temp

Center Pt. air vel.

Injection flowmeter

Stack flow

Sampling flowmeter

Ambient pressure

Ambient humidity

B\&K vapor correction

Back-Gd gas ppb

No. Bk-Gd samples

Ambient Temp, $F$

Instuments Used:

B\&K 1302 Gas Analyzer SN 1788615 Cat2 MTE

TSI 8360 Velocity SN 209060

Omega FMA-2617A flowmeter SN30348

due $11 / 01 / 07$

\begin{tabular}{|c|c|c|}
\hline Start & Finish & \\
\hline 200 & 200 & psig \\
\hline 62 & 63 & $F^{\circ}$ \\
\hline 1070 & 1100 & $\mathrm{fpm}$ \\
\hline 59 & 59 & $\mathrm{sccm}$ \\
\hline-- & -- & $\mathrm{cfm}$ \\
\hline 10 & 10 & Ipm Sierra \\
\hline 1010.0 & 1011.0 & $\mathrm{mb}$ \\
\hline 39 & 37 & $\mathrm{RH}$ \\
\hline $\mathrm{N}$ & $\mathrm{N}$ & $\mathrm{Y} / \mathrm{N}$ \\
\hline $22,25,27,25$ & $28,24,27,24$ & \\
\hline 4 & & $n$ \\
\hline 55 & 59 & \\
\hline
\end{tabular}

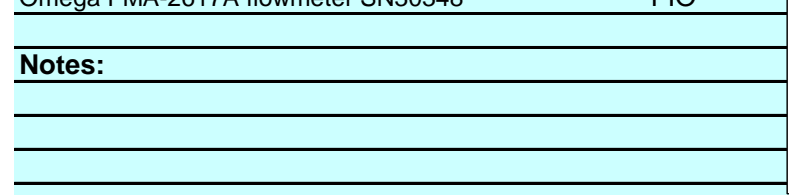

TRACER GAS TRAVERSE DATA FORM

Run No. GT-41

Fan Configuration $\mathbf{A}$ Fan Setting 35

Stack Temp

Start/End Time 1040 -- 112

Center $2 / 3$ from

Points in Center $2 / 3$ Injection Point $\mathbf{A \text { far right }}$ w/ 3M filterete prefilters

$\mathrm{Hz}$

$62.5 \operatorname{deg} \mathrm{F}$

1.10 to: 10.90

$\frac{1.10}{2}$ to: $\frac{10.90}{7}$

2

\begin{tabular}{l|ll} 
& & \\
3 & & Bottom
\end{tabular}

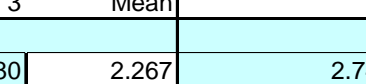

\begin{tabular}{|l|r|r|r|r|r|}
\hline .280 & 2.267 & 2.740 & 2.630 & 2.730 & 2.700 \\
\hline 170 & 2.223 & 2.750 & 2.690 & 2.650 & 2.697 \\
\hline 210 & 2.250 & 2.770 & 2.760 & 2.740 & 2.757 \\
\hline .230 & 2.250 & 2.640 & 2.530 & 2.590 & 2.587 \\
\hline 410 & 2.383 & 2.410 & 2.500 & 2.400 & 2.437 \\
\hline .570 & 2.583 & 2.450 & 2.420 & 2.360 & 2.410 \\
\hline .740 & 2.680 & 2.380 & 2.420 & 2.370 & 2.390 \\
\hline 680 & 2.737 & 2.340 & 2.390 & 2.330 & 2.353 \\
\hline 620 & 2.660 & 2.360 & 2.390 & 2.390 & 2.380 \\
\hline 4 & 2.448 & 2.54 & 2.53 & 2.51 & 2.523 \\
\hline
\end{tabular}


Rev. 0

31-Jul-06

Measurement units ppm SF6

\begin{aligned} Date & $\frac{1 / 23 / 2007}{\text { Tester }} \\$ Stack Dia. & \multicolumn{1}{c}{12 in. } \\ Stack X-Area & 113.1 in. $^{2} \\$ Elevation & Port \\ \cline { 2 - 2 } Distance to disturbance & 53.5 inches \end{aligned}

TRACER GAS TRAVERSE DATA FORM

Run No. GT-42

Fan Configuration $\mathbf{A}$ Fan Setting 35

Stack Temp

Start/End Time 1305 -- 1405

Center $2 / 3$ from

Points in Center $2 / 3$ Injection Point $\mathbf{A}$ far left

w/ 3M filterete prefilters

$\mathrm{Hz}$

$62.5 \operatorname{deg} \mathrm{F}$

1.10

1.10 to: 10.90

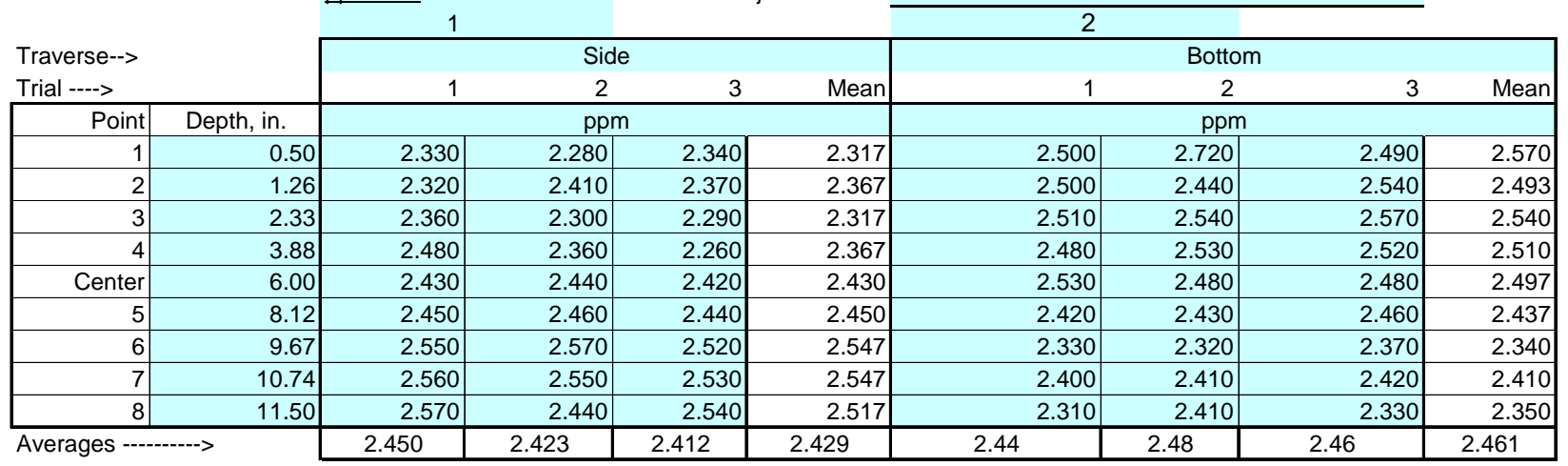

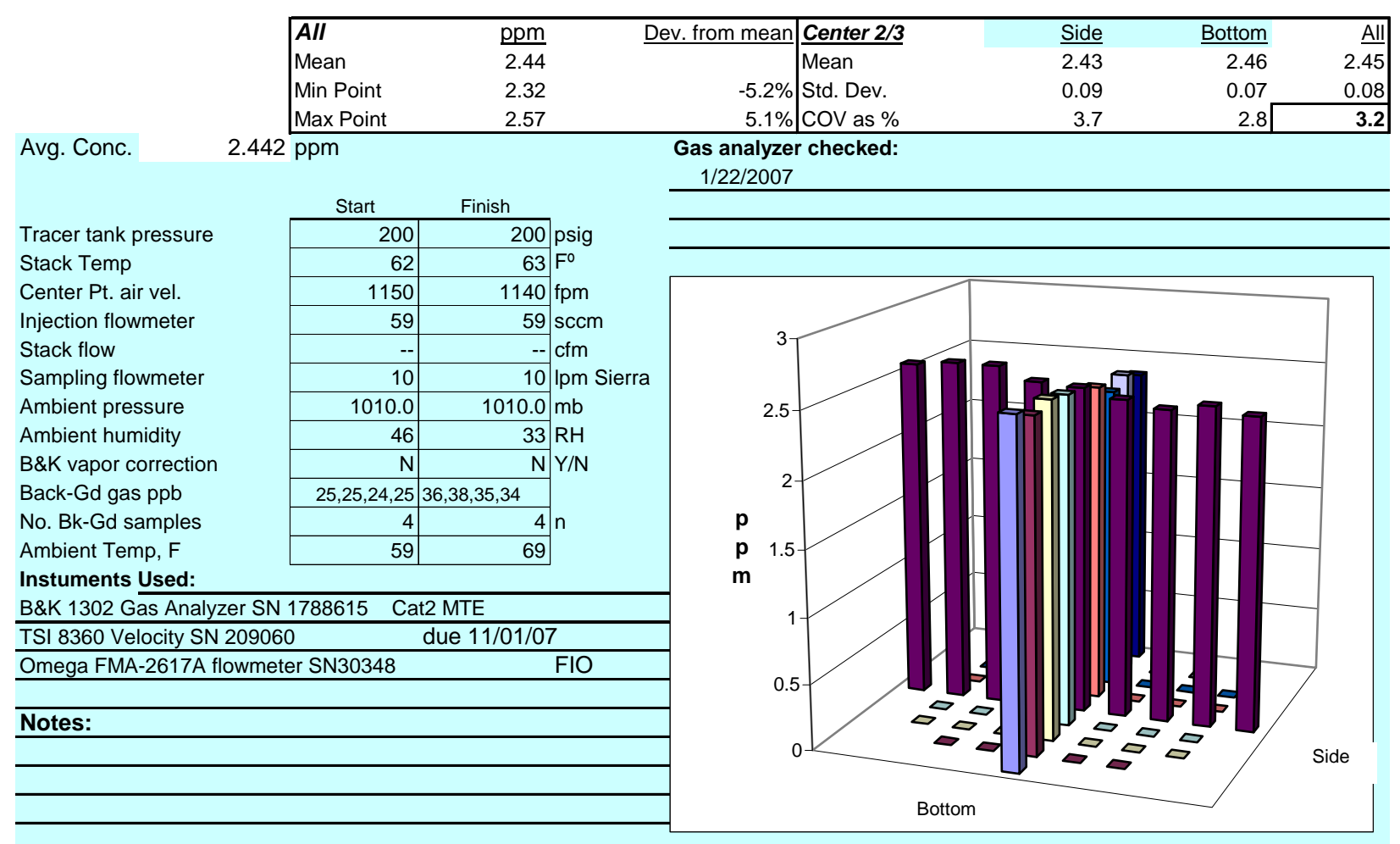

Signature signifies compliance with

Signature verifying data and calculations:

Procedure EMS-JAG-01

Signature/date

Signatures on original data sheet in Test Instruction Package TI-RPP-WTP-465

Reference: CCP-WTPSP-176

(HVC2_gas-dataRev0 (8)).xls

gas-dataRev0.xls

GT42

31 July 2006

$4 / 18 / 2007$ 
Rev. 0

31-Jul-06

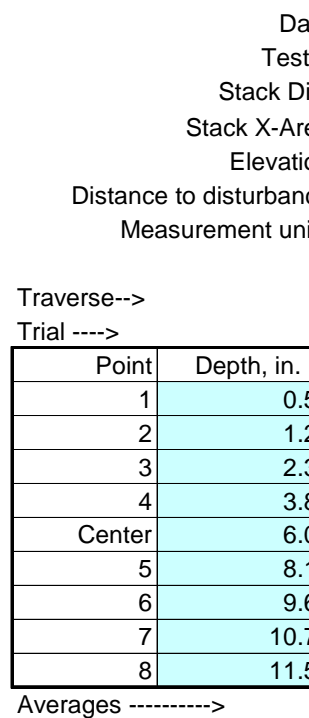

TRACER GAS TRAVERSE DATA FORM

Site HV-C2 Model

Date 1/23/2007

Tester MSP

tack Dia.

\begin{tabular}{c}
\hline \multicolumn{1}{c}{12 in. } \\
\hline 113.1 in. $^{2}$ \\
\hline Port \\
\hline 53.5 inches
\end{tabular}

53.5 inches

ppm SF6
Run No. GT-43

Fan Configuration $\mathbf{A}$ Fan Setting 35

Stack Temp

Start/End Time 1410 -- 1505

Center $2 / 3$ from

Points in Center $2 / 3$ Injection Point $\mathrm{A}$ near left w/ 3M filterete prefilters

$\mathrm{Hz}$

$62 \operatorname{deg} F$

1505

1.10 to: 10.90

$\frac{1.10}{2}$ to: $\frac{10.90}{7}$

1

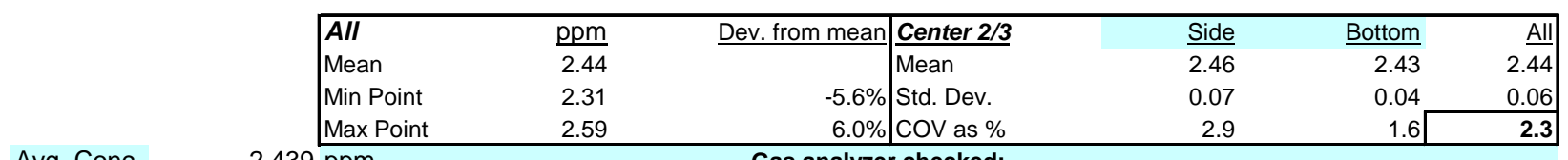

Avg. Conc.

$2.439 \mathrm{ppm}$

Stack Temp

Center Pt. air vel.

Injection flowmeter

Stack flow

Sampling flowmeter

Ambient pressure

Ambient humidity

B\&K vapor correction

Back-Gd gas ppb

No. Bk-Gd samples

Ambient Temp, $\mathrm{F}$

Instuments Used:

B\&K 1302 Gas Analyzer SN 1788615 Cat2 MTE

TSI 8360 Velocity SN 209060

Omega FMA-2617A flowmeter SN30348 FIO

due $11 / 01 / 07$

\section{Notes:}

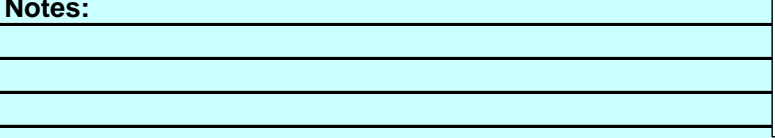

Tracer tank pressure

Gas analyzer checked:

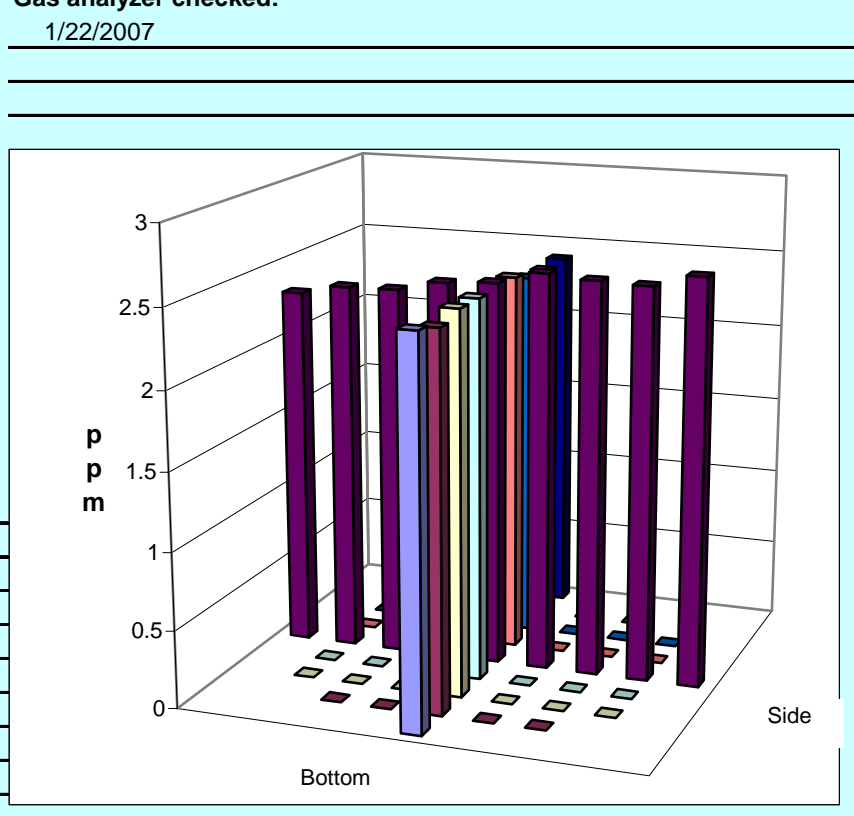

Signature signifies compliance with

Signature verifying data and calculations:

Signature/date

Signatures on original data sheet in Test Instruction Package TI-RPP-WTP-465

Reference: CCP-WTPSP-176

(HVC2_gas-dataRev0 (8)).xls

gas-dataRev0.xIs

GT43

31 July 2006

4/18/2007 
Rev. 0

31-Jul-06

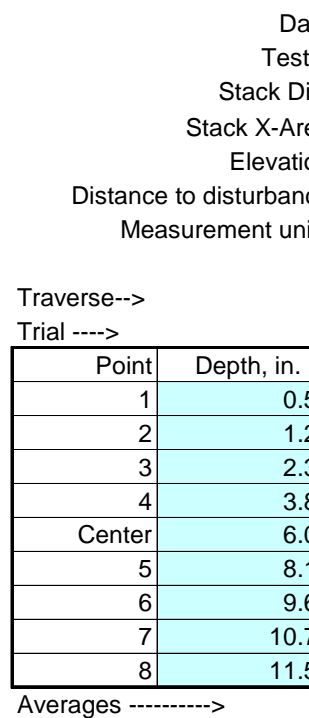

TRACER GAS TRAVERSE DATA FORM

Site HV-C2 Model

Date 1/23/2007

Tester MSP

tack Dia.

\begin{tabular}{c}
\hline$\frac{12 \text { in. }}{113.1 \text { in. }^{2}}$ \\
\hline Port \\
\hline 53.5 inches
\end{tabular}

ppm SF6

1

Run No. GT-44

Fan Configuration $\mathbf{A}$ Fan Setting 35

Stack Temp

Start/End Time 1510 -- 155

Center $2 / 3$ from

Points in Center $2 / 3$ Injection Point $\mathbf{A}$ near right w/ 3M filterete prefilters

$\mathrm{Hz}$

$62 \operatorname{deg} F$

55

1.10 to: 10.90

\begin{tabular}{|r|r|r|r|r|r|r|r|r|r|}
\hline & 2.210 & 2.240 & 2.180 & 2.210 & 2.420 & 2.390 & 2.380 & 2.397 \\
\hline
\end{tabular}

Avg. Conc.

$$
2.419 \mathrm{ppm}
$$

Tracer tank pressure

Stack Temp

Center Pt. air vel.

Injection flowmeter

Stack flow

Sampling flowmeter

Ambient pressure

Ambient humidity

$B \& K$ vapor correction

Back-Gd gas ppb

No. Bk-Gd samples

Ambient Temp, $\mathrm{F}$

Instuments Used:

B\&K 1302 Gas Analyzer SN 1788615 Cat2 MTE

TSI 8360 Velocity SN 209060

Omega FMA-2617A flowmeter SN30348

\begin{tabular}{|c|c|c|}
\hline Start & Finish & \\
\hline 200 & 200 & psig \\
\hline 62 & 62 & $\mathrm{~F}^{\mathrm{O}}$ \\
\hline 1140 & 1150 & $\mathrm{fpm}$ \\
\hline 59 & 59 & $\mathrm{sccm}$ \\
\hline-- & -- & $\mathrm{cfm}$ \\
\hline 10 & 10 & Ipm Sierra \\
\hline 1010.0 & 1010.0 & $\mathrm{mb}$ \\
\hline 34 & 33 & $\mathrm{RH}$ \\
\hline $\mathrm{N}$ & $\mathrm{N}$ & $\mathrm{Y} / \mathrm{N}$ \\
\hline $40,44,41,42$ & $41,39,40,38$ & \\
\hline 4 & 4 & $n$ \\
\hline 66 & 66 & \\
\hline
\end{tabular}

due $11 / 01 / 07$

Notes:

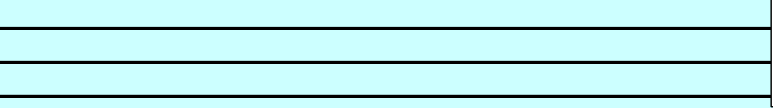

Signature signifies compliance with Procedure EMS-JAG-01

Signature/date

Signatures on original data sheet in Test Instruction Package TI-RPP-WTP-465
Reference: CCP-WTPSP-176

gas-dataRev0.xls

31 July 2006

\begin{tabular}{|c|c|c|c|c|}
\hline Dev. from mean & Center $2 / 3$ & Side & Bottom & All \\
\hline & Mean & 2.45 & 2.41 & 2.43 \\
\hline$-9.0 \%$ & Std. Dev. & 0.21 & 0.07 & 0.15 \\
\hline $11.1 \%$ & cov as $\%$ & 8.6 & 3.0[ & 6.3 \\
\hline
\end{tabular}

FIO

Gas analyzer checked:

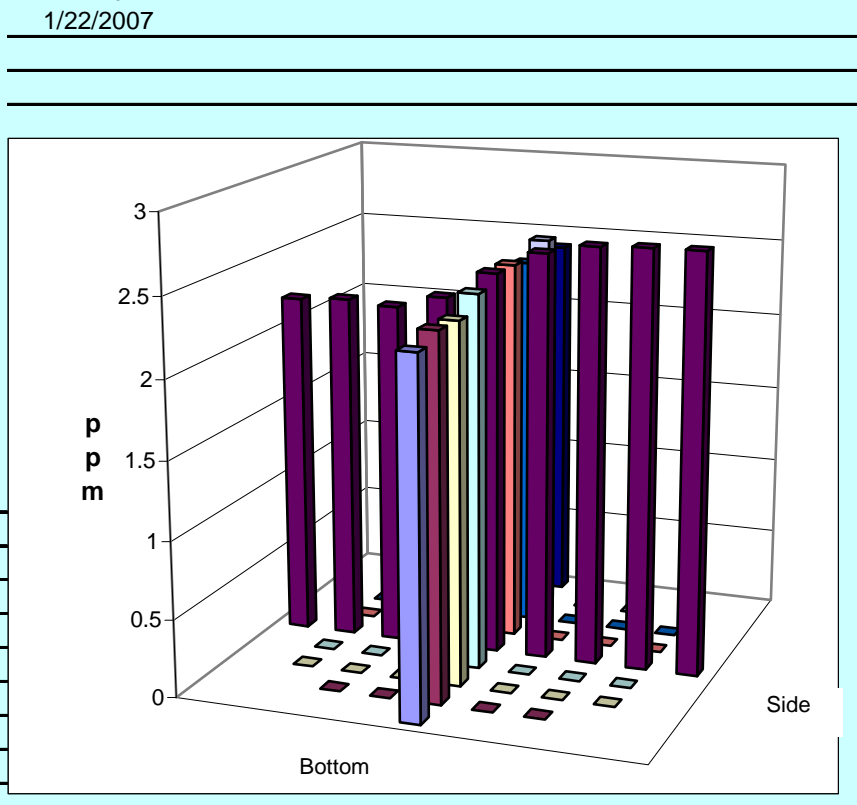

Signature verifying data and calculations: 
Rev. 0

31-Jul-06

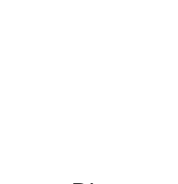

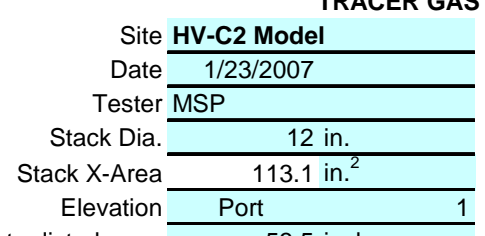

Distance to disturbance Measurement units ppm SF6

TRACER GAS TRAVERSE DATA FORM

\begin{tabular}{l} 
Measurement units ppm SF6 \\
\cline { 4 - 11 }
\end{tabular}

Avg. Conc. 2.113 Mpm Point

\begin{tabular}{|lr}
\hline All & ppm \\
Mean & 2.12 \\
Min Point & 2.02 \\
Max Point & 2.26 \\
\hline
\end{tabular}

Tracer tank pressure

Stack Temp

Center Pt. air vel.

Injection flowmeter

Stack flow

Sampling flowmeter

Ambient pressure

Ambient humidity

B\&K vapor correction

Back-Gd gas ppb

No. Bk-Gd samples

Ambient Temp, $F$

Instuments Used:

B\&K 1302 Gas Analyzer SN 1788615 Cat2 MTE

TSI 8360 Velocity SN 209060

Omega FMA-2617A flowmeter SN30348

due $11 / 01 / 07$

FIO

\section{Notes:}

\begin{tabular}{|c|c|c|}
\hline Start & Finish & \multirow[b]{2}{*}{ psig } \\
\hline 200 & 200 & \\
\hline 63 & 63 & \\
\hline 1350 & 1360 & \multirow{3}{*}{$\begin{array}{l}\mathrm{fpm} \\
\mathrm{sccm} \\
\mathrm{cfm}\end{array}$} \\
\hline 59 & 59 & \\
\hline-- & -- & \\
\hline 10 & 10 & \multirow{2}{*}{$\begin{array}{l}\text { Ipm Sierra } \\
\mathrm{mb}\end{array}$} \\
\hline 1010.0 & 1010.0 & \\
\hline 33 & 34 & \multirow{3}{*}{$\begin{array}{l}\mathrm{RH} \\
\mathrm{Y} / \mathrm{N}\end{array}$} \\
\hline $\mathrm{N}$ & $\mathrm{N}$ & \\
\hline $26,26,25,22$ & $36,38,34,31$ & \\
\hline 4 & 4 & \multirow{2}{*}{$n$} \\
\hline 66 & 63 & \\
\hline
\end{tabular}

Run No. GT-45

Fan Configuration $\mathbf{B}$ Fan Setting 35

Stack Temp

Start/End Time

Center $2 / 3$ from Injection Point $\mathbf{B}$ cente w/ 3M filterete prefilters

$\mathrm{Hz}$

$63 \mathrm{deg} F$

655

10 to: 10.90 
Rev. 0

31-Jul-06

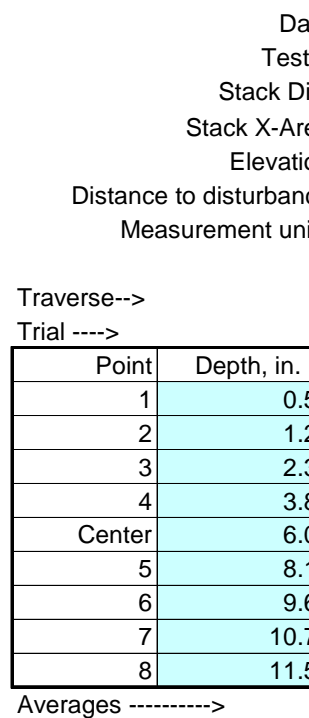

TRACER GAS TRAVERSE DATA FORM

Site HV-C2 Model

Date 1/24/2007

Tester BG Frtiz

Stack Dia.

$\frac{12 \text { in. }}{113.1 \text { in. }^{2}}$

142.25 inches
Run No. GT-46

Fan Configuration B Fan Setting 35

Stack Temp

Start/End Time 9:15/10:00

Center $2 / 3$ from

Points in Center 2/3 Injection Point B Center w/ 3M filterete prefilters

$\mathrm{Hz}$

$60.5 \operatorname{deg} \mathrm{F}$

1.10 to: 10.90

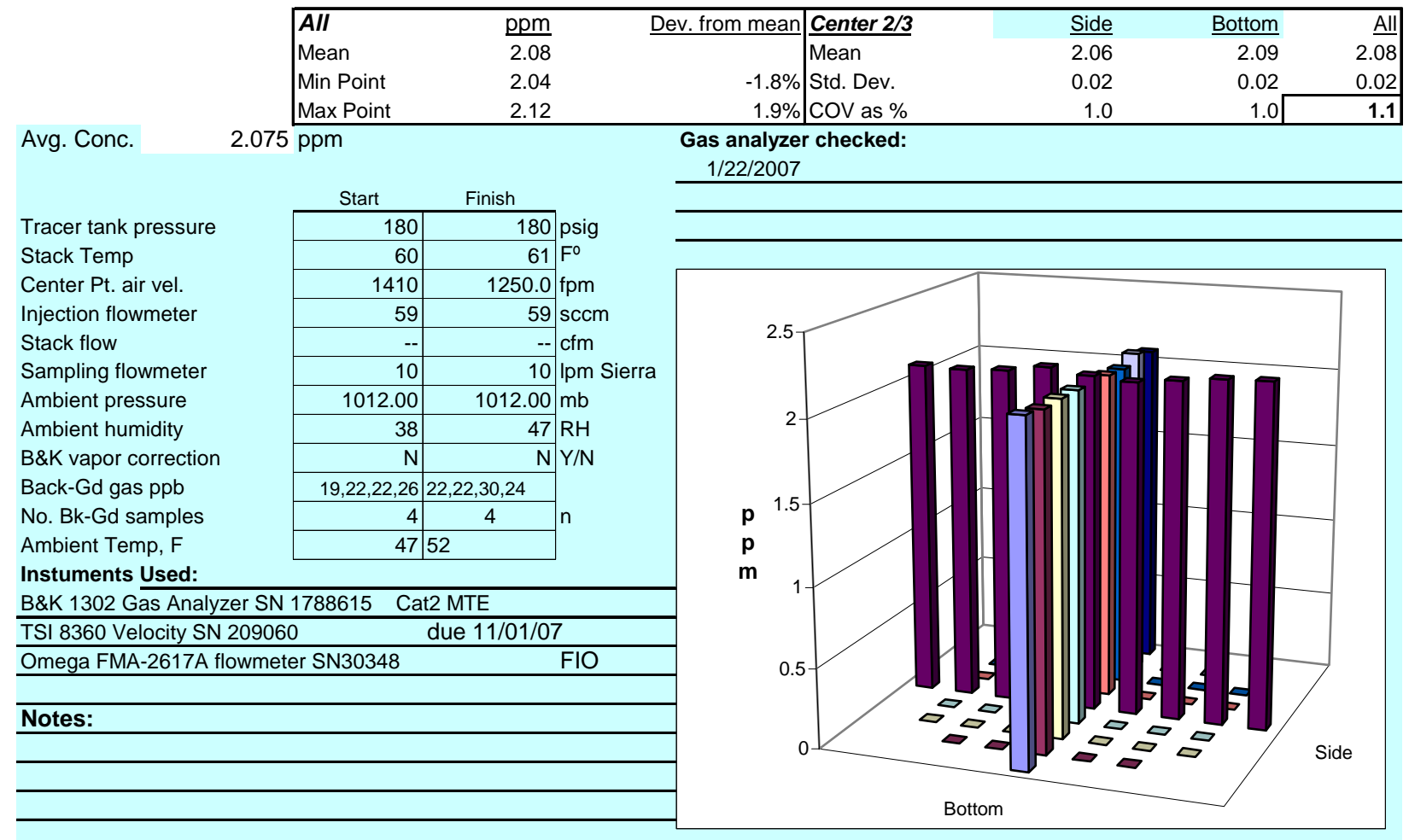

Signature signifies compliance with

Signature verifying data and calculations:

Procedure EMS-JAG-01

Signature/date

Signatures on original data sheet in Test Instruction Package TI-RPP-WTP-465

Reference: CCP-WTPSP-176

(HVC2_gas-dataRev0 (8)).xls

gas-dataRev0.xls

GT46

31 July 2006

$4 / 18 / 2007$ 
Rev. 0

31-Jul-06

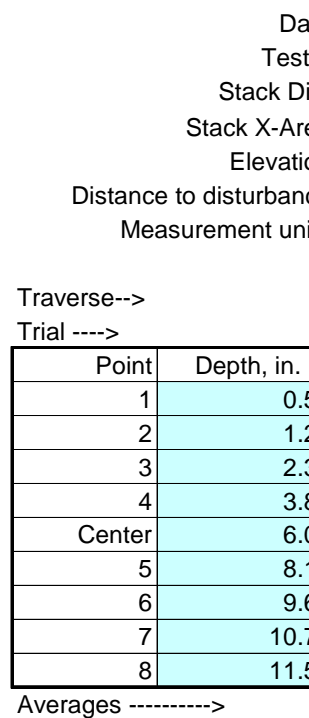

TRACER GAS TRAVERSE DATA FORM

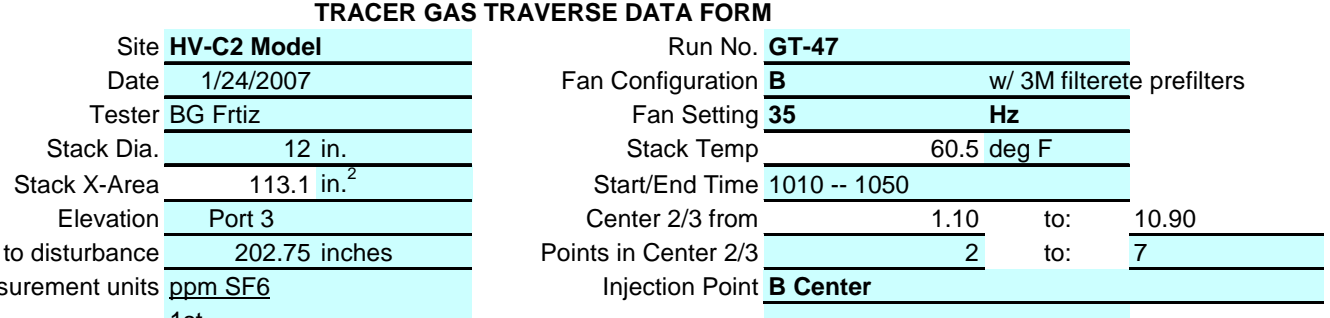

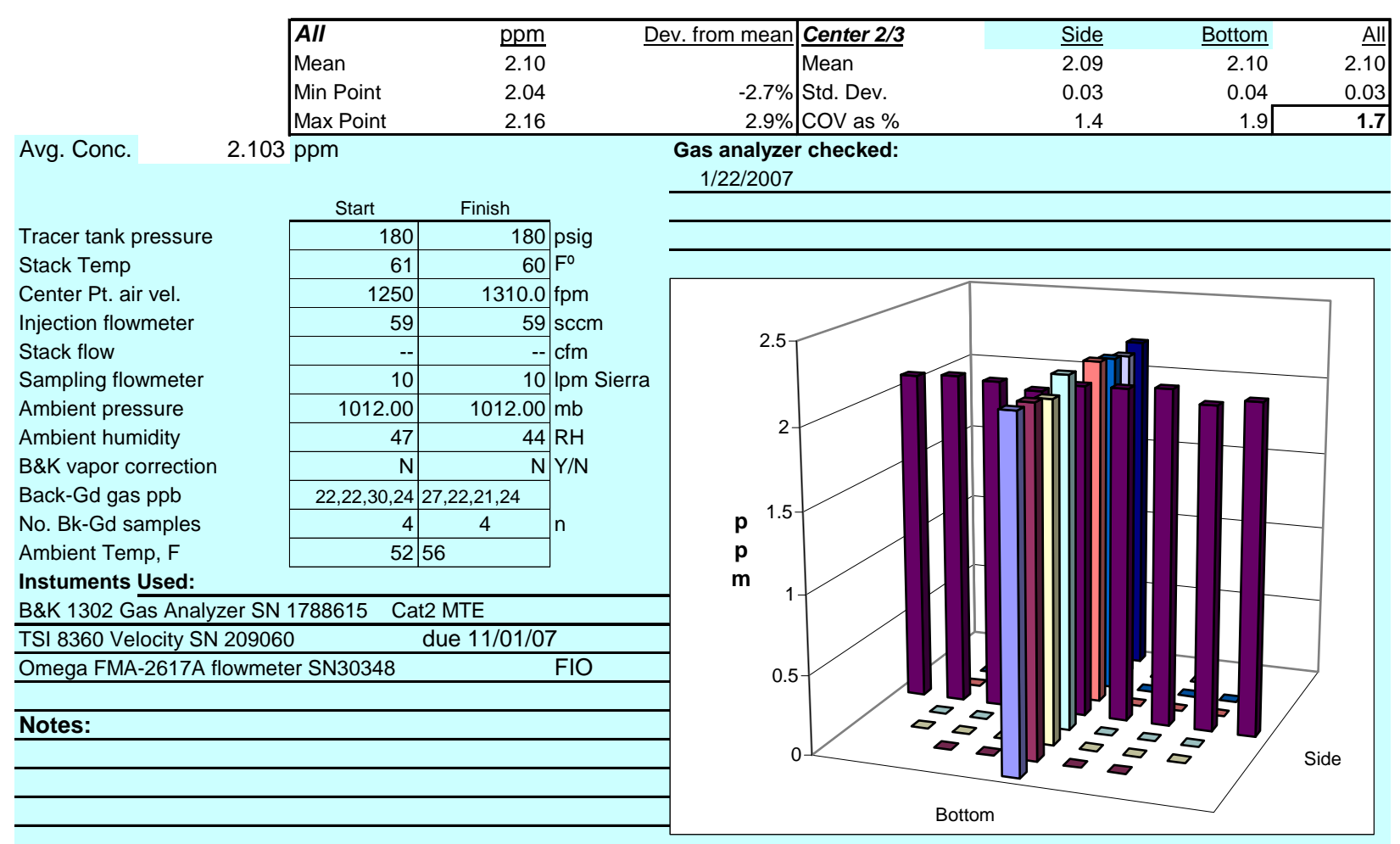

Signature signifies compliance with Signature verifying data and calculations:

Procedure EMS-JAG-01

Signature/date

Signatures on original data sheet in Test Instruction Package TI-RPP-WTP-465

Reference: CCP-WTPSP-176

(HVC2_gas-dataRev0 (8)).xls

gas-dataRev0.xls

GT47

31 July 2006

$4 / 18 / 2007$ 
Rev. 0

31-Jul-06

$\begin{aligned} \text { Site } & \text { HV-C2 Model } \\ \text { Date } & \text { 1/24/2007 } \\ \text { Tester } & \text { JG Droppo } \\ \text { Stack Dia. } & 12 \text { in. } \\ \text { Stack X-Area } & 113.1 \text { in. }^{2} \\ \text { Elevation } & \text { Port 3 }\end{aligned}$

Distance to disturbance Measurement units ppm SF6
TRACER GAS TRAVERSE DATA FORM

Run No. GT-48

Fan Configuration $\mathbf{A \& B}$

Fan Setting 40

Stack Temp

Start/End Time 11:30/13:00

Center $2 / 3$ from

Points in Center $2 / 3$ Injection Point B Center w/ 3M filterete prefilters

$\mathrm{Hz}$

$61 \mathrm{deg} F$

0

1.10 to: 10.90

2 to: $\frac{1}{7}$

$1 \mathrm{st}$

\begin{tabular}{|c|c|c|c|c|c|c|c|c|c|}
\hline \multirow{3}{*}{$\begin{array}{l}\text { Traverse--> } \\
\text { Trial ----> }\end{array}$} & & \multicolumn{8}{|c|}{$1 \mathrm{st}$} \\
\hline & & \multicolumn{4}{|c|}{ Side } & \multicolumn{4}{|c|}{ Bottom } \\
\hline & & 1 & 2 & 3 & Mean & 1 & 2 & 3 & Mean \\
\hline Point & Depth, in. & \multicolumn{4}{|c|}{ ppm } & \multicolumn{4}{|c|}{$\mathrm{ppm}$} \\
\hline 1 & 0.50 & 1.04 & 1.06 & 1.08 & 1.06 & 1.06 & 1.07 & 1.07 & 1.067 \\
\hline 2 & 1.26 & 1.04 & 1.05 & 1.05 & 1.05 & 1.06 & 1.09 & 1.08 & 1.077 \\
\hline 3 & 2.33 & 1.04 & 1.02 & 1.05 & 1.04 & 1.05 & 1.05 & 1.06 & 1.053 \\
\hline 4 & 3.88 & 1.07 & 1.06 & 1.08 & 1.07 & 1.06 & 1.05 & 1.05 & 1.053 \\
\hline Center & 6.00 & 1.05 & 1.05 & 1.03 & 1.04 & 1.06 & 1.04 & 1.05 & 1.050 \\
\hline 5 & 8.12 & 1.05 & 1.06 & 1.04 & 1.05 & 1.03 & 1.02 & 1.02 & 1.023 \\
\hline 6 & 9.67 & 1.04 & 1.07 & 1.07 & 1.06 & 1.03 & 1.03 & 1.04 & 1.033 \\
\hline 7 & 10.74 & 1.04 & 1.04 & 1.05 & 1.04 & 1.05 & 1.06 & 1.05 & 1.053 \\
\hline 8 & 11.50 & 1.05 & 1.06 & 1.04 & 1.05 & 1.02 & 1.02 & 1.03 & 1.023 \\
\hline Averages --- & $-->$ & 1.047 & 1.052 & 1.054 & 1.051 & 1.047 & 1.048 & 1.050 & 1.048 \\
\hline
\end{tabular}

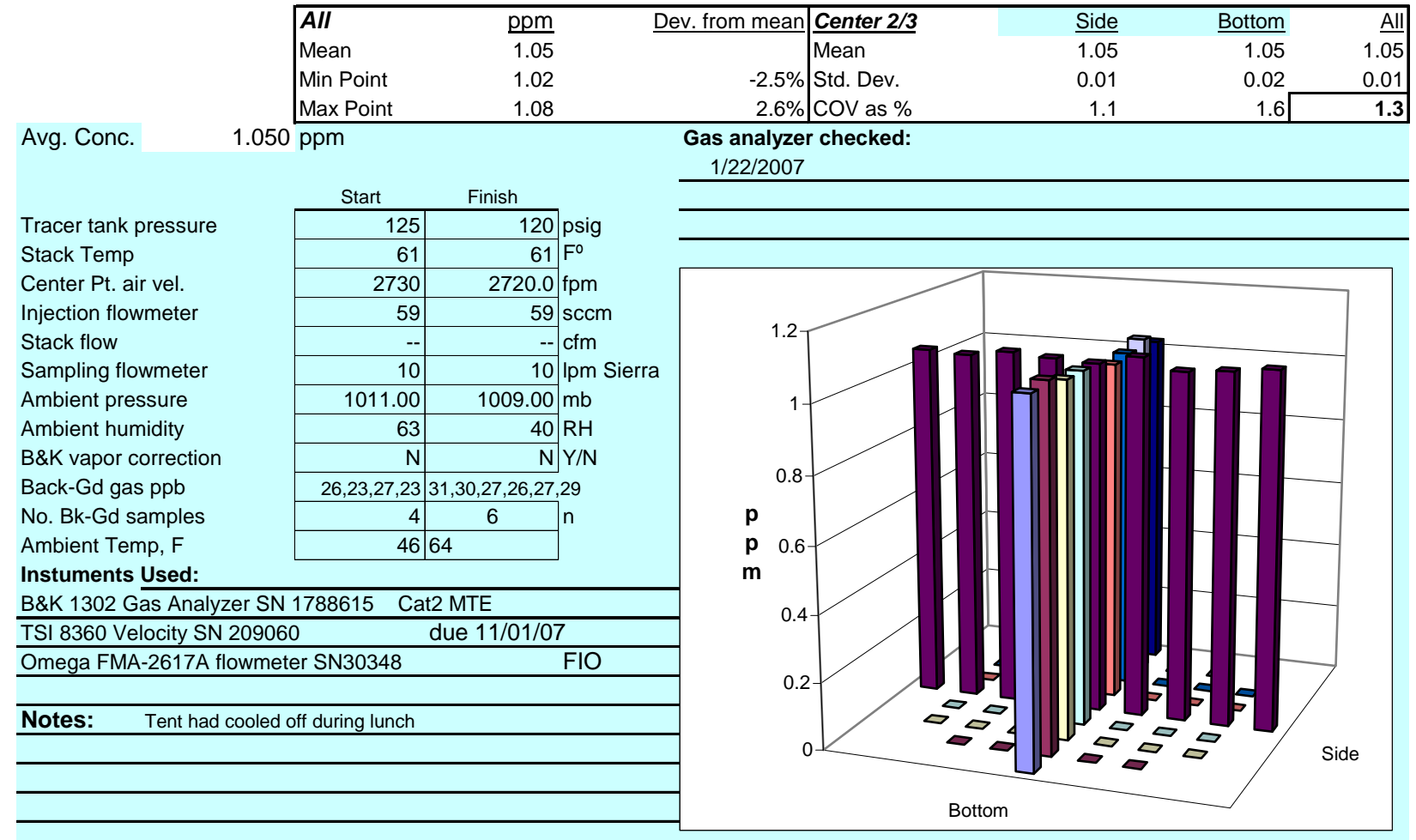

Signature signifies compliance with

Signature verifying data and calculations:

Procedure EMS-JAG-01

Signature/date

Signatures on original data sheet in Test Instruction Package TI-RPP-WTP-465

Reference: CCP-WTPSP-176

(HVC2_gas-dataRev0 (8)).xls

gas-dataRev0.xls

GT48

31 July 2006

$4 / 18 / 2007$ 
Rev. 0

31-Jul-06

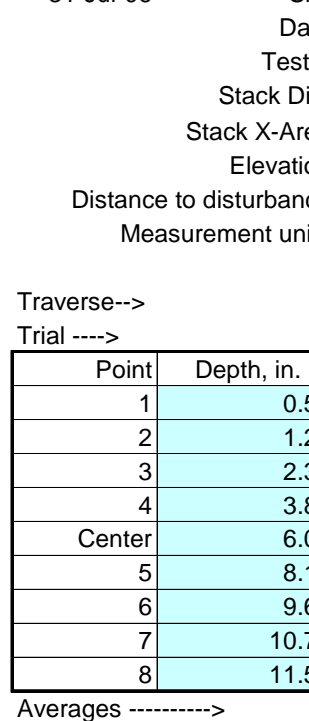

TRACER GAS TRAVERSE DATA FORM

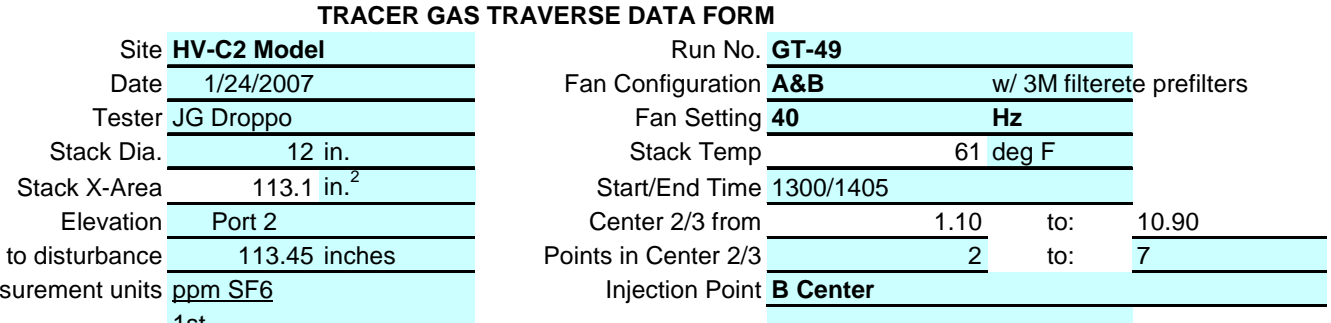

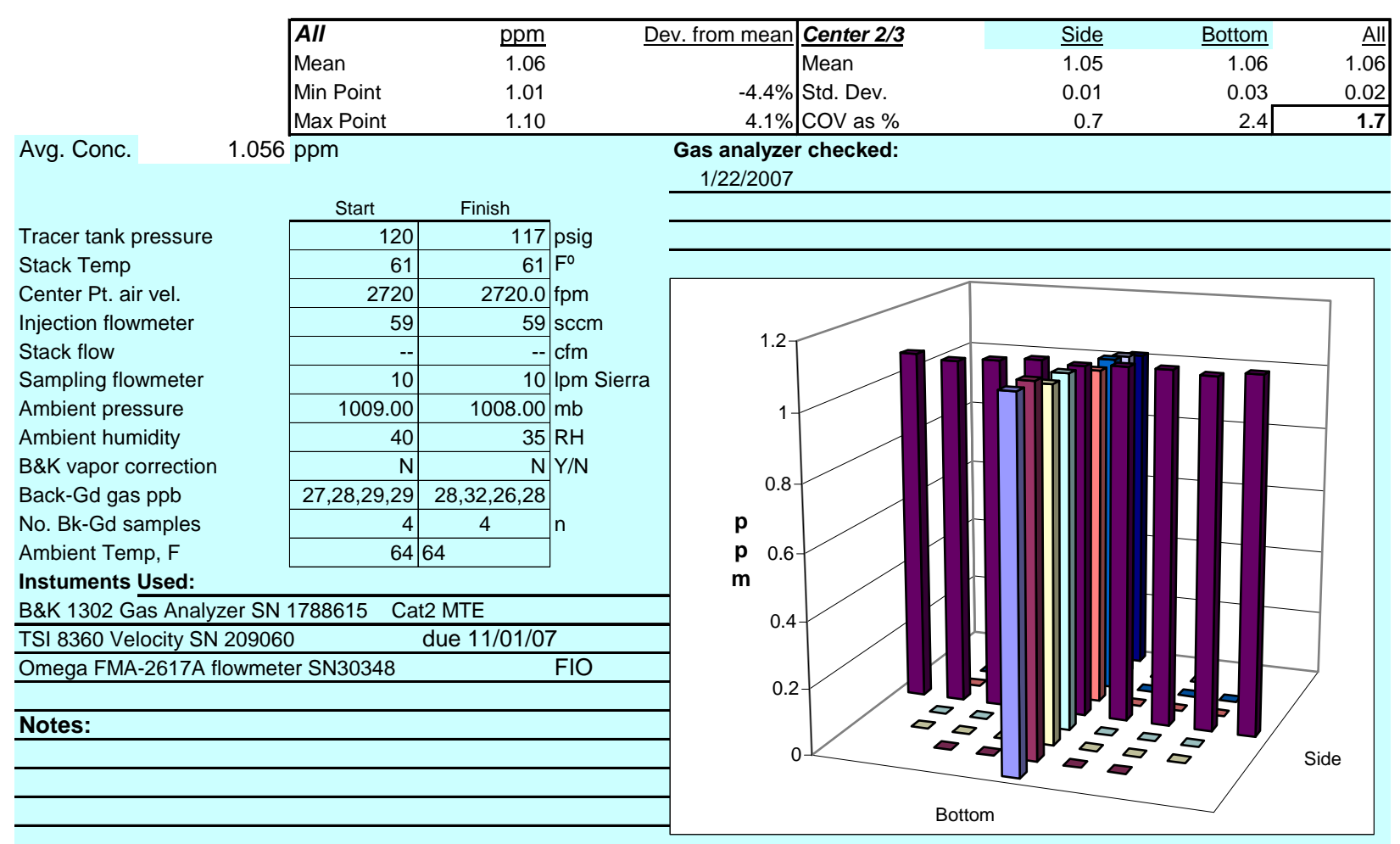

Signature signifies compliance with Signature verifying data and calculations:

Procedure EMS-JAG-01

Signature/date

Signatures on original data sheet in Test Instruction Package TI-RPP-WTP-465

Reference: CCP-WTPSP-176

(HVC2_gas-dataRev0 (8)).xls

gas-dataRev0.xls

GT49

31 July 2006

$4 / 18 / 2007$ 
Rev. 0

31-Jul-06

$\begin{aligned} \text { Site } & \text { HV-C2 Model } \\ \text { Date } & \frac{1 / 24 / 2007}{\text { JG Droppo }} \\ \text { Tester } & \frac{12 \text { in. }}{\text { JG }} \\ \text { Stack Dia. } & \frac{113.1 \text { in. }^{2}}{\text { Stack X-Area }} \\ \text { Elevation } & \text { Port 1 }\end{aligned}$

Distance to disturbance Measurement units ppm SF6
TRACER GAS TRAVERSE DATA FORM

Run No. GT-50

Fan Configuration $\mathbf{A \& B}$

Fan Setting 40

Stack Temp

Start/End Time 1405/1510

Center $2 / 3$ from

Points in Center $2 / 3$ Injection Point $\mathbf{B}$ Center w/ 3M filterete prefilters

$\mathrm{Hz}$

$61 \mathrm{deg} F$

$\frac{1.10}{2}$ to: $\frac{10.90}{7}$

2 to: $\frac{1}{7}$

Ist

\begin{tabular}{|c|c|c|c|c|c|c|c|c|c|}
\hline \multirow{3}{*}{$\begin{array}{l}\text { Traverse--> } \\
\text { Trial ----> }\end{array}$} & & \multicolumn{8}{|c|}{ Ist } \\
\hline & & \multicolumn{4}{|c|}{ Side } & \multicolumn{4}{|c|}{ Bottom } \\
\hline & & \multirow[t]{2}{*}{1} & 2 & \multirow[t]{2}{*}{3} & \multirow[t]{2}{*}{ Mean } & \multirow[t]{2}{*}{1} & 2 & \multirow[t]{2}{*}{3} & \multirow[t]{2}{*}{ Mean } \\
\hline Point & Depth, in. & & ppm & & & & ppm & & \\
\hline 1 & 0.50 & 1.03 & 1.02 & 1.06 & 1.037 & 1.07 & 1.08 & 1.09 & 1.080 \\
\hline 2 & 1.26 & 0.993 & 1.01 & 1.04 & 1.014 & 1.05 & 1.05 & 1.13 & 1.077 \\
\hline 3 & 2.33 & 1.00 & 1.01 & 1.05 & 1.020 & 1.07 & 1.06 & 1.08 & 1.070 \\
\hline 4 & 3.88 & 1.02 & 1.06 & 1.05 & 1.043 & 1.12 & 1.12 & 1.08 & 1.107 \\
\hline Center & 6.00 & 1.09 & 1.07 & 1.06 & 1.073 & 1.13 & 1.08 & 1.11 & 1.107 \\
\hline 5 & 8.12 & 1.08 & 1.11 & 1.12 & 1.103 & 1.07 & 1.05 & 1.08 & 1.067 \\
\hline 6 & 9.67 & 1.04 & 1.11 & 1.08 & 1.077 & 1.07 & 1.06 & 1.07 & 1.067 \\
\hline 7 & 10.74 & 1.06 & 1.11 & 1.12 & 1.097 & 1.07 & 1.08 & 1.02 & 1.057 \\
\hline 8 & 11.50 & 1.090 & 1.090 & 1.080 & 1.087 & 1.06 & 1.05 & 1.05 & 1.053 \\
\hline \multicolumn{2}{|c|}{ Averages ----------> } & 1.045 & 1.066 & 1.073 & 1.061 & 1.079 & 1.070 & 1.079 & 1.076 \\
\hline
\end{tabular}

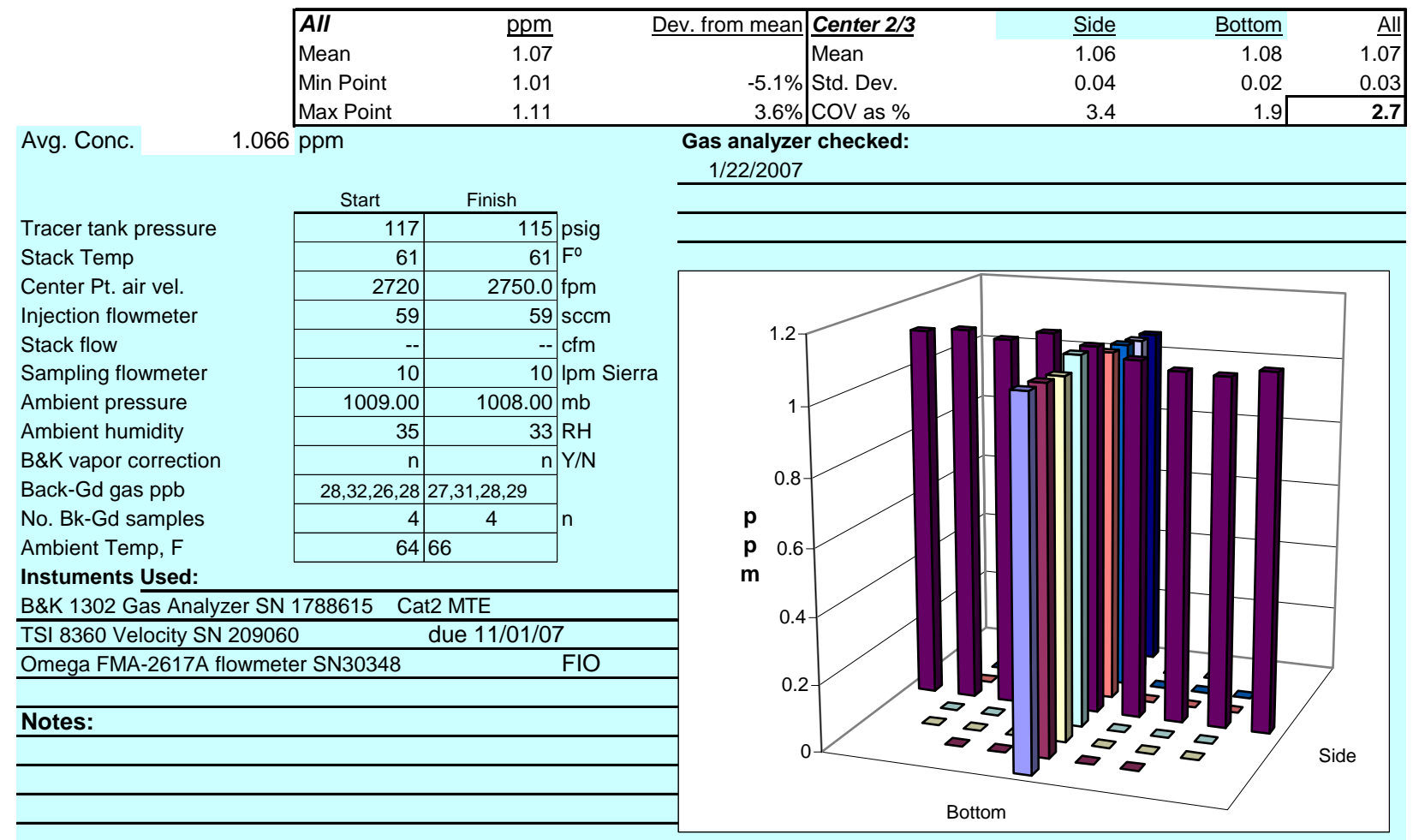

Signature signifies compliance with Signature verifying data and calculations:

Procedure EMS-JAG-01

Signature/date

Signatures on original data sheet in Test Instruction Package TI-RPP-WTP-465

Reference: CCP-WTPSP-176

(HVC2_gas-dataRev0 (8)).xls

gas-dataRev0.xls

GT50

31 July 2006

4/18/2007 
Rev. 0

31-Jul-06

\begin{tabular}{rr}
\multicolumn{1}{c}{} & $\begin{array}{r}\text { Test } \\
\text { Stack Dia } \\
\text { Stack X-Area } \\
\text { Elevatio }\end{array}$ \\
Distance to disturbance \\
Measurement units \\
Traverse--> \\
Trial ----> \\
\hline Point & Depth, in. \\
\hline 1 & 0.50 \\
\hline 2 & 1.26 \\
\hline 3 & 2.33 \\
\hline 4 & 3.88 \\
\hline Center & 6.00 \\
\hline 5 & 8.12 \\
\hline 6 & 9.67 \\
\hline 7 & 10.74 \\
\hline 8 & 11.50 \\
\hline Averages ------->>
\end{tabular}

Site HV-C2 Model

Date 1/26/2007

Tester BGF

tack Dia.

Elevation

\begin{tabular}{l}
$\frac{12 \text { in. }^{2}}{113.1 \text { in. }^{2}}$ \\
\hline Port \\
\hline 113.75 inches $^{2}$
\end{tabular}

113.75 inches

2

TRACER GAS TRAVERSE DATA FORM

Run No. GT-51

Fan Configuration A \& B $\quad$ w/ 3M filterete prefilters

Fan Setting 40

Stack Temp $\mathrm{Hz}$

Start/End Time

Center $2 / 3$ from

Points in Center $2 / 3$ Injection Point $\mathbf{A}$ near left

\begin{tabular}{|c|c|c|}
\hline \multicolumn{2}{|c|}{$61 \mathrm{deg} F$} & \\
\hline \multicolumn{2}{|c|}{$1015-1115$} & \\
\hline 1.10 & to: & 10.90 \\
\hline 2 & to: & 7 \\
\hline & & \\
\hline
\end{tabular}

1

\begin{tabular}{|c|c|c|c|c|c|c|}
\hline \multicolumn{3}{|c|}{ Side } & \multicolumn{4}{|c|}{ Bottom } \\
\hline 2 & 3 & Mean & 1 & 2 & 3 & Mean \\
\hline \multicolumn{3}{|c|}{$\mathrm{ppm}$} & \multicolumn{4}{|c|}{ ppm } \\
\hline .930 & 0.942 & 0.943 & 0.932 & 0.951 & 0.948 & 0.944 \\
\hline 0.965 & 0.971 & 0.966 & 0.921 & 0.927 & 0.965 & 0.938 \\
\hline 0.994 & 0.938 & 0.967 & 0.929 & 0.957 & 0.941 & 0.942 \\
\hline 0.935 & 0.950 & 0.947 & 0.951 & 0.957 & 0.962 & 0.957 \\
\hline .951 & 0.948 & 0.946 & 0.955 & 0.960 & 0.953 & 0.956 \\
\hline 0.966 & 0.961 & 0.959 & 0.971 & 0.975 & 0.970 & 0.972 \\
\hline 0.940 & 0.957 & 0.949 & 0.986 & 0.993 & 0.979 & 0.986 \\
\hline .940 & 0.921 & 0.928 & 0.979 & 1.040 & 0.986 & 1.002 \\
\hline 0.975 & 0.966 & 0.963 & 1.010 & 0.989 & 0.992 & 0.997 \\
\hline 5 & 0.950 & 0.952 & 0.96 & 0.97 & 0.97 & 0.966 \\
\hline
\end{tabular}

Avg. Conc.

\begin{tabular}{|lr}
\hline All & ppm \\
Mean & 0.96 \\
Min Point & 0.93 \\
Max Point & 1.00 \\
\hline
\end{tabular}

Tracer tank pressure

Stack Temp

Injection flowmeter

Stack flow

Ambient pressure

Ambient humidity

B\&K vapor correction

Back-Gd gas ppb

No. Bk-Gd samples

Ambient Temp, $F$

Instuments Used:

B\&K 1302 Gas Analyzer SN 1788615 Cat2 MTE

TSI 8360 Velocity SN 209060

Omega FMA-2617A flowmeter SN30348

due $11 / 01 / 07$

\begin{tabular}{|c|c|c|}
\hline Start & Finish & \\
\hline 180 & 180 & psig \\
\hline 62 & 60 & $F^{\circ}$ \\
\hline 3000 & 3060 & $\mathrm{fpm}$ \\
\hline 59 & 59 & $\mathrm{sccm}$ \\
\hline- & -- & $\mathrm{cfm}$ \\
\hline 10 & 10 & Ipm Sierra \\
\hline 1010.0 & 1010.0 & $\mathrm{mb}$ \\
\hline 34 & 34 & $\mathrm{RH}$ \\
\hline $\mathrm{N}$ & $\mathrm{N}$ & $\mathrm{Y} / \mathrm{N}$ \\
\hline $18,21,22,21$ & $23,22,27,21$ & \\
\hline 4 & & $\mathrm{n}$ \\
\hline 59 & 58 & \\
\hline
\end{tabular}

Notes:

$$
0.960 \mathrm{ppm}
$$

Center Pt. air vel.

Sampling flowmeter

\begin{tabular}{r|l} 
Dev. from mean & Center $\mathbf{2 / 3}$ \\
$-3.3 \%$ & Mean \\
$4.5 \%$ & Ctd. Dev. \\
COV as \%
\end{tabular}

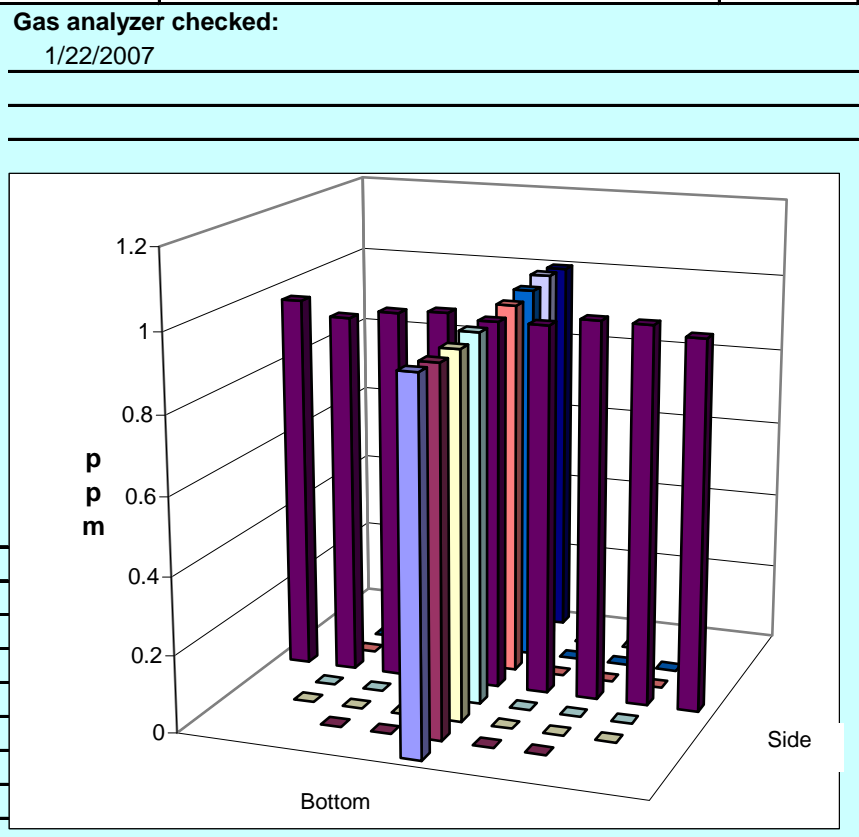

Signature signifies compliance with Procedure EMS-JAG-01

Signature/date

Signatures on original data sheet in Test Instruction Package TI-RPP-WTP-465
Reference: CCP-WTPSP-176

gas-dataRev0.xls

31 July 2006
(HVC2_gas-dataRev0 (8)).xls

GT51

$4 / 18 / 2007$ 
Rev. 0

31-Jul-06

\begin{aligned} Site & HV-C2 Model \\ Date & $\frac{1 / 26 / 2007}{12} \\$ Tester & BGF \\ Stack Dia. & \multicolumn{1}{c}{12 in. } \\ Stack X-Area & 113.1 in. $^{2} \\$ Elevation & Port \end{aligned}

Distance to disturbance Measurement units ppm SF6
TRACER GAS TRAVERSE DATA FORM

Run No. GT-52

Fan Configuration $\mathbf{A}$ \& B Fan Setting 40

Stack Temp

Start/End Time 1145 -- 1245

Center $2 / 3$ from

Points in Center $2 / 3$ Injection Point $\mathbf{B}$ center w/ 3M filterete prefilters

$\mathrm{Hz}$

$61.5 \operatorname{deg} \mathrm{F}$

1.10

1.10

to. $\quad 10.90$

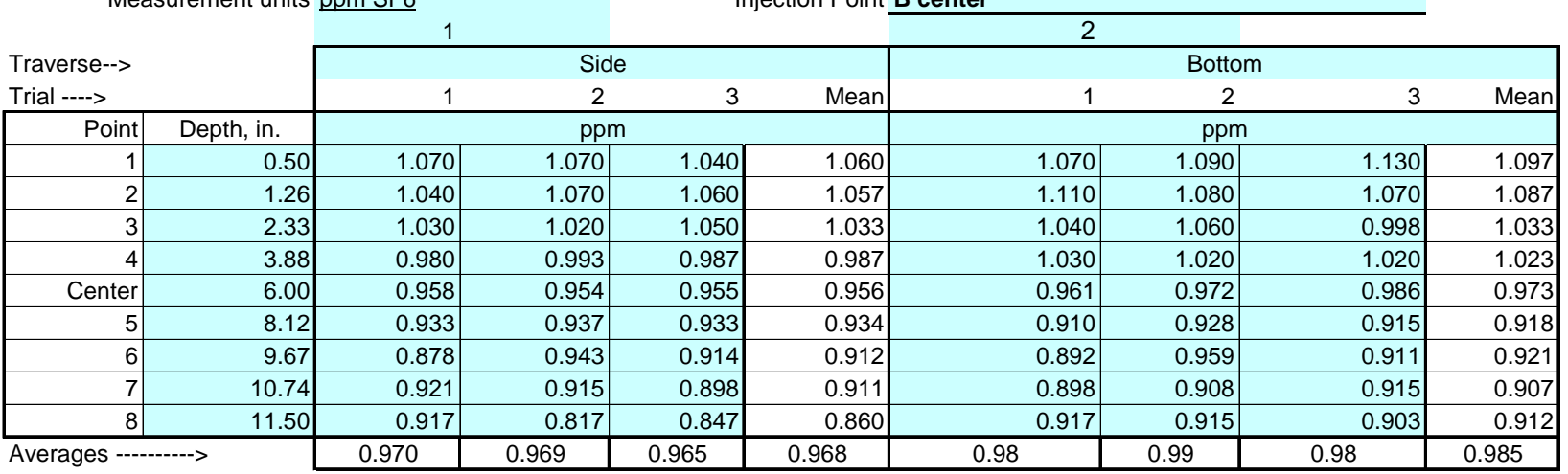

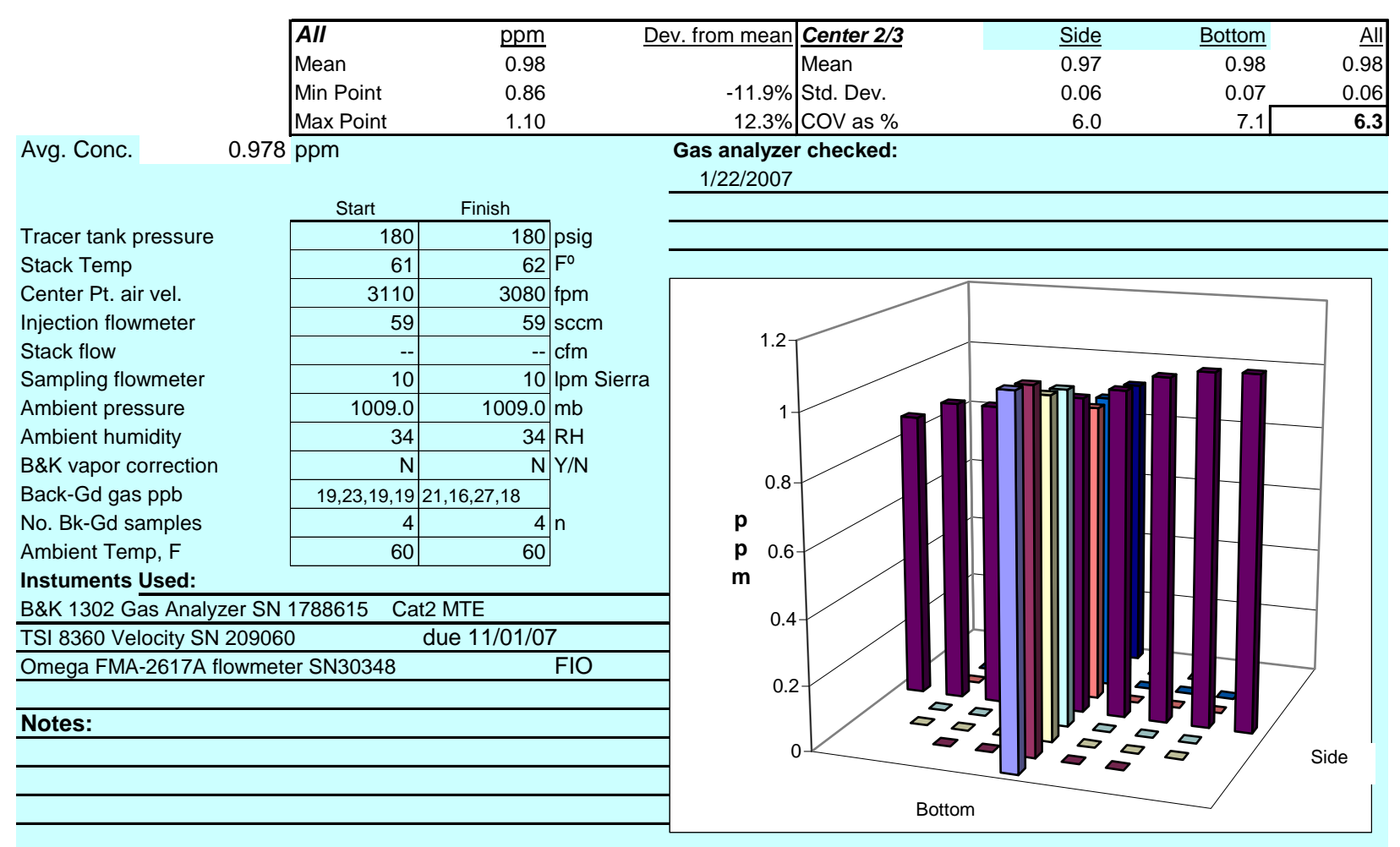

Signature signifies compliance with Signature verifying data and calculations:

Procedure EMS-JAG-01

Signature/date

Signatures on original data sheet in Test Instruction Package TI-RPP-WTP-465

Reference: CCP-WTPSP-176

(HVC2_gas-dataRev0 (8)).xls

gas-dataRev0.xls

GT52

31 July 2006

$4 / 18 / 2007$ 
Rev. 0

31-Jul-06

\begin{tabular}{|c|c|}
\hline $\begin{array}{r}\text { Distanc } \\
\text { Me }\end{array}$ & $\begin{array}{r}\text { Dat } \\
\text { Test } \\
\text { Stack Di } \\
\text { Stack X-Are } \\
\text { Elevatio } \\
\text { to disturbanc } \\
\text { urement uni }\end{array}$ \\
\hline \multicolumn{2}{|l|}{ Traverse--> } \\
\hline Point & Depth, in. \\
\hline 1 & 0.5 \\
\hline 2 & 1.2 \\
\hline 3 & 2.3 \\
\hline 4 & \\
\hline Center & \\
\hline 5 & \\
\hline 6 & \\
\hline 7 & \\
\hline 8 & \\
\hline
\end{tabular}

TRACER GAS TRAVERSE DATA FORM

Site HV-C2 Model

Date 1/26/2007

Tester BGF \& MSP

Stack Dia.

\begin{tabular}{c}
\hline \multicolumn{1}{c}{12 in. } \\
\hline 113.1 in. $^{2}$ \\
\hline Port \\
\hline 53.5 inches
\end{tabular}

53.5 inches

ppm SF6
Run No. GT-53

Fan Configuration A \& B Fan Setting 40

Stack Temp

Start/End Time 1245 -- 1330

Center $2 / 3$ from

Points in Center $2 / 3$ Injection Point $\mathbf{B}$ center w/ 3M filterete prefilters

$\mathrm{Hz}$

$62 \operatorname{deg} F$

330

1.10 to: 10.90

2 to: $\frac{10}{7}$

1.2

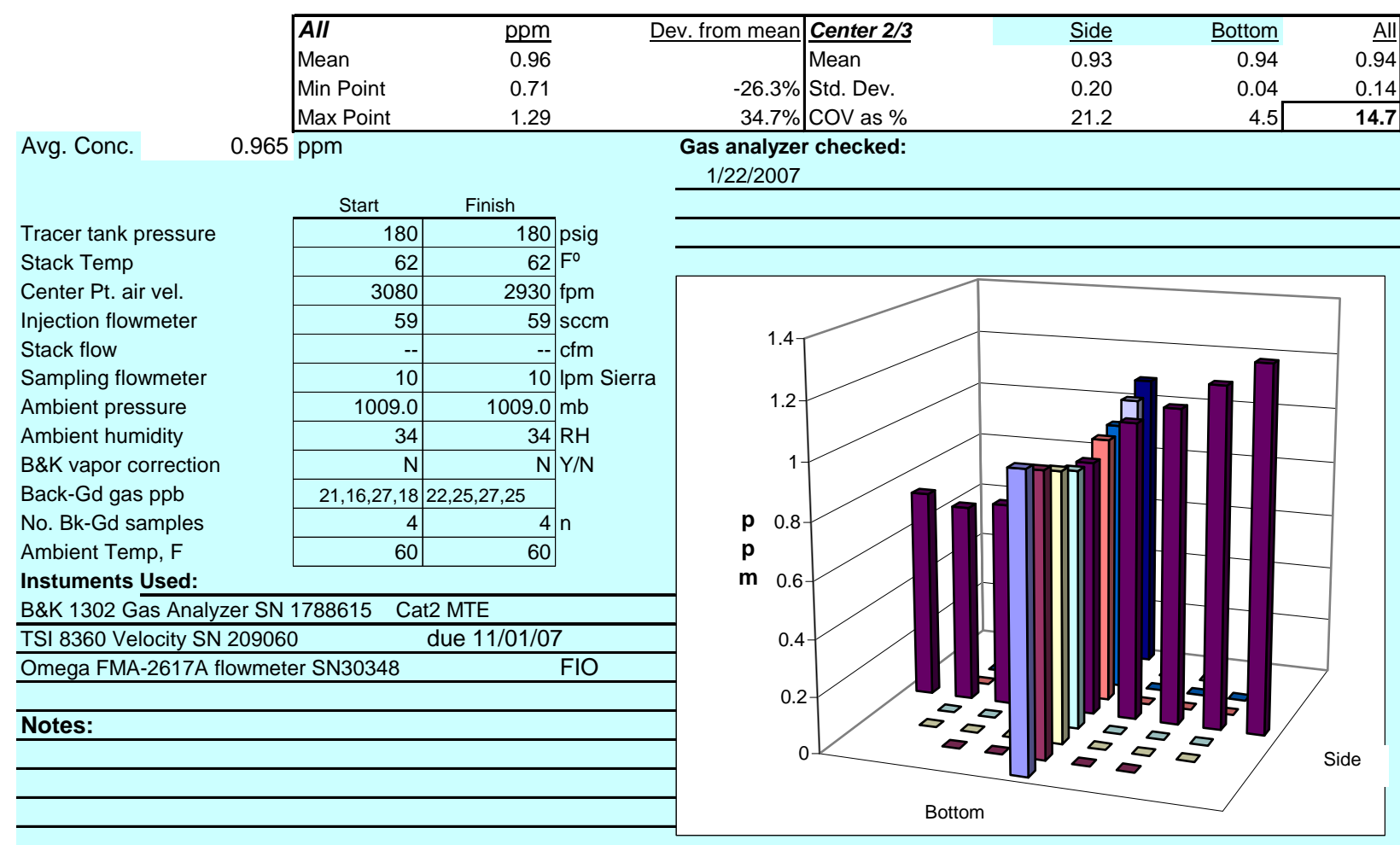

Signature signifies compliance with

Signature verifying data and calculations:

Procedure EMS-JAG-01

Signature/date

Signatures on original data sheet in Test Instruction Package TI-RPP-WTP-465

Reference: CCP-WTPSP-176

(HVC2_gas-dataRev0 (8)).xls

gas-dataRev0.xls

GT53

31 July 2006

$4 / 18 / 2007$ 
Rev. 0

31-Jul-06

\begin{aligned} Site & HV-C2 Model \\ Date & $\frac{1 / 26 / 2007}{M S} \\$ Tester & \\ Stack Dia. & 12 in. \\ Stack X-Area & 113.1 in. $^{2} \\$ Elevation & Port \\ \cline { 2 - 2 } & 174.25 inches $^{2}\end{aligned}$

Distance to disturbance Measurement units ppm SF6
TRACER GAS TRAVERSE DATA FORM

Run No. GT-54

Fan Configuration $\mathbf{A}$ \& $\mathbf{B}$ Fan Setting 40

Stack Temp

Start/End Time 1335 -- 1430

Center $2 / 3$ from

Points in Center $2 / 3$ Injection Point $\mathbf{B}$ center w/ 3M filterete prefilters

$\mathrm{Hz}$

30

$62 \operatorname{deg} F$

1.10

to: 10.90

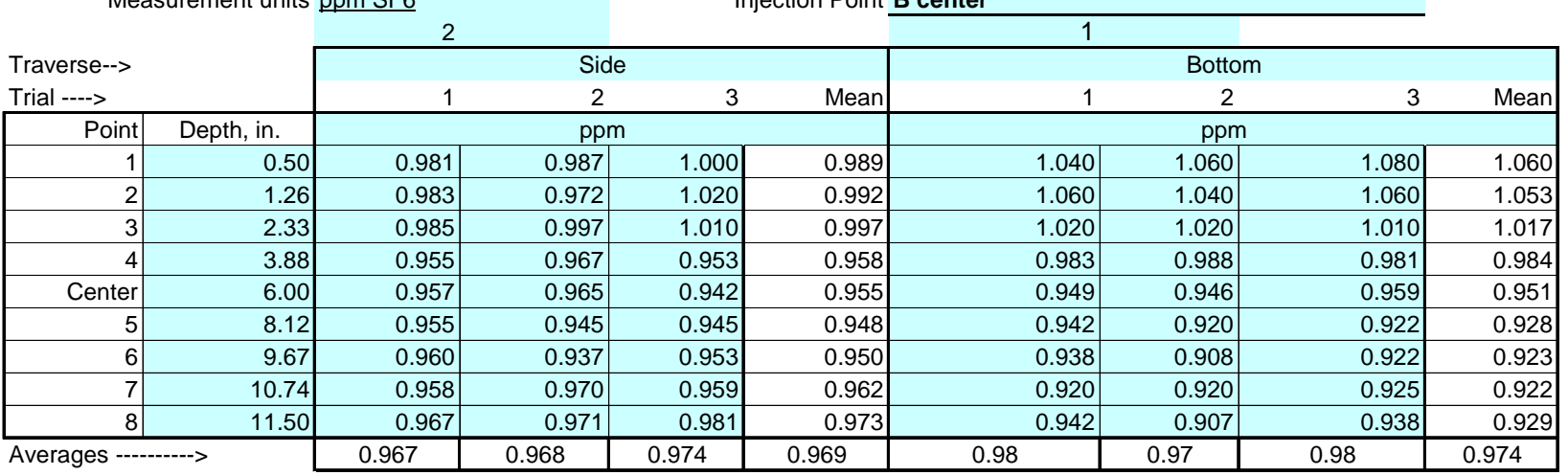

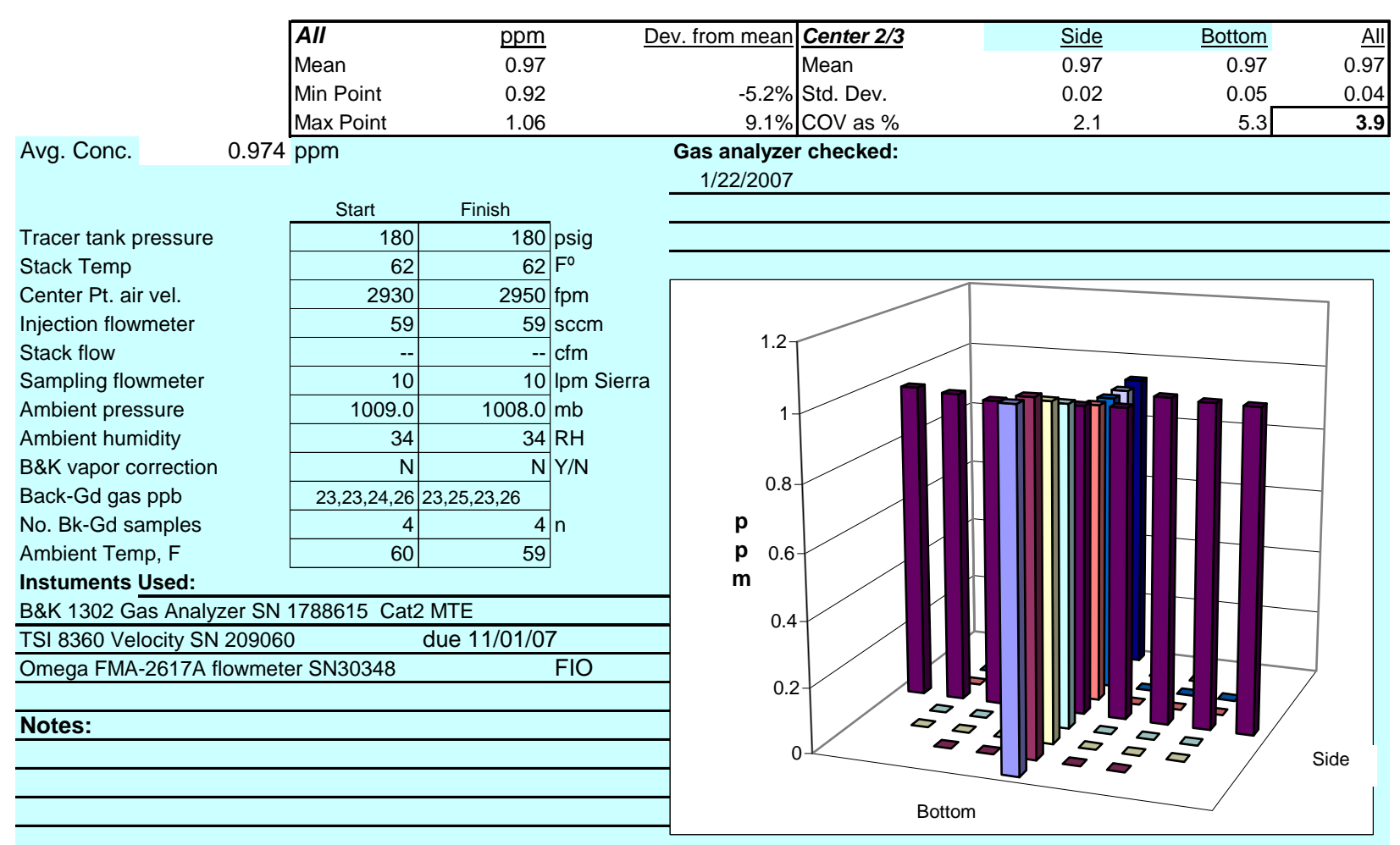

Signature signifies compliance with Signature verifying data and calculations:

Procedure EMS-JAG-01

Signature/date

Signatures on original data sheet in Test Instruction Package TI-RPP-WTP-465

Reference: CCP-WTPSP-176

(HVC2_gas-dataRev0 (8)).xls

gas-dataRev0.xls

GT54

31 July 2006

4/18/2007 
Sulfur hexafluoride Gas Calibration performed on B\&K on

11/17/2006 by Jim Droppo

B\&K Model 1302: Property No. WD17210 Serial No. 1765299

$6.8 \mathrm{ft} \quad$ B\&K sample inlet tube length

1007.45 mbar station pressure

45 deg $\mathrm{F}$ ambient temp analyzer corrects to $20 \operatorname{deg} \mathrm{C}$

54 percent $\mathrm{RH}$

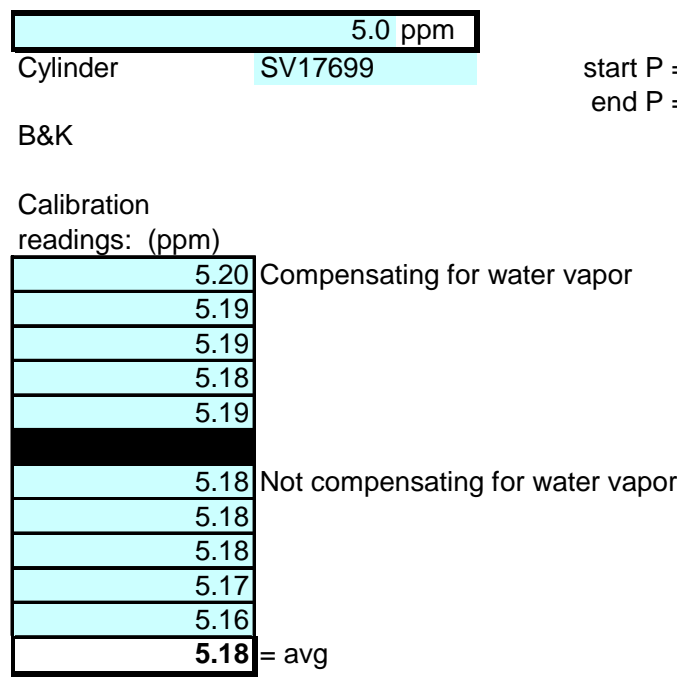

\begin{tabular}{|c|c|c|c|c|}
\hline & & \multicolumn{2}{|c|}{$0.5 \mathrm{ppm}$} & \\
\hline Cylinder & & SV18280 & $\begin{array}{r}\text { start } \mathrm{P}= \\
\text { end } \mathrm{P}=\end{array}$ & $\begin{array}{l}1200 \mathrm{ps} \\
1100 \mathrm{ps}\end{array}$ \\
\hline \multicolumn{5}{|l|}{ B\&K } \\
\hline \multicolumn{5}{|c|}{$\begin{array}{l}\text { Calibration } \\
\text { readings: (ppm) }\end{array}$} \\
\hline \multicolumn{5}{|c|}{0.499 Compensating for water vapor } \\
\hline \multicolumn{5}{|c|}{0.494} \\
\hline \multicolumn{5}{|c|}{0.494} \\
\hline \multicolumn{5}{|c|}{0.496} \\
\hline \multicolumn{5}{|c|}{0.496} \\
\hline \multicolumn{5}{|c|}{0.497 Not compensating for water vapor } \\
\hline \multicolumn{5}{|c|}{0.498} \\
\hline \multicolumn{5}{|c|}{0.497} \\
\hline \multicolumn{5}{|c|}{0.494} \\
\hline \multicolumn{5}{|c|}{0.497} \\
\hline \multicolumn{5}{|c|}{$0.496=$} \\
\hline
\end{tabular}

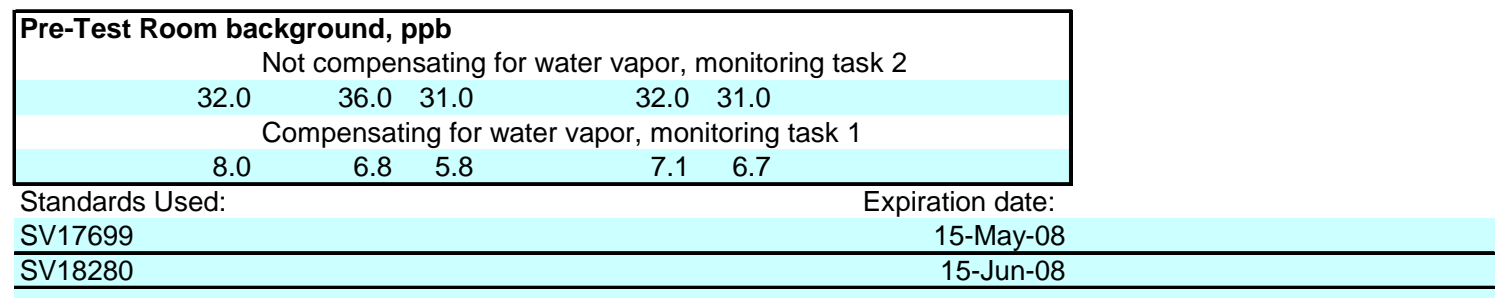

Signature signifies compliance with Signature verifying data and calculations:

Procedure EMS-JAG-01

Signature/date Signatures on original data sheet in Test Instruction Package TI-RPP-WTP-465

Reference: CCP-WTPSP-

BK calibration check data sheets.xls

BcalcheckRev0.xls

Nov17

4 August 2006

$4 / 18 / 2007$ 
Sulfur hexafluoride Gas Calibration performed on B\&K on

11/27/2006 by John Glissmeyer B\&K Model 1302: Property No. WD17210 Serial No. 1765299

Setup:

$6.7 \mathrm{ft} \quad$ B\&K sample inlet tube length

991.2 mbar station pressure

71 deg $\mathrm{F}$ ambient temp analyzer corrects to $20 \operatorname{deg} \mathrm{C}$

24 percent $\mathrm{RH}$

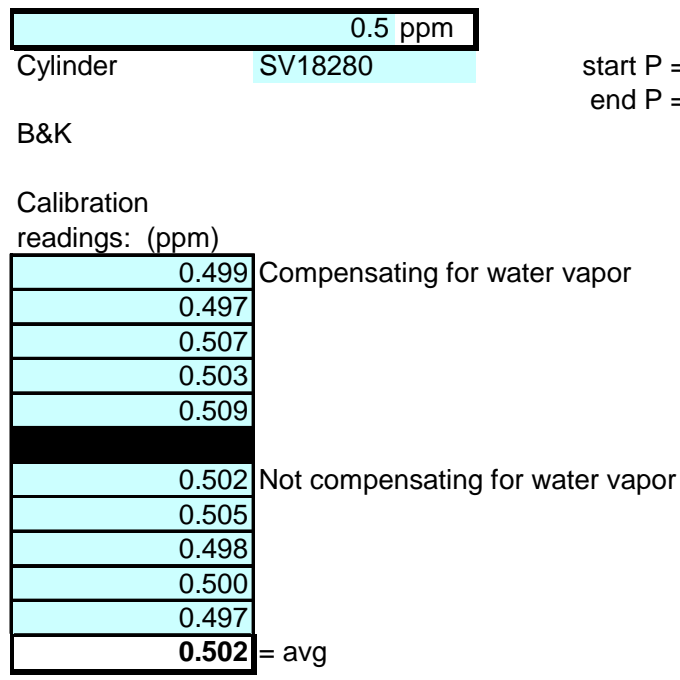

\begin{tabular}{|c|c|c|c|c|}
\hline & & \multicolumn{2}{|c|}{$5.0 \mathrm{ppm}$} & \\
\hline Cylinder & & SV17699 & $\begin{array}{r}\text { start } P= \\
\text { end } P=\end{array}$ & $\begin{array}{r}1000 \mathrm{ps} \\
950 \mathrm{ps}\end{array}$ \\
\hline \multicolumn{5}{|l|}{ B\&K } \\
\hline \multicolumn{5}{|c|}{$\begin{array}{l}\text { Calibration } \\
\text { readings: (ppm) }\end{array}$} \\
\hline \multicolumn{5}{|c|}{5.24 Compensating for water vapor } \\
\hline \multicolumn{5}{|c|}{5.21} \\
\hline \multicolumn{5}{|c|}{5.20} \\
\hline \multicolumn{5}{|c|}{5.21} \\
\hline \multicolumn{5}{|c|}{5.21} \\
\hline \multicolumn{5}{|c|}{ 5.20 Not compensating for water vapor } \\
\hline \multicolumn{5}{|c|}{5.19} \\
\hline \multicolumn{5}{|c|}{5.19} \\
\hline \multicolumn{5}{|c|}{5.18} \\
\hline \multicolumn{5}{|c|}{5.20} \\
\hline \multicolumn{5}{|c|}{5.20} \\
\hline
\end{tabular}

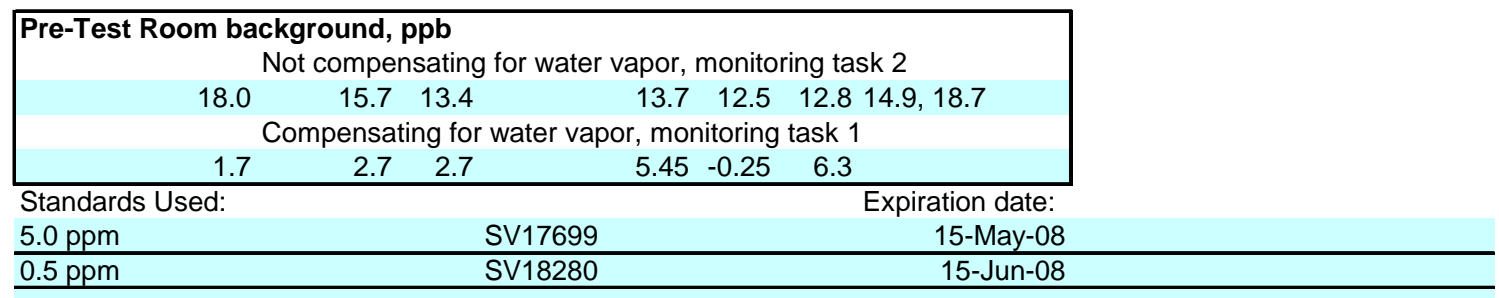

Signature signifies compliance with

Signature verifying data and calculations:

Procedure EMS-JAG-01

Signature/date

Signatures on original data sheet in Test Instruction Package TI-RPP-WTP-465

Reference: CCP-WTPSP-

BK calibration check data sheets.xls

BcalcheckRev0.xls

Nov27

4 August 2006

$4 / 18 / 2007$ 


\begin{tabular}{|c|c|c|c|c|c|c|}
\hline \multicolumn{3}{|c|}{$\begin{array}{l}\text { Sulfur hexafluoride Gas Calibration performed on B\&K on } \\
\text { B\&K Model 1302: Property No. WD17210 Serial No. } 1765299\end{array}$} & \multicolumn{4}{|c|}{$12 / 4 / 2006$ by } \\
\hline Setup: & $\begin{array}{cc}6 \mathrm{ft} \quad \text { B\&K sample inlet } \\
1016.9 \text { mbar station pressure } \\
70 \text { deg F ambient temp } \\
<50 \quad \text { percent } \mathrm{RH}\end{array}$ & $\begin{array}{l}\text { tube length } \\
\text { analyzer cc }\end{array}$ & rrects to $20 \mathrm{deg} C$ & & & \\
\hline & $0.5 \mathrm{ppm}$ & & & & ppm & \\
\hline $\begin{array}{l}\text { Cylinder } \\
\text { B\&K }\end{array}$ & $\begin{aligned} \text { start } P & = \\
\text { end } P & =\end{aligned}$ & $\begin{array}{l}1000 \mathrm{psi} \\
1000 \mathrm{psi}\end{array}$ & $\begin{array}{l}\text { Cylinder } \\
\text { B\&K }\end{array}$ & SV17699 & $\begin{array}{r}\text { start } P= \\
\text { end } P=\end{array}$ & $\begin{array}{l}900 \text { psi } \\
800 \text { psi }\end{array}$ \\
\hline $\begin{array}{l}\text { Calibration } \\
\text { readings: }(\mathrm{ppm})\end{array}$ & & & $\begin{array}{l}\text { Calibration } \\
\text { readings: }(\mathrm{ppm})\end{array}$ & $\left(\frac{10}{10}\right.$ & & \\
\hline $\begin{array}{r}0.499 \\
0.501 \\
0.499 \\
0.495 \\
0.499 \\
\end{array}$ & Compensating for water vapor & & \begin{tabular}{|l|}
5.15 \\
5.16 \\
5.16 \\
5.15 \\
5.16 \\
\end{tabular} & Compens & ting for wat & \\
\hline $\begin{array}{l}0.493 \\
0.493 \\
0.492 \\
0.491 \\
0.492 \\
\mathbf{0 . 4 9 5}\end{array}$ & $\begin{array}{l}\text { Not compensating for water vapor } \\
=\text { avg }\end{array}$ & & $\begin{array}{l}5.16 \\
5.15 \\
5.15 \\
5.15 \\
5.14 \\
\mathbf{5 . 1 5}\end{array}$ & $\begin{array}{l}\text { Not comp } \\
=\text { avg }\end{array}$ & ensating for & vapor \\
\hline
\end{tabular}

\begin{tabular}{|c|c|c|}
\hline \multicolumn{3}{|c|}{ Pre-Test Room background, ppb } \\
\hline \multicolumn{3}{|c|}{ Not compensating for water vapor, monitoring task 2} \\
\hline 11 & 1613 & 11 \\
\hline \multicolumn{3}{|c|}{ Compensating for water vapor, monitoring task 1} \\
\hline 0.918 & $4.10 \quad 2.11$ & 7.83 \\
\hline
\end{tabular}

\begin{tabular}{lrr} 
Standards Used: & & Expiration date: \\
$5.0 \mathrm{ppm}$ SV17699 & Matheson Tri-Gas & 15-May-08 \\
\hline $0.5 \mathrm{ppm}$ SV18280 & Matheson Tri-Gas & 15-Jun-08 \\
\hline
\end{tabular}

\begin{tabular}{|lc|}
\hline $\begin{array}{l}\text { Signature signifies compliance with } \\
\text { Procedure EMS-JAG-01 }\end{array}$ & Signature verifying data and calculations: \\
Signature/date & Signatures on original data sheet in Test Instruction Package TI-RPP-WTP-465 \\
\hline
\end{tabular}

Reference: CCP-WTPSP-185

BcalcheckRev0.xls

4 August 2006EA7C3E
BK calibration check data sheets.xls

Dec04

$4 / 18 / 2007$ 


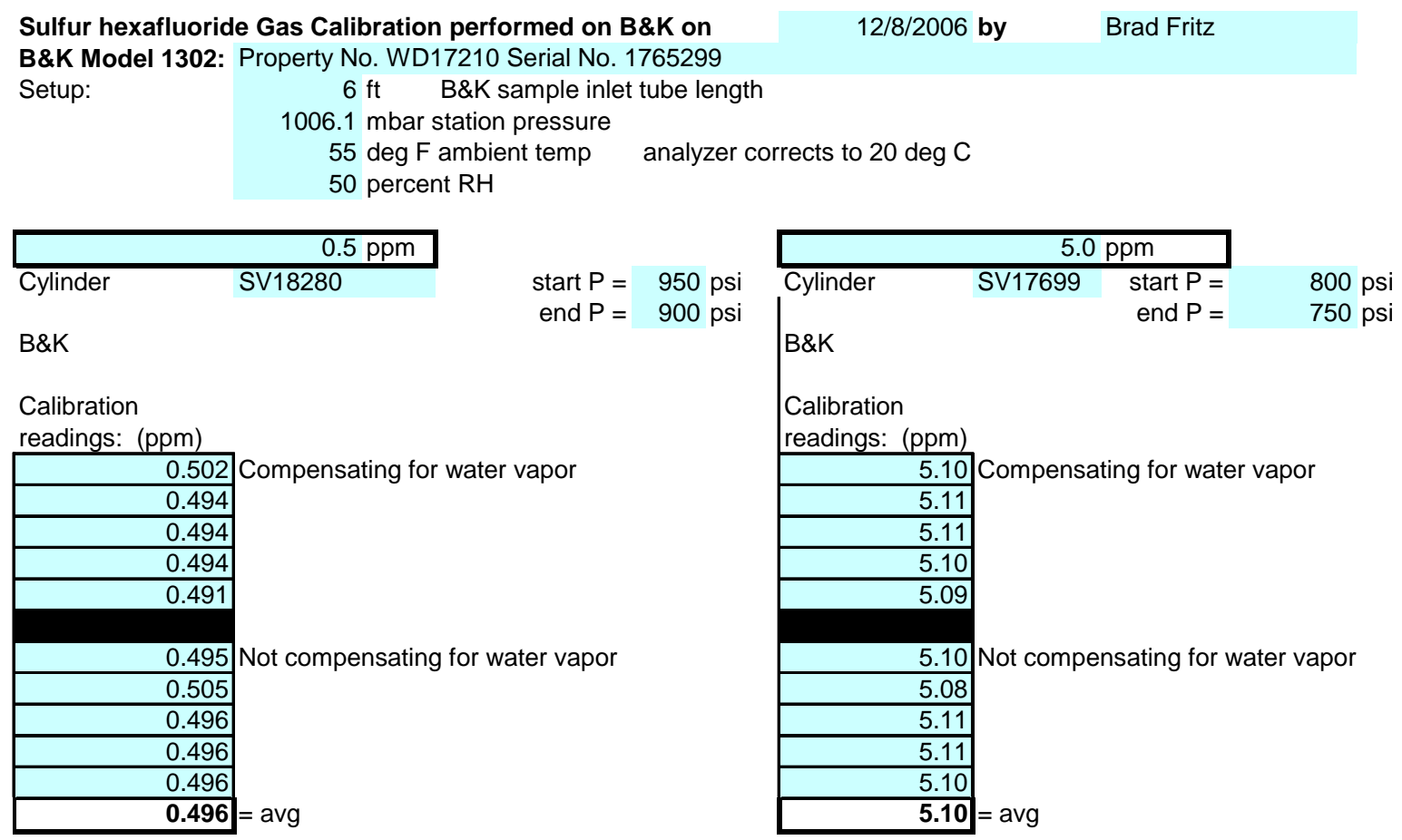

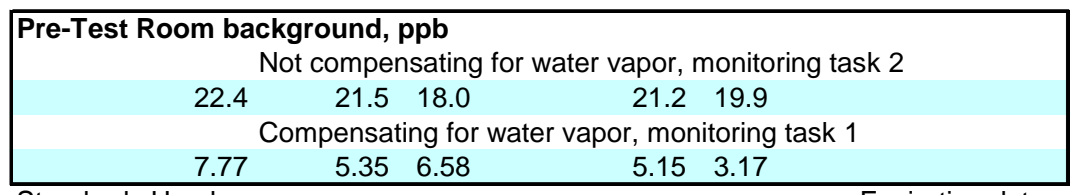

Standards Used: $\quad$ Expiration date:

5.0 ppm SF6 Matheson Tri-Gas 15-May-08

0.5 ppm SF6 Matheson Tri-Gas $15-J u n-08$

Signature signifies compliance with $\quad$ Signature verifying data and calculations:

Procedure EMS-JAG-01

Signature/date Signatures on original data sheet in Test Instruction Package TI-RPP-WTP-465

Reference: CCP-WTPSP-185

BK calibration check data sheets.xls

BcalcheckRev0.xls

Dec08

4 August 2006EA7C3E

$4 / 18 / 2007$ 


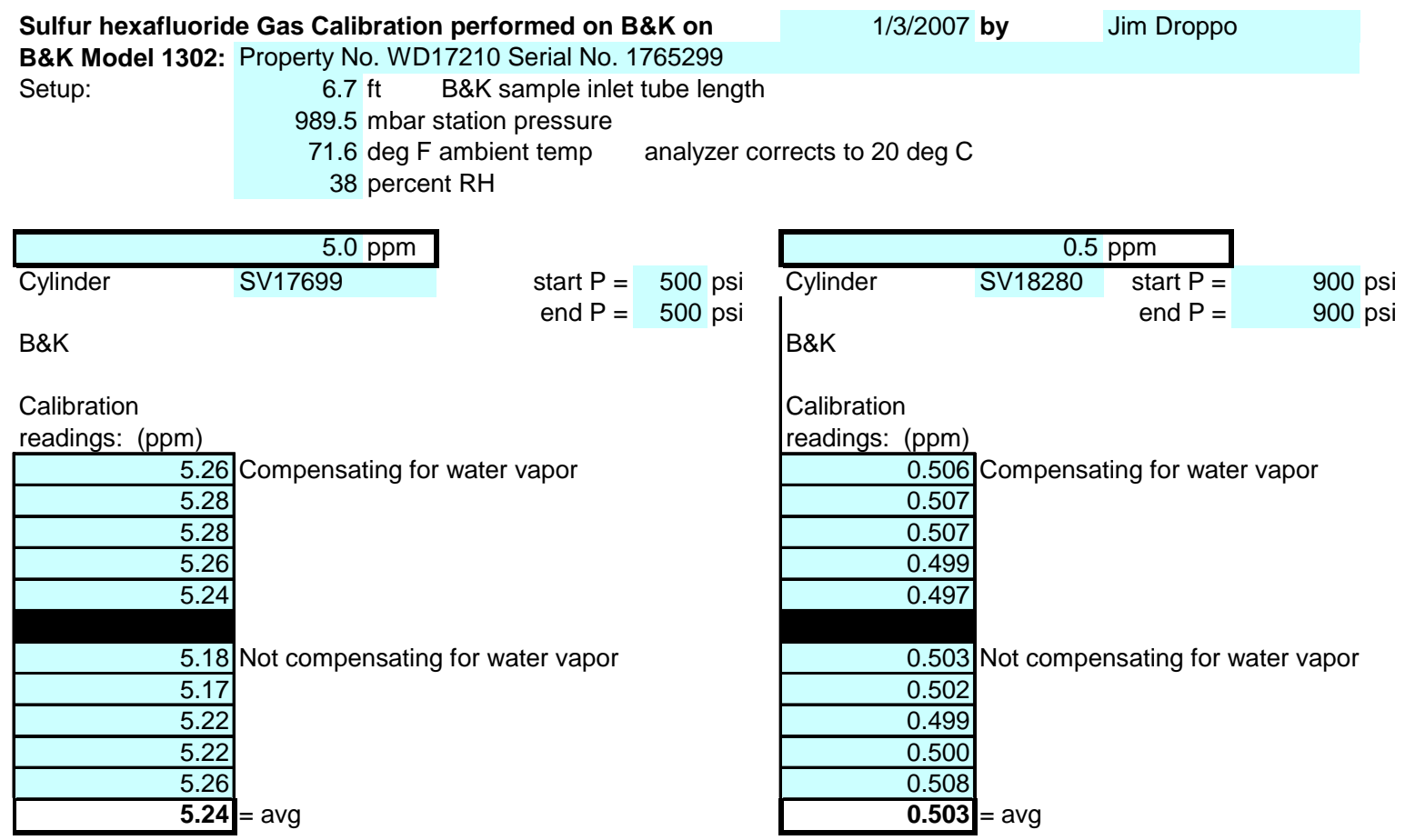

\begin{tabular}{|c|c|c|c|}
\hline \multicolumn{4}{|c|}{ Pre-Test Room background, ppb } \\
\hline \multicolumn{4}{|c|}{ Not compensating for water vapor, monitoring task 2} \\
\hline 30.0 & $27.0 \quad 30.0$ & 31.0 & 29.0 \\
\hline \multicolumn{4}{|c|}{ Compensating for water vapor, monitoring task 1} \\
\hline 0.168 & $0 \quad 0.3$ & 0 & 0 \\
\hline
\end{tabular}

Standards Used: $\quad$ Expiration date:

$5.0 \mathrm{ppm}$ SF6 Matheson Tri-Gas $15-$ May-08

0.5 ppm SF6 Matheson Tri-Gas $15-J u n-08$

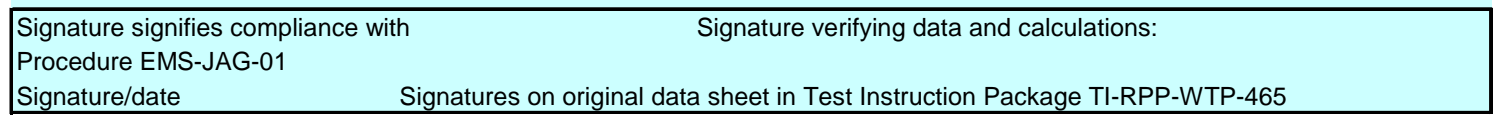

Reference: CCP-WTPSP-185

BK calibration check data sheets.xls

BcalcheckRev0.xls

Jan03

4 August 2006EA7C3E

$4 / 18 / 2007$ 
Sulfur hexafluoride Gas Calibration performed on B\&K on

1/16/2007 by JGD \& MSP

B\&K Model 1302: Property No. WC74247 Serial No. 1804888

Setup:

$6 \mathrm{ft} \quad$ B\&K sample inlet tube length

1015 mbar station pressure

68 deg F ambient temp analyzer corrects to 20 deg C

16 percent $\mathrm{RH}$

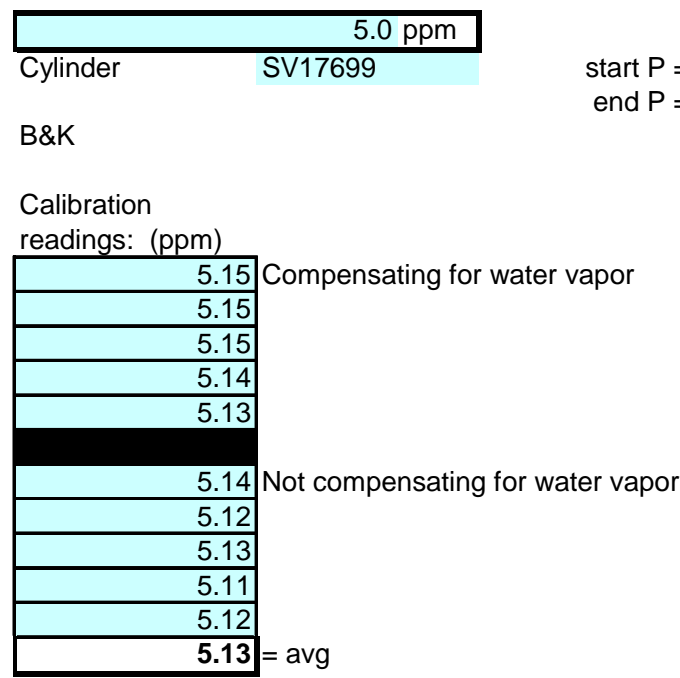

\begin{tabular}{|c|c|c|c|c|}
\hline & & \multicolumn{2}{|c|}{$0.5 \mathrm{ppm}$} & \\
\hline Cylinder & & SV18280 & $\begin{array}{r}\text { start } P= \\
\text { end } P=\end{array}$ & $\begin{array}{l}700 \text { ps } \\
700 \text { ps }\end{array}$ \\
\hline \multicolumn{5}{|l|}{ B\&K } \\
\hline \multicolumn{5}{|c|}{$\begin{array}{l}\text { Calibration } \\
\text { readings: (ppm) }\end{array}$} \\
\hline \multicolumn{5}{|c|}{0.520 Compensating for water vapor } \\
\hline \multicolumn{5}{|c|}{0.512} \\
\hline \multicolumn{5}{|c|}{0.512} \\
\hline \multicolumn{5}{|c|}{0.508} \\
\hline \multicolumn{5}{|c|}{0.509} \\
\hline \multicolumn{5}{|c|}{0.511 Not compensating for water vapor } \\
\hline \multicolumn{5}{|c|}{0.510} \\
\hline \multicolumn{5}{|c|}{0.508} \\
\hline \multicolumn{5}{|c|}{0.509} \\
\hline \multicolumn{5}{|c|}{0.507} \\
\hline \multicolumn{5}{|c|}{$0.511=$} \\
\hline
\end{tabular}

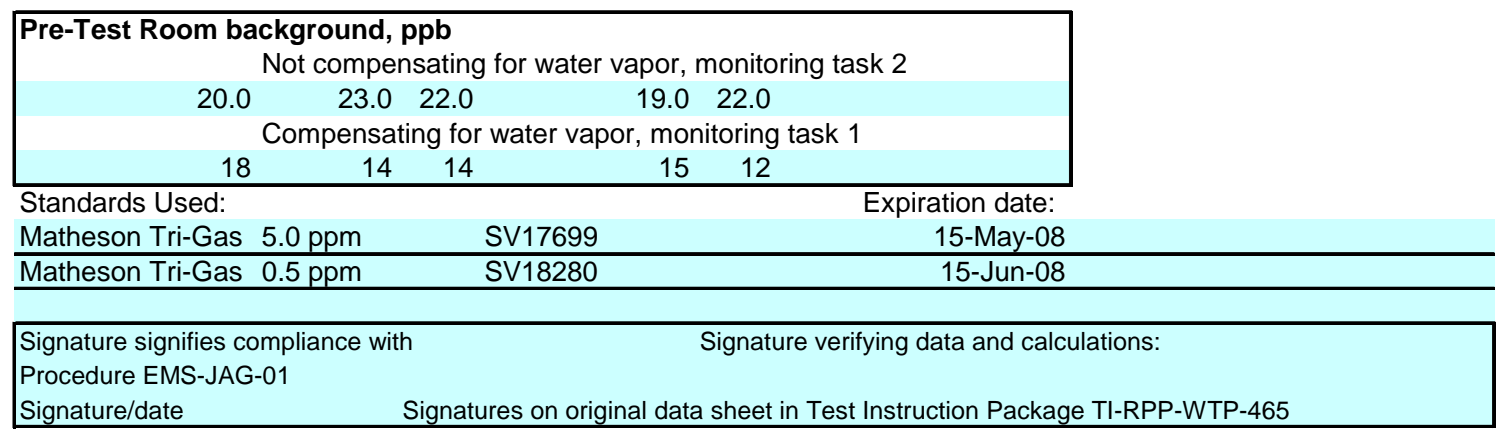

Reference: CCP-WTPSP-185

BK calibration check data sheets.xls

BcalcheckRev0.xls

Jan16_1

4 August 2006EEA7C3E

$4 / 18 / 2007$ 
Sulfur hexafluoride Gas Calibration performed on B\&K on

1/16/2007 by Jim Droppo

B\&K Model 1302: Property No. WC74073 Serial No. 1788615

Setup:

$6 \mathrm{ft} \quad$ B\&K sample inlet tube length

1016.9 mbar station pressure

61.5 deg F ambient temp analyzer corrects to 20 deg C

15.1 percent $\mathrm{RH}$

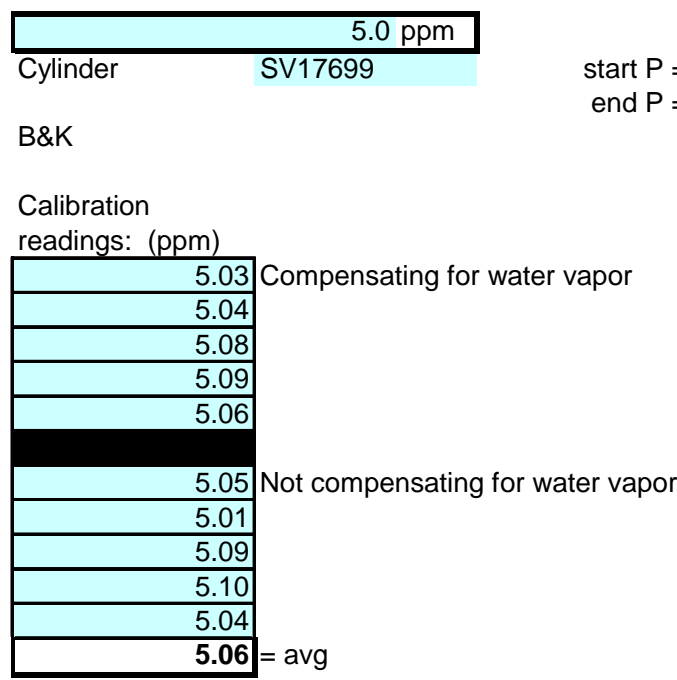

\begin{tabular}{|c|c|c|c|c|}
\hline & & \multicolumn{2}{|c|}{$0.5 \mathrm{ppm}$} & \\
\hline Cylinder & & SV18280 & $\begin{array}{r}\text { start } P= \\
\text { end } P=\end{array}$ & $\begin{array}{l}700 \text { ps } \\
700 \text { ps }\end{array}$ \\
\hline \multicolumn{5}{|l|}{ B\&K } \\
\hline \multicolumn{5}{|c|}{$\begin{array}{l}\text { Calibration } \\
\text { readings: (ppm) }\end{array}$} \\
\hline \multicolumn{5}{|c|}{0.510 Compensating for water vapor } \\
\hline \multicolumn{5}{|c|}{0.509} \\
\hline \multicolumn{5}{|c|}{0.505} \\
\hline \multicolumn{5}{|c|}{0.501} \\
\hline \multicolumn{5}{|c|}{0.507} \\
\hline \multicolumn{5}{|c|}{0.508 Not compensating for water vapor } \\
\hline \multicolumn{5}{|c|}{0.512} \\
\hline \multicolumn{5}{|c|}{0.513} \\
\hline \multicolumn{5}{|c|}{0.511} \\
\hline \multicolumn{5}{|c|}{0.504} \\
\hline \multicolumn{2}{|r|}{0.508} & $=\operatorname{avg}$ & & \\
\hline
\end{tabular}

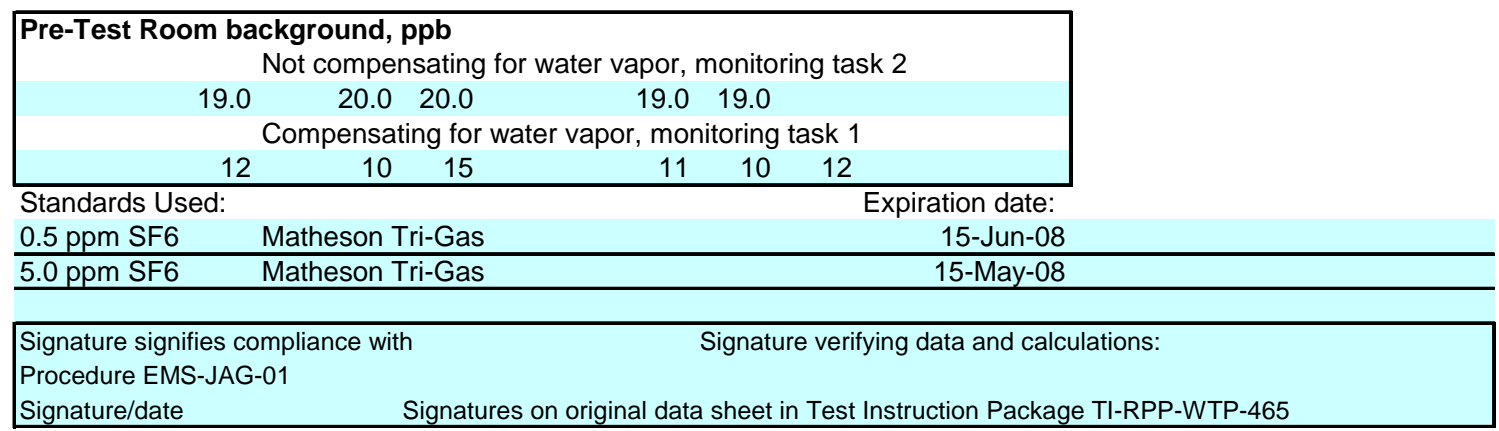

Reference: CCP-WTPSP-185

BK calibration check data sheets.xls

BcalcheckRev0.xls

Jan16_2

4 August 2006EEA7C3E

$4 / 18 / 2007$ 
Sulfur hexafluoride Gas Calibration performed on B\&K on

1/22/2007 by Brad Fritz B\&K Model 1302: Property No. WC74073 Serial No. 1788615

Setup:

$6 \mathrm{ft} \quad B \& K$ sample inlet tube length

1011 mbar station pressure

68 deg $\mathrm{F}$ ambient temp analyzer corrects to 20 deg $\mathrm{C}$

25 percent $\mathrm{RH}$

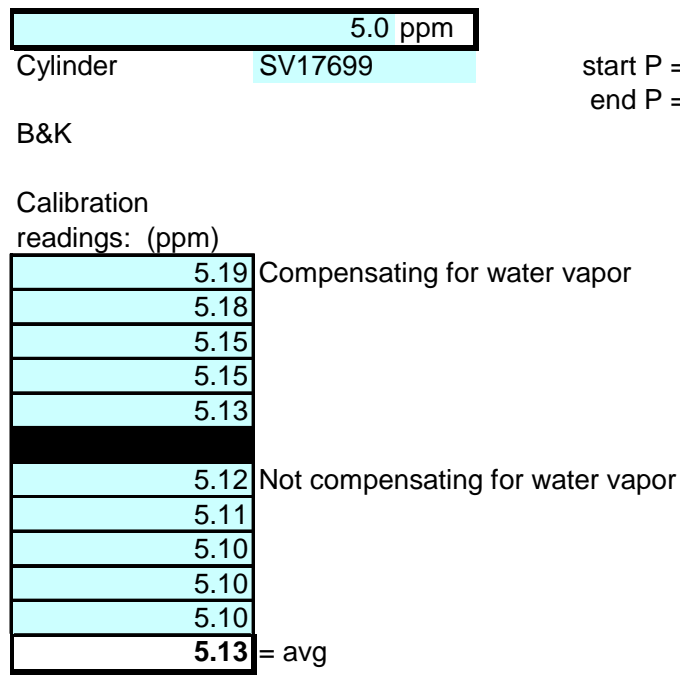

\begin{tabular}{|c|c|c|c|c|}
\hline & & \multicolumn{2}{|c|}{$0.5 \mathrm{ppm}$} & \\
\hline Cylinder & & SV18280 & $\begin{array}{r}\text { start } P= \\
\text { end } P=\end{array}$ & $\begin{array}{l}700 \mathrm{ps} \\
600 \mathrm{ps}\end{array}$ \\
\hline \multicolumn{5}{|l|}{ B\&K } \\
\hline \multicolumn{5}{|c|}{$\begin{array}{l}\text { Calibration } \\
\text { readings: (ppm) }\end{array}$} \\
\hline \multicolumn{5}{|c|}{0.507 Compensating for water vapor } \\
\hline \multicolumn{5}{|c|}{0.508} \\
\hline \multicolumn{5}{|c|}{0.506} \\
\hline \multicolumn{5}{|c|}{0.504} \\
\hline \multicolumn{5}{|c|}{0.506} \\
\hline \multicolumn{5}{|c|}{0.504 Not compensating for water vapor } \\
\hline \multicolumn{5}{|c|}{0.500} \\
\hline \multicolumn{5}{|c|}{0.502} \\
\hline \multicolumn{5}{|c|}{0.499} \\
\hline \multicolumn{5}{|c|}{0.508} \\
\hline \multicolumn{5}{|c|}{$0.504=$} \\
\hline
\end{tabular}

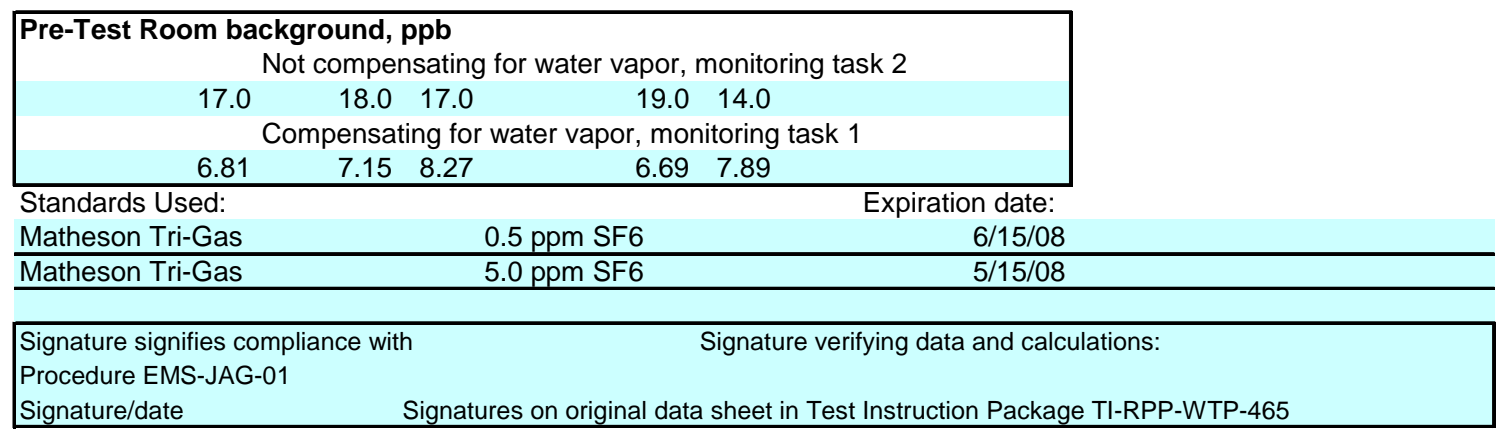

Reference: CCP-WTPSP-185

BK calibration check data sheets.xls

BcalcheckRev0.xls

Jan22

4 August 2006EEA7C3E

$4 / 18 / 2007$ 
Sulfur hexafluoride Gas Calibration performed on B\&K on

1/25/2007 by Brad Fritz

B\&K Model 1302: Property No. WD17210 Serial No. 1765299

Setup:

$6 \mathrm{ft} \quad$ B\&K sample inlet tube length

1009 mbar station pressure

67 deg F ambient temp analyzer corrects to 20 deg C

30 percent $\mathrm{RH}$

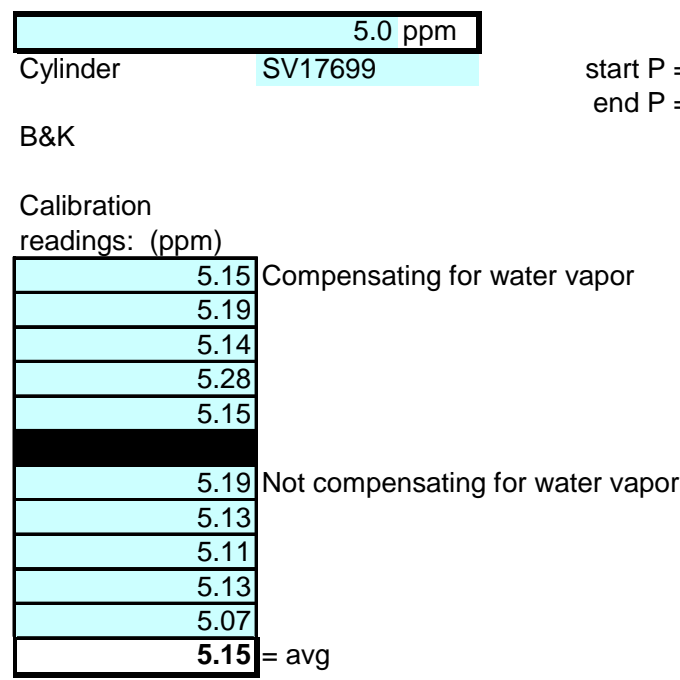

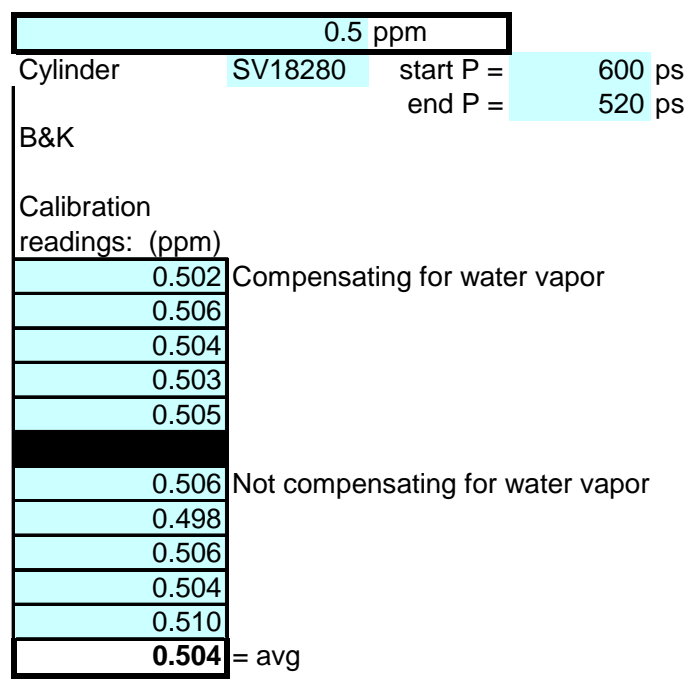

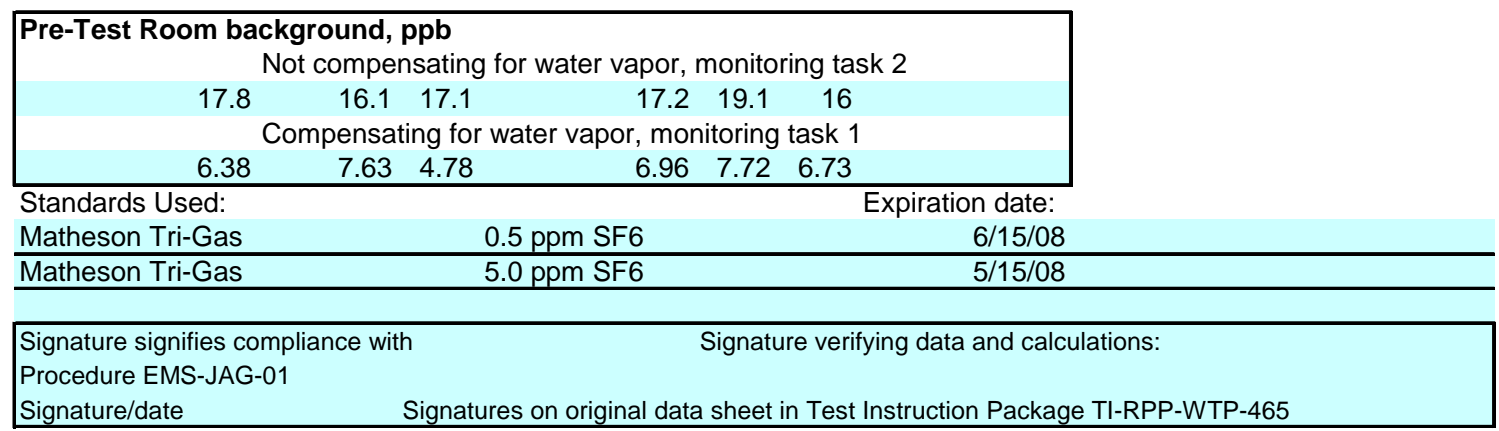

Reference: CCP-WTPSP-185

BK calibration check data sheets.xls

BcalcheckRev0.xls

Jan25

4 August 2006EEA7C3E

$4 / 18 / 2007$ 
Sulfur hexafluoride Gas Calibration performed on B\&K on

2/5/2007 by Brad Fritz B\&K Model 1302: Property No.WC74073 Serial No.1788615

Setup:

$6 \mathrm{ft} \quad$ B\&K sample inlet tube length

1009 mbar station pressure

67 deg F ambient temp analyzer corrects to 20 deg C

40 percent $\mathrm{RH}$

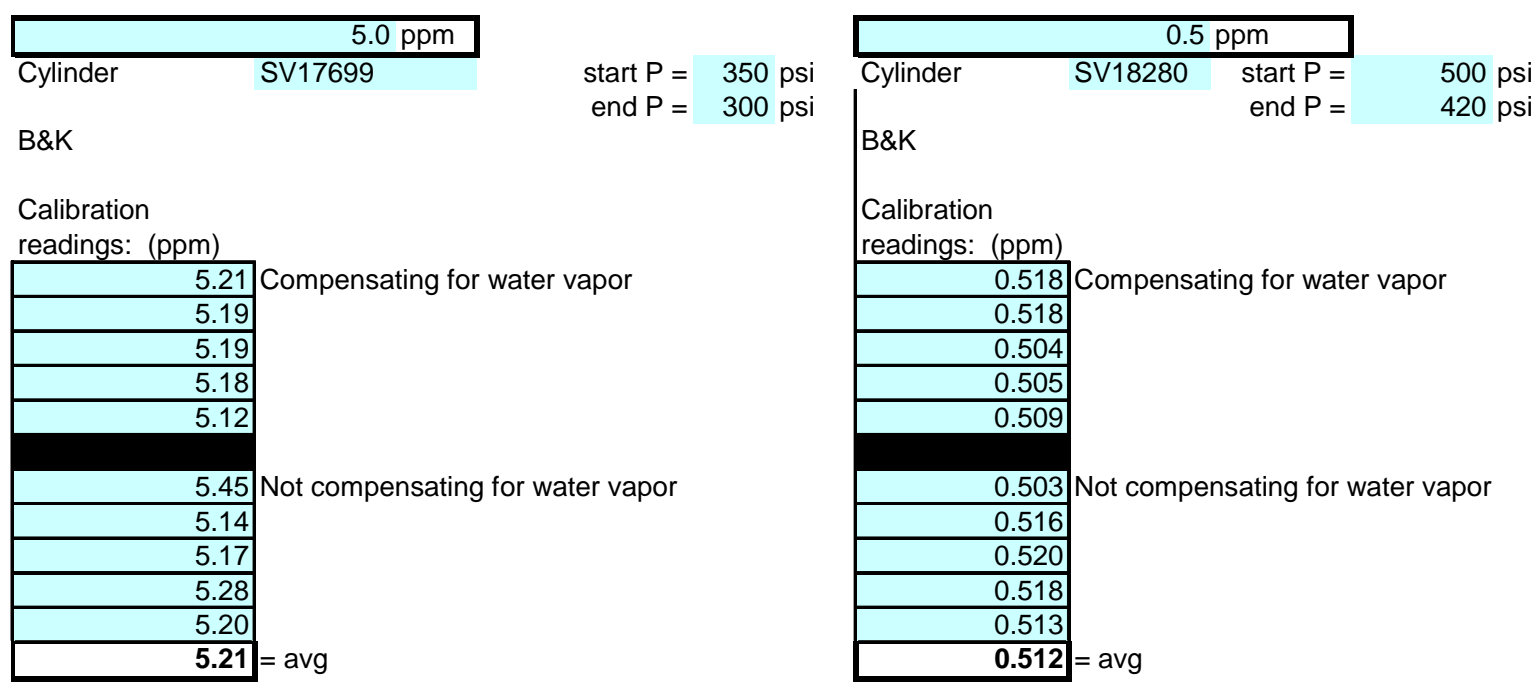

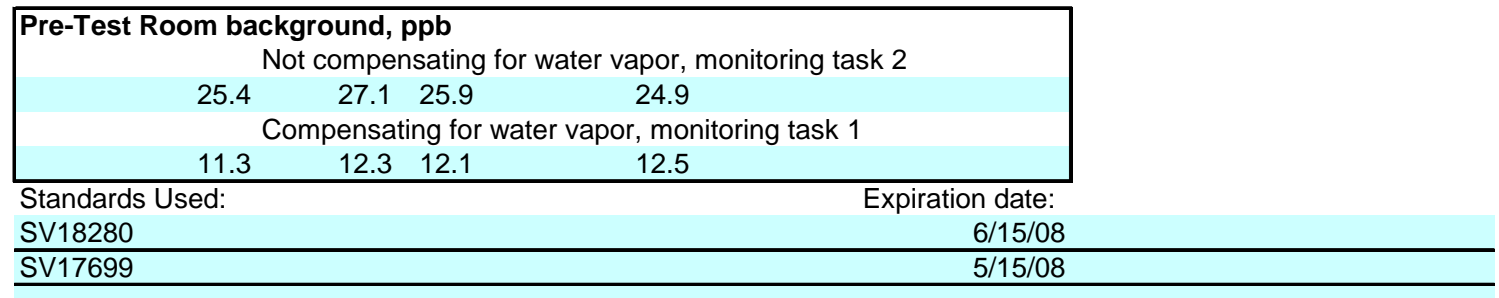

Signature signifies compliance with Signature verifying data and calculations:

Procedure EMS-JAG-01

Signature/date Signatures on original data sheet in Test Instruction Package TI-RPP-WTP-465

Reference: CCP-WTPSP-185

BK calibration check data sheets.xls

BcalcheckRev0.xls

Feb05

4 August 2006EEA7C3E

$4 / 18 / 2007$ 


\section{Appendix F}

\section{Particle-Tracer Uniformity Data Sheets}




\section{Appendix F: Particle-Tracer Uniformity Data Sheets}

Rev. 0

3 Aug. 2006

PARTICLE TRACER TRAVERSE DATA FORM

Site HV-C2 Model

Date 12/6/2006

Tester BGF MSP

Stack Dia

Stack X-Area

Elevation Port 1

Distance to disturbance

Measurement units particles/ft3

Order ----->

Traverse-->

Trial ---->

\begin{tabular}{|r|r|} 
Trial ---> \\
\hline Point & Depth, in. \\
\hline 1 & 0.5 \\
\hline 2 & 1.2 \\
\hline 3 & 2.33 \\
\hline 4 & 3.88 \\
\hline Center & 6.0 \\
\hline 5 & 8.1 \\
\hline 6 & 9.67 \\
\hline 7 & 10.74 \\
\hline 8 & 11.5 \\
\hline
\end{tabular}

Averages --------->>
12 in.

13.1 in. 2

53.5 inches

1 st

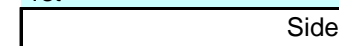

Run No. PT-1

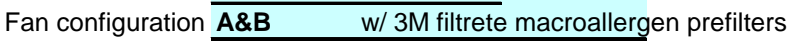

Fan Setting 40

Stack Temp

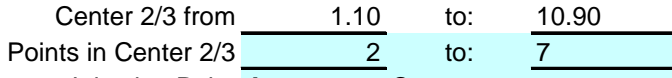

Injection Point $\mathbf{A} \quad$ Center
$\mathrm{Hz}$

2nd

Bottom

\begin{tabular}{cc}
1 & 2 \\
\hline & particles/ft3
\end{tabular}

$3 \quad$ Mean

$1 \quad 2 \quad 3$

3

\begin{tabular}{|l|l|l|l|}
\hline 3325 & 4738 & 4183 & 4082.0 \\
\hline 3773 & 4901 & 4344 & 4339.3 \\
\hline 3691 & 5166 & 4214 & 4357.0 \\
\hline 3731 & 4944 & 4281 & 4318.7 \\
\hline 3290 & 4354 & 3841 & 3828.3 \\
\hline 2530 & 3437 & 2844 & 2937.0 \\
\hline 2273 & 2902 & 2540 & 2571.7 \\
\hline 1311 & 2592 & 2255 & 2314.0 \\
\hline 91.0 & 1792 & 1529 & 1544.0 \\
\hline
\end{tabular}

\begin{tabular}{|c|c|c|c|c|c|c|c|}
\hline$A I I$ & $\mathrm{pt} / \mathrm{ft} 3$ & Dev. from mean & Center 2/3 & Side & Bottom & All & Normlzd \\
\hline Mean & $2 6 \longdiv { 2 6 5 . 1 }$ & & Mean & 1945.3 & 3523.7 & $2734 \overline{.5}$ & 3290.88 \\
\hline Min Point & 599.0 & $-77.4 \%$ & Std. Dev. & 780.7 & 894.3 & 1149.4 & 1059.51 \\
\hline Max Point & 4357.0 & $64.2 \%$ & cov as $\%$ & 40.1 & 25.4 & 42.0 & 32.20 \\
\hline
\end{tabular}

Avg Conc

$$
2593 \mathrm{pt} / \mathrm{ft} 3
$$

Generator Inlet Press

Stack Temp

Centerline vel.

Ambient pressure

Ambient humidity

Ambient temp

Back-Gd aerosol

No. Bk-Gd samples

Compressor output reg

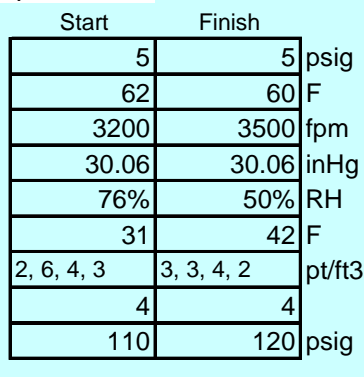

Notes: There was no obvious reason why the count change between runs 1 and 2 on the bottom was so large. Nothing changed in the setups.

Oil Used: $\quad$ FisherBrand 19

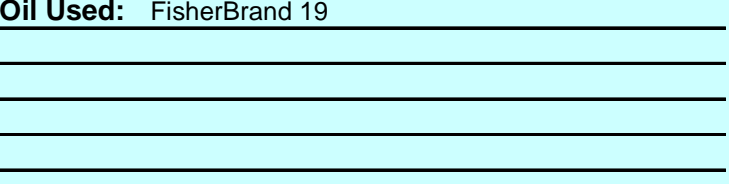

$$
\text { Instuments Used: }
$$

TSI Velocity Calc Plus S/N $209060 \quad 11 / 1 / 2007$

\begin{tabular}{llr}
\hline Met One A2408 & SN 96258675 & $10 / 6 / 2007$ \\
\hline
\end{tabular}

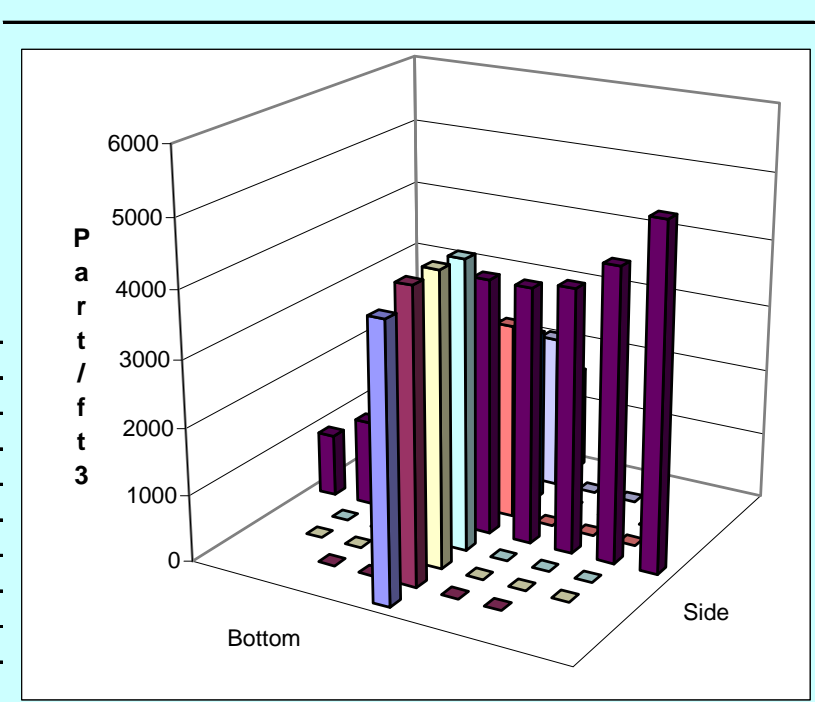

Signature signifies compliance with

Procedure EMS-JAG-02

Signature/date

Signature verifying data and calculations:

Signatures on original data sheet in Test Instruction Package TI-RPP-WTP-466

Reference: CCP-WTPSP-175

(HVC2_part-dataRev0 (7)).xls / PT1

part-dataRev0.xls

$4 / 18 / 2007$

3 August 2006 
Rev. 0

3 Aug. 2006
Site HV-C2 Model

Date 12/6/2006

Tester BGF

Stack Dia. Stack X-Area

Elevation Port 1

Distance to disturbance Measurement units particles/ft3

PARTICLE TRACER TRAVERSE DATA FORM

Run No. PT-2

Fan configuration $\overline{\mathbf{A} \& \mathbf{B}} \quad$ w/ 3M filtrete macroallergen prefilters

Fan Setting $\mathbf{4 0}$

Stack Temp

Start/End Time $1330--1515$

Center $2 / 3$ from

Points in Center $2 / 3$ Injection Point $\mathrm{A}$

\begin{tabular}{|c|c|c|c|}
\hline & 1.10 & to: & 10.90 \\
\hline & 2 & to: & 7 \\
\hline A & \multicolumn{3}{|c|}{ Center } \\
\hline
\end{tabular}

Order ----->

Traverse-->

Trial ---->

\begin{tabular}{|r|r|}
\hline Point & Depth, in \\
\hline 1 & 0.5 \\
\hline 2 & 1.2 \\
\hline 3 & 2.33 \\
\hline 4 & 3.88 \\
\hline Center & 6.0 \\
\hline 5 & 8.1 \\
\hline 6 & 9.67 \\
\hline 7 & 10.7 \\
\hline 8 & 11.5 \\
\hline
\end{tabular}

Averages ---------->

\begin{tabular}{|l|}
\multicolumn{1}{c|}{$2 n$} \\
\end{tabular}
1 st Bottom

Side 12

2

Mean

$12 \quad 3$

\begin{tabular}{|c|c|c|c|c|c|c|}
\hline particte & & & & & 5110 & \\
\hline 886 & 1108 & 1100.3 & 2328 & 1929 & 1814 & 2023.7 \\
\hline
\end{tabular}

\begin{tabular}{|l|r|r|r|r|r|r|r|}
\hline 1130 & 1186 & 984 & 1100.0 & 2409 & 1997 & 2010 & 2138.7 \\
\hline 1197 & 1056 & 993 & 1082.0 & 2571 & 2166 & 2091 & 2276.0 \\
\hline
\end{tabular}

\begin{tabular}{r|r|r|r}
1197 & 1056 & 993 \\
\hline
\end{tabular}

1082.0

1082.0
938.7

$\begin{array}{ll}716 & 1150\end{array}$

$1107 \quad 1028$

766

950

\begin{tabular}{l|r|}
806 & 980.3 \\
\hline
\end{tabular}

\begin{tabular}{|l|r|r|}
618 & 766 & 829 \\
\hline 297 & 548 & 575 \\
\hline 390 & 352 & 398 \\
\hline
\end{tabular}

\begin{tabular}{|l|r|r|}
390 & 352 & 398 \\
\hline 123 & 205 & 189 \\
\hline
\end{tabular}

\begin{tabular}{r|r}
\hline 123 & 2 \\
\hline 65.0 & 797.4 \\
\hline
\end{tabular}

\begin{tabular}{l|r}
\hline 205 & 189 \\
\hline 4 & 759.1 \\
\hline
\end{tabular}

737.7
473.3

380.0

172.3

\begin{tabular}{ll|l}
2571 & 2166 & 2091 \\
\hline
\end{tabular}

\begin{tabular}{|l|l|l|}
2571 & 2166 & 2091 \\
2484 & 2104 & 1970 \\
\hline
\end{tabular}

\begin{tabular}{l|l|l|}
2484 & 2104 & 1970 \\
\hline 1496 & 1744 & 1711 \\
\hline 1096 & 1260 & 1153 \\
\hline
\end{tabular}

\begin{tabular}{r|r|r|}
1496 & 1260 & 1153 \\
\hline 1096 & 982 & 957
\end{tabular}

\begin{tabular}{r|r|r|}
\hline 1096 & 982 & 957 \\
\hline 807 & 774 & 675 \\
\hline 405 & 386 & 562 \\
\hline
\end{tabular}

\begin{tabular}{r|r}
405 & 386 \\
\hline
\end{tabular}

\begin{tabular}{|c|c|c|c|c|c|c|c|}
\hline$A / I$ & $\mathrm{pt} / \mathrm{ft} 3$ & Dev. from mean & Center 2/3 & Side & Bottom & All & Normlzd \\
\hline Mean & 1164.2 & & Mean & 813.1 & 1645.2 & 1229.2 & 1589.59 \\
\hline Min Point & 172.3 & $-85.2 \%$ & Std. Dev. & 290.7 & 618.0 & 633.8 & 564.30 \\
\hline Max Point & 2276.0 & $95.5 \%$ & COV as \% & 35.8 & 37.6 & 51.6 & 35.50 \\
\hline
\end{tabular}

\section{Avg Conc} $1133 \mathrm{pt} / \mathrm{ft} 3$

Generator Inlet Press

Stack Temp

Centerline vel.

Ambient pressure Ambient humidity

Ambient temp

Back-Gd aerosol

No. Bk-Gd samples

Compressor output reg

\begin{tabular}{|c|c|c|}
\hline Start & Finish & \\
\hline 3 & 3 & 3 psig \\
\hline 60 & 60 & $0 \mathrm{~F}$ \\
\hline 3500 & 3380 & 0 fpm \\
\hline 30.06 & 30.06 & $6 \mathrm{inHg}$ \\
\hline $50 \%$ & $70 \%$ & $0 \mathrm{RH}$ \\
\hline 42 & 36 & $6 \mathrm{~F}$ \\
\hline $3,3,4,2$ & $3,5,5,7$ & $\mathrm{pt} / \mathrm{ft} 3$ \\
\hline 4 & 4 & 4 \\
\hline 120 & 110 & 0 psig \\
\hline
\end{tabular}

Notes:

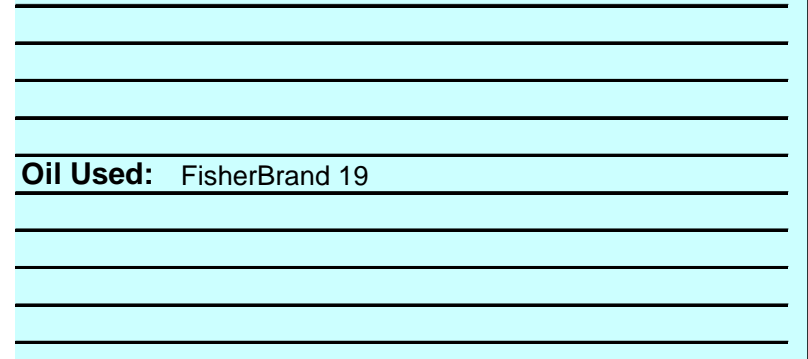

Instuments Used:

TSI Velocity Calc Plus S/N 209060 Cal. Due

35.50

\begin{tabular}{llr} 
TSI Velocity Calc Plus & S/N 209060 & $11 / 1 / 2007$ \\
\hline Met One A2408 & SN 96258675 & $10 / 6 / 2007$ \\
\hline
\end{tabular}

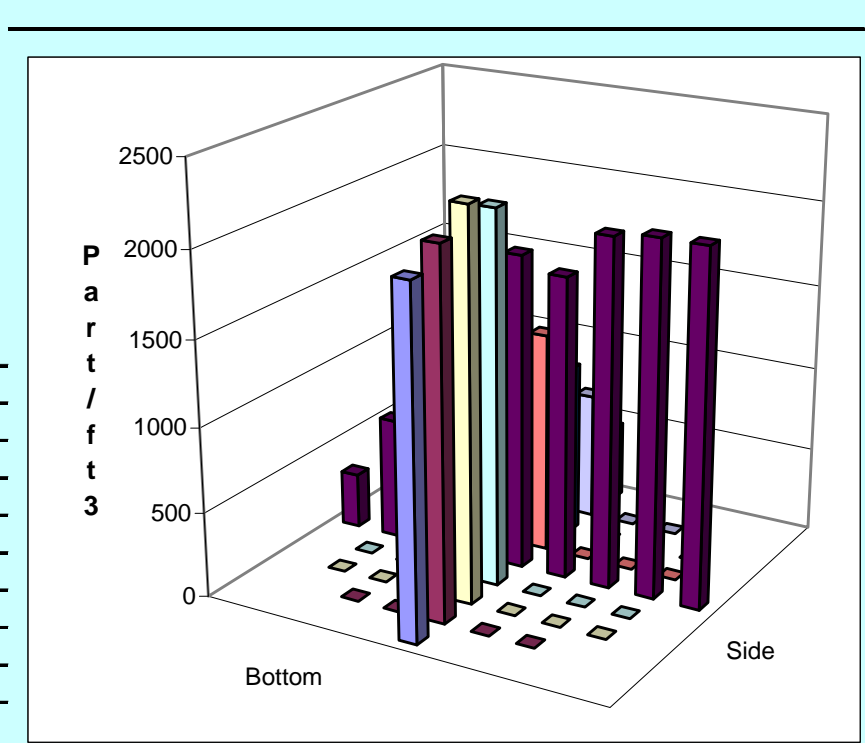

Signature signifies compliance with Procedure EMS-JAG-02

Signature/date

Signature verifying data and calculations:

Reference: CCP-WTPSP-175

part-dataRev0.xls

(HVC2_part-dataRev0 (7)).xls / PT2

3 August 2006

Signatures on original data sheet in Test Instruction Package TI-RPP-WTP-466 
Rev. 0

3 Aug. 2006

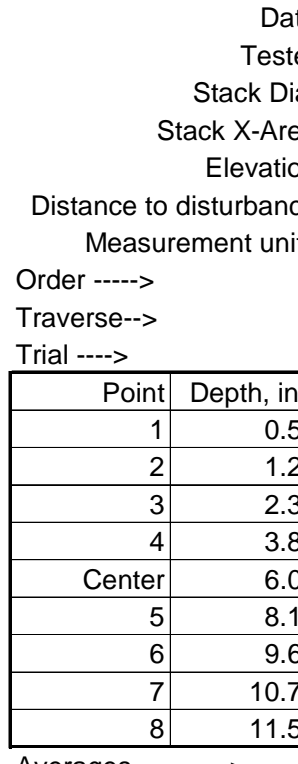

Site $\frac{\text { HV-C2 Model }}{12 / 7 / 2006}$

Tester JGD JAG

\begin{aligned} Stack Dia. & 12 in. \\ Stack X-Area & 113.1 in. 2 \\ Elevation & Port 1 \\ \cline { 2 - 2 } o disturbance & 53.5 inches \end{aligned}

Measurement units particles/ft3

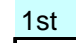

1 st

Side

\begin{tabular}{|l|l|}
\hline in. & \\
\hline .50 & \\
\hline .26 & \\
\hline .33 & \\
\hline .88 & \\
\hline .00 & \\
\hline .12 & \\
\hline .67 & \\
\hline .74 & \\
\hline .50 & \\
\hline
\end{tabular}

\begin{tabular}{|r|r|}
\hline 967 & 7 \\
1115 & 10 \\
\hline 1106 & 11 \\
\hline 1078 & 11 \\
\hline 984 & 1099 \\
\hline 756 & 937 \\
\hline 575 & 701 \\
\hline 434 & 436 \\
\hline 241 & 304 \\
\hline 06.2 & 842.4 \\
\hline
\end{tabular}

PARTICLE TRACER TRAVERSE DATA FORM

Run No. PT-3

Fan configuration $\overline{\mathbf{A} \& \mathbf{B}} \quad \mathrm{w} / 3 \mathrm{M}$ filtrete macroallergen prefilters

Fan Setting $\mathbf{4 0}$

Stack Temp

$1025-1200$

Center $2 / 3$ from

Points in Center $2 / 3$

Injection Point $\overline{\mathbf{A}}$

$\begin{array}{lrl}1.10 & \text { to: } & 10.90 \\ 2 & \text { to: } & \\ \text { Center } & \end{array}$
2nd

$3 \quad$ Mean $12^{\text {Bottom }}$

particles/ft3

$3 \quad$ Mean

\begin{tabular}{|r|r|r|r|}
\hline \multicolumn{4}{|c|}{ particles/ft3 } \\
\hline 1201 & 1810 & 1592 & 1534.3 \\
\hline 1849 & 2143 & 1886 & 1959.3 \\
\hline 2013 & 2339 & 1928 & 2093.3 \\
\hline 1988 & 2152 & 1920 & 2020.0 \\
\hline 1841 & 1866 & 1655 & 1787.3 \\
\hline 1339 & 1280 & 1175 & 1264.7 \\
\hline 862 & 950 & 807 & 906.3 \\
\hline 819 & 736 & 701 & 752.0 \\
\hline 388 & 406 & 347 & 380.3 \\
\hline 377.8 & 1520.2 & 1334.6 & 1410.9 \\
\hline
\end{tabular}

\begin{tabular}{|r|r|}
\hline 780 & \\
\hline 1048 & \\
\hline 1128 & \\
\hline 1149 & \\
\hline 1099 & \\
\hline 937 & \\
\hline 701 & \\
\hline 436 & \\
\hline 304 & \\
\hline 2.4 & \\
\hline
\end{tabular}

\begin{tabular}{|r|}
\hline 1088 \\
1278 \\
1227 \\
1209 \\
1106 \\
950 \\
727 \\
532 \\
\hline 398 \\
\hline 46.1
\end{tabular}

\begin{tabular}{|r|}
\hline 945.0 \\
1147.0 \\
\hline 1153.7 \\
\hline 1145.3 \\
\hline 1063.0 \\
\hline 881.0 \\
\hline 667.7 \\
\hline 467.3 \\
\hline 314.3 \\
\hline 864.9
\end{tabular}

\begin{tabular}{|c|c|c|c|c|}
\hline \multirow[b]{2}{*}{ Avg Conc } & \begin{tabular}{|l}
$A I I$ \\
Mean \\
Min Point \\
Max Point
\end{tabular} & $\begin{array}{r}\mathrm{pt} / \mathrm{ft} 3 \\
1137.9 \\
314.3 \\
2093.3\end{array}$ & $\underline{\mathrm{Dev}}$ & v. from \\
\hline & \multicolumn{2}{|c|}{$1102 \mathrm{pt} / \mathrm{ft} 3$} & & \\
\hline \multirow{3}{*}{\multicolumn{2}{|c|}{$\begin{array}{l}\text { Generator Inlet Press } \\
\text { Stack Temp }\end{array}$}} & Start & Finish & \\
\hline & & 35 & 37 & 7 psig \\
\hline & & 60 & 60 & $\mathrm{~F}$ \\
\hline \multirow{2}{*}{\multicolumn{2}{|c|}{$\begin{array}{l}\text { Centerline vel. } \\
\text { Ambient pressure }\end{array}$}} & 3280 & 3250 & fpm \\
\hline & & 29.99 & 29.96 & $\mathrm{inHg}$ \\
\hline \multicolumn{2}{|c|}{ Ambient humidity } & $31 \%$ & $27 \%$ & $\mathrm{RH}$ \\
\hline \multicolumn{2}{|c|}{ Ambient temp } & $\begin{array}{r}61 \\
\end{array}$ & 64 & $\mathrm{~F}$ \\
\hline \multicolumn{2}{|c|}{ Back-Gd aerosol } & $6,0,2,5$ & $1,4,0,1$ & $\mathrm{pt} / \mathrm{ft} 3$ \\
\hline \multirow{2}{*}{\multicolumn{2}{|c|}{$\begin{array}{l}\text { No. Bk-Gd samples } \\
\text { Compressor output reg }\end{array}$}} & 4 & 4 & 4 \\
\hline & & 115 & 112 & 2 psig \\
\hline
\end{tabular}

Notes:

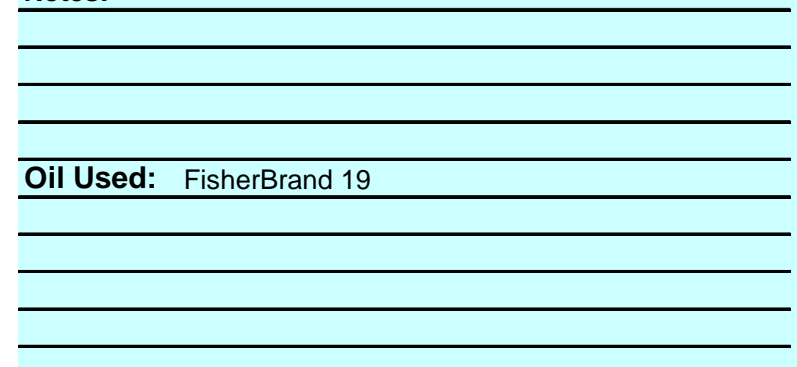

$-72.4 \%$
$84.0 \%$

\begin{tabular}{|lr}
\hline Center 2/3 & Side \\
\hline Mean & 932.1 \\
Std. Dev. & 272.8 \\
CoV as \% & 29.3 \\
\hline
\end{tabular}

\begin{tabular}{|c|c|c|}
\hline Bottom & All & Normlzd \\
\hline 1540.4 & 1236.3 & 1553.87 \\
\hline 558.6 & 527.2 & 491.19 \\
\hline 36.3 & 42.6 & 31.61 \\
\hline
\end{tabular}

Instuments Used:

TSI Velocity Calc Plus S/N $209060 \quad$ 11/1/2007

\begin{tabular}{llr} 
& $11 / 1 / 2007$ \\
\hline Met One A2408 & SN 96258675 & $10 / 6 / 2007$ \\
\hline
\end{tabular}

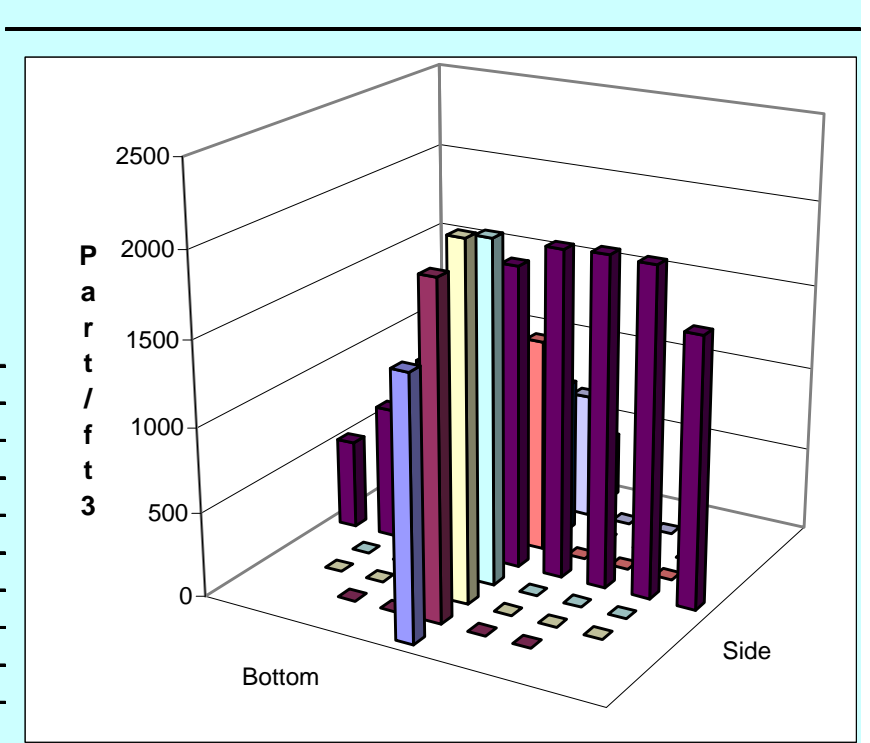

Signature signifies compliance with Procedure EMS-JAG-02

Signature/date

Signature verifying data and calculations:

Reference: CCP-WTPSP-175

part-dataRev0.xls

(HVC2_part-dataRev0 (7)).xls / PT3

3 August 2006

Signatures on original data sheet in Test Instruction Package TI-RPP-WTP-466 
Rev. 0

3 Aug. 2006

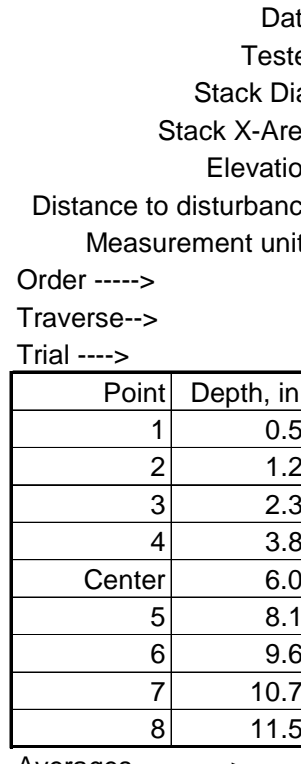

Site HV-C2 Model

Date 12/7/2006

Tester JGD

$\begin{aligned} \text { Stack Dia. } & 12 \text { in. } \\ \text { Stack X-Area } & 113.1 \text { in.2 } \\ \text { Elevation } & \end{aligned}$

53.5 inches

particles/ft3

2nd
CER TRAVERSE DATA FORM

Run No. PT-4

Fan configuration $\overline{A \& B} \quad$ w/ 3M filtrete macroallergen prefilters

Fan Setting $\mathbf{4 0}$

Stack Temp

Center 2/3 from

Points in Center $2 / 3$

Injection Point $\frac{}{\mathbf{A}}$ Center

1.10

$\frac{10}{2}$ to:

to: $\quad 10.90$

1st $\quad$ Bottom

\begin{tabular}{|l|r|r|r|r|r|r|r|r|r|} 
& 333 & 239 & 309 & 293.7 & 502 & 481 & 455 & 479.3 \\
\hline Averages -------> & 968.7 & 964.2 & 970.9 & 967.9 & 2206.4 & 2115.6 & 1996.6 & 2106.2 \\
\hline
\end{tabular}

\begin{tabular}{|c|c|c|c|c|c|c|c|}
\hline$A / I$ & $\mathrm{pt} / \mathrm{ft} 3$ & Dev. from mean & Center 2/3 & $\underline{\text { Side }}$ & Bottom & All & Normlzd \\
\hline Mean & 1537.1 & & Mean & 1031.9 & 2268.1 & 1650.0 & 2159.87 \\
\hline Min Point & 293.7 & $-80.9 \%$ & Std. Dev. & 333.5 & 886.8 & 908.7 & 760.56 \\
\hline Max Point & 3106.3 & $102.1 \%$ & COV as $\%$ & 32.3 & 39.1 & 55.1 & 35.21 \\
\hline
\end{tabular}

Avg Conc $1492 \mathrm{pt} / \mathrm{ft} 3$

Generator Inlet Press

Stack Temp

Centerline vel.

Ambient pressure

Ambient humidity

Ambient temp

Back-Gd aerosol

No. Bk-Gd samples

Compressor output reg

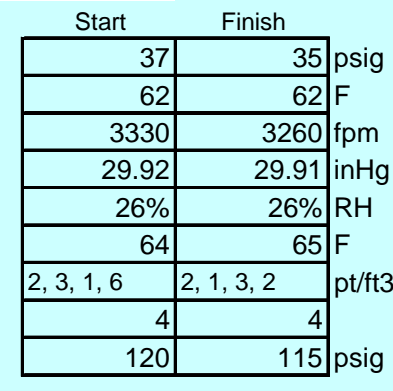

Notes:

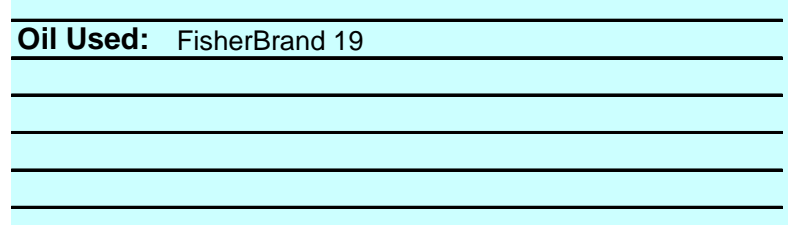

Instuments Used:

TSI Velocity Calc Plus S/N $209060 \quad 11 / 1 / 2007$

\begin{tabular}{lrr}
\hline Met One A2408 & $11 / 1 / 2007$ \\
\hline
\end{tabular}

$\longrightarrow$

Signature signifies compliance with Procedure EMS-JAG-02

Signature/date

Signature verifying data and calculations:

Reference: CCP-WTPSP-175

part-dataRev0.xls

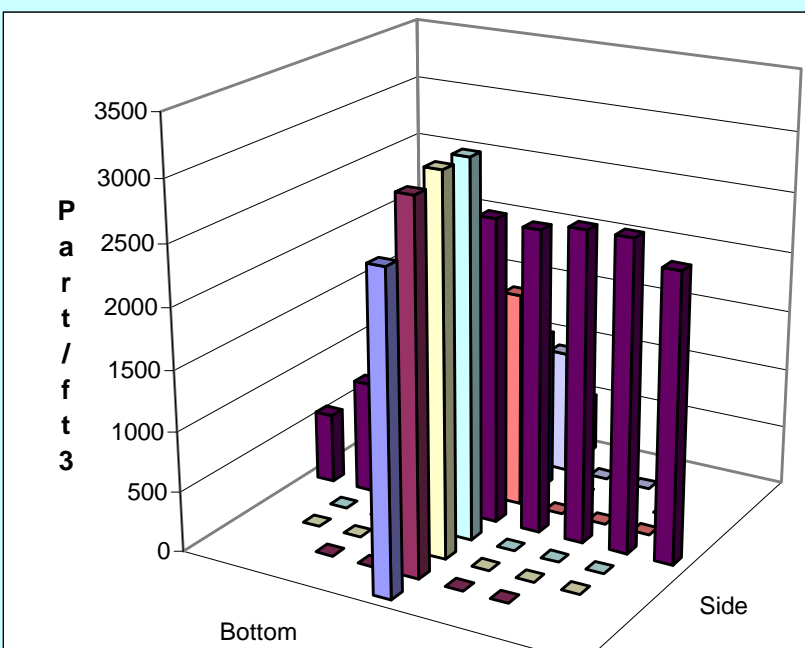

3 August 2006

(HVC2_part-dataRev0 (7)).xIs / PT4 $4 / 18 / 2007$ 
Rev. 0

3 Aug. 2006

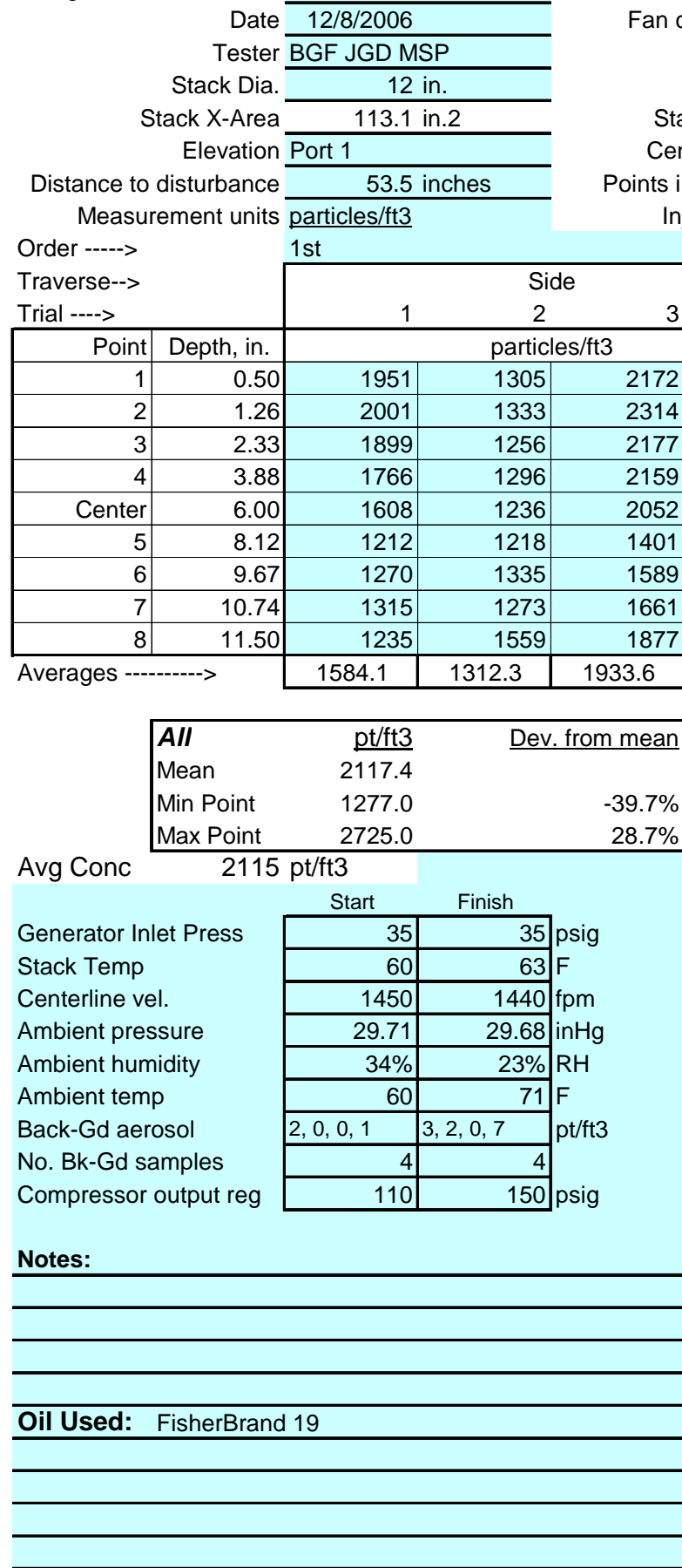

Signature signifies compliance with Procedure EMS-JAG-02

Signature/date

Signature verifying data and calculations:

Signatures on original data sheet in Test Instruction Package TI-RPP-WTP-466

Reference: CCP-WTPSP-175

part-dataRev0.xls

3 August 2006
Run No, PT-5

an configuration $\mathbf{A}$

Fan Setting 35

Stack Temp

Center $2 / 3$ from

in Center $2 / 3$

Injection Point $\mathbf{A}$

1.10

w/ 3M filtrete macroallergen prefilters

$\mathrm{Hz}$

\begin{tabular}{r|r|r|r|r|}
\cline { 2 - 5 } Mean & \multicolumn{4}{|c|}{ Bottom } \\
\hline & \multicolumn{4}{|c|}{ particles/ft3 } \\
\hline 1809.3 & 2699 & 2549 & 2643 & 2630.3 \\
\hline 1882.7 & 2625 & 2695 & 2644 & 2654.7 \\
\hline 1777.3 & 2577 & 2698 & 2531 & 2602.0 \\
\hline 1740.3 & 2754 & 2703 & 2493 & 2650.0 \\
\hline 1632.0 & 2691 & 2687 & 2525 & 2634.3 \\
\hline 1277.0 & 2647 & 2607 & 2486 & 2580.0 \\
\hline 1398.0 & 2795 & 2666 & 2673 & 2711.3 \\
\hline 1416.3 & 2745 & 2717 & 2713 & 2725.0 \\
\hline 1557.0 & 2368 & 2407 & 2533 & 2436.0 \\
\hline 1610.0 & 2655.7 & 2636.6 & 2582.3 & 2624.9 \\
\hline
\end{tabular}

\begin{tabular}{lrrrrr|}
\hline Center 2/3 & & Side & Bottom & $\underline{\text { All }}$ & Normlzd \\
Mean & 1589.1 & 2651.0 & 2120.1 & 2608.06 \\
Std. Dev. & 227.3 & 53.0 & 573.4 & 255.81 \\
COV as \% & 14.3 & 2.0 & $\mathbf{2 7 . 0}$ & $\mathbf{9 . 8 1}$ \\
\hline
\end{tabular}

Instuments Used:

Cal. Due

TSI Velocity Calc Plus S/N $209060 \quad 11 / 1 / 2007$

\begin{tabular}{llr}
\hline Met One A2408 & SN 96258675 & $11 / 6 / 2007$ \\
\hline
\end{tabular}

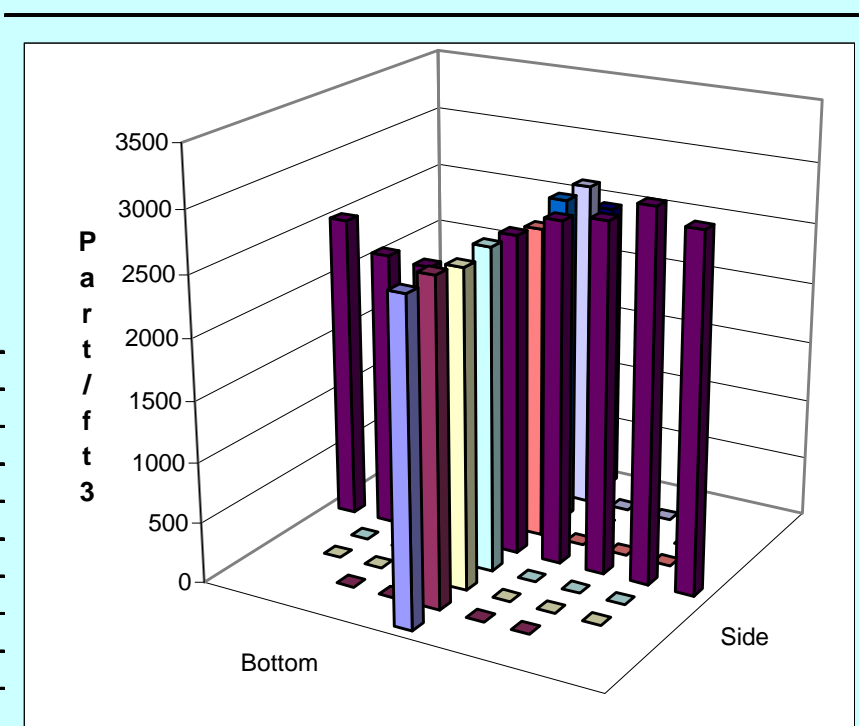

Bottom

(HVC2_part-dataRev0 (7)).xls / PT5 $4 / 18 / 2007$ 
Rev. 0

3 Aug. 2006 Site $\frac{\text { HV-C2 Model }}{12 / 8 / 2006}$

Tester BGF JGD MSP

Stack Dia

12 in.

Stack X-Area

Elevation Port 1

Distance to disturbance Measurement units particles/ft3

Order ----->

Traverse-->

Trial ---->

\begin{tabular}{|r|r|}
\hline Point & Depth, in. \\
\hline 1 & 0.5 \\
\hline 2 & 1.2 \\
\hline 3 & 2.33 \\
\hline 4 & 3.8 \\
\hline Center & 6.0 \\
\hline 5 & 8.1 \\
\hline 6 & 9.67 \\
\hline 7 & 10.7 \\
\hline 8 & 11.5 \\
\hline
\end{tabular}

Averages ----------> 2nd

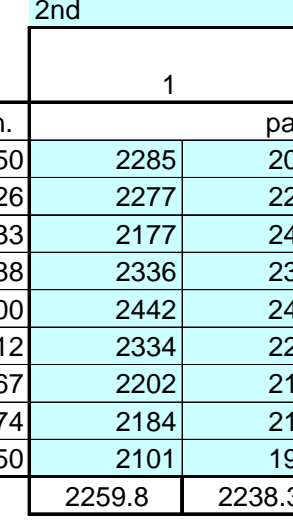

Run No. PT-6

Fan configuration $\mathbf{B}$

Fan Setting 35

Stack Temp

Start/End Time 1040 -- 1245

Center 2/3 from

Points in Center 2/3

Injection Point $\mathbf{B}$ w/ 3M filtrete macroallergen prefilters

$\mathrm{Hz}$

$60 \operatorname{deg} \mathrm{F}$

1.10 to: 10.90

2 to: 7 Center $1 \mathrm{st}$

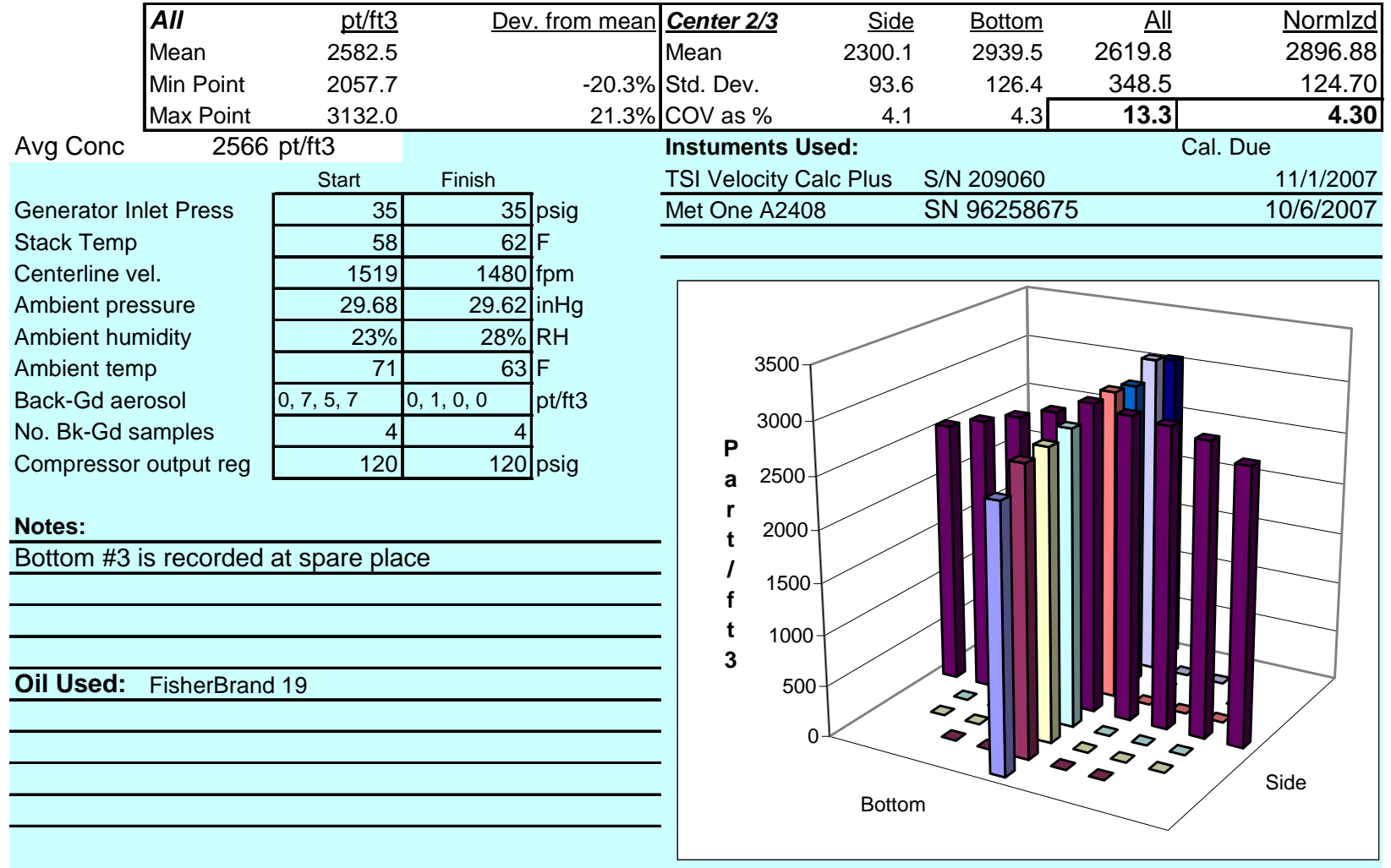

Signature signifies compliance with Procedure EMS-JAG-02

Signature/date

Reference: CCP-WTPSP-175

part-dataRev0.xls

3 August 2006
Signature verifying data and calculations:

Signatures on original data sheet in Test Instruction Package TI-RPP-WTP-466

(HVC2_part-dataRev0 (7)).xIs / PT6 $4 / 18 / 2007$ 
Rev. 0

3 Aug. 2006

PARTICLE TRACER TRAVERSE DATA FORM

Site HV-C2 Model

Date 12/8/2006

Tester JGD MSP

Stack Dia.

Stack X-Area

Elevation Port 1

Distance to disturbance Measurement units particles/ft 3

12 in.

53.5 inches

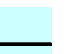

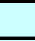

Fan configuration A \& B

Fan Setting $\mathbf{4 0}$

Stack Temp

Start/End Time

Center $2 / 3$ from

Points in Center $2 / 3$

Injection Point $\mathbf{A}$

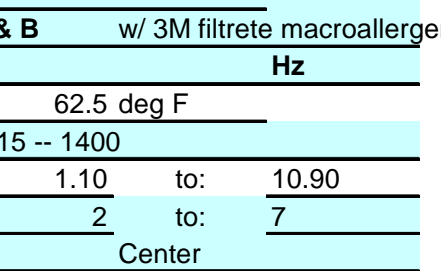
2nd

Center
Order ----->

Traverse-->

Trial ---->

\begin{tabular}{|c|c|}
\hline Point & Depth, in. \\
\hline 1 & 0.5 \\
\hline 2 & 1.2 \\
\hline 3 & 2.3 \\
\hline 4 & 3.8 \\
\hline Center & 6.0 \\
\hline 5 & 8.1 \\
\hline 6 & 9.6 \\
\hline 7 & 10.7 \\
\hline 8 & 11.5 \\
\hline
\end{tabular}

1st

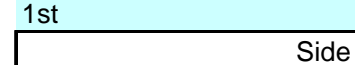

\begin{tabular}{|c|c|}
\hline \multicolumn{2}{|c|}{1} \\
\hline 1811 & $\frac{\mathrm{pa}}{17}$ \\
\hline 2424 & 22 \\
\hline $24 \angle 4$ & $\angle 2$ \\
\hline 2566 & 25 \\
\hline 2386 & 23 \\
\hline 2059 & 21 \\
\hline 1549 & 15 \\
\hline 1105 & 12 \\
\hline 879 & 8 \\
\hline 396 & 3 \\
\hline 1686.1 & 1664.6 \\
\hline
\end{tabular}

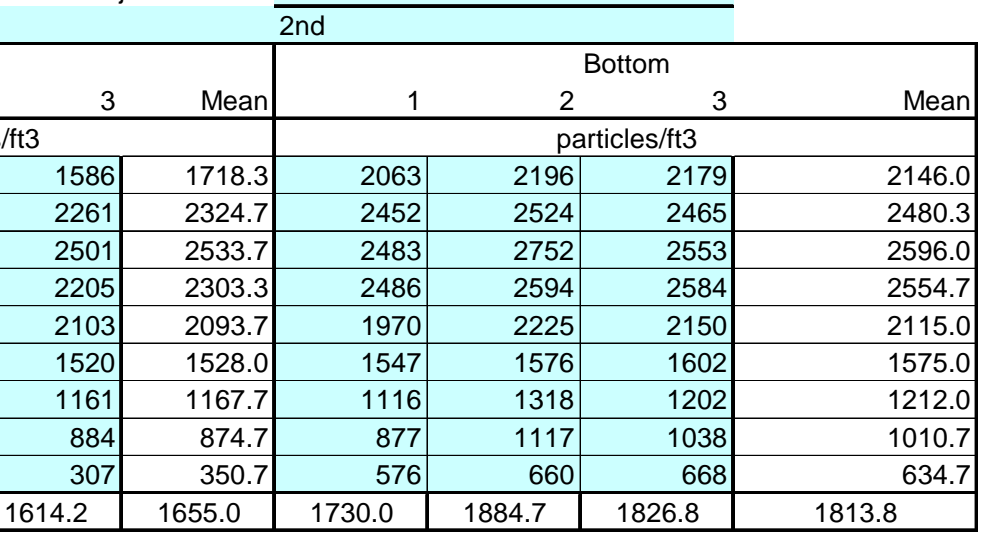

Averages ---------->

\begin{tabular}{|c|c|}
\hline \multicolumn{2}{|c}{2} \\
\hline \multicolumn{2}{|c}{ particles/ft3 } \\
\hline 1758 & \\
\hline 2289 & \\
\hline 2534 & \\
\hline 2319 & \\
\hline 2119 & \\
\hline 1515 & \\
\hline 1237 & \\
\hline 861 & \\
\hline 349 & \\
\hline 64.6 & 161 \\
\hline
\end{tabular}

$$
\mathrm{pt} / \mathrm{ft} 3
$$

\begin{abstract}
Dev. from mean
\end{abstract}

$-79.8 \%$
$49.7 \%$

Center $2 / 3$
Mean
Std. Dev.
COV as \%

$\begin{array}{r}\text { Side } \\ 1832.2 \\ 642.4 \\ 35.1 \\ \hline\end{array}$

\begin{tabular}{rrr|}
$\frac{\text { Bottom }}{1934.8}$ & \multicolumn{1}{c}{ All } & Normlzd \\
665.5 & 1883.5 & 1892.86 \\
\cline { 2 - 3 } 34.4 & 630.6 & 632.98 \\
\hline
\end{tabular}

Avg Conc

Min Point

1734.4
350.7

Max Point $\quad 2596.0$

Instuments Used:

TSI Velocity Calc Plus S/N 209060

Cal. Due

Generator Inlet Press

Stack Temp

Centerline vel.

Ambient pressure

Ambient humidity

Ambient temp

Back-Gd aerosol

No. Bk-Gd samples

Compressor output reg

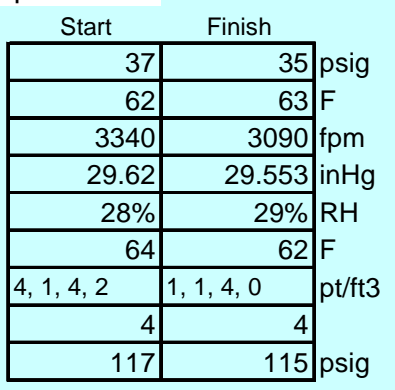

Notes:

Same probe used on top and bottom

Analyzer turned on end for side port

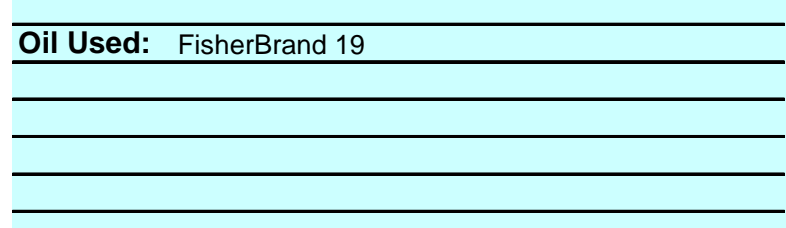

\begin{tabular}{llr} 
TSI Velocity Calc Plus & S/N 209060 & $11 / 1 / 2007$ \\
\hline Met One A2408 & SN 96258675 & $10 / 6 / 2007$ \\
\hline & & \\
\hline
\end{tabular}

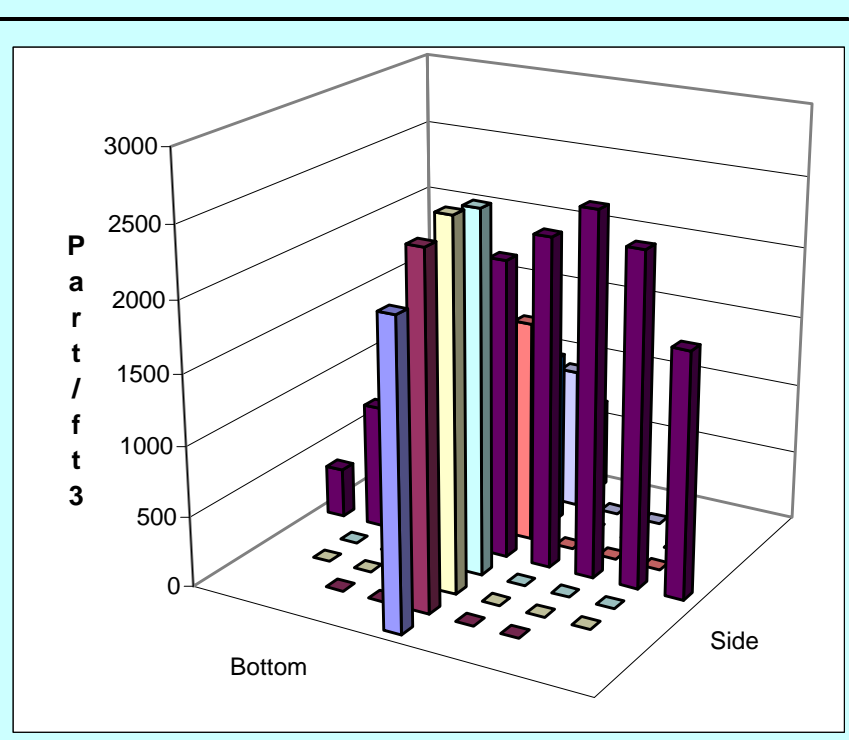

Signature signifies compliance with Procedure EMS-JAG-02

Signature/date

Signature verifying data and calculations:

Reference: CCP-WTPSP-175

part-dataRev0.xIs

(HVC2_part-dataRev0 (7)).xls / PT7

part-dataRev0.
3 August 2006

Signatures on original data sheet in Test Instruction Package TI-RPP-WTP-466 
Rev. 0

3 Aug. 2006

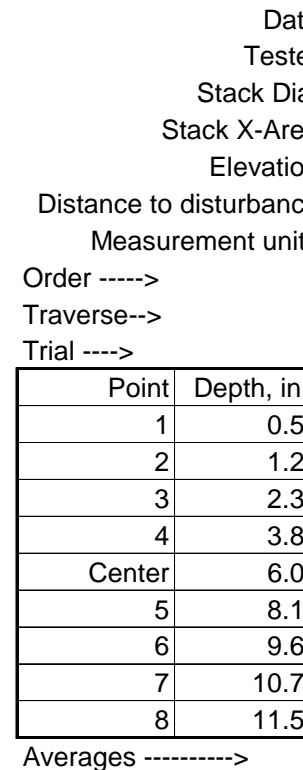

PARTICLE TRACER TRAVERSE DATA FORM

Site HV-C2 Model

Date 12/11/2006

ester JGD MSP

X-Area 113.1 in. 2

Port 2

113.75 inches

$$
\text { particles/ft3 }
$$

$1 \mathrm{st}$

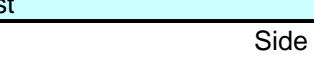

Run No. PT-8

Fan configuration $\begin{array}{ll}\mathbf{A} \& \mathbf{B} & \text { w/ 3M filtrete macroallergen prefilters }\end{array}$

Fan Setting $\mathbf{4 0}$

Stack Temp

Center $2 / 3$ from

Points in Center $2 / 3$

Injection Point $\mathbf{A}$
$62.5 \mathrm{deg}$ $\mathrm{Hz}$

1.10

2 to:

to: $\quad 10.90$ Center 2nd

r|rom

\begin{tabular}{|c|c|c|c|c|c|c|c|}
\hline 1 & 2 & 3 & Mean & 1 & 2 & 3 & Mean \\
\hline \multicolumn{4}{|c|}{ particles/ft3 } & \multicolumn{4}{|c|}{ particles/ft3 } \\
\hline 2014 & 2064 & 2113 & 2063.7 & 1498 & 1530 & 1800 & 1609.3 \\
\hline 2133 & 2141 & 2227 & 2167.0 & 1783 & 1821 & 2180 & 1928.0 \\
\hline 2264 & 2213 & 2270 & 2249.0 & 1947 & 1991 & 2407 & 2115.0 \\
\hline 2266 & 2473 & 2348 & 2362.3 & 2110 & 2128 & 2560 & 2266.0 \\
\hline 2220 & 2212 & 2261 & 2231.0 & 2333 & 2284 & 2685 & 2434.0 \\
\hline 1816 & 1862 & 1813 & 1830.3 & 2121 & 2141 & 2539 & 2267.0 \\
\hline 1415 & 1402 & 1429 & 1415.3 & 1768 & 2064 & 2262 & 2031.3 \\
\hline 1082 & 1138 & 1256 & 1158.7 & 1588 & 1654 & 1956 & 1732.7 \\
\hline 951 & 978 & 929 & 952.7 & 1343 & 1229 & 1742 & 1438.0 \\
\hline 95.7 & 1831.4 & 1849.6 & 1825.6 & 1832.3 & 1871.3 & 2236.8 & 1980.1 \\
\hline
\end{tabular}

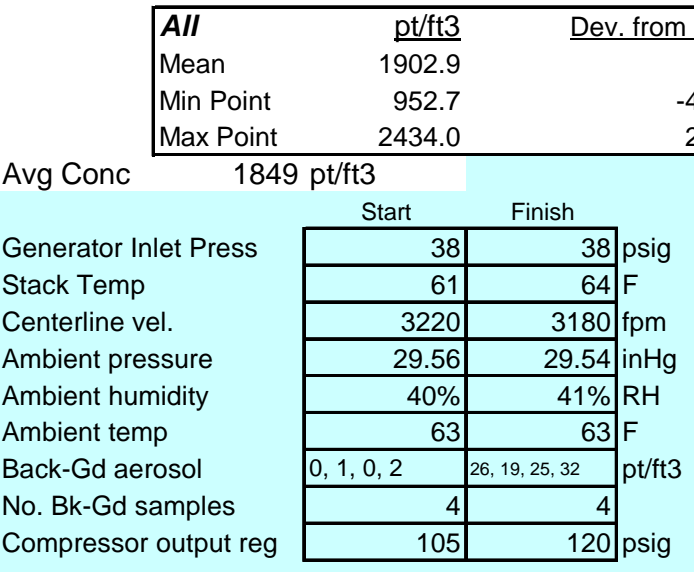

Notes:

Started raining at Bottom transect \#2.

\section{Oil Used: $\quad$ FisherBrand 19}

\begin{tabular}{r|l} 
& Center $2 / 3$ \\
$-49.9 \%$ & Mean \\
$27.9 \%$ & Std. Dev. \\
COV as \%
\end{tabular}

$\begin{array}{r}\text { Side } \\ 1916.2 \\ 466.2 \\ 24.3 \\ \hline\end{array}$

\begin{tabular}{rrr|}
\hline Bottom & $\underline{\text { All }}$ & $\frac{\text { Normlzd }}{2100.58}$ \\
2110.6 & 2013.4 & 381.20 \\
236.5 & 369.2 & $\mathbf{1 8 . 1 5}$ \\
\cline { 2 - 3 } & $\mathbf{1 8 . 3}$ &
\end{tabular}

Instuments Used:

Cal. Due

\begin{tabular}{llr} 
TSI Velocity Calc Plus S/N 209060 & $11 / 1 / 2007$ \\
\hline
\end{tabular}

\begin{tabular}{llr}
\hline Met One A2408 & SN 96258675 & $11 / 6 / 2007$ \\
\hline
\end{tabular}

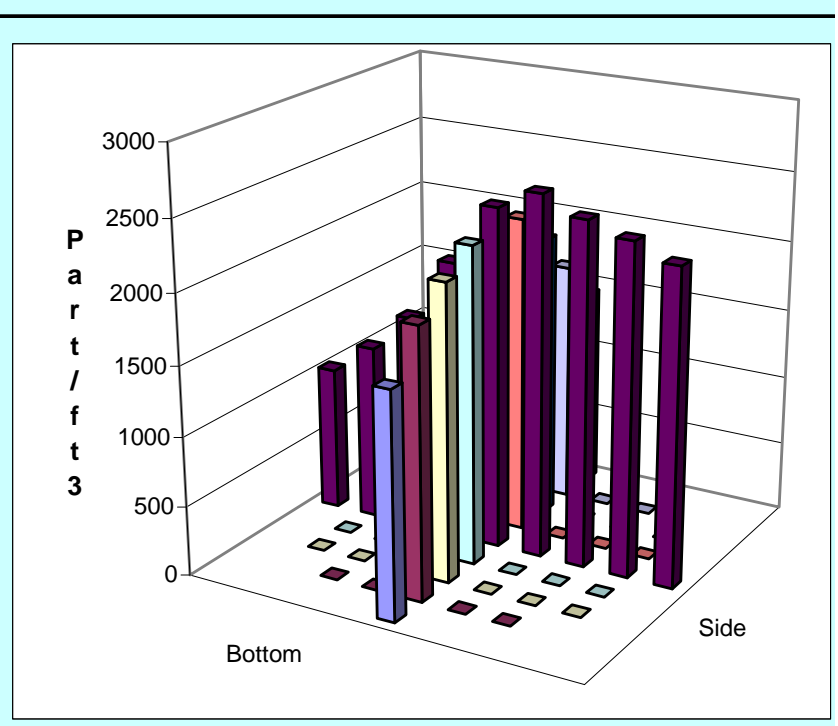

Signature signifies compliance with

Procedure EMS-JAG-02

Signature verifying data and calculations:

Signature/date

Signatures on original data sheet in Test Instruction Package TI-RPP-WTP-466

Reference: CCP-WTPSP-175

(HVC2_part-dataRev0 (7)).xls / PT8 part-dataRev0.xls $4 / 18 / 2007$

3 August 2006 
Rev. 0

3 Aug. 2006

PARTICLE TRACER TRAVERSE DATA FORM

Site HV-C2 Model

Date 12/8/2006

Tester JGD MSP

Stack Dia

Stack X-Area

Elevation Port 3

Distance to disturbance Measurement units particles/ft3

Order ----->

Traverse-->

Trial ---->

\begin{tabular}{|r|r|r|r|r|} 
Trial --->> & \multicolumn{3}{c|}{1} & particles/ft3 \\
\hline Point & Depth, in. & & 981 & 956 \\
\hline 1 & 0.50 & 1260 & 1281 & 1259 \\
\hline 2 & 1.26 & 1280 & 1457 & 1505 \\
\hline 3 & 2.33 & 1345 & 1309 & 1355 \\
\hline 4 & 3.88 & 1586 & 1392 & 1341 \\
\hline Center & 6.00 & 1472 & 943 & 1199 \\
\hline 5 & 8.12 & 1342 & 841 & 1139 \\
\hline 6 & 9.67 & 1133 & 627 & 957 \\
\hline 7 & 10.74 & 978 & 608 & 776 \\
\hline 8 & 11.50 & 839 & 1048.8 & 1165.2 \\
\hline Averages ---------> & 1248.3 & &
\end{tabular}

Run No. PT-9

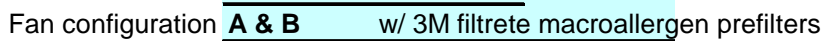

Fan Setting 40

Stack Temp

Start/End Time 1640

Center $2 / 3$ from

Points in Center $2 / 3$ Injection Point $\mathbf{A}$

$1640--1900$

$\mathrm{Hz}$

\begin{tabular}{lrrr} 
& 1.10 & to: & 10.90 \\
\hline & 2 & to: & 7 \\
Center & &
\end{tabular}
$1 \mathrm{st}$ Bottom

(nean

\begin{tabular}{ll|llll}
3 & Mean & 1 & 2 & 3 & Mean \\
\hline & & particles $/ 3$ &
\end{tabular}

\begin{tabular}{l|l|}
1065.7 \\
\hline
\end{tabular}

\begin{tabular}{|l|l|l|l|l|}
\hline 7 & 1042 & 1094 & 1031 & 1055.7 \\
\hline 7 & 1263 & 1262 & 1276 & 1267.0 \\
\hline 7 & 1390 & 1485 & 1483 & 1452.7 \\
\hline 7 & 1578 & 1567 & 1522 & 1555.7 \\
\hline 1699 & 1721 & 1748 & 1722.7 \\
\hline 7 & 1719 & 1662 & 1685 & 1688.7 \\
\hline 0 & 1520 & 1575 & 1583 & 1559.3 \\
\hline 0 & 1191 & 1349 & 1381 & 1349.3 \\
\hline & 1413.3 & 1425.8 & 1429.6 & 1155.0 \\
\hline
\end{tabular}

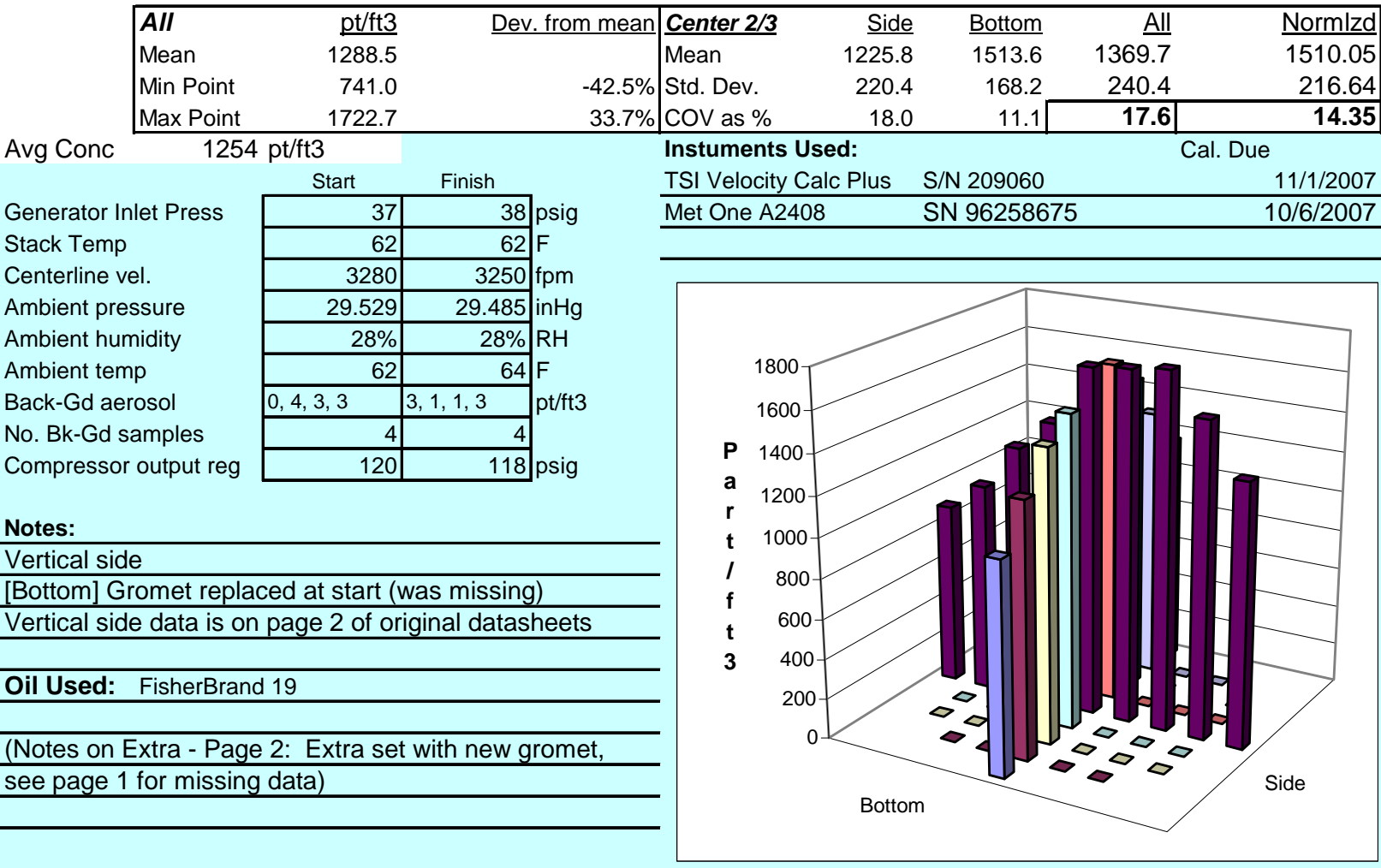

Signature signifies compliance with

Procedure EMS-JAG-02

Signature/date

Reference: CCP-WTPSP-175

part-dataRev0.xls

3 August 2006
Signature verifying data and calculations:

Signatures on original data sheet in Test Instruction Package TI-RPP-WTP-466 
Rev. 0

3 Aug. 2006

PARTICLE TRACER TRAVERSE DATA FORM

\section{Site HV-C2 Model}

Date 12/18/2006

Tester MSP

Stack Dia.

Stack X-Area

Elevation Port 1

Distance to disturbance

Measurement units particles/ft3
Run No. PT-10

Fan configuration $\mathbf{A}$

Fan Setting 35

Stack Temp

Center $2 / 3$ fror

Points in Center $2 / 3$

Injection Point $\mathbf{A}$

$\begin{array}{lll}\text { Order }-----> & \text { 2nd } & \\ \text { Traverse--> } & & \text { Side }\end{array}$

Trial ---->

\begin{tabular}{|r|r|}
\hline Point & Depth, in. \\
\hline 1 & 0.50 \\
\hline 2 & 1.26 \\
\hline 3 & 2.33 \\
\hline 4 & 3.88 \\
\hline Center & 6.00 \\
\hline 5 & 8.12 \\
\hline 6 & 9.67 \\
\hline 7 & 10.74 \\
\hline 8 & 11.50 \\
\hline
\end{tabular}

Averages ---------->

1.50

113.1 in. 2

13.75 inches

\begin{tabular}{lrrr} 
& 1.10 & to: & 10.90 \\
\hline & 2 & to: & 7 \\
\hline A & Center &
\end{tabular}

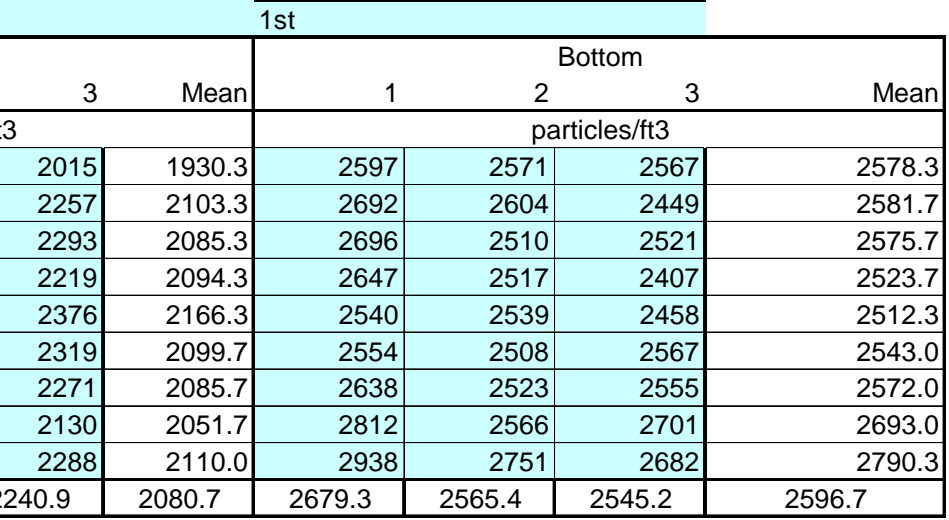

\begin{tabular}{|c|c|c|c|c|}
\hline & $\begin{array}{l}\text { AII } \\
\text { Mean } \\
\text { Min Point } \\
\text { Max Point } \\
\end{array}$ & $\begin{array}{r}\mathrm{pt} / \mathrm{ft} 3 \\
2338.7 \\
1930.3 \\
2790.3\end{array}$ & Dev. & fro \\
\hline Avg Conc & 233 & $\mathrm{pt} / \mathrm{ft} 3$ & & \\
\hline & & Start & Finish & \\
\hline Generator Inl & let Press & 40 & 40 & \\
\hline Stack Temp & & 58 & 57 & \\
\hline Centerline ve & & 1210 & 1120.0 & fpm \\
\hline Ambient pres & ssure & 30.08 & 30.07 & \\
\hline Ambient hum & hidity & $31 \%$ & $33 \%$ & $\mathrm{RH}$ \\
\hline Ambient tem & & 62 & 60 & \\
\hline Back-Gd aer & osol & $4,0,1,2$ & $1,0,1,1$ & \\
\hline No. Bk-Gd s & nples & 4 & 4 & \\
\hline Compressor & utput reg & 120 & 125 & \\
\hline
\end{tabular}

Notes:

Used same probe on side and bottom transects. Analyzer turned on end for the side port measurements Both shutters @ $45 \mathrm{deg}$

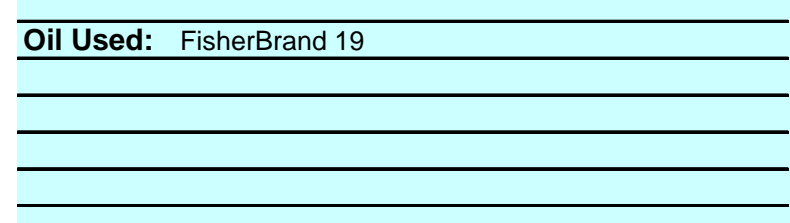

$19.3 \%$ COV as \%

$\begin{array}{r}\text { Side } \\ 2098.0 \\ 34.6 \\ 1.6 \\ \hline\end{array}$

\begin{tabular}{rrrr}
$\frac{\text { Bottom }}{2571.6}$ & \multicolumn{1}{c}{ All } & Normlzd \\
59.8 & 2334.8 & 2502.38 \\
\cline { 2 - 3 } 2.3 & 250.2 & 86.93 \\
\hline
\end{tabular}

Instuments Used:

Cal. Due

TSI Velocity Calc Plus S/N 209060

$11 / 1 / 2007$

\begin{tabular}{lll}
\hline Met One A2408 & SN 96258675 & $10 / 6 / 2007$ \\
\hline
\end{tabular}

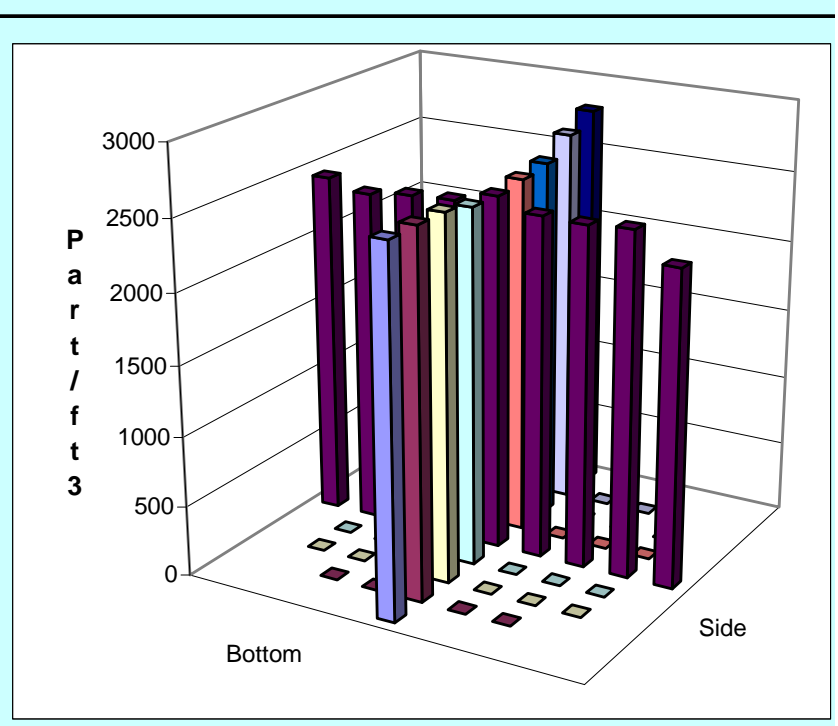

Signature signifies compliance with

Signature verifying data and calculations:

Procedure EMS-JAG-02

Signature/date

Signatures on original data sheet in Test Instruction Package TI-RPP-WTP-466

Reference: CCP-WTPSP-175

part-dataRev0.xls

(HVC2_part-dataRev0 (7)).xls / PT10

3 August 2006

4/18/2007 
Rev. 0

3 Aug. 2006
PARTICLE TRACER TRAVERSE DATA FORM

Site HV-C2 Model

Date $12 / 19 / 2006$

Tester JGD

Stack Dia. Stack X-Area

Elevation Port 1

Distance to disturbance

Measurement units particles/ft 3

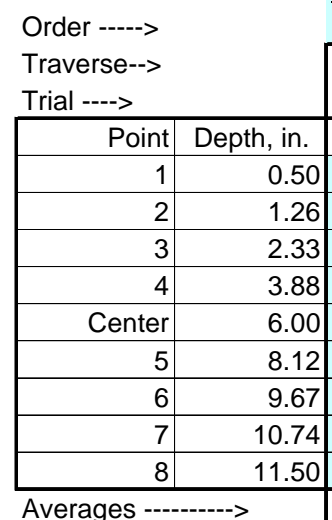

$1 \mathrm{st}$

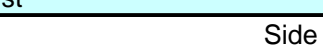

Stack Temp
Run No. PT-11

Fan configuration $\overline{\mathbf{A} \& \mathbf{B}} \quad \mathrm{w} / 3 \mathrm{M}$ filtrete macroallergen prefilters

Fan Setting $\underline{40}$

Start/End Time $1000--1230$

Center $2 / 3$ from

Points in Center $2 / 3$ Injection Point $\mathbf{A}$

\begin{tabular}{lrll}
\hline & 1.10 & to: & 10.90 \\
\hline $\mathbf{A}$ & Center & \\
\hline 2nd & & &
\end{tabular}

2nd

\begin{tabular}{|c|c|c|c|c|c|c|c|}
\hline 4 & 5 & 6 & Mean & 1 & 2 & 3 & Mean \\
\hline \multicolumn{4}{|c|}{ particles/ft3 } & \multicolumn{4}{|c|}{ particles/ft3 } \\
\hline 896 & 896 & 910 & 900.7 & 641 & 446 & 500 & 529.0 \\
\hline 1154 & 1142 & 1199 & 1165.0 & 711 & 602 & 607 & 640.0 \\
\hline 1130 & 1134 & 1294 & 1186.0 & 817 & 706 & 672 & 731.7 \\
\hline 1232 & 1269 & 1303 & 1268.0 & 909 & 834 & 794 & 845.7 \\
\hline 1302 & 1308 & 1327 & 1312.3 & 1161 & 1035 & 1041 & 1079.0 \\
\hline 1553 & 1613 & 1685 & 1617.0 & 1364 & 1202 & 1258 & 1274.7 \\
\hline 1824 & 1952 & 2136 & 1970.7 & 1734 & 1606 & 1510 & 1616.7 \\
\hline 2014 & 2084 & 2239 & 2112.3 & 1854 & 1706 & 1667 & 1742.3 \\
\hline 1660 & 1748 & 1710 & 1706.0 & 1135 & 1310 & 1382 & 1275.7 \\
\hline 1418.3 & 1460.7 & 1533.7 & 1470.9 & 1147.3 & 1049.7 & 1047.9 & 1081.6 \\
\hline
\end{tabular}

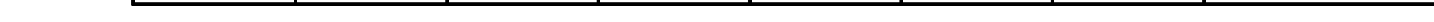

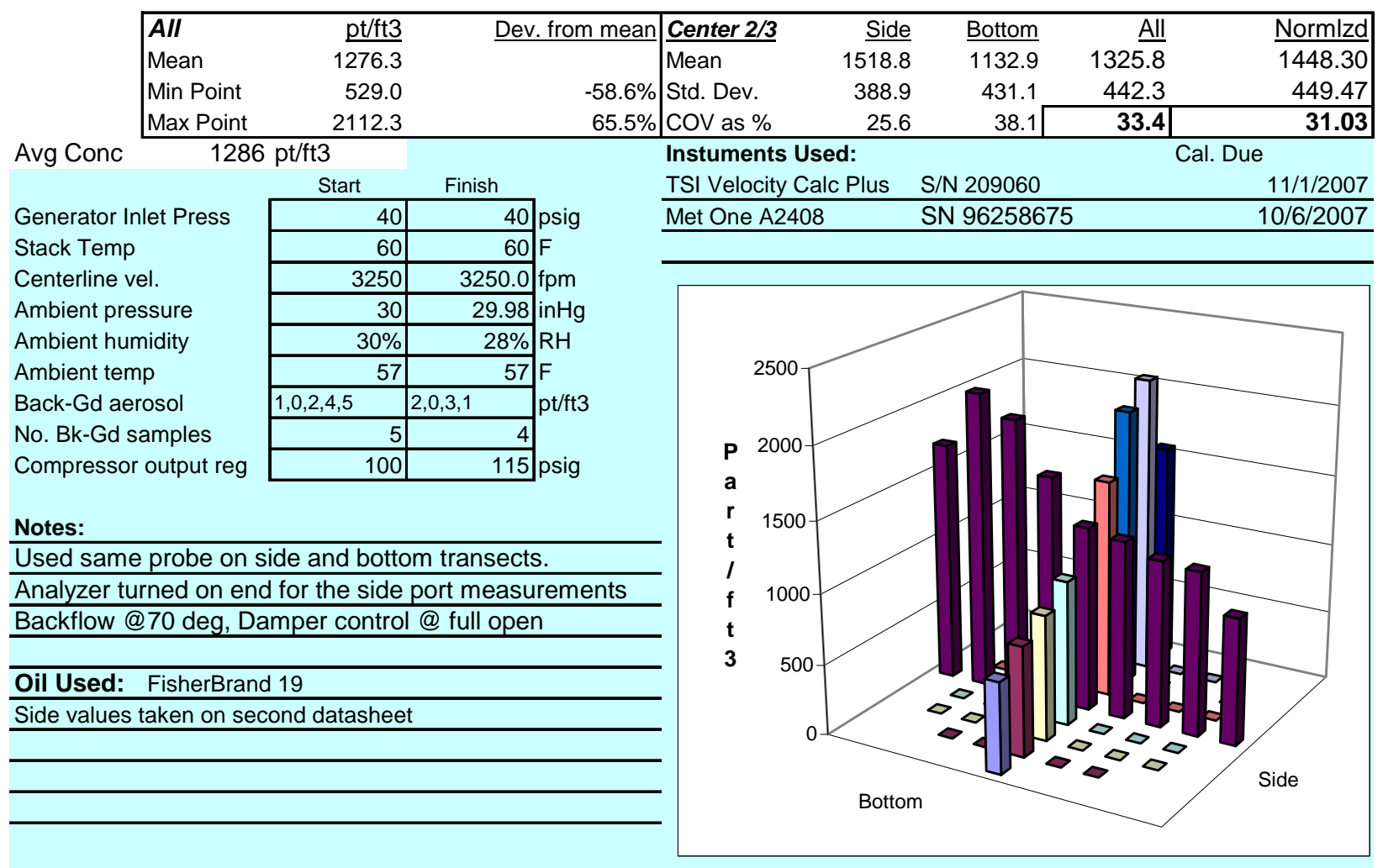

Signature signifies compliance with Procedure EMS-JAG-02

Signature/date

Reference: CCP-WTPSP-175

part-dataRev0.xls

3 August 2006
Signature verifying data and calculations:

Signatures on original data sheet in Test Instruction Package TI-RPP-WTP-466

(HVC2_part-dataRev0 (7)).xIs / PT11 $4 / 18 / 2007$ 
Rev. 0

3 Aug. 2006

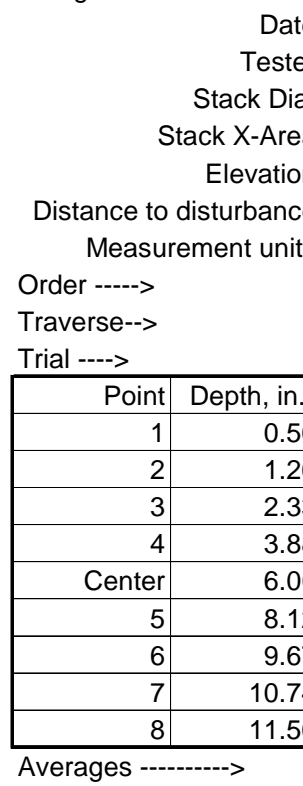

\section{PARTICLE TRACER TRAVERSE DATA FORM}

Site HV-C2 Model

Date 12/19/2006

ester MSP

$\frac{12 \text { in. }}{113.1 \text { in. } 2}$

113.75 inches particles/ft3

2nd

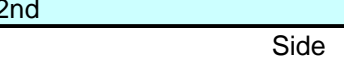

Run No. PT-12

Fan configuration $\overline{\mathbf{A} \& \mathbf{B}} \quad \mathrm{w} / 3 \mathrm{M}$ filtrete macroallergen prefilters

Fan Setting $\underline{40}$

Stack Temp

Start/End Time 1330 -- 1540

Center 2/3 from

Points in Center $2 / 3$

Injection Point $\mathbf{A}$

\begin{tabular}{|c|c|c|}
\hline 0 & & $\mathrm{~Hz}$ \\
\hline \multicolumn{2}{|c|}{$59.5 \operatorname{deg} F$} & \\
\hline \multicolumn{3}{|c|}{$330--1540$} \\
\hline 1.10 & to: & 10.90 \\
\hline 2 & to: & 7 \\
\hline & & \\
\hline
\end{tabular}

1 st

Bottom

\begin{tabular}{|c|c|c|c|c|c|c|c|}
\hline 1 & 2 & 3 & Mean & 1 & 2 & 3 & Mean \\
\hline \multicolumn{4}{|c|}{ particles/ft3 } & \multicolumn{4}{|c|}{ particles/ft3 } \\
\hline 1052 & 1066 & 1093 & 1070.3 & 975 & 976 & 958 & 969.7 \\
\hline 1091 & 1158 & 1133 & 1127.3 & 1109 & 1060 & 1031 & 1066.7 \\
\hline 1188 & 1210 & 1168 & 1188.7 & 1181 & 1265 & 1165 & 1203.7 \\
\hline 1361 & 1290 & 1314 & 1321.7 & 1402 & 1466 & 1304 & 1390.7 \\
\hline 1157 & 1283 & 1301 & 1247.0 & 1669 & 1465 & 1484 & 1539.3 \\
\hline 1276 & 1284 & 1289 & 1283.0 & 1692 & 1694 & 1728 & 1704.7 \\
\hline 1250 & 1264 & 1301 & 1271.7 & 1805 & 1765 & 1808 & 1792.7 \\
\hline 1298 & 1338 & 1360 & 1332.0 & 1826 & 1799 & 1817 & 1814.0 \\
\hline 1200 & 1206 & 1192 & 1199.3 & 1718 & 1682 & 1595 & 1665.0 \\
\hline 1208.1 & 1233.2 & 1239.0 & 1226.8 & 1486.3 & 1463.6 & 1432.2 & 1460.7 \\
\hline
\end{tabular}

\begin{tabular}{|c|c|c|c|c|c|c|c|}
\hline$A I I$ & $\mathrm{pt} / \mathrm{ft} 3$ & Dev. from mean & Center 2/3 & Side & Bottom & All & Normlzd \\
\hline Mean & $13 \overline{1343.7}$ & & Mean & 1253.0 & 1501.7 & 1377.4 & 1524.23 \\
\hline Min Point & 969.7 & $-27.8 \%$ & Std. Dev. & 73.2 & 293.0 & 242.4 & 209.65 \\
\hline Max Point & 1814.0 & $35.0 \%$ & Cov as $\%$ & 5.8 & 19.5 & 17.6 & 13.75 \\
\hline
\end{tabular}

Avg Conc $1338 \mathrm{pt} / \mathrm{ft} 3$

\begin{tabular}{|c|c|c|}
\hline \multirow[b]{2}{*}{ Generator II } & Start & Finish \\
\hline & 40 & 40 psig \\
\hline Stack Temp & 60 & $59 \mathrm{~F}$ \\
\hline Centerline vel. & 3130 & $3170.0 \mathrm{fpm}$ \\
\hline Ambient pressure & 29.99 & $29.96 \mathrm{inHg}$ \\
\hline Ambient humidity & $43 \%$ & 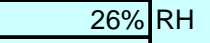 \\
\hline Ambient temp & 54 & $57 \mathrm{~F}$ \\
\hline Back-Gd aerosol & $1,4,0,5$ & $2,1,1,4$ \\
\hline Bk-Gd samples & 4 & 4 \\
\hline Compressor output reg & 105 & 115 \\
\hline
\end{tabular}

Notes:

Used same probe on side and bottom transects.

Analyzer turned on end for the side port measurements

Dampers: full open; $70 \mathrm{deg}$

Oil Used: $\quad$ FisherBrand 19

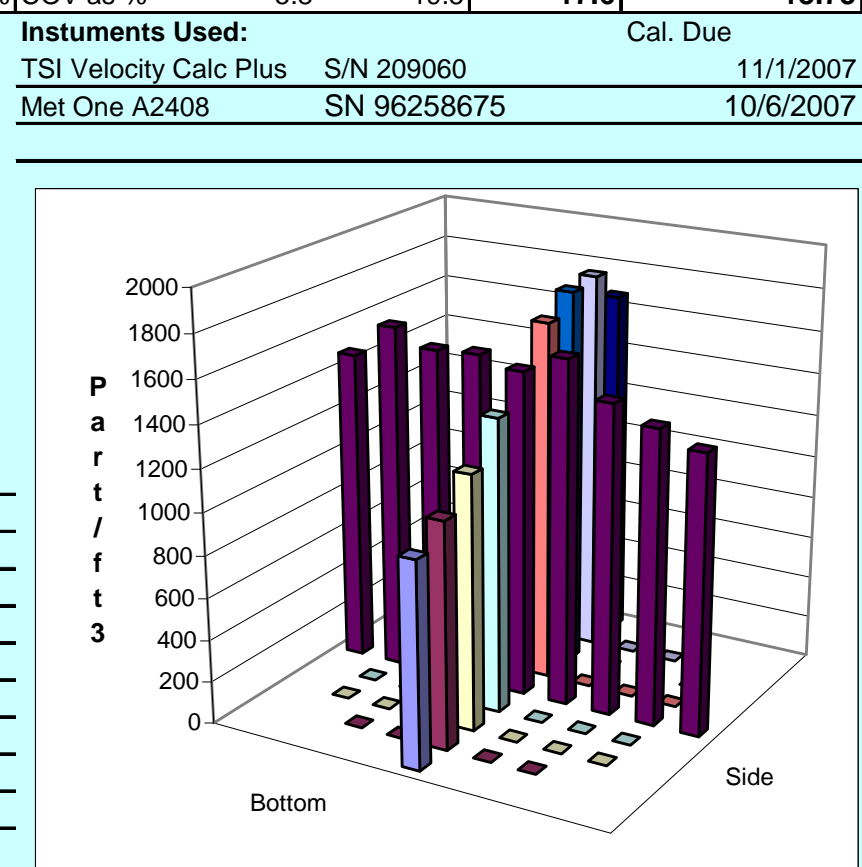

Signature signifies compliance with Procedure EMS-JAG-02

Signature/date

Signature verifying data and calculations:

Reference: CCP-WTPSP-175

part-dataRev0.xls

(HVC2_part-dataRev0 (7)).xls / PT12

3 August 2006

Signatures on original data sheet in Test Instruction Package TI-RPP-WTP-466

$4 / 18 / 2007$ 
Rev. 0

3 Aug. 2006

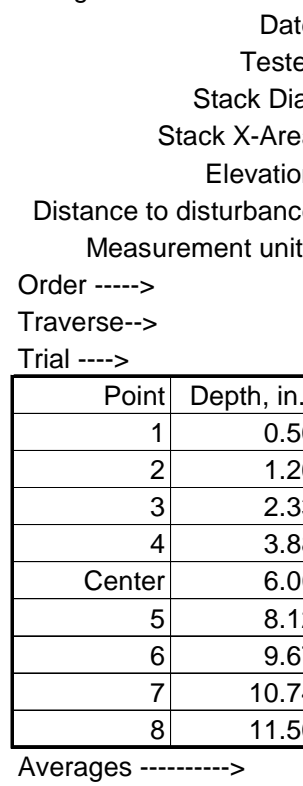

\section{PARTICLE TRACER TRAVERSE DATA FORM}

Site HV-C2 Model

Date 12/20/2006

ester MSP

Dia. 12 in. 113.1 in. 2

Port 3

174.25 inches particles/ft3

1 st

1 st
Run No. PT-13

Fan configuration $\overline{\mathbf{A \& B}} \quad \mathrm{W} / 3 \mathrm{M}$ filtrete macroallergen prefilters

Fan Setting $\underline{40}$

Stack Temp

Start/End Time 1350 -- 1550

Center 2/3 from

Points in Center $2 / 3$ Injection Point $\mathbf{A}$

Hz
$\mathrm{Hz}$

\begin{tabular}{lrrr} 
& 1.10 & to: & 10.90 \\
\hline & 2 & to: & 7 \\
\hline A & & Center & \\
\hline 2nd & &
\end{tabular}

2nd

\begin{tabular}{|c|c|c|c|c|c|c|}
\hline \multicolumn{7}{|c|}{ 2nd } \\
\hline \multicolumn{3}{|c|}{ Side } & \multicolumn{4}{|c|}{ Bottom } \\
\hline 2 & 3 & \multirow[t]{2}{*}{ Mean } & \multicolumn{2}{|l|}{1} & 3 & Mean \\
\hline \multicolumn{2}{|c|}{ articles/ft3 } & & \multicolumn{4}{|c|}{ particles/ft3 } \\
\hline 949 & 936 & 936.0 & 957 & 961 & 894 & 937.3 \\
\hline 950 & 1028 & 1052.0 & 1028 & 970 & 1039 & 1012.3 \\
\hline 1112 & 1107 & 1114.7 & 1166 & 1188 & 1173 & 1175.7 \\
\hline 1080 & 1202 & 1154.3 & 1301 & 1240 & 1345 & 1295.3 \\
\hline 161 & 1060 & 1120.0 & 1358 & 1440 & 1386 & 1394.7 \\
\hline 1037 & 1061 & 1053.7 & 1446 & 1563 & 1444 & 1484.3 \\
\hline 1016 & 967 & 990.3 & 1501 & 1427 & 1477 & 1468.3 \\
\hline 992 & 972 & 976.3 & 1310 & 1360 & 1279 & 1316.3 \\
\hline 869 & 736 & 819.3 & 1141 & 1206 & 1142 & 1163.0 \\
\hline 4 & 1007.7 & 1024.1 & 1245.3 & 1261.7 & 1242.1 & 1249.7 \\
\hline
\end{tabular}

\begin{tabular}{|lr}
\hline All & $\mathrm{pt} / \mathrm{ft} 3$ \\
Mean & 1136.9 \\
Min Point & 819.3 \\
Max Point & 1484.3 \\
\hline
\end{tabular}

Avg Conc $1122 \mathrm{pt} / \mathrm{ft} 3$

\begin{tabular}{r|r|}
\hline 853 & 869 \\
\hline
\end{tabular}

Generator Inlet Press

Stack Temp

Centerline vel.

Ambient pressure

Ambient humidity

Ambient temp

Back-Gd aerosol

No. Bk-Gd samples

Compressor output reg

\begin{tabular}{|c|c|c|}
\hline Start & Finish & \\
\hline 40 & 40 & psig \\
\hline 62 & 62 & $2 \mathrm{~F}$ \\
\hline 3110 & 3010.0 & $\mathrm{fpm}$ \\
\hline 29.86 & 29.83 & $3 \mathrm{inHg}$ \\
\hline $22 \%$ & $22 \%$ & $\mathrm{RH}$ \\
\hline 61 & 61 & $1 \mathrm{~F}$ \\
\hline $7,10,7,2$ & $2,3,9,1$ & $\mathrm{pt} / \mathrm{ft}$ \\
\hline 4 & 4 & \\
\hline 110 & 110 & psig \\
\hline
\end{tabular}

Notes:

Used same probe on side and bottom transects.

Analyzer turned on end for the side port measurements Dampers: full open; $70 \mathrm{deg}$

Oil Used: $\quad$ FisherBrand 19

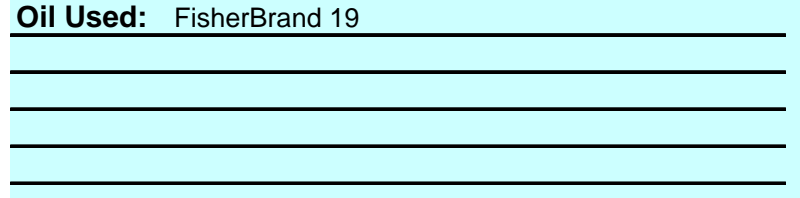

\begin{tabular}{r|l} 
Dev. from mean & Center $\mathbf{2 / 3}$ \\
\hline$-27.9 \%$ & Mean \\
$30.6 \%$ & Std. Dev. \\
COV as \%
\end{tabular}

$\begin{array}{r}\text { Side } \\ 1065.9 \\ 67.3 \\ 6.3 \\ \hline\end{array}$

\begin{tabular}{rrrr|}
\hline Bottom & & All & \\
\hline 1306.7 & 1186.3 & Normlzd \\
168.1 & 175.3 & 128.04 \\
& $\mathbf{1 4 . 8}$ & $\mathbf{9 . 7 2}$ \\
\hline
\end{tabular}

Instuments Used:

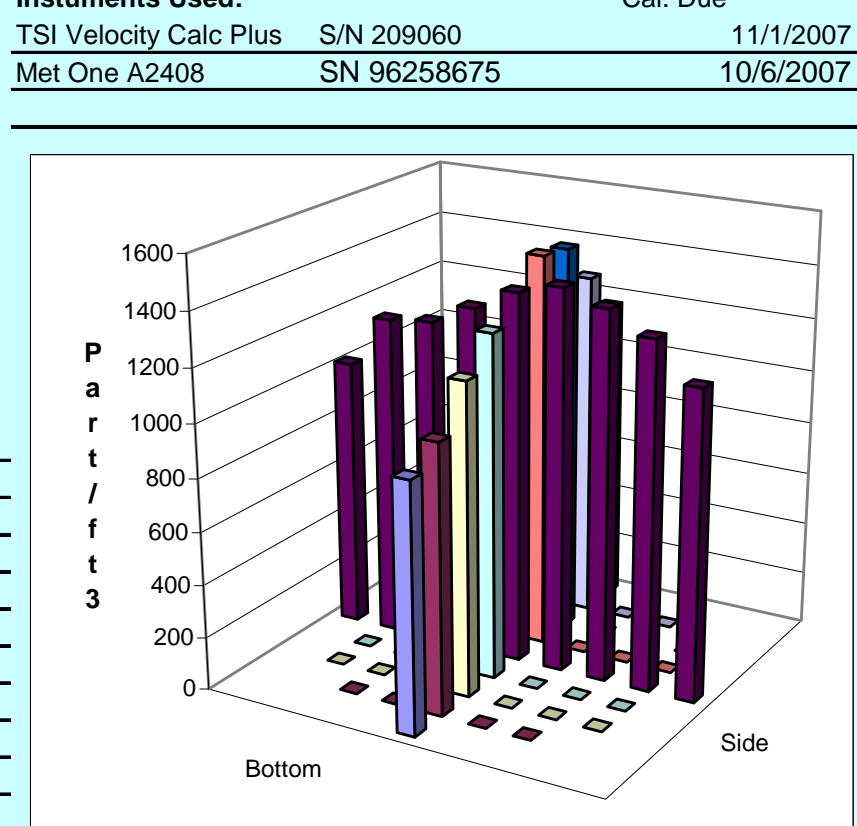

Signature signifies compliance with Procedure EMS-JAG-02

Signature/date

Reference: CCP-WTPSP-175

part-dataRev0.xls

3 August 2006
Signature verifying data and calculations:

Signatures on original data sheet in Test Instruction Package TI-RPP-WTP-466

(HVC2_part-dataRev0 (7)).xIs / PT13 $4 / 18 / 2007$ 
Rev. 0

3 Aug. 2006

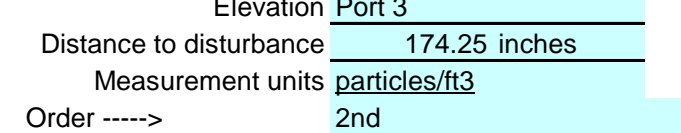

174.25 inches

2nd

Order ----->

Site HV-C2 Model

Date 12/20/2006

Tester MSP

Stack Dia. Stack X-Area

Elevation

12 in

113.1 in. 2

Traverse-->

Trial ---->

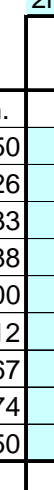

Side 12

\section{RR TRAVERSE DATA FORM}

Fan configuration \begin{aligned} Run No & w/ 3M filtrete macroallergen prefilters \\ \cline { 2 - 2 } & \end{aligned}

Fan Setting 35

Stack Temp

Start/End Time $1550--1750$

Center $2 / 3$ from

Points in Center $2 / 3$

Injection Point $\mathbf{A}$

\begin{tabular}{lll}
\multicolumn{3}{c}{$\mathrm{Hz}$} \\
$62.5 \operatorname{deg} \mathrm{F}$ & \\
$\frac{1.10}{2} \quad$ to: & $\frac{10.90}{7}$ \\
to: & $\mathbf{7}$ \\
Center &
\end{tabular}

\begin{tabular}{|c|c|c|c|c|c|c|c|}
\hline & \multicolumn{4}{|c|}{$1 \mathrm{st}$} \\
\hline \multicolumn{4}{|c|}{ Side } & \multicolumn{4}{|c|}{ Bottom } \\
\hline 1 & 2 & 3 & Mean & 1 & 2 & 3 & Mean \\
\hline \multicolumn{4}{|c|}{ particles/ft3 } & \multicolumn{4}{|c|}{ particles/ft3 } \\
\hline 2388 & 2031 & 1469 & 1962.7 & 2348 & 2367 & 2269 & 2328.0 \\
\hline 2323 & 2183 & 1482 & 1996.0 & 2389 & 2509 & 2338 & 2412.0 \\
\hline 1931 & 2170 & 1512 & 1871.0 & 2521 & 2639 & 2383 & 2514.3 \\
\hline 1979 & 1980 & 1684 & 1881.0 & 2461 & 2480 & 2424 & 2455.0 \\
\hline 2156 & 1799 & 1688 & 1881.0 & 2384 & 2350 & 2415 & 2383.0 \\
\hline 2148 & 1821 & 1620 & 1863.0 & 2393 & 2354 & 2475 & 2407.3 \\
\hline 2065 & 1744 & 1663 & 1824.0 & 2453 & 2158 & 2317 & 2309.3 \\
\hline 2328 & 2090 & 1780 & 2066.0 & 2362 & 2039 & 2542 & 2314.3 \\
\hline 2103 & 1821 & 2194 & 2039.3 & 2298 & 2106 & 2675 & 2359.7 \\
\hline 7.9 & 1959.9 & 1676.9 & 1931.6 & 2401.0 & 2333.6 & 2426.4 & 2387.0 \\
\hline
\end{tabular}

Averages ---------->

2157.9

\begin{tabular}{|c|c|c|c|c|c|c|c|}
\hline All & $\mathrm{pt} / \mathrm{ft} 3$ & Dev. from mean & Center $2 / 3$ & Side & Bottom & All & Normlzd \\
\hline Mean & 2159.3 & & Mean & 1911.7 & 2399.3 & 2155.5 & 2410.62 \\
\hline Min Point & 1824.0 & $-15.5 \%$ & Std. Dev. & 86.1 & 73.2 & 264.4 & 90.03 \\
\hline Max Point & 2514.3 & $16.4 \%$ & cov as $\%$ & 4.5 & 31 & 12.3 & 373 \\
\hline
\end{tabular}

Avg Conc $2163 \mathrm{pt} / \mathrm{ft} 3$

$16.4 \%$ COV as \%

4.5

Instuments Used:

Generator Inlet Press

Stack Temp

Centerline vel.

Ambient pressure

Ambient humidity

Ambient temp

Back-Gd aerosol

No. Bk-Gd samples

Compressor output reg

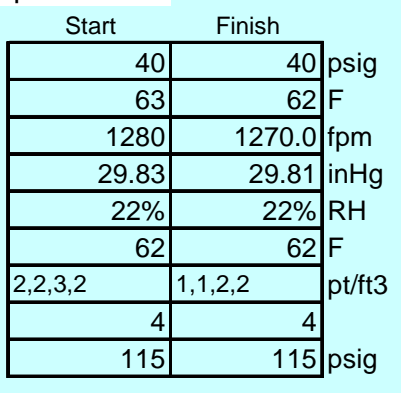

Notes:

Used same probe on side and bottom transects.

Analyzer turned on end for the side port measurements

Dampers: full open; $70 \mathrm{deg}$

Oil Used: $\quad$ FisherBrand 19

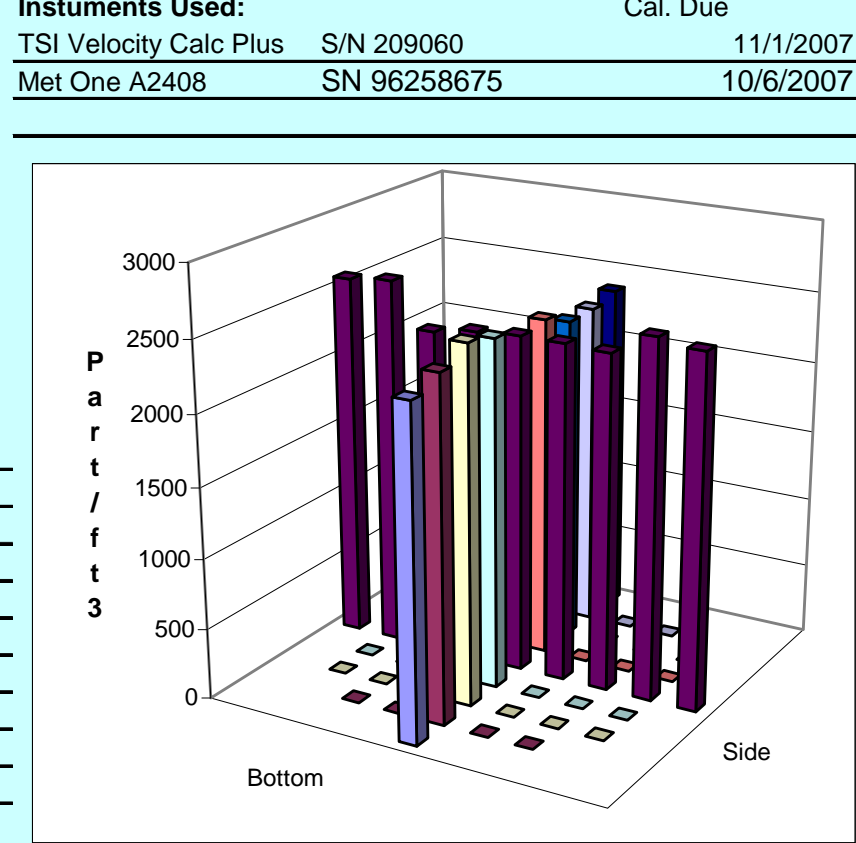

Signature signifies compliance with Procedure EMS-JAG-02

Signature/date

Signature verifying data and calculations:

Reference: CCP-WTPSP-175

part-dataRev0.xls

(HVC2_part-dataRev0 (7)).xls / PT14

3 August 2006

Signatures on original data sheet in Test Instruction Package TI-RPP-WTP-466

$4 / 18 / 2007$ 
Rev. 0

3 Aug. 2006

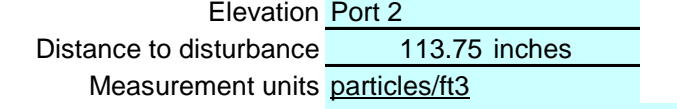

Elevation Port 2

PARTICLE TRACER TRAVERSE DATA FORM

Site HV-C2 Model

Date 12/21/2006

Tester MSP

Stack Dia.

12 in.

Stack X-Area

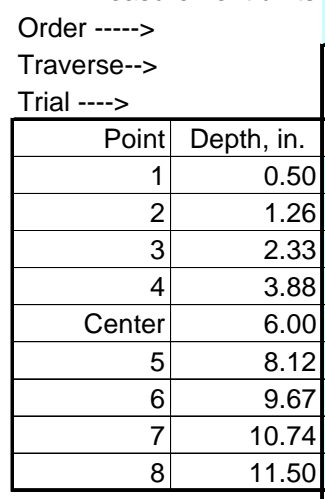

Averages --------->

$1 \mathrm{st}$
Run No. PT-15

Fan configuration $\mathbf{A}$

Fan Setting 35

Stack Temp

Start/End Time

Center $2 / 3$ from

Points in Center $2 / 3$

Injection Point $\mathbf{A}$

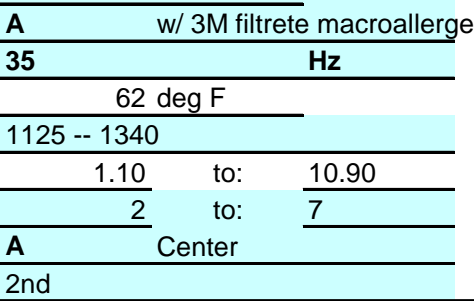

\begin{tabular}{|c|r|r|r|r|r|r|}
\begin{tabular}{|c|} 
Side \\
2
\end{tabular} & 3 & Mean & \multicolumn{5}{|c|}{ Bottom } \\
\hline $\begin{array}{r}\text { particles/ft3 } \\
2979\end{array}$ & 3009 & 2987.7 & 3345 & 2488 & 2876 & Mean \\
\hline 2810 & 2974 & 2956.7 & 3456 & 2624 & 3209 & 2903.0 \\
\hline 2900 & 2959 & 2964.0 & 3437 & 2562 & 3441 & 3096.3 \\
\hline 2978 & 3031 & 3010.0 & 3541 & 2581 & 3543 & 3146.7 \\
\hline 2957 & 2948 & 2956.7 & 3755 & 2731 & 3301 & 3221.7 \\
\hline 2854 & 2973 & 2927.7 & 3875 & 2570 & 3308 & 3251.0 \\
\hline 2829 & 2911 & 2842.3 & 3808 & 2490 & 3165 & 3154.3 \\
\hline 2782 & 2820 & 2816.3 & 3818 & 2438 & 2955 & 3070.3 \\
\hline 2734 & 2842 & 2800.0 & 3579 & 2585 & 2938 & 3034.0 \\
\hline 2869.2 & 2940.8 & 2917.9 & 3623.8 & 2563.2 & 3192.9 & 3126.6 \\
\hline
\end{tabular}

\begin{tabular}{|lr}
\hline All & $\mathrm{pt} / \mathrm{ft} 3$ \\
Mean & 3022.3 \\
Min Point & 2800.0 \\
Max Point & 3262.3 \\
\hline
\end{tabular}

Avg Conc $3011 \mathrm{pt} / \mathrm{ft} 3$

Generator Inlet Press

Stack Temp

Centerline vel.

Ambient pressure

Ambient humidity

Ambient temp

Back-Gd aerosol

No. Bk-Gd samples

Compressor output reg

\begin{tabular}{|c|c|c|}
\hline Start & Finish & \\
\hline 40 & 40 & 0 psig \\
\hline 62 & 62 & $2 \mathrm{~F}$ \\
\hline 1320 & 1320.0 & $0 \mathrm{fpm}$ \\
\hline 29.72 & 29.68 & $8 \mathrm{inHg}$ \\
\hline $26 \%$ & $31 \%$ & $\mathrm{RH}$ \\
\hline 60 & 63 & $3 \mathrm{~F}$ \\
\hline $2,6,4,0$ & $0,0,0,2$ & $\mathrm{pt} / \mathrm{ft} 3$ \\
\hline 4 & 4 & 4 \\
\hline 110 & 110 & 0 psig \\
\hline
\end{tabular}

\begin{tabular}{r|l} 
Dev. from mean & Center 2/3 \\
\hline$-7.4 \%$ & Mean \\
$7.9 \%$ & Std. Dev. \\
COV as \%
\end{tabular}

$\begin{array}{r}\text { Side } \\ 2924.8 \\ 70.0 \\ 2.4 \\ \hline\end{array}$

\begin{tabular}{rrrr}
\hline Bottom & $\underline{\text { All }}$ & Normlzd \\
\hline 3171.8 & 3048.3 & 3199.50 \\
75.1 & 145.9 & 78.65 \\
2.4 & $\mathbf{4 . 8}$ & $\mathbf{2 . 4 6}$ \\
\hline
\end{tabular}

Instuments Used:

Cal. Due

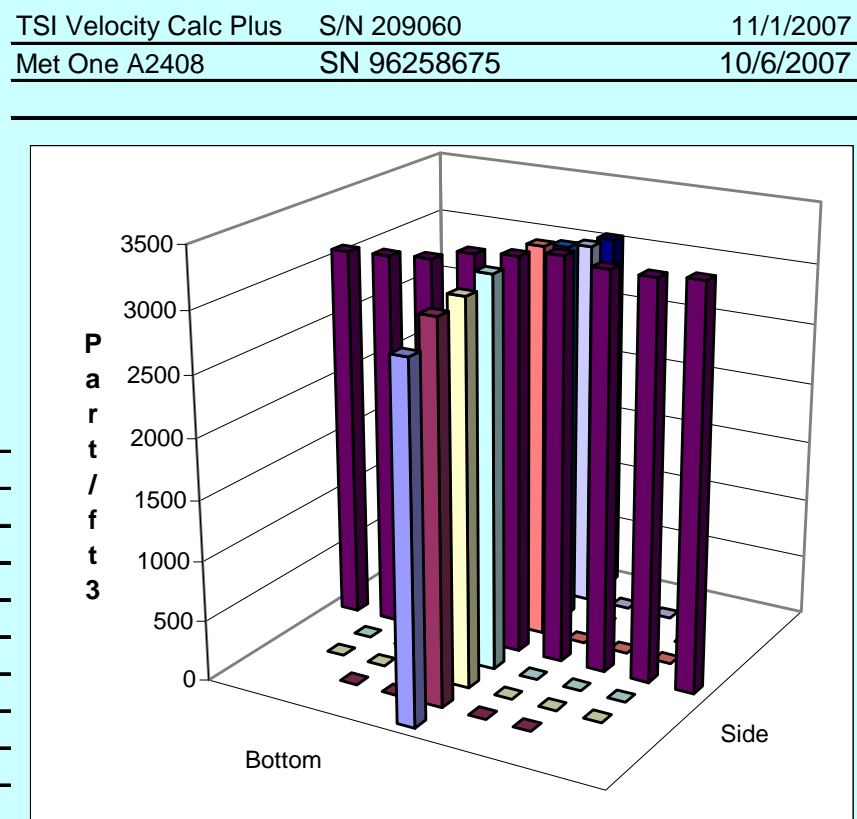

Notes:

Used same probe on side and bottom transects.

Analyzer turned on end for the side port measurements

Dampers: full open; $70 \mathrm{deg}$

Re-ran bottom \#2 and \#3

Oil Used: FisherBrand 19

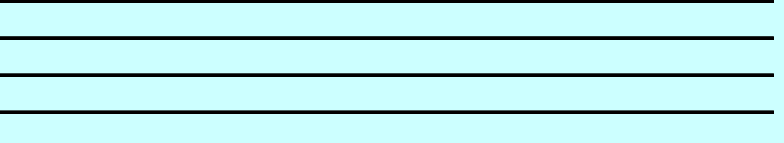

Signature signifies compliance with Procedure EMS-JAG-02

Signature/date

Signature verifying data and calculations:

Signatures on original data sheet in Test Instruction Package TI-RPP-WTP-466

Reference: CCP-WTPSP-175

part-dataRev0.xls

(HVC2_part-dataRev0 (7)).xls / PT15

3 August 2006

$4 / 18 / 2007$ 
Rev. 0

3 Aug. 2006
PARTICLE TRACER TRAVERSE DATA FORM

Site HV-C2 Model Date 12/21/2006

Tester MSP

Stack Dia. Stack X-Area

Elevation Port 1

Distance to disturbance Measurement units particles/ft3

Order ----->

Traverse-->

Trial ---->
12 in. 113.1 in. 2

2nd

$2 \mathrm{n}$

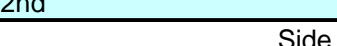

53.5 inches
Run No. PT-16

Fan configuration $\mathbf{A}$

Fan Setting 35

Stack Temp

Start/End Time

Center $2 / 3$ from

Points in Center 2/3 Injection Point $\mathbf{A}$ $1 \mathrm{st}$ $\mathrm{w} / 3 \mathrm{M}$ filtrete macroallergen prefilters $\mathrm{Hz}$ $62 \operatorname{deg} \mathrm{F}$

$1500--1645$

\begin{tabular}{rrrr} 
& 1.10 & to: & 10.90 \\
\hline & 2 & to: & 7 \\
\hline A & Center & \\
\hline $15 t$ & &
\end{tabular}

\begin{tabular}{|r|r|r|r|r|}
\hline & \multicolumn{4}{|c|}{ Bottom } \\
\hline & 1 & \multicolumn{4}{|c|}{$2^{\mid}$particles/ft3 } \\
\hline 1188.0 & 1112 & 1217 & 1241 & 1190.0 \\
\hline 1122.3 & 1249 & 1240 & 1202 & 1230.3 \\
\hline 1051.0 & 1005 & 1240 & 1232 & 1159.0 \\
\hline 1005.0 & 1113 & 1264 & 1165 & 1180.7 \\
\hline 1025.3 & 1252 & 1231 & 1183 & 1222.0 \\
\hline 988.3 & 1423 & 1367 & 1406 & 1398.7 \\
\hline 1023.0 & 1584 & 1611 & 1705 & 1633.3 \\
\hline 1085.0 & 1770 & 1759 & 1897 & 1808.7 \\
\hline 1083.7 & 2019 & 1783 & 2099 & 1967.0 \\
\hline 1063.5 & 1391.9 & 1412.4 & 1458.9 & 1421.1 \\
\hline
\end{tabular}

\begin{tabular}{l|r}
1128.3 & 1084.2 \\
\hline
\end{tabular}

\begin{tabular}{rrrrr} 
Side & Bottom & $\underline{\text { All }}$ & Normlzd \\
1042.9 & 1376.1 & 1209.5 & 1309.49 \\
47.0 & 253.0 & 245.9 & 189.14 \\
\cline { 3 - 5 } 4.5 & 18.4 & $\mathbf{2 0 . 3}$ & $\mathbf{1 4 . 4 4}$ \\
\hline
\end{tabular}

Avg Conc $1257 \mathrm{pt} / \mathrm{ft} 3$

Generator Inlet Press

Stack Temp

Centerline vel.

Ambient pressure

Ambient humidity

Ambient temp

Back-Gd aerosol

No. Bk-Gd samples

Compressor output reg

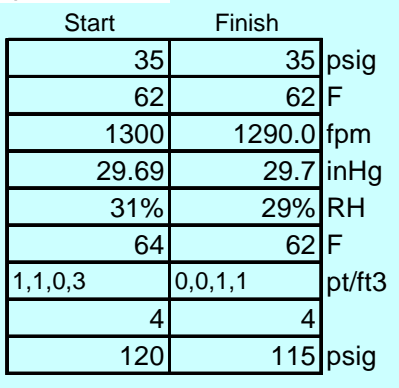

Notes:

Used same probe on side and bottom transects.

Analyzer turned on end for the side port measurements

Dampers: full open; $70 \mathrm{deg}$

Oil Used: $\quad$ FisherBrand 19

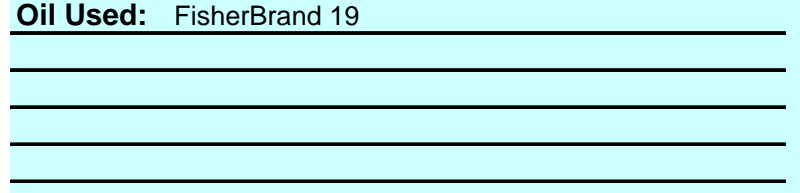

\begin{tabular}{|l} 
Center 2/3 \\
Mean \\
Std. Dev. \\
COV as \%
\end{tabular}

Instuments Used:
Cal. Due

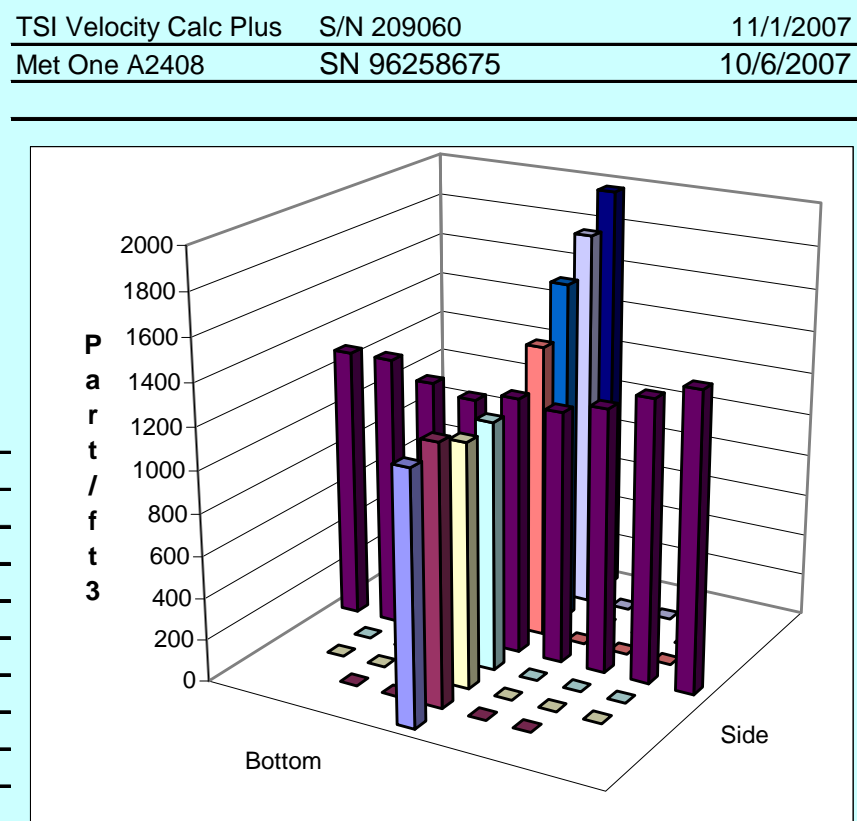

Signature signifies compliance with Procedure EMS-JAG-02

Signature/date

Reference: CCP-WTPSP-175

part-dataRev0.xls

3 August 2006
Signature verifying data and calculations:

Signatures on original data sheet in Test Instruction Package TI-RPP-WTP-466

(HVC2_part-dataRev0 (7)).xIs / PT16 $4 / 18 / 2007$ 
Rev. 0

3 Aug. 2006

\begin{tabular}{|c|c|}
\hline \multirow{2}{*}{\multicolumn{2}{|c|}{ Tester MSP }} \\
\hline & \\
\hline Stack Dia. & $12 \mathrm{in.}$ \\
\hline Stack X-Area & 113.1 in.2 \\
\hline \multicolumn{2}{|c|}{ Elevation Port 1} \\
\hline Distance to disturbance & 82 inches \\
\hline
\end{tabular}

PARTICLE TRACER TRAVERSE DATA FORM

Run No. PT-17

Fan configuration $\begin{array}{ll}\mathbf{B} & \mathrm{w} / 3 \mathrm{M} \text { filtrete macroallergen prefilters }\end{array}$

Fan Setting 35

Fan Setting 35

Start/End Time

Center $2 / 3$ from

Points in Center $2 / 3$

Injection Point $\mathbf{B}$

\begin{tabular}{|c|c|c|}
\hline \multicolumn{3}{|c|}{$\mathrm{w} / 3 \mathrm{M}$ filtrete macroallerge } \\
\hline 35 & & $\mathrm{~Hz}$ \\
\hline \multicolumn{3}{|c|}{$58 \operatorname{deg} F$} \\
\hline \multicolumn{3}{|c|}{$1030--1215$} \\
\hline 1.10 & to: & 10.90 \\
\hline 2 & to: & 7 \\
\hline B & nter & \\
\hline
\end{tabular}

\author{
2nd
}

1 st

Traverse-->

Trial ---->

Trial ---->
\begin{tabular}{|r|r|}
\hline Point & Depth, in. \\
\hline 1 & 0.50 \\
\hline 2 & 1.2 \\
\hline 3 & 2.33 \\
\hline 4 & 3.88 \\
\hline Center & 6.00 \\
\hline 5 & 8.1 \\
\hline 6 & 9.67 \\
\hline 7 & 10.7 \\
\hline 8 & \multicolumn{2}{|c|}{11.5} \\
\hline
\end{tabular}

\begin{tabular}{|l|l}
\hline & \\
\hline 50 & \\
\hline 6 & \\
33 & \\
\hline 38 & \\
\hline 2 & \\
\hline 67 & \\
74 & \\
\hline 0 & \\
\hline
\end{tabular}

(20)

Side

2nd

\begin{tabular}{|l|l|l|l|l|l|l|l|l|}
\hline Averages --------> & 1327.7 & 1325.3 & 1338.4 & 1330.5 & 1508.7 & 1491.4 & 1455.9 & 1485.3 \\
\cline { 2 - 8 }
\end{tabular}

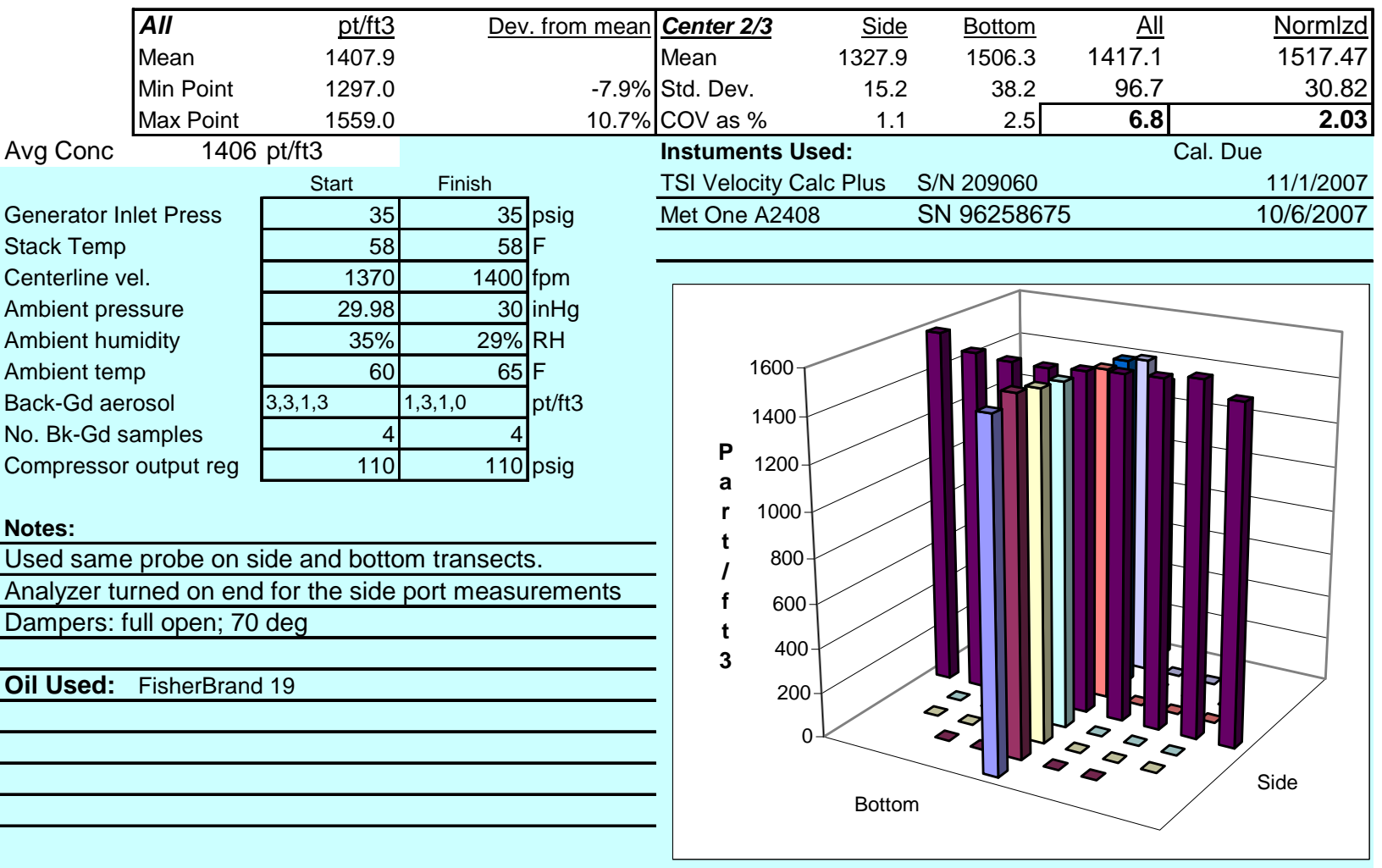

Signature signifies compliance with Procedure EMS-JAG-02

Signature/date

Signature verifying data and calculations:

Reference: CCP-WTPSP-175

part-dataRev0.xls

Signatures on original data sheet in Test Instruction Package TI-RPP-WTP-466

3 August 2006

(HVC2_part-dataRev0 (7)).xls / PT17 $4 / 18 / 2007$ 
Rev. 0

3 Aug. 2006

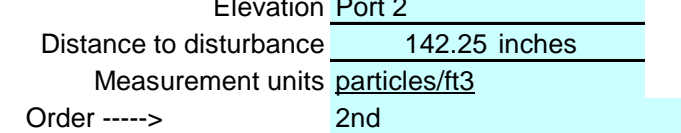

2nd

Order ----->

Site HV-C2 Model

Date 12/22/2006

Tester MSP

Stack Dia. Stack X-Area

Elevation

113.1 in. 2

Traverse-->

Trial ---->
12 in.

PARTICLE TRACER TRAVERSE DATA FORM

Run No. PT-18

Fan configuration $\mathbf{B}$

Fan Setting 35

Stack Temp

Start/End Time

Center 2/3 from

Points in Center $2 / 3$

Injection Point

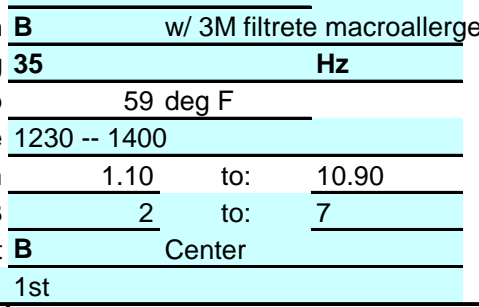

\begin{tabular}{|c|c|c|c|c|c|c|c|c|c|}
\hline Trial ----> & & \multirow{2}{*}{\multicolumn{4}{|c|}{$\frac{2}{\text { particles/ft3 }}$}} & \multicolumn{4}{|c|}{$2 \quad 3$} \\
\hline Point & Depth, in. & & & & & & & icles/ft3 & \\
\hline 1 & 0.50 & 1054 & 1156 & 1083 & 1097.7 & 1249 & 1316 & 1361 & 1308.7 \\
\hline 2 & 1.26 & 1089 & 1207 & 1158 & 1151.3 & 1431 & 1348 & 1399 & 1392.7 \\
\hline 3 & 2.33 & 1173 & 1227 & 1142 & 1180.7 & 1390 & 1309 & 1527 & 1408.7 \\
\hline 4 & 3.88 & 1108 & 1133 & 1206 & 1149.0 & 1368 & 1313 & 1461 & 1380.7 \\
\hline Center & 6.00 & 1126 & 1142 & 1139 & 1135.7 & 1321 & 1306 & 1473 & 1366.7 \\
\hline 5 & 8.12 & 1138 & 1127 & 1137 & 1134.0 & 1328 & 1269 & 1371 & 1322.7 \\
\hline 6 & 9.67 & 1170 & 1146 & 1179 & 1165.0 & 1327 & 1270 & 1403 & 1333.3 \\
\hline 7 & 10.74 & 1086 & 1118 & 1150 & 1118.0 & 1279 & 1198 & 1305 & 1260.7 \\
\hline 8 & 11.50 & 1030 & 1146 & 1092 & 1089.3 & 1207 & 1175 & 1317 & 1233.0 \\
\hline Averages --- & & 1108.2 & 1155.8 & 1142.9 & 1135.6 & 1322.2 & 1278.2 & 1401.9 & 1334.1 \\
\hline
\end{tabular}

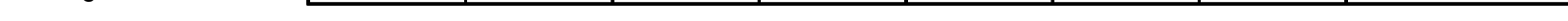

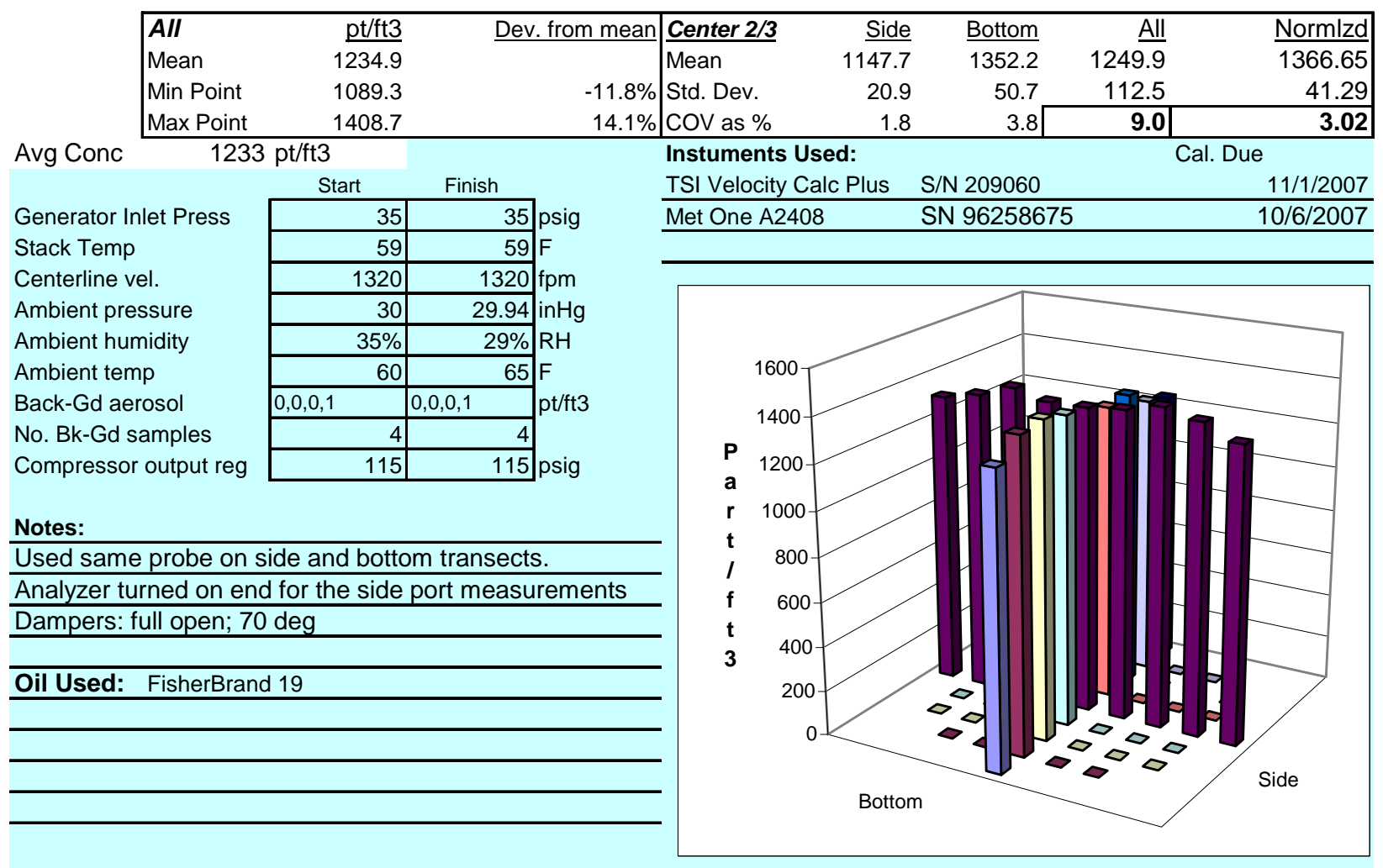

Signature signifies compliance with Procedure EMS-JAG-02

Signature/date

Reference: CCP-WTPSP-175

part-dataRev0.xls

3 August 2006
Signature verifying data and calculations:

Signatures on original data sheet in Test Instruction Package TI-RPP-WTP-466

(HVC2_part-dataRev0 (7)).xIs / PT18 $4 / 18 / 2007$ 
Rev. 0

3 Aug. 2006

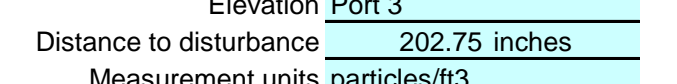

PARTICLE TRACER TRAVERSE DATA FORM

Site HV-C2 Model

Date 12/22/2006

Tester MSP

Stack Dia. Stack X-Area

Elevation Port 3

Order ----->

Traverse-->

Trial ---->

$1 \mathrm{st}$
Run No. PT-19

Fan configuration $\mathbf{B}$

Fan Setting 35

Stack Temp

Start/End Time 1415 -- 1550

Center $2 / 3$ from

Points in Center $2 / 3$

Injection Point $\mathrm{B}$ $\mathrm{w} / 3 \mathrm{M}$ filtrete macroallergen prefilters $\mathrm{Hz}$

\begin{tabular}{lrrl}
\hline & 1.10 & to: & 10.90 \\
\hline & 2 & to: & 7 \\
\hline B & Center & \\
\hline 2nd & &
\end{tabular}

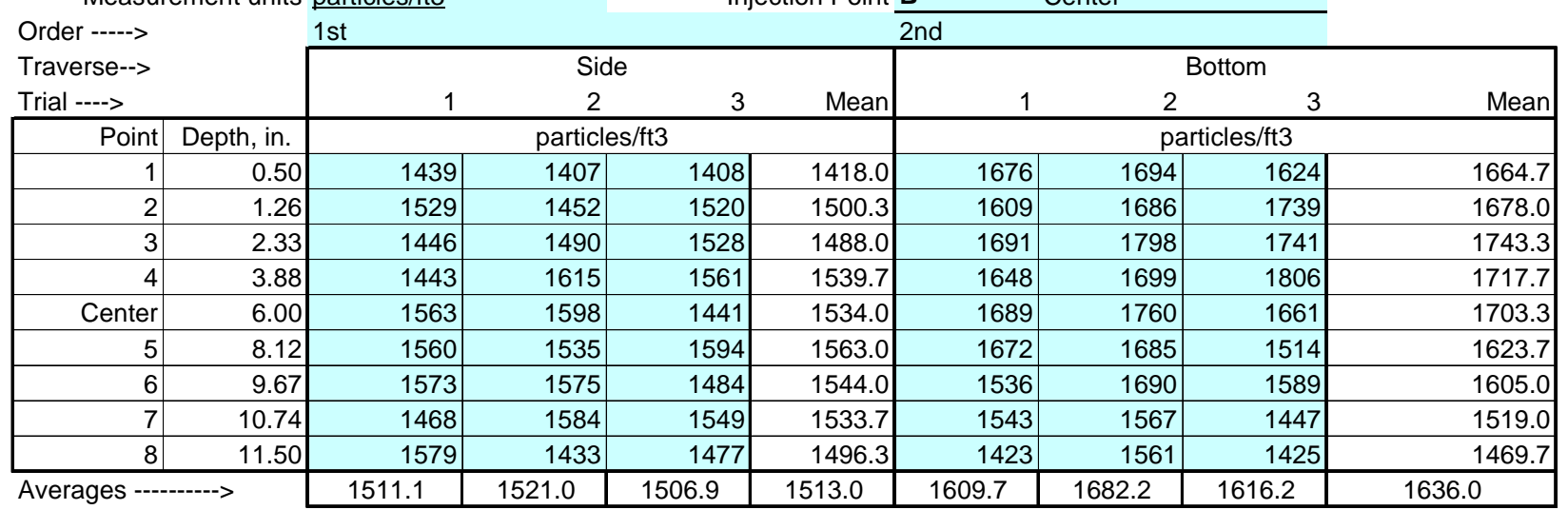

Averages ---------

\begin{tabular}{l|r}
1511.1 & 1521.0 \\
\hline
\end{tabular}

\begin{tabular}{|c|c|c|c|c|c|c|c|}
\hline AII & $\mathrm{pt} / \mathrm{tt} 3$ & Dev. from mean & Center $2 / 3$ & Side & Bottom & All & Normlzd \\
\hline Mean & 1574.5 & & Mean & 1529.0 & 1655.7 & 1592.3 & 1676.72 \\
\hline Min Point & 1418.0 & $-9.9 \%$ & Std. Dev. & 26.0 & 78.1 & 86.3 & 60.60 \\
\hline Max Point & 1743.3 & $10.7 \%$ & cov as $\%$ & 1.7 & 4.7 & 5.4 & 3.61 \\
\hline
\end{tabular}

Avg Conc $1569 \mathrm{pt} / \mathrm{ft} 3$

\begin{tabular}{|c|c|c|c|}
\hline \multirow[b]{2}{*}{ Generator Inlet Press } & Start & Finish & \\
\hline & 35 & 35 & psig \\
\hline Stack Temp & 60 & 59 & $F$ \\
\hline Centerline vel. & 1410 & 1410 & fpm \\
\hline Ambient pressure & 29.94 & 30 & $\mathrm{inHg}$ \\
\hline Ambient humidity & $29 \%$ & $30 \%$ & $\mathrm{RH}$ \\
\hline Ambient temp & 65 & 65 & $F$ \\
\hline Back-Gd aerosol & $1,4,0,0$ & $0,0,1,0$ & $\mathrm{pt} / \mathrm{ft} 3$ \\
\hline o. Bk-Gd samples & 4 & 4 & \\
\hline ompressor output reg & 120 & 120 & psig \\
\hline
\end{tabular}

Notes:

Used same probe on side and bottom transects.

Analyzer turned on end for the side port measurements

Dampers: full open; $70 \mathrm{deg}$

Oil Used: $\quad$ FisherBrand 19

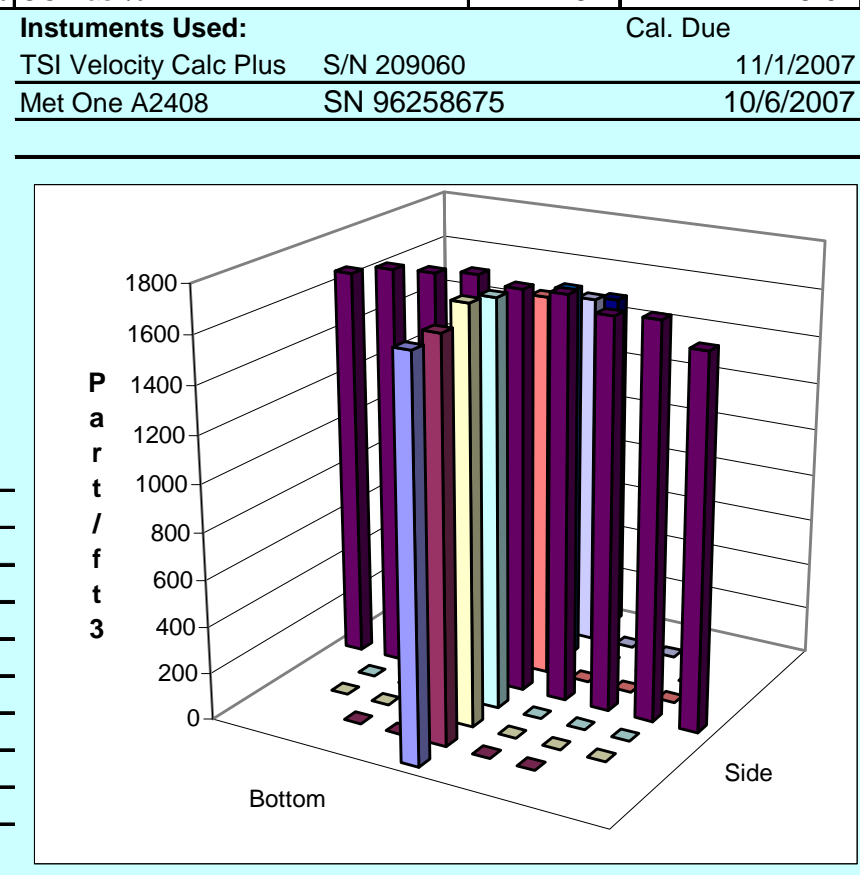

Signature signifies compliance with Procedure EMS-JAG-02

Signature/date

Signature verifying data and calculations:

Reference: CCP-WTPSP-175

part-dataRev0.xls

(HVC2_part-dataRev0 (7)).xls / PT19

3 August 2006

Signatures on original data sheet in Test Instruction Package TI-RPP-WTP-466

$4 / 18 / 2007$ 
Rev. 0

3 Aug. 2006

PARTICLE TRACER TRAVERSE DATA FORM

Site HV-C2 Model

Date 1/2/2007

Tester BGF

Stack Dia. Stack X-Area

Elevation Port 3

Distance to disturbance

Measurement units particles/ft3

Order ----->

Traverse-->

Trial ---->
12 in. 113.1 in. 2

174.25 inches

2nd

$2 \mathrm{nd}$

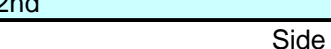

Run No. PT-20

Fan configuration $\overline{\mathbf{A} \& \mathbf{B}} \quad \mathrm{w} / 3 \mathrm{M}$ filtrete macroallergen prefilters

Fan Setting 40

Stack Temp

Start/End Time $1245-1445$

Center $2 / 3$ from

Points in Center $2 / 3$ Injection Point $\mathbf{B}$

\begin{tabular}{lrrl}
\hline & 1.10 & to: & 10.90 \\
\hline & 2 & to: & 7 \\
\hline B & Center & \\
\hline $1 \mathrm{st}$ & & &
\end{tabular}

\begin{tabular}{|c|c|c|c|c|c|}
\hline \multicolumn{2}{|c|}{1} & \multicolumn{4}{|c|}{ st } \\
\hline \multirow[b]{2}{*}{3} & \multirow[b]{2}{*}{ Mean } & \multicolumn{4}{|c|}{ Bottom } \\
\hline & & 1 & 2 & 3 & Mean \\
\hline t3 & & \multicolumn{4}{|c|}{ particles/ft3 } \\
\hline 1425 & 1416.7 & 1947 & 1477 & 1634 & 1686.0 \\
\hline 1628 & 1640.0 & 2166 & 1424 & 1575 & 1721.7 \\
\hline 1710 & 1757.0 & 2109 & 1532 & 1570 & 1737.0 \\
\hline 1659 & 1677.7 & 2157 & 1467 & 1528 & 1717.3 \\
\hline 1621 & 1655.3 & 2192 & 1617 & 1734 & 1847.7 \\
\hline 1749 & 1659.3 & 2151 & 2168 & 2345 & 2221.3 \\
\hline 1737 & 1628.0 & 1959 & 2129 & 2269 & 2119.0 \\
\hline 1650 & 1510.3 & 1740 & 1848 & 1906 & 1831.3 \\
\hline 1216 & 1149.0 & 1503 & 1430 & 1602 & 1511.7 \\
\hline 1599.4 & 1565.9 & 1991.6 & 1676.9 & 1795.9 & 1821.4 \\
\hline
\end{tabular}

Averages --------->

.50

\begin{tabular}{|r|r|r|}
\hline \multicolumn{3}{|c}{ particles/ft3 } \\
\hline 1523 & 1302 & 142 \\
\hline 1534 & 1758 & 162 \\
\hline 1634 & 1927 & 171 \\
\hline 1642 & 1732 & 165 \\
\hline 1623 & 1722 & 162 \\
\hline 1564 & 1665 & 174 \\
\hline 1613 & 1534 & 173 \\
\hline 1453 & 1428 & 165 \\
\hline 1126 & 1105 & 121 \\
\hline 1523.6 & 1574.8 & 1599.4 \\
\hline
\end{tabular}

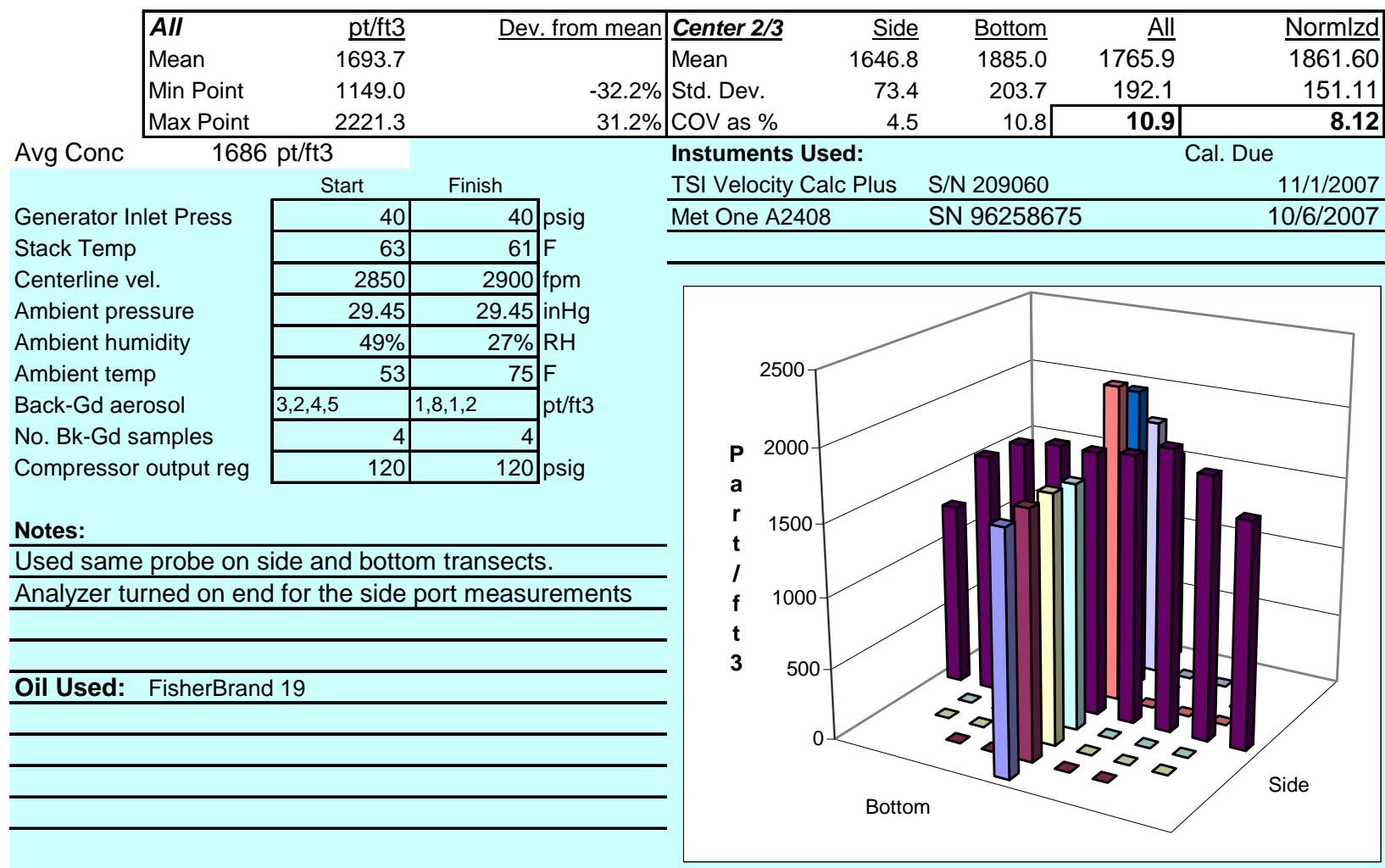

Signature signifies compliance with Procedure EMS-JAG-02

Signature/date

Signature verifying data and calculations:

Reference: CCP-WTPSP-175

part-dataRev0.xls

(HVC2_part-dataRev0 (7)).xIs / PT20
$4 / 18 / 2007$

3 August 2006

Signatures on original data sheet in Test Instruction Package TI-RPP-WTP-466 
Rev. 0

3 Aug. 2006
PARTICLE TRACER TRAVERSE DATA FORM

\begin{tabular}{|c|c|}
\hline \multicolumn{2}{|c|}{ Site HV-C2 Model } \\
\hline Date & $1 / 3 / 2007$ \\
\hline \multicolumn{2}{|c|}{ Tester BGF } \\
\hline Stack Dia. & $12 \mathrm{in.}$ \\
\hline Stack X-Area & 113.1 in. 2 \\
\hline \multicolumn{2}{|c|}{ Elevation Port 2} \\
\hline o disturbance & 113.75 inches \\
\hline
\end{tabular}

$$
\text { Fan Setting } \underline{40}
$$

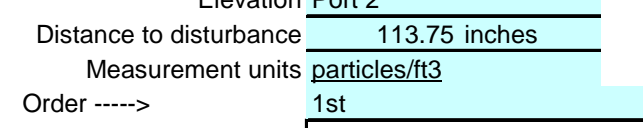

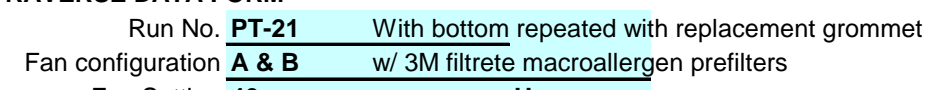

$\begin{aligned} \text { Stack Temp } & 63 \mathrm{deg} F \\ \text { Start/End Time } & 1230--1330\end{aligned}$

Center $2 / 3$ from $\frac{1230-1330}{1.10}$

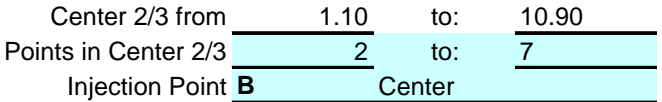

Traverse-->

Trial ---->

\begin{tabular}{|c|c|c|c|c|c|c|c|c|c|}
\hline \multirow{2}{*}{\multicolumn{2}{|c|}{ Trial ----> }} & \multicolumn{4}{|c|}{ siue } & \multicolumn{4}{|c|}{ DUtuII } \\
\hline & & 1 & 2 & 3 & Mean & 1 & 2 & 3 & Mean \\
\hline Point & Depth, in. & \multicolumn{4}{|c|}{ particles/ft3 } & \multicolumn{4}{|c|}{ particles/ft3 } \\
\hline 1 & 0.50 & 1128 & 1086 & 1111 & 1108.3 & 1676 & 1682 & 1720 & 1692.7 \\
\hline 2 & 1.26 & 1141 & 1110 & 1186 & 1145.7 & 1697 & 1910 & 1988 & 1865.0 \\
\hline 3 & 2.33 & 1160 & 1056 & 1204 & 1140.0 & 1902 & 1981 & 2030 & 1971.0 \\
\hline 4 & 3.88 & 1178 & 1041 & 1175 & 1131.3 & 1996 & 2036 & 2055 & 2029.0 \\
\hline Center & 6.00 & 1150 & 1033 & 1099 & 1094.0 & 2018 & 2058 & 2046 & 2040.7 \\
\hline 5 & 8.12 & 982 & 992 & 931 & 968.3 & 1887 & 2125 & 2020 & 2010.7 \\
\hline 6 & 9.67 & 916 & 962 & 919 & 932.3 & 1897 & 2032 & 2014 & 1981.0 \\
\hline 7 & 10.74 & 914 & 825 & 950 & 896.3 & 1868 & 1884 & 1886 & 1879.3 \\
\hline 8 & 11.50 & 800 & 780 & 924 & 834.7 & 1601 & 1586 & 1789 & 1658.7 \\
\hline Averages --- & $---->$ & 1041.0 & 987.2 & 1055.4 & 1027.9 & 1838.0 & 1921.6 & 1949.8 & 1903.1 \\
\hline
\end{tabular}

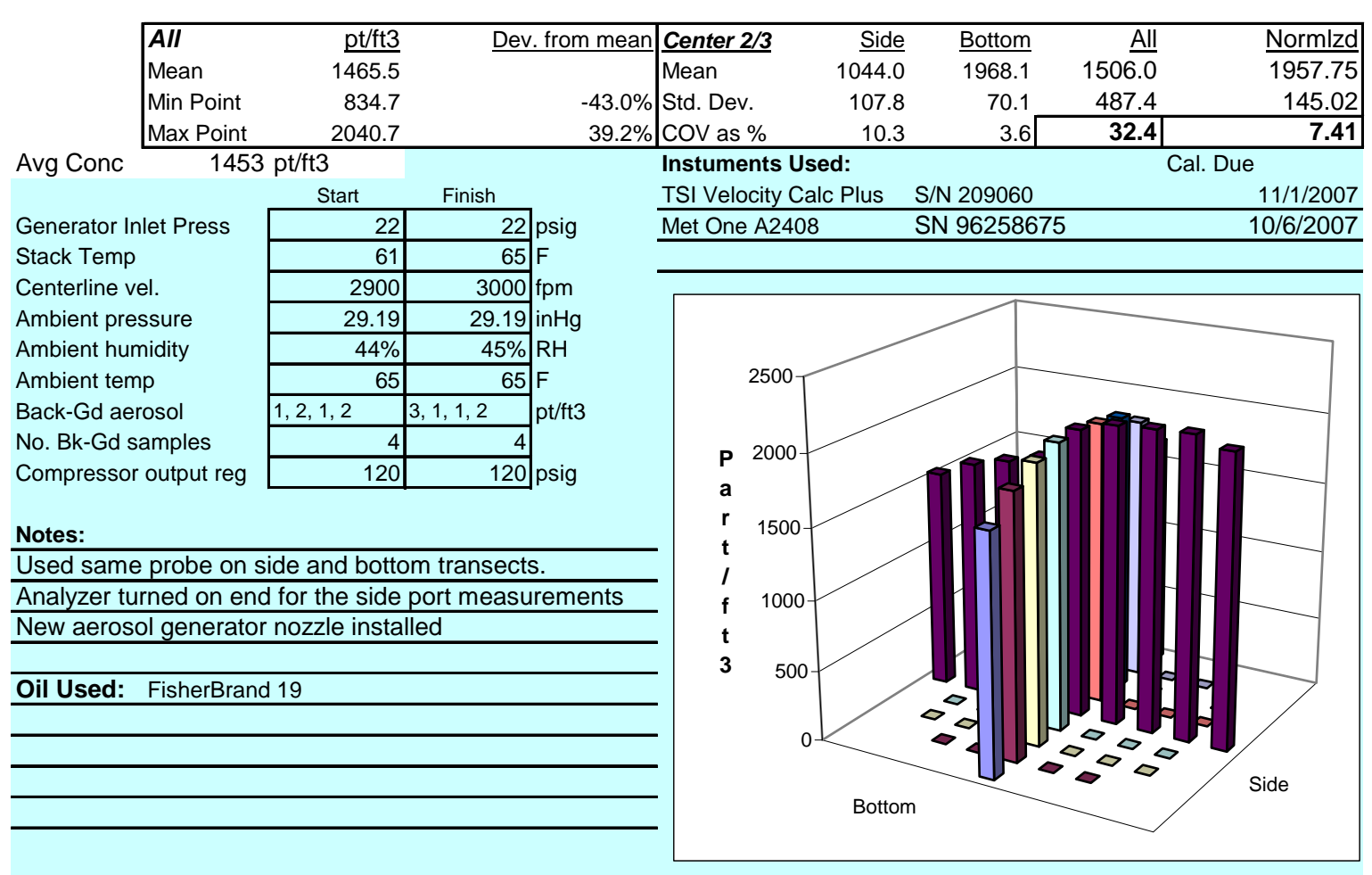

Signature signifies compliance with Procedure EMS-JAG-02

Signature/date

Reference: CCP-WTPSP-175

part-dataRev0.xIs

3 August 2006
Signature verifying data and calculations:

Signatures on original data sheet in Test Instruction Package TI-RPP-WTP-466 
Rev. 0

3 Aug. 2006

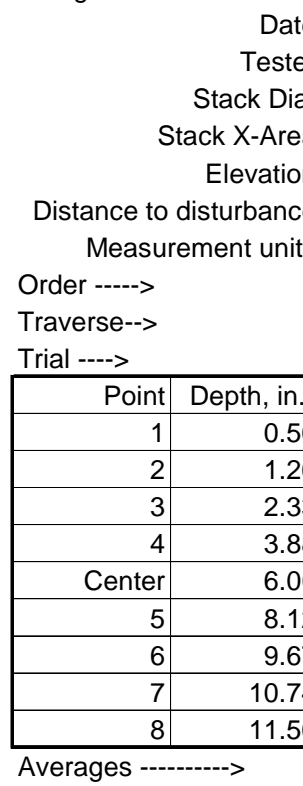

\section{PARTICLE TRACER TRAVERSE DATA FORM}

Site HV-C2 Model

Date 1/3/2007

Tester BGF

$\frac{12 \text { in. }}{113.1 \mathrm{in.} 2}$

Port 1

53.5 inches

$$
\text { particles/ft3 }
$$

2nd

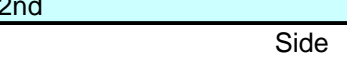

Run No. PT-22

Fan configuration $\mathbf{A}$ \& B $\quad$ w/ 3M filtrete macroallergen prefilters

Fan Setting 40

Stack Temp

Start/End Time $1330--1445$

Center 2/3 from

Points in Center $2 / 3$ Injection Point $\mathbf{B}$

\begin{tabular}{lrrr}
\hline & 1.10 & to: & 10.90 \\
\hline & 2 & to: & ( \\
\hline B & Center & \\
\hline Ist & & &
\end{tabular}

\begin{tabular}{|c|c|c|c|c|c|}
\hline \multicolumn{2}{|c|}{$1 \mathrm{~s}$} & \multicolumn{4}{|l|}{ st } \\
\hline \multirow[b]{2}{*}{3} & \multirow[b]{2}{*}{ Mean } & \multicolumn{4}{|c|}{ Bottom } \\
\hline & & 1 & 2 & 3 & Mean \\
\hline t3 & & \multicolumn{4}{|c|}{ particles/ft3 } \\
\hline 1907 & 1910.7 & 1848 & 1734 & 1740 & 1774.0 \\
\hline 2026 & 2084.7 & 2037 & 2422 & 2163 & 2207.3 \\
\hline 1853 & 1827.0 & 1910 & 2308 & 2120 & 2112.7 \\
\hline 1534 & 1701.3 & 1849 & 2149 & 1996 & 1998.0 \\
\hline 1231 & 1401.7 & 1880 & 2014 & 1960 & 1951.3 \\
\hline 1170 & 1340.3 & 2244 & 2272 & 2039 & 2185.0 \\
\hline 1347 & 1399.7 & 2463 & 2218 & 2303 & 2328.0 \\
\hline 1471 & 1412.3 & 2552 & 2471 & 2461 & 2494.7 \\
\hline 1141 & 1126.0 & 1533 & 1562 & 1557 & 1550.7 \\
\hline 1520.0 & 1578.2 & 2035.1 & 2127.8 & 2037.7 & 2066.9 \\
\hline
\end{tabular}

\begin{tabular}{|l|l|l}
\hline & 1587.8 & 1626.8 \\
\hline
\end{tabular}

\begin{tabular}{|c|c|c|}
\hline \multirow[t]{2}{*}{1} & \multicolumn{2}{|c|}{2} \\
\hline & \multicolumn{2}{|c|}{ particles/f } \\
\hline 1833 & 1992 & \\
\hline 1938 & 2290 & \\
\hline 1838 & 1790 & \\
\hline 1783 & 1787 & \\
\hline 1540 & 1434 & \\
\hline 1467 & 1384 & \\
\hline 1430 & 1422 & \\
\hline 1390 & 1376 & \\
\hline 1071 & 1166 & \\
\hline 1587.8 & 1626.8 & \\
\hline
\end{tabular}

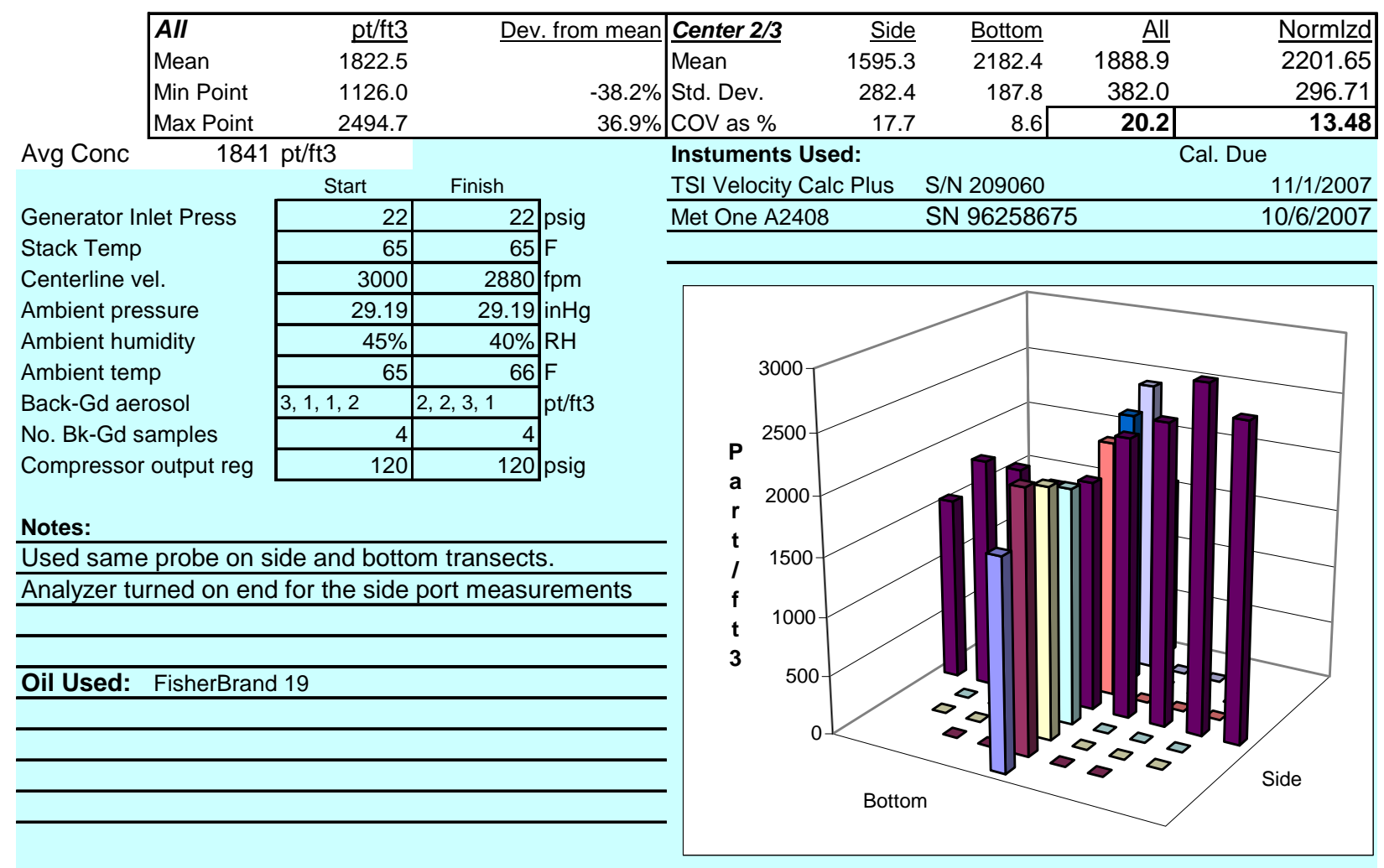

Signature signifies compliance with Procedure EMS-JAG-02

Signature/date

Reference: CCP-WTPSP-175

part-dataRev0.xls
Signature verifying data and calculations:

Signatures on original data sheet in Test Instruction Package TI-RPP-WTP-466

(HVC2_part-dataRev0 (7)).xls / P22 $4 / 18 / 2007$ 
PNNL-16611

WTP-RPT-158, Rev. 0

\section{Distribution}

No. of

Copies

No. of

No. of

Copies

Copies

OFFSITE

\section{ONSITE}

3 Pacific Northwest National Laboratory

J. A. Glissmeyer K3-54

D. E. Kurath P7-28

Project Office P7-28

1 Bechtel National Incorporated
C. Icayan
H4-02

Distr. 1 